\title{
Propeller Tip-Vortex Cavitation and its Broadband Noise
}

- JOHAN BOSSCHERS -

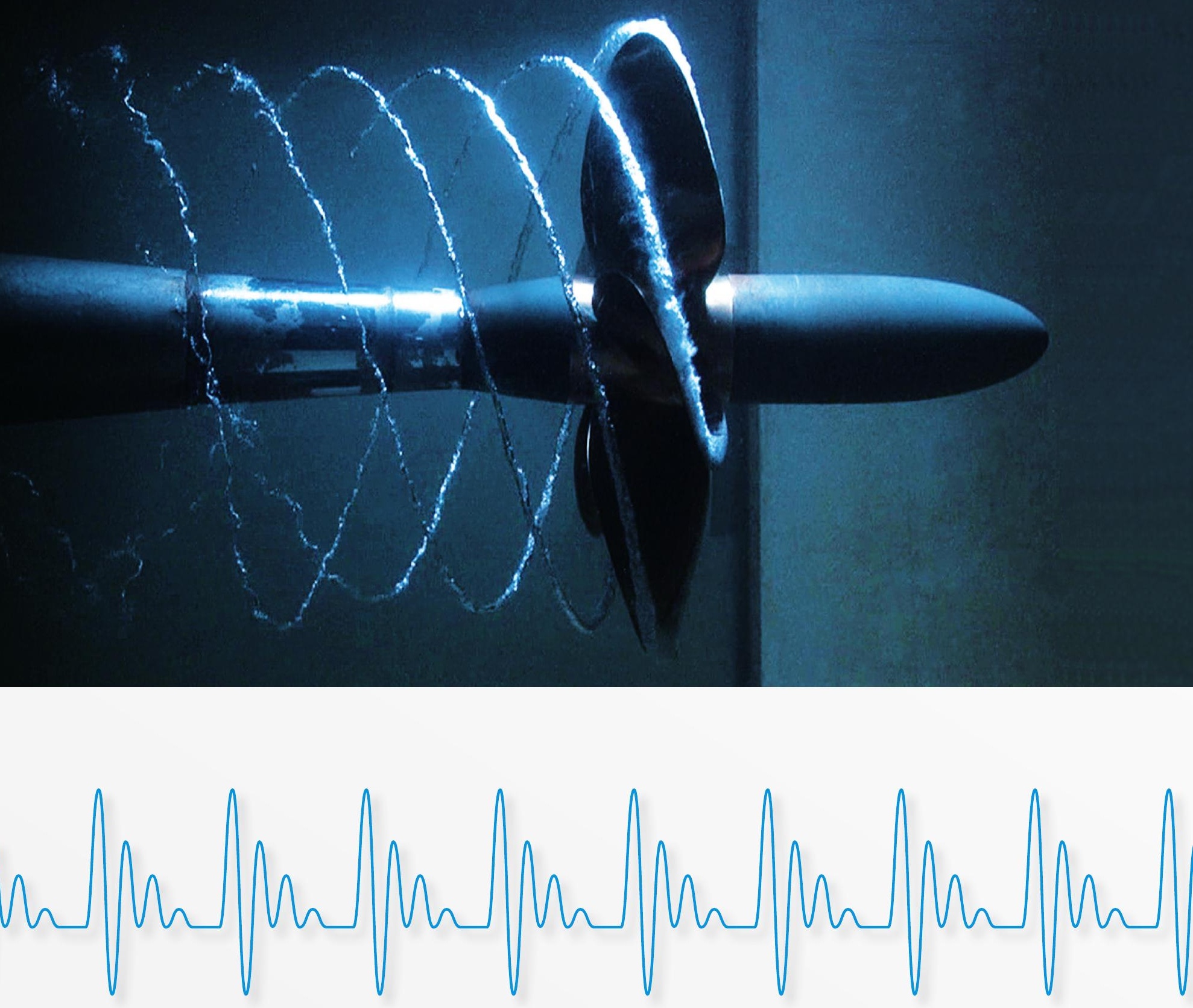



Propeller Tip-Vortex Cavitation and its Broadband Noise

J. Bosschers 
Samenstelling promotiecommissie:

Prof. dr. G.P.M.R. Dewulf, Technische Universiteit Twente, voorzitter

Prof. dr. ir. H.W.M. Hoeijmakers, Technische Universiteit Twente, promotor

Prof. dr. ir. T.J.C. van Terwisga, Technische Universiteit Delft, promotor

Prof. dr. ir. A. de Boer, Technische Universiteit Twente

Prof. dr. ir. C.H. Venner Technische Universiteit Twente

Prof. dr. ir. D.M.J. Smeulders Technische Universiteit Eindhoven

Prof. dr. R. Bensow

Prof. dr. S.L. Ceccio University of Michigan

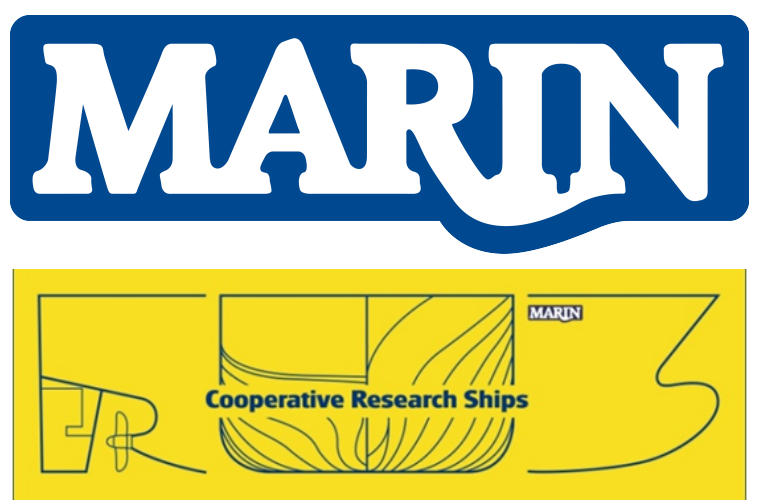

Propeller Tip-Vortex Cavitation and its Broadband Noise

J. Bosschers

ISBN: 978-94-92679-52-9 (print)

Printed by Print Service Ede BV.

Cover design by Ina Louwrink, cover photo by MARIN

The research presented in this thesis was partly funded by the Maritime Research Institute Netherlands (MARIN) and partly sponsored by the Cooperative Research Ships (CRS).

Copyright (c) 2018 by J. Bosschers, Renkum, The Netherlands. All rights reserved. 


\title{
PROPELLER TIP-VORTEX CAVITATION AND ITS BROADBAND NOISE
}

\section{PROEFSCHRIFT}

\author{
ter verkrijging van \\ de graad van doctor aan de Universiteit Twente, \\ op gezag van de Rector Magnificus \\ prof. dr. T.T.M. Palstra, \\ volgens besluit van het College voor Promoties \\ in het openbaar te verdedigen \\ op vrijdag 21 september 2018 om 14:45 uur
}

door

Johan Bosschers

geboren op 5 april 1967

te Holten, Nederland 
Dit proefschrift is goedgekeurd door de promotoren:

Prof. dr. ir. H.W.M. Hoeijmakers

Prof. dr. ir. T.J.C. van Terwisga 


\title{
Summary
}

\author{
Propeller Tip-Vortex Cavitation and its Broadband Noise \\ by \\ Johan Bosschers
}

Comfort on board has become an important aspect in the design of ships over the last decades, especially comfort for passengers on cruise vessels and for owners of yachts. Noise and vibration need to be minimized, which poses design constraints on the propeller with respect to cavitation. Cavitation on propellers for ships requiring high levels of comfort should be minimized, leaving often only a tip-vortex cavity. However, tip-vortex cavitation is known to be a cause of broadband pressure fluctuations on the hull above the propeller, typically in a frequency range from 30 to $100 \mathrm{~Hz}$, which has led to vibration problems for some ships. Broadband noise of cavitation is also of relevance for the underwater radiated noise emitted by the ship, not only from the perspective of the acoustic signature of military ships, but also because of the impact of noise on fish and marine mammals.

The objective of the present $\mathrm{PhD}$ thesis was to develop prediction methods for broadband noise, including hull-pressure fluctuations, by developed propeller tip-vortex cavitation. However, developed tip-vortex cavitation and its broadband noise are far from understood, because most research on vortex cavitation has been focussed on its inception. Therefore, fundamental aspects of a cavitating vortex were investigated, aiming at understanding the mechanisms involved in the generation of broadband noise. This has been achieved by theoretical and computational studies of the kinematics, dynamics and acoustics of vortex cavitation and the analysis of experimental data. The knowledge and formulations obtained were used for the development of a semi-empirical prediction method. The formulations were also used to develop a novel methodology for model tests to correct for the Reynolds-number scale effect on the radius of the vortex-cavity and its broadband noise.

The kinematics of the flow around a vortex cavity was investigated by deriving an analytical solution for the radial distribution of the azimuthal velocity and pressure from NavierStokes equations for axisymmetric incompressible flow. After extending this vortex model with a semi-empirical formulation to account for vorticity roll-up, published experimental data could be matched well. The results show that the -by approximation zero-shear-stress- boundary condition at the cavity interface is realistic. Remarkable was that the measured relation between cavity radius and cavitation number could only be reproduced if the measured increase in viscous core radius in cavitating flow was not taken into account. It was also found that the analytical vortex model becomes independent of vortex strength and Reynolds number when the ratio of cavity radius and viscous core radius is presented as a function of the ratio of ca- 
vitation number and cavitation inception number. The vortex model suggests that the effect of viscosity on cavity radius reduces with increasing cavity radius.

The dynamics of a vortex cavity was first studied by numerically solving the NavierStokes equations for axisymmetric, unsteady, and incompressible flow. The results show that the collapse of the cavity is inertia driven and show the presence of a resonance frequency. The dynamics in 3-D flow was investigated by analysing the dispersion relation that describes the propagation of waves on the vortex-cavity interface. The results of the analytical formulation for potential flow with an ad-hoc correction for viscosity show acceptable agreement with published experimental data. Several criteria for resonance have been proposed. However, resonance has not been demonstrated because all criteria correspond to neutrally stable waves.

The acoustics of a vortex cavity was investigated by studying the analytical formulation for the radiated noise by vibrations of the wall of a cylinder of finite length. The far-field formulation is similar to that of a monopole, but at the distance where the hull-pressure fluctuations are measured, the situation is slightly more complicated. An analytical formulation was developed for the effect on the hull-pressure spectrum of the variation, from one blade passage to the other, of amplitude and phase of the pressure signal.

The mechanisms of broadband noise generated by vortex-cavitation were analysed and reviewed using the analytical formulations derived in the fundamental studies. It was shown that the transient oscillatory dynamics of the vortex cavity, in combination with variability between blade passages, generates the broadband hump. An important excitation source of a vortex cavity is the shedding of vapour from the sheet cavity into the vortex cavity. It was also shown that the collapse of the closure-vortex cavity, generated by the side-entrant jet of the sheet, produces broadband noise.

A semi-empirical prediction method for broadband noise by propeller tip-vortex cavitation was developed by combining the results of the fundamental studies with experimental data obtained from model tests and sea trials. This new method predicts broadband hull-pressure fluctuations as well as underwater radiated noise. It makes use of results of a boundary element method that computes the flow on the propeller operating in the ship's wake field. Despite its simplicity, the method gives good results, also for ships that were not used to determine the empirical parameters from fits.

A new methodology for model tests was developed and evaluated to correct for the viscous-scale effect on the radius of the vortex cavity and, thereby, on its broadband noise. The methodology makes use of a formulation for a vortex model in which only the cavitation number and cavitation inception number are required to evaluate this scale effect. A semiempirical relation was used to relate the ratio of the cavity radius on model-scale and that on full-scale to a difference in noise level. The first results of the methodology are encouraging but more detailed validation studies are required.

In general, a basic understanding of developed vortex cavitation and its associated broadband noise has been obtained, but several details need to be further investigated. 


\title{
Samenvatting
}

\section{Tipwervelcavitatie op Schroeven en haar Breedbandig Geluid}

\author{
door
}

\section{Johan Bosschers}

In de laatste paar decennia is comfort aan boord belangrijk geworden in het ontwerpproces van schepen, vooral comfort voor passagiers op cruiseschepen en voor eigenaren van jachten. Geluid en trillingen moeten worden geminimaliseerd, hetgeen ontwerpeisen stelt aan de schroef met betrekking tot cavitatie. Schroeven voor schepen die moeten voldoen aan strenge eisen voor comfort hebben minimale cavitatie en laten vaak alleen tipwervelcavitatie zien. Het is echter bekend dat tipwervelcavitatie breedbandige drukfluctuaties kan veroorzaken op de huid van de romp boven de schroef, hetgeen tot trillingsproblemen van enkele schepen heeft geleid. Bovendien is breedbandig cavitatiegeluid van belang voor het onderwater-uitgestraalde geluid van het schip; voor militaire schepen vanwege de akoestische signatuur, en, meer in het algemeen, vanwege de invloed van geluid op vissen en zeezoogdieren.

Het doel van dit proefschrift was het ontwikkelen van voorspellingsmethoden voor breedbandig geluid, inclusief de drukken op de huid van de romp, veroorzaakt door ontwikkelde tipwervelcaviteiten van de schroef. Echter, ontwikkelde tipwervelcavitatie en haar breedbandig geluid zijn nog verre van begrepen, omdat het meeste onderzoek aan wervelcavitatie gericht is geweest op het inceptieproces. Daarom zijn eerst fundamentele aspecten van een caviterende wervel onderzocht, gericht op het begrijpen van de mechanismen die een rol spelen bij het genereren van breedbandig geluid. Hiertoe zijn theoretische studies verricht van de kinematica, dynamica en akoestiek van wervelcavitatie. Verder zijn experimentele gegevens geanalyseerd. De verkregen kennis en formuleringen zijn gebruikt voor de ontwikkeling van een semi-empirische voorspellingsmethode. De formuleringen zijn ook gebruikt voor de ontwikkeling van een nieuwe methodologie voor modelproeven om te corrigeren voor het schaaleffect van Reynoldsgetal op de straal van de wervelcaviteit en het gemeten breedbandig geluid.

De kinematica van de stroming om een caviterende wervel is onderzocht door een analytische oplossing van de Navier-Stokes-vergelijkingen af te leiden die de radiale verdeling van de azimutale snelheid en druk beschrijft voor een as-symmetrische onsamendrukbare stroming. Dit wervelmodel is uitgebreid met een semi-empirische formulering voor de invloed van het oprollen van de vorticiteitslaag in de wervelkern. Hierdoor werd een goede overeenkomst gevonden met gepubliceerde meetdata. De resultaten tonen aan dat de gebruikte randvoorwaarde voor de schuifspanning op de rand van de caviteit -die bij benadering nul wordt- realistisch is. Opmerkelijk was dat de gemeten relatie tussen de straal van de caviteit en het cavitatiegetal alleen kon worden gereproduceerd als de gemeten toename in de caviterende stroming 
van de straal van de viskeuze kern niet mee werd genomen. Het bleek ook dat het analytische wervelmodel onafhankelijk wordt van de wervelsterkte en het Reynoldsgetal wanneer de verhouding van de straal van de caviteit en de straal van de viskeuze kern wordt gepresenteerd als functie van de verhouding van cavitatiegetal en cavitatiegetal voor inceptie. Het wervelmodel suggereert dat het effect van de viscositeit op de straal van de caviteit afneemt als de straal van de caviteit toeneemt.

De dynamica van een wervelcaviteit is eerst bestudeerd door de Navier-Stokes-vergelijkingen numeriek op te lossen voor een as-symmetrische, instationaire en onsamendrukbare stroming. De resultaten laten zien dat de implosie van de caviteit door traagheid wordt gedreven. De dynamica voor een 3D-stroming werd onderzocht door analyse van de dispersierelatie die de voortplanting beschrijft van golven op de rand van de wervelcaviteit. De resultaten van de analytische formulering voor een potentiaalstroming, uitgebreid met een ad-hoc correctie voor effect van viscositeit, laten een aanvaardbare overeenkomst zien met gepubliceerde experimentele gegevens. Er zijn verschillende criteria voor resonantie geformuleerd. Er is echter geen resonantie aangetoond omdat alle criteria overeen komen met neutraal stabiele golven.

De akoestiek van een wervelcaviteit is onderzocht door de analytische formulering voor het uitgestraalde geluid te bestuderen voor trillingen van de wand van een cylinder van eindige lengte. De formulering voor het verre veld is vergelijkbaar met die voor een monopool, maar op de afstand waar de cavitatie-geïnduceerde huiddrukken worden gemeten, is de situatie gecompliceerder. Ook is er een analytische formulering ontwikkeld voor het effect op het spectrum van de huiddrukken van gelijktijdige variatie van amplitude en fase van het druksignaal tussen opeenvolgende bladdoorgangen.

De mechanismen van breedbandig geluid gegenereerd door wervelcavitatie zijn geanalyseerd en beoordeeld met behulp van de analytische formuleringen afgeleid in de fundamentele studies. Er is aangetoond dat de breedbandige bult in het spectrum wordt genereert door de kortstondige oscillerende wervelcaviteit, in combinatie met variabiliteit van het druksignaal tussen bladpassages. Een belangrijke excitatiebron van de wervelcavitatie is het transport van damp van vliescavitatie naar de wervelcaviteit. Er is ook aangetoond dat de wervelcaviteit die wordt gegenereerd door de stroming om de gekromde zijrand van vliescavitatie bijdraagt aan breedbandig geluid.

Een semi-empirische voorspellingsmethode voor breedbandig geluid door tipwervelcavitatie op een schroef is ontwikkeld door de resultaten van de fundamentele studies te combineren met beschikbare experimentele gegevens verkregen uit modelproeven en proeven uitgevoerd op zee. Deze nieuwe methode voorspelt de breedbandige drukfluctuaties op de scheepshuid evenals het onderwater uitgestraalde geluid. De methode maakt gebruik van resultaten van een randelementmethode die de stroming berekent om de schroef die roteert in het volstroomveld van het schip. Ondanks zijn eenvoud geeft de methode goede resultaten, ook voor schepen die niet werden gebruikt om de waarden van de empirische parameters te bepalen.

Een nieuwe methodologie is ontwikkeld en geëvalueerd voor het corrigeren van resultaten van modelproeven voor het schaaleffect van de viskeuze kern op de straal van de wervel- 
caviteit en daarmee op het gegenereerde breedbandig geluid. De methodologie maakt gebruik van een formulering voor een wervelmodel waarin alleen het cavitatiegetal en het cavitatieinceptiegetal nodig zijn om het schaaleffect vast te stellen. Een semi-empirische relatie is gebruikt om de verhouding van de straal van de caviteit op modelschaal en die op ware grootte te relateren aan een verschil in geluidsniveau. De eerste resultaten van de methodologie zijn bemoedigend, maar meer gedetailleerde validatiestudies zijn vereist.

Over het geheel genomen is er basisbegrip verkregen van ontwikkelde wervelcavitatie en de generatie van breedbandig geluid, maar meerdere details moeten verder worden onderzocht. 
Page intentionally left blank 


\section{Contents}

Summary $\quad$ v

Samenvatting vii

Contents $\quad$ xiv

List of Symbols $\quad$ Xv

1 Introduction 1

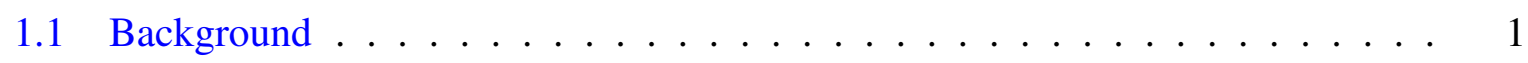

1.2 Problem definition $\ldots \ldots \ldots \ldots \ldots \ldots$

1.3 Research objectives . . . . . . . . . . . . . . . . . . 4

1.4 Outline of the thesis . . . . . . . . . . . . . . . 5

2 Characteristics $\quad 7$

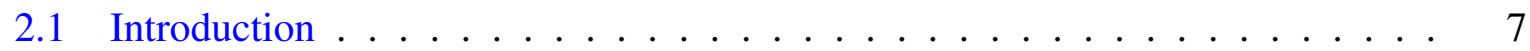

2.2 Non-cavitating vortices . . . . . . . . . . . . . . . 8

2.3 Cavitation inception . . . . . . . . . . . . . . . . . . . 12

2.4 Vortex cavitation . . . . . . . . . . . . . . . . . . . . 13

2.5 Hull-pressure fluctuations . . . . . . . . . . . . . . . . . . 17

2.6 Underwater radiated noise . . . . . . . . . . . . . . . . 21

2.7 Conclusions . . . . . . . . . . . . . . . . . . . 23

3 Kinematics $\quad 25$

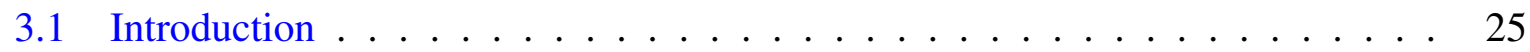

3.2 An analytical solution for the flow around a vortex cavity . . . . . . . 26

3.2 .1 Derivation . . . . . . . . . . . . . . . . 26

3.2 .2 Analysis .......................... 30

3.3 A semi-empirical model for a cavitating vortex . . . . . . . . . . 32

3.3 .1 Formulation . . . . . . . . . . . . . . . 32

3.3.2 Comparison with experimental data . . . . . . . . . . . 35

3.3 .3 Discussion . . . . . . . . . . . . . . . . . 41

3.4 Conclusions . . . . . . . . . . . . . . . . . . . . 44 
4 Dynamics $\quad 47$

4.1 Introduction . . . . . . . . . . . . . . . . . . 47

4.2 Dynamics of a vortex cavity in 2-D viscous flow . . . . . . . . . . . . . . 49

4.2.1 Governing equations and computational procedure . . . . . . . . . . 49

4.2.2 Results for constant free-stream pressure . . . . . . . . . . . . . 54

4.2.3 Results for a change in free-stream pressure . . . . . . . . . . . . 57

4.3 The dispersion relation for a 3-D columnar vortex cavity $\ldots \ldots$. . . . . . 60

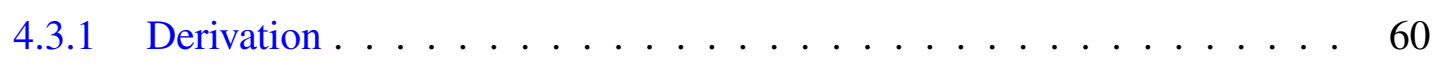

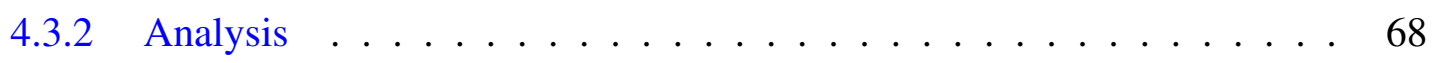

4.3.3 Comparison with experimental data . . . . . . . . . . . . . 74

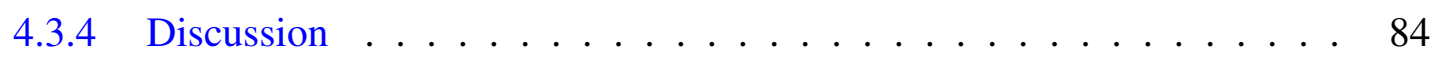

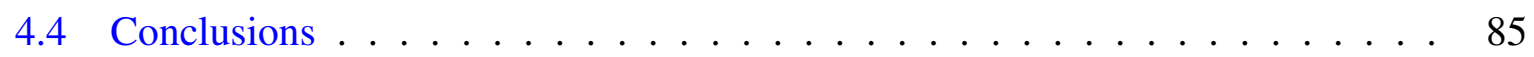

5 Acoustics 87

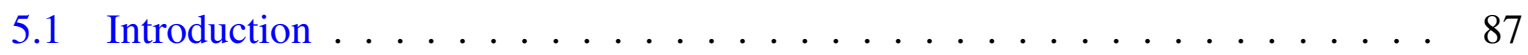

5.2 Analytical formulations for acoustic sources . . . . . . . . . . . 88

5.2.1 Pulsating and vibrating sphere . . . . . . . . . . . . . . . . 88

5.2.2 Infinite length vortex cavity . . . . . . . . . . . . . 90

5.2.3 Finite length cylinder composed of distributed sources . . . . . . . . 92

5.2.4 Finite length cylinder with prescribed vibration . . . . . . . . . . 93

5.2.5 Analysis and discussion . . . . . . . . . . . . . . . 98

5.3 Spectral analysis . . . . . . . . . . . . . . . . . . 104

5.3 .1 Background . . . . . . . . . . . . . . . . . . 104

5.3.2 Examples of Fourier transform pairs . . . . . . . . . . . . . . 105

5.3.3 Formulation for effect of variability between blade passages . . . . . . 107

5.4 Scaling of cavitation noise . . . . . . . . . . . . . . . 113

5.4.1 Hull-pressure fluctuations at blade rate frequencies . . . . . . . . . . 113

5.4.2 Broadband hull-pressure fluctuations . . . . . . . . . . . . 113

5.4.3 Radiated noise: high-frequency formulation . . . . . . . . . . . . 115

5.4.4 Radiated noise: low-frequency formulation . . . . . . . . . . . 116

5.4 .5 Discussion . . . . . . . . . . . . . . . . 117

5.5 Scattering by hull and free surface . . . . . . . . . . . . . . 118

5.5.1 Analytical formulation for the solid boundary factor . . . . . . . . . 118

5.5.2 Analytical formulations for Lloyd's mirror . . . . . . . . . . . . . 123

5.6 Conclusions . . . . . . . . . . . . . . . . . 125

6 Mechanisms of vortex-cavitation noise 127

6.1 Introduction . . . . . . . . . . . . . . . . . 127

6.2 Narrowband noise . . . . . . . . . . . . . . . . . . . . . 128

6.3 Broadband hull-pressure data . . . . . . . . . . . . 133 
6.3.1 Background . . . . . . . . . . . . . . . . . . . 133

6.3.2 Hull-pressure data synchronized with high-speed video . . . . . . . 134

6.3 .3 Variability between blade passages . . . . . . . . . . . 136

6.3.4 Spatial distribution over hull surface . . . . . . . . . . . . . . 141

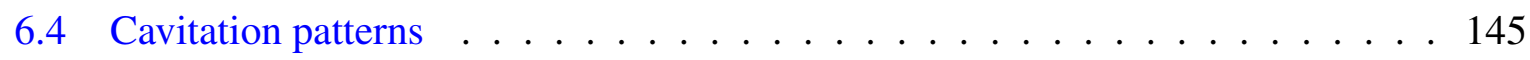

6.5 Excitation of vortex-cavity dynamics . . . . . . . . . . . . . . . . 149

6.6 Review of mechanisms of broadband cavitation noise . . . . . . . . . 154

7 Semi-empirical prediction of broadband noise $\quad 157$

7.1 Introduction . . . . . . . . . . . . . . . . . 157

7.2 Estimation of cavity size . . . . . . . . . . . . . . . . . 159

7.3 Estimation of the centre frequency and level . . . . . . . . . . . . 163

7.4 Spectral shape . . . . . . . . . . . . . . . . . . . . . . 167

7.5 Results . . . . . . . . . . . . . . . . . . . . . . . . . . 169

7.5.1 Hull-pressure fluctuations . . . . . . . . . . . . . . . . . . . . 169

7.5.2 Underwater radiated noise . . . . . . . . . . . . . . . . 171

7.5.3 Scaling with ship speed . . . . . . . . . . . . . . . . . 173

7.6 Discussion . . . . . . . . . . . . . . . . . . . . . 175

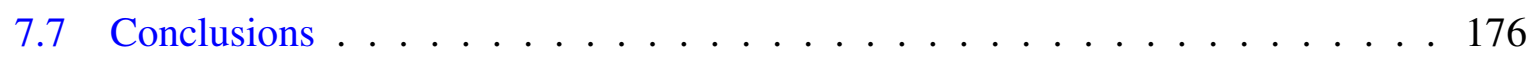

8 Experimental prediction of broadband noise 179

8.1 Introduction . . . . . . . . . . . . . . . . . . . . . . 179

8.2 Scaling of vortex-cavity size with Reynolds number . . . . . . . . . . . . 182

8.3 Scaling of vortex-cavity noise with cavity size $\ldots \ldots \ldots \ldots \ldots$

8.4 Results . . . . . . . . . . . . . . . . . . . . . . 188

8.4.1 Published hull-pressure data of a face-side vortex cavity . . . . . 188

8.4.2 Radiated noise of a small research vessel . . . . . . . . . . . . 190

8.4.3 Hull pressures of a twin-screw vessel . . . . . . . . . . . . 193

8.4.4 Hull pressures of the Combi Freighter . . . . . . . . . . . . . . 194

8.5 Discussion . . . . . . . . . . . . . . . . . . . . . . 196

8.6 Conclusions . . . . . . . . . . . . . . . . . . . . 197

9 Concluding remarks 199

9.1 Introduction . . . . . . . . . . . . . . . . . . . . . . . . 199

9.2 Conclusions on mechanisms of vortex-cavitation noise $\ldots \ldots \ldots \ldots . \ldots 200$

9.3 Conclusions on the semi-empirical prediction method . . . . . . . . . 201

9.4 Conclusions on the experimental prediction method . . . . . . . . . 202

9.5 Practical implications of the studies performed . . . . . . . . . . 203

9.6 Recommendations . . . . . . . . . . . . . . . . . . . . . . 203

$\begin{array}{ll}\text { Bibliography } & 205\end{array}$ 
Acknowledgements

About the author

221

List of Publications

223 


\section{List of Symbols}

\section{Roman letters}

\begin{tabular}{|c|c|c|}
\hline Symbol & Unit & Property \\
\hline$a$ & [m] & radius of sphere \\
\hline$a_{n}$ & {$[\mathrm{~Pa}]$} & pressure amplitude of pulse number $n$ \\
\hline$B$ & {$[\mathrm{~m}]$} & wing span \\
\hline$C_{L}$ & {$[-]$} & lift coefficient \\
\hline$c$ & [m] & chord length \\
\hline$c_{0}$ & & chord length at the root of the wing \\
\hline$c$ & {$[\mathrm{~m} / \mathrm{s}]$} & speed of sound \\
\hline$c_{g}$ & {$[\mathrm{~m} / \mathrm{s}]$} & group velocity \\
\hline$c_{p}$ & {$[\mathrm{~m} / \mathrm{s}]$} & phase velocity \\
\hline$D$ & {$[\mathrm{~m}]$} & propeller diameter \\
\hline$E_{1}$ & {$[-]$} & Exponential integral \\
\hline$f$ & {$[-]$} & $\begin{array}{l}\text { multiplication factor for } r_{v} \text { and } q \text { in the cavitating modified } \\
\text { Lamb-Oseen vortex, Eq. (3.35) }\end{array}$ \\
\hline$f$ & {$[\mathrm{~Hz}]$} & frequency \\
\hline $\begin{array}{l}f_{b p} \\
f_{c}\end{array}$ & & $\begin{array}{l}\text { blade-passage frequency } \\
\text { centre frequency }\end{array}$ \\
\hline$G$ & {$[1 / \sqrt{\mathrm{Hz}}]$} & unit-amplitude density spectrum, Section 5.3.3 \\
\hline$G$ & {$[1 / \mathrm{Hz}]$} & unit-amplitude Fourier transform, Section 5.3.2 \\
\hline$g$ & {$\left[\mathrm{~m} / \mathrm{s}^{2}\right]$} & acceleration of gravity \\
\hline$H_{H}$ & {$[-]$} & Shape of source-level spectrum in decibel as function of frequency \\
\hline $\begin{array}{c}H_{h} \\
H_{s}\end{array}$ & & $\begin{array}{l}\text { Shape of hump in spectrum } \\
\text { Shape of spectrum as prescribed by the slope at low and } \\
\text { high frequency }\end{array}$ \\
\hline$H_{n}^{(1)}$ & {$[-]$} & $\begin{array}{l}\text { Bessel function of the third kind, also called Hankel function } \\
\text { of the first kind, and order } n\end{array}$ \\
\hline$H_{n}^{(1) \prime}$ & & first derivative of $H_{n}^{(1)}$ with respect to its argument \\
\hline $\mathrm{He}$ & {$[-]$} & Helmholtz number, $\mathrm{He}=k a$ \\
\hline$h$ & [m] & vertical distance below water surface \\
\hline$h_{i}$ & {$[\mathrm{~m}]$} & grid size of refinement case $i$ \\
\hline$h_{n}^{(1)}$ & {$[-]$} & $\begin{array}{l}\text { spherical Bessel function of the third kind, also called spherical } \\
\text { Hankel function of order } n\end{array}$ \\
\hline I & {$[-]$} & identity matrix \\
\hline & {$[-]$} & imaginary unit \\
\hline
\end{tabular}




\begin{tabular}{|c|c|c|}
\hline$J$ & {$[-]$} & (apparent) propeller advance ratio, $J=V_{s} / n D$ \\
\hline$K_{n}$ & {$[-]$} & $\begin{array}{l}\text { modified Bessel function of the second kind, also called } \\
\text { MacDonald function, and order } n\end{array}$ \\
\hline$K_{n}^{\prime}$ & & first derivative of $K_{n}$ with respect to its argument \\
\hline$K_{n}^{\prime \prime}$ & & second derivative of $K_{n}$ with respect to its argument \\
\hline$K_{T}$ & [-] & propeller thrust coefficient, $K_{T}=T / \rho n^{2} D^{4}$ \\
\hline$K_{\sigma}$ & {$[-]$} & non-dimensional stiffness term, Eq. (4.71) \\
\hline k & {$[\mathrm{rad} / \mathrm{m}]$} & wavenumber vector, $\mathbf{k}=\left(k_{z}, k_{r}\right)$ \\
\hline$k$ & {$[\mathrm{rad} / \mathrm{m}]$} & acoustic wavenumber, $k=|\mathbf{k}|=2 \pi / \lambda=\omega / c$ \\
\hline$k_{m}$ & {$[\mathrm{rad} / \mathrm{m}]$} & mode $m$ of wavenumber $k_{z}$, Eq. (5.30) \\
\hline$k_{p} k_{p, \max }$ & {$[-]$} & $\begin{array}{l}\text { non-dimensional pressure amplitude density, Eq. (5.97) } \\
\text { maximum level of broadband } k_{p}\end{array}$ \\
\hline$k_{r}$ & {$[\mathrm{rad} / \mathrm{m}]$} & wavenumber in radial direction, $k_{r}=\sqrt{k^{2}-k_{z}^{2}}$ \\
\hline$k_{z}$ & {$[\mathrm{rad} / \mathrm{m}]$} & wavenumber in axial direction, $k_{z}=2 \pi / \lambda_{z}$ \\
\hline$L$ & {$[-]$} & half-length of cylinder \\
\hline$m$ & {$[-]$} & McCormick scaling factor for cavitation inception, Eq. (2.2) \\
\hline$n$ & {$[-]$} & wavenumber in azimuthal direction \\
\hline$n$ & {$[-]$} & pulse index, Section 5.3.3 \\
\hline$n$ & [rps] & propeller shaft rotation rate \\
\hline$P_{a}$ & [W] & acoustic power \\
\hline$P_{n}$ & {$[-]$} & Legendre polynomial \\
\hline$P_{\text {pot }}$ & {$[\mathrm{W}]$} & power due to change in potential energy \\
\hline$P_{0.7}$ & {$[\mathrm{~m}]$} & propeller pitch at $70 \%$ of tip radius \\
\hline$p$ & {$[-]$} & constant in the modified Lamb-Oseen vortex \\
\hline$p$ & {$[-]$} & apparent order of convergence \\
\hline$p$ & {$[\mathrm{~Pa}]$} & pressure \\
\hline$p_{\text {min }}$ & & minimum pressure in the fluid \\
\hline$p_{T}$ & & pressure change due to surface tension \\
\hline$p_{v}$ & & vapour pressure \\
\hline$\hat{p}_{s}$ & {$[\mathrm{~Pa} \mathrm{~m}]$} & root-source factor, Eq. (5.8) \\
\hline$q$ & {$[-]$} & constant in the cavitating modified Lamb-Oseen vortex \\
\hline$R$ & [m] & coordinate in radial direction, spherical coordinate system \\
\hline$R$ & [m] & propeller tip radius \\
\hline$R_{c}$ & [m] & radius of curvature of the hull surface \\
\hline $\operatorname{Re}$ & {$[-]$} & Reynolds number \\
\hline $\operatorname{Re}_{v}$ & & vortex Reynolds number, $\operatorname{Re}_{\mathrm{v}}=\Gamma_{\infty} / 2 \pi v$ \\
\hline $\operatorname{Re}_{n}$ & & Reynolds number defined as $\operatorname{Re}_{\mathrm{n}}=n D^{2} / v$ \\
\hline$r$ & [m] & coordinate in radial direction, cylindrical coordinate system \\
\hline$r_{c}$ & & cavity radius \\
\hline$r_{v}$ & & viscous core radius \\
\hline$\hat{r}_{m}^{F}$ & {$\left[\mathrm{~m}^{2}\right]$} & Fourier transform of radial perturbation, Eq. (5.33) \\
\hline$s$ & [m] & radial distance to cavity interface, $s=r-r_{c}$ \\
\hline$T$ & {$[\mathrm{~N}]$} & propeller thrust \\
\hline$T$ & {$[\mathrm{~Pa} / \mathrm{m}]$} & surface tension \\
\hline$T$ & {$[\mathrm{~s}]$} & time of one oscillatory period \\
\hline
\end{tabular}




\begin{tabular}{|c|c|c|}
\hline$T_{\omega}$ & {$[-]$} & $\begin{array}{l}\text { non-dimensional term containing contribution of surface } \\
\text { tension to the dispersion relation, Eq. (4.73) }\end{array}$ \\
\hline$t$ & [s] & time \\
\hline $\mathbf{u}$ & {$[\mathrm{m} / \mathrm{s}]$} & velocity vector \\
\hline $\mathbf{u}_{\mathbf{s}}$ & & interface velocity vector \\
\hline $\mathbf{U}$ & {$[\mathrm{m} / \mathrm{s}]$} & free-stream velocity vector, cylindrical coordinate system \\
\hline$U$ & {$[\mathrm{~m} / \mathrm{s}]$} & time-averaged velocity in radial direction \\
\hline$u$ & {$[\mathrm{~m} / \mathrm{s}]$} & $\begin{array}{l}\text { velocity in radial direction, radial and cylindrical coordinate } \\
\text { system }\end{array}$ \\
\hline$u_{s}$ & & interface velocity in radial direction \\
\hline$V$ & {$[\mathrm{~m} / \mathrm{s}]$} & $\begin{array}{l}\text { time-averaged velocity in azimuthal direction, cylindrical } \\
\text { coordinate system }\end{array}$ \\
\hline$V_{s}$ & {$[\mathrm{~m} / \mathrm{s}]$} & ship speed, can also be given in knots \\
\hline$v$ & {$[\mathrm{~m} / \mathrm{s}]$} & velocity in azimuthal direction, cylindrical coordinate system \\
\hline$v_{t}$ & {$[\mathrm{~m} / \mathrm{s}]$} & velocity magnitude in transverse plane \\
\hline$W$ & {$[\mathrm{~m} / \mathrm{s}]$} & time-averaged velocity in axial direction \\
\hline We & {$[-]$} & Weber number, Eq. (4.74) \\
\hline$w$ & {$[\mathrm{~m} / \mathrm{s}]$} & velocity in axial direction, cylindrical coordinate system \\
\hline$x$ & {$[\mathrm{~m}]$} & coordinate in horizontal direction \\
\hline$y$ & {$[\mathrm{~m}]$} & coordinate in vertical direction \\
\hline$Z$ & {$[-]$} & number of blades of propeller \\
\hline$z$ & [m] & coordinate in axial direction, cylindrical coordinate system \\
\hline$z_{r}$ & {$[\mathrm{~m}]$} & $\begin{array}{l}\text { vertical distance between reflecting (image) acoustic source } \\
\text { and ship hull }\end{array}$ \\
\hline$z_{s}$ & [m] & vertical distance between acoustic source and ship hull \\
\hline
\end{tabular}

\section{Greek letters}

\begin{tabular}{|c|c|c|}
\hline Symbol & Unit & Property \\
\hline$\alpha$ & {$\left[{ }^{\circ}\right]$} & angle of attack \\
\hline$\alpha$ & {$[-]$} & parameter to weight the spectral shapes $H_{h}$ and $H_{s}$ \\
\hline$\hat{\alpha}$ & {$[-]$} & constant in the modified Lamb-Oseen vortex \\
\hline$\alpha_{l}$ & {$[-]$} & slope at low frequency of $H_{S}$ \\
\hline$\alpha_{h}$ & {$[-]$} & slope at high frequency of $H_{S}$ \\
\hline$\beta$ & {$[-]$} & parameter in the cavitating (modified) Lamb-Oseen vortex \\
\hline$\Gamma$ & {$\left[\mathrm{m}^{2} / \mathrm{s}\right]$} & circulation \\
\hline$\Gamma(n)$ & {$[-]$} & Gamma function for positive integer $n$ \\
\hline$\gamma$ & {$[-]$} & constant of Euler-Mascheroni, $\gamma \approx 0.5772$ \\
\hline$\gamma$ & {$[1 / \mathrm{m}]$} & axial wavenumber in the spectral domain \\
\hline $\bar{\gamma}$ & & wavenumber $\gamma$ at point of stationary phase \\
\hline$\Delta_{T}$ & {$[-]$} & Dirac comb, Eq. (5.90) \\
\hline$\Delta f$ & {$[\mathrm{~Hz}]$} & frequency resolution bandwidth of the spectrum \\
\hline$\Delta t_{i}$ & {$[\mathrm{~s}]$} & time step of case $i$ \\
\hline$\eta$ & {$[\mathrm{m}]$} & local cavity radius \\
\hline$\eta_{a c}$ & {$[-]$} & acoustic efficiency, Eq. (5.105) \\
\hline
\end{tabular}




\begin{tabular}{|c|c|c|}
\hline$\theta$ & [rad] & $\begin{array}{l}\text { coordinate in azimuthal direction, radial and cylindrical } \\
\text { coordinate system }\end{array}$ \\
\hline$\vartheta$ & [rad] & coordinate in polar direction, radial coordinate system \\
\hline$\kappa$ & {$[-]$} & non-dimensional wavenumber \\
\hline & & $\kappa=k_{r} r_{c}$ when used in $H_{n}^{(1)}(\kappa)$ \\
\hline & & $\kappa=k_{z} r_{c}$ when used in $K_{n}(\kappa)$ \\
\hline$\kappa$ & {$[1 / \mathrm{m}]$} & interface curvature \\
\hline$\kappa_{1}$ & {$[-]$} & empirical exponent to relate $k_{p, \max }$ to $r_{c} / D$, Eq. (7.7) \\
\hline$\lambda$ & [m] & wavelength \\
\hline$\lambda$ & {$[-]$} & geometric scale factor used in model test \\
\hline$\mu$ & {$[\mathrm{kg} /(\mathrm{m} \mathrm{s})]$} & dynamic viscosity \\
\hline$v$ & {$\left[\mathrm{~m}^{2} / \mathrm{s}\right]$} & kinematic viscosity \\
\hline$\pi$ & {$[-]$} & ratio of a circle's circumference to its diameter, $\pi=3.1416 \ldots$ \\
\hline$\rho$ & {$\left[\mathrm{kg} / \mathrm{m}^{3}\right]$} & density \\
\hline$\sigma$ & {$[-]$} & cavitation number, $\sigma=\left(p_{\text {ref }}-p_{v}\right) / 0.5 \rho V_{\text {ref }}^{2}$ \\
\hline$\sigma_{i}$ & & cavitation inception number \\
\hline$\sigma_{n}$ & & cavitation number using $V_{\text {ref }}=n D$ \\
\hline$\sigma_{W}$ & & cavitation number using $V_{\text {ref }}=W_{\infty}$ \\
\hline$\sigma$ & {$[-]$} & standard deviation \\
\hline$\sigma_{a}$ & {$[\mathrm{~m}]$} & standard deviation of the amplitude variation \\
\hline$\sigma_{\tau}$ & {$[\mathrm{s}]$} & standard deviation of the time variation \\
\hline$\sigma_{\theta}$ & [rad] & standard deviation of the phase variation \\
\hline$\varsigma$ & {$[-]$} & constant to define $r_{v}$ at $\partial v / \partial r=0$ in vortex model, $\varsigma=1.2564 \ldots$ \\
\hline$\varsigma_{1}$ & {$[-]$} & empirical parameter to define roll-up region in vortex model \\
\hline$\varsigma_{2}$ & {$[-]$} & parameter to define $r_{v}$ at $\partial v / \partial r=0$ in vortex model \\
\hline$\tau_{n}$ & {$[\mathrm{~s}]$} & time shift of pulse $n$ \\
\hline $\bar{\tau}$ & {$[\mathrm{Pa}]$} & stress tensor \\
\hline$\varphi$ & {$\left[\mathrm{m}^{2} / \mathrm{s}\right]$} & velocity potential \\
\hline$\phi$ & {$\left[\mathrm{m}^{2} / \mathrm{s}\right]$} & velocity potential that is only a function of $r$ \\
\hline$\Omega$ & {$[\mathrm{rad} / \mathrm{s}]$} & angular velocity at the cavity interface, $\Omega=V_{c} / r_{c}$ \\
\hline$\omega$ & {$[\mathrm{rad} / \mathrm{s}]$} & angular frequency \\
\hline$\omega^{*}$ & & corrected angular frequency, Eq. (5.17) \\
\hline$\omega_{z}$ & {$[1 / \mathrm{s}]$} & vorticity in axial direction \\
\hline
\end{tabular}

Superscripts, on any variable, $x$ :

\begin{tabular}{ll}
\hline Symbol & Property \\
\hline$\dot{x}$ & derivative of $x$ with respect to time \\
$\ddot{x}$ & second derivative of $x$ with respect to time \\
$\bar{x}$ & mean value of $x$ \\
$x^{\prime}$ & perturbation of $x$ \\
$\hat{x}$ & peak amplitude of $x$ in time \\
$\tilde{x}$ & non-dimensional value of $x$ \\
$x^{ \pm}$ & mode number $x$ with sign corresponding to the branch of the dispersion relation \\
\hline
\end{tabular}


Subscripts, on any variable, $x$ :

\begin{tabular}{ll}
\hline Symbol & Property \\
\hline$x_{c}$ & pertaining to the liquid side of the cavity interface \\
$x_{l}$ & pertaining to the liquid phase of the fluid \\
$x_{m}$ & model-scale value \\
$x_{n}$ & value at outer edge of computational domain \\
$x_{\text {ref }}$ & reference value \\
$x_{s}$ & ship-scale value \\
$x_{v}$ & pertaining to the vapour phase of the fluid \\
$x_{z}$ & component of $x$ in axial direction, cylindrical coordinate system \\
$x_{\theta}$ & component of $x$ in azimuthal direction, cylindrical coordinate system \\
$x_{\infty}$ & value at large distance, in upstream or radial direction \\
$x_{1 \mathrm{~Hz}}$ & spectral value of $x$ for a frequency resolution bandwidth of $1 \mathrm{~Hz}$ \\
$x_{1 / 3}$ & spectral value of $x$ for a frequency resolution bandwidth of $1 / 3 \mathrm{octave}$ \\
\hline
\end{tabular}

\section{Acronyms}

\begin{tabular}{ll}
\hline Abbreviation & Meaning \\
\hline AR & Aspect Ratio \\
BEM & Boundary Element Method \\
BPF & Blade Passage Frequency \\
CDL & Crossing Dispersion Lines \\
CFD & Computational Fluid Dynamics \\
CFL & Courant-Friedrichs-Lewy \\
CPP & Controllable Pitch Propeller \\
CPSD & Cross-Power Spectral Density \\
CRS & Cooperative Research Ships \\
DO & Dissolved Oxygen content \\
DWB & Depressurized Wave Basin \\
EXCALIBUR & EXcitation CALculation with Improved BURton \& miller method (BEM com- \\
& puter code) \\
ETV & Empirical cavitating Tip Vortex method \\
FFT & Fast Fourier Transform \\
HPF & Hull-Pressure Fluctuations \\
HSV & High-Speed Video \\
IMO & International Maritime Organization \\
ISO & International Standards Organization \\
ITTC & International Towing Tank Conference \\
LDV & Laser Doppler Velocimetry \\
LM & Lloyd's Mirror \\
MARIN & MAritime Research Institute Netherlands \\
OASPL & OverAll Sound Pressure Level \\
PIV & Particle Image Velocimetry \\
PL & Propagation Loss \\
PROCAL & PROpropeller CALculation method (BEM computer code) \\
&
\end{tabular}


PSD Power Spectral Density

RNL Radiated Noise Level

rms root-mean-square

rpm revolutions per minute

rps revolutions per second

SBF

SL

Solid Boundary Factor

SPL

Source Level

URN

Sound Pressure Level

ZGV

Underwater Radiated Noise

ZPV

Zero Group Velocity

Zero Phase Velocity 


\section{Chapter 1}

\section{Introduction}

This chapter provides the motivation, the problem definition, and the objectives for the conducted research study. It also presents the outline of the thesis.

\subsection{Background}

Exploring the world from aboard a ship has become a popular way of spending holiday time over the last decades. It has led to an enormous increase in the number as well as the size of cruise ships and luxury yachts. However, passengers and yacht owners expect similar comfort levels on board as in luxury hotels. Starting with the DNV Comfort class in 1995, most classification societies have developed comfort class rules for ships. These class rules also describe crew comfort on board cargo ships. With the introduction of comfort class rules, the noise and vibration levels on board ships have decreased significantly (de Lorenzo and Biot, 2006).

Noise generated by a ship is not only relevant on board but also underwater. The underwater radiated noise (URN) by the ship is of importance for, e.g., the acoustic signature of military vessels, for the operation of onboard equipment that requires low self-noise such as sonar, and for fishery research vessels. Also, there is a growing concern about the effect of shipping noise on the behaviour of marine mammals and fish and on the masking of sound generated by these animals (Götz et al., 2009). Marine mammals and fish depend on sound for communication, prey finding and observation of the underwater world. Underwater noise in the ocean by anthropogenic sources has increased significantly over the last 50 years, with the increase in low frequency noise partly related to the large increase in ship size and shipping traffic in that period (Hildebrand, 2009).

The concern about the effect of shipping noise on marine mammals and fish has led to the development of non-mandatory 'Guidelines for the Reduction of Underwater Noise from Commercial Shipping' by the IMO (MEPC 66/17, 2013). It has also led to the inclusion of underwater noise by shipping in the environmental descriptors to achieve Good Environmental Status of the European Seas in the EU Marine Strategy Framework Directive (2008/56/EC). Guidelines for regulation of URN from commercial shipping were jointly developed by the EU FP7 projects AQUO and SONIC (Baudin and Mumm, 2015), but regulations involving limiting noise levels are not yet in place. However, starting with the DNV-GL Silent class notation in 

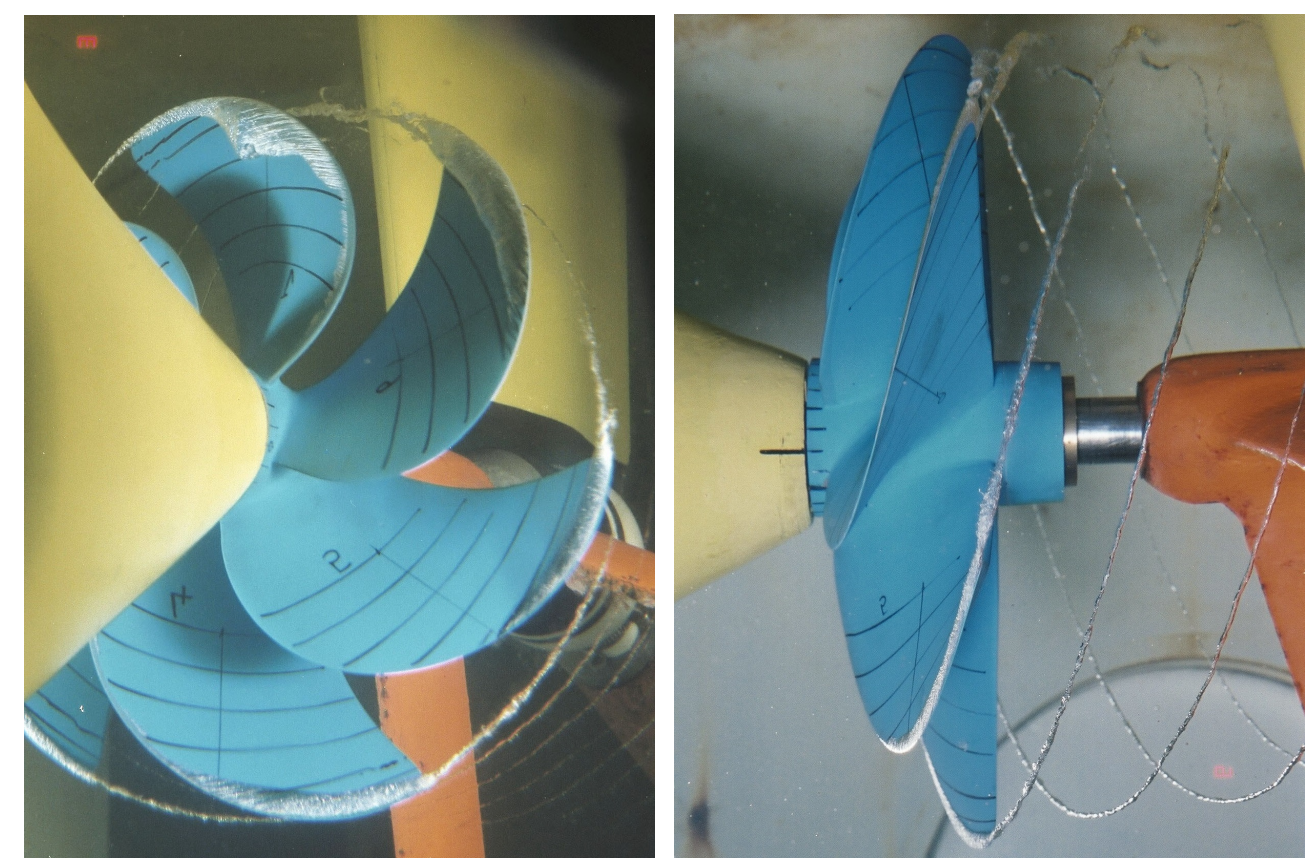

Figure 1.1: Photographs of a propeller with sheet and tip-vortex cavitation operating in the wake of a ship hull mounted in the cavitation tunnel of MARIN. Flow from left to right.

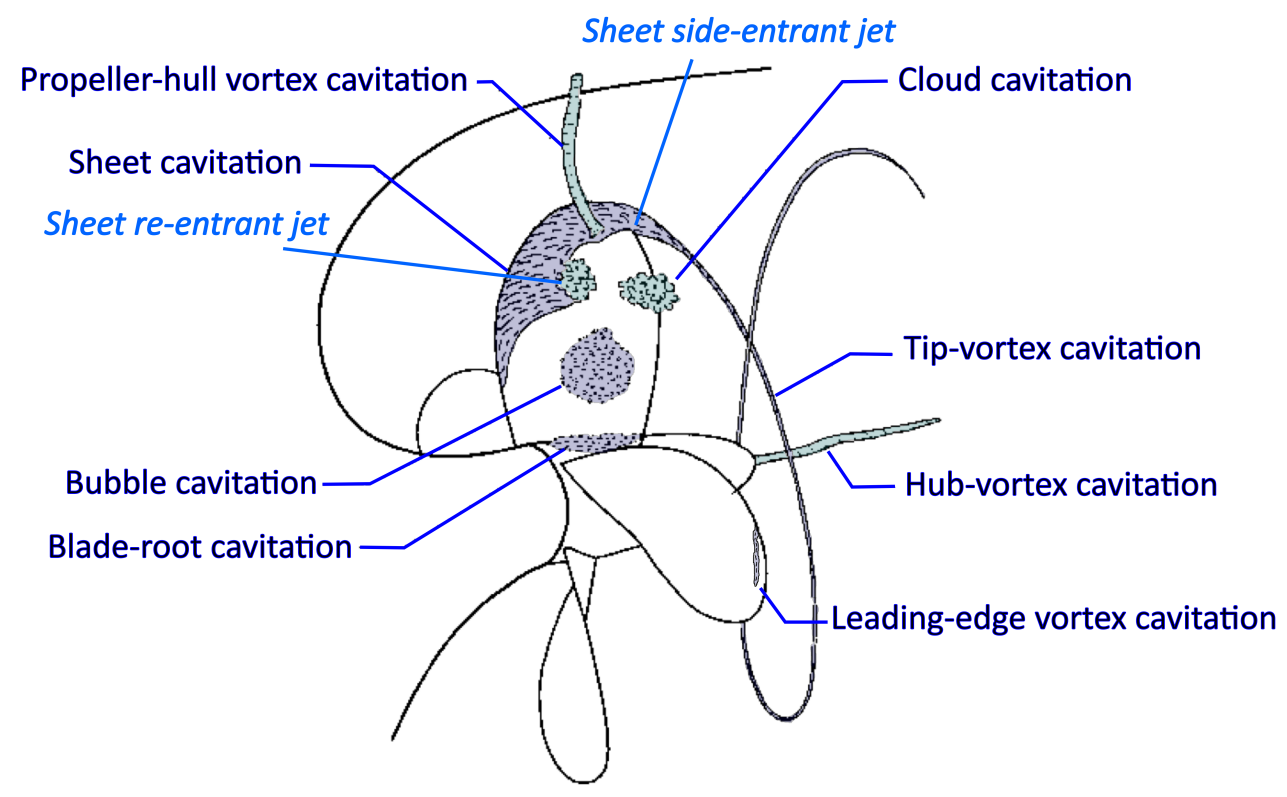

Figure 1.2: Illustration of possible cavitation patterns on ship propellers. Adapted from ITTC procedure 7.5-02-03-03.2.

2010, also BV, RINA, and LR now offer class rules that include limits on the URN levels for ships.

If present, cavitation on the propeller blades is an important contributor to ship noise. Cavitation is the vapour phase of a liquid due to the rupture of that liquid. It can be initiated 


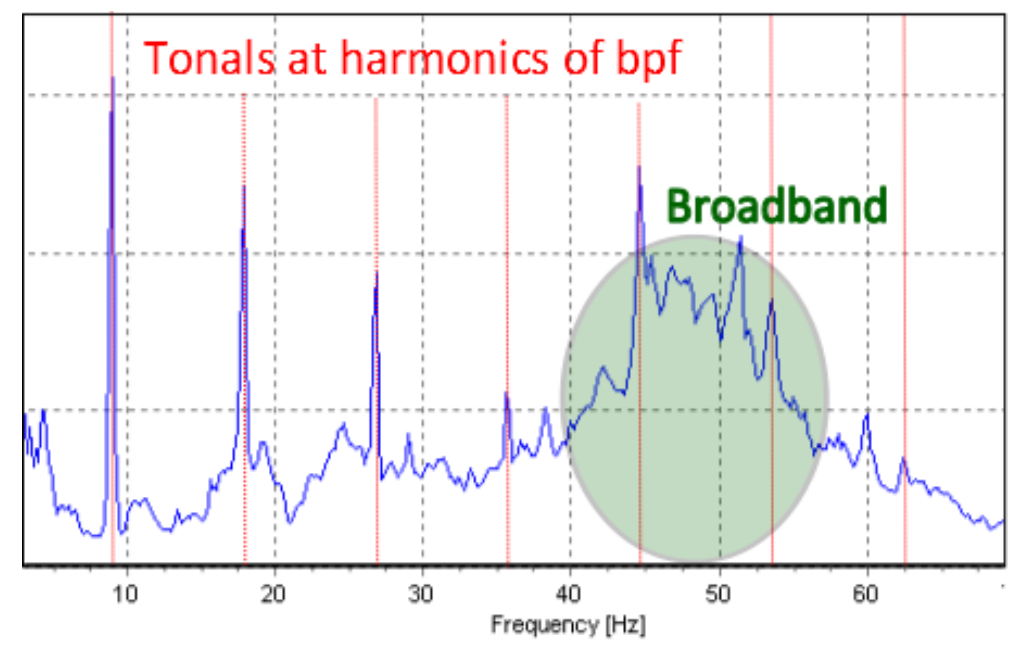

Figure 1.3: Example of an amplitude spectrum (linear scale) of propeller-induced hull pressures on a twin-screw vessel. Adapted from van Wijngaarden et al. (2005).

by setting up a tension in the liquid or by depositing energy in it (Lauterborn and Ohl, 1997). The energy deposit can be applied by heat or light. The tension can be applied by a pressure reduction due to fluid flow, in which case one speaks of hydrodynamic cavitation, or by sound waves, in which case one speaks of acoustic cavitation. On ship propellers, it is hydrodynamic cavitation that is present. It occurs when the pressure drops below vapour pressure provided sufficient nuclei are present. Cavitation can also occur at other locations at the ship such as struts and rudders, but, from the point of view of noise, cavitation on the propeller is usually most relevant. The type, extent and dynamics of cavitation on the propeller are determined by the hydrodynamic design of ship hull and propeller geometry and by the operating conditions of ship and propeller.

In principle, we can distinguish between three types of developed cavitation: sheet cavitation, vortex cavitation and bubble cavitation. Sheet cavitation occurs when the pressure has its minimum value on a surface and the location of the cavity detachment point remains fixed to that surface such that the phase discontinuity forms a sheet. Vortex cavitation occurs when the pressure has its minimum in the centre of a vortex and the phase discontinuity forms a rotating tube. Bubble cavitation describes the cavitation patterns for which the phase discontinuity has approximately the shape of a sphere. It occurs when the pressure has its minimum in the fluid or on a surface but such that a sheet or vortex cavity does not occur. The most common cavitation patterns on ship propellers are sheet and tip-vortex cavitation, see Figure 1.1 for an example. An illustration of the various possible cavitation patterns on ship propellers is shown in Figure 1.2. The collapse of sheet cavitation through its re-entrant jet and side-entrant jet leads to a cloud of cavitating bubbles and vortices. Developed vortex cavitation can occur at the tip and leading edge of the blade, and aft of the hub. If the propeller loading is very high, a vortex is generated between the hull and the propeller blade. This propeller-hull vortex may also cavitate.

In the hydrodynamic design phase of ship and propeller, the analysis of propeller cavitation hindrance for on board noise and vibration usually focusses on the low-frequency hullpressure fluctuations (HPF) on the surface of the hull above the propeller. For these locations, the repetitive blade passages generates tonals at the blade passage frequency (BPF) and har- 
monics thereof. Usually, the results are presented as the pressure amplitude of these blade rate tonals, typically up to the fourth harmonic of the BPF. However, some ships have experienced vibration problems at frequencies located in between the harmonics of the BPF. Analysis of the HPF spectrum, of which an example is presented in Figure 1.3, showed that within a small frequency range a broadband 'hump' was present. Such a broadband hump is most relevant when the tonals are of small amplitude, which is occurring when the cavity pattern on the propeller blade is dominated by tip-vortex cavitation rather than sheet cavitation.

The URN of propeller cavitation is usually presented for a frequency range varying between $\mathrm{BPF}$ and $25 \mathrm{kHz}$ or higher. This spectrum is at low frequencies very similar to Figure 1.3, while at higher frequencies only broadband noise is present. The focus in the analysis of URN is usually on the broadband levels.

\subsection{Problem definition}

The ships that suffered from vibration problems due to broadband excitation were twin-screw vessels with stringent noise and vibration requirements, such as cruise vessels, ferries and yachts. The design of the propellers for such ships is such that cavitation is avoided as much as possible. However, avoiding cavitation in the tip area of the propeller blade requires significant unloading of the blade tip which is only possible at the expense of propeller efficiency. As a low efficiency is undesirable because of fuel cost, the propellers for these ships do show the presence of a cavitating tip vortex, possibly in combination with some sheet cavitation near the tip.

Traditionally, the prediction and analysis of propeller-induced HPF focusses on frequencies that are harmonics of the BPF. Relatively little knowledge is available on the low-frequency broadband character of the HPF and on the mechanisms involved. Most of the research on cavitation in relation to erosion and noise has been focussed on sheet and bubble cavitation as these cavitation patterns are more harmful than vortex cavitation. Research on vortex cavitation has mostly been focussed on the inception of cavitation, this due to its relevance for the acoustic signature of military ships. Computational tools for propeller design based on potential flow theory usually account for sheet cavitation but often lack a model for vortex cavitation.

For the reasons mentioned above, there is a lack of fundamental knowledge on developed vortex cavities and its related broadband noise. This leads to uncertainties in the design of propellers and in the application of model tests to predict HPF and URN for the case of vortex cavitation.

\subsection{Research objectives}

The problem definition described above, has led to the following research objectives:

1. Determine the mechanisms that lead to broadband noise by vortex cavitation.

2. Develop a computational method for the prediction of broadband noise due to tip-vortex cavitation on ship propellers and evaluate the results of model tests on this topic. 
With broadband noise, we denote here broadband hull-pressure fluctuations (HPF) as well as underwater radiated noise (URN), but the focus of the present thesis is on broadband HPF.

The first objective requires fundamental research on vortex cavitation due to a lack of knowledge in this area. For the present thesis, it was decided to focus on theoretical studies as it was expected that this would give the largest gain in knowledge on vortex cavitation. An important research question for a model basin is how important viscous effects are for vortex cavities as the Reynolds number in the model test is smaller than for the ship propeller. Since the publication by McCormick (1962), it is well-known that viscous effects are very important for the minimum pressure in a non-cavitating vortex and, therefore, for the inception of cavitation. However, very little is known on the effect of viscosity on developed vortex cavities.

The second objective aims to apply the results of the fundamental research to the consultancy services of the Maritime Research Institute Netherlands (MARIN) in the prediction of HPF and URN by propeller cavitation. To a large extent, these services rely on the use of model tests in MARIN's Depressurized Wave Basin (DWB), in which broadband noise by cavitating vortices has been measured for quite some time. However, the interpretation of the results of these measurements needs to be further improved. Next to model tests, also computational predictions are made but no model for tip-vortex cavitation noise was available at the start of the present study.

The last decade has shown a rapid progress in the prediction of propeller cavitation by using computational fluid dynamics (CFD). In spite of this progress, capturing cavitating vortices is still a challenge, specifically capturing the vortex dynamics and resulting radiated noise. Therefore, it was decided not to use CFD in the present thesis but to develop a semi-empirical method. This method is to be used in combination with a boundary-element method (BEM), which is still the daily workhorse in the maritime industry for the analysis of cavitating propellers operating in the wake of a ship.

To limit the scope of work, it was decided to disregard the amplitudes at the higher harmonics of the blade passage frequency despite its high importance for ship vibrations. Also, the inception of vortex cavitation and the noise generated at inception is not further investigated here. Furthermore, the focus is on tip-vortex cavitation leaving aside the other forms of vortex cavitation given in Figure 1.2, as far as possible. The description of propeller tip-vortex cavitation also requires sheet cavitation to be considered, because a sheet cavity in the tip area is often directly connected to the tip-vortex cavity while it may also form a vortex cavity by itself.

\subsection{Outline of the thesis}

The outline of the thesis is shown in Figure 1.4. The thesis is divided into four parts. The first part presents the background information, the second part describes the fundamental research performed, the third part describes the applied research, and the thesis closes with concluding remarks. The derivation and, where possible, validation of prediction formulas for a vortex cavity and its broadband noise are presented in the part describing the fundamental research. In each chapter, a box is drawn around the formulation that is used in the applied research or that is the end result of the derivation. The key formulations and figures are also referred to in the conclusions of each chapter. 


\begin{tabular}{|c|c|}
\hline \multicolumn{2}{|c|}{$\begin{array}{l}\text { Background information of } \\
\text { vortex cavitation and broadband noise: } \\
\text { 1. Introduction } \\
\text { 2. Characteristics }\end{array}$} \\
\hline $\begin{array}{l}\text { Fundamental research } \\
\text { on vortex cavitation: } \\
\text { 3. Kinematics } \\
\text { 4. Dynamics } \\
\text { 5. Acoustics }\end{array}$ & $\begin{array}{l}\text { Applied research on } \\
\text { broadband noise: } \\
\text { 6. Mechanisms } \\
\text { 7. Semi-empirical prediction } \\
\text { 8. Experimental prediction }\end{array}$ \\
\hline 9. Conclua & emarks \\
\hline
\end{tabular}

Figure 1.4: Outline of the chapters in the present thesis.

In Chapter 2, the characteristics of vortex cavitation, HPF, and URN are described. This chapter includes a brief discussion on the inception of vortex cavitation which is, however, not further considered in this work. Various aspects of non-cavitating vortices are also presented.

Chapters 3 through 5 describe the more fundamental research performed on vortex cavitation and its radiated noise. It starts in Chapter 3 with the derivation of an analytical model to describe the kinematics of a vortex cavity in two-dimensional viscous flow. The model is extended towards a semi-empirical formulation that is capable of describing available experimental data of a cavitating vortex. The dynamic behaviour of vortex cavitation is studied in Chapter 4. It contains a computational study of the unsteady behaviour of a vortex cavity in two-dimensional viscous flow. For three-dimensional flow, the unsteady behaviour of the vortex cavity is studied by deriving an analytical formulation for the dispersion relation of the propagation of inertial waves on the cavity interface. The results of the analytical relation are compared with available experimental data. In Chapter 5, various aspects of HPF and URN, including its signal analysis, are studied from a theoretical point of view. This chapter also includes various analytical formulations for the noise generated by the dynamics of a vortex cavity using formulations for vibrating cylinders.

The results of the fundamental research are applied in the studies described in Chapters 6 through 8. Chapter 6 presents the mechanisms of broadband HPF and URN by vortex cavitation. The development of a semi-empirical prediction method is described in Chapter 7 , and Chapter 8 is related to the experimental prediction. It presents a methodology to account for viscous scale-effects on vortex cavitation in model tests by either adjusting the cavitation number in the basin or by applying additional corrections to the measured noise spectrum.

The concluding remarks are presented in Chapter 9. 


\section{Chapter 2}

\section{Characteristics}

This chapter describes the characteristics of cavitating tip vortices, of propeller-induced hullpressure fluctuations, and of underwater radiated noise. It also includes a brief description of some aspects of non-cavitating vortices and of the inception of vortex cavitation.

\subsection{Introduction}

Vortices have been a topic of intense research in fluid dynamics since the formulation of the basic vorticity theorems by Helmholtz (1858). Vortices can be described as a connected fluid region with high concentration of vorticity compared to its surroundings (Wu et al., 2005), with vorticity defined as the rotation of the velocity vector. Vorticity can be generated by shear flow, curvature of the flow, Coriolis force or baroclinity. Vortices dominate turbulent flow through a mixture of chaotic motions and coherent structures, which are (vortical) flow structures with spatial and temporal coherence. Lifting bodies such as wings and propellers generate vortices through the roll-up of vortex sheets generated by the velocity difference between the suction side and the pressure side of the body. These vortices should be taken into account when analysing the forces on the body, and they may lead to other fluid dynamic phenomena such as noise. Dedicated books are available on vorticity and vortices. For the present work use has been made of Saffman (1992), Green (1995), and Wu et al. (2005).

As described by Young (1989), the phenomenon of cavitation was first described by Newton in 1704. Between 1895 and 1897, the investigation of racing of steam turbines driving ship propellers led to papers by Barnaby and Thornycroft, and Barnaby and Parsons in which cavitation was first mentioned and discussed. The word cavitation was suggested by R.E. Froude. In 1895, Parsons performed the first model tests in a very small cavitation tunnel, that had to be filled with hot water to generate cavitation on the propeller. In 1910, he built the first real cavitation tunnel at the University of Newcastle. The circular test section had a diameter of $91 \mathrm{~cm}$ and the diameter of the propellers that were tested was $30 \mathrm{~cm}$ (Burrill, 1951). These investigations were related to thrust-breakdown of the ship propeller due to cavitation. This phenomenon was unknown in those days but is well predicted nowadays. Current research on cavitation for ship propellers, pumps, and turbines is related to erosion and to noise and vibration. Cavitation can also be used for cleaning and for fragmentation of biological cells in medical applications. De- 
tailed information on cavitation and cavitation noise can be found in textbooks such as Young (1989), Leighton (1992), Franc and Michel (2004) and Brennen (2009). The textbook by Kuiper (2010) specifically deals with propeller cavitation, while propeller cavitation noise is discussed by Ross (1987) and Blake (1986). Carlton (2007) treats practically all aspects involved in ship propellers, including cavitation.

This chapter describes the characteristics of vortex cavitation as generated by ship propellers and its associated broadband noise by first reviewing relevant aspects of non-cavitating vortices in Section 2.2 and of cavitation inception in Section 2.3. Section 2.4 discusses vortex cavities. The cavitation induced hull-pressure fluctuations (HPF) and the underwater radiated noise (URN) are described in Sections 2.5 and 2.6, respectively. Section 2.7 summarizes the most relevant aspects for the present thesis. The focus in all sections is on describing the physics to provide background information for the studies performed in the following chapters.

\subsection{Non-cavitating vortices}

A large body of literature is available on the structure and the development of wing-tip vortices, describing experimental, theoretical and computational research. This section briefly describes several aspects of the physics of non-cavitating vortices and provides some references in which more detailed descriptions and more literature can be found.

The load distribution on the wing leads to a trailing sheet of vorticity in the wake of the wing. Downstream the wing, this vortex sheet rolls-up around the centre of vorticity close to the end of the sheet. This process can be described by inviscid flow theory (Moore and Saffman, 1973). The roll-up of vorticity results in the tip vortex schematically shown in Figure 2.1, somewhat similar as shown in Phillips (1981). The region with individual vortex layers and merged vortex layers by diffusion is denoted here as the vorticity roll-up region with potential flow outside this region. At the vortex centre, the azimuthal velocity should be zero. This boundary condition can only be fulfilled through the action of viscosity resulting in solid body rotation of the fluid in the centre. Here, a laminar flow region may exist for an isolated vortex. Between the region with solid body rotation and the vorticity roll-up region, a turbulent viscous flow region exists. This region, combined with the region of solid body rotation, is designated the viscous core. The size of this core is usually defined as the radius at which the azimuthal velocity has its maximum value, but the region in which viscosity has an effect on the flow is larger. As shown in Figure 2.1, the distribution of the azimuthal velocity leads to a minimum in pressure at the centre of the vortex. The roll-up of vorticity is accompanied by an increase of the axial velocity component. At the vortex centre, however, an axial-flow deficit can occur due to the wake of the boundary layer on the wing. The roll-up of a vortex in turbulent flow is described by Phillips (1981). Staufenbiel (1984) has computed the roll-up of a wing tip vortex using conservation of rotational energy, and Rule and Bliss (1998) have related the structure of the rolled-up vortex to parameters of the wing loading. The formation of the tip vortex above a rectangular wing is shown in Figure 2.2. The graph with the total pressure loss, caused by viscous shear stresses, clearly shows the vortex sheet and the viscous core.

Measurements have shown that vortices are very persistent. Downstream the wing tip, the viscous core of the vortex grows very gradually despite the turbulent flow on the wing. Zeman 

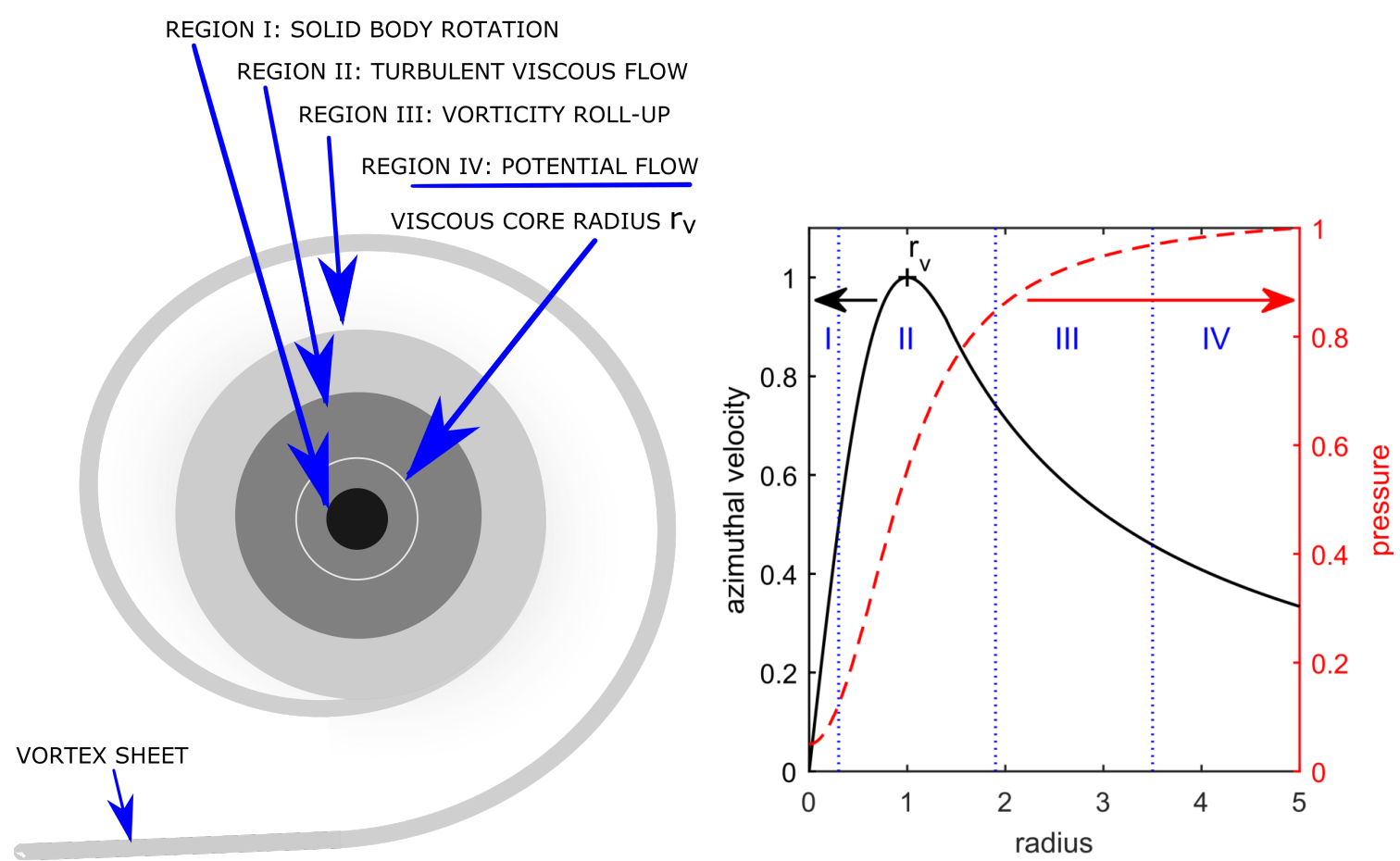

Figure 2.1: (Left) Sketch showing the roll-up of the vortex sheet and the different flow regions. (Right) Scaled circumferential-average (of the upper sector in the left graph) of the azimuthal velocity on the left $y$-axis and of the pressure on the right $y$-axis, the numbers refer to the flow regions.

(1995) has shown by CFD computations that the Reynolds shear stress is suppressed in the centre of the vortex. This is explained by analysing the Richardson number, Ri, for streamline curvature, as derived by Bradshaw (1969) in analogy with the gradient Richardson number that describes the ratio of a buoyancy term and a shear term in relation to absorption and production of turbulence, respectively. Bradshaw replaced the buoyancy term by a term related to flow curvature (centrifugal effects). The original definition by Bradshaw was rewritten by Holzäpfel et al. (2002) into a formulation in a cylindrical coordinate system which is better suited to describe the characteristics of vortices,

$$
\mathrm{Ri}=\frac{2 v}{r^{2}} \frac{\partial(v r)}{\partial r} /\left[r \frac{\partial(v / r)}{\partial r}\right]^{2},
$$

with $v$ the azimuthal velocity and $r$ the radial distance from the vortex centre. This definition of the Richardson number leads to a value of zero for $\mathrm{Ri}$ for those radii where circulation is constant and to a value of two at the viscous core. Ri increases to infinity in the centre region where the flow is described by solid body rotation. Cotel (2002) has shown that the flow is laminar where the Richardson number is larger than a critical vortex Reynolds number, $\mathrm{Ri}>\mathrm{Re}_{v}^{1 / 4}$, where $\operatorname{Re}_{v}$ is the Reynolds number based on vortex circulation. Devenport et al. (1996) showed that velocity fluctuations in the vortex core are due to the motion of the core by turbulence from the surrounding flow field and scale such that the core is laminar. Their measurements 


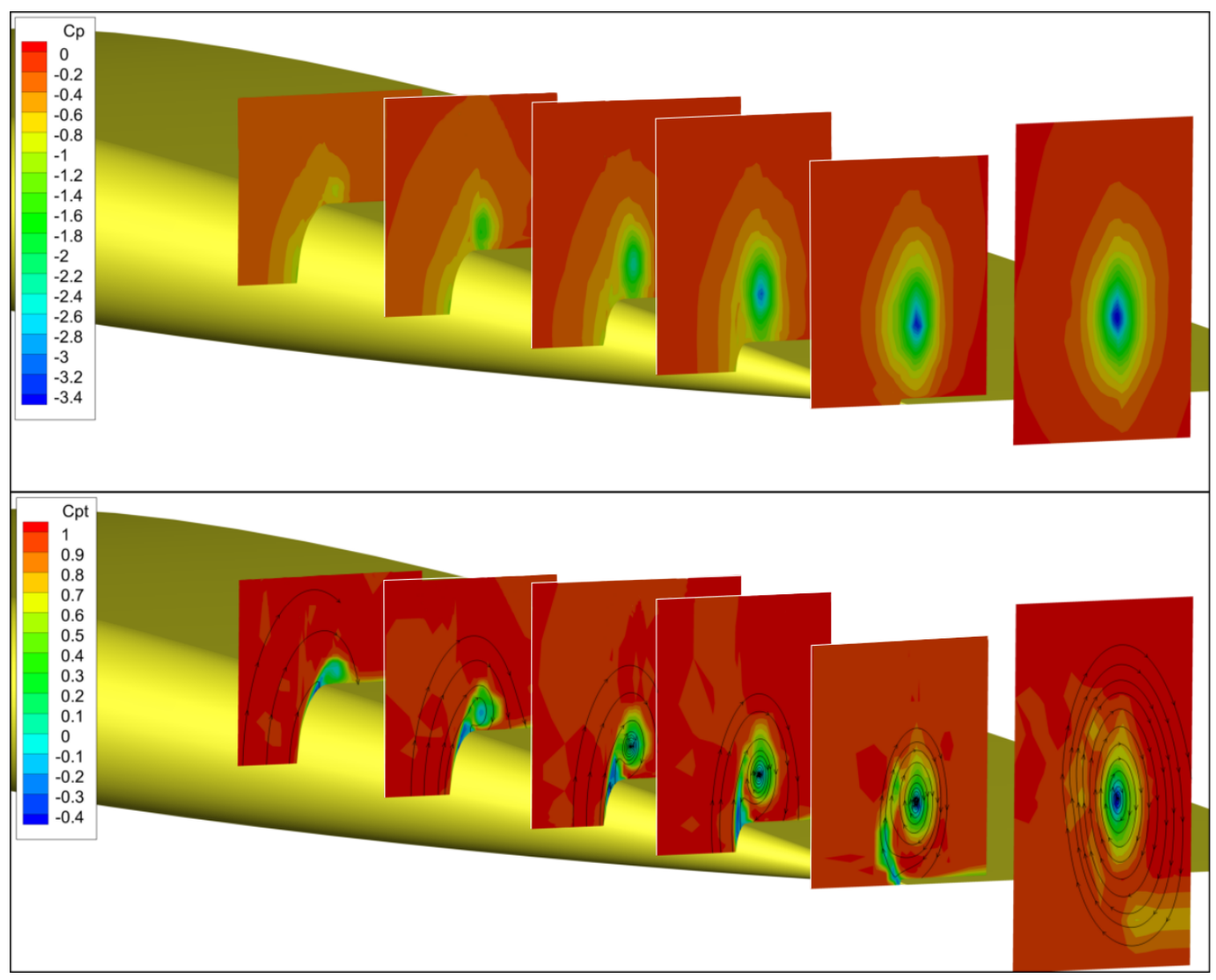

Figure 2.2: (Top) Contour plot of the pressure coefficient $C_{p}$ and (bottom) the total pressure coefficient $C_{p t}$ showing the vortex formation above the tip of a rectangular wing at $10 \mathrm{deg}$ angle of attack, $\mathrm{Re}=4.6 \times 10^{6}$. Experimental data by Chow et al. (1997). $C_{p}=\left(p-p_{\infty}\right) / 0.5 \rho W_{\infty}^{2}, C_{p t}=\left(p+0.5 \rho|\mathbf{u}|^{2}-p_{\infty}\right) / 0.5 \rho W_{\infty}^{2}$.

were performed in the far wake at a distance more than five chord lengths downstream the wing leading edge. The effect of low-frequency vortex motions, vortex wandering or meandering, has also been analysed in detail by Devenport et al. (1996). Vortex wandering is likely due to unsteadiness of the flow in the test-facility,

The tip-vortex formation at the tip of a rotating blade is very similar to that at a wing tip as shown for a rotor blade by Tung et al. (1981). However, rather than forming a longitudinal vortex structure, the trailing tip vortices of the rotor blade form a helical structure as illustrated in Figure 1.1. Detailed flow field measurements in the wake of a marine propeller have been published by Jessup (1989), Chesnakas and Jessup (1998), Di Felice et al. (2000), Di Felice et al. (2004) and Felli et al. (2011). The various models that describe the roll-up of the vortex sheet for a wing cannot directly be applied to rotating blades due to the variation of sectional speed with radius and the helical structure of the tip vortex.

Rayleigh (1917) showed that an axisymmetric, swirling, inviscid flow becomes unstable when the square of circulation $\Gamma$ decreases in radial direction, i.e. $\partial \Gamma^{2} / \partial r<0$. This is designated centrifugal instability. The extension of the analysis to the case with axial flow is 
discussed by Ash and Khorrami (1995) and the extension to non-axisymmetric instabilities is discussed by Billant and Gallaire (2005). However, concentrated vortices are generally stable with respect to this centrifugal instability, though the vortex core may act as a waveguide for Kelvin waves (Rossi, 2000).

Lord Kelvin (Thomson, 1880) showed that, at the transition from a rotating fluid to a fluid described by a potential flow vortex, hence at the viscous core radius of a Rankine vortex, stable transverse waves exist that propagate in axial and in azimuthal direction. Fabre et al. (2006) have shown that for an isolated vortex the wave modes are damped in case viscosity is taken into account. However, these so-called Kelvin waves are responsible for vortex instability in the presence of an external strain field. This has been considered in a large body of literature that is briefly reviewed in Fabre et al. (2006).

The interaction between counter-rotating vortices, such as the tip vortices of the wing of an aircraft, leads to a long-wave cooperative or Crow instability (Crow, 1970; Saffman, 1992; Steij1, 2001). Jacquin et al. (2003) mention that the amplification of the Kelvin displacement mode, which ultimately leads to a break-up of the vortex, is due to the superposition of three effects. The first effect is the displacement of the vortex in the strain field by the other vortex when the other vortex is not disturbed. The second effect is the self-influence when the vortex is displaced, and the third effect is the change in induction velocity by the other vortex when that is displaced. Such an interaction has also been observed in the wake of marine propellers in the absence of a rudder (Felli et al., 2011). A theoretical study on the interaction of helical tip vortices and the hub vortex has been published by Okulov and Sørensen (2007).

Under the influence of a strain field due to, for instance, another vortex, vortices may be subjected to the so-called short-wave or elliptic instability (Widnall et al., 1974; Moore and Saffman, 1975; Tsai and Widnall, 1976). A literature review on this topic has been given by Kerswell (2002). Experimental evidence on the elliptic instability leading to the long-wave Crow instability of two counter-rotating vortices is provided by Leweke and Williamson (1998). The elliptic instability is associated with two modes of Kelvin waves having the same axial wave length, a difference in frequency equal to the frequency of the imposed strain field, and with a difference in azimuthal wave number equal to two. The elliptic instability is also present in the merging process of two co-rotating vortices (Meunier et al., 2005). These interactions of vortices involve two displacement modes of the centreline (Le Dizès and Laporte, 2002). Lacaze et al. (2007) show that these two centreline displacement modes only occur in the absence of axial flow. With increasing axial flow, other modes, including a volume variation mode and an elliptical deformation mode, become more unstable. An example of such an interaction between two co-rotating vortices on marine propulsors is the merging of a tip vortex and a tip-leakage vortex on a ducted propeller (Oweis et al., 2006). A review on the dynamics and instabilities of a pair of co-rotating as well as counter-rotating vortices is provided by Leweke et al. (2016).

Leading-edge vortices generated at the leading edge of swept wings may breakdown or 'burst' above a certain angle of attack. An internal stagnation point is formed on the vortex axis, followed by reversed flow in a region of limited axial extent (Leibovich, 1978). The region with reversed flow can be close to axisymmetric, which is referred to as bubble vortex breakdown, or completely asymmetric, which is referred to as spiral vortex breakdown. Reviews of the flow structure and theoretical and computational prediction methods are given by Leibovich (1978), Escudier (1988), Delery (1994) and Lucca-Negro and O’Doherty (2001). Critical conditions 
for the occurrence of vortex breakdown include the ratio of axial and azimuthal velocities. Moet et al. (2005) show that bursting can occur at the intersection of two waves propagating in opposite direction. Leading-edge vortices are also present in the flow field around propellers with high skew, but there are no reports of vortex breakdown. Spiral vortex breakdown has been observed in the cavitating tip vortex of a marine propeller passing a rudder (Felli et al., 2010).

\subsection{Cavitation inception}

The inception of cavitation is a complex process requiring the presence of nuclei in the fluid. A review of inception mechanisms has been given by Rood (1991) and, more recently but for sheet cavitation only, by van Rijsbergen (2016). Most of the research on vortex cavitation has been concentrated on its inception, see e.g. Arndt (2002). In the following, the mechanisms involved in the inception of tip-vortex cavitation are briefly discussed, focusing on the minimum pressure in the centre of the vortex and the effect of nuclei. Even though solid particles can also initiate cavitation, most of the research on nuclei has been focussed on free gas bubbles.

It was first shown by McCormick (1962) that the inception of cavitation of a wing-tip vortex depends on the thickness of the boundary-layer on the pressure side of the wing tip. The cavitation inception number $\sigma_{i}$ was found to increase with increasing Reynolds number $\mathrm{Re}$ and increasing angle of attack and was found to depend significantly on the undissolved gas content. McCormick found that the viscous core size is not governed by induced drag and that the completely rolled-up vortex sheet is not representative of the inception condition. A semi-empirical relation was devised that provides a relation between the cavitation inception number and the Reynolds number. This relation between vortex cavitation inception number and Reynolds number has been confirmed by others, see e.g. Platzer and Souders (1980); Billet and Holl (1981) and Fruman et al. (1992). The general inception-scaling relation for a wing is given by (Arndt, 1981) as

$$
\sigma_{i}=K C_{L}^{2} \operatorname{Re}^{m}
$$

with $\sigma_{i}$ the cavitation inception number, $K$ a proportionality constant, $C_{L}$ the lift-coefficient of the wing and Re the Reynolds number based on chord length and free-stream velocity. The value for the parameter $m$ can be related to the growth of a turbulent boundary layer, but it is often determined by fitting experimental data. This scaling relation is also being used to correct the cavitation inception speed of propellers measured in model tests at a Reynolds number that is different from that at full scale. A review of published semi-empirical values for $m$ is given by the 21st ITTC Cavitation Committee (1996). Shen et al. (2009) have proposed a model in which $m$ is dependent on Reynolds number. At MARIN, $m=0.35$ is used (Noordzij, 1977).

Tip-vortex inception usually occurs on or immediately downstream of the wing tip, typically within a distance of one chord length. At the position of inception, the vortex is highly threedimensional and only further downstream the flow approaches symmetry about the vortex axis. The vortex structure and roll-up for wings in relation to cavitation inception was measured by Higuchi et al. (1987); Stinebring et al. (1991); Falçao De Campos (1992); Maines and Arndt (1997b). For propellers, such flow field measurements have been published by Jessup (1989) and Chesnakas and Jessup (1998). Cavitation inception models including vortex roll-up for wings have been published by Astolfi et al. (1999) and del Pino et al. (2011). 
The inception of tip-vortex cavitation requires the presence of nuclei in the flow. A nucleus, typically a small gas bubble, becomes unstable and starts to grow exponentially when the pressure falls below the Blake threshold pressure (Franc and Michel, 2004). For a quiescent fluid, the Blake threshold pressure $p_{b}$ is given by

$$
p_{b}=p_{v}-\sqrt{\frac{32 T^{3}}{27 r_{b}^{3} p_{g}}},
$$

with $p_{v}$ and $T$ the vapour pressure and surface tension of the liquid, respectively, and $r_{b}$ and $p_{g}$ the initial radius and initial partial gas pressure of the spherical bubble, respectively. However, for the inception of vortex cavitation both the trajectory of the bubble and its dynamic behaviour, described by the Rayleigh-Plesset equation, needs to be taken into account (Chahine, 1995; Choi et al., 2004; Oweis et al., 2005). At inception, the bubble becomes a cylinder. Hsiao and Chahine (2008) show that CFD does not reproduce the empirical values for $m$ if the minimum pressure in the vortex is considered. Only if nuclei are taken into account, the classical scaling rule for $m$ is retrieved. The nuclei-size distribution leads to an effective tensile strength of the water. The effect of this tensile strength on the inception of tip-vortices has been investigated by, among others, Arndt and Keller (1992); 21st ITTC Cavitation Committee (1996) and Gindroz et al. (1996). Using model-scale and large-scale inception measurements, Gowing and Shen (2001) have developed an empirical correction formula for the effect of nuclei on the inception of propeller tip vortex cavitation. A review on the effect of water quality in cavitation test facilities is given by the 23rd ITTC Specialist Committee on Water Quality and Cavitation (2002). Cavitation inception on propellers at MARIN is controlled by the application of leadingedge roughness and electrolysis (Kuiper, 1981; van Terwisga et al., 1999).

It is remarked that the inception of cavitation can be determined visually as well as acoustically. The growth and collapse of nuclei in the low pressure region generates noise which can be used to define inception. Acoustic inception of vortex cavitation has been used by, among others, Briançon-Marjollet and Merle (1996), and studied in detail by Choi and Ceccio (2007) and Chang and Ceccio (2011).

The inception of tip-vortex cavitation can be affected by the tip geometry (Platzer and Souders, 1979; Kuiper et al., 2006), injection of mixture of water and polymer in the tip vortex (Fruman and Aflalo, 1989; Chahine et al., 1993; Chang et al., 2011) or mounting a flexible thread on the tip of the propeller (Park et al., 2014).

\subsection{Vortex cavitation}

As there are many types of vortices in the flow around marine propellers, there are also many types of vortex cavities, see e.g. Arndt (2002) for a review and Franc and Michel (2004) for an introduction to vortex cavitation. Some of these vortex cavities are sketched in Figure 1.2. Developed vortex cavitation is discussed in detail in the following chapters distinguishing between the kinematics and the dynamics of a vortex cavity. These chapters include a short review of relevant literature. Therefore, the present section will only briefly discuss some general aspects of vortex cavitation as known from observations. The discussion includes sheet cavitation as that may also generate a vortex cavity. 


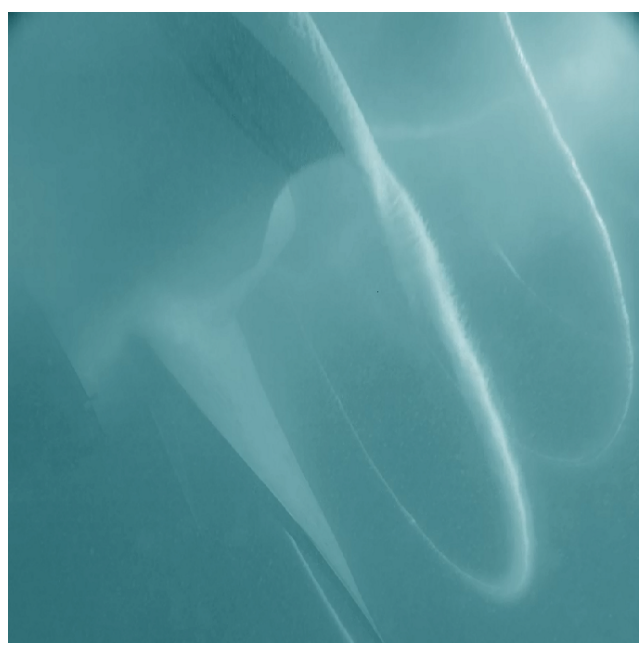

a) Single-screw bulk carrier

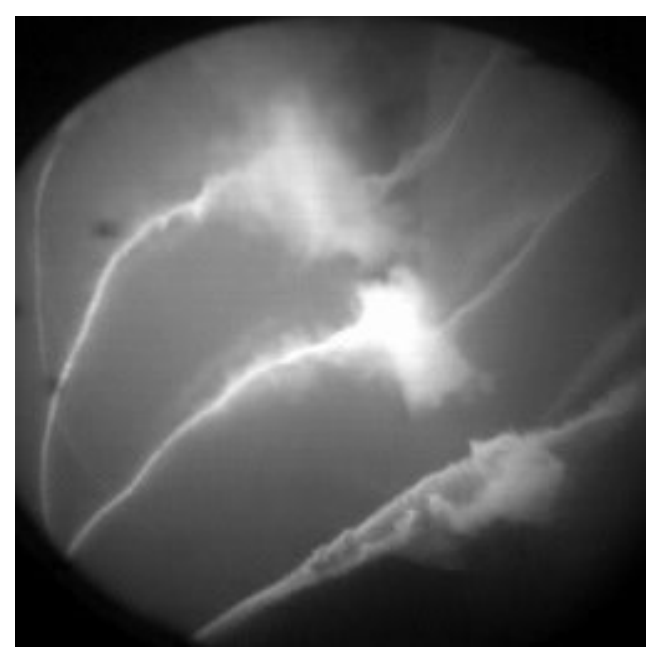

b) Single-screw container vessel

Figure 2.3: High-speed video images made by MARIN of full-scale cavitating tip-vortices. Image a) was obtained within the EU FP7 GRIP project (grant agreement 284905), image b) was obtained in the EU FP5 EROCAV project (grant agreement G3RDCT-2000-00268).

Cavitating vortices generated at the blade of marine propellers are persistent in the near field of the propeller in the absence of a collapsing sheet cavity or other vortex structures, see Figures 1.1 and 2.3a. Only far downstream of the propeller, a cooperative instability may occur as discussed in Section 2.2, and as shown in Figure 2.4. The vortex-cavity interface may, however, deviate from a cylindrical shape. It can have an elliptical cross-section or it may show the presence of nodes as discussed by, amongst others, Weitendorf (1976). The tip-vortex cavity of a foil in steady conditions can show oscillations, which can lead to a strong tonal noise (Maines and Arndt, 1997a). The same foil was also used by Pennings (2016) to generate a cavitating tip-vortex of which the perturbations were observed using high-speed video. These will be discussed in more detail in Chapter 4.

The most commonly observed cavitation on marine propellers is a combination of sheet cavitation and tip-vortex cavitation as shown in Figure 1.1. However, at the closure region of a sheet cavity, another vortex may form that is designated closure vortex (Bark and Bensow, 2013). In the cavity closure region, the sheet-cavity interface curves and becomes upstream directed. Due to the curvature of this interface, vorticity is generated. The upstream directed flow of the liquid next to the interface forms the re-entrant jet which, upon break-up of the sheet cavity, forms a vortex cavity. If the flow becomes more span wise directed, it can be referred to as a side-entrant jet (Foeth et al., 2008). The collapse of sheet cavitation by its re-entrant and side-entrant jet results into cloud cavitation, or more general secondary cavity structures (Bark and Bensow, 2013), containing a mixture of bubbles and vortices, as illustrated in Figure 1.2. It is remarked that cloud cavitation can also be generated by a sheet cavity through a so-called condensation shock (Ganesh et al., 2014).

Sheet cavitation, of which the chordwise extent increases towards the tip, generates a sideentrant jet. When the sheet cavity extent in radial direction is small and the chordwise and radial extent changes gradually in time, the side-entrant jet merges with the tip vortex forming a stable 


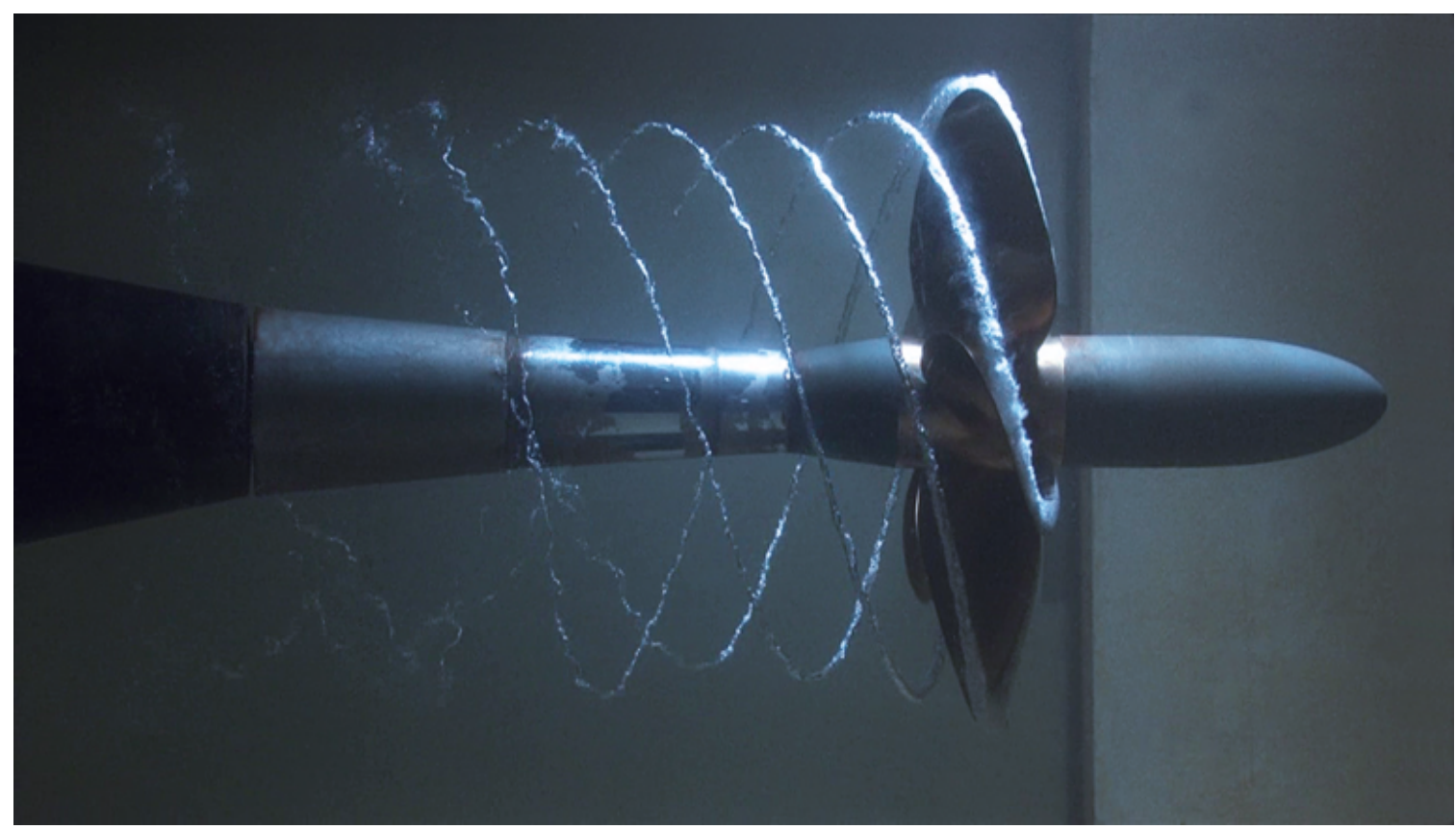

Figure 2.4: Photograph of cavitating helical tip vortices of a propeller in open water condition in cavitation tunnel of MARIN.

vortex cavity downstream of the blade, see Figure 1.1. However, when the extent of the sheet cavity changes very rapidly, such as on a propeller of a typical single-screw vessel, it leads to a collapse of the cavity, or, if present, of the cavity formed by the closure vortex.

A cavity collapse leads to a cloud of bubbles (and vortex cavities). If the collapse occurs at the tip and downstream of the blade, this cloud is intersecting the tip-vortex cavity, see Figure 2.3b. Unfortunately, this has been referred to as a bursting tip-vortex cavity by English (1979). The 'bursting' tip vortex cavity has been discussed in several papers. Oshima et al. (1986) showed that the collapse and rebounds of the cavitating vortex were related to the collapse of the sheet cavity on the blade. For a screen generated wake with large variation in velocity, the amplitudes of the pressure fluctuations at the higher-order blade rate tonals were decreasing with increasing thrust coefficient and were showing local maximum amplitudes for a certain cavitation number. Kuiper (2001) discusses that there is no relation between 'vortex cavity burst' and the 'burst' observed for non-cavitating leading-edge vortices as discussed in Section 2.2. Konno et al. (2002) mention that the sheet cavity on a blade was affected by pressure pulses emitted by the collapse of a cavitating vortex of a neighbouring blade. The sheet cavity then became unstable and its vapour volume was shed into the cavitating vortex. At the trailing edge of the sheet, a vortex cavity was formed which interacted with the tip-vortex cavity leading to a collapse and rebounds forming a cluster of bubbles. The tip-vortex cavity could be stabilised by increasing the propeller loading or by reducing the sheet cavity extent. Van Wijngaarden et al. (2005) discuss various mechanisms for the collapse of a cavitating vortex, such as the interaction with other vortices and the interaction with the sheet cavity and its side-entrant jet, which forms a vortex cavity if it is oriented along the propeller leading edge. The presence of a secondary vortex originating from the sheet was also found by Lücke (2006) to be responsible for the collapse of the cavitating tip vortex. Full-scale cavitation observations of this interaction between sheet and vortex cavitation are given by van Wijngaarden et al. (2005) and 


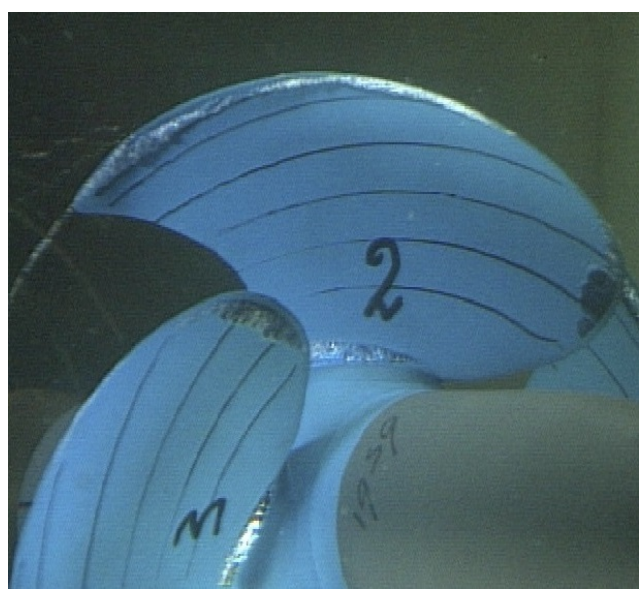

a) Cavitating tip vortex and leading-edge vortex

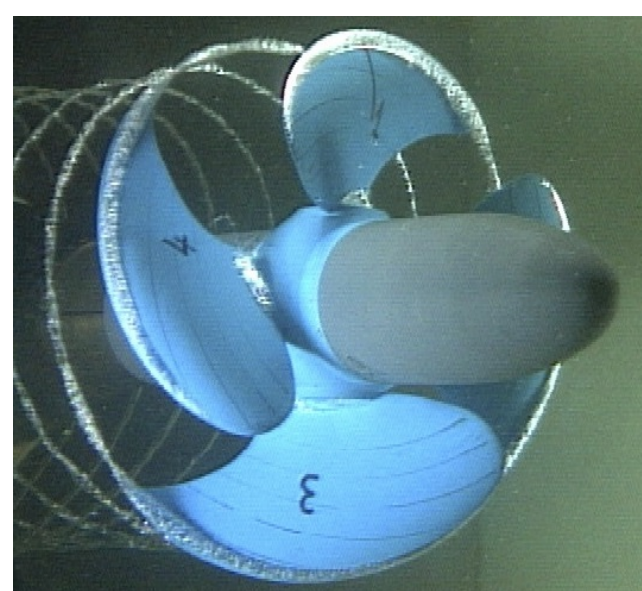

b) Sheet-cavity closure vortex, merged with tip vortex cavity

Figure 2.5: Images of cavitation pattern on a skewed propeller blade in open water conditions in cavitation tunnel of MARIN.

Carlton and Fitzsimmons (2006). Based on all these discussions and analyses of model-scale and full-scale videos, we prefer to denote this cavity pattern as a collapse of the closure-vortex cavity instead of a bursting tip-vortex cavity. The closure-vortex cavity is discussed in more detail in Section 6.4.

Propellers with weakly loaded tips and high skew may feature a cavitating leading-edge vortex as observed in Figure 2.5. Although it can clearly be observed on propellers in open water conditions (and on highly swept wings), it can be difficult to distinguish from sheet cavitation with a closure-vortex cavity, especially for propellers operating in a wake field. This aspect is discussed in more detail in Section 6.4. Cavitating leading-edge vortices on delta wings can have a cylindrical cavity structure as shown by Ganesh et al. (2014) but the vorticity sheet feeding the vortex may also cavitate as shown by Brandner and Walker (2003).

There are situations in which several vortices are generated at the tip of the propeller. An example of two co-rotating vortices is the combination of a leading-edge vortex cavity and a tip-vortex cavity. Such vortices will ultimately merge, although the interaction between the two may lead to the break-up of the cavity structure of one of them. Another example of two co-rotating vortices is the combination of a closure-vortex cavity and a tip-vortex cavity. Two counter-rotating vortices can occur on controllable-pitch propellers with skew and tipunloading when operating at reduced pitch in order to obtain low ship speed (Okamura et al., 1994; Carlton, 2015). For such propellers, a leading-edge vortex arises on the face of the blade tip. Overall, the propeller is still producing thrust, so a tip vortex is also generated on the back of the propeller. The interaction of these counter-rotating vortex cavities appears to be similar to the interaction described in terms of elliptical and cooperative instabilities for non-cavitating vortices, see Section 2.2. The weaker of the two vortices will break-up and may form cavitating ring vortices around the stronger vortex, see Figure 2.6a. Cavitating vortex structures may also appear in the shear layer between the two vortices, as shown in Figure 2.6b. 


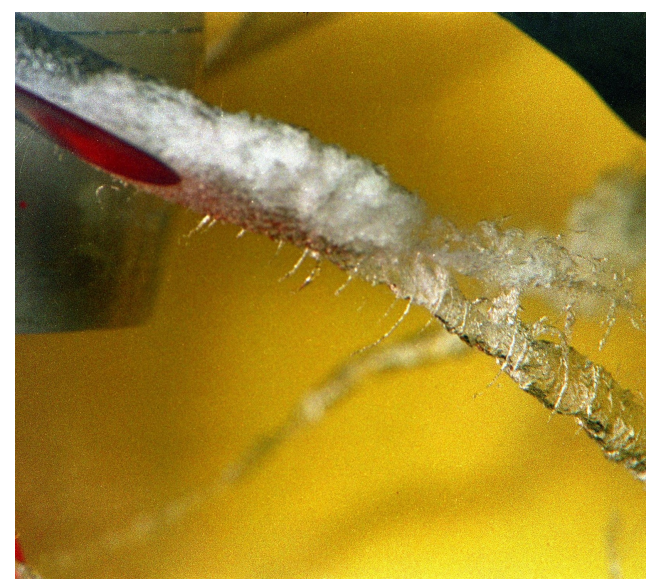

a) Cavitating ring vortices

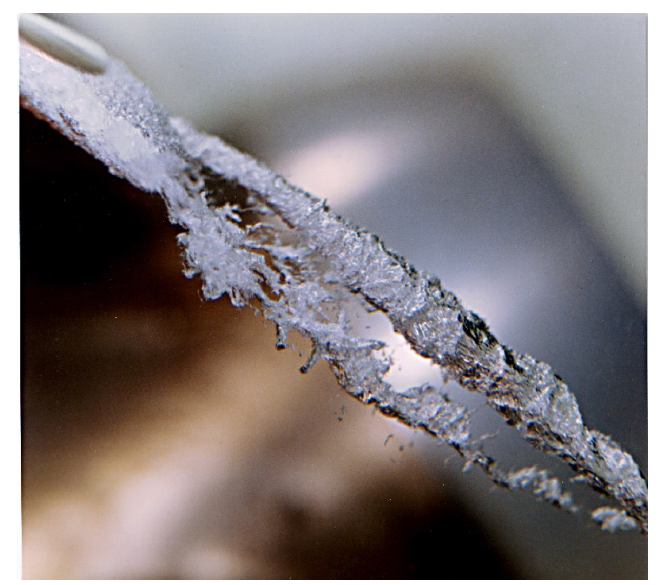

b) Cavitating shear layer

Figure 2.6: Images of cavitation patterns due to vortex-vortex interaction in cavitation tunnel of MARIN.

\subsection{Hull-pressure fluctuations}

Cavitation tests at MARIN on ship propellers are usually performed in the Depressurized Wave Basin (DWB). The DWB is $240 \mathrm{~m}$ long, $18 \mathrm{~m}$ wide, and $8 \mathrm{~m}$ deep, and the basin pressure can be reduced to $2500 \mathrm{~Pa}$ to achieve the correct cavitation number. Most of the cavitation tests are performed with the large towing carriage, Figure $2.7 \mathrm{a}$, but for the underwater radiated noise measurements use is made of a silent towing carriage, Figure $2.7 \mathrm{~b}$. The low-frequency hull-excitation force induced by the cavitating propeller is predicted by measuring the hullpressure fluctuations (HPF) utilizing approximately 20 pressure transducers in the hull above the propeller, see Figure 2.8. These pressure transducers are distributed over an area of length and width approximately equal to the propeller diameter. The aspects affecting HPF measurements have been reviewed by van Wijngaarden (2011). This section presents only a brief review with focus on procedures and results for the DWB and for broadband HPF.

An example of the spectrum of HPF for a cavitating propeller is given in Figure 1.3. This spectrum also contains contributions from other sources than cavitation which can be determined by measurements with a non-cavitating propeller. Such a spectrum is composed of broadband contributions due to the electronic noise of the transducer and conditioner, and due to the self-noise from the turbulent boundary layer on the hull. Both are considered as background noise levels as they are not related to the propeller. The periodic passage of the propeller blade below the transducers leads to blade rate tonals in the spectrum, which are tonals at harmonics of the blade passage frequency (BPF). These tonals are due to the thickness and loading of the propeller blade (Denny, 1967; Breslin, 1970; Breslin et al., 1982; Lafeber et al., 2009). The tonal is very pronounced at the BPF but its amplitude decreases rapidly at the 2nd and higher harmonics of the BPF. The amplitudes of the higher harmonics depend on the number of blades and on the propeller-hull clearance.

In 1969 at the $12^{\text {th }}$ ITTC, Takahashi and Ueda reported that cavitation on the propeller increases the HPF significantly (Takahashi and Ueda, 1969). This led to the measurement of HPF in cavitation tunnels, also in the Cavitation Tunnel of MARIN (van Manen, 1972). Van 


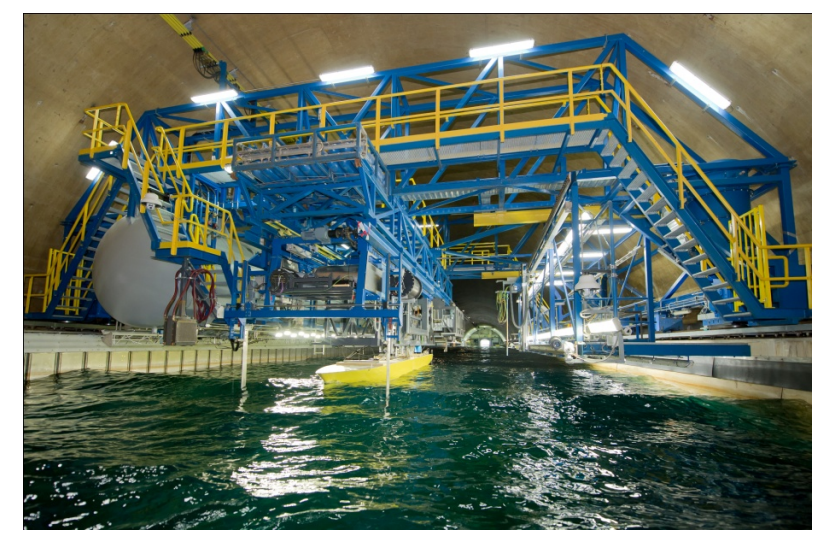

a) Large towing carriage

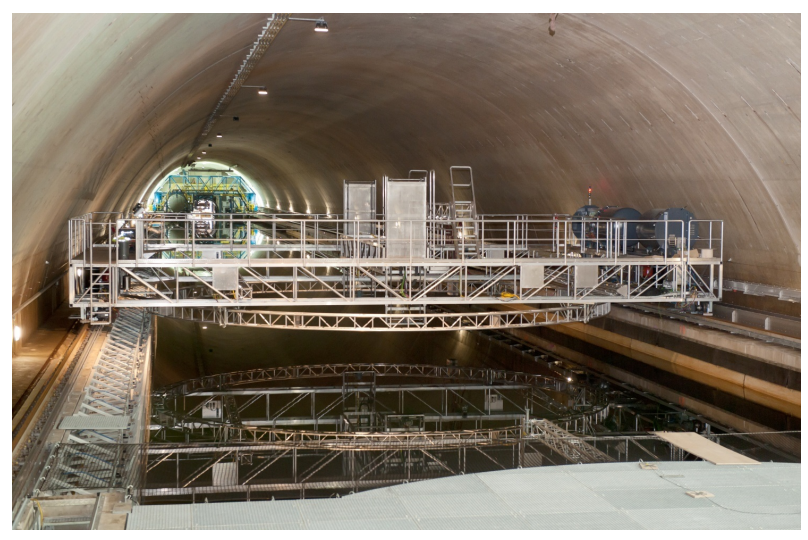

b) Silent towing carriage

Figure 2.7: Photographs of the towing carriages in the DWB.

Oossanen and van der Kooij (1973) have measured in that tunnel the HPF for numerous Bseries propellers operating in the wake of a single-screw vessel. In the 1970s, the DWB came into operation (Kuiper, 1974). The DWB was at that time known as the Depressurized Towing Tank, sometimes also referred to as Vacuum Tank. The procedure for the HPF measurements and a comparison of model-test results with full-scale data has been described by van der Kooij (1979). Experimental and numerical procedures to predict HPF were described by the 23rd ITTC Specialist Committee on Cavitation Induced Pressures (2002). Predictions of cavitation extents and cavitation-induced pressure fluctuations by various computational methods were compared to experimental data in Vaz et al. (2015).

Many aspects are of importance when performing measurements of HPF on model-scale configurations:

- An important aspect is the simulation of the correct wake field in which the propeller operates. In model tests, this wake field is different from that at full scale due to the difference in Reynolds number. In the DWB, most often use is made of a relatively large-size geometrically-scaled ship model. However, with help of CFD, it is nowadays also possible to design the aft geometry of a single-screw ship such that in the model test the full-scale wake field is obtained, thereby eliminating this scale effect (Schuiling et al., 2011; van Wijngaarden, 2011). Such a procedure has not yet been applied for twin-screw vessels. However, the scale effect on the wake of a twin-screw vessel is much smaller than that of a single-screw vessel.

- The propeller operating point in terms of advance ratio $J$ is normally defined such that a prescribed mean thrust-coefficient is obtained. As the wake-deficit in the model test is normally higher than at full scale, a small increase of the advance ratio is required. Instead of having identical mean thrust-coefficient at model scale and full scale, one can also adjust the advance ratio such that the local blade thrust-coefficient is identical in the sector of the propeller disc where cavitation is present (Holtrop and Kuiper, 2003). This is especially relevant for large and slender single-screw vessels.

- The non-dimensional pressure margin against cavitation is given by the cavitation number. Due to the variation in vertical direction of the hydrostatic pressure over the propeller disc, the cavitation number also varies in the propeller disc. The pressure in the model-test facility is usually selected such that the cavitation number at a certain position in the propeller 


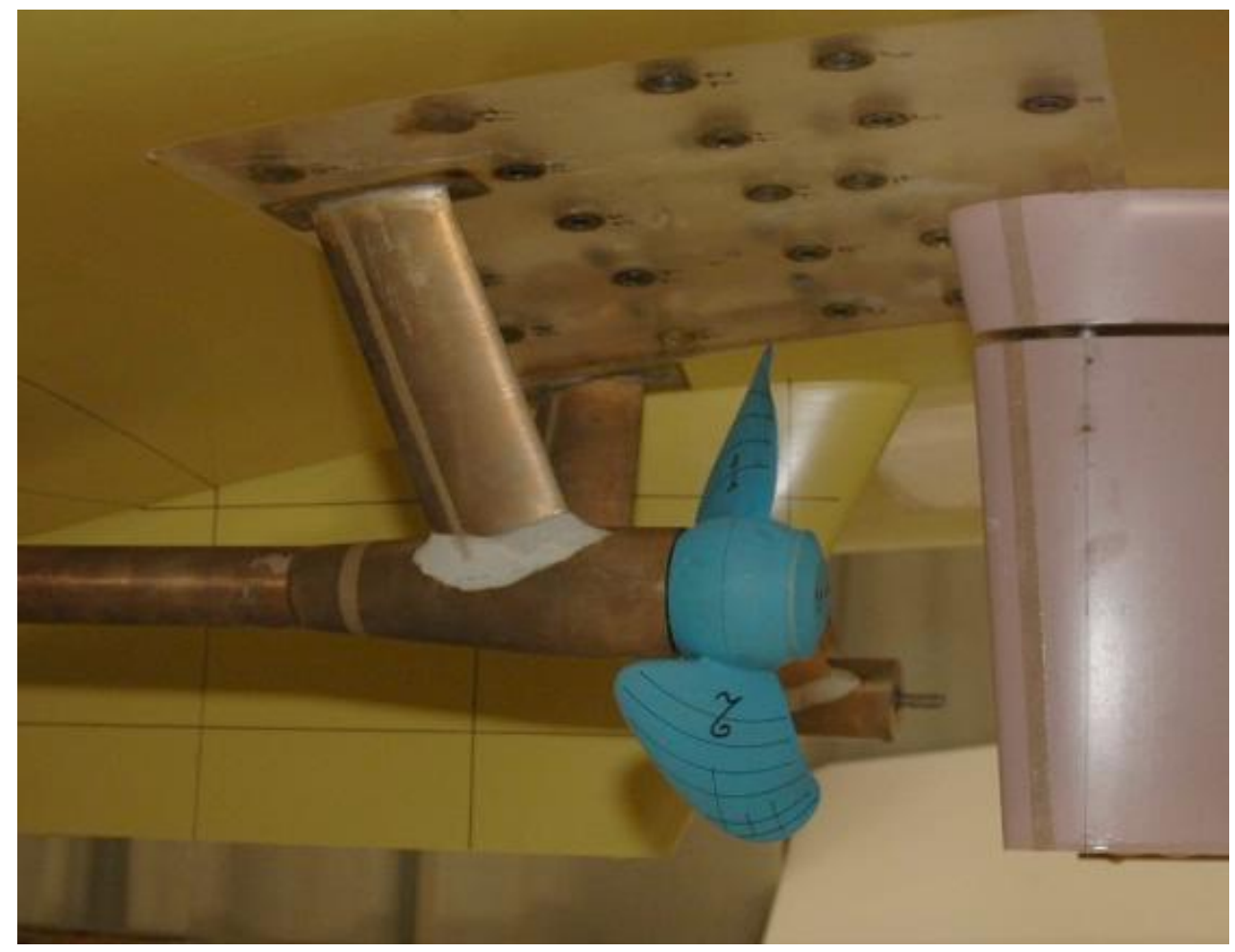

Figure 2.8: Example of aft part of ship hull equipped with pressure transducers to measure cavitation-induced hull excitation in the DWB.

disc equals the cavitation number at the corresponding position for the ship. Only when the Froude number is identical for model and ship, with Froude number based on propeller diameter and propeller tip speed, the variation of the cavitation number over the entire propeller disc is identical for model test and ship. This situation holds for the DWB.

- As discussed in Section 2.3, the inception of cavitation requires nuclei of relevant size and sufficient concentration. The measurement and control of nuclei are both difficult aspects in testing propeller cavitation. Very often, only the concentration of total dissolved gas is measured and controlled. The topic is discussed in more detail by the 23rd ITTC Specialist Committee on Water Quality and Cavitation (2002). At MARIN, the nuclei are provided by the application of leading-edge roughness and electrolysis (Kuiper, 1981). A nuclei measurement method based on Interferometric Particle Imaging (IPI) is in development (Birvalski and van Rijsbergen, 2018).

- The compressibility of the interior of the cavity depends on the non-condensable gas content of the water. With increasing gas content, a cushioning of the collapse of cavities is observed thereby reducing the amplitudes of the HPF. The effect of gas content on results of cavitation tests has been reported by several researchers (Lövik, 1981; Bark, 1985; Johannsen, 1998; Bosschers and van Wijngaarden, 2012), but as the dissolved gas content also has an effect on the nuclei, and thereby cavitation inception, the interpretation of the effect of dissolved gas on hull pressure measurements can be difficult.

- The scattering of the cavitation-induced pressure waves by the ship hull and free surface affects the pressure amplitudes on the hull. The effect is characterised by the Helmholtz number which is different for model test and full scale. However, directly above the pro- 
peller and at the low frequencies at which the HPF are measured, the scale effect is usually negligible. A simple model to analyse this scattering effect is provided in Section 5.5.1.

- A possible disturbing factor in the measurement of cavitation-induced HPF is the hull pressure caused by the vibration of the ship hull. The topic can be of importance for full scale as well as for the model test. A method to correct for the effect of model vibrations on hull pressures in the DWB is described by van Wijngaarden (2011).

- The scaling of the tonals of the HPF is through the dynamic pressure only. Hence, the hull pressures scale with $\rho n^{2} D^{2}$ with $\rho$ the density of the fluid, $n$ the shaft rotation rate and $D$ the propeller diameter. The frequencies of the blade rate tonals scale with $n$. The scaling of broadband noise is discussed in more detail in Section 5.4.

The HPF are often only analysed with respect to the blade rate tonals, typically up to the fourth harmonic. Comparison of model-test results obtained in the DWB with full-scale data are provided by, among others, van der Kooij (1979); Ligtelijn et al. (1992); Hämäläinen and van Heerd (1998); Ligtelijn et al. (2004); Hämäläinen et al. (2005) and van Wijngaarden (2011). In general, the amplitude of the first harmonic shows good agreement provided the appropriate wake field is applied for fast single-screw vessels, as discussed above. The higher harmonics are often somewhat overpredicted. In Johannsen et al. (2012), it is shown that, for a large slender container vessel, the hull-pressure fluctuations at the first four harmonics obtained in the DWB are in good agreement with data measured in the large cavitation tunnel HYKAT of HSVA ${ }^{1}$ as well as at full scale.

The broadband part of the spectrum is usually only relevant from a ship vibration point of view if the blade-rate tonals have small amplitudes. This occurs when the propeller blade shows only a small amount of sheet cavitation. Such propellers are usually propellers of twin-screw vessels with stringent noise and vibration requirements.

One of the first examples of vibration hindrance due to broadband hull excitation is the cruise vessel QE2, as reported by Brubakk and Smogeli (1988). The broadband HPF were found to increase downstream of the propeller with a maximum value of about $0.7 \mathrm{kPa}$ and were found to be related to the cavitating tip vortex. The vibration problems were solved with a new propeller design in which the tip vortex was considerably reduced in strength by unloading the blade tip. Since then, considerable effort has been put in further reduction of the HPF levels by improving hull and propeller design. For example, the spectrum of hull pressures of a ferry that satisfies the highest comfort class requirements shows amplitudes at the 2nd and higher blade-rate frequency (and broadband part) that are smaller than $0.05 \mathrm{kPa}$ (Hämäläinen et al., 2005). The spectrum shows a small amount of broadband noise in the frequency range between 35 and $70 \mathrm{~Hz}$ caused by a thin cavitating tip vortex.

Broadband spectra of HPF as measured on model scale for twin-screw vessels were presented by Friesch (1998); Fréchou et al. (2000); Kuiper (2001); van Terwisga et al. (2007) and Bosschers (2009a) and the topic is briefly addressed by the 23rd ITTC Specialist Committee on Cavitation Induced Pressures (2002). Consensus is that broadband HPF is related to the cavitating tip vortex and that variability of the cavitation dynamics between blade passages is of importance, though the physical mechanisms are still not fully understood. Pennings et al. (2016) show that the frequency of the oscillations of the volume of a propeller tip-vortex ca-

\footnotetext{
${ }^{1}$ Hamburgische Schiffbau Versuchanstalt / the Hamburg Ship Model Basin, Germany
} 
vity, determined by high-speed video, is identical to the most dominant frequency in the noise spectrum. The latter was determined by an auto-correlation analysis. The tests were performed using a screen generated wake in a cavitation tunnel.

The relation between broadband noise and tip-vortex cavitation is illustrated by the CFD results of Fujiyama and Nakashima (2017). They compute the HPF for the single-screw vessel Seiun-Maru for two different propeller geometries. Computational results, obtained at a modeltest Reynolds number and corrected by a scaling rule for URN, showed good agreement with full-scale measurement data with respect to the broadband part. However, computations at the full-scale Reynolds number, in which sheet cavitation was predicted but the tip-vortex cavity was not captured, showed a significant underprediction of the levels of the broadband noise, especially for the propeller of conventional planform. These results show that, for this ship, the broadband hump of the spectrum, centred around $50 \mathrm{~Hz}$, is mainly due to tip-vortex cavitation.

Very large broadband noise levels are known to occur when a controllable-pitch propeller is operating at reduced pitch setting in order to achieve low ship speed. These broadband noise levels are due to the interaction of two counter-rotating vortices as discussed in Section 2.4. Okamura et al. (1994) present the broadband noise spectrum as measured in a cavitation tunnel, Berghult (2000) has developed an empirical formulation for the broadband noise levels as function of pitch offset and blade area ratio, and Carlton (2015) shows the interacting cavitating vortices and resulting vibration level at full-scale.

Whereas the HPF are usually measured up to a frequency of 100 to $200 \mathrm{~Hz}$ at full scale, they have also been measured up to 20 to $40 \mathrm{kHz}$, which is the frequency range for URN measurements, see e.g. Bark (1985). Measurements of HPF for this frequency range are usually presented in $1 / 3$ octave band levels in order to focus on the broadband character of the spectrum. The results of such measurements can be used to determine the URN source levels of the cavitating propeller (Newman and Abrahamsen, 2007; Foeth and Bosschers, 2016).

The comfort level of a ship is not expressed in terms of its HPF, but by its vibration and noise levels. Most class rules are based on the ISO procedure 6954 for the analysis of vibration levels. The procedure released in 1984 poses a limit on the maximum repetitive value of the vibrations in the range up to $100 \mathrm{~Hz}$. This maximum repetitive value can also be obtained from the maximum value in a spectrum when an appropriate crest factor is applied. The maximum value is usually found at frequencies that correspond to blade rate harmonics. The 1984 procedure was updated in 2000. This new procedure poses a limit on the overall frequency-weighted rms value of the vibrations in the range between $1 \mathrm{~Hz}$ and $80 \mathrm{~Hz}$. Due to this update, the broadband content of the HPF has also become more important.

\subsection{Underwater radiated noise}

In the 1970s and 1980s, the underwater radiated noise (URN) of cavitating ship propellers has received considerable attention due its importance for the signature of naval vessels. However, after this period, the research related to this signature has shifted towards delaying cavitation inception as much as possible. The procedure for the URN measurements in the DWB and some typical results have been reported by van der Kooij and de Bruijn (1984). The measurement setup consists of two hydrophones mounted on a pole in the basin with the ship model sailing over 
the hydrophones.

All discussed aspects of the measurement of hull-pressure fluctuations are also relevant for the measurements of URN. In addition, the following aspects are of importance:

- For the model test of URN, the background noise is much more of an issue than for HPF. The background noise in the DWB consists of the noise from the towing carriage, the propeller drive train, and the electronic noise of the measurement equipment. The URN measurements during sea trials also show a contribution due to machinery noise, especially at low frequencies. This may mask cavitation noise.

- Due to the larger distance between propeller and measurement transducer, usually a hydrophone, the correction for propagation loss can be an issue when the reverberation of the facility affects the noise measurement. The effect can be investigated by transfer function measurements. In the DWB, it is especially the reflection of sound at the free-surface, the so-called Lloyd's mirror effect, that needs to be taken into account when determining the acoustic source level of the cavitating propeller at low frequencies. The comparison of source levels from model-tests with results of sea trials also requires correcting the sea trial data for the Lloyd's mirror effect. This correction needs to be applied to both data sets due to the difference in Helmholtz number between model test sea trial. Sea trials performed in acoustic shallow water require more sophisticated correction procedures for propagation loss than the formula for spherical spreading loss used for deep-water measurements.

- The scaling of the URN spectra is somewhat more complicated than the scaling of HPF data. Two formulations for scaling of noise are being used by model basins, but they lead to different noise levels. This aspect is discussed in more detail in Chapter 5.

The relevance of these aspects for the DWB in its present status have been presented by Bosschers et al. (2013); Lafeber et al. (2015); Lloyd et al. (2018). More detailed reviews on URN measurements are provided by the 27th ITTC Specialist Committee on Hydrodynamic Noise (2012) and the 28th ITTC Specalist Committee on Hydrodynamic Noise (2017) which have also developed guidelines for URN measurements. Procedures for full-scale noise measurements are described by ISO 17208-1, while the class societies BV, DNV-GL, LR and RINA also prescribe the measurement procedure in their URN class rules.

Validation studies of URN measurements in the DWB have been reported by van der Kooij and de Bruijn (1984); Ligtelijn et al. (1992); Nethercote et al. (1992); Lafeber and Bosschers (2016) and Lloyd et al. (2018). Overall, the agreement between results of model tests and results of sea trials is within $5 \mathrm{~dB}$, with maximum differences up to $10 \mathrm{~dB}$. This is considered to be within the combined measurement uncertainty of model test and sea trial (28th ITTC Specalist Committee on Hydrodynamic Noise, 2017). Similar results have been obtained for URN measurements in cavitation tunnels (Seol et al., 2015; Aktas et al., 2016; Tani et al., 2016). Unfortunately, observations of cavitation for sea trials are often missing or of insufficient quality due to poor visibility. This hampers the identification of causes of differences between model-test results and full-scale data.

Compared to full scale, the inception of vortex cavitation is delayed in the model test due to the lower Reynolds number, see also Section 2.3. This can cause significant differences in HPF and URN levels for propellers with tip-vortex cavitation in case the model test is performed at identical cavitation number as for the ship (28th ITTC Specalist Committee on Hydrodynamic 
Noise, 2017). This aspect is discussed in more detail in Chapter 8, in which a novel procedure is proposed to perform such model tests.

\subsection{Conclusions}

The characteristics of vortex cavitation have been described by a literature review on physical aspects of non-cavitating vortices, of cavitating vortices, and of the inception of a vortex cavity. Literature on the kinematics and dynamics of vortex cavitation is described in Chapters 3 and 4, respectively. A review of aspects involved in model tests with the aim to predict HPF and URN has also been provided in the present chapter.

Knowledge of the instability mechanisms of non-cavitating vortices is well-developed. These instabilities can be explained by, sometimes called, Kelvin waves that propagate on the surface of the viscous core. Dedicated computational methods have been developed to study these mechanisms. Kelvin waves also exist on the interface of a vortex cavity which will be discussed in detail in Chapter 4.

The roll-up of a vortex sheet into a tip vortex of a wing has been described by various models. The roll-up of vorticity into the tip vortex of a propeller behaves similarly, but these models cannot directly be applied to propellers. However, most likely such a roll-up model is not required to predict the size of a tip-vortex cavity close to the propeller tip.

The inception of vortex cavitation depends on the tensile strength of the fluid and is known to be strongly affected by the Reynolds number. The tensile strength depends on the size and concentration of nuclei, and on the path and dynamics of the nuclei entering the vortex core. Therefore, the tensile strength is difficult to determine. Usually, it is only the gas content in the test-facility that is monitored and controlled. The effect of Reynolds number on the inception of vortex cavitation is well-known, it does have a theoretical basis, but the scaling parameter involved has often been determined empirically.

The fundamental knowledge on developed vortex cavitation is rather limited, as shown in more detail in the chapters to follow, because most research has been related to the inception of vortex cavitation. Often, due to a lack of detailed quantitative information, only the appearance of the cavity has been described. The increase in quantitative information through CFD and detailed flow field measurements leads to further development of the nomenclature used to describe cavitation. In that respect, the vortex formation by sheet cavitation deserves attention. In the present thesis, the term closure-vortex cavity is used to denote the vortex cavity generated by the sheet cavity. This is described in more detail in Section 6.4. It is hypothesized here that the collapse of the closure-vortex cavity describes the phenomenon of the so-called 'bursting tip-vortex cavity'.

A brief description of the various aspects involved in measuring HPF and URN at model scale is provided. Broadband HPF is known to be related to tip-vortex cavitation, but details of mechanisms involved have not been studied. The variability of the cavitation from one blade passage to the next has been mentioned to be of importance. A review of literature on URN by vortex cavitation is not presented here due to the focus in the present study on broadband HPF. Chapters 6 through 8 refer to some literature on this topic. 
Page intentionally left blank 


\section{Chapter 3}

\section{Kinematics}

This chapter discusses models that describe the kinematics of a cavitating vortex with the focus on the azimuthal velocity distribution ${ }^{1}$. An analytical solution for a columnar cavitating vortex is presented, which can be interpreted as a cavitating Lamb-Oseen vortex. This vortex model is extended with a semi-empirical formulation to take vorticity roll-up into account. The resulting model is fitted to available experimental data for cavitating as well as non-cavitating flow. The results are used in Chapter 4 to analyse the dynamics of vortex cavities and in Chapters 7 and 8 to develop prediction methods for the radiated noise of vortex cavities.

\subsection{Introduction}

Cavitating vortices can be observed on lifting bodies at locations where the pressure in the vortex is lower than the vapour pressure, provided that sufficient nuclei are present. On a ship propeller, tip vortex cavities are often present. Vortex cavitation can also be observed in hydraulic machinery and in flows with concentrated vorticity such as turbulent jets, wakes and shear layers. A review of vortex cavitation is given by Arndt (2002). The dynamic behaviour of cavitation can lead to noise and vibration issues as well as to erosion, which is why for ship propellers cavitation needs to be controlled and considered during the design process.

Research on cavitation has been focused on bubble and sheet cavitation rather than on vortex cavitation as the former two occur more often and generally lead to larger detrimental effects. Bubble and sheet cavitation are dominated by inertia and the effect of viscosity is small. However, in the core of a vortex, viscous effects dominate the flow and determine the minimum pressure in that region. The low pressure drives cavitation inception, which therefore depends strongly on Reynolds number (McCormick, 1962; Fruman et al., 1995; Arndt, 2002; Shen et al., 2009). Research on cavitating vortices has often been focused on inception, which also includes the growth and collapse of natural nuclei in the vortex core (Hsiao and Chahine, 2005; Choi and

\footnotetext{
${ }^{1}$ This chapter has been published as: Bosschers, J. (2018). An analytical and semi-empirical model for the viscous flow around a vortex cavity. International Journal of Multiphase Flow, 105:122-133. The boundary condition at the vortex-cavity interface was first published in: Bosschers, J., Janssen, A.A., and Hoeijmakers, H.W.M. (2008). Similarity solutions for viscous cavitating vortex cores. Journal of Hydrodynamics, Vol. 20, No. 6.
} 
Ceccio, 2007).

Much less research has been devoted to developed vortex cavitation. The velocity field surrounding a developed vortex cavity has been measured by Falçao De Campos (1992) and by van Rijsbergen and Kuiper (1997) using Laser Doppler Velocimetry. The results showed that away from the cavity the velocity distribution of the cavitating vortex is identical to that of the non-cavitating vortex. Near the cavity interface, the velocity distribution could not be accurately measured due to the unsteadiness of the location of the cavity. Detailed flow measurements in cavitation tunnels using Particle Image Velocimetry around a wing-tip vortex cavity were performed by Pennings et al. (2015b) and Dreyer (2015). These measurements showed that near the cavity interface the azimuthal velocity decreases compared to the velocity of the noncavitating vortex, leading to a maximum in azimuthal velocity at some distance from the edge of the cavity. The overall velocity distribution resembles the analytical formulation for a cavitating vortex given by Bosschers (2009c).

A model for the azimuthal velocity can be used to obtain a relation between the size of a vortex cavity and the cavitation number. Kuiper (1981) and van Rijsbergen and Kuiper (1997) investigated a semi-empirical model, while a heuristic modification of the Lamb-Oseen vortex model was investigated by Arakeri et al. (1988) and Choi and Ceccio (2007). A computational analysis utilizing simplified Navier-Stokes equations representing a similarity solution for a cavitating vortex-for which the circulation and cavity size grow parabolically in streamwise direction-has been presented by Bosschers et al. (2008a). However, such a vortex cavity has not been observed on marine propellers. A cavitating variant of the Lamb-Oseen vortex has been developed by Bosschers (2009c) but the model has only been analysed from a theoretical point of view. The Lamb-Oseen vortex is an analytical solution of the Navier-Stokes equations for axisymmetric flow describing the azimuthal velocity distribution (Lamb, 1932).

There is a lack of knowledge on the structure of the flow around developed vortex cavities. Especially, the effect of viscosity on a developed vortex cavity is not well-established. In the present study, this topic is investigated by further elaborating on the analytical formulation for the cavitating Lamb-Oseen vortex presented by Bosschers (2009c). The model is extended with an empirical relation to account for vorticity roll-up, and is also extended to describe cavitation using an approach similar as the one for the cavitating Lamb-Oseen vortex. The resulting velocity distribution is compared with and fitted to experimental data of Pennings et al. (2015b) for non-cavitating and cavitating vortices. Finally, a new scaling relation between cavity size and cavitation number is proposed that collapses data for a range of vortex strengths and viscous core sizes.

\subsection{An analytical solution for the flow around a vortex cavity}

\subsubsection{Derivation}

Consider the continuity equation and the Navier-Stokes equations for incompressible, isothermal, axisymmetric and unsteady flow in the cylindrical coordinate system $(r, \theta, z)$ with velocity components $(u, v, w)$ in radial, azimuthal and axial direction, respectively. Pressure is denoted by $p$, density by $\rho$, dynamic viscosity by $\mu$ and time by $t$. The flow is considered axisym- 
metric, so derivatives in azimuthal direction are zero. Furthermore, the velocity component in axial direction $w$ is constant and the derivatives in axial direction are also considered zero. The governing equations are then given by

$$
\begin{gathered}
\frac{1}{r} \frac{\partial}{\partial r}(u r)=0 \\
\frac{\partial u}{\partial t}+u \frac{\partial u}{\partial r}-\frac{v^{2}}{r}=-\frac{1}{\rho} \frac{\partial p}{\partial r}+\frac{\mu}{\rho} \frac{\partial}{\partial r}\left[\frac{1}{r} \frac{\partial(u r)}{\partial r}\right] \\
\frac{\partial v}{\partial t}+u \frac{\partial v}{\partial r}+\frac{u v}{r}=\frac{\mu}{\rho} \frac{\partial}{\partial r}\left[\frac{1}{r} \frac{\partial(v r)}{\partial r}\right]
\end{gathered}
$$

corresponding to the continuity equation and the momentum equations in radial and azimuthal direction. From Eq. (3.1) it is clear that the diffusion term in Eq. (3.2) is zero. The momentum equations can be further simplified by assuming that the radial velocity component $u$ and its gradients are an order of magnitude smaller than the azimuthal velocity $v$. Thus, Eqs. (3.2) and (3.3) become

$$
\begin{aligned}
& \frac{v^{2}}{r}=\frac{1}{\rho} \frac{\partial p}{\partial r} \\
& \frac{\partial v}{\partial t}=\frac{\mu}{\rho} \frac{\partial}{\partial r}\left[\frac{1}{r} \frac{\partial(v r)}{\partial r}\right],
\end{aligned}
$$

from which it is observed that the partial differential equations for the radial and azimuthal velocity are decoupled and that the pressure only depends on the azimuthal velocity.

The continuity equation can directly be integrated leading to

$$
u(r, t)=\frac{c_{u}}{r},
$$

with $c_{u}$ a constant that is determined by the boundary conditions. The boundary condition at $r \rightarrow \infty$ and at the centre of the non-cavitating vortex is that the radial velocity equals zero. It then follows that $c_{u}=0$ and the radial velocity $u(r, t)$ is zero throughout the flow field. Equations (3.4) and (3.5) are then an exact representation of the flow.

For $r \rightarrow \infty$, the azimuthal velocity is described by a potential flow vortex with circulation $\Gamma_{\infty}$

$$
v(r \rightarrow \infty, t)=\Gamma_{\infty} / 2 \pi r .
$$

The azimuthal velocity should be zero at $r=0$ and the solution is the Lamb-Oseen vortex (Lamb, 1932),

$$
v(r, t)=\frac{\Gamma_{\infty}}{2 \pi r}\left\{1-\exp \left[-\frac{r^{2}}{4 v_{l} t}\right]\right\},
$$

where $v_{l}=\mu / \rho$ denotes the kinematic viscosity of the liquid. For small $r$, solid-body rotation occurs, resulting in

$$
v(r \rightarrow 0, t)=\frac{\Gamma_{\infty}}{2 \pi} \frac{r}{4 v_{l} t}+\mathscr{O}\left(r^{3}\right) .
$$

Instead of using the Navier-Stokes equations for unsteady flow, the Lamb-Oseen vortex model can also be derived from the steady Navier-Stokes equations by assuming that all vortex-induced 
velocity components are small with respect to the free-stream velocity (Newman, 1959; Batchelor, 1964). The distribution of the azimuthal velocity is identical to Eq. (3.8) but for the transformation $t=z / W_{\infty}$, with $W_{\infty}$ the free-stream axial velocity.

The boundary conditions for a cavitating vortex are given by the jump relations for mass and momentum across the vapour-liquid interface (Bosschers et al., 2008a). These jump relations are obtained by applying the Reynolds transport theorem for conservation of mass and momentum to the total volume of fluid and vapour with the boundary of the interface moving with velocity vector $\mathbf{u}_{\mathbf{s}}$. The equations are given in vector notation by

$$
\begin{gathered}
\llbracket \rho\left(\mathbf{u}-\mathbf{u}_{s}\right) \cdot \mathbf{n} \rrbracket=0 \\
\llbracket \rho \mathbf{u}\left[\left(\mathbf{u}-\mathbf{u}_{\mathbf{s}}\right) \cdot \mathbf{n}\right]-\bar{\tau} \cdot \mathbf{n} \rrbracket=T\left(\kappa_{z}+\kappa_{\theta}\right) \mathbf{n},
\end{gathered}
$$

where $\mathbf{n}$ denotes the unit normal vector at the interface, $\bar{\tau}$ the stress tensor, $\llbracket . \rrbracket$ denotes the jump across the vapour-liquid interface, $T$ denotes the surface tension and $\kappa_{z}$ and $\kappa_{\theta}$ correspond to the interface curvature in axial and azimuthal direction, respectively. For the present vortex the curvatures correspond to $\kappa_{z}=0$ and $\kappa_{\theta}=1 / r_{c}$ with $r_{c}$ the radius of the cavity interface. The stress tensor for incompressible flow is given by

$$
\bar{\tau}=-p \mathbf{I}+\mu\left[\nabla \mathbf{u}+(\nabla \mathbf{u})^{T}\right] .
$$

The density and dynamic viscosity of water vapour at a temperature of $20^{\circ} \mathrm{C}$ are $\rho_{v}=$ $0.017 \mathrm{~kg} \mathrm{~m}^{-3}$ and $\mu_{v}=9.7 \times 10^{-6} \mathrm{Pas}$, respectively, while the corresponding values for liquid water are $\rho_{l}=998.6 \mathrm{~kg} \mathrm{~m}^{-3}$ and $\mu_{l}=1.0 \times 10^{-3} \mathrm{Pas}^{2}$. The density ratio of vapour to liquid is then given by $\rho_{v} / \rho_{l}=1.7 \times 10^{-5}$. Substitution of this ratio into Eq. (3.10) leads to the following relation for the radial velocity $u_{c}$ in the liquid at $r_{c}$,

$$
u_{c}\left(r_{c}, t\right)=u_{s}\left(1-\frac{\rho_{v}}{\rho_{l}}\right)+u_{v} \frac{\rho_{v}}{\rho_{l}} \approx u_{s}=\frac{d r_{c}}{d t}
$$

where it is assumed that the radial velocity $u_{v}$ at the vapour part of the cavity interface is of the same order of magnitude as $u_{s}$. Equation (3.13) shows that the mass transfer through the interface can be neglected and the interface is a streamline.

Equation (3.13) also simplifies Eq. (3.11) and if the viscosity ratio of vapour and liquid of $\mu_{v} / \mu_{l}=9.7 \times 10^{-3}$ is taken as negligibly small-valid if the shear strain in the liquid and vapour are of the same order of magnitude-we find that the tangential stress in the liquid at the interface equals zero. The boundary conditions at the cavity interface are then given by

$$
\frac{p_{c}-2 \mu_{l}\left(\frac{\partial u}{\partial r}\right)_{c}+\frac{T}{r_{c}}=p_{v},}{\left(\frac{\partial v}{\partial r}\right)_{c}-\frac{v_{c}}{r_{c}}=0,}
$$

where subscript $c$ denotes the liquid side of the cavity interface and subscript $v$ the vapour side.

\footnotetext{
${ }^{2}$ The values are obtained from http://webbook.nist.gov/.
} 
It is observed that the azimuthal velocity in the core of the vortex, described by the solid body rotation of Eq. (3.9), satisfies Eq. (3.15). As Eq. (3.15) is also a valid boundary condition at $r=0$ for the non-cavitating vortex, there is a smooth transition in the azimuthal velocity from non-cavitating to cavitating flow. If it is assumed that the flow in the vapour part of the vortex cavity is also described by solid body rotation, Eq. (3.15) is an exact boundary condition for the flow at the cavity interface and the cavity interface becomes a contact surface. Small temporal variations of the size of the cavity cross-section keep the radial velocity component small, so we can neglect the viscous contribution to Eq. (3.14). Because the present interest is on welldeveloped vortex cavities, the effect of surface tension can also be neglected in Eq. $(3.14)^{3}$ which leads to

$$
p_{c}(t)=p_{v}
$$

The simplified boundary conditions at the cavity interface are given by Eqs. (3.13), (3.15) and (3.16). A general analytical solution for the azimuthal velocity distribution that satisfies Eq. (3.5) reads

$$
v(r)=\frac{\Gamma_{\infty}}{2 \pi r}\left[1-\beta \exp \left(-\varsigma r^{2} / r_{v}^{2}\right)\right]
$$

in which $\beta$ is a constant which depends on the boundary condition, with $\beta=1$ for non-cavitating flow. Compared to Eq. (3.8), the exponent is rewritten by introducing a viscous core radius $r_{v}(t)=\sqrt{4 v_{l} \zeta t}$ which changes $v(r, t)$ into $v(r)$ with $r_{v}$ a prescribed parameter. The constant $\varsigma=1.2564$ is introduced so that for non-cavitating flow the azimuthal velocity has its maximum at $r=r_{v}$. For a cavitating vortex, $\beta$ is found by substitution of Eq. (3.17) in the boundary condition (3.15), which gives

$$
\beta=\frac{r_{v}^{2}}{r_{v}^{2}+\varsigma r_{c}^{2}} \exp \left(\varsigma r_{c}^{2} / r_{v}^{2}\right)
$$

The expression for the azimuthal velocity of a cavitating vortex now becomes

$$
v(r)=\frac{\Gamma_{\infty}}{2 \pi r}\left\{1-\frac{r_{v}^{2}}{r_{v}^{2}+\varsigma r_{c}^{2}} \exp \left[-\varsigma \frac{\left(r^{2}-r_{c}^{2}\right)}{r_{v}^{2}}\right]\right\},
$$

from which the velocity $v_{c}$ at the cavity radius $r=r_{c}$ is obtained,

$$
v_{c}\left(r_{c}\right)=\frac{\Gamma_{\infty}}{2 \pi}\left(\frac{\varsigma r_{c}}{r_{v}^{2}+\varsigma r_{c}^{2}}\right) .
$$

Substitution of Eq. (3.19) in Eq. (3.4) and integration from radius $r$ to infinity gives the relation for the pressure,

$$
\begin{array}{r}
p(r)-p_{\infty}=-\frac{\rho \Gamma_{\infty}^{2}}{(2 \pi r)^{2}}\left\{\frac{1}{2}-\beta \exp \left(-\varsigma r^{2} / r_{v}^{2}\right)+\frac{\beta^{2}}{2} \exp \left(-2 \varsigma r^{2} / r_{v}^{2}\right)\right. \\
\left.+\frac{\beta \varsigma r^{2}}{r_{v}^{2}} E_{1}\left(\frac{\varsigma r^{2}}{r_{v}^{2}}\right)-\frac{\beta^{2} \varsigma r^{2}}{r_{v}^{2}} E_{1}\left(\frac{2 \varsigma r^{2}}{r_{v}^{2}}\right)\right\},
\end{array}
$$

\footnotetext{
${ }^{3} \mathrm{Using} T=0.073 \mathrm{~N} / \mathrm{m}$ and $p_{v}=1700 \mathrm{~Pa}$, the effect of surface tension for the smallest considered cavity size in this chapter, $r_{c}=0.4 \mathrm{~mm}$, is $10 \%$ of $p_{v}$.
} 
where $E_{1}(x)=\int_{x}^{\infty}[\exp (-u) / u] d u$ corresponds to the exponential integral and $p_{\infty}$ to the pressure at infinity. The pressure at the cavity interface is found by substituting $r=r_{c}$ and this pressure has to be equal to the vapour pressure according to the boundary condition (3.16). This gives a relation between the pressure difference $\left(p_{\infty}-p_{v}\right)$ and the cavity radius $r_{c}$. Using Eq. (3.14) instead of Eq. (3.16) would lead to a small change in this relation. In absence of cavitation, the minimum pressure $p_{\min }$ occurs at $r=0$ and is given by

$$
p(r=0)-p_{\infty}=p_{\min }-p_{\infty}=-\frac{\rho \Gamma_{\infty}^{2}}{4 \pi^{2} r_{v}^{2}} \varsigma \ln 2 .
$$

Introducing a reference velocity $V_{\text {ref }}$, the cavitation inception number $\sigma_{i}$ is defined as

$$
\sigma_{i}=\frac{p_{\infty}-p_{\min }}{\frac{1}{2} \rho V_{\text {ref }}^{2}}=\left(\frac{\Gamma_{\infty}}{\pi V_{\text {ref }} r_{v}}\right)^{2} \frac{1}{2} \varsigma \ln 2,
$$

while the cavitation number $\sigma$ is defined as

$$
\sigma=\frac{p_{\infty}-p_{v}}{\frac{1}{2} \rho V_{\mathrm{ref}}^{2}}
$$

Finally, the radial velocity component is obtained by combining Eqs. (3.6) and (3.13) which results in

$$
u(r, t)=\frac{r_{c}}{r} \frac{d r_{c}}{d t}
$$

\subsubsection{Analysis}

The analytical expressions derived above will now be analysed in more detail. The requirement that $\beta$, given by Eq. (3.18), is a constant that does not depend on time is only satisfied if

$$
r_{c}(t)=c_{1} r_{v}(t)=2 c_{1} \sqrt{\varsigma v_{l} t}
$$

with $c_{1}$ a non-dimensional proportionality constant. Equation (3.26) shows a temporal growth of the cavity and viscous core radius due to diffusion. This growth also leads to a temporal variation of the difference between the pressure at $r=r_{c}$, where $p=p_{v}$, and the pressure at $r=\infty$. However, as both pressures are assumed constant, the temporal variation of the cavitating vortex solution is considered unrealistic. Therefore, the derived solution will only be interpreted as an instantaneous solution for the flow in a columnar vortex at a given time or a given value of the viscous core radius $r_{v}$. The vortex behaviour in 2-D axisymmetric unsteady flow was studied in more detail by Bosschers $(2009 \mathrm{c})$, in which it was shown that, for constant outer pressure, the cavity decreases in size when the viscous core radius increases due to diffusion.

The vorticity and circulation distribution follow directly from Eq. (3.19). The axial vorticity $\omega_{z}$ is given by

$$
\omega_{z}(r)=\frac{1}{r} \frac{\partial(r v)}{\partial r}=\frac{\Gamma_{\infty}}{\pi} \frac{\varsigma}{r_{v}^{2}+\varsigma r_{c}^{2}} \exp \left[-\varsigma\left(r^{2}-r_{c}^{2}\right) / r_{v}^{2}\right]
$$

and the circulation $\Gamma$ by

$$
\Gamma(r)=\Gamma_{\infty}\left[1-\frac{r_{v}^{2}}{r_{v}^{2}+\varsigma r_{c}^{2}} \exp \left[-\varsigma\left(r^{2}-r_{c}^{2}\right) / r_{v}^{2}\right]\right]
$$


The vorticity has its maximum at the cavity radius $r=r_{c}$ with its value decreasing with increasing cavity size.

Another interesting quantity for vortices is the angular momentum. However, in 2-D flow this quantity is not well-defined and, therefore, the vortical angular momentum $A_{z}$ is investigated (Saffman, 1992). For the cavitating vortex, $A_{z}$ is given by

$$
A_{z}=-\rho \iint_{S} r^{2} \omega_{z} d S=-2 \pi \rho \int_{r_{c}}^{\infty} r^{3} \omega_{z} d r=-\frac{\rho \Gamma_{\infty} r_{v}^{2}}{\varsigma} .
$$

It is remarkable that the cavity size $r_{c}$ does not appear in Eq. (3.29), from which it is concluded that the vortical angular momentum for cavitating flow is identical to that for non-cavitating flow if the viscous core size $r_{v}$ does not change due to the presence of cavitation. The angular momentum is not further discussed here.

A comparison between the results for an inviscid non-cavitating vortex and the results for a viscous vortex, for which the non-dimensional cavity size $r_{c} / r_{v}$ varies from zero to two, is presented in Fig. 3.1. The azimuthal velocity is non-dimensionalized by $v_{\text {ref }}$ which is chosen as the maximum azimuthal velocity of the non-cavitating viscous vortex. This maximum velocity is located at $r=r_{v}$. The velocity distribution for the cavitating vortex only differs from that for the non-cavitating situation near the cavity, this in order to satisfy the boundary condition of zero shear stress at $r=r_{c}$. This boundary condition leads to a vorticity layer, which can also be considered as a viscous boundary layer, around the cavity. The thickness of this layer decreases with increasing cavity size, and the model suggests that the effect of viscosity on the vortex cavity becomes small when the cavity size becomes larger than the viscous core size. The effect of cavitation on the pressure distribution is small.

Note that $r_{v}$, used as the reference length, is defined as the viscous core radius for noncavitating flow. The actual size of the region in which viscous forces are relevant can be defined as the radius where the circulation $\Gamma$ is a certain percentage of $\Gamma_{\infty}$. For non-cavitating flow, the radial location where $\Gamma=0.995 \Gamma_{\infty}$ is approximately two times $r_{v}$. In the presence of cavitation, it is better to interpret $r_{v}$ as the distance, or the time period, during which the growth of the viscous core by diffusion occurs when starting from a potential flow solution, as $r_{v}=\sqrt{4 v_{l} \varsigma t}$. So the actual thickness of the vorticity layer depends on $r_{v}$ and on $r_{c}$. Also, the value for $r_{v}$ may change by the effect of cavitation. For instance, the cavity pushes the vorticity layer outward and it may affect the growth of this vorticity layer. This has not been considered in the presented theoretical analysis.

The relation between $r_{c}$ and $\sigma$ becomes independent of $\Gamma_{\infty}$ and $r_{v}$ if it is presented as a relation between the ratios $r_{c} / r_{v}$ and $\sigma / \sigma_{i}$. These ratios are obtained by dividing Eq. (3.21) by Eq. (3.22). The relation can also be computed for a non-cavitating vortex by defining the cavity radius as the radius where the pressure equals the vapour pressure. Figure 3.1d shows that the difference between the relation for non-cavitating and the one for cavitating flow is very small. This suggests that the pressure (or azimuthal velocity) distribution of the non-cavitating vortex can be used to predict the cavity size as function of cavitation number. 


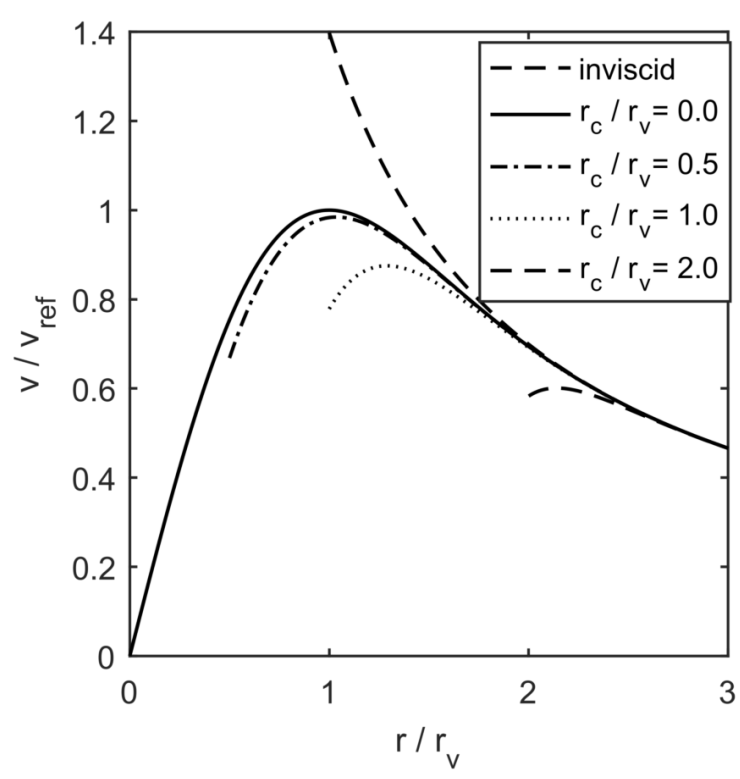

a) Azimuthal velocity distribution

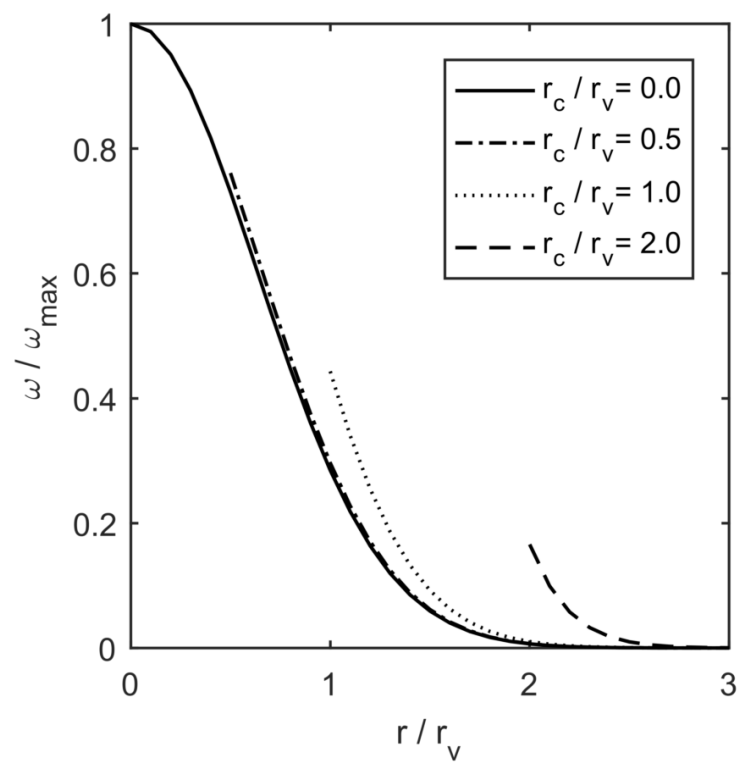

c) Vorticity distribution

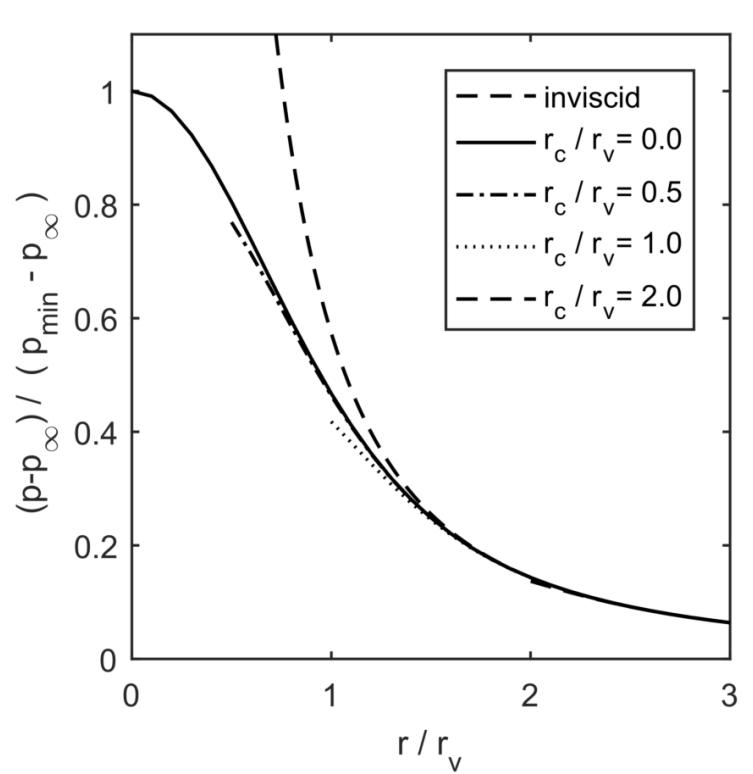

b) Pressure distribution

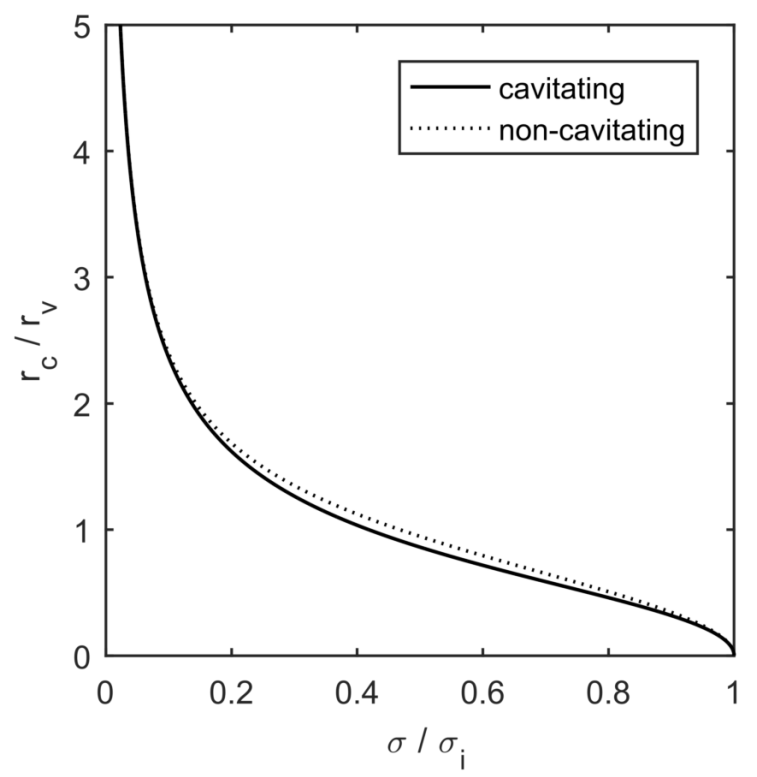

d) Cavity size against cavitation number

Figure 3.1: Radial distribution of azimuthal velocity, pressure, and vorticity for different cavity size $r_{c}$ of the cavitating Lamb-Oseen vortex. The relation between cavity size $r_{c}$ and cavitation number $\sigma$ is also presented.

\subsection{A semi-empirical model for a cavitating vortex}

\subsubsection{Formulation}

The vortex trailing from the tip of a wing or a propeller blade is generated by the roll-up of vorticity generated in the wake associated with the spanwise circulation distribution on the wing or blade (Phillips, 1981; Green, 1995). In the roll-up process, the vorticity layer spirals around 
the centre of the vortex and the turns of the spiral eventually merge into the viscous vortex core giving a continuous distribution of vorticity as function of radius and azimuth angle. However, in the near wake and outside the viscous core region, layers of vorticity can still exist. The Lamb-Oseen vortex describes a potential flow vortex for which the vorticity is distributed by diffusion only. The (circumferentially averaged) azimuthal velocity distribution of the vorticity layer due to the roll-up process is therefore not included in the Lamb-Oseen vortex.

Literature describes various semi-empirical vortex models that better represent an azimuthal velocity distribution during roll-up; see, for instance, Gerz et al. (2002) for a review. However, the expressions for most of these vortices are not easily transformed into an expression for a cavitating vortex. The vortex model that is most closely related to the Lamb-Oseen vortex is the Proctor vortex (Proctor et al., 2010), which is given by

$$
v(r)=\left\{\begin{array}{ccc}
1.0939 \frac{\Gamma_{\infty}}{2 \pi r}\left\{1-\exp \left[-\varsigma_{1}\left(1.4 r_{v} / B\right)^{0.75}\right]\right\} & \\
\times\left\{1-\exp \left[-\varsigma\left(r / r_{v}\right)^{2}\right]\right\} & & r \leq 1.4 r_{v} \\
\frac{\Gamma_{\infty}}{2 \pi r}\left\{1-\exp \left[-\varsigma_{1}(r / B)^{0.75}\right]\right\} & r>1.4 r_{v}
\end{array}\right.
$$

This model uses an additional length scale $B$, equal to the full wing span, to account for the vorticity roll-up region. The tuning factor for the vorticity roll-up region is $\varsigma_{1}$ for which Proctor et al. use $\varsigma_{1}=10$. A disadvantage of this model is that it is described by two formulas that need to be matched at $r=1.4 r_{v}$.

Following a similar approach as used in the Proctor vortex, a new model is introduced here in which the velocity distribution is described by a single formula for the entire range of the radial coordinate. A single formula has the advantage that continuity of the velocity and its derivatives is implicit, i.e., it does not need to be enforced. The azimuthal velocity distribution of this modified Lamb-Oseen vortex is given by

$$
v(r)=\frac{\Gamma_{\infty}}{2 \pi r}\left\{1-\hat{\alpha} \exp \left[-\varsigma_{1}\left(\frac{r}{B}\right)^{p}\right]\right\}\left\{1-\exp \left[-\varsigma_{2}\left(\frac{r}{r_{v}}\right)^{2}\right]\right\} .
$$

The non-dimensional parameters $\varsigma_{1}$ and $p$ can be used to tune the velocity distribution. The parameter $\hat{\alpha}$, that should have a value between zero and one, has been added to maintain a solid body rotation in the region close to the vortex centre. A disadvantage of Eq. (3.31) is that $v(r)$ does not automatically have its maximum value at $r=r_{v}$ in the case $\varsigma_{2}=\varsigma$ for arbitrary values of $\hat{\alpha}, \varsigma_{1}$ and $p$. Therefore, $\varsigma_{2}$ needs to be adjusted. A simple update of $\varsigma_{2}$ is given by $\varsigma_{2}^{\text {new }}=\varsigma_{2}\left[r\left(v_{\max }\right) / r_{v}\right]^{2}$ with $r\left(v_{\max }\right)$ the radius at which $v(r)$ has its maximum value. For the results presented in the following, a maximum of two updates were sufficient to obtain the maximum value of the distribution of $v(r)$ at $r_{v}$.

Another disadvantage of Eq. (3.31) is that, for larger values of $\hat{\alpha}$, the circulation distribution inside the viscous core also shows larger differences with the universal inner-scaled circulation distribution of Hoffmann and Joubert (1963) and Phillips (1981); see also Birch (2012). For $\hat{\alpha}=0.5$, the difference within this circulation distribution at $r / r_{v}=0.1$ is already $16 \%$ but this difference in circulation is hardly noticed in the velocity distribution. The Lamb-Oseen vortex, as well as the Proctor vortex, have a circulation distribution that is in agreement with this universal circulation distribution. The radial distribution of the azimuthal velocity and that 


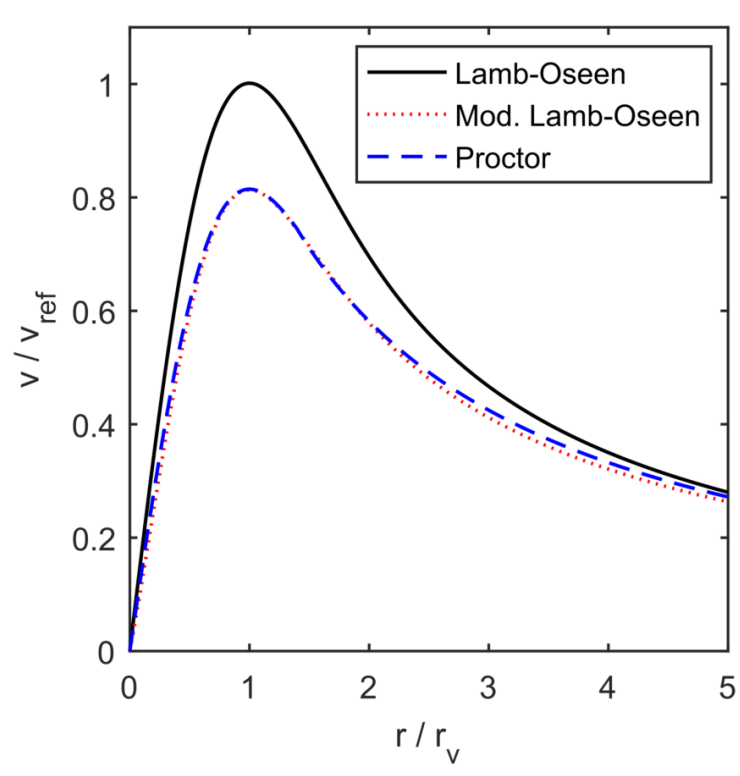

a) Azimuthal velocity

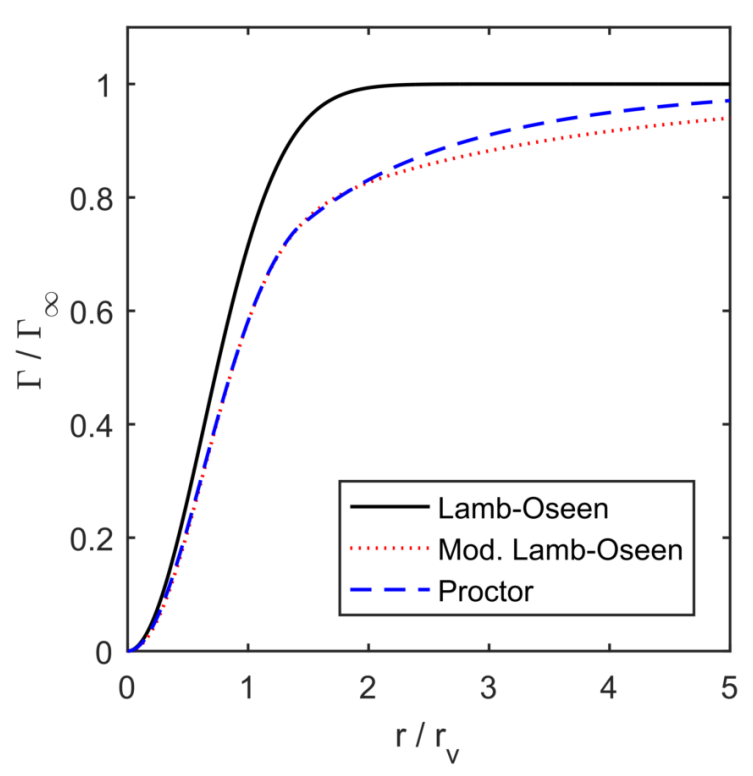

b) Circulation

Figure 3.2: Radial distribution of the azimuthal velocity and of the circulation for the various vortex models. The values for the parameters are $\hat{\alpha}=0.5, B / r_{v}=20, p=0.75, \varsigma_{1}=$ 6.

of the circulation for the Lamb-Oseen, Proctor and modified Lamb-Oseen vortex are given in Fig. 3.2. Parameters $B$ and $p$ were taken identical for the latter two vortices. For a given value of $\hat{\alpha}, \varsigma_{1}$ was adjusted such that the velocity distribution is in reasonable agreement with that of the Proctor vortex.

For the case of the cavitating vortex, Equation (3.31) has been modified by introducing a parameter $\beta$, as in Eq. (3.17), with $r_{c}$ added to the argument of the exponential function to simplify the expression for $\beta$. The radial distribution of the azimuthal velocity then becomes

$$
v(r)=\frac{\Gamma_{\infty}}{2 \pi r}\left\{1-\hat{\alpha} \exp \left[-\varsigma_{1}\left(\frac{r}{B}\right)^{p}\right]\right\}\left\{1-\beta \exp \left[-\varsigma_{2} \frac{r^{q}-r_{c}^{q}}{r_{v}^{q}}\right]\right\} .
$$

The parameter $q$ has been introduced, with default value $q=2$, to allow a different distribution of the azimuthal velocity in the vorticity layer for cavitating conditions than for the non-cavitating condition. The value of $\beta$ is obtained from the boundary condition given by Eq. (3.15), which results in

$$
\beta=\frac{2(1-\bar{\alpha}) B^{p} r_{v}^{q}-\bar{\alpha} \varsigma_{1} p r_{c}^{p} r_{v}^{q}}{2(1-\bar{\alpha}) B^{p} r_{v}^{q}-\bar{\alpha} \varsigma_{1} p r_{c}^{p} r_{v}^{q}+(1-\bar{\alpha}) B^{p} q \varsigma_{2} r_{c}^{q}},
$$

with

$$
\bar{\alpha}=\hat{\alpha} \exp \left[-\varsigma_{1}\left(\frac{r_{c}}{B}\right)^{p}\right] .
$$

Here, it is assumed that the roll-up process of the shear layer is not effected by cavitation. However, any effect can easily be included by multiplying $\hat{\alpha}$ by $\exp \left[\varsigma_{1}\left(r_{r} / B\right)^{p}\right]$ in which $r_{r}$ is a user-defined parameter. This effect is not considered further here and $r_{r}=0.0$ has been chosen for all results presented. 


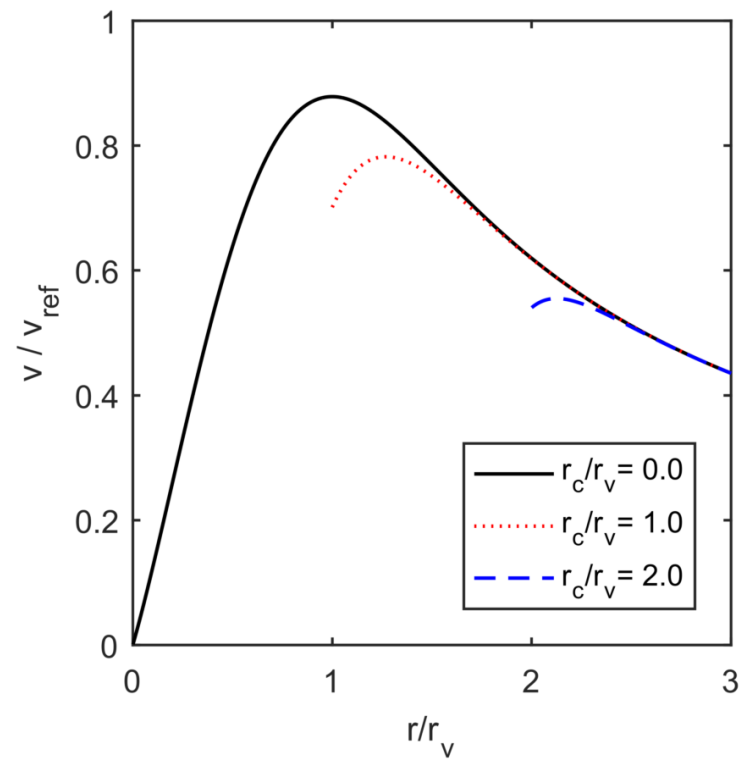

a) $r_{v, \text { cav }}=r_{v}, q=2.0$

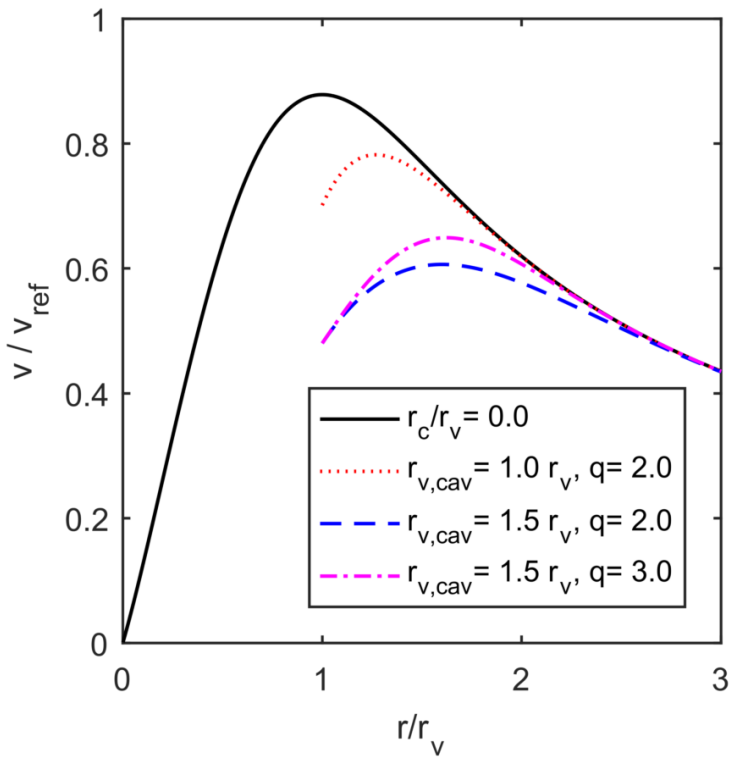

b) Varying $r_{v, c a v}$ and $q$ for $r_{c} / r_{v}=1.0$

Figure 3.3: Radial distribution of the azimuthal velocity for the cavitating modified LambOseen vortex, $\hat{\alpha}=0.5, B / r_{v}=10, \varsigma_{1}=5, p=0.75$.

The effect of cavitation on the azimuthal velocity distribution is presented in Fig. 3.3. Keeping all parameters of the vortex model identical, it is seen in Fig. 3.3a that the effect of cavitation on the modified Lamb-Oseen vortex is identical to that on the Lamb-Oseen vortex, see Fig. 3.1. The effect of a change in parameters $r_{v}$ and $q$, which determine the thickness and distribution of the vorticity layer next to the cavity interface, is presented in Fig. 3.3b.

\subsubsection{Comparison with experimental data}

The velocity distributions of a non-cavitating and of a cavitating tip vortex trailing from a wing of elliptical planform have been measured by Pennings et al. (2015b) using Stereoscopic Particle Image Velocimetry (SPIV). The wing has a half span $B / 2$ of $0.150 \mathrm{~m}$ and a chord length $c_{0}$ at the root of $0.1256 \mathrm{~m}$. The measurements have been performed in a cavitation tunnel at a free-stream velocity $W_{\infty}$ of $6.8 \mathrm{~m} / \mathrm{s}$. For these conditions, a typical viscous core radius $r_{v}$ is 1 $\mathrm{mm}$. The pixel size of the cameras is $7 \mu \mathrm{m}$ with an image format of $3248 \mathrm{x} 4875$ pixels. The stereo cross-correlation was initially performed with various pixel areas with the smallest area consisting of $48 \times 48$ pixels, resulting in a vector spacing of $0.35 \mathrm{~mm}$. Because there was some vortex wandering present (about $0.3 \mathrm{~mm}$ in all cross-flow directions), particle images taken at the same vortex location were selected and processed. The correlation maps of the individual particle images were summed to obtain a single vector field. The applied interrogation area for the results presented here was $12 \times 12$ pixels, which gives a vector spacing of $83 \mu \mathrm{m}$. Use was made of the sum of correlations approach, which not only gives a higher resolution of the flow in the viscous core but also gives a higher maximum azimuthal velocity. The images were also analysed for the size of the cavitating vortex.

The velocity field has been measured for three angles of attack, but the initial focus is on 
Table 3.1: Conditions for the tests in the cavitation tunnel by Pennings et al. (2015b) at $7^{\circ}$ angle of attack. Measured were cavity size $r_{c}$, cavitation number $\sigma_{W}$, dissolved oxygen content $D O$, lift-coefficient $C_{L}$ and Reynolds number $R e$ based on chord length $c_{0}$ at the root.

\begin{tabular}{c|ccccc}
\hline & $100 r_{c} / c_{0}$ & $\sigma_{W}$ & $D O[\mathrm{mg} / \mathrm{l}]$ & $C_{L}$ & $R e$ \\
\hline Case 1 & 0.00 & 4.10 & 2.6 & 0.573 & $9.3 \times 10^{5}$ \\
Case 2 & 0.52 & 2.03 & 2.6 & 0.576 & $9.4 \times 10^{5}$ \\
Case 3 & 0.89 & 1.70 & 2.6 & 0.575 & $9.4 \times 10^{5}$ \\
Case 4 & 1.22 & 1.26 & 2.6 & 0.581 & $9.3 \times 10^{5}$ \\
\hline
\end{tabular}

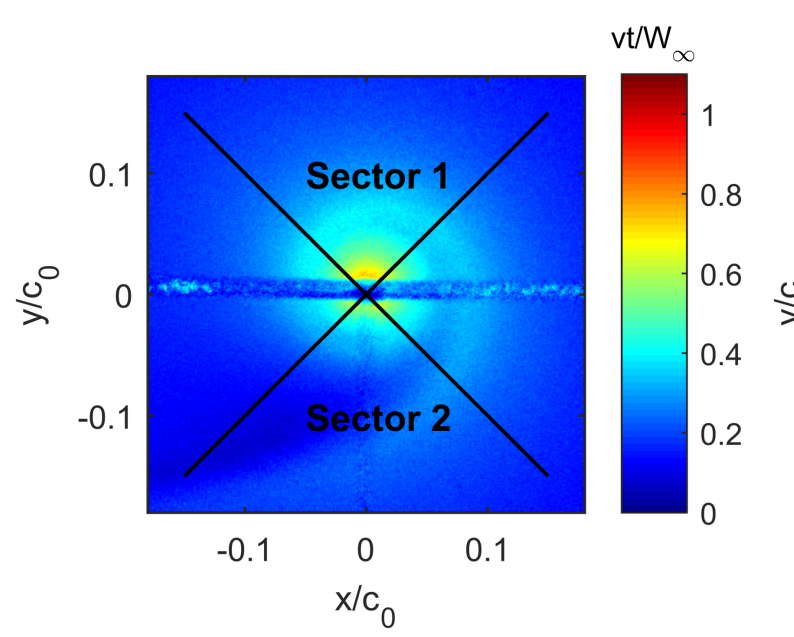

a) Transverse velocity magnitude

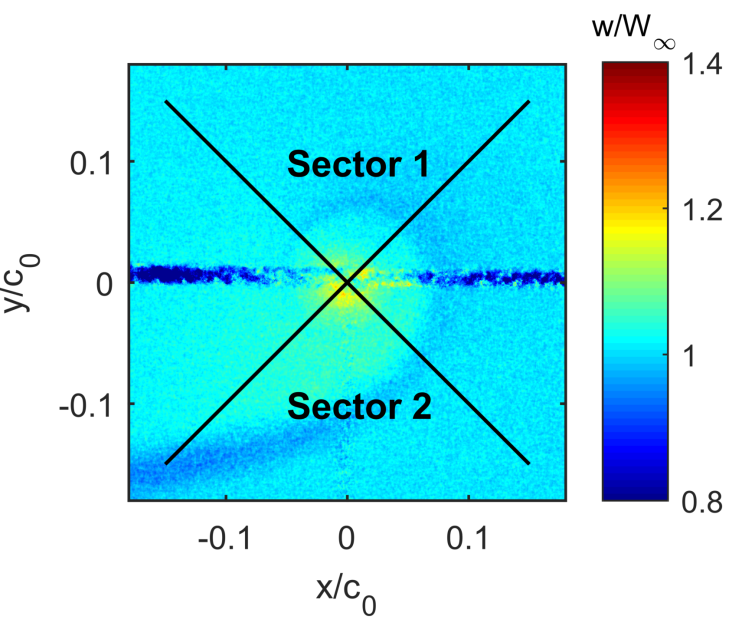

b) Axial velocity

Figure 3.4: Contour plot of the measured transverse and axial velocity components in a crossplane $z / c_{0}=1.14$ intersecting the cavitating tip vortex (Pennings et al., 2015b). The velocity components are defined in the tunnel frame of reference. The test condition is Case 3 of Table 3.1.

results obtained for an angle of attack of $7^{\circ}$. For this case, the measurements were performed in several cross-flow planes downstream of the trailing edge, but here only results are considered in a plane at a distance of about one root chord length behind the tip, $z / c_{0}=1.14$. At this distance the initial roll-up of the vorticity layer, that leads to inception of vortex cavitation, is completed and the streamwise change in vortex strength is small as shown by e.g. Astolfi et al. (1999).

Contour plots of the transverse and axial velocities, as measured in the cavitation tunnel frame of reference, are given in Fig. 3.4 for a condition for which the radius of the vortex cavity is about $1.1 \mathrm{~mm}$. Figure 3.4 shows the shadow of the cavity at $y / c_{0}=0$. Two sectors are defined in the contour plot with Sector 1 defined in the upper part and Sector 2 in the lower part of the cross-flow plane. In the latter sector and outside the vortex core, the remnants of the free vorticity layer can clearly be recognized. The included angle of both sectors is 90 degrees and the circumferentially averaged results for this angle are presented for each sector separately. 
The azimuthal and axial velocity distributions are given in Fig. 3.5 for various cavity sizes with the conditions given in Table 3.1. For Sector 1, cavitation only affects the velocity distribution in the region outside the cavity, as in the analytical solution, while for Sector 2 larger effects are observed. However, the velocity measurements in Sector 2 are considered to be less accurate than those in Sector 1 due to the shadow in Sector 2 of the cavity by the light source positioned above Sector 1 (Pennings et al., 2015b).

Both the maximum velocity and the velocity at the cavity interface are significantly different for the two sectors. This difference is reduced if the velocities are rotated around the y-axis and $\mathrm{x}$-axis in the tunnel frame of reference to align the z-axis (flow stream direction) with the direction of the vortex centre line. Analysis of high-speed video data, such as reported in Pennings et al. (2015a), shows that the mean line of the vortex cavity at $z / c_{0}=1.14$ has an angle of $3^{\circ}$ in the horizontal $x-z$ plane and an angle of $1^{\circ}$ in the vertical y-z plane. After applying this coordinate transformation, the distributions of the azimuthal and axial velocity for Sector 1 and Sector 2 are much more similar, see Fig. 3.6. This frame of reference is referred to as the vortex frame of reference. Although the effect of cavitation on the distribution of the azimuthal velocity agrees well with the analytical solution, the thickness of the vorticity layer next to the cavity is much larger in the experimental data than in the analytical solution.

The distribution of the axial velocity is presented in Fig. 3.5 and 3.6 because this velocity component has an effect on the azimuthal velocity distribution through the transformation from the tunnel frame of reference to the vortex frame of reference. The distribution of the axial velocity in non-cavitating flow shows, in general, a jet-like behaviour, which is in good agreement with the data presented by Giuni and Benard (2011). They show that the magnitude of the axial velocity at the vortex centre scales linearly with a non-dimensional circulation parameter $\left(\Gamma_{\infty} / W_{\infty} B\right) \sqrt{A R}$, which for the experimental case discussed here equals 0.15 . The parameter $A R$ denotes the aspect ratio of the elliptical wing, $A R=4 B / \pi c_{0}=3.04$. In the viscous core of the vortex, a reduction of the axial velocity is observed that is most likely the wake of the boundary layer on the wing. Similarity solutions for 3-D steady cavitating flow show that, contrary to the azimuthal velocity, the axial velocity distribution does not change significantly due to the occurrence of cavitation (Bosschers et al., 2008a). In these similarity solutions, roll-up of vorticity layers is modelled but a reduction of the axial velocity due to the wake of the boundary layer on the foil is not included. The measurement data shows that in the presence of cavitation the axial velocity is reduced in the vorticity layer next to the cavity suggesting a similar mechanism as for the velocity reduction in non-cavitating flow. Outside this vorticity layer, the axial velocity distribution is close to the one for the case without cavitation. In the present study, no effort was made to model the axial (and radial) velocity distribution. The focus is on the azimuthal velocity distribution as this is the dominant term in the radial component of the momentum equation from which the pressure distribution is calculated (Hommes et al., 2015).

The distribution of the circumferential-averaged azimuthal velocity of Sectors 1 and 2 in non-cavitating flow was fitted with the modified Lamb-Oseen vortex by prescribing $\hat{\alpha}=0.8$ and $B=300 \mathrm{~mm}$. In the fitting process, the origin of the vortex model was allowed to vary due to the uncertainty in the vortex centre position in the measurements. The vortex strength $\Gamma_{\infty}$ was prescribed from the measured azimuthal velocity distribution at a large distance from the centre of the vortex. Its value corresponds to $52 \%$ of the maximum circulation on the wing computed from the measured lift coefficient, assuming an elliptical spanwise distribution of the circulation for which $\Gamma_{\max }=0.5 C_{L} c_{0} W_{\infty}$. The fitting procedure resulted in $\varsigma_{1}=98.6, p=1.13$, 


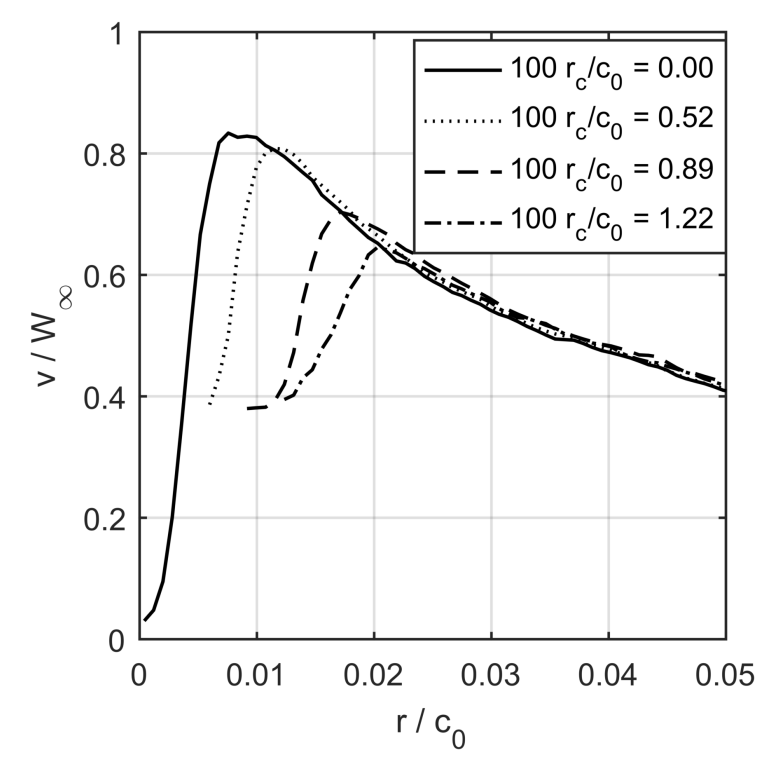

a) Azimuthal velocity in Sector 1

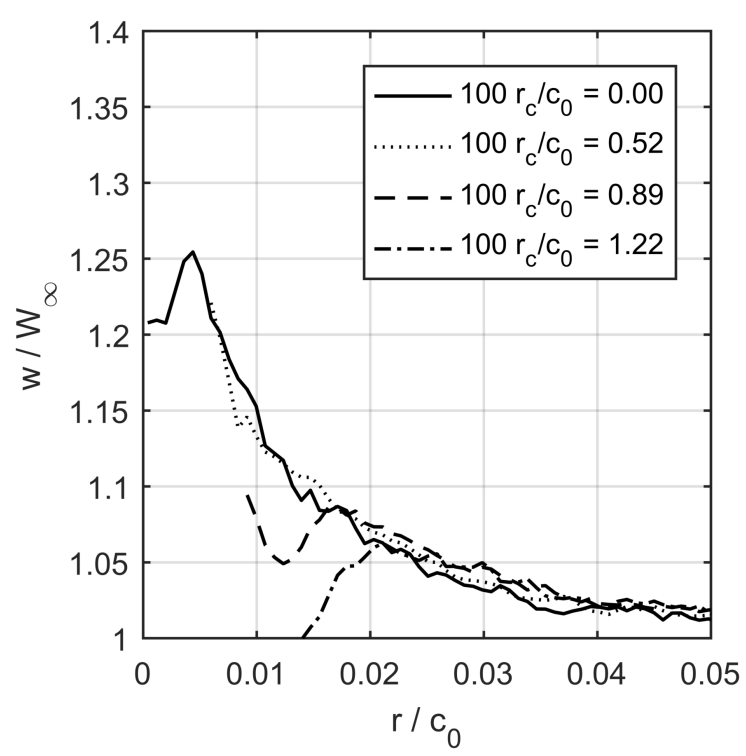

c) Axial velocity in Sector 1

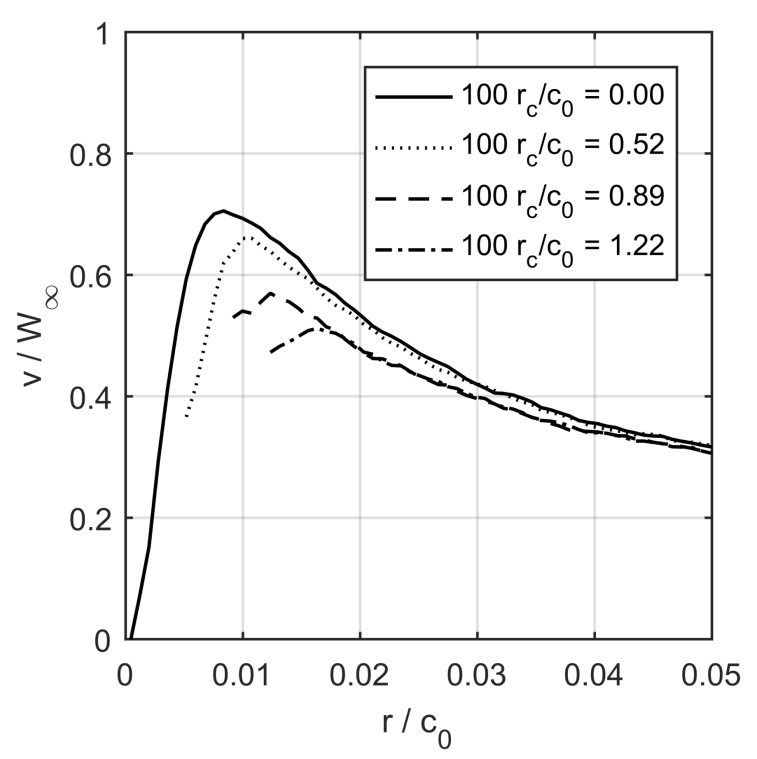

b) Azimuthal velocity in Sector 2

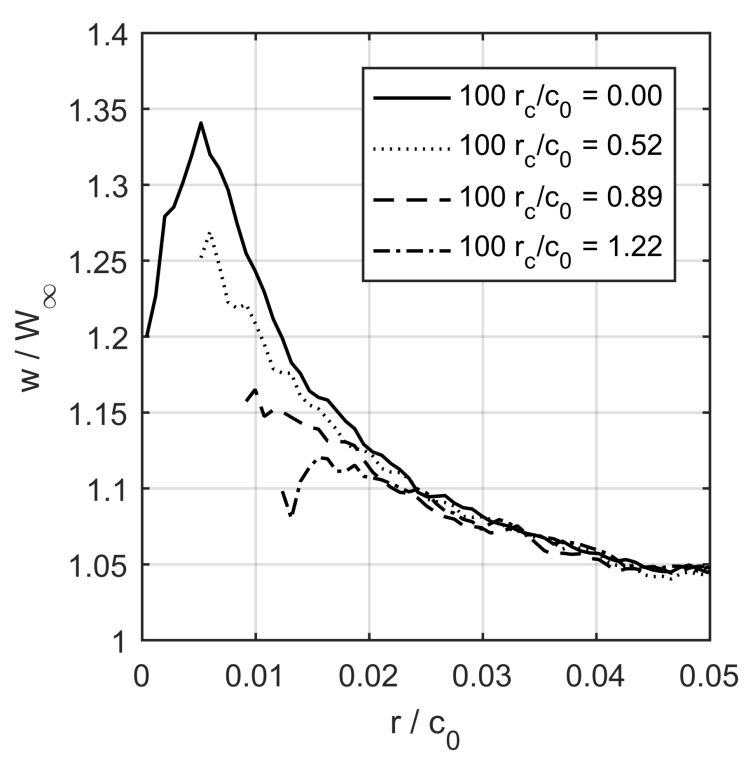

d) Axial velocity in Sector 2

Figure 3.5: Distributions of circumferential-averaged measured velocities in the tunnel frame of reference in Sector 1 (left column) and Sector 2 (right column) for non-cavitating and cavitating conditions as given in Table 3.1.

and $r_{v}=1.04 \mathrm{~mm}$ with $\varsigma_{2}=1.91$ so that the maximum of the azimuthal velocity is located at $r=r_{v}$. These parameters were then also used for the cavitating conditions. The resulting azimuthal velocity distribution for a number of cavity sizes is compared with the measured data in Fig. 3.7. Results are also shown for a larger value of $r_{v}$ and $q$, with $r_{v, c a v}=f r_{v}$ and $q_{c a v}=f q$. The introduced multiplication factor $f$ had to be increased with increasing cavity size to obtain a good correlation with the measurements. The experimental data is inconclusive regarding the effect of cavitation on the vortex roll-up as a change in velocity in the roll-up region is only observed in Sector 2. However, results in this sector are considered less accurate than those in 


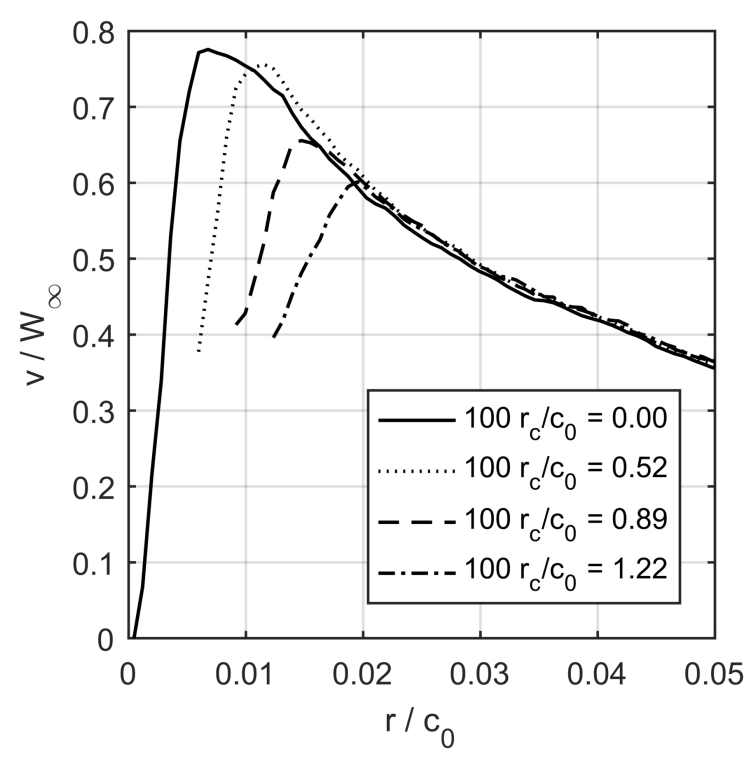

a) Azimuthal velocity in Sector 1

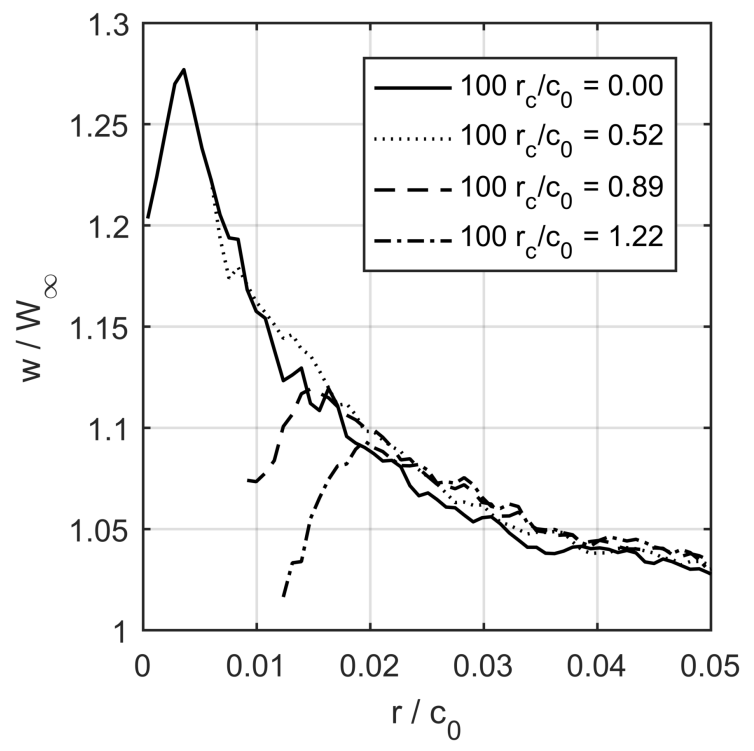

c) Axial velocity in Sector 1

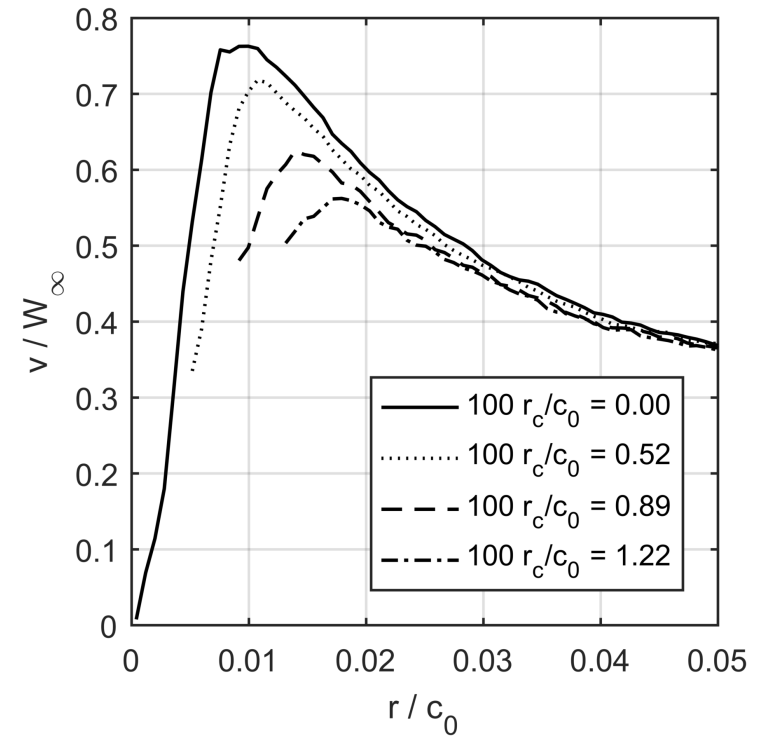

b) Azimuthal velocity in Sector 2

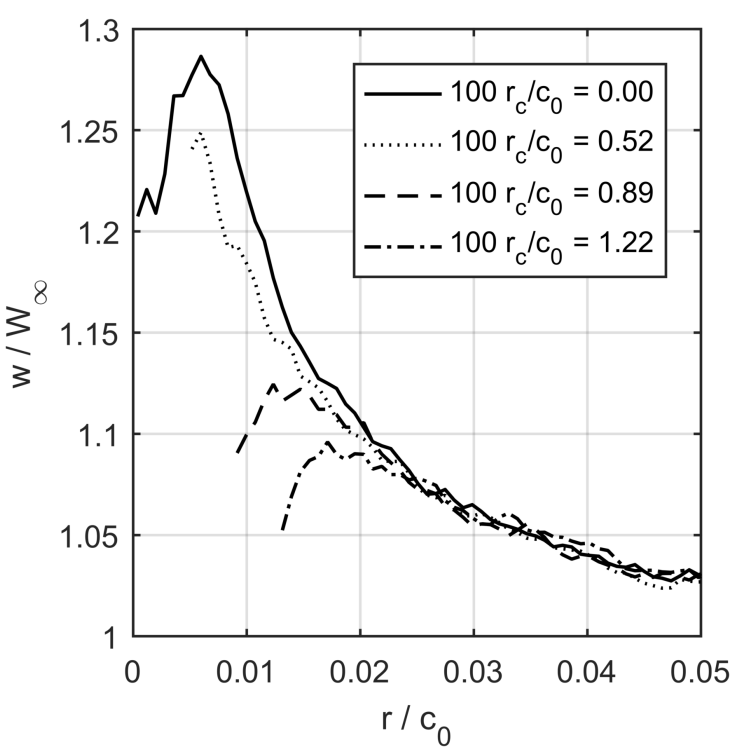

d) Axial velocity in Sector 2

Figure 3.6: Distributions of circumferential-averaged measured velocities in the vortex frame of reference in Sector 1 (left column) and Sector 2 (right column) for non-cavitating and cavitating conditions as given in Table 3.1.

Sector 1, as discussed before. Therefore, no effort was made to adjust the vortex model in this respect.

The fitted azimuthal velocity distribution are integrated to obtain the pressure at the cavity interface which results in the relation between cavity size and cavitation number. For a noncavitating vortex, a cavity size can be estimated from the radius at which the pressure equals the vapour pressure. For this purpose, only the azimuthal velocity in Sector 1 was considered, since in the far field the velocity distribution in this sector could be represented by a potential 


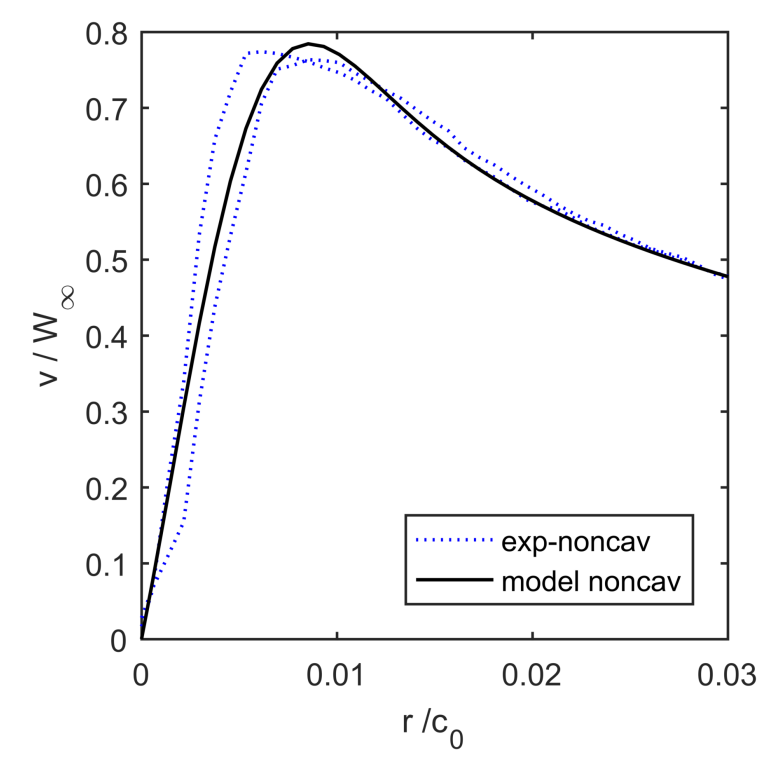

a) Cavity size $r_{c} / c_{0}=0.0$

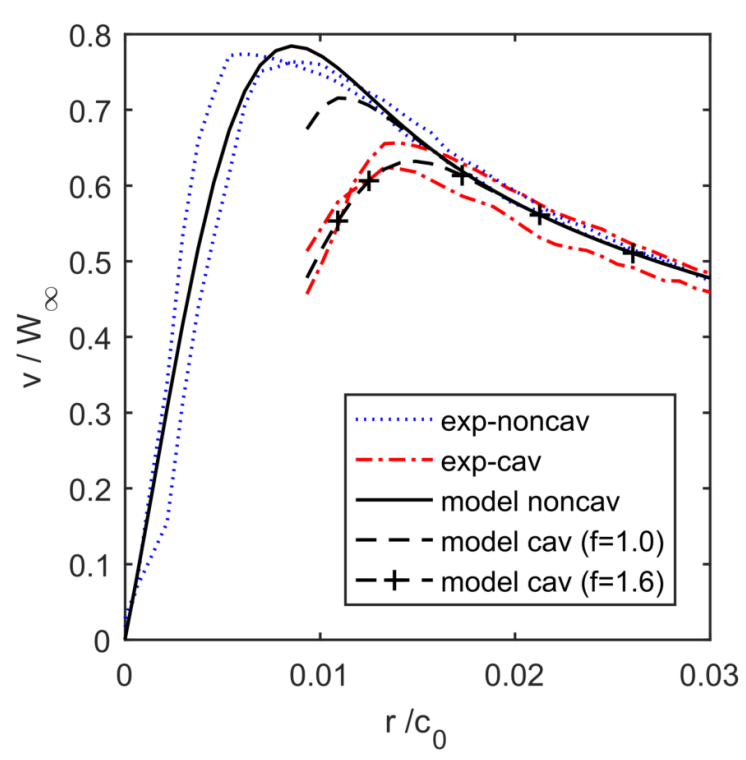

c) Cavity size $100 r_{c} / c_{0}=0.89$

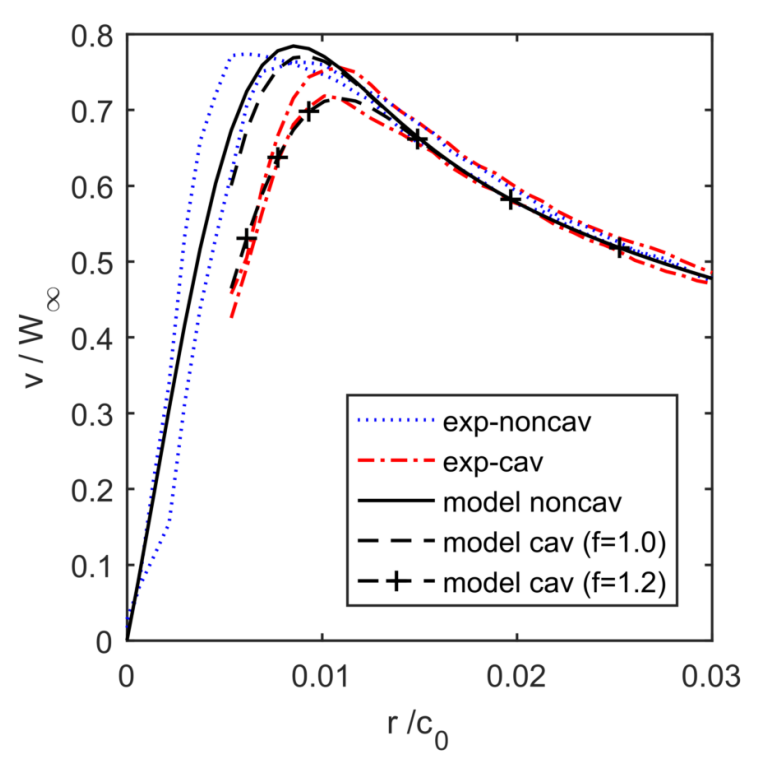

b) Cavity size $100 r_{c} / c_{0}=0.52$

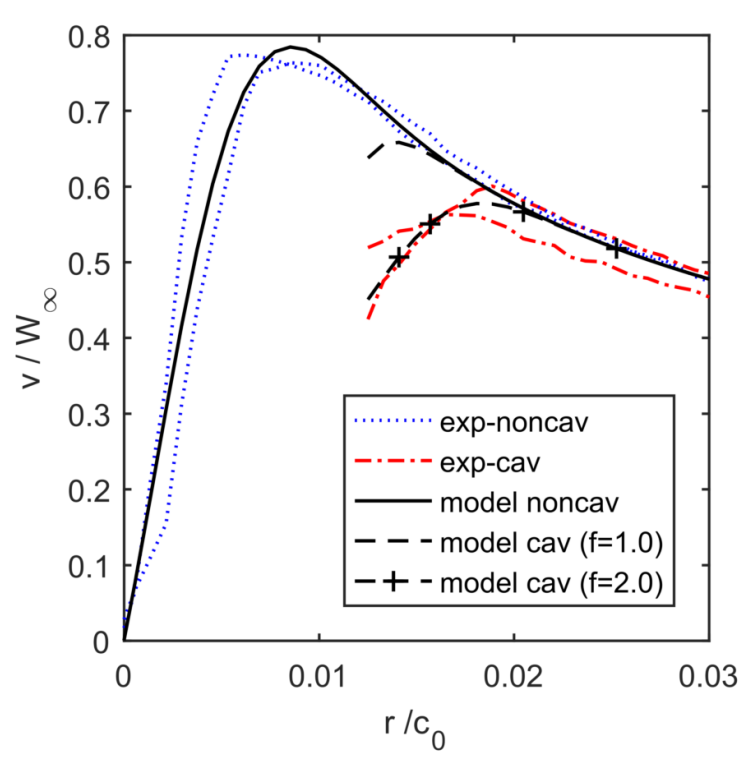

d) Cavity size $100 r_{c} / c_{0}=1.22$

Figure 3.7: Measured and fitted distributions of the azimuthal velocity for non-cavitating and cavitating conditions as given in Table 3.1. The measured velocities are given in the vortex frame of reference and for Sectors 1 and 2 separately. The fit is applied to the average of the two sectors for non-cavitating flow condition. Parameter $f$ is the correction factor for $r_{v}$ and $q$.

flow vortex. Results are presented in Fig. 3.8 for the fitted velocity distribution in the tunnel frame of reference for angles of attack equal to $5^{\circ}, 7^{\circ}$ and $9^{\circ}$. The experimental data, reported in Pennings et al. (2015b), is obtained from the stereo PIV data and high speed video data and is referred to as (spiv) and (hsv), respectively. The vortex strength in the tunnel frame of reference, taken as $75 \%$ of the maximum value of the circulation distribution on the wing for all three angles of attack, is significantly larger than when using the vortex frame of reference 
for which a value of $52 \%$ was found. With the value of the circulation from the vortex frame of reference, the cavity size is underestimated. At larger distances from the vortex centre the velocity distribution in the tunnel frame of reference is considered to be representative for the vortex induced velocities as, at these larger distances, the flow is forced to be aligned with the tunnel walls.

The parameters for the vortex model are obtained from a fitting procedure applied to the velocity distribution of the non-cavitating vortex for each angle of attack separately. The mean values, with prescribed $\hat{\alpha}=0.8$ and $B=300 \mathrm{~mm}$, are $\varsigma_{1}=57.5, p=1.13$ and $r_{v}=1.21 \mathrm{~mm}$ with $\varsigma_{2}=1.81$ so that the maximum of the azimuthal velocity is located at $r=r_{v}$. For the different angles of attack, these parameters vary by less than $15 \%$ and do not show a trend. The variability is therefore considered to correspond to the uncertainty of the measurement data and that of the non-linear fitting procedure. It was seen that the velocity distribution is in much better agreement with experimental data when the values for $r_{v}$ and $q$ increase in case of cavitation. The increase is modelled by an empirical relation derived for the cases presented in Fig. 3.7,

$$
r_{v, \mathrm{cav}}=r_{v} f\left(r_{c} / r_{v}\right)=r_{v} \sqrt{1+2.214\left(r_{c} / r_{v}\right)^{2}}
$$

with the same correction $f\left(r_{c} / r_{v}\right)$ also applied to $q$. This correction gives an improvement for the cases with small cavity size at an angle of attack of $5^{\circ}$ and $7^{\circ}$ but gives a deterioration for the larger cavity sizes. At the angle of attack of $9^{\circ}$, the correction gives a deterioration for all cavity sizes.

Figure 3.9 presents the data using the non-dimensional relation for the vortex cavity scaling discussed in Section 3.2.2. Because cavitation inception was not determined in the measurements, the cavitation inception number of the model has also been used for the experimental data, so the effect of nuclei and surface tension is not included. The experimental data forms two trend lines. One trend line, for small cavity size at $5^{\circ}$ and $7^{\circ}$ angle of attack, shows good agreement with the model in which the viscous core size was changed due to cavitation. The other trend line, for larger cavity size at $5^{\circ}$ and $7^{\circ}$ angle of attack and all cases at $9^{\circ}$ angle of attack, shows a good fit with the model for which the viscous core size was kept constant. The relation for the cavitating Lamb-Oseen vortex is also plotted in this graph, but this gives a poor fit to the experimental data. The Lamb-Oseen vortex predicts a too large pressure drop in the viscous core of the vortex, shifting the curve to smaller values of $\sigma / \sigma_{i}$ for larger cavity size. It is remarked that the presented relation for the Lamb-Oseen vortex is completely independent of vortex strength and viscous core size while the relation for the modified Lamb-Oseen vortex shows a very small variability with viscous core size.

\subsubsection{Discussion}

A new model for a cavitating vortex has been presented that well matches experimental data for the azimuthal velocity distribution. The model uses a zero shear stress boundary condition for the azimuthal velocity, but gives only a good fit to experimental data if the viscous core size and the exponent $q$ in the model increases with cavity size. The cause for this increase is unknown, but it might be due to an increase in boundary layer thickness on the foil or an increase in growth rate of the viscous core. 


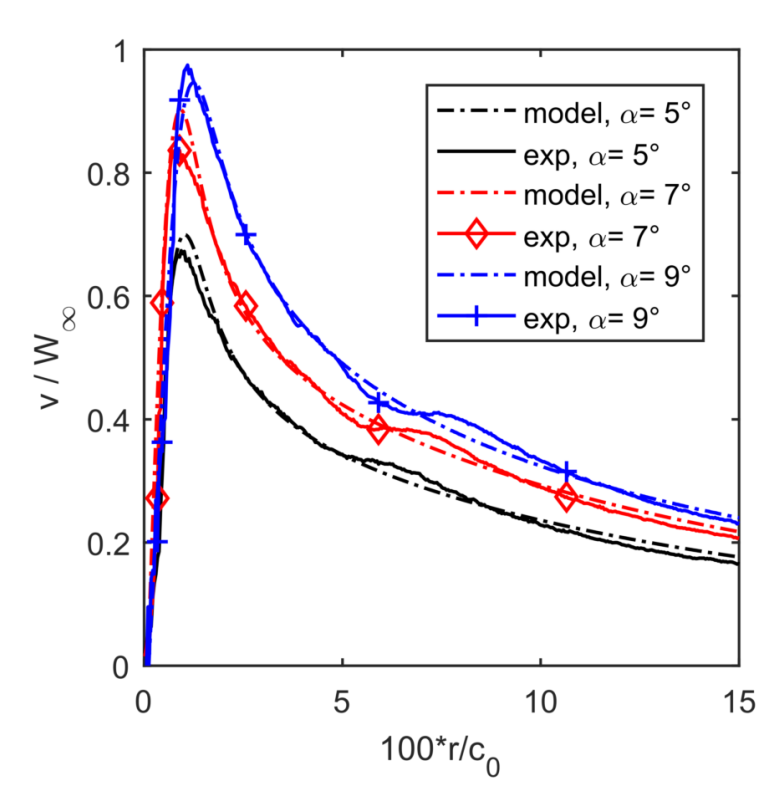

a) Azimuthal velocity

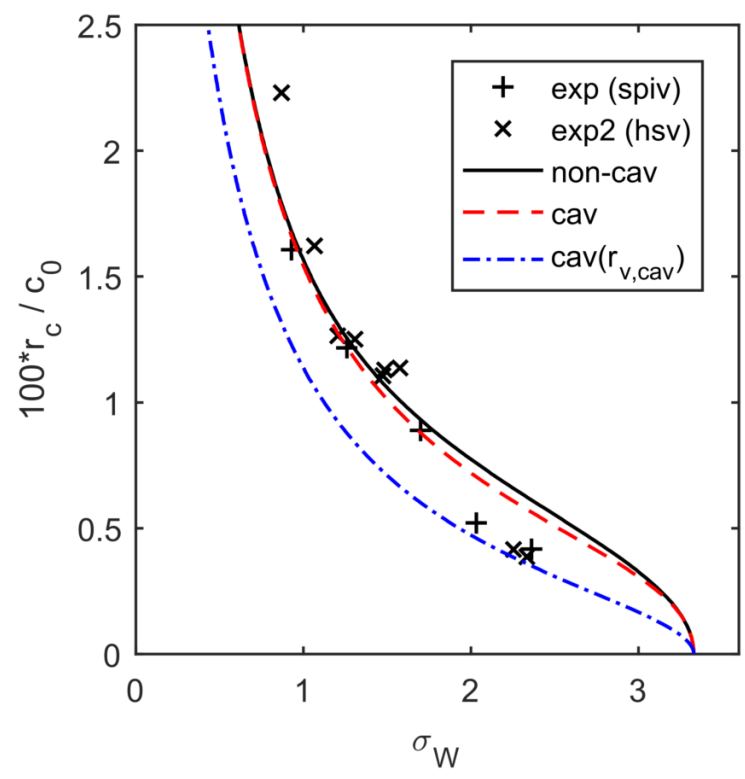

c) Cavity size, $\alpha=7^{\circ}$

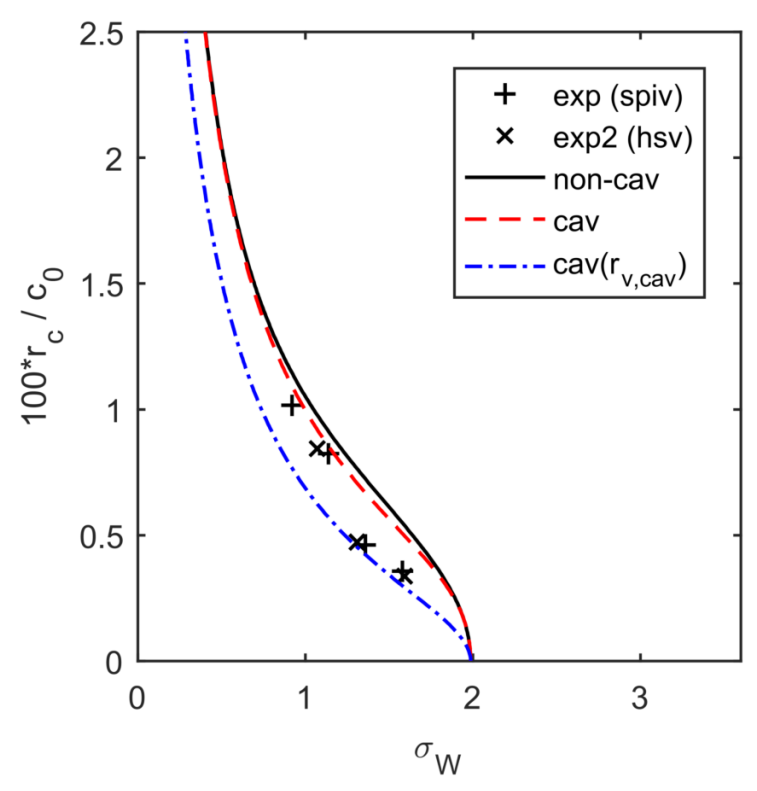

b) Cavity size, $\alpha=5^{\circ}$

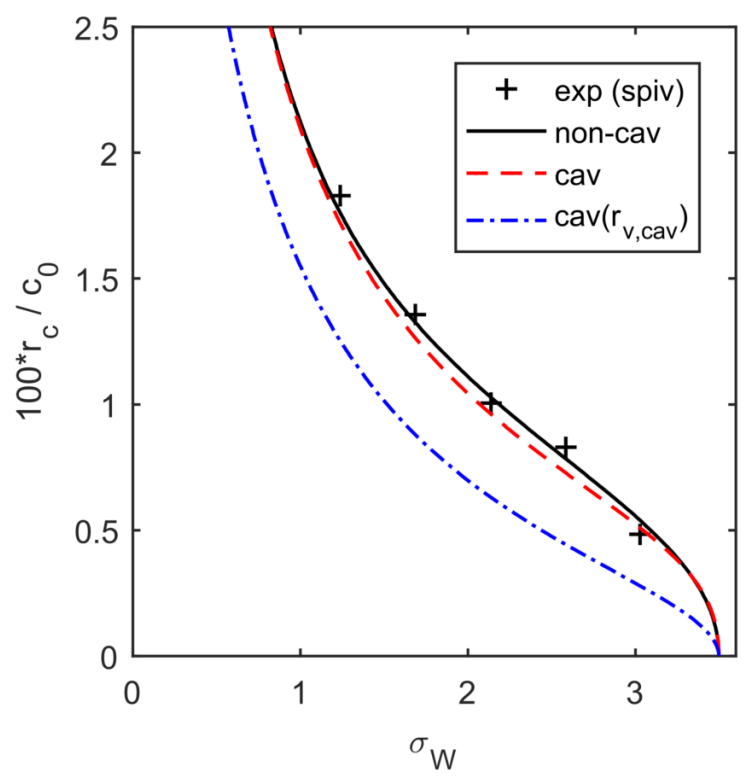

d) Cavity size, $\alpha=9^{\circ}$

Figure 3.8: Measured (Sector 1, tunnel frame of reference) and fitted distributions of the azimuthal velocity distribution for non-cavitating flow and the variation of cavity size with cavitation number at $\alpha=5^{\circ}, 7^{\circ}$ and $9^{\circ}$.

The relation between cavitation number and cavity size has been computed by integrating the azimuthal velocity distribution, neglecting the contribution of the mean axial and radial velocity components and the contribution of the Reynolds stresses. This aspect has been investigated by Hommes et al. (2015) using the experimental dataset of Chow et al. (1997) in which the mean and turbulent velocity components as well as the pressure was measured. It was concluded that the contribution of the mean axial and radial velocity components was neg- 


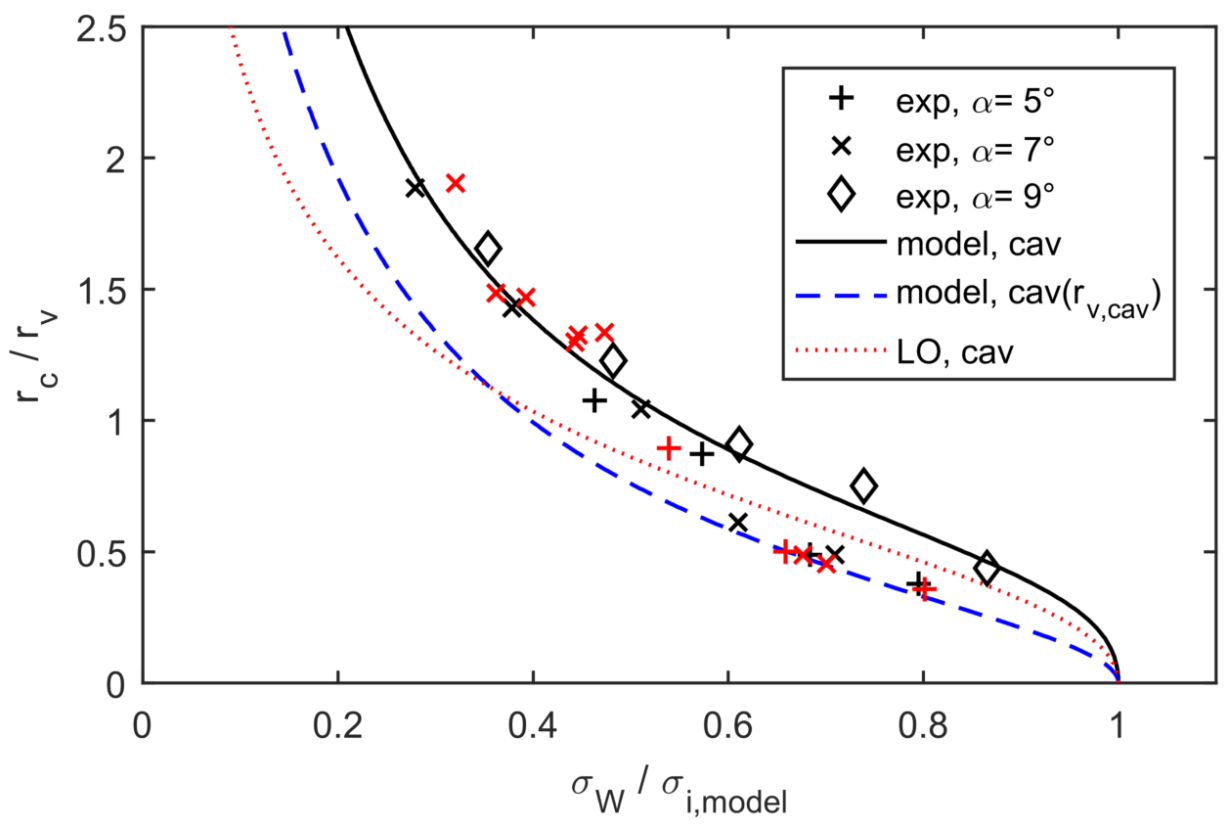

Figure 3.9: Comparison between measurements and results of vortex models using the proposed scaling relation between cavity size and cavitation number, 'model, cav' denotes the modified cavitating Lamb-Oseen vortex with constant viscous core size, 'model, $\operatorname{cav}\left(r_{v, \text { cav }}\right)$ ' denotes the modified cavitating Lamb-Oseen vortex with corrected viscous core size using Eq. (3.35), 'LO, cav' denotes the cavitating Lamb-Oseen vortex with constant viscous core size. Symbols in black denote SPIV data, symbols in red denote HSV data.

ligible and that the Reynolds stresses reduce the pressure by a few percent near the centre of the vortex only. Note that the effect of turbulence on the mean azimuthal flow is automatically taken into account through the fitting procedure. The azimuthal velocity distribution of the measurement data was taken from the tunnel frame of reference, while it was shown that closer to the vortex centre the vortex frame of reference gives a better representation of this velocity distribution. However, incorporating a smooth transition between the two frames of reference in the azimuthal velocity distribution gave a negligible change in cavitation number. Hence, it is expected that the error in the prediction of the cavitation number from the fitted azimuthal velocity distribution for given cavity size is small.

The comparison between the measured and the predicted relation between cavity size and cavitation number shows good correspondence for small cavity size when corrections are applied to the viscous core size. This shows that, for these conditions, the method to compute the cavitation number is sufficiently accurate. For larger cavity size this model underpredicts the cavity size. Analysis of video images shows that for the small cavity sizes the vortex cavity is detached from the foil while for the larger cavity size the vortex cavity is attached to the tip of the foil. At $9^{\circ}$ angle of attack the vortex cavity was always attached to the foil. Hence, there is a consistent relation between the accuracy of the prediction of the cavity size and the occurrence of attachment to or detachment from the foil. The physical mechanisms that explain the change in cavity size could not be determined from the experimental data.

In cavitation tunnel measurements, the vortex cavity size increases due to diffusion of non- 
condensable gas, with the growth rate depending on gas content (Briançon-Marjollet and Merle, 1996). For the presented experimental test set-up, the effect of gas content on cavity size has been presented by Pennings et al. (2015a). Results shown here are for the smallest applied oxygen content in the facility, which gave the smallest cavity size. The dissolved oxygen content was $2.5,2.6$ and $2.3 \mathrm{mg} / 1$ for angles of attack of $5^{\circ}, 7^{\circ}$ and $9^{\circ}$, respectively. Unfortunately, the growth rate of the cavity size in time has not been investigated. Therefore, it is also unknown whether the vortex-cavity attachment to or detachment from the foil has an effect on the diffusion of non-condensable gas. This aspect needs to be further investigated.

When the vortex cavity is attached to the foil, the model with constant viscous core size gives an accurate representation of the relation between cavity size and cavitation number despite significant differences in the azimuthal velocity distributions. Because the boundary condition for the cavity has a very small effect on this relation, the model for non-cavitating flow also gives an accurate representation of this relation. It needs to be further investigated if these surprising results are generally valid.

The presented scaling relation between vortex cavity size and cavitation number is, from a theoretical point of view, for an isolated vortex independent of Reynolds number and vortex strength. The scaling parameters involved are the viscous core size and the cavitation inception number. However, the estimation of the viscous core size requires flow field measurements and the cavitation inception number should be corrected for the tensile strength of water such that it correspond to the minimum pressure inside the vortex (Gindroz et al., 1996). Hence, validation of the presented relation requires very detailed measurements. Unfortunately, cavitation inception was not measured by Pennings et al. (2015a) and Pennings et al. (2015b), so in this respect the relation could not be validated.

\subsection{Conclusions}

An analytical solution exists for the flow around a vortex cavity that satisfies the Navier-Stokes equations for 2-D axisymmetric flow (Eq. 3.19). The solution fulfils a boundary condition at the cavity interface that prescribes zero shear stress (Eq. 3.15), and the vortex can be interpreted as a cavitating Lamb-Oseen vortex. The boundary conditions at the cavity interface are obtained by simplifying the condition of continuity of stress. Even though the solution was derived for unsteady flow, it describes an instantaneous flow field only. The analytical solution, as well as the boundary condition at the cavity interface, are an important new finding of the present study.

The analytical solution, extended with a tunable term to account for the effect of vorticity roll-up, shows a good match with published experimental data (Eq. 3.32 and Figure 3.7). The experimental data consists of the azimuthal velocity distribution of a non-cavitating and cavitating tip vortex. The comparison shows that the applied boundary condition at the cavity interface is realistic. For cavitating flow, the fit of the model with experimental data required an increase in the size of the viscous core, the cause of which needs to be further investigated. A disadvantage of the vortex model is that the circulation near the vortex centre is not correctly represented.

The relation between cavitation number and cavity size is well predicted by the vortex model for small cavity size, but a good prediction for large cavity size is only obtained if the 
viscous core size is kept equal to the value for non-cavitating flow (Figure 3.8). The relation between cavitation number and cavity size was obtained by integrating the radial distribution of the azimuthal velocity. The condition of small cavity size was associated with the vortex cavity detached from the foil, whereas for large cavity size the vortex cavity was attached to the foil. A good prediction for large cavity size was also obtained for the non-cavitating vortex model. This aspect is not understood and needs to be further investigated. The effect of diffusion of non-condensable gas in the cavity needs to be monitored during the experiment for this purpose.

The analytical and semi-empirical model show that, for constant size of the viscous core, the effect of viscosity on the cavity size becomes small when the cavity size becomes larger than the viscous core size (Figures 3.1 and 3.3). Given the statements above, it is not clear if this also holds for the experimental data.

The function that describes the ratio of cavity size and viscous core size with respect to the ratio of cavitation number and cavitation inception number is essentially independent of tip vortex strength, hence loading, and viscous core size, hence Reynolds number (Figure 3.9). The experimental data gives some support to the correctness of this relation, but the cavitation inception number was taken from the model and the relation for small cavity size was different from the relation for large cavity size as discussed above. Therefore, further validation of this scaling relation is required.

The focus of the presented research has been on modelling the azimuthal velocity distribution of a cavitating vortex. The model still needs to be extended with a relation for the axial and radial velocity distribution but this requires more information on the vorticity roll-up and streamwise variation of the flow. 
Page intentionally left blank 


\section{Chapter 4}

\section{Dynamics}

This chapter discusses some aspects of the dynamics of a vortex cavity. The effect of viscosity is analysed first by numerically solving the Navier Stokes equations for 2-D, axisymmetric, incompressible, and laminar flow. Next, a dispersion relation is derived for a vortex cavity in 3-D axisymmetric inviscid compressible flow, but with a simple ad-hoc correction for the effect of viscosity. The relation is compared with available experimental data ${ }^{1}$. The inclusion of the effect of viscosity, for which use has been made of results obtained in Chapter 3, is a novel aspect for the formulations for 2-D and 3-D flow.

\subsection{Introduction}

Computational results for unsteady flow around a 2-D vortex cavity have been presented by Ligneul et al. (1983), Ligneul (1988) and Chahine (1995). The methodology of Chahine, based on the Navier-Stokes equations for incompressible flow, has also been used in Choi et al. (2009). The formulation can be interpreted as the '2-D vortex cavity' variant of the 2-D Rayleigh-Plesset equation that describes the dynamic behaviour of a spherical bubble. Ligneul et al. (1983) and Ligneul (1988) use a vortex model for inviscid flow, which does not require a boundary condition for the azimuthal velocity at the cavity interface. The boundary condition for the azimuthal velocity at the cavity interface used in Chahine (1995) is that the velocity equals zero. The methodology of Chahine (1995) is also adopted in the present work, but the boundary condition as derived in Chapter 3 will be used, which requires that the tangential component of the shear stress at the cavity interface equals zero. Results for this boundary condition have been presented in Bosschers (2009c) and Bosschers (2010) and results are reproduced here. The 2-D vortex model has been used by Ligneul et al. (1983), Ligneul (1988) and Berger et al. (2016) to predict the far-field radiated noise produced by propeller tip vortex cavitation. In the present study, the focus is on the effect of viscosity on the cavity size, on the cavity dynamics and on the resonance frequency of the cavity motion. The governing equations and the results are presented in Section 4.2. This section also contains an analysis of the vortex dynamics in terms of energy balance.

\footnotetext{
${ }^{1}$ Section 4.3 is an update of the theory and results presented in Pennings, P.C., Bosschers, J., Westerweel, J., and van Terwisga, T.J.C. (2015). Dynamics of isolated vortex cavitation. J. Fluid Mech., 778:288-313.
} 
The behaviour of a vortex cavitation bubble at inception has been studied by Choi and Ceccio (2007) and Choi et al. (2009). Choi and Ceccio (2007) examined the growth, oscillation and collapse of a laser-initiated bubble in the centre of a vortex. The dynamics of the vortex bubble was not well described by the vortex parameters like circulation, viscous core size and cavitation number alone, but also depended on the details of the process of bubble growth. This finding was confirmed by computational results presented in Choi et al. (2009). They also show that the frequency of oscillation is of the order of the rotational frequency of the viscous core.

The first 3-D analysis of the flow around a cavitating vortex was presented by Lord Kelvin (Thomson, 1880), who analysed the dispersion relation of surface waves on a hollow columnar vortex using small perturbation solutions of incompressible potential flow without a free-stream velocity. A free-stream velocity was added by Ackeret (1930) to derive a relation for the wave length of a standing wave, which is stationary in time, with an axially symmetric deformation for the analysis of the hub vortex of a water turbine. The scattering by a cavitating line vortex due to a line and a point acoustic source was examined by FfowcsWilliams and O'Shea (1970). The analysis was obtained for compressible flow and for axisymmetric modes only. A linear stability analysis of a vortex, of which the core contains a gas of arbitrary density, was performed by Uberoi et al. (1972) and this analysis was extended to a non-linear analysis by Narain and Uberoi (1973). Both analyses show that when the density of the gas in the core is infinitesimally small the solution is always stable. The analysis by Lord Kelvin was extended to compressible flow by Morozov (1974) and Koop and Krohn (1978). Whereas these analyses were all carried out in the frequency domain, Keller and Escudier (1980) derive the dispersion relation for incompressible flow in the time domain to find a relation for the wave length of a standing wave for a cavitating vortex in a cylindrical tank. Pylkkänen $(1981,1982)$ performed both a linear and a non-linear small-perturbation analysis of the stability of cavitating vortices in inviscid incompressible flow. He included the effect of a small region of vorticity outside the cavity. Unstable solutions were not found. Another analytical study was performed by Roberts (2003) who examined solutions for compressible flow.

The practical application of the dispersion relation has been discussed in a few publications. Ackeret (1930) shows that the analytical relation is in good agreement with one experimental data point for the wave length of a standing wave. A similar analysis was made by Weitendorf (1976) for propeller tip vortex cavitation. Keller and Escudier (1980) analyse the length of the standing wave for a cavitating vortex in a cylindrical tank of small axial extent with the criterion that the group velocity is zero for this condition. Good agreement with experimental data is obtained for the largest cavity diameters but the agreement for the smallest cavity diameters is poor. Viscous effects are mentioned as an explanation for this difference.

Experimental evidence of the existence of a dispersion relation for deformations of a cavitating vortex was provided by Pennings et al. (2015a). The results of these experiments were compared with the results of an analytical formulation for incompressible inviscid flow with an ad-hoc correction for viscous flow, as initially presented in Bosschers (2008, 2009b). Here, the analytical formulation applied is described and further elaborated in Section 4.3. The predicted results are compared in Section 4.3.4 to the experimental data of Pennings (2016) presenting findings additional to those presented in Pennings et al. (2015a).

The application of the dispersion relation to analyse resonance frequencies in the radiated noise of cavitating vortices is presented in Section 6.2. The conclusions on the dynamics of 
vortex cavities are presented in Section 4.4.

\subsection{Dynamics of a vortex cavity in 2-D viscous flow}

\subsubsection{Governing equations and computational procedure}

The analysis of the dynamic behaviour of a 2-D cavity is performed for incompressible, axisymmetric unsteady flow, similar as in Section 3.2. The governing equations are the continuity and Navier-Stokes equations, expressed in a cylindrical coordinate system $(r, \theta, z)$ with velocity components $(u, v, w)$ in radial, circumferential and axial direction, respectively, while time is denoted by $t$. It is furthermore assumed that the flow is two-dimensional. Hence, $w$ is constant and all derivatives in axial direction are zero. The governing equations are given by

$$
\begin{gathered}
\frac{1}{r} \frac{\partial}{\partial r}(u r)=0 \\
\frac{\partial u}{\partial t}+u \frac{\partial u}{\partial r}-\frac{v^{2}}{r}=-\frac{1}{\rho} \frac{\partial p}{\partial r}+\frac{\mu}{\rho} \frac{\partial}{\partial r}\left[\frac{1}{r} \frac{\partial(u r)}{\partial r}\right], \\
\frac{\partial v}{\partial t}+u \frac{\partial v}{\partial r}+\frac{u v}{r}=\frac{\mu}{\rho} \frac{\partial}{\partial r}\left[\frac{1}{r} \frac{\partial(v r)}{\partial r}\right],
\end{gathered}
$$

corresponding to the continuity equation and the momentum equations in radial and azimuthal direction. From Eq. (4.1) it is clear that the diffusion term in Eq. (4.2) is zero. The boundary conditions for $u(r, t), v(r, t)$ and $p(r, t)$ at the cavity interface $r=r_{c}(t)$ are derived from the jump relations for mass and momentum as given in Section 3.2.1 and are given by

$$
\begin{array}{r}
u_{c}=\frac{d r_{c}}{d t}=\dot{r}_{c} \\
p_{c}-2 \mu\left(\frac{\partial u}{\partial r}\right)_{c}=p_{v}, \\
\left(\frac{\partial v}{\partial r}\right)_{c}-\frac{v_{c}}{r_{c}}=0 .
\end{array}
$$

Subscript $c$ corresponds to the value at the cavity interface, $p_{v}$ corresponds to the vapour pressure and the dot corresponds to a time derivative. In Eq. (4.4) the mass transfer through the interface is neglected, and in Eq. (4.5) the surface tension is neglected. The effect of vapour and gas inside the cavity on normal and tangential stress is neglected in Eqs. (4.5) and (4.6).

At the far-field boundary, inviscid flow is assumed. The variation of the radial velocity component is obtained by combining Eq. (4.4) with Eq. (4.1) to yield

$$
u(r, t)=\frac{\dot{r}_{c} r_{c}}{r} .
$$

The computational method only solves for the liquid part of the flow field and for $r_{c}$. The difficulty of the change in the time-dependent location of the boundary $r_{c}$ is circumvented by 
introducing the coordinate transformation $(r, t) \rightarrow(s, \breve{t})$ with $s=r-r_{c}(t)$ and $\breve{t}=t$. The resulting system of equations, in which Eq. (4.7) is substituted in Eq. (4.2) and (4.3), is then given by

$$
\begin{gathered}
r_{c} \ddot{r}_{c}+\dot{r}_{c}^{2}-\left(\frac{r_{c} \dot{r}_{c}}{r_{c}+s}\right)^{2}-v^{2}+\frac{r_{c}+s}{\rho} \frac{\partial p}{\partial s}=0 \\
\frac{\partial v}{\partial \breve{t}}-\dot{r}_{c} \frac{\partial v}{\partial s}+\frac{r_{c} \dot{r}_{c}}{r_{c}+s}\left(\frac{\partial v}{\partial s}+\frac{v}{r_{c}+s}\right)-v_{l}\left\{\frac{\partial^{2} v}{\partial s^{2}}+\frac{1}{r_{c}+s} \frac{\partial v}{\partial s}-\frac{v}{\left(r_{c}+s\right)^{2}}\right\}=0
\end{gathered}
$$

where the kinematic viscosity of the liquid is denoted by $v_{l}$. The partial time derivative with respect to $\breve{t}$ corresponds to the (material) derivative following the motion of the grid in the $(r, t)$ domain,

$$
\frac{\partial v}{\partial \breve{t}}=\frac{d v}{d t}=\frac{\partial v}{\partial t}+\frac{\partial r}{\partial t} \frac{\partial v}{\partial r}=\frac{\partial v}{\partial t}+\dot{r}_{c} \frac{\partial v}{\partial s} .
$$

By integrating Eq. (4.8) with respect to $s$ from $s=0$ to $s=s_{n}$, the number of unknowns in the pressure distribution can be reduced to its values at the inner boundary $s=s_{c}=0$ and outer boundary $s=s_{n}$. This gives

$$
\left(r_{c} \ddot{r}_{c}+\dot{r}_{c}^{2}\right) \ln \left(\frac{r_{c}+s_{n}}{r_{c}}\right)+\frac{\left(r_{c} \dot{r}_{c}\right)^{2}}{2\left(r_{c}+s_{n}\right)^{2}}-\frac{\dot{r}_{c}^{2}}{2}-\int_{0}^{s_{n}} \frac{v^{2}}{r_{c}+s} d s+\frac{p_{n}-p_{c}}{\rho}=0 .
$$

The boundary conditions at the cavity interface are given by

$$
\begin{gathered}
v_{c}-r_{c}\left(\frac{\partial v}{\partial s}\right)_{c}=0, \\
p_{c}=p_{v}-2 \mu \frac{\dot{r}_{c}}{r_{c}} .
\end{gathered}
$$

At the outer boundary, the boundary condition for the azimuthal velocity is given by the formulation for a potential flow vortex and for the radial velocity by Eq. (4.7),

$$
\begin{gathered}
v_{n}=\frac{\Gamma_{\infty}}{2 \pi r_{n}}=\frac{\Gamma_{\infty}}{2 \pi\left(r_{c}+s_{n}\right)}, \\
u_{n}=\frac{\dot{r}_{c} r_{c}}{r_{c}+s_{n}} .
\end{gathered}
$$

The vortex strength $\Gamma_{\infty}$ is prescribed for all computations.

The boundary condition for the pressure $p_{n}$ is obtained by integrating Eq. (4.2) with respect to $r$ between $r=r_{n}$ and $r=r_{\infty}$ while assuming potential flow. The velocity components $v_{\infty}$ and $u_{\infty}$ vanish when $r_{\infty} \rightarrow \infty$. This gives

$$
p_{n}=p_{\infty}-\frac{1}{2} \rho\left(v_{n}^{2}+u_{n}^{2}\right)+\rho\left(r_{c} \ddot{r}_{c}+\dot{r}_{c}^{2}\right) \ln \left(\frac{r_{\infty}}{r_{n}}\right) .
$$

The last term in Eq. (4.16) is problematic as it does not vanish for $r_{\infty}$. The term corresponds to the pressure perturbation by the cavity dynamics which is described by a monopole in 2$\mathrm{D}$ potential flow. Because the term does not vanish, it should be included in the boundary 
condition for $p_{n}$. Instead of using $p_{\infty}$, use is made of a reference pressure $p_{\text {ref }}$, which is defined as the pressure in a quiescent fluid. The boundary condition is then given by

$$
p_{n}=p_{\text {ref }}-\frac{1}{2} \rho\left(v_{n}^{2}+u_{n}^{2}\right)+\rho\left(r_{c} \ddot{r}_{c}+\dot{r}_{c}^{2}\right) \ln \left(r_{\text {ref }} / r_{n}\right) .
$$

The parameter $r_{\text {ref }}$ has been introduced to keep dimensions consistent with $r_{\text {ref }}=1 \mathrm{~m}$ so the cavity dynamics does not affect the pressure at $r=r_{\text {ref. }}$. In the following, $\breve{p}_{n}$ is used to simplify the expression, with $\breve{p}_{n}$ given by

$$
\breve{p_{n}}=p_{\text {ref }}-\frac{1}{2} \rho\left(v_{n}^{2}+u_{n}^{2}\right)
$$

Substituting Eq. (4.17) into Eq. (4.11) gives

$$
\left(r_{c} \ddot{r}_{c}+\dot{r}_{c}^{2}\right) \ln \left(r_{\mathrm{ref}} / r_{c}\right)+\frac{\left(r_{c} \dot{r}_{c}\right)^{2}}{2\left(r_{c}+s_{n}\right)^{2}}-\frac{\dot{r}_{c}^{2}}{2}-\int_{0}^{s_{n}} \frac{v^{2}}{r_{c}+s} d s+\frac{\breve{p}_{n}-p_{c}}{\rho}=0
$$

which is the equation to be solved in combination with Eq. (4.9). Eq. (4.19) now requires the boundary condition for $\breve{p_{n}}$ instead of $p_{n}$.

Note that previous research on the system of equations for this 2-D cavitating vortex equation did not use the boundary condition Eq. (4.17), but used ${ }^{2}$

$$
p_{n}=p_{\infty}-\frac{1}{2} \rho\left(v_{n}^{2}+u_{n}^{2}\right) .
$$

The pressure at the outer boundary is then artificially prescribed and the solution will depend on the location of the outer boundary. Only if $r_{n}=1$, Eq. (4.20) is identical to Eq. (4.17) with $p_{\infty}=p_{\text {ref. }}$ This condition is applied in the computational model for which the results are shown here.

The resulting equations are solved using a Crank-Nicholson discretisation scheme which is implicit and second order accurate in time. The discretisation scheme for the first spatial derivative is second order accurate for arbitrary grid spacing while the scheme for the second spatial derivative is only second order accurate for equidistant grid spacing. A Newton iteration method is employed to solve the non-linear system of equations. The initial solution at each time step is obtained from a second order accurate explicit Runge-Kutta method.

The solutions for the 2-D vortex cavity show the presence of a resonance frequency. This resonance frequency can be derived from Eq. (4.19) if an azimuthal velocity distribution is assumed and if we assume relatively small perturbations of $r_{c}$ such that the formulation can be linearised. A solution for the resonance frequency is only found if the integral of the azimuthal velocity distribution can be linearised as well. As the cavitating Lamb-Oseen vortex model discussed in Chapter 3 is too complicated for this purpose, the non-cavitating vortex model of Rosenhead (1931), also introduced by Burnham and Hallock (1982), is used. The azimuthal

\footnotetext{
${ }^{2}$ It was noted by H.W.M. Hoeijmakers that the time derivative of the potential is missing in this (Bernoulli) equation. This time derivative corresponds to the last term in Eq. (4.17).
} 
velocity distribution and resulting pressure distribution are given by

$$
\begin{gathered}
v(r, t)=\frac{\Gamma_{\infty}}{2 \pi} \frac{r}{r_{v}^{2}+r^{2}} \\
p_{\infty}-p(r, t)=\rho \int_{r}^{\infty} \frac{v^{2}}{r} d r=\frac{\rho}{2}\left(\frac{\Gamma_{\infty}}{2 \pi}\right)^{2} \frac{1}{r_{v}^{2}+r^{2}},
\end{gathered}
$$

with $r_{v}$ the size of the viscous core. It is assumed here that the expressions for the non-cavitating vortex can also be used to describe the cavitating vortex with the values at the cavity interface given by substituting $r=r_{c}$. The validity of this assumption has been discussed in Section 3.3.3. We now use $r_{c}(t)=\bar{r}_{c}+\hat{r}_{c} \exp (-i \omega t)$, with $\omega$ the resonance frequency of the small disturbance of amplitude $\hat{r}_{c}$ with respect to the mean cavity size $\bar{r}_{c}$. The equations are linearised using $\hat{r}_{c} / \bar{r}_{c} \ll 1$. The velocity integral in Eq. (4.22), which gives the pressure difference between $r=r_{\infty}$ and $r=r_{c}$, then reads

$$
\rho \int_{r_{c}}^{\infty} \frac{v^{2}}{r} d r=\frac{\rho}{2}\left(\frac{\Gamma_{\infty}}{2 \pi}\right)^{2} \frac{1}{r_{v}^{2}+\bar{r}_{c}^{2}}\left[1-\frac{2 \bar{r}_{c}}{r_{v}^{2}+\bar{r}_{c}^{2}} \hat{r}_{c} \exp (-i \omega t)\right] .
$$

Substituting Eq. (4.23) into Eq. (4.19) leads to two equations, one equation for $\bar{r}_{c}$ and one for $\hat{r}_{c}$. The equation for $\bar{r}_{c}$ gives a relation between $\Gamma_{\infty}$ and $\left(\bar{p}_{\infty}-p_{c}\right)$ which is substituted into the equation for $\hat{r}_{c}$. The equation for $\hat{r}_{c}$ is linearised for small $\hat{r}_{c} / \bar{r}_{c}$, and, using $p_{c}=p_{v}, r_{\infty}=r_{\text {ref }}$, and $\bar{p}_{\infty}=p_{\text {ref }}$, it becomes

$$
\rho \bar{r}_{c}^{2} \ln \left(r_{\mathrm{ref}} / \bar{r}_{c}\right) \omega^{2}-\frac{2 \bar{r}_{c}^{2}}{r_{v}^{2}+\bar{r}_{c}^{2}}\left(p_{\mathrm{ref}}-p_{v}\right)=0 .
$$

In this expression, the first term is proportional to the added mass $m_{a}$ of the cylindrical vortex cavity,

$$
m_{a}=\rho 2 \pi \bar{r}_{c}^{2} \ln \left(r_{\text {ref }} / \bar{r}_{c}\right) .
$$

The second term in Eq. (4.24) can be interpreted as the stiffness while the equation does not contain a term describing damping. The resonance frequency is given by

$$
\left(\frac{\omega \bar{r}_{c}}{W}\right)^{2}=K_{\sigma} \frac{1}{\ln \left(r_{\mathrm{ref}} / \bar{r}_{c}\right)}
$$

with the stiffness coefficient $K_{\sigma}$ defined as

$$
K_{\sigma}=\frac{\sigma_{W}}{1+\left(r_{v} / \bar{r}_{c}\right)^{2}}
$$

and with $W$ a reference velocity that is introduced to non-dimensionalise the pressure difference such that the cavitation number $\sigma_{W}=\left(p_{\text {ref }}-p_{v}\right) / \frac{1}{2} \rho W^{2}$ is obtained. The viscous correction term can also be written as the ratio of either velocity or square root of pressure at the cavity interface divided by the value for a potential flow vortex. Using Eqs. (4.21) and (4.22), the stiffness coefficient can also be written as

$$
K_{\sigma}=\left(\frac{v_{c}}{W}\right)^{2}=K_{v_{c}}
$$


with $v_{c}$ the azimuthal velocity at the cavity interface. Subscript $v_{c}$ has been introduced in the stiffness coefficient to distinguish $K_{v_{c}}$ from $K_{\sigma}$. For a potential flow vortex, as well as for the Rosenhead vortex, we find $K_{\sigma}=K_{v_{c}}$, but this may not be generally valid.

The formulation for potential flow is obtained from Eq. (4.26) by taking $r_{v}=0$, which gives

$$
\begin{gathered}
\frac{\omega \bar{r}_{c}}{W}=\sqrt{\sigma_{W}} \frac{1}{\sqrt{\ln \left(r_{\mathrm{ref}} / \bar{r}_{c}\right)}}, \\
\frac{\omega \bar{r}_{c}}{W}=\frac{\Gamma_{\infty}}{2 \pi \bar{r}_{c} W} \frac{1}{\sqrt{\ln \left(r_{\mathrm{ref}} / \bar{r}_{c}\right)}} .
\end{gathered}
$$

The use of boundary condition (4.20) in combination with Eq. (4.11) leads to an expression for the resonance frequency that, for potential flow, is given by

$$
\frac{\omega \bar{r}_{c}}{W}=\frac{\Gamma_{\infty}}{2 \pi \bar{r}_{c} W} \frac{1}{\sqrt{\ln \left(r_{\infty} / \bar{r}_{c}\right)}} .
$$

The resonance frequency $\omega$ now depends on the location $r_{\infty}$ of the outer boundary instead of $r_{\text {ref. }}$ Equation (4.31) is the one given by Franc and Michel (2004).

It is illustrative to interpret the vortex cavity dynamics in terms of the energy balance, similar as performed for the Rayleigh-Plesset equation by e.g. Franc and Michel (2004). If we take the limit $s_{n} \rightarrow \infty$, the second term in Eq. (4.19) can be neglected and the equation becomes

$$
\left(r_{c} \ddot{r}_{c}+\dot{r}_{c}^{2}\right) \ln \left(r_{\mathrm{ref}} / r_{c}\right)-\frac{\dot{r}_{c}^{2}}{2}-\int_{r_{c}}^{r_{n}} \frac{v^{2}}{r} d r+\frac{p_{n}-p_{v}}{\rho}=0 .
$$

The first two terms can be written as

$$
\left(r_{c} \ddot{r}_{c}+\dot{r}_{c}^{2}\right) \ln \left(r_{\mathrm{ref}} / r_{c}\right)-\frac{\dot{r}_{c}^{2}}{2}=\frac{1}{2 r_{c} \dot{r}_{c}} \frac{d}{d t}\left[r_{c}{ }^{2} \dot{r}_{c}^{2} \ln \left(r_{\mathrm{ref}} / r_{c}\right)\right] .
$$

Inserting Eq. (4.33) in Eq. (4.32) and using the vortex model of potential flow for $v$ leads to

$$
\frac{d}{d t}\left[\rho r_{c}^{2} \dot{r}_{c}^{2} \ln \left(r_{\mathrm{ref}} / r_{c}\right)\right]-\rho\left(\frac{\Gamma_{\infty}}{2 \pi}\right)^{2} \frac{\dot{r}_{c}}{r_{c}}+2 r_{c} \dot{r}_{c}\left(p_{n}-p_{v}\right)=0,
$$

which can be written as

$$
\frac{d}{d t}\left[\rho r_{c}^{2} \dot{r}_{c}^{2} \ln \left(r_{\mathrm{ref}} / r_{c}\right)\right]+\frac{d}{d t}\left[\rho r_{c}^{2} \ln \left(r_{\mathrm{ref}} / r_{c}\right)\left(\frac{\Gamma_{\infty}}{2 \pi r_{c}}\right)^{2}\right]+\frac{d}{d t}\left[r_{c}^{2}\left(p_{n}-p_{v}\right)\right]=0 .
$$

Introducing $v_{c}=\Gamma_{\infty} / 2 \pi r_{c}$, this equation is simplified by using Eq. (4.25) for the added mass $m_{a}$ resulting in

$$
\frac{d}{d t}\left[m_{a}\left(\dot{r}_{c}^{2}+v_{c}^{2}\right)\right]+\frac{d}{d t}\left[2 \pi r_{c}^{2}\left(p_{n}-p_{v}\right)\right]=0
$$

in which the first term corresponds to the change in kinetic energy, given by the product of the added mass and the velocities at the cavity interface and the second term to the change in potential energy. 


\subsubsection{Results for constant free-stream pressure}

First, several cases will be analysed in which the free-stream pressure $p_{\text {ref }}$ is kept constant. All results have been computed with the outer boundary located at $r_{n}=1.0 \mathrm{~m}$, a vortex strength $\Gamma_{\infty}=1.0 \mathrm{~m}^{2} \mathrm{~s}^{-1}$ and a viscous core radius in non-cavitating flow $r_{v}=0.01 \mathrm{~m}$. The density equals $\rho=1000 \mathrm{~kg} \mathrm{~m}^{-3}$ and the molecular viscosity equals $\mu=1.0 \times 10^{-3} \mathrm{Pas}$. The azimuthal velocity distribution is initialized by the analytical solution of the cavitating Lamb-Oseen vortex (Section 3.2.1). With the user prescribed initial cavity radius, the pressure difference $\left(p_{\text {ref }}-p_{v}\right)$ is computed from this solution and this pressure difference is kept constant in the numerical simulation unless stated otherwise.

The grid sensitivity of the solution of the system of equations is investigated first for a prescribed variation of $r_{c}$. The azimuthal velocity distribution can directly be obtained by solving Eq. (4.9) which reduces to a linear equation for this situation. Results are presented for a grid composed of 513 vertices and 513 time steps. The grid spacing $\Delta s / r_{n}$ equals $4.0 \times 10^{-6}$ near the cavity interface and gradually increases to $1.9 \times 10^{-3}$ at the outer boundary. The variation of $r_{c}$ with time is prescribed by one period, $T$, of a cosine function such that the minimum value is $10 \%$ of the initial value, $r_{c}(t) / r_{v}=1.0-0.9\left[\frac{1}{2}-\frac{1}{2} \cos (\omega T)\right]$. For all results presented, the viscous core size for non-cavitating flow, $r_{v}$, is used to non-dimensionalise the radial position $r$ and the cavity radius $r_{c}$.

Figure 4.1 presents the prescribed variation of $r_{c}(t)$, together with the computed variation with time of the viscous core radius and the azimuthal velocity at the cavity interface. As usual, the viscous core radius is defined as the radius at which the maximum azimuthal velocity is obtained. The strong increase of the azimuthal velocity at the cavity interface shows that the collapse of the cavity is inertia driven, which was already suggested by Franc and Michel (2004). With decreasing $r_{c}$, the region between the cavity interface and the viscous core decreases. The radial distribution of the azimuthal velocity is presented in Figure 4.2 for two time steps, corresponding to the first time step and the time step at which the cavity radius reaches its minimum value. The figure clearly shows that, when $r_{c}$ becomes close to its minimum value, the effect of viscosity is limited to a very thin region in which the solution is adjusted to match the zero shear stress boundary condition (4.6).

A solution verification study has been carried out according to ASME V\&V 20-2009 (2009) using systematic grid and time step refinement. The convergence rate of the discretisation error is investigated by assuming the numerical solution for variable $\phi$ at refinement step $i$ to behave as

$$
\phi\left(h_{i}\right)=\phi_{e}+\alpha\left(h_{i} / h_{1}\right)^{p}
$$

with $\phi_{e}$ the Richardson extrapolated value, $h_{i}$ the step size and $h_{1}$ a reference (finest) step size. The analysis consists of finding the three unknowns $\left(\phi_{e}, \alpha, p\right)$ of Eq. (4.21) for which the leastsquares approach of ASME V\&V 20-2009 (2009) has been adopted. The uncertainty for each numerical solution is given by the Grid Convergence Index (GCI) according to Eça and Hoekstra (2002) and is presented by error bars using a factor of safety $F_{s}=1.25$. Geometrically similar grids have been used. The analysis is made for the time step at which the cavity has its minimum size. Results are presented in Figure 4.3a for the grid refinement study (using 513 time steps) and in Figure $4.3 \mathrm{~b}$ for the time step refinement study (using 513 vertices). For the grid nearly second order accuracy is found, while the order of convergence for the time step is smaller 

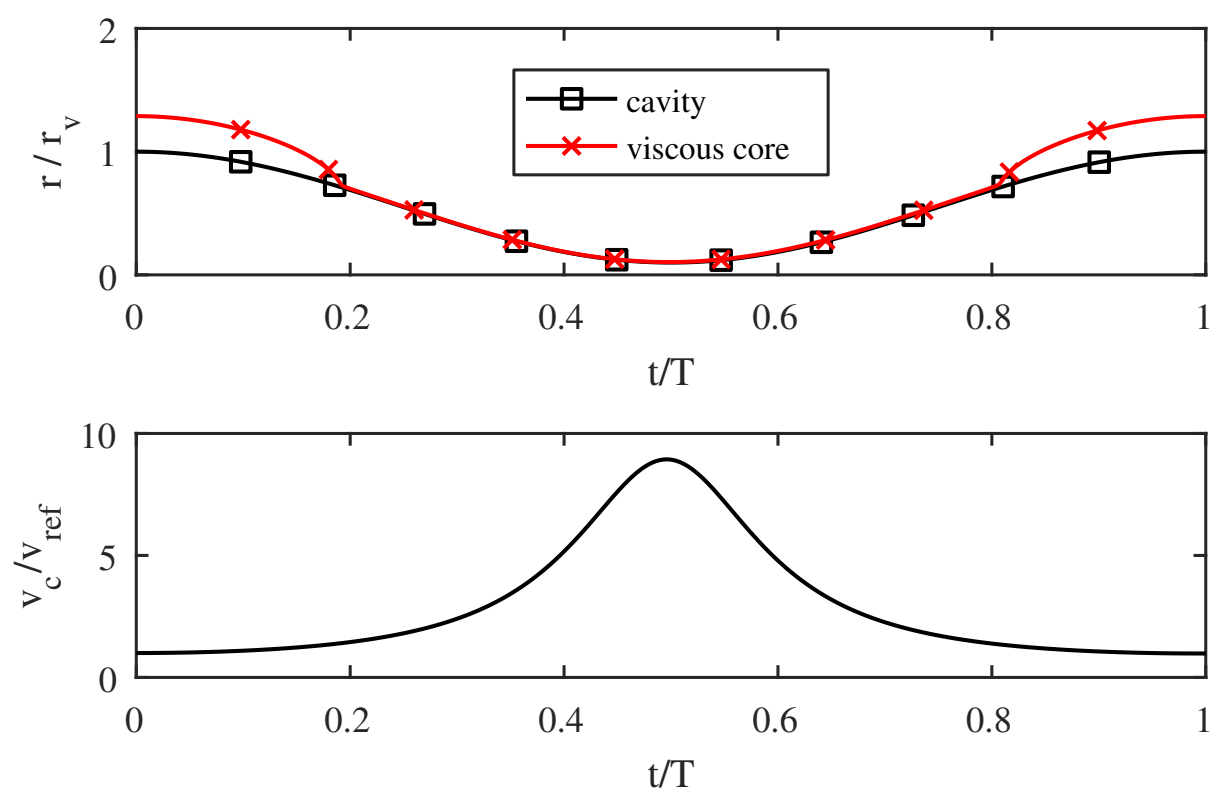

Figure 4.1: Results for a prescribed cavity motion with $T=16 \mathrm{~ms}$. Top figure shows the temporal behaviour of the viscous core and the (prescribed) cavity radius. Bottom figure shows the variation of the azimuthal velocity at the cavity interface. Parameter $r_{\mathrm{V}}$ corresponds to the viscous core size for non-cavitating flow and $v_{\text {ref }}$ corresponds to $v_{c}$ at $t=0$.

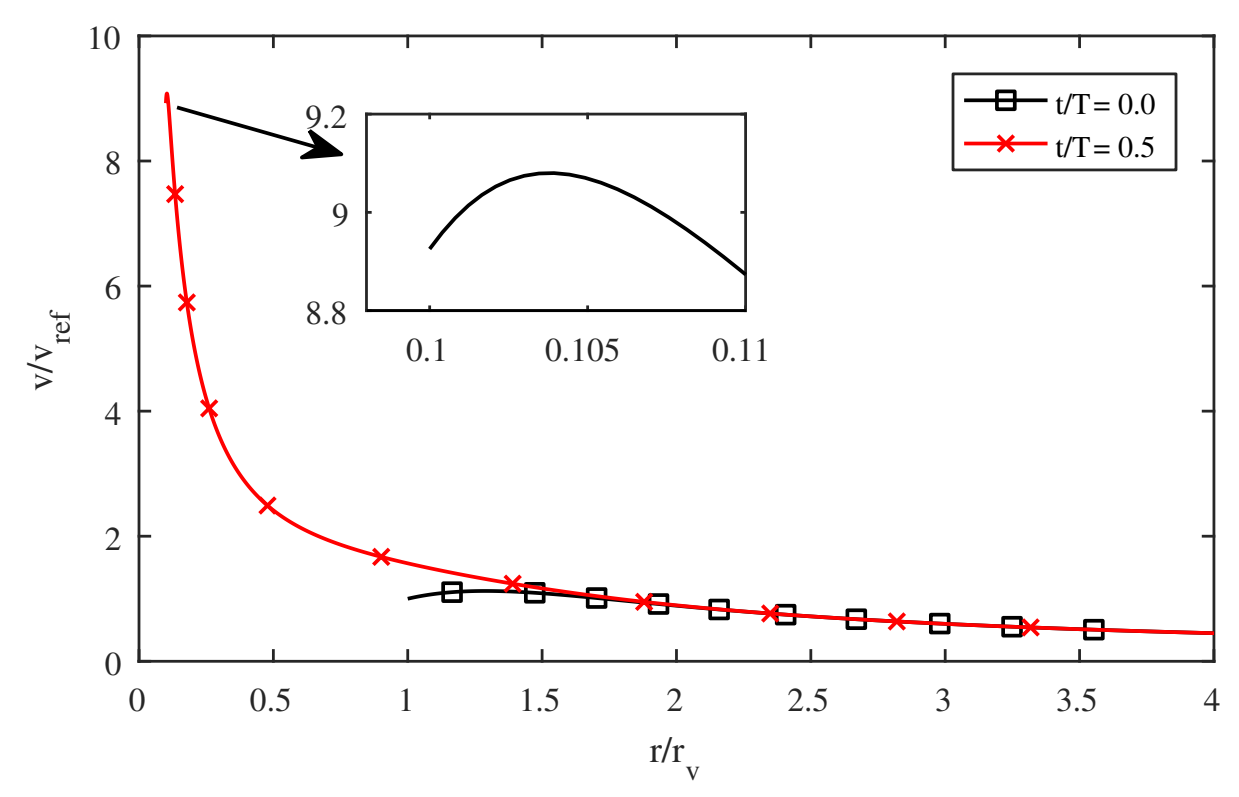

Figure 4.2: Radial distribution of the azimuthal velocity for two time instants for the prescribed cavity radius as a function of time.

at $p=1.33$. It is concluded that the uncertainty in maximum azimuthal velocity presented in Figure 4.2, for which $h_{i} / h_{1}=4$ and $\Delta t_{i} / \Delta t_{1}=4$, is $0.06 \%$ due to grid resolution and $0.03 \%$ due to time step resolution, respectively. 


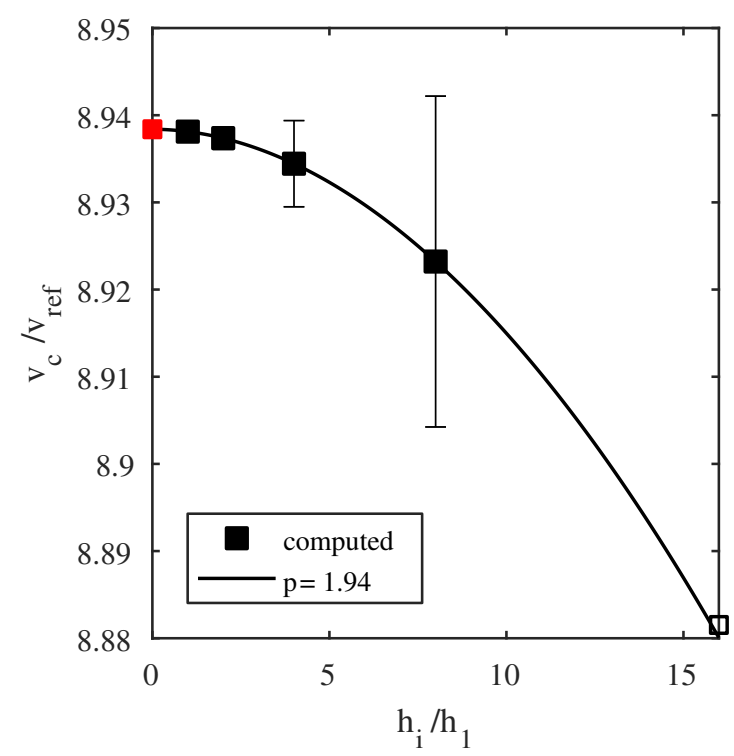

a) Grid refinement, $\Delta t / \Delta t_{1}=4$

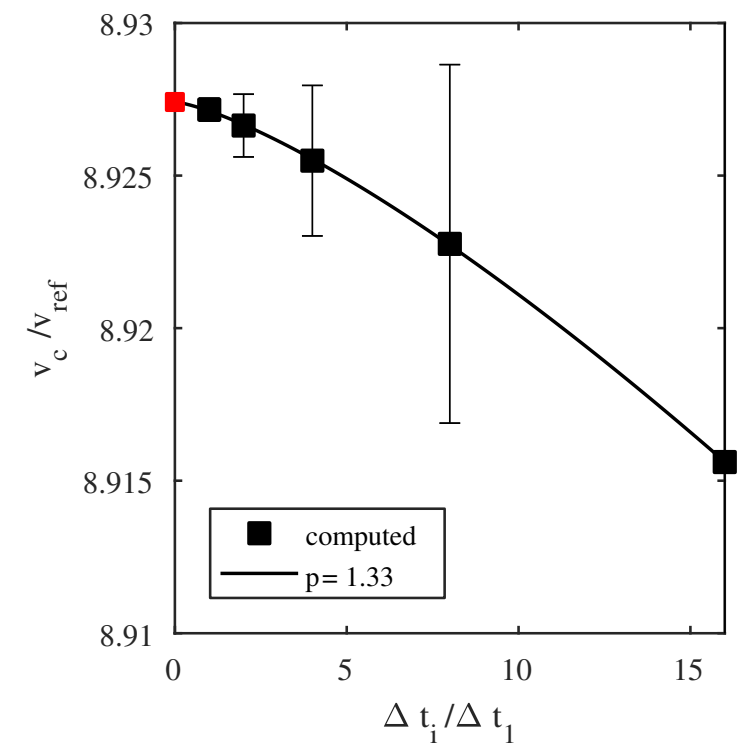

b) Time-step refinement, $h / h_{1}=4$

Figure 4.3: Sensitivity studies for the azimuthal velocity at the cavity interface for the prescribed cavity radius as function of time at $t / T=0.5$. Finest grid $h_{1}$ corresponds to 2049 vertices, finest time step $\Delta t_{1}$ corresponds to 2049 steps per cycle.

Next, the resonance frequency of a cavitating vortex is investigated by solving the complete system of equations for a steady state initial solution and keeping the vortex strength and reference pressure constant. The resonance frequency is easily excited by setting the reference pressure to a value of $99 \%$ of the equilibrium value. A variable time step procedure was used by comparing the temporal discretisation error between a second-order explicit Runge-Kutta method and a second-order Heun method. The time step was adjusted until the difference in solution was equal to a specified tolerance. The obtained solution was then used to initialize the non-linear system of equations that was solved with the implicit approach discussed earlier. This adaptive time stepping procedure is sometimes referred to as Milne's approach (Butcher, 2008). The grid consists of 257 vertices. For all computations, the number of time steps within one oscillatory period is in the order of $10^{3}$. An example of the history in cavity size is presented in Figure 4.4a. Due to the inertia of the fluid, an oscillatory motion occurs around a new equilibrium for the cavity size which slowly decreases in time due to diffusion. The change in cavity size with respect to the initial value becomes smaller for smaller initial cavities.

The resonance frequency is computed from the time difference between the occurrence of the positive and of the negative extrema of the time derivative of the cavity radius. A solution verification study showed that the uncertainty in the frequency for the variable time step procedure for the smallest and largest cavity radius is less than $0.1 \%$. The variation of the resonance frequency with initial cavity size for fixed viscous core size is presented in Figure 4.4b. Doubling the grid size gave a difference in frequency of less than $0.06 \%$. Results show that the resonance frequency approaches the analytical inviscid flow solution for cavity sizes larger than the viscous core size (for non-cavitating flow) while a strong reduction in frequency is observed for smaller cavity radii.

For the resonance frequency in Figure $4.4 \mathrm{~b}$ described by Eq. (4.30), the vortex strength as 


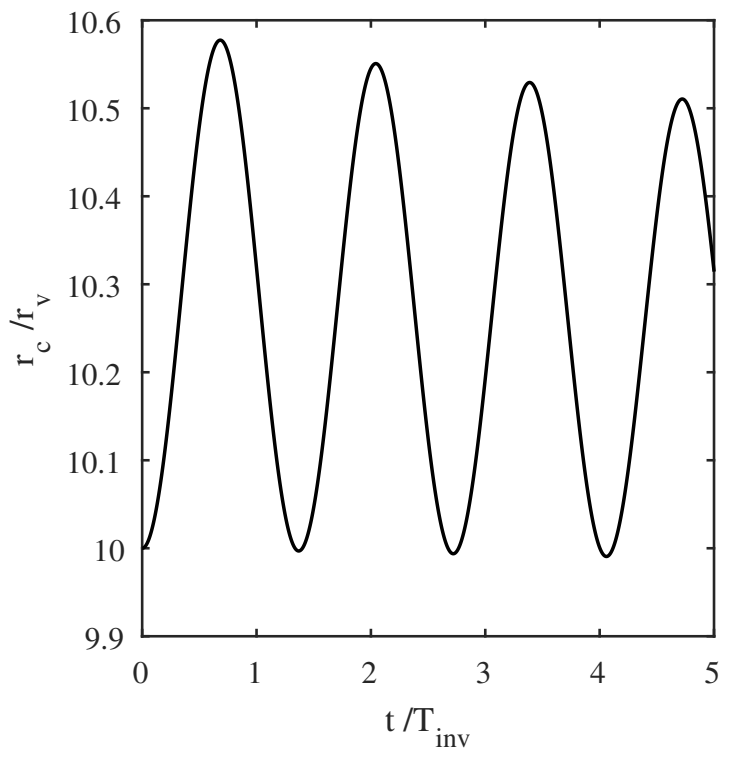

a) Typical time trace

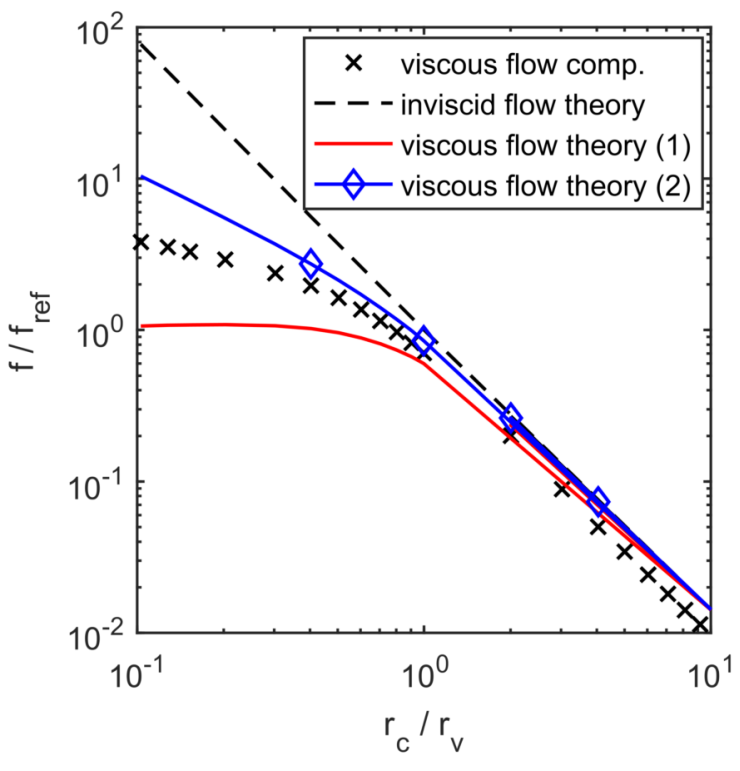

b) Resonance frequency

Figure 4.4: Results of unsteady flow computations. The reference frequency $f_{\text {ref }}$ is for inviscid flow theory at $\bar{r}_{c} / r_{\mathrm{v}}=1$. Results for 'inviscid flow theory', viscous flow theory (1)' and 'viscous flow theory (2)' correspond to Eqs. (4.30), (4.26) and (4.29), respectively. Parameter $r_{\mathrm{v}}$ corresponds to the viscous core size for non-cavitating flow.

prescribed in the computation is used. The cavity size and cavitation number used in Eqs. (4.26) and (4.29) correspond to the mean cavity size and cavitation number of the computation. This relation depends on the viscous core size. The computed solution is in between the two analytical formulations for viscous flow theory. It shows that the additional correction on the stiffness is required but that the applied vortex model for non-cavitating flow leads to a correction that is too large.

The computed resonance frequency in Bosschers (2009c), in which the coordinate transformation $s=r / r_{c}$ was used, does agree with the analytical solution for inviscid flow for large cavity size. The reason for the discrepancy between the results presented here and those presented in Bosschers (2009c) requires further investigation.

\subsubsection{Results for a change in free-stream pressure}

In this section, we analyse the effect of an increase of $p_{\text {ref }}$ which results in a reduction in $r_{c}$. As already shown in Figure 4.1, the reduction in $r_{c}$ is accompanied by a strong reduction of the thickness of the vorticity layer around the cavity. Numerical tests revealed that the solution was unstable when an insufficient number of grid points were present in this region or when the time step was too large. The results presented here have been obtained using in total 1025 vertices and a time step proportional to the inverse of the interface velocity ${ }^{3}$ with a maximum value of $10^{-6} \mathrm{~s}$. For the initial size of the cavity, $r_{c} / r_{v}=1.0$ was used. The reference pressure

\footnotetext{
${ }^{3}$ This suggests the presence of a CFL condition in relation to the moving boundary, but this was not further investigated.
} 
at the outer boundary increases within $0.5 \mathrm{~ms}$ to a value of $153 \%$ of the initial value. This pressure corresponds to a non-dimensional cavity radius $r_{c} / r_{v}=0.65$ if the analytical solution of Eq. (3.21) is used. Results for the cavity radius are presented in Figure 4.5a, upper graph. The cavity radius quickly decreases and then oscillates around an non-dimensional value of about $r_{c} / r_{v}=0.55$. The parameter $T$, used to non-dimensionalise time, is the period that corresponds to the resonance frequency given by Eq. (4.29) with the cavity size and reference pressure taken from the initial solution resulting in $T=0.01 \mathrm{~s}$.

The variation in time of the azimuthal velocity at the cavity interface $v_{c}$ is presented in Figure $4.5 \mathrm{~b}$, top graph. The radial variation of the azimuthal velocity for three time steps is presented in Figure 4.6a. When $r_{c}$ has its smallest value, at $t / T=0.418$, the azimuthal velocity distribution resembles that of a potential flow solution and the effect of the vorticity layer at the cavity is negligible, similar to results presented in Figure 4.2. However, when the cavity size obtains its largest value, at $t / T=0.704$, the distribution of the azimuthal velocity changes significantly in the vorticity layer and becomes convex curved instead of concave. At this time step, two inflexion points are present on either side of the maximum of the azimuthal velocity.

Figure $4.6 \mathrm{~b}$ shows the phase space of the cavity motion. The egg-shape of the curve shows that the extrema of the interface velocity occur for a cavity size that is smaller than the mean value indicating that the velocity of the cavity interface changes more rapidly for small cavity size than for large cavity size. This demonstrates that the instantaneous frequency increases with decreasing cavity size, thereby following the same trend as observed in Figure 4.4b. The curve is symmetric around $\dot{r}_{c}=0$ showing that the absolute value of the velocity of the cavity interface in the growth phase is identical to that in the collapse phase.

The collapse of a 2-D vortex can be compared with the collapse of a 2-D bubble. The equation of motion for a 2-D bubble corresponds to that of a 2-D vortex as described by Eqs. (4.11) and (4.36) but with the azimuthal velocity identical to zero. The integral of the azimuthal velocity in Eq. (4.11) can be interpreted as a reduction of the pressure between the outer boundary and the cavity interface. For a stationary cavity, this pressure reduction equals the difference between outer pressure and vapour pressure. Increasing the pressure difference leads to a smaller cavity and a decrease leads to a larger cavity. The variation of the integral with time, denoted as $p_{h}$, is presented in the bottom graph of Figure $4.5 \mathrm{~b}$. Following the initial increase of $p_{\text {ref }}$, the cavity reduces in size, which leads to a rapid increase of $p_{h}$. The bottom graph of Figure 4.5a shows the scaled acceleration term of the cavity size, denoted by $p_{a 1}$. This term is initially negative accelerating the collapse, but rapidly becomes positive due to the positive value of $p_{h}$, thereby dampening the cavity collapse after which a rebound occurs.

The collapse of a 2-D vortex thus occurs less fast than the collapse of a 2-D bubble. However, it is remarked that a vortex cavity may exist longer in a flow than a bubble cavity because the azimuthal velocity gives a reduction of the pressure in the vortex core. The resonance of the vortex cavity occurs without the presence of non-condensable gas in the cavity as required for resonance of a bubble cavity.

The conclusion that the velocity integral dampens the collapse was already drawn in Franc and Michel (2004) for an inviscid cavitating vortex. The present results confirm this for a viscous cavitating vortex. It is also stated in Franc and Michel (2004) that an infinite pressure difference is required for the complete radial collapse of a cavitating vortex. This statement is probably based on the observation that, for potential flow, the stiffness of the system tends to in- 

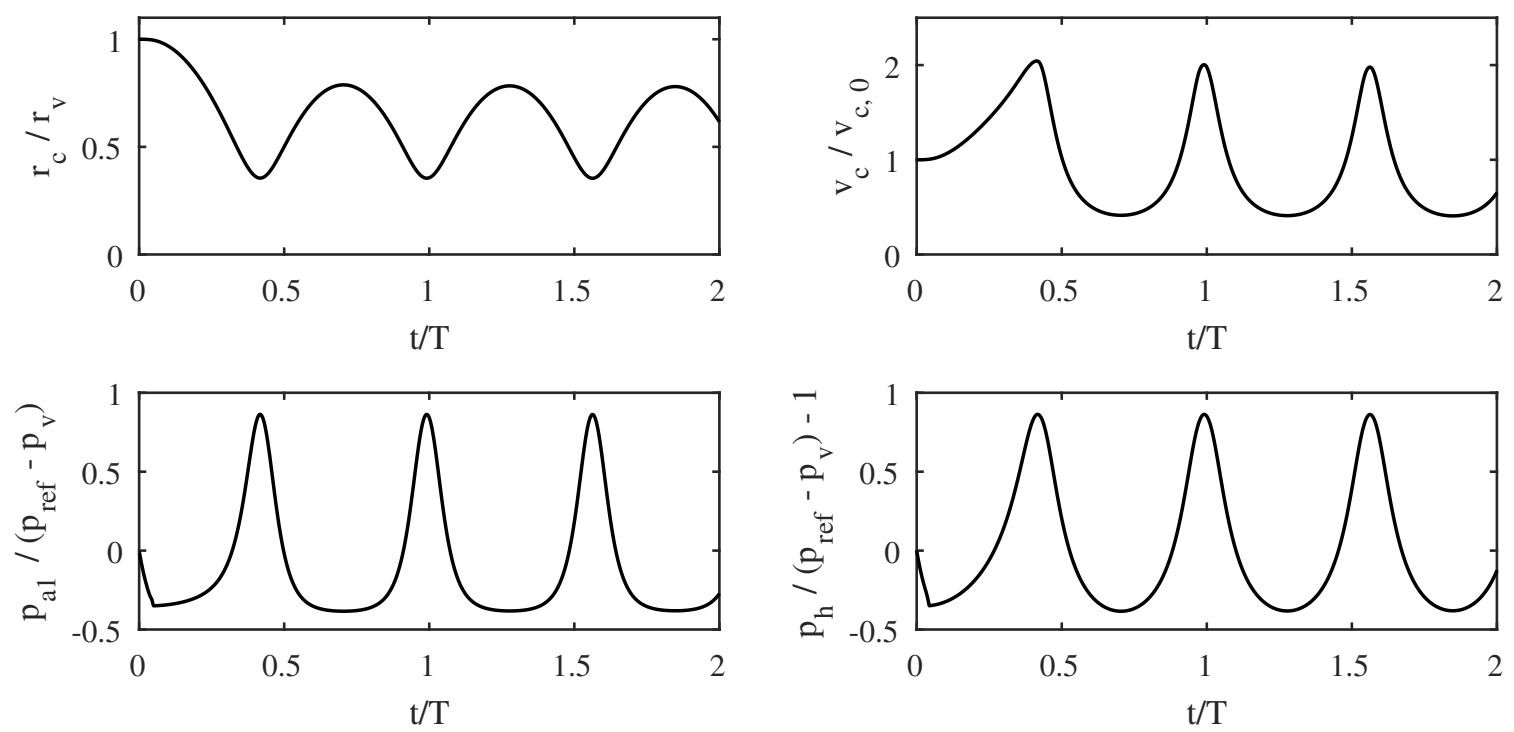

a) Top:cavity size

Bottom: pressure due to radial motion

b) Top: azimuthal velocity at cavity

Bottom: pressure due to azimuthal velocity

Figure 4.5: Temporal variation of various parameters induced by a change in reference pressure at the outer boundary, $p_{a 1}=\rho \ddot{r}_{c} r_{c} \ln \left(r_{\mathrm{ref}} / r_{c}\right), p_{h}=\int_{0}^{s_{n}} v^{2} /\left(r_{c}+s\right) d s$.

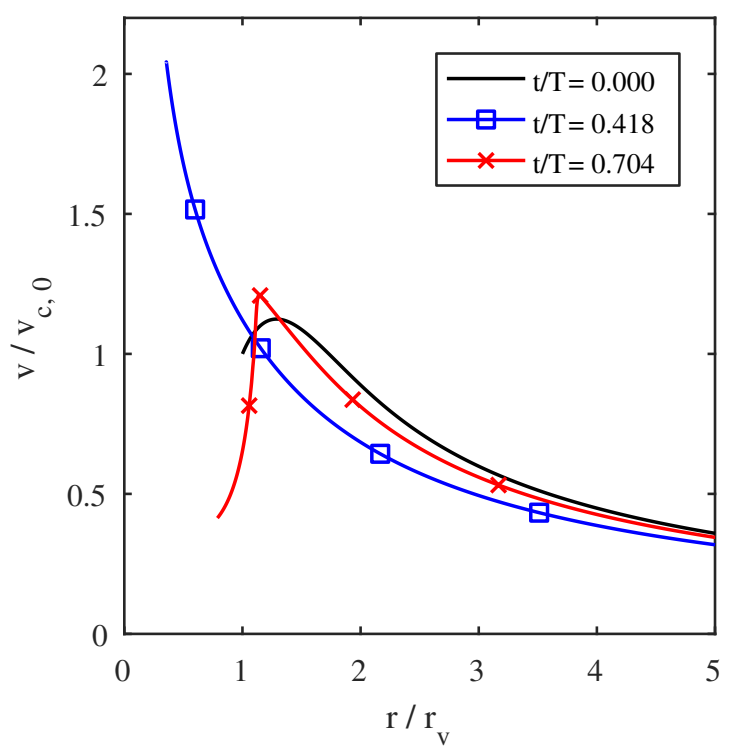

a) Velocity distribution

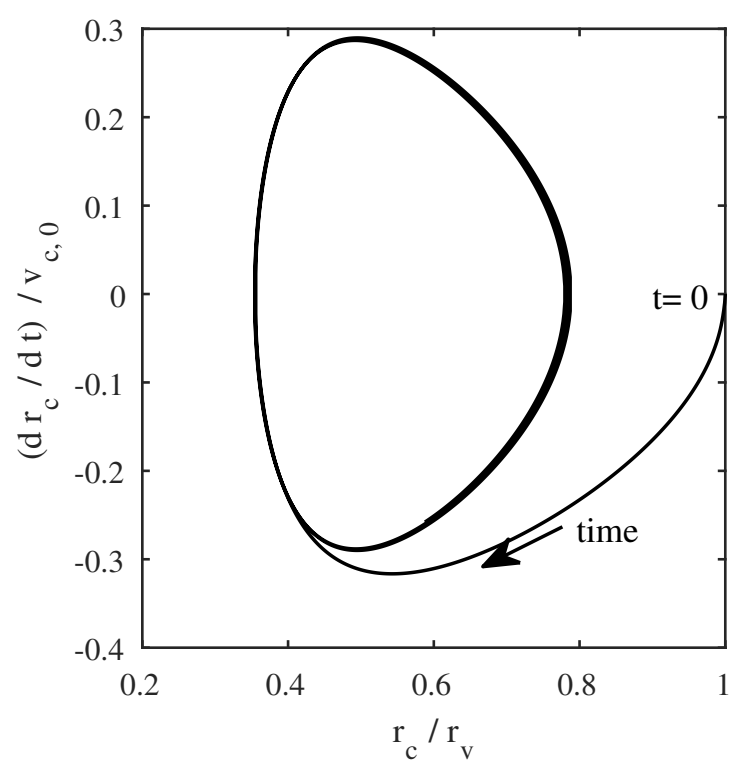

b) Phase space

Figure 4.6: Distribution of the azimuthal velocity at three time steps and the phase space plot, both due to a change in reference pressure at the outer boundary.

finity when the cavity size approaches zero. The present computations show that this conclusion also holds for viscous flow as Figures 4.5 and 4.6 show that in the first phase of the collapse the motion is inertia driven and the distribution of the azimuthal velocity resembles that of a 
potential flow vortex. Unfortunately, due to lack of convergence of the numerical procedure, no results could be obtained for a full collapse of the cavity. This lack of convergence might be related to the apparent presence of a CFL-condition for the relation between the radial velocity of the interface, the time step and the grid size near the interface, that was suggested by computational studies. The very large azimuthal velocity near the cavity interface during the collapse in combination with the zero shear-stress boundary condition leads to a very thin vorticity layer around the cavity as shown in Figure 4.2. This requires a very small grid size near the cavity interface. During the collapse, the radial velocity of the interface increases in magnitude and very small time steps were required. The lack of convergence requires further research.

A deficiency of the present method is that the flow inside the cavity has been neglected. Even though it was shown in Chapter 3 that this is a valid assumption for steady flow conditions, it is expected that in the final phase of the collapse, featuring a very large azimuthal velocity at the cavity interface, this assumption is no longer valid. Also, at the end of the collapse, i.e. in absence of cavitation, the azimuthal velocity has to be zero at the centre of the vortex, but the corresponding physical mechanism required for this appears to be absent in the present computational model.

It needs to be remarked that the collapse of a 3-D tip vortex cavity typically only occurs within a limited axial extent of the cavity. Therefore, the change in azimuthal velocity and the pressure perturbation due to the change in azimuthal velocity is much smaller for 3-D flow than for 2-D flow. As this pressure perturbation determines the stiffness of the system, it is concluded that the stiffness in 2-D flow is larger than in 3-D flow. Therefore, we need to be careful in drawing general conclusions on vortex cavity dynamics from the 2-D flow simulations.

\subsection{The dispersion relation for a 3-D columnar vortex cavity}

\subsubsection{Derivation}

The starting point for the derivation of the dispersion relation is the convected Helmholtz equation for a disturbance velocity potential $\varphi^{\prime}$ (Rienstra and Hirschberg, 2006),

$$
\nabla^{2} \varphi^{\prime}-\frac{1}{c^{2}}\left(\frac{\partial}{\partial t}+\mathbf{U} \cdot \nabla\right)^{2} \varphi^{\prime}=0
$$

in which $c$ corresponds to the speed of sound, $\mathbf{U}$ to the mean velocity vector and $t$ to time. Both the mean velocity and the velocity perturbation need to be irrotational and isentropic, i.e. $\nabla \times \mathbf{U}=0$ and $\nabla \times \mathbf{u}^{\prime}=0$, so that $\mathbf{u}^{\prime}=\nabla \varphi^{\prime}$, with $\mathbf{u}^{\prime}$ the velocity perturbation. Furthermore, the mean density and speed of sound need to be uniform. A cylindrical coordinate system $(r, \theta, z)$ is adopted with a harmonic variation of the disturbance potential given by

$$
\varphi^{\prime}(r, \theta, z, t)=\phi(r) \exp \left[i\left(k_{z} z+n \theta-\omega t\right)\right]
$$

in which $\phi(r)$ corresponds to a potential that is only a function of radius $r, k_{z}$ corresponds to the axial wavenumber, $n$ to the azimuthal wavenumber which must be an integer, and $\omega$ to the angular frequency. The mean velocity contains an azimuthal velocity component $V$, an axial 
velocity component $W$, but the radial velocity component equals zero, $\mathbf{U}=(0, V, W)$, which gives

$$
\frac{d^{2} \phi}{d r^{2}}+\frac{1}{r} \frac{d \phi}{d r}+\left[-k_{z}^{2}-\frac{n^{2}}{r^{2}}+\frac{1}{c^{2}}\left(\omega-W k_{z}-\frac{n V}{r}\right)^{2}\right] \phi=0 .
$$

Introducing a potential flow vortex for the azimuthal velocity component gives $V=\Gamma_{\infty} /(2 \pi r)$, which leads to

$$
\begin{aligned}
\frac{d^{2} \phi}{d r^{2}}+\frac{1}{r} \frac{d \phi}{d r}+ & {\left[-k_{z}^{2}+\frac{1}{c^{2}}\left(2 \omega-W k_{z}\right)^{2}-\right.} \\
& \left.\frac{1}{r^{2}}\left\{n^{2}+\frac{1}{c^{2}}\left[\left(\omega-W k_{z}\right)\left(\frac{n \Gamma_{\infty}}{2 \pi}\right)\right]\right\}+\frac{1}{r^{4}}\left(\frac{n \Gamma_{\infty}}{2 \pi c}\right)^{2}\right] \phi=0,
\end{aligned}
$$

Introducing

$$
\begin{aligned}
k_{r}^{2} & =\frac{1}{c^{2}}\left(\omega-W k_{z}\right)^{2}-k_{z}^{2} \\
v^{2} & =n^{2}+\frac{1}{c^{2}}\left\{2\left(\omega-W k_{z}\right)\left(n \frac{\Gamma_{\infty}}{2 \pi}\right)\right\} \\
\gamma^{2} & =\left(\frac{n \Gamma_{\infty}}{2 \pi c}\right)^{2}
\end{aligned}
$$

leads to

$$
\frac{d^{2} \phi}{d r^{2}}+\frac{1}{r} \frac{d \phi}{d r}+\left(k_{r}^{2}-\frac{v^{2}}{r^{2}}+\frac{\gamma^{2}}{r^{4}}\right) \phi=0
$$

The parameter $k_{r}$ is the acoustic wavenumber component in radial direction. With the substitution $r=\sqrt{\gamma / k_{r}} \exp (x)$, Eq. (4.45) becomes Mathieu's modified differential equation,

$$
\frac{d^{2} \phi}{d x^{2}}-\left(v^{2}-2 \gamma k_{r} \cosh 2 x\right) \phi=0
$$

Unfortunately, this equation does not have a closed form analytical solution. However, for the current field of application, the speed of sound $c$ is very large relative to the mean velocity $\mathbf{U}$, and we therefore assume

$$
\begin{aligned}
& v=n, \\
& \gamma=0 .
\end{aligned}
$$

For the mode $n=0$, this approximation is exact. Equation (4.45) then becomes a cylinder equation,

$$
\frac{d^{2} \phi}{d r^{2}}+\frac{1}{r} \frac{d \phi}{d r}+\left(k_{r}^{2}-\frac{n^{2}}{r^{2}}\right) \phi=0
$$

Considering that the propagation direction of the acoustic waves is only in outward direction, i.e. reflections are neglected, the solution for the disturbance potential is given by a Hankel function of the first kind, $H_{n}^{(1)}$,

$$
\varphi^{\prime}(r, \theta, z, t)=\hat{\phi} H_{n}^{(1)}\left(k_{r} r\right) \exp \left[i\left(k_{z} z+n \theta-\omega t\right)\right],
$$




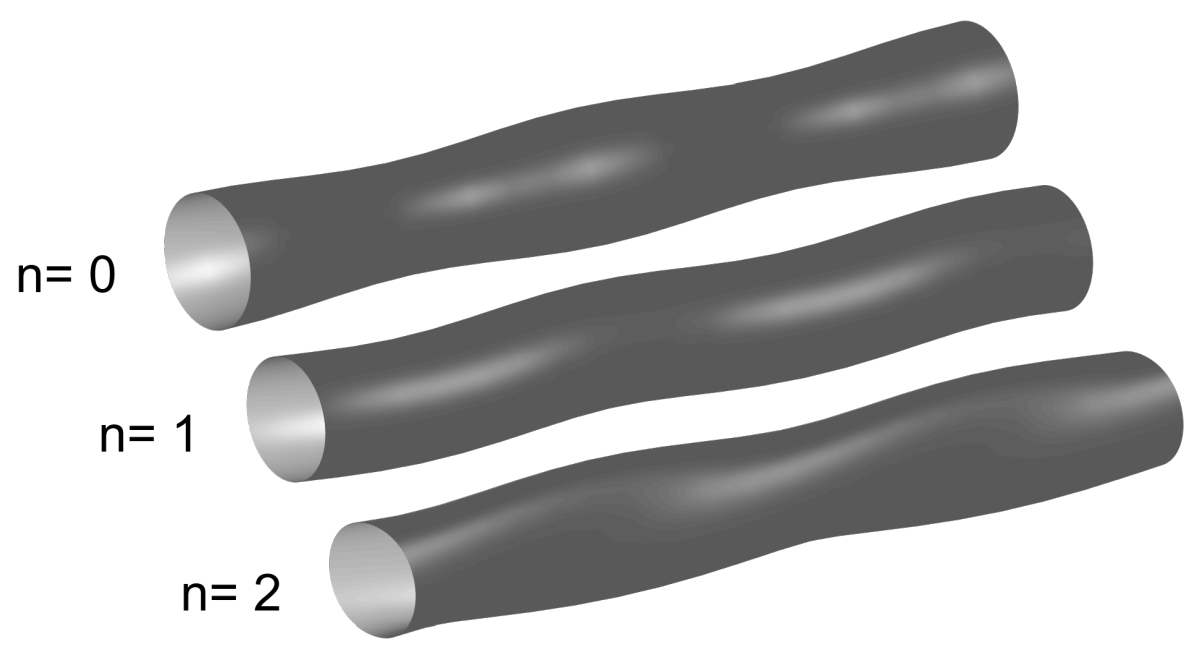

Figure 4.7: Deformation modes of the vortex cavity.

in which $\hat{\phi}$ is the amplitude of the disturbance potential. Note that $r$ and $k_{r}$ are always positive.

The distortion of the cavitating vortex with average radius $r_{c}$ is described by a number of modes characterized by $k_{z}, n, \omega$ and amplitude $\hat{r}$. For small amplitudes, the local cavity radius $\eta$ is given by ${ }^{4}$

$$
\eta(r, \theta, z, t)=r_{c}+r^{\prime}=r_{c}+\hat{r} \exp \left[i\left(k_{z} z+n \theta-\omega t\right)\right] .
$$

The mode $n=0$ corresponds to a breathing mode and involves volume variations. Mode $n=1$ corresponds to a serpentine mode, also called bending mode, helical mode or displacement mode, as it leads to a displacement of the vortex centre line only. Mode $n=2$ is the bell mode also called double helix or fluted mode, and leads to an elliptical shape of the vortex cross-section. These three modes are visualised in Figure 4.7. The distortions are transversely propagating inertial waves and are referred to as Kelvin waves (Saffman, 1992).

The dispersion relation is obtained by using a small-perturbation analysis for the kinematic and dynamic boundary condition. A perturbation in cavity radius will result in a perturbation velocity given by the spatial derivative of the perturbation potential $\varphi^{\prime}$. The derivations by Thomson (1880) and Morozov (1974) use a potential flow formulation for the mean azimuthal velocity component $V$, but we show here that, at least for $n=0$, the boundary conditions can also be derived for viscous mean flow ${ }^{5}$.

We assume a zero mean radial velocity component $U$ and a constant mean axial velocity component $W$. The mean velocity for potential flow, in which a vortex with circulation $\Gamma_{\infty}$ is present, then reads

$$
\mathbf{U}=(U, V, W)=\left(0, \frac{\Gamma_{\infty}}{2 \pi r}, W\right)
$$

For viscous flow, the situation is more complicated. Neglecting the flow inside the cavity, the azimuthal velocity component in a viscous flow has to satisfy a zero shear stress boundary

\footnotetext{
${ }^{4}$ To simplify the equations, the mean cavity size is denoted here by $r_{c}$ whereas in Section 4.2 the mean cavity size was denoted by $\bar{r}_{c}$.

${ }^{5}$ This is considered an ad-hoc correction as it was assumed in the derivation of the convected Helmholtz equation that the mean flow is irrotational.
} 
condition at the cavity interface. This leads to a different velocity distribution near the cavity interface in comparison to the non-cavitating vortex, as shown in Chapter 3. If the change in viscous core size due to cavitation is not taken into account, this change in velocity leads to a small change in pressure at the cavity interface from which it is concluded that a formulation for the azimuthal velocity distribution of a non-cavitating vortex can also be used to find the relation between cavity size and pressure. The validity of this approach was shown in Section 3.3.2 although it was also stated that this is not understood. For the azimuthal velocity distribution, we will use the model of Rosenhead (1931) due its simple expression for the pressure distribution. The formulations for this vortex model were already given by Eqs. (4.21) and (4.22), but the formulation for the velocity distribution is repeated here for convenience,

$$
V(r)=\frac{\Gamma_{\infty}}{2 \pi} \frac{r}{r_{v}^{2}+r^{2}}
$$

with $r_{v}$ the radius of the viscous core.

The kinematic boundary condition for $f=r-\eta$ is given by

$$
\frac{D f}{D t}=\frac{\partial f}{\partial t}+\left(\mathbf{U}+\nabla \varphi^{\prime}\right) \cdot \nabla f=0
$$

which results in

$$
\frac{\partial r}{\partial t}=\left.\frac{\partial \varphi^{\prime}}{\partial r}\right|_{\eta}=\frac{\partial \eta}{\partial t}+\left(W_{c}+\frac{\partial \varphi^{\prime}}{\partial x}\right) \frac{\partial \eta}{\partial x}+\left(V_{c}+\frac{\partial \varphi^{\prime}}{r_{c} \partial \theta}\right) \frac{\partial \eta}{r_{c} \partial \theta}
$$

where $W_{c}$ and $V_{c}$ correspond to the mean axial and azimuthal velocity at the cavity interface, respectively. Using Eq. (4.50) for $\varphi^{\prime}$ and retaining only the linear terms of the perturbations in the potential and cavity radius gives,

$$
\hat{\phi} k_{r} H_{n}^{(1) \prime}\left(k_{r} r_{c}\right)=\left(-i \omega+W_{c} i k_{z}+V_{c} \frac{i n}{r_{c}}\right) \hat{r},
$$

where the prime denotes the derivative of the Hankel function with respect to its argument $k_{r} r_{c}$. This gives a first relation between $\hat{\phi}$ and $\hat{r}$,

$$
\frac{\hat{\phi}}{\hat{r}}=\frac{i\left(W_{c} k_{z}+V_{c} \frac{n}{r_{c}}-\omega\right)}{k_{r} H_{n}^{(1) \prime}\left(k_{r} r_{c}\right)} .
$$

The dynamic boundary condition states that the pressure at the cavity interface equals the vapour pressure subtracted with the contribution due to surface tension. For potential flow, the Bernoulli equation can be used, just like Thomson (1880) and Morozov (1974) did. Here, we investigate whether a solution for the mean viscous flow of a vortex can be applied by starting from the radial component of the momentum equation. Neglecting diffusion, the radial momentum equation is given by

$$
-\frac{1}{\rho} \frac{\partial p}{\partial r}=\frac{\partial}{\partial t} \frac{\partial \varphi^{\prime}}{\partial r}+\left(\mathbf{U}+\nabla \varphi^{\prime}\right) \cdot \nabla \frac{\partial \varphi^{\prime}}{\partial r}-\frac{\left(V+\frac{\partial \varphi^{\prime}}{r \partial \theta}\right)^{2}}{r} .
$$


If higher-order terms of the perturbation velocity are neglected, the equation reads

$$
-\frac{1}{\rho} \frac{\partial p}{\partial r}=\frac{\partial}{\partial t} \frac{\partial \varphi^{\prime}}{\partial r}+W \frac{\partial}{\partial z} \frac{\partial \varphi^{\prime}}{\partial r}+V \frac{\partial}{r \partial \theta} \frac{\partial \varphi^{\prime}}{\partial r}-\frac{V^{2}}{r}-\frac{2 V}{r} \frac{\partial \varphi^{\prime}}{r \partial \theta} .
$$

Integrating the left-hand-side between $r=\eta$ and $r=\infty$ gives

$$
-\int_{\eta}^{\infty} \frac{1}{\rho} \frac{\partial p}{\partial r} d r=-\frac{p_{\infty}-p_{\eta}}{\rho}
$$

The pressure in the fluid at the cavity interface is given by

$$
p_{\eta}=p_{v}-p_{T}
$$

where $p_{v}$ corresponds to the vapour pressure and $p_{T}$ to the contribution of surface tension. The contribution of surface tension $T$ at $r=\eta$ is (Batchelor, 1967),

$$
\begin{aligned}
p_{T}=T \nabla^{2} f & =T\left(\frac{\partial^{2}}{\partial z^{2}}+\frac{\partial^{2}}{\partial r^{2}}+\frac{1}{r} \frac{\partial}{\partial r}+\frac{1}{r^{2}} \frac{\partial^{2}}{\partial \theta^{2}}\right)(r-\eta), \\
& =T\left(k_{z}^{2}+\frac{1}{\eta^{2}} n^{2}\right) r^{\prime}+T \frac{1}{\eta} \\
& \approx \frac{T}{r_{c}}\left[1+\left(n^{2}+k_{z}^{2} r_{c}^{2}-1\right) \frac{r^{\prime}}{r_{c}}\right] .
\end{aligned}
$$

The first two terms in the right-hand-side of Eq. (4.59) can be integrated directly,

$$
\int_{\eta}^{\infty}\left(\frac{\partial}{\partial t} \frac{\partial \varphi^{\prime}}{\partial r}+W \frac{\partial}{\partial z} \frac{\partial \varphi^{\prime}}{\partial r}\right) d r=i \omega \varphi^{\prime}(\eta)-W_{c} i k_{z} \varphi^{\prime}(\eta) .
$$

Integrating the third and fifth (last) term in the right-hand-side of Eq. (4.59), while introducing $v^{\prime}=\partial \varphi^{\prime} / r \partial \theta$, gives

$$
\begin{aligned}
\int_{\eta}^{\infty}\left(V \frac{\partial}{r \partial \theta} \frac{\partial \varphi^{\prime}}{\partial r}-\frac{2 V}{r} \frac{\partial \varphi^{\prime}}{r \partial \theta}\right) d r & =\int_{\eta}^{\infty}\left[V\left(\frac{\partial v}{\partial r}-\frac{v^{\prime}}{r}\right)\right] d r \\
& =\left.V v^{\prime}\right|_{\eta} ^{\infty}-\int_{\eta}^{\infty}\left[v^{\prime}\left(\frac{\partial V}{\partial r}+\frac{V}{r}\right)\right] d r \\
& =-V(\eta) v^{\prime}(\eta) \quad \forall V=\frac{\Gamma_{\infty}}{2 \pi r} \\
& \approx-V_{c} \frac{i n \varphi^{\prime}}{r_{c}}
\end{aligned}
$$

where partial integration has been applied and the potential flow solution for $V$ is used because no analytical solution for $V$ could be generated for a viscous flow. Hence, the contribution of the viscous core to the integral in the second line of Eq. (4.64) is neglected. For mode $n=0$ we have $v^{\prime}=0$ and the integral is identical to zero. 
The integral of the fourth term in Eq. (4.59) is taken using the azimuthal velocity distribution of Eq. (4.53) which leads to

$$
\begin{aligned}
-\int_{\eta}^{\infty} \frac{V^{2}}{r} d r & =-\frac{1}{2}\left(\frac{\Gamma_{\infty}}{2 \pi}\right)^{2} \frac{1}{r_{v}^{2}+\eta^{2}} \\
& \approx-\frac{1}{2}\left(\frac{\Gamma_{\infty}}{2 \pi}\right)^{2} \frac{1}{r_{v}^{2}+r_{c}^{2}}\left(1-2 \frac{r^{\prime}}{r_{c}} \frac{r_{c}^{2}}{r_{v}^{2}+r_{c}^{2}}\right) .
\end{aligned}
$$

For the situation without perturbation, the pressure drop between a location at large distance from the cavity and at the cavity radius is given by

$$
p_{\infty}-\left(p_{v}-\frac{T}{r_{c}}\right)=\frac{\rho}{2}\left(\frac{\Gamma_{\infty}}{2 \pi}\right)^{2} \frac{1}{\left(r_{v}^{2}+r_{c}^{2}\right)} .
$$

In most experiments, the pressure drop is well-known but the distribution of the azimuthal velocity is unknown. For that reason it is preferred to write the contribution of the cavity perturbation in Eq. (4.65) as a perturbation of the pressure drop,

$$
\begin{aligned}
\frac{1}{2}\left(\frac{\Gamma_{\infty}}{2 \pi}\right)^{2} \frac{2}{r_{v}^{2}+r_{c}^{2}} \frac{r^{\prime}}{r_{c}} \frac{r_{c}^{2}}{r_{v}^{2}+r_{c}^{2}} & =\frac{2}{\rho}\left(p_{\infty}-p_{v}+\frac{T}{r_{c}}\right) \frac{r^{\prime}}{r_{c}} \frac{r_{c}^{2}}{r_{v}^{2}+r_{c}^{2}}, \\
& =\frac{2}{\rho}\left(p_{\infty}-p_{v}+\frac{T}{r_{c}}\right)^{*} \frac{r^{\prime}}{r_{c}}, \\
& \approx \frac{2}{\rho}\left[\left(p_{\infty}-p_{v}\right)^{*}+\frac{T}{r_{c}}\right] \frac{r^{\prime}}{r_{c}},
\end{aligned}
$$

where the asterisk indicates that an additional correction for viscous flow effects needs to be applied to the pressure drop when the cavity radius is of the same order as the viscous core radius.

The sum of all terms in Eq. (4.59) containing terms linear in the perturbations can now be considered by combining Eqs. (4.62), (4.63), (4.64) and (4.67) which gives

$$
\frac{T}{\rho r_{c}}\left(n^{2}+k_{z}^{2} r_{c}^{2}-1\right) \frac{r^{\prime}}{r_{c}}=\left(i \omega \varphi^{\prime}-W_{c} i k_{z} \varphi^{\prime}\right)-\left(V_{c} \frac{i n \varphi^{\prime}}{r_{c}}\right)-\frac{2}{\rho}\left[\left(p_{\infty}-p_{v}\right)^{*}+\frac{T}{r_{c}}\right] \frac{r^{\prime}}{r_{c}},
$$

from which the second equation for the relation between $\hat{\phi}$ and $\hat{r}$ is derived

$$
\frac{\hat{\phi}}{\hat{r}}=\frac{\frac{T}{r_{c}}\left(n^{2}+k_{z}^{2} r_{c}^{2}+1\right)+2\left(p_{\infty}-p_{v}\right)^{*}}{i \rho r_{c}\left(W_{c} k_{z}+V_{c} \frac{n}{r_{c}}-\omega\right) H_{n}^{(1)}\left(k_{r} r_{c}\right)}
$$

The dispersion relation is obtained by combining the kinematic boundary condition (4.57) and the dynamic boundary condition (4.69),

$$
\omega^{ \pm}\left(k_{z}, n\right)=W_{c} k_{z}+\Omega n \pm \frac{W_{\infty}}{r_{c}} \sqrt{K_{\sigma}} \sqrt{\frac{-k_{r} r_{c} H_{n}^{(1) \prime}\left(k_{r} r_{c}\right)}{H_{n}^{(1)}\left(k_{r} r_{c}\right)}} T_{\omega}
$$


in which $\Omega=V_{c} / r_{c}$ represents the cavity angular velocity. Following the discussion in Section 4.2.1, the pressure drop $\left(p_{\infty}-p_{v}\right)^{*}$ can be considered as a stiffness term which, in nondimensional form, is denoted by $K_{\sigma}$,

$$
K_{\sigma}\left(\sigma_{W}, r_{c}, r_{v}\right)=\frac{\left(p_{\infty}-p_{v}\right)^{*}}{\frac{1}{2} \rho W_{\infty}^{2}}=\sigma_{W} \frac{r_{c}^{2}}{r_{v}^{2}+r_{c}^{2}}
$$

with the cavitation number $\sigma_{W}$ defined as

$$
\sigma_{W}=\frac{p_{\infty}-p_{v}}{\frac{1}{2} \rho W_{\infty}^{2}}
$$

For potential flow we find $K_{\sigma}=\sigma_{W}$. Similar as given by Eq. (4.28), $K_{\sigma}$ can also be defined by the square of the non-dimensional azimuthal velocity at the cavity interface. Parameter $T_{\omega}$ contains the contribution of the surface tension,

$$
T_{\omega}\left(k_{z}, n\right)=\sqrt{1+\frac{1}{K_{\sigma} \mathrm{We}}\left(n^{2}+k_{z}^{2} r_{c}^{2}+1\right)}
$$

with the Weber number We defined as

$$
\mathrm{We}=\frac{\rho W_{\infty}^{2} r_{c}}{T} .
$$

The non-dimensional form of Eq. (4.70) is given by

$$
\widetilde{\omega}^{ \pm}(\kappa, n)=\frac{\omega^{ \pm} r_{c}}{W_{\infty}}=\widetilde{W}_{c} k_{z} r_{c}+\widetilde{V}_{c} n \pm \sqrt{K_{\sigma}} \sqrt{\frac{-\kappa H_{n}^{(1) \prime}(\kappa)}{H_{n}^{(1)}(\kappa)}} T_{\omega}
$$

with non-dimensional wavenumber $\kappa=k_{r} r_{c}$, non-dimensional axial velocity $\widetilde{W}_{c}=W_{c} / W_{\infty}$ and non-dimensional azimuthal velocity $\widetilde{V}_{c}=V_{c} / W_{\infty}$.

Each vibration mode contains two branches, hence two frequencies, one corresponding to the plus and one to the minus sign in the right-hand-side of Eq. (4.75). In the following, this sign is also used in the identification of the mode (e.g. $n=0^{-}$and $n=0^{+}$). The contribution of the surface tension is only relevant for very small cavity size at which condition the viscous correction also becomes relevant. Unless stated otherwise, the contribution of surface tension is neglected in the following, i.e. $T_{\omega}=1$.

The criterion for a sound wave to occur is that the radial wavenumber squared, defined by Eq. (4.42), is larger than zero. If the axial velocity $W$ is small with respect to the speed of sound, the criterion reads

$$
\left|\frac{c_{p, z}}{c}\right|=\left|\frac{k}{k_{z}}\right|>1
$$

where $c_{p, z}=\omega / k_{z}$ corresponds to the axial phase velocity and $k$ corresponds to the acoustic wavenumber in the fluid, $k=\omega / c$.

We now consider the situation that the phase velocity of the axial deformation is much smaller than the speed of sound, equivalent to the condition that the acoustic wavenumber is 
much smaller than the axial wavenumber, which gives $k_{r}^{2} \cong-k_{z}^{2}$. In that situation, the radial wavenumber becomes imaginary and the Hankel function reduces to a modified Bessel function of the second kind, also called MacDonald function, $K$. The wave in radial direction then turns into an evanescent wave. This results in a non-dimensional dispersion relation for low frequencies as

$$
\widetilde{\omega}^{ \pm}(\kappa, n)=\frac{\omega^{ \pm} r_{c}}{W_{\infty}}=\widetilde{W}_{c} \kappa+\widetilde{V}_{c} n \pm \sqrt{K_{\sigma}} \sqrt{\frac{-|\kappa| K_{n}^{\prime}(|\kappa|)}{K_{n}(|\kappa|)}} T_{\omega}
$$

with $\kappa$ redefined as $\kappa=k_{z} r_{c} \cong-i k_{r} r_{c}$. Equation (4.77) will be used in the remainder of this chapter. It is remarked that $k_{r}$ is always positive but $k_{z}$ may have positive as well as negative values. Because the equations are defined for $k_{r}$ and $k_{z}$ squared, the absolute value of $k_{z}$ needs to be taken for the argument of $K_{n}$ and of the square root term.

The term with the square root in Eq. (4.77) can be written as the dispersive component $\widetilde{\omega}_{d}$,

$$
\widetilde{\omega}_{d}=\sqrt{\frac{-|\kappa| K_{n}^{\prime}(|\kappa|)}{K_{n}(|\kappa|)}} T_{\omega}
$$

and Eq. (4.77) reads

$$
\widetilde{\omega}^{ \pm}(\kappa, n)=\widetilde{W}_{c} \kappa+\widetilde{V}_{c} n \pm \sqrt{K_{\sigma}} \widetilde{\omega}_{d} .
$$

If the vortex cavity is located in unbounded flow and in the absence of $\widetilde{\omega}_{d}$, the frequency is given by $\widetilde{\omega}(\kappa, n)=\widetilde{W}_{c} \kappa+\widetilde{V}_{c} n$. Any perturbation in axial and azimuthal direction is then convected with the velocity at the cavity interface which is a transformation between an inertial coordinate system and a coordinate system moving with the perturbation of the cavity.

It is remarked that, both for the potential flow vortex and for the Rosenhead vortex, $K_{\sigma}=$ $\widetilde{V}_{c}^{2}$. However, as also mentioned in Section 4.2.1, it still needs to be proven that this relation is generally valid. Because the mode $n=0$ has the most interest and $\sigma_{W}$ is usually known in experiments while $\widetilde{V}_{c}$ is unknown, preference is given to the use of $K_{\sigma}$ in Eq. (4.77).

The dispersion relation requires the actual azimuthal velocity at the cavity interface for modes $n=1$ and $n=2$. In the present study, the following models will be used

$$
\begin{array}{ll}
\widetilde{V}_{c}=\frac{\Gamma_{\infty}}{2 \pi W_{\infty} r_{c}}=\sqrt{\sigma_{W}} ; & \text { potential flow vortex } \\
\widetilde{V}_{c}=\frac{\Gamma_{\infty}}{2 \pi W_{\infty} r_{c}}\left\{\frac{\varsigma r_{c}^{2}}{r_{v}^{2}+\zeta r_{c}^{2}}\right\} ; & \text { cav. Lamb - Oseen vortex } \\
\hline
\end{array}
$$

The details of the cavitating Lamb-Oseen (LO) vortex are presented in Chapter 3. It is noted that for $\zeta=1$ the velocity of the cavitating LO vortex is equal to the velocity of the non-cavitating Rosenhead vortex.

At present, no analytical models are available for the axial velocity $W_{c}$ at the cavity interface, but this velocity component has been measured by Pennings (2016) and results have been presented in Section 3.3.2. Unless stated otherwise, $W_{c}=W_{\infty}$ is used. 
The modified Bessel function can be simplified for limiting values of the axial wavenumber, see Abramowitz and Stegun (1972), which results in to the following dispersion relations,

$$
\begin{array}{ll}
\widetilde{\omega}^{ \pm}(\kappa, n)=\widetilde{W}_{c} \kappa \pm \sqrt{K_{\sigma}} \sqrt{-1 /(\ln |\kappa|-\ln 2+\gamma)}, & \forall n=0, \kappa \ll 1 \\
\widetilde{\omega}^{ \pm}(\kappa, n)=\widetilde{W}_{c} \kappa+\widetilde{V}_{c} n \pm \sqrt{K_{\sigma}} \sqrt{n}, & \forall n>0, \kappa \ll 1 \\
\widetilde{\omega}^{ \pm}(\kappa, n)=\widetilde{W}_{c} \kappa+\widetilde{V}_{c} n \pm \sqrt{K_{\sigma}} \sqrt{|\kappa|}, & \forall n \geq 0, \kappa \gg 1
\end{array}
$$

where $\gamma$ corresponds to constant of Euler-Mascheroni $(\gamma \approx 0.5772)$, hence $(\ln 2-\gamma)=0.1159$. There exists no solution for $\widetilde{\omega}$ that is independent of $\kappa$ for $n=0$ and $\kappa \rightarrow 0$. Equation (4.82) has a resemblance to Eq. (4.26) for the resonance frequency in 2-D flow but with the logarithmic term for $r_{\text {ref }}$ replaced by $1 / k_{z}$.

For the propagation velocity of the wave, we should make a distinction between the phase velocity and the group velocity. The phase velocity, $c_{p}$, is the velocity at which the wave crests and troughs propagate, and is defined as $c_{p}=\omega / k$. The group velocity, $c_{g}$, is the velocity at which the wave energy propagates, and is defined as $c_{g}=\partial \omega / \partial k$. The phase velocity $c_{p, z}$ and group velocity $c_{g, z}$ in axial direction are obtained from Eq. (4.70) and read in non-dimensional form,

$$
\begin{gathered}
\bar{c}_{p, z}^{ \pm}(\kappa, n)=\frac{c_{p, z}^{ \pm}}{W_{\infty}}=\frac{\omega^{ \pm}}{W_{\infty} k_{z}}=\widetilde{W}_{c}+\frac{\widetilde{V}_{c} n}{\kappa} \pm \frac{\sqrt{K_{\sigma}}}{\kappa} \sqrt{\frac{-|\kappa| K_{n}^{\prime}(|\kappa|)}{K_{n}(|\kappa|)}} \\
\bar{c}_{g, z}^{ \pm}(\kappa, n)=\frac{c_{g, z}^{ \pm}}{W_{\infty}}=\frac{\partial \omega^{ \pm}}{W_{\infty} \partial k_{z}}= \\
\widetilde{W}_{c} \pm \frac{\sqrt{K_{\sigma}}}{2|\kappa|} \sqrt{\frac{-|\kappa| K_{n}^{\prime}(|\kappa|)}{K_{n}(|\kappa|)} \times} \\
\\
{\left[1+\frac{|\kappa| K_{n}^{\prime \prime}(|\kappa|)}{K_{n}^{\prime}(|\kappa|)}-\frac{|\kappa| K_{n}^{\prime}(|\kappa|)}{K_{n}(|\kappa|)}\right]}
\end{gathered}
$$

In the following, $c_{p, z}$ and $c_{g, z}$ will be referred to as $c_{p}$ and $c_{g}$, respectively.

\subsubsection{Analysis}

In absence of $\widetilde{\omega}_{d}$ in Eq. (4.77) and for unbounded flow, all perturbations are convected with the velocity at the cavity interface, and we only have positive values for the axial and azimuthal wavenumber. However, if the domain is bounded, negative wavenumbers may be required to satisfy additional conditions for the perturbation at the downstream boundary of the domain. The waves with negative wavenumbers are generated by reflections of waves with positive wavenumber at the downstream boundary, that is downstream in the direction of $\widetilde{W}_{c}$ and $\widetilde{V}_{c}$. As the azimuth angle $\theta$ is periodic, the downstream boundary of the wave in azimuthal direction can be located at an identical location as the upstream boundary.

The dispersive term $\widetilde{\omega}_{d}$ in Eq. (4.77) is presented in Figure 4.8. The modified Bessel function of the second kind $K_{n}$ is symmetric with respect to $n$, i.e. $K_{-n}(\kappa)=K_{n}(\kappa)$, so results are identical for positive and negative azimuthal wavenumbers. Only positive values are allowed for the argument of the square root term and $K_{n}$. The dispersion term $\widetilde{\omega}_{d}$ is always real valued and the perturbations are therefore neutrally stable. 


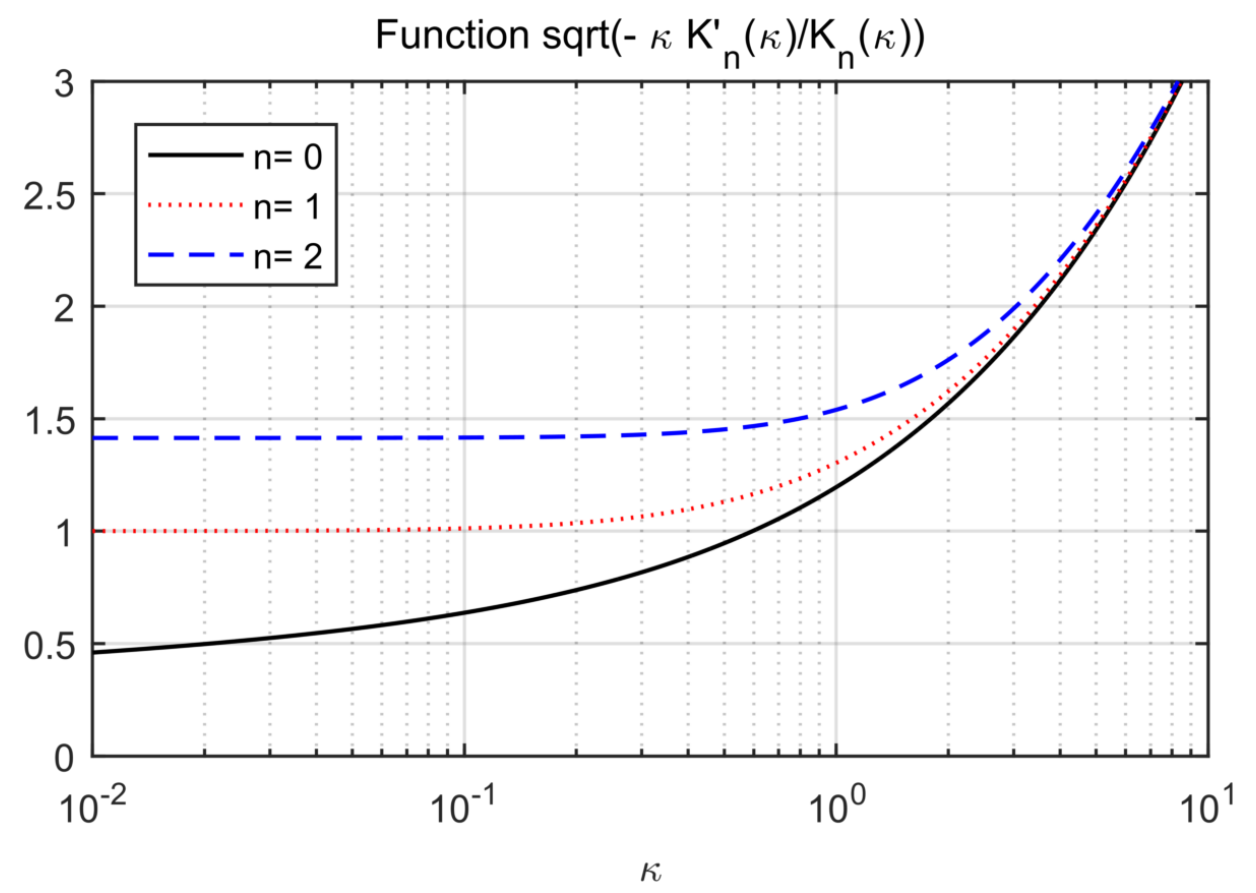

Figure 4.8: Variation of the dispersive term $\widetilde{\omega}_{d}$ in the dispersion relation Eq. (4.77) with nondimensional axial wavenumber $\kappa$.

The analytical dispersion relation, presented as function of axial wavenumber and frequency for given azimuthal wavenumber, leads to results in four quadrants if positive as well as negative axial wavenumbers are considered. However, the dispersion curves are symmetric about the origin if also negative values for the azimuthal modes are considered, so two quadrants are sufficient to present the full dispersion diagram. The 2-D Fast Fourier Transform (FFT) of measurement data of cavity deformations from the spatial-time domain to the wavenumberfrequency domain also gives results in four quadrants but two quadrants are symmetric about the origin as the deformations are real valued. So the measurement data is uniquely described by two quadrants and one can choose between presenting the upper two quadrants (positive frequency, positive and negative wavenumber) and presenting the right-most two quadrants (positive wavenumber, positive and negative frequency). Because wave propagation in time can only have one direction, we prefer to show the results for positive frequency only and for both positive and negative axial wavenumbers. A negative axial wavenumber then indicates that the axial phase velocity is directed upstream. The presented results of the analytical dispersion relation, initially computed for positive axial wavenumber only, are obtained by the transformation,

$$
\begin{aligned}
k_{z}^{p} & =k_{z} \operatorname{sgn}(\omega), \\
\omega^{p} & =|\omega| .
\end{aligned}
$$

where superscript $p$ denotes the presented value. Note that in the sign function we have to exclude $\omega=0$. Previous presentations of the dispersion relation, such as in Bosschers (2008) and Pennings et al. (2015a), have shown the results in the other two quadrants, i.e. positive wavenumber and positive and negative frequency, which is more consistent with the analytical expression. However, for the practical interpretation of the results, the quadrants shown here are preferred. 


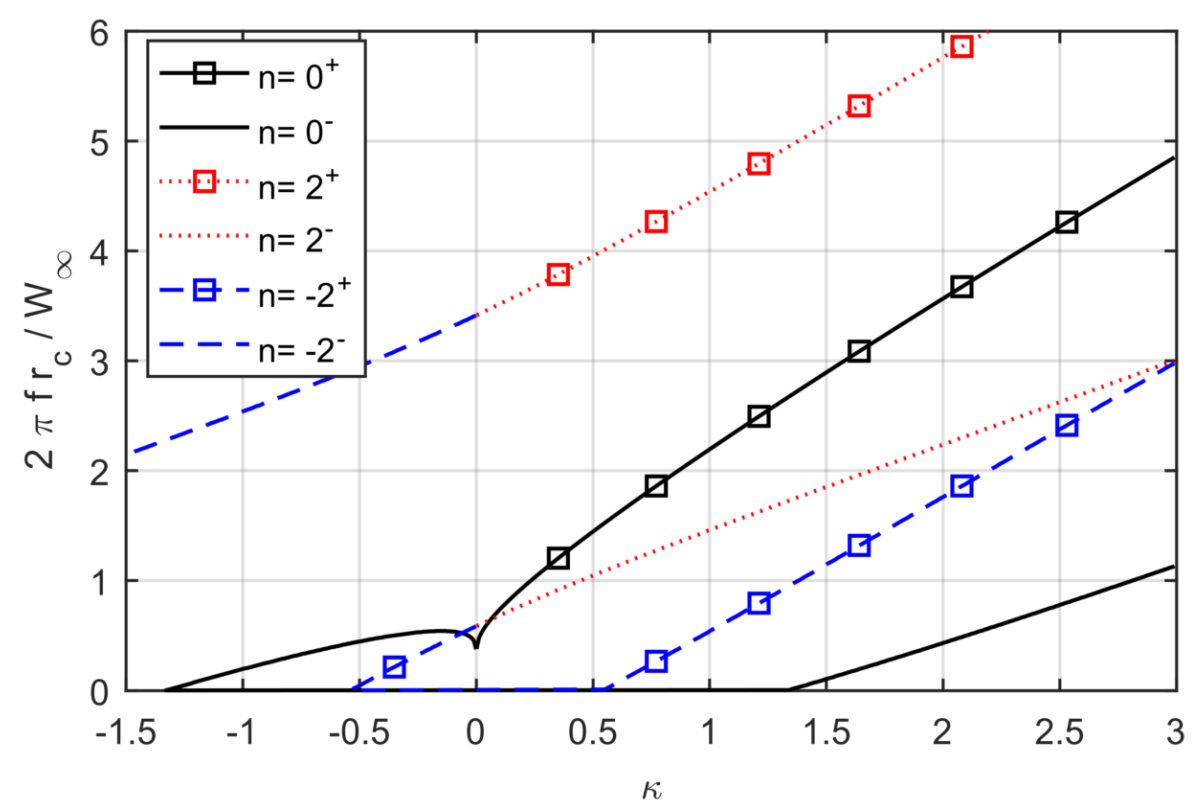

Figure 4.9: Non-dimensional dispersion diagram for the cavity deformation modes $n=0^{ \pm}$and $n= \pm 2^{ \pm}$, described by Eq. (4.77), $\sigma_{W}=1.0$, potential flow vortex.

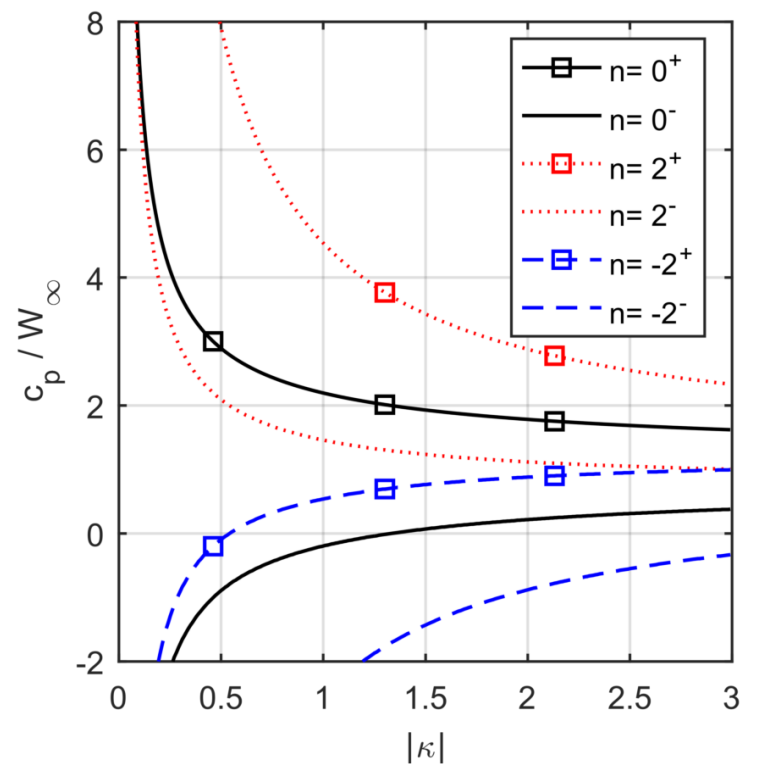

a) Phase velocity in axial direction

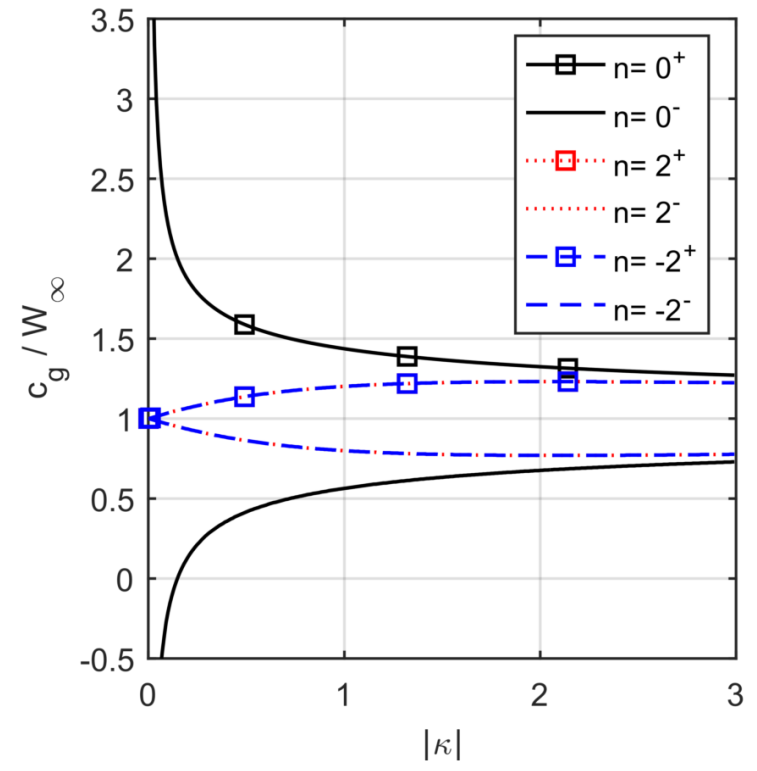

b) Group velocity in axial direction

Figure 4.10: Variation of the non-dimensional phase velocity and group velocity with absolute value of the non-dimensional wavenumber $\kappa$ for $n=0^{ \pm}$and $n= \pm 2^{ \pm}$and for $\sigma_{W}=1.0$, potential flow vortex.

An example of the dispersion relation of Eq. (4.77) is given in Figure 4.9 for $n=0^{ \pm}$and $n= \pm 2^{ \pm}$and in Figure 4.11 for $n= \pm 1^{ \pm}$. The corresponding phase velocity and group velocity for these two cases, both in axial direction, are presented in Figure 4.10 and Figure 4.12, respectively. For $|\kappa| \rightarrow 0$, the frequency for both modes $n=0^{ \pm}$very slowly approaches zero, 


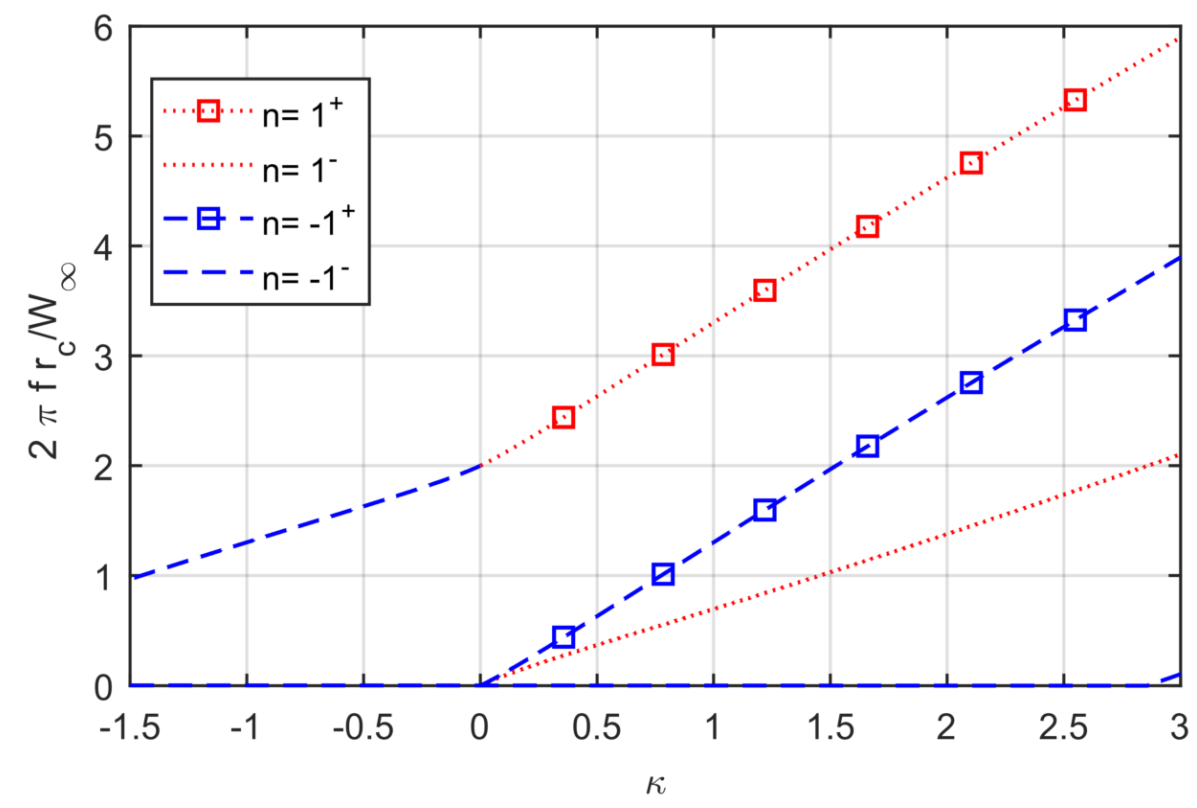

Figure 4.11: Non-dimensional dispersion diagram for the cavity deformation modes $n= \pm 1^{ \pm}$, described by Eq. (4.77), $\sigma_{W}=1.0$, potential flow vortex.

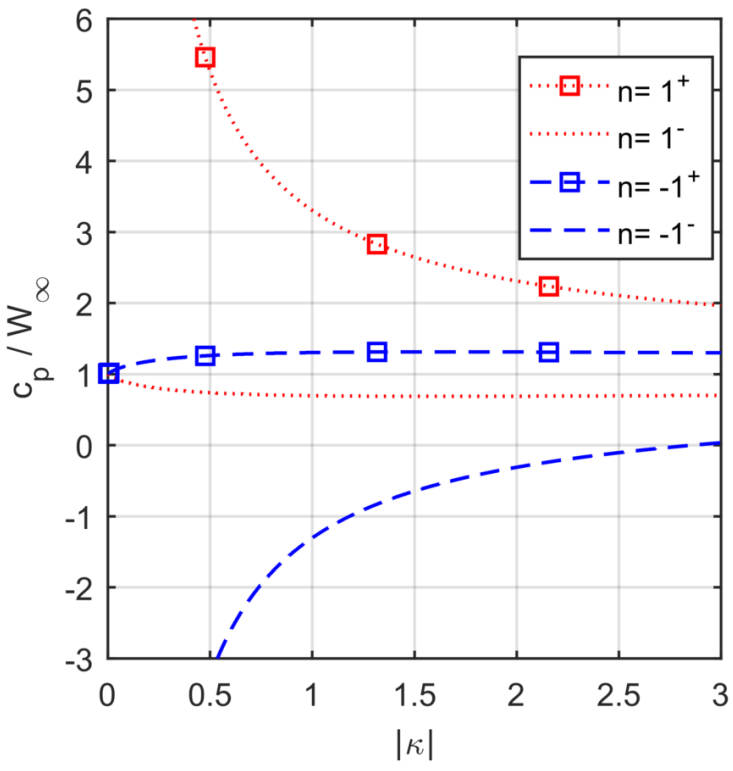

a) Phase velocity in axial direction

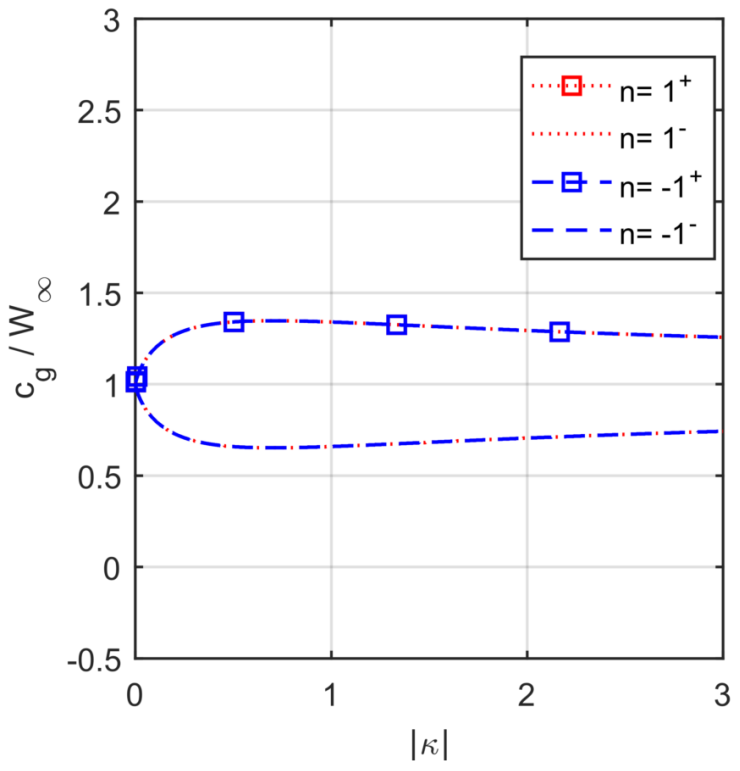

b) Group velocity in axial direction

Figure 4.12: Variation of the non-dimensional phase velocity and group velocity with absolute value of the non-dimensional wavenumber $\kappa$ for $n= \pm 1^{ \pm}$and for $\sigma_{W}=1.0$, potential flow vortex. Note that the absolute value of the wave number is used.

as also concluded from Eq. (4.82). The frequency for $\kappa=0$ in Figure 4.9 is determined by the smallest value used for $\kappa$. The graph of the phase velocity as well as that of the group velocity show that the lines are symmetric with respect to the free-stream axial (convection) velocity for all deformation modes. For the phase velocity, symmetry occurs between positive and negative 

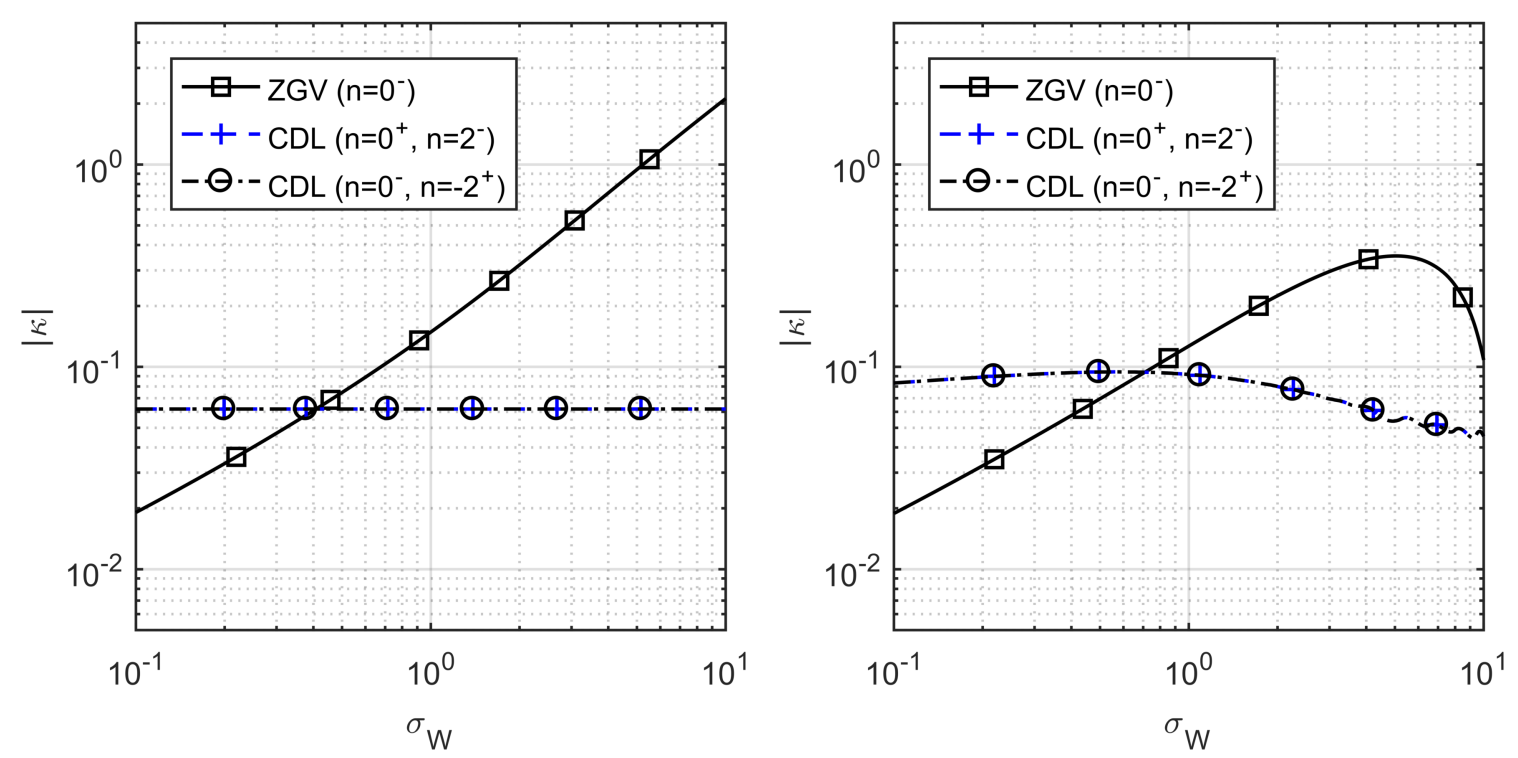

a) Potential flow vortex

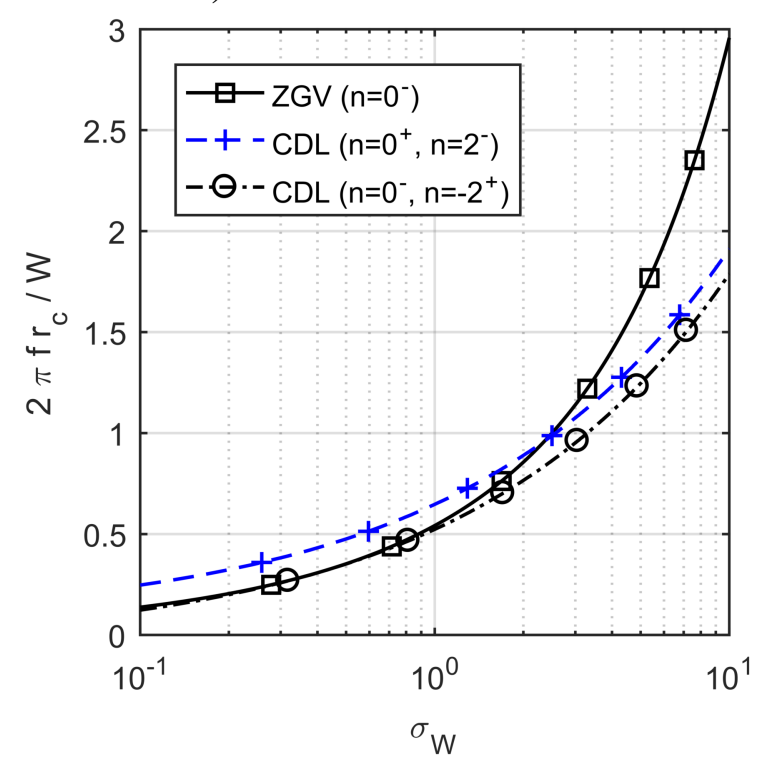

b) Cavitating Lamb-Oseen vortex $\left(\sigma_{i}=12\right)$

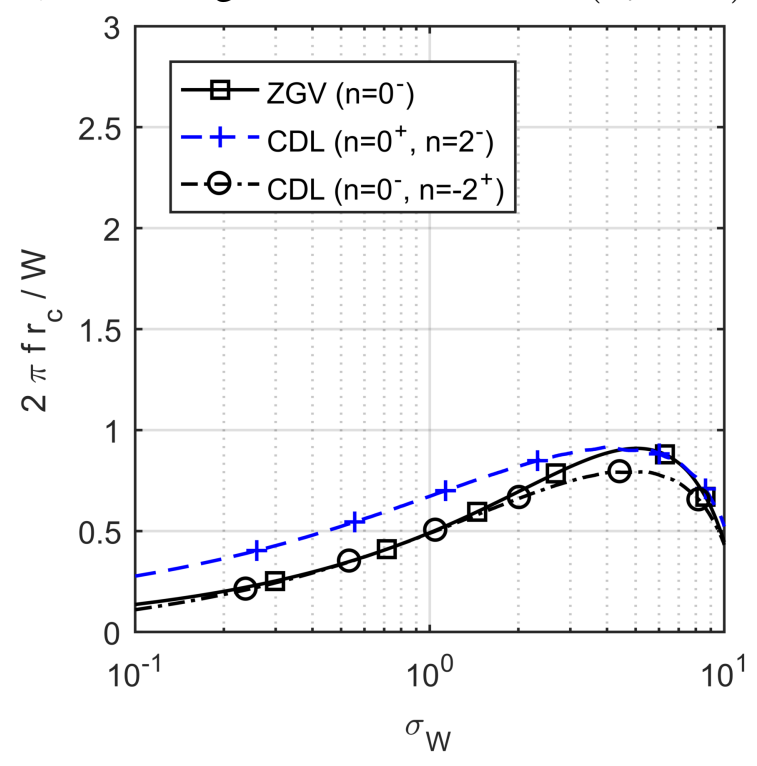

c) Potential flow vortex

d) Cavitating Lamb-Oseen vortex $\left(\sigma_{i}=12\right)$

Figure 4.13: Axial wavenumber and frequency as function of cavitation number for zero axial group velocity (ZGV) and crossing dispersion lines (CDL).

modes, i.e. $n= \pm 1$, while for the group velocity the symmetry occurs between two branches of each mode, i.e. $n=1^{ \pm}$.

The axial wavenumber and phase velocity $c_{p}$ for mode $n=0^{-}$is negative for small $|\kappa|$ indicating upstream directed disturbances and becomes positive for large $|\kappa|$. A zero value of $c_{p}$ corresponds to a stationary deformation (i.e. no temporal variation). The $c_{p}$ for mode $n=1^{-}$ has a minimum value which decreases with increasing $\sigma_{W}$ and which passes through zero for $\sigma_{W}$ above $\approx 10.3$. For larger values of $\sigma_{W}, c_{p}$ becomes zero at two values of $\kappa$, but cavitation inception at such high values of $\sigma_{W}$ is rare. In the limit of infinitely large $|\kappa|$, the $c_{p}$ of all modes approach the free-stream velocity. Negative phase velocities are also observed for the 


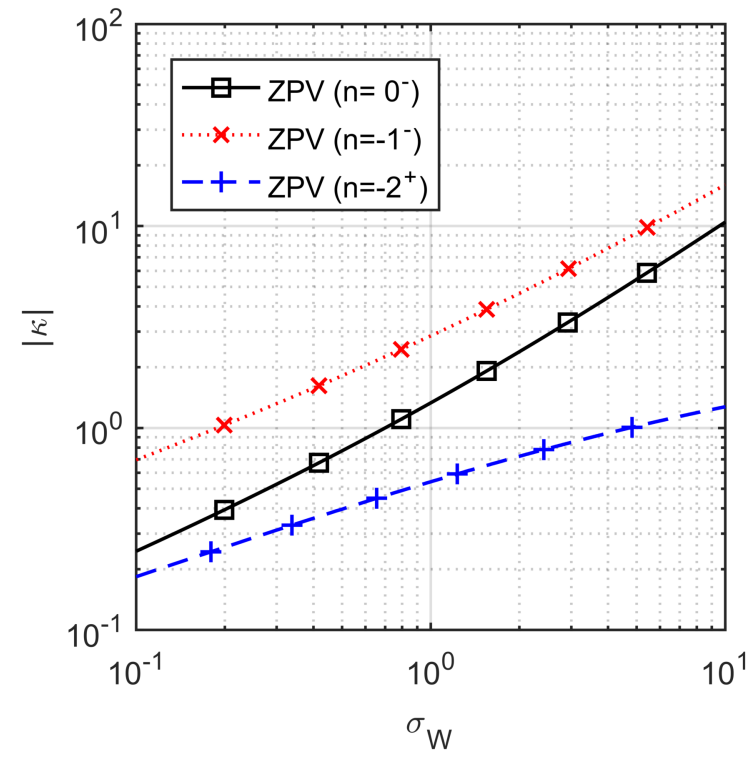

a) Potential flow vortex

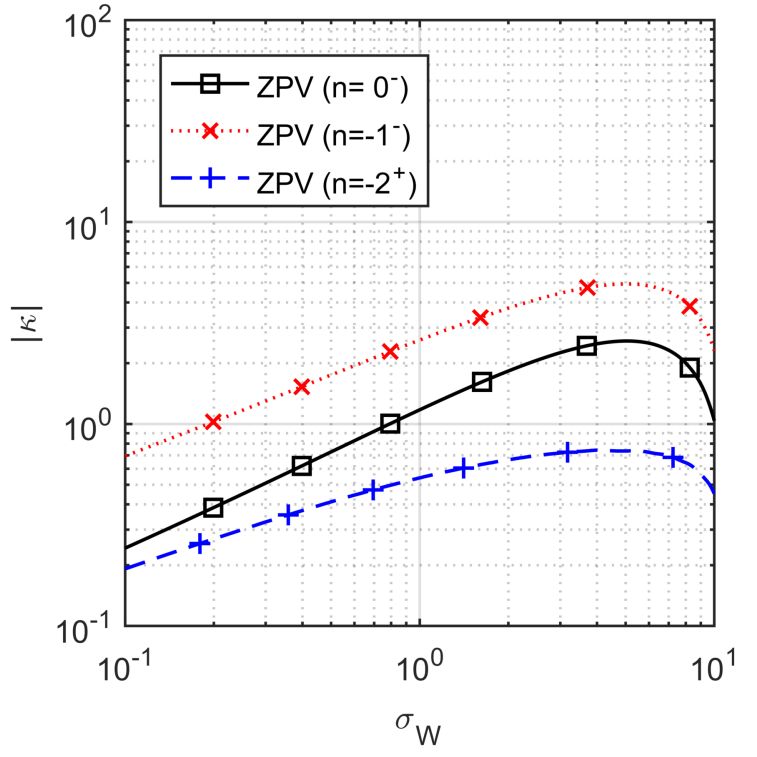

b) Cavitating Lamb-Oseen vortex $\left(\sigma_{i}=12\right)$

Figure 4.14: Axial wavenumber as function of cavitation number for zero phase velocity (ZPV) in axial direction.

mode $n=-1^{-}$.

The group velocity $c_{g}$ is independent of the sign of $n$ but depends on the sign of the branch. The $c_{g}$ for all modes approaches the free-stream velocity for infinitely large $\kappa$. For very small $\kappa$, only the $c_{g}$ of the modes $n= \pm 1$ and $n= \pm 2$ approaches the free-stream velocity. The $c_{g}$ for the mode $n=0^{-}$passes through zero for a value of $\kappa$ that varies with $\sigma_{W}$. The minimum value for $c_{g}$ of the modes $n=1^{-}$and $n=2^{-}$decreases with increasing cavitation number, and $c_{g}$ becomes zero above $\sigma_{W} \approx 8.3$ for mode $n=1^{-}$and above $\sigma_{W} \approx 20$ for mode $n=2^{-}$.

The dispersion relation shows various interesting aspects, such as the condition of zero axial group velocity (ZGV) for $n=0^{-}$, implying that energy for this value of $\kappa$ is not propagated which might lead to resonance. Another special location is the crossing of dispersion lines (CDL) for modes $n=0^{+}$and $n=2^{-}$and for modes $n=0^{-}$and $n=-2^{+}$. At such a crossing, energy can be exchanged between the two modes which might lead to unstable behaviour of the cavity. The values for $\kappa$ for these conditions are presented in Figure 4.13, top graph, and the corresponding frequencies are presented in the bottom graph. Results are presented for a potential flow vortex in the left two graphs and for the cavitating Lamb-Oseen vortex, Eq. (4.81), in the right two graphs. The results for the viscous flow vortex include the correction for viscous effects on the stiffness coefficient $K_{\sigma}$. The controlling factor for the viscous effects is the ratio $r_{c} / r_{v}$. In Chapter 3 it was shown that this ratio is directly related to the ratio $\sigma_{W} / \sigma_{W, i}$ with $\sigma_{W, i}$ the cavitation inception number. The results show that at low $\sigma_{W}$ the results for the potential flow vortex and for the viscous flow vortex are identical. At higher $\sigma_{W}$, significant differences are observed with smaller values for the frequency in the viscous flow situation compared to the potential flow situation. There is an axial wavenumber for which the frequency of the ZGV is identical to the frequency of the CDL for $n=0^{-}$and $n=-2^{+}$with a range of wavenumbers for which the frequencies are very close. This particular wavenumber does not depend very much on the viscous flow correction. 
Table 4.1: Conditions for the cavitation tunnel tests of Pennings (2016) at $7^{\circ}$ angle of attack describing mean cavity size $r_{c}$, cavitation number $\sigma_{W}$, tunnel velocity $W_{\infty}$, dissolved oxygen content $D O$, lift-coefficient $C_{L}$ and Reynolds number $R e$ based on chord length at the root $c_{0}$.

\begin{tabular}{l|ccccccc}
\hline & $r_{c}[\mathrm{~mm}]$ & $100 r_{c} / c_{0}$ & $\sigma_{W}$ & $W_{\infty}[\mathrm{m} / \mathrm{s}]$ & $D O[\mathrm{mg} / \mathrm{l}]$ & $C_{L}$ & $R e$ \\
\hline case A & 0.48 & 0.38 & 2.33 & 6.26 & 2.3 & 0.58 & $8.9 \times 10^{5}$ \\
case B & 2.78 & 2.21 & 0.87 & 6.32 & 2.7 & 0.57 & $9.1 \times 10^{5}$ \\
\hline
\end{tabular}

The values for $\kappa$ for which the phase velocity becomes zero are given in Figure 4.14 for the potential flow vortex and for the viscous flow vortex. Trends are similar as found for zero group velocity.

\subsubsection{Comparison with experimental data}

The results of the analytical model have been compared with the experimental data of Pennings (2016). A first comparison between the analytical dispersion relation and this experimental data was presented in Pennings et al. (2015a). Other experimental datasets of cavitating vortices, such as presented by Briançon-Marjollet and Merle (1996) and Maines and Arndt (1997a), have only been analysed with respect to resonance frequencies in the radiated noise spectra. A comparison between predictions with the present analytical dispersion relation and results from these data sets is made in Section 6.2.

The test set-up of Pennings (2016) consists of a half-wing of elliptical planform with an aspect ratio $\mathrm{AR}=3$ positioned at various angles of attack in the cavitation tunnel of Delft University of Technology. The wing has a chord length at the root of $c_{0}=0.1256 \mathrm{~m}$ and semi-span of $0.150 \mathrm{~m}$ with the tip positioned in the centre of the test section. The geometry and testconditions are identical to those used for the PIV measurements described in Section 3.3.2. Two high-speed video cameras have been used to observe the cavitating tip vortex, located above and aside the test-section. The camera resolution was $1920 \times 1080$ pixels, the frame rate was $5 \times 10^{3}$ frames per second, and total recording time was five seconds. The top-view camera observes the cavitating vortex between the blade tip at $z / c_{0}=0.0$ and $z / c_{0}=2.48$ with an equivalent pixel size of $0.16 \mathrm{~mm}$. The side-view camera observes the cavitating vortex between $z / c_{0}=1.12$ to $z / c_{0}=2.60$ with an equivalent pixel size of $0.10 \mathrm{~mm}$. Shadowgraph images of the cavitating vortex were generated using two backlight LED panels. The edge of the cavity was detected using the gradient in light intensity (Canny edge detector) from which the cavity centreline and diameter was determined by Pennings.

Only two cases have been analysed here: The first case, designated Case A, is for a cavitation number just below the value for inception in which the cavity has a cylindrical shape. The second case, Case B, is for a cavitation number for which the cavity is much more developed resulting in a twisted elliptical shape. Table 4.1 presents the conditions, and Figure 4.15 shows the top view and side view of the vortex cavity for these two cases. Case B corresponds to Condition a of Pennings et al. (2015a).

The analysis of the deformations of the cavity diameter and centreline was performed by 


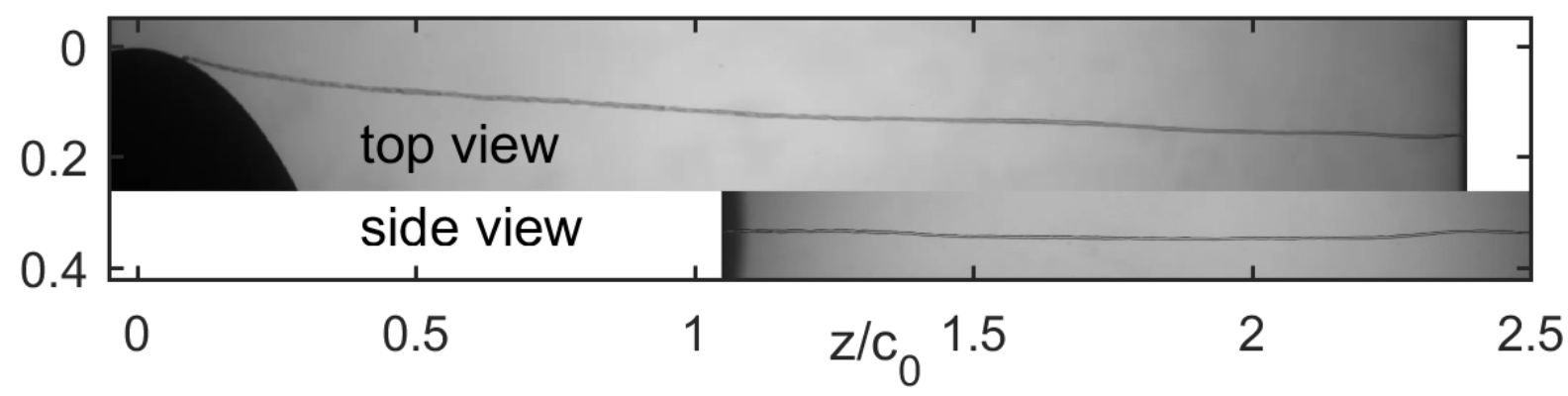

a) Case $\mathrm{A}$

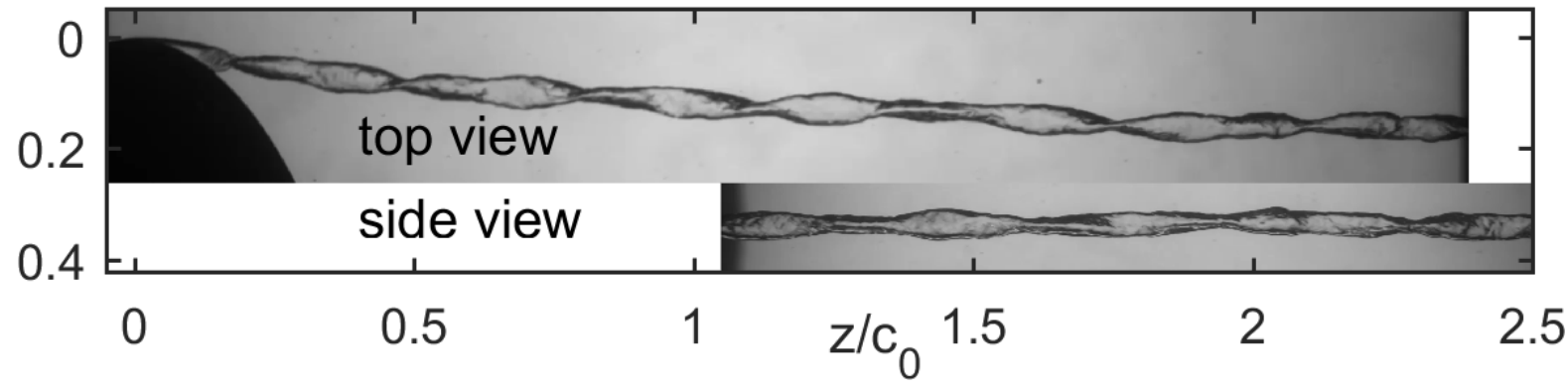

b) Case B

Figure 4.15: Top and side view images of the vortex cavity, the top view shows the pressure side of the wing. Flow is from left to right. High speed video recordings by Pennings (2016).

first interpolating the data for the top view camera at each time step to the axial coordinate of the side view camera. Because the image quality of the cameras was less near the edges, the view length was reduced by $1.26 \mathrm{~cm}$ for the right side of the top-view camera and by $1.26 \mathrm{~cm}$ for the left side of the side-view camera. The time-averaged data for each streamwise station was subtracted such that only the perturbations to the mean value remain. The data was then transformed to the wavenumber-frequency domain by a 2-D Fast Fourier Transform (FFT). Both the cross-spectral density and the spectral coherence have been computed.

The coherence between the perturbations of the two camera views was computed by subdividing the data series in segments of size $25 \% T_{\max }$ by $50 \% Z_{\max }$, with $T_{\max }$ the recording time which equals five seconds and $Z_{\max }$ the overlapping axial cavity length well observed by both cameras which equals $0.145 \mathrm{~m}$. The segments for the FFT were $75 \%$ overlapping in time and space and a 2-D Hann-window was used for each segment. The total number of segments was 48, and the FFT was computed using 32768 samples in time and 2048 samples in space. These numbers of samples are larger than the available number of samples, so zero-padding was applied. The resolution in the spectrum is $\Delta k_{z}=31.6 \mathrm{~m}^{-1}$ and $\Delta f=0.15 \mathrm{~Hz}$. For Case A, this results in non-dimensional values $\Delta \kappa=0.015$ and $\Delta \widetilde{\omega}=7.3 \times 10^{-5}$. Note that the actual resolution is coarser as zero-padding is applied.

The cross-power spectral density (CPSD) was computed from the FFT for each data set separately using a single segment to maximize the resolution. It is presented in decibel values as

$$
\operatorname{CPSD}\left(k_{z}, f\right)=120+10 \log _{10}\left[\frac{G_{\text {top }}\left(k_{z}, f\right) G_{\text {side }}^{*}\left(k_{z}, f\right)}{r_{c}^{2}}\right]
$$


where $G\left(k_{z}, f\right)$ corresponds to the 2-D FFT transform of $r^{\prime}$, and * denotes the complex conjugate. The phase angle was computed from the coherence analysis. The average spatial and temporal mean cavity radius of both views has been used to non-dimensionalise the axial wavenumber $k_{z}$ and frequency $f$, while the tunnel velocity $W_{\infty}$ has been used as reference velocity. All values are given in Table 4.1.

Before discussing the wavenumber-frequency diagrams, the variation and spectra of the temporal mean and the spatial mean values for the diameter variation are presented. Figure 4.16 shows for both views in the upper two graphs the streamwise variation of the temporal mean cavity radius and the corresponding wavenumber spectrum. The lower two graphs show the temporal variation of the spatial mean cavity radius and the corresponding frequency spectrum. The spectra are given by non-dimensional power spectral densities in decibels, similar as for the CPSD. The graphs on the left show the results for Case A and the graphs on the right show the results for Case B.

For Case A in Figure 4.16, the two views show a difference in time-averaged cavity size of $100 r_{c} / c_{0}=0.08$, which corresponds to $r_{c}=0.1 \mathrm{~mm}$. This difference is of the same order as the magnitude of the pixel size and can be interpreted as the measurement uncertainty for the mean cavity size. For Case A, the wavenumber spectrum is rather flat, indicating a smooth cylindrical shape for the mean cavity diameter. In the time domain, a tonal is present at $\widetilde{\omega}=0.021$ and at the harmonic thereof. This tonal most likely corresponds to the blade passage frequency of the four bladed impeller of the tunnel that is close to $47 \mathrm{~Hz}$ (Pennings et al., 2015a), so $\widetilde{\omega}=0.023$. Also, a small broadband hump is present at $\widetilde{\omega} \approx 0.4$.

Case B in Figure 4.16 shows a twisted shape for the temporal mean with the maxima in the top view located at the similar axial location as the minima in the side view. One full period of this twist then corresponds to twice the difference in viewing angle. As the difference in viewing angle of both cameras is $90^{\circ}$, one period corresponds to $180^{\circ}$, hence it is equal to the elliptical shape of $n=2$. The non-dimensional wavenumber of this shape is $\kappa=0.42$, which corresponds to a non-dimensional wave length $\lambda / c_{0}=0.33$. The top view also shows a second hump at $\kappa=0.74$ which can be a harmonic of the first hump but the spectral resolution is not high enough to determine the actual wavenumber of the peaks. The temporal variation shows a tonal at $\widetilde{\omega} \approx 0.12$, again most likely due to the blade passage frequency of the impeller which is close to $47 \mathrm{~Hz}$. The spectrum also shows a broadband hump at $\widetilde{\omega}=0.407$, which corresponds to a frequency of $158 \mathrm{~Hz}$. This broadband hump is approximately $20 \mathrm{~dB}$ higher than the average hump of the two views for Case A.

The wavenumber-frequency spectrum of cavity diameter variations for Case A is presented in Figure 4.17. The flow conditions that are required for the predicted curves have been obtained by tuning the parameters in the prediction model to the experimental data, see Table 4.2. The value for the stiffness coefficient was obtained by considering the slope and wavenumber at $\widetilde{\omega}=0$ for mode $n=0^{-}$. The axial and azimuthal velocities at the cavity interface were obtained by matching the experimental curves for the mode $n=-1^{+}$and $n=1^{-}$. The effect of surface tension is taken into account but the effect is small, as the maximum value of $T_{\omega}$ equals $T_{\omega}=$ 1.026 for mode $n=2$.

The best way to compare the prediction with experiment is by analysing the phase, of which the values are only shown if the coherence value is larger than 0.4. Furthermore, only the absolute value of the phase, that varies between $-180^{\circ}$ and $180^{\circ}$, is shown which simplifies 

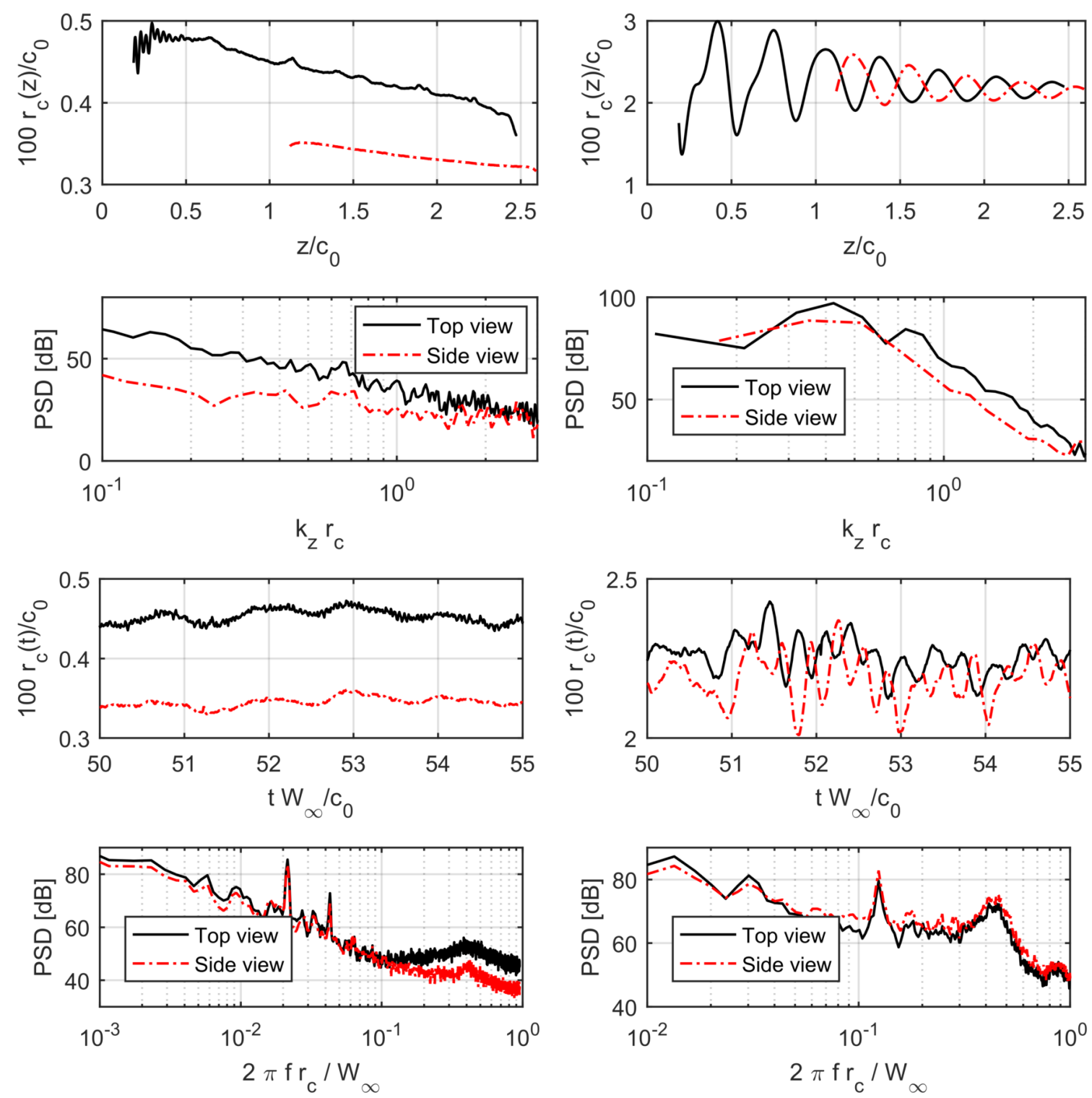

a) Case $\mathrm{A}$

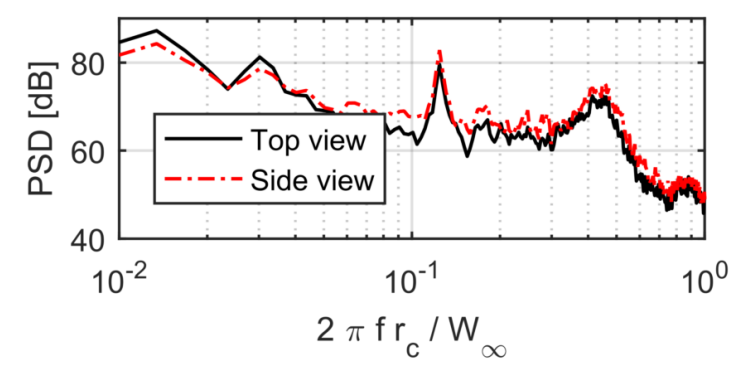

b) Case B

Figure 4.16: Analysis of the time-averaged (upper two rows) and spatial-averaged (lower two rows) variation of the mean cavity diameter of the experimental data by Pennings (2016). Left column shows results for Case A, right column shows results for Case B.

the identification of the modes. The graph for the CPSD has a higher resolution and provides information on the relative magnitude of the deformation of the cavity. The agreement for mode $n=0$ is quite good with only a small underprediction of the frequency for zero group velocity at $\kappa \approx-0.1$. In the experiment, the phase between the two views near the lines $n=0$ is about $0^{\circ}$, which is in agreement with theory. The phase in the experimental data for the lines $n=-1^{+}$ and $n=1^{-}$is also about $0^{\circ}$. This suggests that the centreline deformation is accompanied by a cross-section deformation, although the line for $n=1^{-}$can also correspond to a convection line for which the group velocity equals the tunnel velocity. In the experiment, the phase for 
line $n=-2^{+}$shows an angle close to $180^{\circ}$, hence matching the prediction. However, the experimental data might consist of two separate lines with a slope that is slightly different from the slope of the predicted curve. The experimental data also shows a dispersion curve in the upper-left corner of the graph for which no equivalent prediction curve is available, although mode $n=-1^{-}$is nearby. The experimental data for mode $n=0^{-}$shows coherent data at positive wavenumbers only for $\kappa<1$.

The wavenumber-frequency spectrum of cavity diameter variations for Case B is presented in Figure 4.18. Table 4.2 presents the values for the predicted dispersion lines obtained from tuning. The twisted cavity shape manifests itself by two dispersion lines for mode $n=2^{-}$ and $n=-2^{+}$. The stationary deformation is generated by the amplitudes at $\kappa= \pm 0.4$ and frequency zero. A peak at this wavenumber was also seen in Figure 4.16. The amplitude is largest at these wavenumbers but it decreases quickly when moving along the dispersion line in positive wavenumber direction. The experimental data also clearly shows the presence of the dispersion line for mode $n=0^{-}$, whereas the line for the mode $n=0^{+}$cannot be identified in the experimental data. Several other lines are observed in the experimental data. The lines with phase of $0^{\circ}$ might correspond to harmonics of the dispersion line $n=0^{-}$, while lines with phase of $180^{\circ}$ might correspond to harmonics of $n=-2^{+}$. The disturbance caused by the impeller, resulting in a disturbance of the mean cavity diameter at $\widetilde{\omega} \approx 0.11$ is expected to be convected with the free-stream velocity with the dispersion line passing through the origin. Such a line is present in the graph of the CPSD.

The fitted values for $\widetilde{W}_{c}, \widetilde{V}_{c}$ and $K_{\sigma}$ are compared in Table 4.2 to values from simple prediction formulas and to velocities measured by SPIV for nearby cases. Results for these cases are similar to those discussed in Section 3.3.2. For Case A as well as for Case B, the fitted values for $\widetilde{W}_{c}$ are in good agreement with measurement data. However, the fitted values for $\widetilde{V}_{c}$ are approximately $80 \%$ and $40 \%$ larger than measured for Case A and B, respectively. The velocities predicted by the modified cavitating Lamb-Oseen vortex, fitted to experimental data in Section 3.3.2, are almost identical to the measured values, hence differences to the fitted values for the dispersion relation are also almost identical. Therefore, results for this vortex model are presented in Table 4.2. Similar as in Figure 4.4b, two simple formulations for the non-cavitating vortex have been used to predict $\widetilde{V}_{c}$ and $K_{\sigma}$. Both formulations make use of the cavitation number instead of a vortex strength. Formulation 2 is the Rosenhead vortex used in the derivation of the dispersion relation, and Formulation 1 can be interpreted as its inviscid flow variant. The fitted values for $\widetilde{V}_{c}$ are $26 \%$ higher and $33 \%$ smaller than the values for Formulation 2 for Case $\mathrm{A}$ and B, respectively. The differences with respect to Formulation 1 are larger, especially for Case A. Equation (4.28), and Formulations 1 and 2, show that $K_{\sigma}=\widetilde{V}_{c}^{2}$. For the fitted values, $\widetilde{V}_{c}^{2}=0.52$ and $\widetilde{V}_{c}^{2}=0.34$ for Case A and Case B, respectively. These values are only $7 \%$ and $6 \%$ smaller than the fitted values for $K_{\sigma}$ for Case A and Case B, respectively, showing that the stiffness coefficient can indeed be computed from the azimuthal velocity at the cavity interface.

The wavenumber-frequency spectra for the perturbations of the cavity centreline are presented in Figure 4.19 and Figure 4.20 for Case A and Case B, respectively. The coefficients for the predicted curves are identical to those for the variation of the diameter. For Case A, the predicted lines shows a reasonable match with experiment, but the phase of the experimental data differs from the expected $90^{\circ}$. The dispersion diagram for Case B shows a complex behaviour and a poor correlation between prediction and experiment although the phase angles of the experimental data is $90^{\circ}$. A number of additional convection lines are drawn defined by 
Table 4.2: Overview of values of the non-dimensional velocities at the cavity interface, and of the stiffness coefficient $K_{\sigma}$ for the cases presented in Table 4.1. For both cases, the viscous core radius equals $r_{v}=1.2 \mathrm{~mm}$. Experimental data of Pennings (2016).

\begin{tabular}{c|cccc}
\hline & Experiment* $^{*}$ & Formulation 1 $^{1}$ & Formulation $2^{2}$ & Fitted \\
\hline Case A & & & & \\
$\widetilde{W}_{c}$ & 1.25 & - & - & 1.20 \\
$\widetilde{V}_{c}$ & 0.4 & 1.53 & 0.57 & 0.72 \\
$K_{\sigma}$ & - & 2.33 & 0.32 & 0.56 \\
\hline Case B & & & & \\
$\widetilde{W}_{c}$ & $\approx 1$ & - & - & 0.95 \\
$\widetilde{V}_{c}$ & 0.42 & 0.93 & 0.86 & 0.58 \\
$K_{\sigma}$ & - & 0.87 & 0.73 & 0.36 \\
\hline
\end{tabular}

*: Experimental values for Case A, $r_{c} / c_{0}=0.38$, are based on SPIV data for $r_{c} / c_{0}=0.42$, and for Case $\mathrm{B}, r_{c} / c_{0}=2.21$, on data for $r_{c} / c_{0}=1.61$.

1: $\widetilde{V}_{c}=\sqrt{\sigma_{W}}, K_{\sigma}=\sigma_{W}$.

2: $\widetilde{V}_{c}=\sqrt{\sigma_{W}} \sqrt{r_{c}^{2} /\left(r_{c}^{2}+r_{v}^{2}\right)}, K_{\sigma}=\sigma_{W} r_{c}^{2} /\left(r_{c}^{2}+r_{v}^{2}\right)$.

$\widetilde{\omega}=c_{g}\left(\kappa-\kappa_{0}\right)$, all at constant group velocity $c_{g}$ of $95 \%$ of the tunnel velocity. The lines are described by $\kappa_{0}=0.42 m$ with $m=-2,-1,1$ and 2 . The wavenumbers at zero frequency most likely correspond to the wavenumbers of the stationary deformation for $n=0$ and $n=2$. 


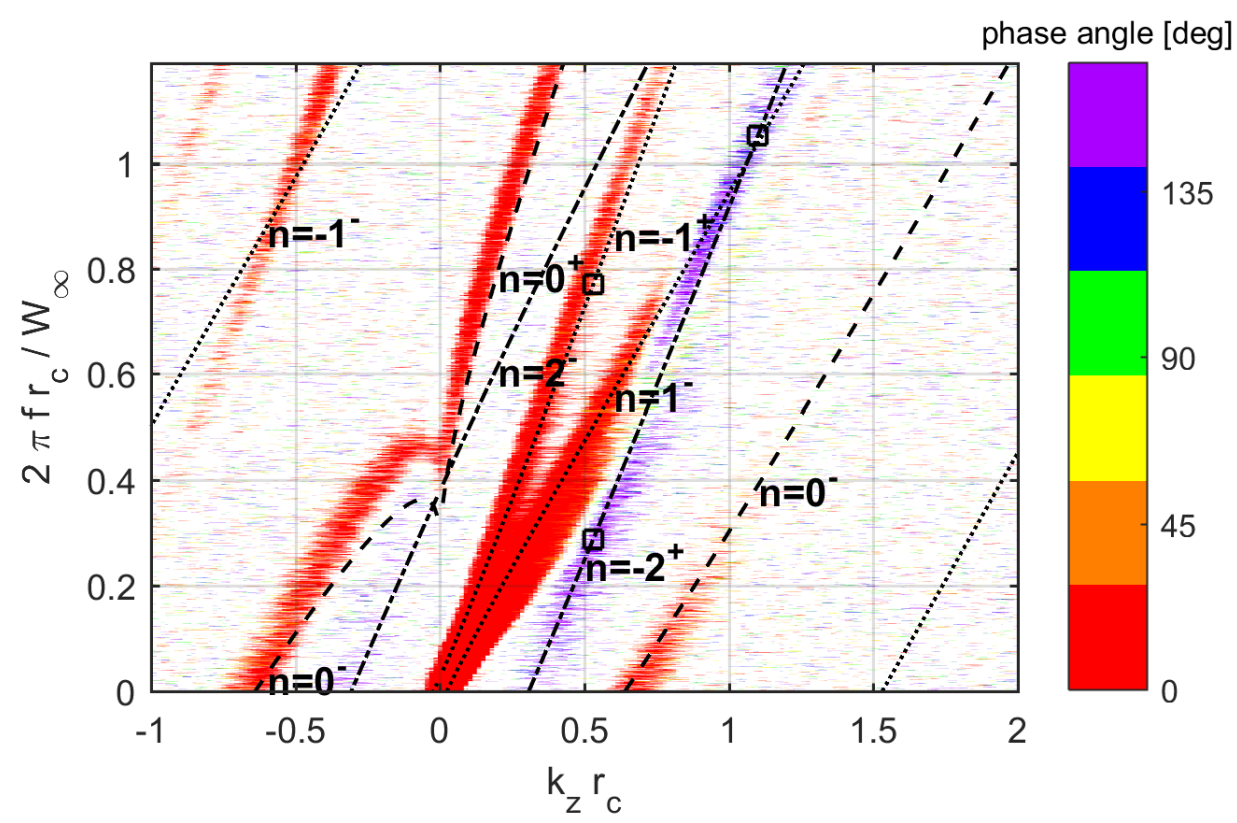

a) Phase difference between two camera views for coherence larger than 0.4

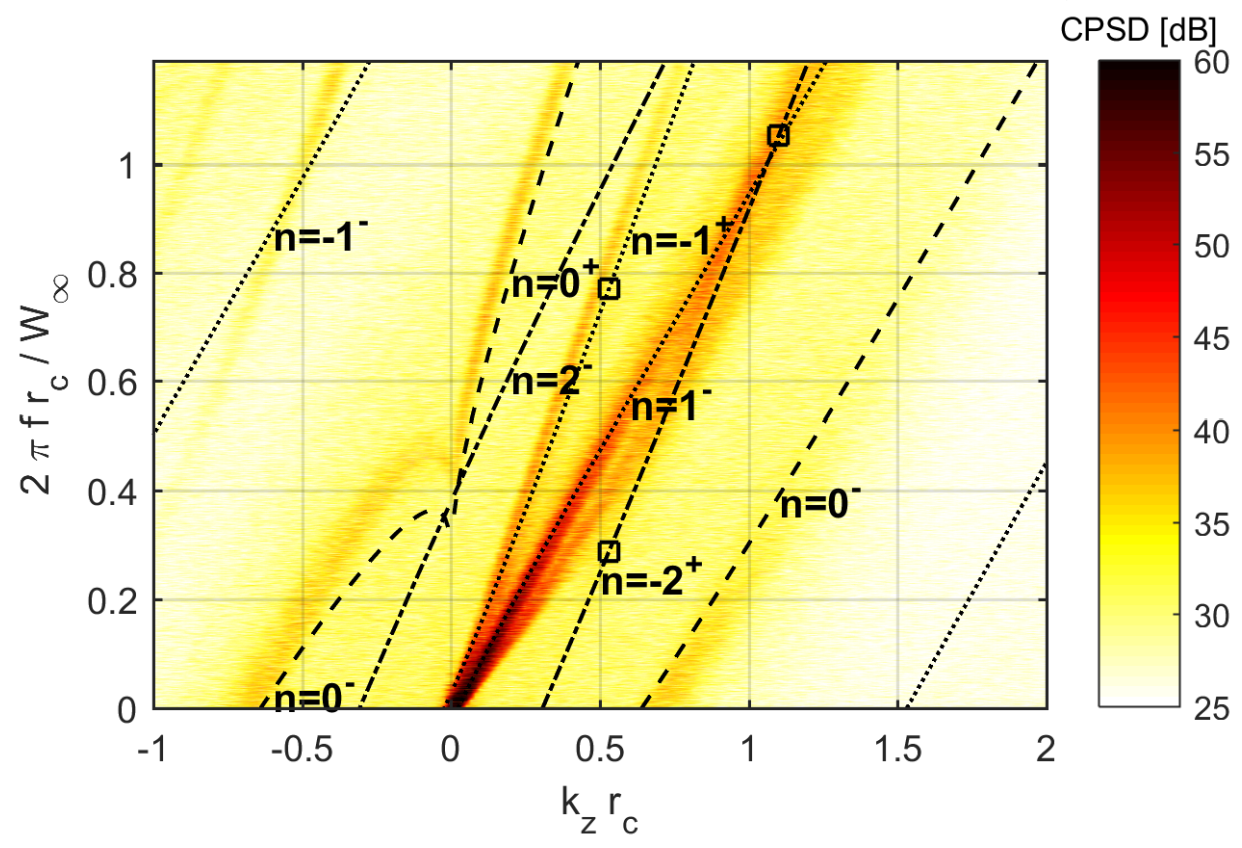

b) Cross-power spectral density

Figure 4.17: Wavenumber-frequency diagram of the variation of the cavity diameter, case A. Coefficients for the prediction are obtained by a fit. 


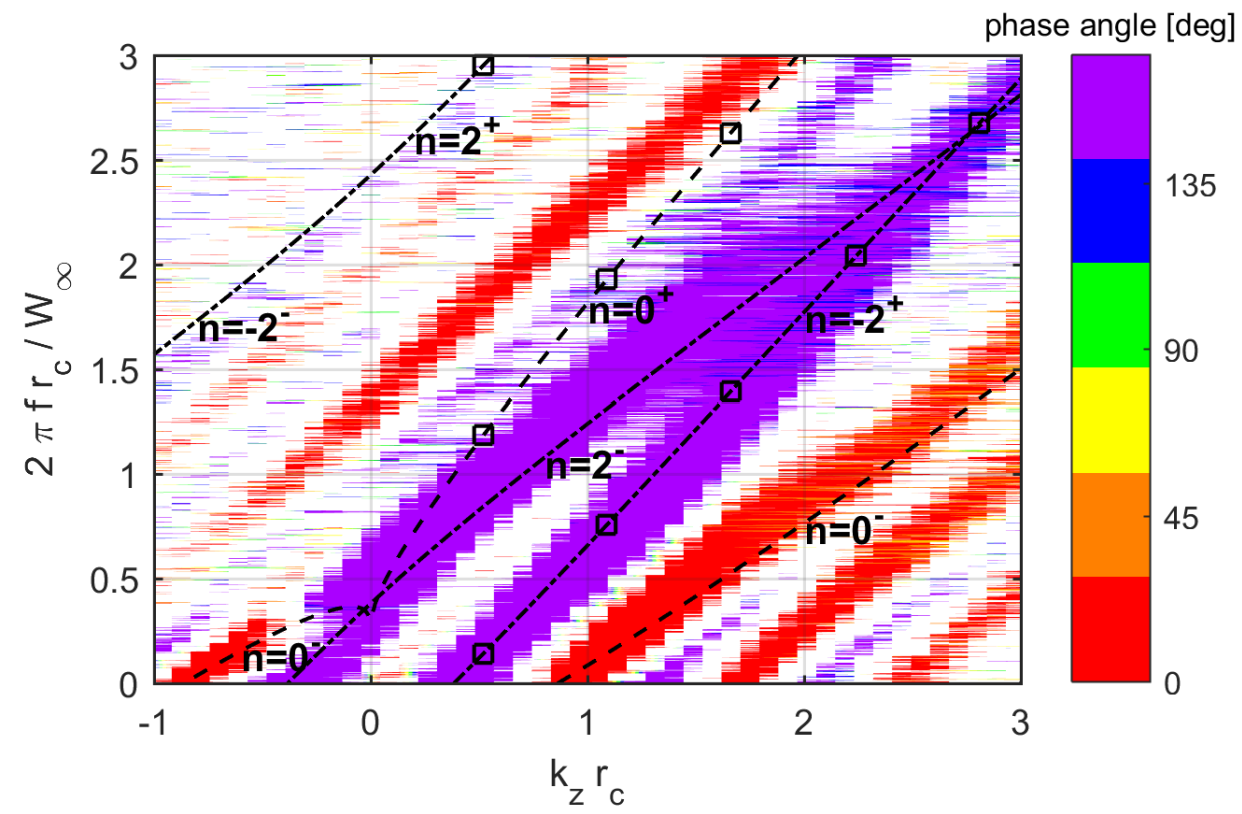

a) Phase difference between two camera views for coherence larger than 0.4

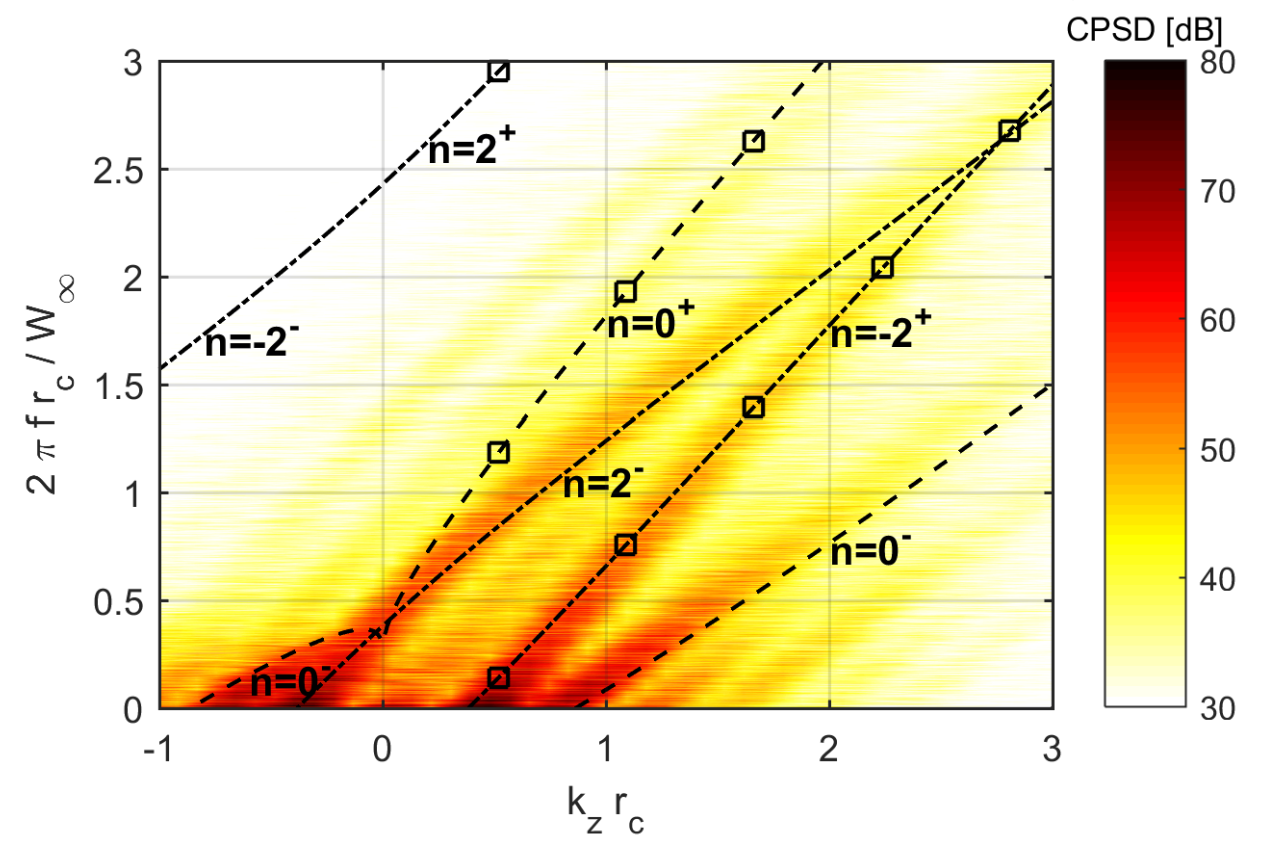

b) Cross-power spectral density

Figure 4.18: Wavenumber-frequency diagram of the variation of the cavity diameter, case B. Coefficients for the prediction are obtained by a fit. 


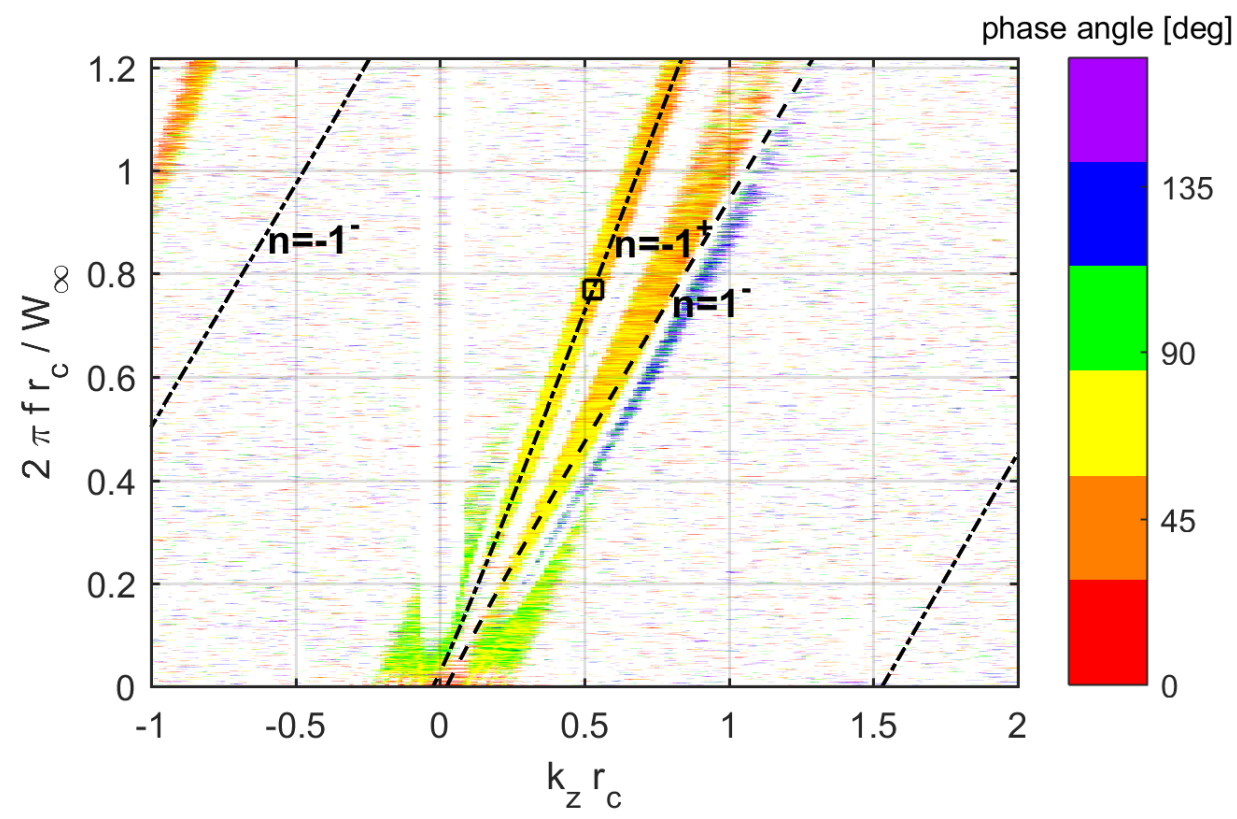

a) Phase difference between two camera views for coherence larger than 0.4

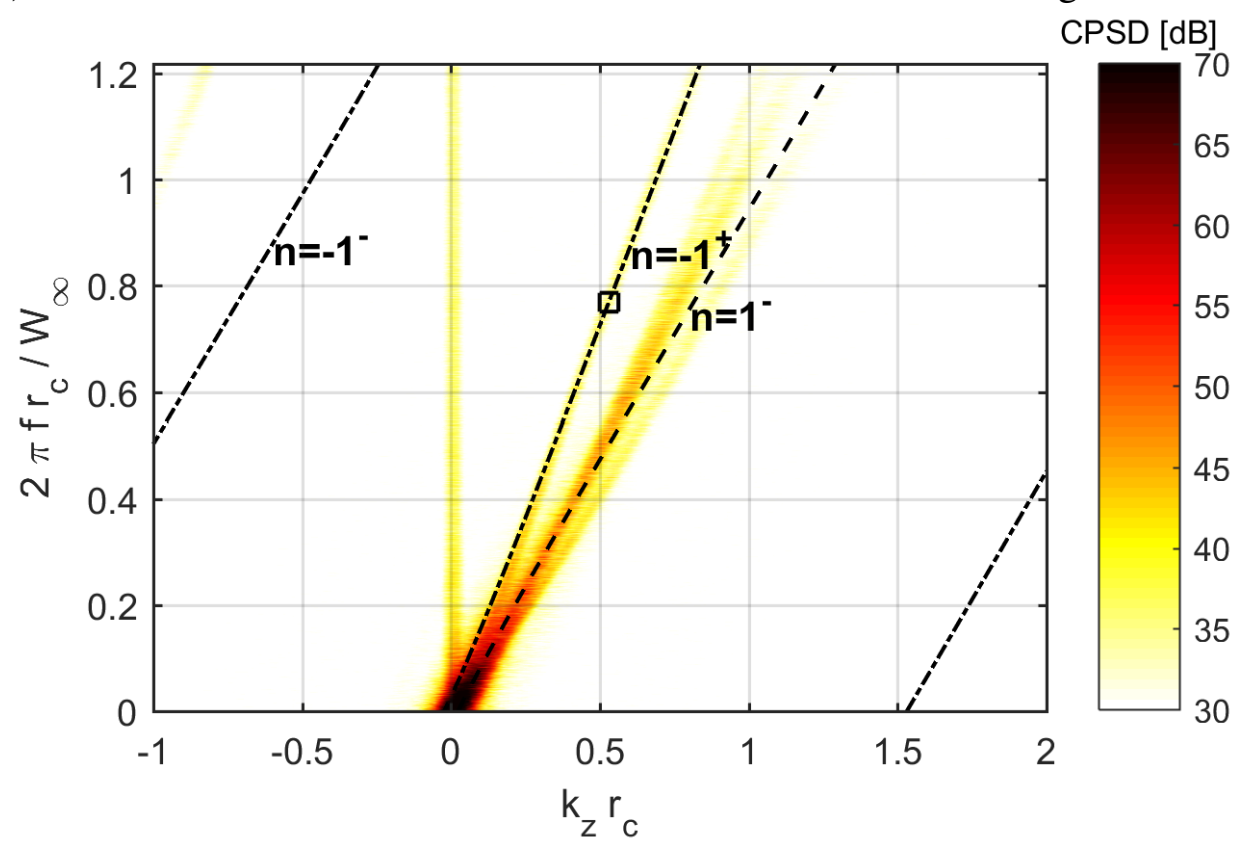

b) Cross-power spectral density

Figure 4.19: Wavenumber-frequency diagram of the variation of the cavity centreline, case A. Coefficients for the prediction are obtained by a fit. 


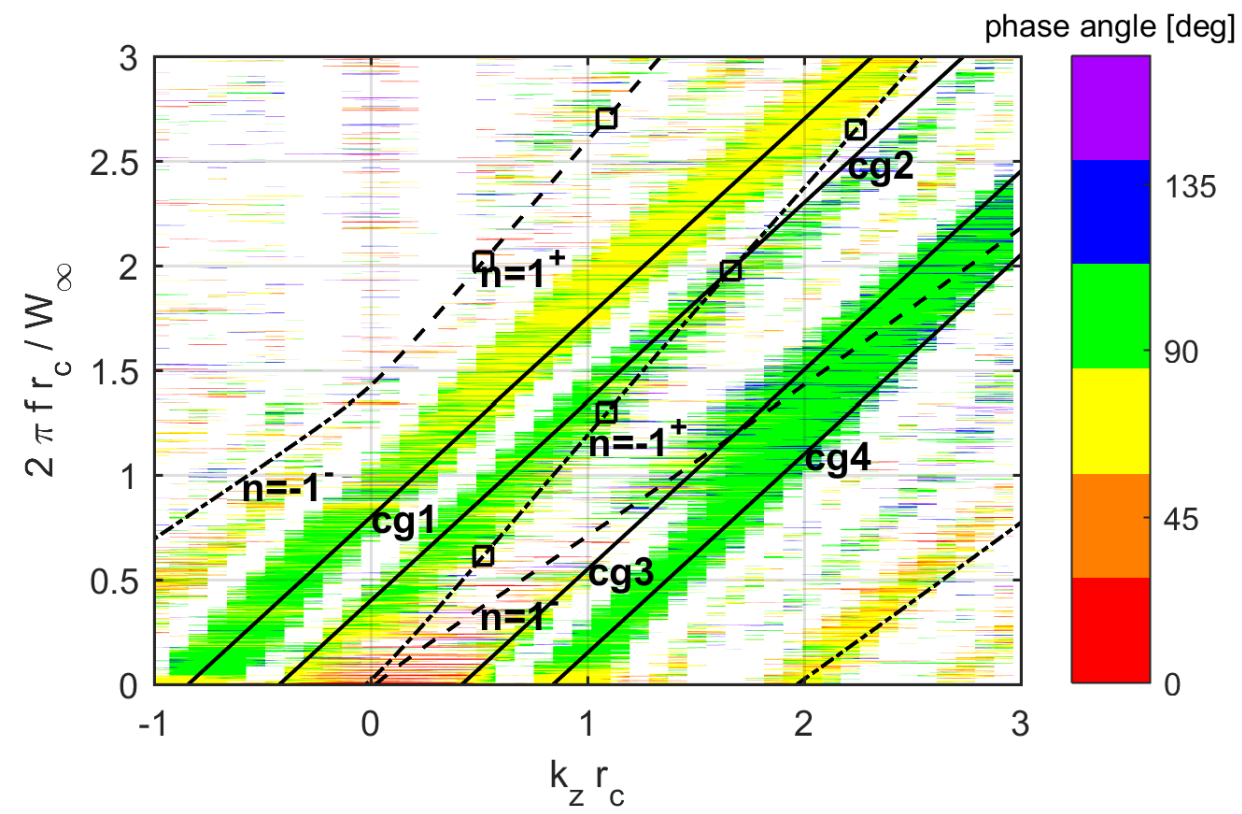

a) Phase difference between two camera views for coherence larger than 0.4

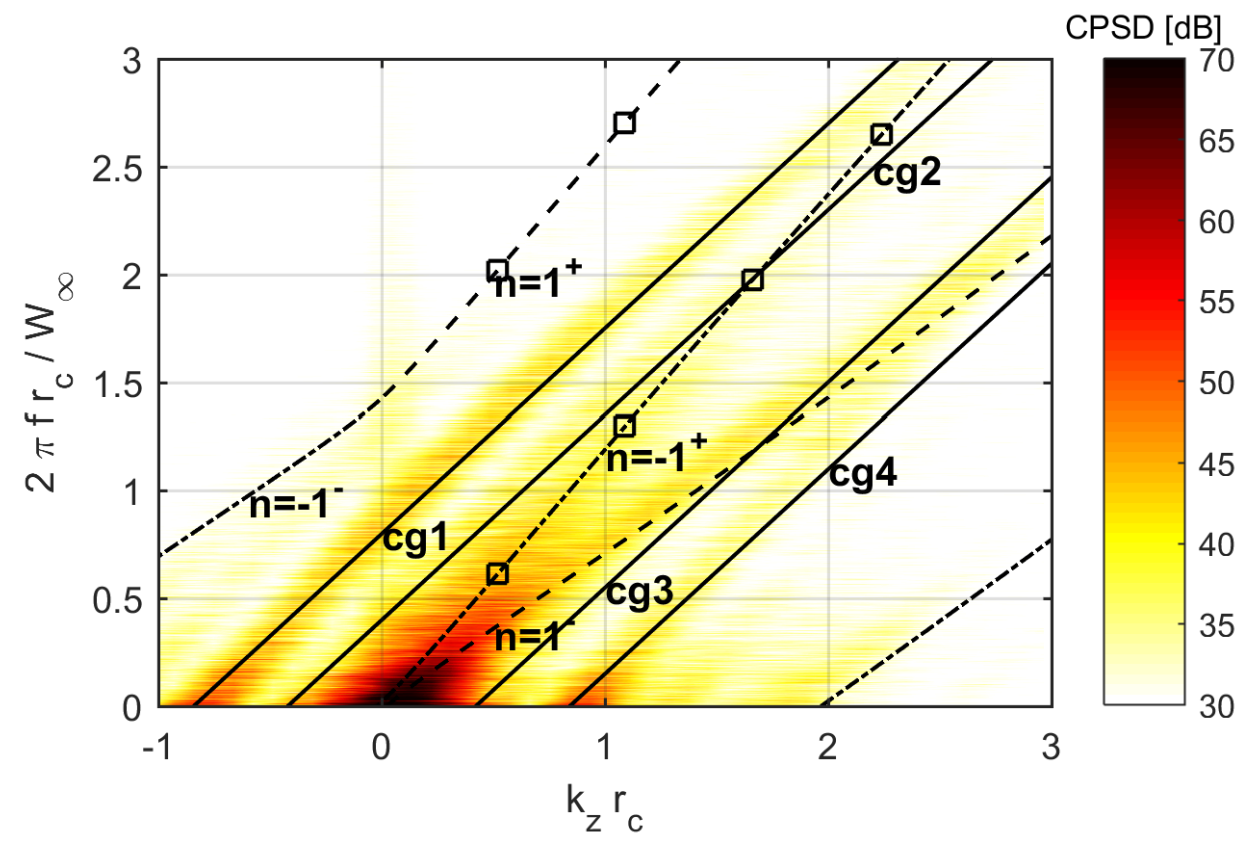

b) Cross-power spectral density

Figure 4.20: Wavenumber-frequency diagram of the variation of the cavity centreline, case B. Coefficients for the prediction are obtained by a fit. Lines $\operatorname{cg} 1$ through $\operatorname{cg} 4$ are lines of constant group speed fitted to the experiments. 


\subsubsection{Discussion}

The dynamics of a cavitating vortex in 3-D flow has been studied by analysing the dispersion relation for an infinitely long vortex. The relation obtained is compared with experimental data of Pennings (2016), see also Pennings et al. (2015a). The experimental data consists of high speed video observations of a cavitating vortex trailing from the tip of a wing of elliptical planform at $7^{\circ}$ angle of attack inside a cavitation tunnel. Two cases were analysed, one case for which the cavity size was smaller than the viscous core size and for which the cavity crosssection was cylindrical, and one case for which the cavity size was larger than the viscous core size and for which the cavity cross-section was elliptical. A distinction has been made for perturbations of the cavity diameter, $n=0$ and $n=2$, and for perturbations of the cavity centreline, $n=1$.

Overall, the analytical dispersion relation for $n=0$ could be well fitted to the experimental data although it was not possible to match the wavenumber at zero frequency as well as the frequency of the condition of zero group velocity (ZGV). For the results presented, the fit was applied to the wavenumber at zero frequency. The fitted values of the axial velocity were in very good agreement with measured velocities of Pennings (2016), but the fitted values for the azimuthal velocity were significantly larger than measured. However, considering the assumptions in the derivation of the stiffness coefficient, the agreement is considered good. The overall trend of the $n=0$ dispersion line, including the hook shape at the $\mathrm{ZGV}$, is well captured by the analytical formulation.

The mode $n=2$ cannot be clearly observed in case A with the cylindrical cavity crosssection, but is very dominant in case B for the elliptical cavity cross-section. For the cavity on the surface of the foil, no perturbation can propagate in azimuthal direction. The perturbation then forms either a standing wave in azimuthal direction with nodes at the surface of the foil or a stationary deformation that does not change in time. However, a standing wave of elliptical shape requires two modes with opposite azimuthal wavenumber and identical axial wavenumber. The dispersion diagram shows that this requirement can not be fulfilled due to the mean axial flow velocity at the cavity interface. So on the foil, only a stationary deformation exists with wavenumber given by modes $n=2^{-}$and $n=-2^{+}$at frequency zero. Due to the realvalued perturbation, the solution on both axes of the wavenumber-frequency diagram needs to be symmetric and solutions for both modes exist. The introduction of the mode $n=-2^{+}$is a new finding with respect to the results described in Pennings et al. (2015a). Downstream of the blade tip, where the overlapping fields of view of the cameras are analysed, this stationary deformation is no longer required and the perturbations can propagate along the dispersion curve. The axial wavenumber of the stationary deformation corresponds to the wavenumber of the centres of the hump in the wavenumber spectra presented in Figure 4.16. For Case A the hump is very small, but for Case B the hump can clearly be identified. A pronounced stationary deformation is also observed for mode $n=0$. The wavenumber of this deformation corresponds to small humps in Figure 4.16 for Case A as well Case B.

The elliptical cross-section observed for Case B is considered to be caused by the attachment of the vortex cavity on the blade, as the blade acts as a mirror for the region of vorticity. This is further discussed in Section 6.4.

The two spectra for the spatial-averaged variation of the cavity diameter in Figure 4.16 
each show a pronounced peak and a broadband hump. In the dispersion diagram, this spectrum corresponds to the values at $\kappa=0$. The disturbance corresponding to the peak that is considered to be due to the rotating impeller of the tunnel is probably convected with the tunnel velocity. Then, the line $n=1^{-}$in Figure 4.17 for Case A corresponds to a convection line. For Case B such a convection line can also be identified in the CPSD but has not been drawn in the graph as the coherence is smaller than 0.4 . The centre frequencies of the broadband humps at $\widetilde{\omega}=0.4$ in Figure 4.16 correspond to the frequencies of the dispersion lines of $n=0^{-}$and $n=0^{+}$ at $\kappa=0$ for Case $\mathrm{A}$ and $\mathrm{B}$ or to the frequency of the line $n=2^{-}$for Case B. However, the analytical dispersion line for mode $n=0$ does not have a limiting solution for $\kappa \rightarrow 0$ as shown by Eq. (4.82). For Case A, we expect that only mode $n=0$ is present. Hence, the experimental data suggests that a limiting solution for $\kappa \rightarrow 0$ does exist and, therefore, that the analytical formulation does not provide the correct trend. Limitations of the analytical formulation are that the vortex cavity is infinite instead of semi-infinite, and that a transient behaviour and viscous effects are not taken into account. Hence, the behaviour for $\kappa \rightarrow 0$ requires further attention, for the experiment as well as for the analytical model.

The results for the cavity centreline, involving modes for $n=1$, show a reasonable comparison for Case A. For Case B however, several additional lines needed to be introduced, for which the group speed equals the tunnel speed. This suggests that the origin of the perturbations was coming from the unsteady character of the flow in the cavitation tunnel of Delft University of Technology.

Whereas the equations for 2-D flow show the presence of a resonance frequency, the equations for 3-D flow show no direct criterion for a specific resonance frequency. Nevertheless, several criteria derived from the analytical dispersion relation have been proposed. These criteria are the zero group velocity (ZGV) criterion for mode $n=0^{-}$and the crossing of the mode $n=0^{-}$with mode $n=-2^{+}$and of the mode $n=0^{+}$with mode $n=2^{-}$. The corresponding wavenumbers are in close proximity so a combination of the criteria may also occur. All these criteria involve the variation of the cavity volume which is an efficient noise source as will be shown in Section 5.2. The modes $n=-2^{+}$and $n=2^{-}$appear when the vortex cavity is more developed and becomes attached to the wing surface. Other fields of application have shown that both the ZGV criterion and the criterion of crossing dispersion lines are valid. This is discussed in Section 6.2.

Finally, it needs to be remarked that the prediction method and the FFT analysis assume that the solution is periodic. In the measurements, a variation of the perturbations with downstream distance is observed. Such a variation leads to broadband peaks in the FFT as shown in Section 5.3.2. Furthermore, the dispersion diagram obtained from the measurements shows the presence of harmonics of the analytical dispersion curves. These harmonics indicate that the actual deformations have a more complex shape than the trigonometric functions assumed in the FFT and analytical formulation.

\subsection{Conclusions}

Computations for a 2-D vortex cavity in incompressible viscous flow show that the collapse is inertia driven, which gives an increase of the azimuthal velocity at the cavity interface. The 
increase in azimuthal velocity reduces the pressure at the cavity interface which dampens the collapse, and which leads to a rebound (Figures 4.2 and 4.5). In the rebound, however, a large variation of the azimuthal velocity occurs within the thin viscous layer around the cavity (Figure 4.6a).

The computations for 2-D viscous flow suggest that a full collapse of a vortex cavity can not occur. This confirms a similar statement made by Franc and Michel (2004) on the basis of 2-D inviscid flow. However, the flow inside the cavity has been neglected in the employed model. Even though it was shown in Chapter 3 that this is a valid assumption for steady flow conditions, it is expected that in the final phase of the collapse, with a very large azimuthal velocity at the cavity interface, this assumption is no longer valid. Also, the collapse of a 3-D tip-vortex cavity of a propeller only occurs for a limited axial extent. Therefore, we need to be careful in interpreting conclusions from the presented 2-D simulations from the point of view of a 3-D vortex cavity.

The computed resonance frequency of a 2-D vortex cavity can reasonably well be predicted from the added mass and the cavitation number, which can be interpreted as a stiffness coefficient (Eq. 4.26 and Figure 4.4b). Instead of the cavitation number, to which a correction for viscous effects needs to be applied, the stiffness coefficient can also be defined from the azimuthal velocity at the cavity interface. Viscous effects reduce the resonance frequency when the initial cavity size becomes smaller than the viscous core size for non-cavitating flow. For this situation, further research is required to improve the prediction of the resonance frequency.

The dispersion relation of inertial waves propagating on the cavity interface of a 3-D vortex agrees well with experimental data (Figures 4.17 through 4.20 ). This relation has been derived for potential flow with an ad-hoc correction for viscous effects on the stiffness coefficient (Eq. 4.77). Negative values for both the axial and azimuthal wavenumbers have been introduced. The fitted values for the parameters in the dispersion relation were in better agreement with predicted values for viscous flow than for potential flow, showing the relevance of these corrections (Table 4.2). The wavenumber-frequency diagram obtained from the measurement data shows more dispersion lines than the predictions (Figures 4.17 through 4.20). The additional lines were expected to correspond to, for instance, harmonics of the predicted dispersion lines, and to disturbances convected by the tunnel speed. The presented results provide a further understanding of the dispersion relation as presented in Pennings et al. (2015a).

A criterion for resonance could not be derived from the dispersion relation for a 3-D vortex cavity because all modes are neutrally stable (Eq. 4.77). Instead, several criteria have been proposed, being the condition of zero group velocity for the mode $n=0^{-}$, and the condition of crossing dispersion lines for branches of the modes $n=0$ and $n=2$.

Future studies on the dispersion relation could be devoted to an analysis for full-viscous flow and analysis of a semi-infinite vortex instead of an infinite vortex. The behaviour for $\kappa \rightarrow 0$ requires further attention, for the experiment as well as for the analytical model. 


\section{Chapter 5}

\section{Acoustics}

This chapter discusses several aspects of the acoustics of a vortex cavity, including its signal analysis. Analytical formulations are derived and briefly discussed. The formulations will be used in Chapter 6 to analyse experimental data and in Chapter 7 and 8 to develop prediction methods for broadband hull-pressure fluctuations and underwater radiated noise due to cavitating vortices

\subsection{Introduction}

Analytical formulations for the radiated noise due to perturbations of cavitating vortices have been discussed in literature by several authors. FfowcsWilliams and O'Shea (1970) discuss aspects of the acoustics of perturbations on a vortex of infinite length. Morozov (1974) presents a general theoretical relation for the radiated noise of a finite length cylindrical vortex using continuously distributed monopoles from which it is concluded that the far-field noise is radiated from the edge of the vibrating part of the vortex. Computational methods to predict the far-field radiated noise by cavitating vortices have been developed by, among others, Ligneul et al. (1983), Ligneul (1988), Szantyr (2006) and Berger et al. (2016). These methods use 2-D Rayleigh-Plesset-like equations, such as presented in Section 4.2, to solve for the dynamics of segments of the cavitating vortex followed by a far-field noise prediction by integrating over all segments.

Computational methods for the similar problem of predicting the radiated noise due to structural vibrations of axi-symmetric bodies of varying diameter have been proposed by Pond (1966), Stepanishen and Chen (1992), Nhieu and Ywanne (1994) and Stepanishen (1997). Most of these methods use slender body theory in which singularities can be put on the centre line of the body allowing for arbitrary axial variation of the diameter and of the perturbation. However, for a cavitating vortex we expect that we may neglect the variation in diameter in streamwise direction and use can be made of analytical formulations presented by Junger (1981) and Junger and Feit (1986) that describe the noise due to vibrations of a cylinder. Their derivation is reproduced in Section 5.2.4. The solution for a finite length cylinder will be compared to solutions for a pulsating and a vibrating sphere, and for an infinite length cylinder. These are presented in Section 5.2.1 and 5.2.2, respectively. The radiated noise generated by a finite length body 
composed of continuously distributed sources is presented in Section 5.2.3. This formulation provides additional information on the distance from the vortex beyond which the far field formulations are valid. This approach has a resemblance to the approach presented by Morozov (1974). The various formulations are discussed in Section 5.2.5.

The measured broadband hull-pressure fluctuations (HPF) and underwater radiated noise (URN) are usually presented as a power density spectrum or a power spectrum computed by a Fourier transform. For the interpretation of this spectrum we have to consider the effect of a series of cavity collapses in time due to a series of propeller-blade passages through the wake peak where the collapse occurs. The spectrum of the noise due to the collapse of a single cavitating vortex is characterised by a hump centred around what is possibly a resonance frequency of the cavitating vortex. The origin of the hump is more easily understood by considering the time-frequency transform pairs of some basic functions. These are presented in Section 5.3.2. In case of multiple collapses due to multiple blade passages, the pressure signals are periodic with blade passage frequency though small variations between blade passages are present. A formulation to predict the consequence of these variations in amplitude and phase separately has been presented by MacFarlane (1949). Section 5.3.3 presents an extension of this formulation by considering the combined variation of amplitude and phase. Results of this formulation for two experimental data sets showing the variation in amplitude and phase are presented in Section 6.3.3.

The semi-empirical prediction method that is presented in Chapter 7 uses both model-scale and ship-scale measurement data. Chapter 8 proposes a novel procedure to predict noise levels due to vortex cavitation measured at model scale. Both require scaling laws for the levels and frequencies in the noise spectra. Two existing scaling laws, one for low frequencies and one for high frequencies, are presented and discussed in Section 5.4.

For propeller-induced HPF, the effect of scattering by the ship hull needs to be taken into account. A novel simple formulation has been derived for this purpose that is presented in Section 5.5.1. The low-frequency URN by the propeller is affected by the sea-surface reflections. This so-called Lloyd's mirror effect is briefly discussed in Section 5.5.2. As we only deal with noise measurements in relative close proximity to the acoustic source, the effect of spatial variations of mean density and speed of sound in water is not considered here. Furthermore, deep water is assumed so the effect of the sea bottom on the URN is not taken into account.

The main conclusions of this chapter are provided in Section 5.6

\subsection{Analytical formulations for acoustic sources}

\subsubsection{Pulsating and vibrating sphere}

It is illustrative to compare the radiated noise of a vortex cavity with a cavitating bubble. Therefore, the radiated noise of a cavitating bubble, represented by a pulsating and vibrating sphere in an otherwise quiescent fluid, is presented first. The procedure to generate the solution is adopted from Blackstock (2000) and Jacobsen and Juhl (2011).

A general solution for the Helmholtz equation for the outward-radiated complex-valued 
pressure, $p^{\prime}$, as a function of time $t$ in spherical coordinates $(R, \theta, \vartheta)$, with $R$ the radial distance, $\theta$ the azimuth angle, $\theta \in[0,2 \pi]$, and $\vartheta$ the polar angle, $\vartheta \in[0, \pi]$, is given by

$$
p^{\prime}(R, \theta, t)=\sum_{n} A_{n} h_{n}^{(1)}(k R) P_{n}(\cos \theta) \exp (-i \omega t),
$$

with $A_{n}$ the amplitude for mode $n, h_{n}^{(1)}(k R)$ the complex-valued spherical Hankel function of the first kind, $P_{n}(\cos \theta)$ the Legendre polynomial, $\omega$ the angular frequency ${ }^{1}$ and $k$ the acoustic wavenumber with $k=\omega / c$ in which $c$ corresponds to the speed of sound. The solution has no dependency on $\vartheta$. The radial component $u^{\prime}$ of the acoustic particle velocity is related to $p^{\prime}$ by making use of a velocity potential and is given by

$$
u^{\prime}=\frac{1}{i \rho \omega} \frac{\partial p^{\prime}}{\partial R}
$$

with $\rho$ the density of the fluid. The pressure amplitude is denoted by $\hat{p}$ with $p^{\prime}(R, \theta, t)=$ $\hat{p}(R, \theta) \exp (-i \omega t)$. The pressure amplitude can be complex valued.

The first mode, $n=0$, is a pulsating mode with the Legendre polynomial and the spherical Hankel function ${ }^{2}$ given by, respectively,

$$
\begin{aligned}
P_{0}(\cos \theta) & =1, \\
h_{0}^{(1)}(k R) & =-i \frac{\exp (i k R)}{k R} .
\end{aligned}
$$

Substituting these relations in Eq. (5.1), the equation for the velocity, Eq. (5.2), becomes for the first mode

$$
u^{\prime}(R, t)=\frac{p^{\prime}(R, t)}{\rho c}\left(1+i \frac{1}{k R}\right)
$$

For the velocity, the boundary condition at the sphere with radius $a$ is $u^{\prime}(R=a, t)=U_{0} \exp (-i \omega t)$. Substitution in Equation (5.5) provides the relation for the modal amplitude $A_{0}$. The pressure then reads

$$
p^{\prime}(R, t)=-\frac{i \rho c k a^{2} U_{0}}{R} \frac{\exp (-i k a)}{1-i k a} \exp [i(k R-\omega t)] .
$$

For a compact source, for which the Helmholtz number $\mathrm{He}=k a \ll 1$ (Rienstra and Hirschberg, 2006), the formulation for the pressure reads

$$
p^{\prime}(R, \theta, \vartheta, t)=-\frac{i \rho c k Q_{0}}{4 \pi R} \exp [i(k R-\omega t)] \quad \forall k a \ll 1,
$$

in which the volume velocity $Q_{0}=4 \pi a^{2} U_{0}$ has been introduced. The pressure always decays as $1 / R$ and the solution is a monopole. In practical applications, the pressure amplitude is defined as

$$
\hat{p}(R)=\frac{\hat{p}_{s}}{R} \exp [i k R]
$$

\footnotetext{
${ }^{1}$ Note that often a different sign is used in front of $\omega$, but the adopted notation is consistent with Chapter 4 .

${ }^{2}$ http://dlmf.nist.gov/10.49, visited August 14, 2018.
} 
with $\hat{p}_{s}$ the root source factor (ISO 18405, 2017), with dimensions Pa m, corresponding to $\hat{p}_{s}=-i \rho c k Q_{0} / 4 \pi$.

For the scaling of cavitation noise, it is useful to consider the acoustic efficiency $\eta_{a c}{ }^{3}$,

$$
\eta_{a c}=\frac{P_{a}}{\omega E_{k i n}}=\frac{\int_{S} \frac{1}{2} \operatorname{Re}\left(\hat{p}^{*} \hat{\mathbf{u}}\right) \cdot \mathbf{n} d S}{\omega \frac{1}{2} m_{a} \frac{1}{2} U_{0}^{2}},
$$

where $P_{a}$ corresponds to the acoustic power in the far field and $E_{k i n}$ to the time-averaged kinetic energy. $P_{a}$ is computed from an integral of the acoustic intensity over a sphere. The acoustic intensity is defined as the real-valued part of the product of the complex conjugate of $p^{\prime}$, denoted by ${ }^{*}$, and the acoustic particle velocity vector $\mathbf{u}^{\prime}$. Parameter $m_{a}$ denotes the mass of the vibrating body for which we have to use here the added mass of the sphere. For the pulsating sphere, for which $m_{a}=\rho 4 \pi a^{3}$, the acoustic efficiency is given by

$$
\eta_{a c}=\frac{1}{k a} \frac{(k a)^{2}}{1+(k a)^{2}},
$$

which gives $\eta_{a c}=k a$ for small values of $k a$ and $\eta_{a c}=1 /(k a)$ for large values of $k a$.

Similar relations can be derived for the second mode $n=1$, which corresponds to a vibrating sphere with boundary condition $\hat{u}(R=a, \vartheta)=U_{0} \cos \vartheta$. The Legendre polynomial and the spherical Hankel function are given by

$$
\begin{aligned}
P_{1}(\cos \vartheta) & =\cos \vartheta \\
h_{1}^{(1)}(k R) & =-\frac{\exp (i k R)}{k R}\left(1-\frac{1}{i k R}\right) .
\end{aligned}
$$

For a compact source, the relation for the $n=1$ mode of the pressure is then given by

$$
p^{\prime}(R, \vartheta, t)=-\frac{k a}{2} \cos \vartheta \frac{\rho c k Q_{0}}{4 \pi R}\left(1-\frac{1}{i k R}\right) \exp [i(k R-\omega t)] \quad \forall k a \ll 1 .
$$

The pressure shows a directivity according to $\cos \vartheta$ and decays as $1 / R$ only in the far field which is defined as $k R \gg 1$. In the far field, the amplitude is a factor $\frac{k a}{2} \cos \vartheta$ smaller than that of the pulsating sphere with the same volume-velocity $Q_{0}$. The solution is that of a dipole with axis in the direction $\vartheta=0$.

\subsubsection{Infinite length vortex cavity}

The solution for the pressure radiated by a vibrating cylindrical vortex cavity of infinite length follows from the relations derived in Section 4.3.1 which were performed in a cylindrical coordinate system $(z, r, \theta)$. The acoustic pressure perturbation $p^{\prime}$ is given by the linearised Bernoulli equation,

$$
p^{\prime}=-\rho\left(\frac{\partial}{\partial t}+\mathbf{U} \cdot \nabla\right) \varphi^{\prime}
$$

\footnotetext{
${ }^{3}$ This definition of acoustic efficiency is sometimes, e.g. Fahy and Gardonio (2007), referred to as the radiation loss factor. However, acoustic efficiency is often used in literature on cavitation noise, e.g. Baiter (1985) and Ross (1987).
} 
where $\mathbf{U}$ and $\varphi^{\prime}$ denote the mean velocity and the acoustic velocity potential, respectively. The spatial and temporal variation of the vortex cavity radius with mean value $r_{c}$ is given by

$$
\eta(r, \theta, z, t)=r_{c}+\hat{r} \exp \left[i\left(k_{z} z+n \theta-\omega t\right)\right]
$$

with $k_{z}$ the wavenumber in axial direction, $n$ the mode or wavenumber in azimuthal direction and $\hat{r}$ the amplitude of the variation of the cavity size. Using Eq. (4.50) and (4.57), we find for the pressure in cylindrical coordinates

$$
p^{\prime}(z, r, \theta, t)=\rho \omega^{* 2} \frac{H_{n}^{(1)}\left(k_{r} r\right)}{k_{r} H_{n}^{(1) \prime}\left(k_{r} r_{c}\right)} \hat{r} \exp \left[i\left(k_{z} z+n \theta-\omega t\right)\right],
$$

in which $H_{n}^{(1)}\left(k_{r} r\right)$ corresponds to the Hankel function of the first kind for mode $n$. Parameter $k_{r}$ is the wavenumber in radial direction, $k_{r}=+\sqrt{k^{2}-k_{z}^{2}}$. The prime in $H_{n}^{(1) \prime}\left(k_{r} r_{c}\right)$ indicates that the derivative of the function with respect to its argument is taken. The corrected angular frequency $\omega^{*}$ is defined as

$$
\omega^{* 2}=\left(W_{\infty} k_{z}+\frac{\Gamma_{\infty} n}{2 \pi r^{2}}-\omega\right)\left(W_{c} k_{z}+\frac{V_{c} n}{r_{c}}-\omega\right) .
$$

with $W_{\infty}$ the free-stream axial velocity, $W_{c}$ and $V_{c}$ the mean axial and mean azimuthal velocity at the cavity interface, respectively, and $\Gamma_{\infty}$ the vortex strength. In the following, use is made of the pressure amplitude $\hat{p}$ related to the acoustic pressure as $p^{\prime}(z, r, \theta, t)=\hat{p}(r) \exp \left[i\left(k_{z} z+n \theta-\omega t\right)\right]$.

Assuming a compact source $\left(k_{r} r_{c} \ll 1\right)$, the formulation for the pressure amplitude can be simplified in the far field $\left(k_{r} r \gg 1\right)$ as

$$
\begin{aligned}
\hat{p}(r)=-i \rho \omega^{* 2} \hat{r} \frac{r_{c}}{2} \sqrt{\frac{2 \pi}{k_{r} r}} \exp \left[i\left(k_{r} r-\frac{1}{4} \pi\right)\right], & \forall n=0, k_{r} r_{c} \ll 1, k_{r} r \gg 1 \\
\hat{p}(r)=-i \rho \omega^{* 2} \hat{r} \frac{r_{c}}{n \Gamma(n)}\left(\frac{k_{r} r_{c}}{2}\right)^{n} \sqrt{\frac{2 \pi}{k_{r} r}} \times & \\
\exp \left[i\left(k_{r} r-\frac{1}{2} n \pi-\frac{1}{4} \pi\right)\right], & \forall n>0, k_{r} r_{c} \ll 1, k_{r} r \gg 1
\end{aligned}
$$

with $\Gamma(n)$ the Gamma function. The pressure decays as $1 / \sqrt{r}$, which corresponds to a cylindrical propagation loss.

In the formulation for the radiated pressure, we have to consider a low-frequency region and a high-frequency region. At high frequencies, defined as $k>k_{z}, k_{r}$ is a real number and the formulation for the pressure describes a propagating wave. At low frequencies, such as the frequency range in which propeller-induced HPF are usually measured, $k$ is smaller than $k_{z}$ which results in

$$
k_{r}^{2}=k^{2}-k_{z}^{2} \approx-k_{z}^{2}
$$

As the radial wavenumber now becomes an imaginary number, $k_{r}=i k_{z}$, the Hankel function changes into the modified Bessel function of the second kind $K$ and the pressure amplitude reads

$$
\hat{p}(r)=\rho \omega^{* 2} \hat{r} \frac{K_{n}\left(\left|k_{z} r\right|\right)}{\left|k_{z}\right| K_{n}^{\prime}\left(\left|k_{z} r_{c}\right|\right)}
$$


where the absolute value of $k_{z}$ is used to allow for negative numbers. For a compact source and $n=0$, the pressure amplitude in the far field is given by

$$
\hat{p}(r)=-\rho \omega^{* 2} \hat{r} \frac{r_{c}}{2} \sqrt{\frac{2 \pi}{k_{z} r}} \exp \left(-k_{z} r\right) \quad \forall n=0, k_{z} r_{c} \ll 1, k_{z} r \gg 1 .
$$

For the higher order modes, the pressure amplitude of a compact source in the far field becomes

$$
\hat{p}(r)=-\rho \omega^{* 2} \hat{r}\left(\frac{r_{c}}{n \Gamma(n)}\right)\left(\frac{k_{z} r_{c}}{2}\right)^{n} \sqrt{\frac{2 \pi}{k_{z} r}} \exp \left(-k_{z} r\right) \quad \forall n>0, k_{z} r_{c} \ll 1 k_{z} r \gg 1 .
$$

Hence, it is concluded that the pressure wave for $k_{r}=i k_{z}$ is an evanescent wave as the spreading loss shows an exponential decay for all modes.

The frequency where $k=k_{z}$ is the critical or lowest coincidence frequency (Fahy and Gardonio, 2007). The efficiency of the modes $n>0$ is a factor $\left(k_{z} r_{c} / 2\right)^{n} / n$ ! smaller than the efficiency of the mode $n=0$ as $\Gamma(n)=(n-1)$ !.

\subsubsection{Finite length cylinder composed of distributed sources}

The next solution considered briefly is a cylinder that vibrates over a finite length only and that is represented by an array of continuously distributed sources on the axis. The line array has length $2 L$ and is oriented along the z-axis with its centre at $z=0$. The pressure amplitude, defined in a spherical coordinate system with $p^{\prime}(R, \vartheta, t)=\hat{p}(R, \vartheta) \exp (-i \omega t)$, is then given by

$$
\hat{p}(R, \vartheta)=\int_{-L}^{L} S(z) \frac{\exp \left[i k R_{z}(z, R, \vartheta)\right]}{R_{z}(z, R, \vartheta)} \exp [i \Phi(z)] \mathrm{d} z
$$

with $S(z)$ the axial distribution of the source amplitude, $\Phi(z)$ the axial distribution of the phase angle and $R_{z}(z, R, \vartheta)$ the distance from the field point at $(R, \vartheta)$ to location $z$ on the vortex. In the following, both the cylindrical coordinate system $(z, r, \theta)$ and the spherical coordinate system $(R, \theta, \vartheta)$ will be used, see Figure 5.1.

The distance $R_{z}$ between a field point at $(R, \vartheta)$ and location $(z, r=0)$ on the vortex can be written as

$$
\begin{aligned}
R_{z}(z, R, \vartheta) & =R \sqrt{1-2 \frac{z}{R} \cos \vartheta+\left(\frac{z}{R}\right)^{2}} \\
& \approx R-z \cos \vartheta+\frac{1}{2} \frac{z^{2}}{R} \sin ^{2} \vartheta \quad \forall z / R \ll 1
\end{aligned}
$$

The region where $R / L \gg 1$ is defined as the far field. The directivity of the source is determined by the term $R_{z}$ in the numerator of Eq. (5.24) and thereby by the second and third term in the second line of Eq. (5.25). However, the integral involving the second term over the interval $[-L, L]$ equals zero leaving only the third term. If the third term becomes larger than $1 / 16$ of the wave length $\lambda$, equal to a change in phase angle smaller than $2 \pi / 16$, the field point is located in 


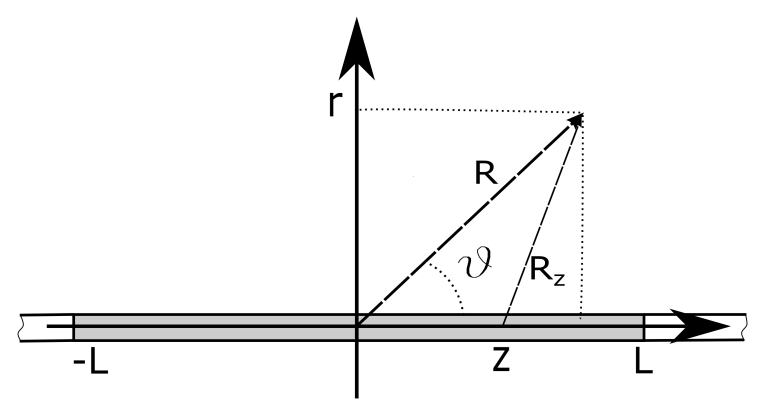

Figure 5.1: Cavitating vortex vibrating over length $2 L$ in a cylindrical coordinate system $(z, r, \theta)$ and spherical coordinate system $(R, \theta, \vartheta)$. The azimuth angle $\theta$ is identical for both systems but is not shown in the figure.

the so-called Fresnel region, and when it becomes smaller it is located in the Fraunhofer region (Milligan, 1995). Hence, in the Fraunhofer region the contribution of this third term can be neglected. The largest value for the dividing radius $R_{F F}$ between the two regions is for $z=L$ and $\vartheta=\pi / 2$, and is given by

$$
R_{F F}=\frac{16 L^{2}}{2 \lambda}=\frac{k}{\pi}(2 L)^{2},
$$

with the Fresnel region located at smaller distances and the Fraunhofer region at larger distances.

A simple idealized situation is when the amplitude and phase of the source distribution are constant over the vibrating cylinder with $S(z)=S / 2 L$ and $\Phi(z)=0$. The amplitude of the pressure in the Fraunhofer region is then given by

$$
\begin{aligned}
\hat{p}(R, \vartheta) & =\frac{\exp (i k R)}{R} \frac{S}{2 L} \int_{-L}^{L} \exp (i k z \cos \vartheta) \mathrm{d} z \\
& =\frac{S \exp (i k R)}{R} \frac{\sin (k L \cos \vartheta)}{k L \cos \vartheta} .
\end{aligned}
$$

The last part of the right-hand-side, which is the sinc function with argument $k L \cos \vartheta$, determines the directivity of the radiated pressure. It has its maximum value of one at $\vartheta=\pi$, so perpendicular to the vibrating cylinder. In the Fresnel region the directivity becomes dependent on the distance $R$.

\subsubsection{Finite length cylinder with prescribed vibration}

\section{Far field formulation}

We now consider a vibrating vortex cavity of finite length with prescribed perturbation of the cavity radius. The formulation for the radiated noise of such a cavity can be derived using the approach described by Junger (1981) and Junger and Feit (1986). They provide an analytical formulation for the far-field radiated noise of structural vibrations of a cylinder of finite extent. The starting point is the equation for the radiated pressure of an infinite length vortex, for 
which we use the general formulation with the Hankel function, Eq. (5.16), repeated here for convenience,

$$
p^{\prime}(z, r, \theta, t)=\rho \omega^{* 2} \frac{H_{n}^{(1)}\left(k_{r} r\right)}{k_{r} H_{n}^{(1) \prime}\left(k_{r} r_{c}\right)} \hat{r} \exp \left[i\left(k_{z} z+n \theta-\omega t\right)\right] .
$$

We consider a cylinder that only vibrates on a finite part $z \in[-L, L]$ with boundary conditions such that no perturbations are present at $|z|=L$. For a cavitating vortex, this boundary condition is not necessarily valid, but for the collapse and rebound of a vortex cavity the deformation is maximum at the collapse point and decreases with distance from this point. Hence, as a limiting situation, this condition is useful to consider.

For this cylinder, the propagating wave in axial direction is replaced by a standing wave and, as only specific axial wavenumbers are allowed due to the boundary condition discussed above, $k_{z}$ is written as $k_{m}$. The formulation for the perturbation of the cavity radius can be written as

$$
r_{m}^{\prime}(z, \theta, t)= \begin{cases}\hat{r} \cos \left(k_{m} z\right) \exp [i(n \theta-\omega t)], & |z|<L \\ 0, & |z|>L\end{cases}
$$

with

$$
k_{m}=\left(\frac{1}{2}+m\right) \pi / L
$$

In Eq. 5.30, $m$ is an integer with $m \geq 0$ which results into

$$
\begin{aligned}
& \cos \left(k_{m} L\right)=0, \\
& \sin \left(k_{m} L\right)=(-1)^{m} .
\end{aligned}
$$

The length of the vibrating part of the cylinder equals $2 L$, and therefore the relative wave length corresponds to

$$
\frac{\lambda_{m}}{2 L}=\frac{2 \pi}{k_{m} 2 L}=\frac{1}{\left(\frac{1}{2}+m\right)}=\frac{2}{(1+2 m)} .
$$

For the mode $m=0$ half a wave length fits on the vibrating part of the cylinder and for the mode $m=1$ this part contains 1.5 wave lengths. The mode $m=0$ is considered representative of the collapse of a vortex cavity.

The Fourier transform of the amplitude variation of the cavity perturbation in axial direction is then given by

$$
\begin{aligned}
\hat{r}_{m}^{F}(\gamma) & =\hat{r} \int_{-L}^{L} \cos \left(k_{m} z\right) \exp (-i \gamma z) \mathrm{d} z \\
& =\hat{r} \frac{2 k_{m}(-1)^{m} \cos (\gamma L)}{k_{m}^{2}-\gamma^{2}} .
\end{aligned}
$$

The superscript $F$ is introduced to indicate that the function is the Fourier transform of the time domain solution. Note that the unit of $\hat{r}_{m}^{F}$ is $\mathrm{m}^{2}$. The azimuthal wavenumber does not depend on the $\mathrm{z}$-coordinate and is therefore not considered in the Fourier transform. Some specific values 
for $\hat{r}_{m}^{F}$ are given by

$$
\begin{aligned}
& \hat{r}_{m}^{F}(\gamma=0)=(-1)^{m} \frac{2 \hat{r}}{k_{m}} \\
& \hat{r}_{m}^{F}\left(\gamma=k_{m}\right)=L \hat{r} \\
& \hat{r}_{m}^{F}(\gamma \rightarrow \infty)=0 .
\end{aligned}
$$

The average perturbation value then reads

$$
\hat{r}_{m, \text { avg }}=\frac{\hat{r}}{2 L} \int_{-L}^{L} \cos \left(k_{m} z\right) d z=\frac{\hat{r}}{L k_{m}}(-1)^{m}
$$

This average value corresponds to the value of $\hat{r}_{m}^{F}$ at $\gamma=0$ divided by the integration length $2 L$.

Introducing the Fourier transform of the amplitude variation in the formulation for the pressure gives

$$
p^{\prime}(z, r, \theta, t)=\frac{\rho}{2 \pi} \sum_{n} \omega_{m n}^{* 2} \exp [i(n \theta-\omega t)] \int_{-\infty}^{\infty} \frac{H_{n}^{(1)}\left(k_{r} r\right)}{k_{r} H_{n}^{(1) \prime}\left(k_{r} r_{c}\right)} \hat{r}_{m}^{F}(\gamma) \exp (i \gamma z) \mathrm{d} \gamma
$$

with $k_{r}^{2}=k^{2}-\gamma^{2}$. The formulation can be simplified by considering the far field approximation of the Hankel function,

$$
H_{n}^{(1)}\left(k_{r} r\right)=\sqrt{\frac{2}{\pi k_{r} r}}(-i)^{n} \exp \left(i k_{r} r-i \pi / 4\right), \quad \forall k_{r} r \gg 1 .
$$

In the far field, it is also more useful to replace the cylindrical coordinates by spherical coordinates,

$$
\begin{aligned}
& r=R \sin \vartheta \\
& z=R \cos \vartheta
\end{aligned}
$$

The resulting pressure perturbation in the far field then reads

$$
\begin{aligned}
p^{\prime}(R, \theta, \vartheta, t)=\frac{\rho}{\sqrt{2 \pi^{3} R \sin \vartheta}} \sum_{n m} & \omega_{m n}^{* 2}(-i)^{n} e^{-i \pi / 4} \exp [i(n \theta-\omega t)] \times \\
& \int_{-\infty}^{\infty} \frac{\exp \left[i R\left(k_{r} \sin \vartheta+\gamma \cos \vartheta\right)\right]}{k_{r}^{3 / 2} H_{n}^{(1) \prime}\left(k_{r} r_{c}\right)} \hat{r}_{m}^{F}(\gamma) \mathrm{d} \gamma, \quad k_{r} r \gg 1
\end{aligned}
$$

The relevant contribution to the pressure in the far field is obtained by applying the method of stationary phase (Lighthill, 1978; Junger and Feit, 1986; James and Skelton, 1997). This method states that, if the modulus of the pressure integral changes slowly with wavenumber, while the phase changes rapidly, the resulting contribution in the far field is relatively small because of cancellation of neighbouring regions of opposite phase and almost equal amplitude. The main contribution is then coming from the region of integration in which the phase changes slowly with wavenumber. 
The method of stationary phase is presented for the integral

$$
I(R, k, \vartheta)=\int_{-\infty}^{\infty} \Phi(\gamma) \exp [i R \Psi(\gamma, k, \vartheta)] \mathrm{d} \gamma
$$

with $\Phi(\gamma)$ the modulus, $R$ a distance and $\Psi(\gamma, k, \vartheta)$ the wavenumber distribution which determines the distribution of the phase. The stationary phase approximation of this integral is given by (Junger and Feit, 1986; James and Skelton, 1997)

$$
I(R, k, \vartheta)=\frac{\sqrt{2 \pi} \Phi(\bar{\gamma}) \exp [ \pm i \pi / 4+i R \Psi(\bar{\gamma}, k, \vartheta)]}{\sqrt{R\left|\partial^{2} \Psi(\bar{\gamma}, k, \vartheta) / \partial \gamma^{2}\right|}} \quad \forall R \gg 1
$$

The sign in the numerator corresponds to the sign of $\partial^{2} \Psi(\bar{\gamma}, k, \vartheta) / \partial \gamma^{2}$. The point of stationary phase $\gamma=\bar{\gamma}$ is defined by

$$
\partial \Psi(\bar{\gamma}) / \partial \gamma=0
$$

which is only valid under the condition that both the first and second derivative of $\Psi$ and $\Phi$ with respect to $\gamma$ are continuous in the interval $\gamma \in[-\infty, \infty]$ and only one point of stationary phase exists in this interval.

For the pressure perturbation, the modulus and phase components are given by, respectively,

$$
\begin{gathered}
\Phi(\gamma, k)=\frac{\hat{r}_{m}^{F}(\gamma)}{\left(k^{2}-\gamma^{2}\right)^{3 / 4} H_{n}^{(1) \prime}\left(\sqrt{k^{2}-\gamma^{2}} r_{c}\right)}, \\
\Psi(\gamma, k, \vartheta)=\left(\sqrt{k^{2}-\gamma^{2}} \sin \vartheta+\gamma \cos \vartheta\right) .
\end{gathered}
$$

The point of stationary phase is given by

$$
\frac{\partial \Psi}{\partial \gamma}=\left(-\frac{\gamma \sin \vartheta}{\sqrt{k^{2}-\gamma^{2}}}+\cos \vartheta\right)=0
$$

from which it follows that

$$
\bar{\gamma}= \pm k \cos \vartheta
$$

Because the distribution of the amplitude is even with $z$, the Fourier transform is also even and two even solutions for the point of stationary phase are obtained. In the following only the solution with the plus sign is considered. The second derivative of the phase then reads

$$
\begin{aligned}
\frac{\partial^{2} \Psi(\bar{\gamma}, k, \vartheta)}{\partial \gamma^{2}} & =-\sin \vartheta\left(\frac{1}{\sqrt{k^{2}-\gamma^{2}}}+\frac{\gamma^{2}}{\left(k^{2}-\gamma^{2}\right)^{3 / 2}}\right) \\
& =-\frac{1}{k \sin ^{2} \vartheta} . \quad \forall \gamma=\bar{\gamma}
\end{aligned}
$$

The modulus and the phase for the point of stationary phase are then given by

$$
\begin{aligned}
\Phi(\bar{\gamma}, k, \vartheta) & =\frac{\hat{r}_{m}^{F}(k \cos \vartheta)}{\left(k^{2} \sin ^{2} \vartheta\right)^{3 / 4} H_{n}^{(1) \prime}\left(k r_{c} \sin \vartheta\right)} \\
\Psi(\bar{\gamma}, k) & =k
\end{aligned}
$$


Due to the minus sign of the second derivative of the phase, a minus sign is used in the numerator of Eq. (5.43) and the integrand equals

$$
I(R, k, \vartheta)=\frac{\sqrt{2 \pi} r_{m}^{F}(k \cos \vartheta) \exp (i k R-i \pi / 4)}{k \sqrt{R \sin \vartheta} H_{n}^{(1) \prime}\left(k r_{c} \sin \vartheta\right)} .
$$

The method of stationary phase shows that in the far field only the axial wavenumber $\bar{\gamma}=k \cos \vartheta$ contributes to the radiated pressure. In the plane $z=R \cos \vartheta=0$, the point of stationary phase corresponds to $\bar{\gamma}=0$. So the radiated pressure in the far field at the plane $z=0$ is only due to the average perturbation, all other components of the perturbation spectrum cancel. For the point of stationary phase, it is also seen that the radial wavenumber squared, given by $k_{r}^{2}=k^{2}-\bar{\gamma}^{2}$, is always positive so the radial wavenumber does not become an imaginary number as was the case for the infinite length vortex.

The stationary phase approximation of Eq. (5.41) now becomes

$$
\begin{aligned}
\hat{p}(R, \vartheta, \theta)=-\frac{\rho \exp (i k R)}{\pi k R \sin \vartheta} \sum_{m n} \omega_{m n}^{* 2}(-i)^{n+1} \frac{\hat{r}_{m}^{F}(k \cos \vartheta)}{H_{n}^{(1) \prime}\left(k r_{c} \sin \vartheta\right)} \exp (i n \theta) \\
\forall R / L \gg 1, k R \sin \vartheta \gg 1 .
\end{aligned}
$$

in which parameter $L$ has been used to non-dimensionalise the criterion for the stationary phase approximation as $\gamma$ scales with the inverse of $L$. An alternative non-dimensionalisation parameter is the wavenumber $k$, which results in the criterion $k R \gg 1$. This criterion is similar to the criterion for the far field approximation of the Hankel function, $k R \sin \vartheta \gg 1$, that has already been used. This aspect will be further discussed later.

For the derivative of the Hankel function we now assume a compact source, $k r_{c} \ll 1$,

$$
H_{n}^{(1) \prime}\left(k r_{c} \sin \vartheta\right)=\frac{i n !}{\pi \varepsilon_{n}}\left(\frac{2}{k r_{c} \sin \vartheta}\right)^{n+1} \quad \forall k r_{c} \sin \vartheta \ll 1,
$$

with $\varepsilon_{0}=1$ and $\varepsilon_{n}=2, \forall n>0$.

Using Eqs. (5.33) and (5.54), the far field formulation for the pressure amplitude, given in Eq. (5.53), reads

$$
\begin{aligned}
& \hat{p}(R, \vartheta, \theta)=\frac{\rho r_{c} \hat{r} \exp (i k R)}{R} \sum_{m n} \omega_{m n}^{* 2}\left(\frac{-i k r_{c} \sin \vartheta}{2}\right)^{n} \exp (i n \theta) \times \\
& \frac{\varepsilon_{n} k_{m}(-1)^{m} \cos (k L \cos \vartheta)}{n !\left(k_{m}^{2}-k^{2} \cos ^{2} \vartheta\right)} \quad \forall R / L \gg 1, k R \sin \vartheta \gg 1, k r_{c} \sin \vartheta \ll 1,
\end{aligned}
$$

Equation (5.55) is the end result for the far field radiated pressure of a vortex with perturbations on a finite part only.

The collapse of a cavitating vortex can be represented by mode $n=0$ and $m=0$ using a value $2 L$ for the cavity length that is directly affected by the collapse. In the far field the pressure amplitude of Eq. (5.55) is then formulated as

$$
\hat{p}(R, \vartheta)=\rho \frac{r_{c} \hat{r}}{k_{0}} \frac{\exp (i k R)}{R} \omega_{00}^{* 2}\left[\frac{\left(k_{0} L\right)^{2} \cos (k L \cos \vartheta)}{\left(k_{0} L\right)^{2}-(k L \cos \vartheta)^{2}}\right], \quad \forall n=0,
$$


with $k_{0}=\pi /(2 L)$ and $\omega_{00}^{*}=\left(W_{\infty} k_{0}-\omega\right)$. In Eq. (5.56) the term between square brackets in the right-hand-side determines the directivity of the radiated pressure. The highest pressure is observed at $\vartheta=\pi / 2$ for which this term equals one for all wavenumbers. The pressure amplitude is then given by

$$
\hat{p}(R, \vartheta=\pi / 2)=\rho \frac{r_{c} \hat{r} 2 L}{\pi} \frac{\exp (i k R)}{R} \omega_{00}^{* 2}, \quad \forall n=0 .
$$

\section{Near-field formulation}

The formulation for the near field is only considered for $n=0$ for which the Hankel function equals

$$
H_{0}^{(1)}\left(k_{r} r\right)=\frac{2 i}{\pi} \ln \left(k_{r} r\right) \quad \forall k_{r} r \ll 1 .
$$

Hence, there is no change in phase and the method of stationary phase cannot be applied. It was already noted that $k_{r}$ becomes an imaginary number for $\gamma>k$. The Hankel function then turns into a modified Bessel function of the second kind $K$ which leads to an evanescent wave in the far field. In the near field, however,

$$
H_{0}^{(1)}\left(i\left|k_{r}\right| r\right)=-\frac{2 i}{\pi} K_{0}\left(\left|k_{r}\right| r\right)=\frac{2 i}{\pi} \ln \left(\left|k_{r}\right| r\right) \quad \forall\left|k_{r}\right| r \ll 1 .
$$

Hence, in the near field, the real and imaginary values of $k_{r}$ contribute equally to the pressure levels. Inserting Eqs. (5.33), (5.54) and (5.58) in Eq. (5.38) results in the near-field formulation for the pressure

$$
\begin{aligned}
p^{\prime}(R, \vartheta, t)=\frac{\rho}{2 \pi} r_{c} \hat{r} \sum \omega_{m 0}^{* 2} \exp [-i \omega t] \int_{-\infty}^{\infty} \ln \left(\sqrt{\left|k^{2}-\gamma^{2}\right|} R \sin \vartheta\right) \times \\
\frac{2 k_{m}(-1)^{m} \cos (\gamma L)}{k_{m}^{2}-\gamma^{2}} \exp (i \gamma R \cos \vartheta) d \gamma
\end{aligned}
$$

The equation cannot be further simplified. Perpendicular to the vortex, the dependency of the phase on $\gamma$ disappears and the pressure amplitude for mode $m=0$ is given by

$$
\hat{p}(R, \vartheta=\pi / 2)=\frac{\rho}{2 \pi} r_{c} \hat{r} \omega_{00}^{* 2} \int_{-\infty}^{\infty} \ln \left(\sqrt{\left|k^{2}-\gamma^{2}\right|} R\right) \frac{2 k_{0} \cos (\gamma L)}{k_{0}^{2}-\gamma^{2}} d \gamma
$$

\subsubsection{Analysis and discussion}

The behaviour of the pressure perturbations generated by the vibrations of the cavitating vortex will now be analysed for the various formulations presented in the previous sections. For a typical, ship scale, propeller we take for the resonance frequency and for the diameter and length of the cavitating vortex collapse region $f=48 \mathrm{~Hz}, k=0.2 \mathrm{~m}^{-1}, 2 r_{c}=0.05 \mathrm{~m}$ and $2 L=0.25 \mathrm{~m}$, respectively. The value for the length is a very crude estimate. For a propeller diameter of $5 \mathrm{~m}$ and a propeller-hull clearance of $25 \%$ of the propeller diameter, the minimum distance between 


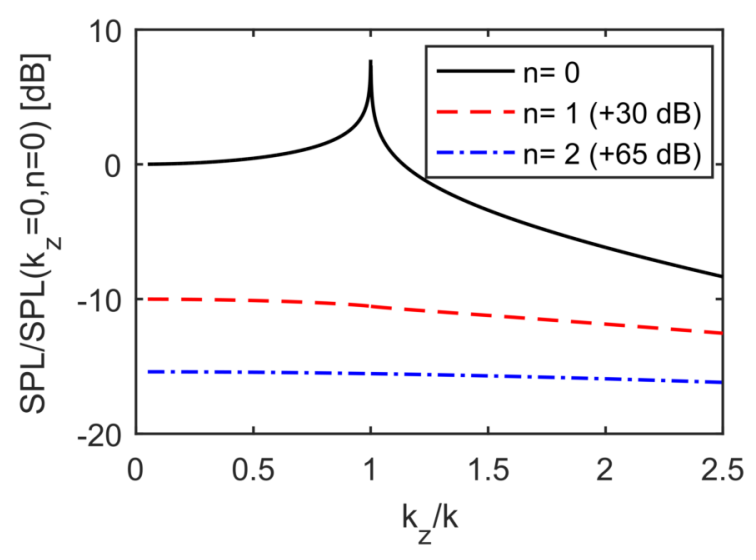

a) $k r=0.25$

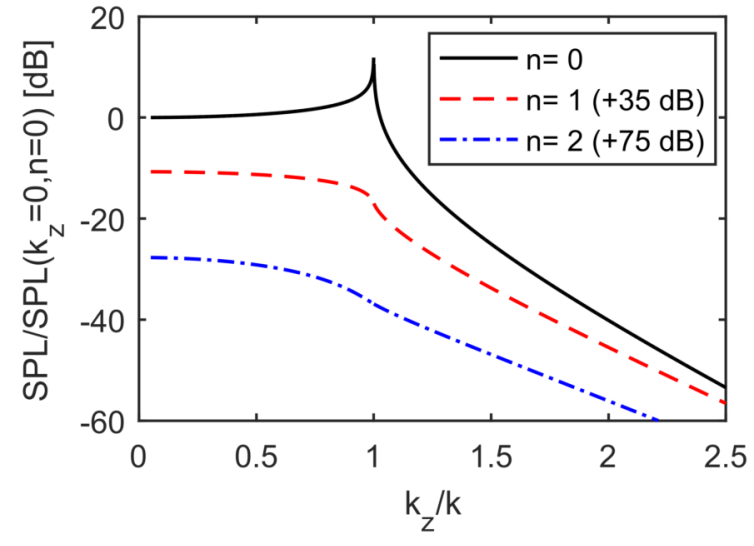

b) $k r=2.5$

Figure 5.2: Variation of normalized pressure level with axial wavenumber for an infinite length vortex prescribing $k=0.2 \mathrm{~m}^{-1}$ and $k r_{c}=0.005$. The SPL-values for $n=1$ and $n=2$ have been increased by the number given in the legend.

cavity and hull is $1.25 \mathrm{~m}$. The measurement of URN is usually performed at large distance from the hull which is $\mathscr{O}(100 \mathrm{~m})$. Results are presented as Sound Pressure Level, SPL, defined as $\mathrm{SPL}=10 \log _{10}\left(\hat{p}_{\mathrm{rms}}^{2} / p_{\text {ref }}^{2}\right)$, with the reference pressure defined as $p_{\text {ref }}=1 \mu \mathrm{Pa}$.

First, the infinite length vortex is studied with respect to the dependency on the axial wavenumber $k_{z}$. Using Eq. (5.16), $k_{z}$ is varied while prescribing $k=0.2 \mathrm{~m}^{-1}$ and $r_{c}=0.025 \mathrm{~m}$ for $r=1.25 \mathrm{~m}$ and $r=12.5 \mathrm{~m}$ resulting in $k r=0.25$ and $k r=2.5$, respectively. The results are presented in Figure 5.2. The mode $n=0$ shows a sharp resonance peak at the critical frequency for which $k=k_{z}$, whereas this peak is not present for the modes $n=1$ and $n=2$. For smaller values of $k_{z}$, or frequencies above the critical frequency, a propagating wave is present while for larger values of $k_{z}$, or frequencies below the critical frequency, the wave becomes of the evanescent type and the sound pressure levels rapidly decrease with distance. The decrease of acoustic efficiency with increasing $n$ is also apparent from Figure 5.2.

For the finite length vortex, the criteria for the validity of Eq. (5.55) for the radiated pressure are (i) that it can be considered as a compact acoustic source, $k r_{c} \ll 1$, (ii) that the field points for which the pressure is computed are in the far field such that the far field approximation of the Hankel function is valid, $k R \sin \vartheta \gg 1$, (iii) that the stationary phase approximation can be used, $R / L \gg 1$, and (iv) that the field points are in the Fraunhofer region, approximately defined by $R / L>k L$. The criterion for the Fraunhofer region was derived in Section 5.2.3, and is assumed to also hold for the formulation given in Section 5.2.4.

For the typical numbers given, the cavity collapse can be considered as a compact source up to a frequency of $1.8 \mathrm{kHz}$ (using $k r_{c}=1 / 5$ ). At this frequency the Fraunhofer region starts at approximately $R=0.12 \mathrm{~m}$, so all hull locations are in the Fraunhofer region.

The criterion for the far field is less easily fulfilled. Using a criterion $R / L=10$ for the stationary phase approximation, the distance $R$ should be larger than $1.25 \mathrm{~m}$ which is just satisfied in the example. However, the far field formulation of the Hankel function starts approximately ${ }^{4}$

\footnotetext{
${ }^{4}$ At $k R=1$ the error of the far-field formulation of the Hankel function is $0.3 \mathrm{~dB}$ in magnitude and 6 deg in phase, at $k R=5$ the errors are $0.02 \mathrm{~dB}$ and $1 \mathrm{deg}$, respectively.
} 


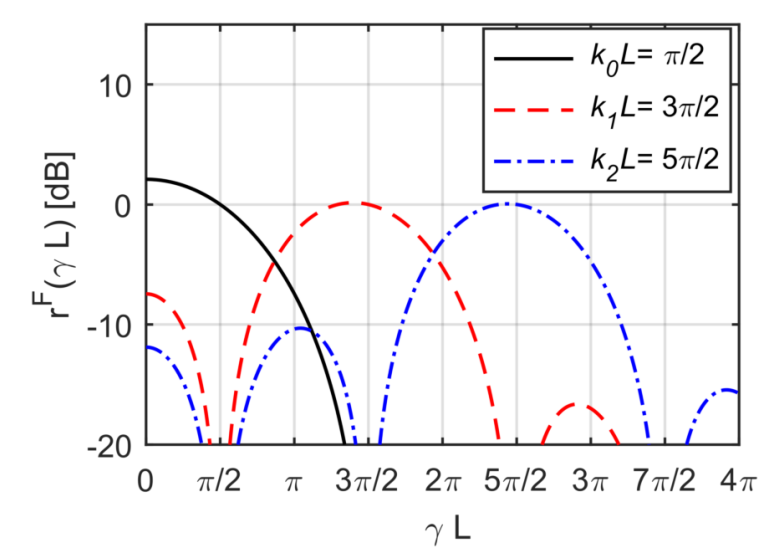

Figure 5.3: Fourier transform of the cavity radius perturbation, presented as $20 \log _{10}\left[\hat{r}_{m}^{F}(\gamma L) / \hat{r} L\right]$, Eq. (5.33), for $m=0,1,2$ in $k_{m}$.

at $k R>1$ which gives $R>5 \mathrm{~m}$ for the example. Hence, the far-field formulation can be used for the measurement of URN but the behaviour at typical locations for hull-pressure measurements needs to be further studied.

In model tests, the geometric dimensions are a geometric scale factor $\lambda$ smaller than ship scale and the frequencies, assuming Froude scaling for the cavitation test, are a factor $\sqrt{\lambda}$ larger. The values for $k r_{c}$ and $k L$ are then a factor $\sqrt{\lambda}$ smaller than at ship scale, so the criteria of compactness and the far field being a Fraunhofer region are more easily satisfied. However, the value for $k R$ is also a factor $\sqrt{\lambda}$ smaller so the far field formulation for the Hankel function does not hold for the hull pressure measurements at model scale.

The behaviour of Eq. (5.38), showing the contribution of $\gamma$ to the pressure, without using the far field formulation for the Hankel function, is studied next. The distribution of $\hat{r}_{m}^{F}(\gamma L)$ with $\gamma$, Eq. (5.33), is presented in Figure 5.3 for various values of $k_{m}$. For $m=0$ the maximum value occurs at $\gamma=0$ but for larger values of $m$, the maximum moves to $\gamma=k_{m}$ with the value at $\gamma=0$ decreasing.

We will now focus on results of Eq. (5.38) for mode $n=0$ and $m=0$ and in direction $\vartheta=\pi / 2$. The values of the full-scale example discussed above are used, so $2 r_{c}=0.05 \mathrm{~m}$, $k=0.2 \mathrm{~m}^{-1}$ and $2 L=0.25 \mathrm{~m}$. Results are presented in Figure $5.4 \mathrm{a}$ and $\mathrm{b}$ for three values of $R$ that correspond to full-scale values. Figure $5.4 \mathrm{c}$ and $\mathrm{d}$ present results for the corresponding model-scale value using a geometric scale factor $\lambda=20$ and using an acoustic wavenumber that is a factor $\sqrt{\lambda}$ larger than full scale. The values for $R$ at full scale are $R=1.25,12.5$ and $62.5 \mathrm{~m}$, resulting in values of $k R=0.25,2.5$ and 12.6, respectively. The values for $k R$ for the model scale situation are then a factor $\sqrt{\lambda}$ smaller than at full scale. The magnitudes in Figure 5.4 represent the source strength in decibels for cylindrical propagation loss, obtained by multiplying $\hat{p}$ with $\sqrt{R}$ and are made non-dimensional with the magnitude for $\gamma=0$ at $k R=$ 0.25 at full scale and $k R=0.06$ at model scale. The values of $\gamma$ are non-dimensionalised with $k$. All values for $k R$ show identical behaviour for $\gamma<k$ and show a peak at the critical frequency for which $\gamma=k$. At small values of $k R, \gamma>k$ also contributes to the pressure, but at larger values for $k R$, say $k R>1$, the contribution rapidly decreases due to the evanescent wave character. The phase hardly varies with $\gamma$ for small values of $k R$ but rapidly oscillates for $k R>10$. Hence, for $k R>10$ only the region near the stationary phase location at $\gamma=0$ contributes to the far field 
pressure. For $1<k R<10$ the range $\gamma \in[0, k]$ contributes and for $k R<1$ the whole range of $\gamma$ contributes.

The stationary phase method has shown that the combination of the cylindrical spreading loss of the Hankel function and the integral over $\gamma$, which varies with $1 / \sqrt{R}$, leads to a spherical spreading loss. However, the stationary phase method cannot be used at low values of $k R$ as discussed above and therefore the consequence for the spreading loss is analysed next. The computed pressures at varying $R$ have been converted to source levels using spherical spreading loss and normalized by the source level for the largest value of $R$. The analysis was made for three values of $L$ using $k=0.2 \mathrm{~m}^{-1}$ with $k L=2.5 \times 10^{-2}$ corresponding to the full-scale reference values given above. Figure 5.5a presents the results for full scale and Figure 5.5b presents the results for model scale. At full scale, spherical spreading loss is observed for $k R>\approx 0.05$ for the reference value of $k L$ and at model scale spherical spreading loss occurs for $k R>\approx 0.2$. It is then concluded that at full scale for $k=0.2 \mathrm{~m}^{-1}$ spherical spreading loss can be assumed for all hull locations, but at model scale, with $k=0.9 \mathrm{~m}^{-1}$, this cannot be assumed. However, there is some uncertainty as the effective length of the cavity collapse region is unknown and has been estimated. Also, the validity of the formulation used for the pressure perturbation due to a cavity collapse still needs to be proven. If spherical spreading loss is assumed, the source level is underestimated at small values of $k R$.

Far-field criteria may also include the criterion that the noise propagation can be described by a plane wave (Junger and Feit, 1986). This condition requires $k R \gg 1$ which is not satisfied for ship and model scale HPF in the typical frequency range considered. This aspect is relevant for the scattering of the pressure by the hull which will be described in Section 5.5.1.

The pressure amplitudes of the higher order modes in the formulation of the finite-length vortex, and of the sphere, scale, approximately, by $\left(k r_{c} / 2\right)^{n}$. If we consider again a frequency of $50 \mathrm{~Hz}$ and a cavity diameter of $5 \mathrm{~cm}$, mode $n=1$ is $51 \mathrm{~dB}$ less efficient than mode $n=0$, see also Figure 5.2. So it is clear that at low frequencies all the noise radiated by the cavity collapse is caused by mode $n=0$, being cavity volume variations. Only at a frequency of approximately $20 \mathrm{kHz}$ the acoustic efficiency of mode $n=1$ equals the efficiency of mode $n=0$. For model scale conditions, the acoustic efficiency of the higher order modes is even less as the value of $k r_{c}$ is a factor $\sqrt{\lambda}$ smaller than for full scale.

The spectrum of $\hat{r}_{m}^{F}(\gamma L)$ defined by Eq. (5.33) varies strongly with $k_{m}$ as illustrated in Figure 5.3. This variation is relevant for the directivity as the point of stationary phase is given by $\gamma=k \cos \vartheta$. For mode $m=0$, for which $k_{0} L=\pi / 2$, all power is concentrated near $\gamma=0$ and gradually decreases with increasing $\gamma$. For modes $m=1$ and $m=2$, however, a large variation of the power near $\gamma=0$ is observed. This suggests a high directivity as the location of stationary phase moves to larger axial wavenumbers when $\vartheta$ becomes larger or smaller than $\pi / 2$.

The stationary phase approximation shows that the far field radiated noise at $\vartheta=\pi / 2$ is only dependent on the spatially averaged amplitude. If multiple wave lengths are present on the cavity length, $m>0$, the contributions from neighbouring sections cancel except near the edges of the vibrating part of the cavity which thus generate the far field noise. This conclusion was already stated by Morozov (1974) for cavitating vortices and has been confirmed by computational results presented by Nijland (2006).

The directivity of the radiated pressure in $\vartheta$-direction is only observed for the formulations 


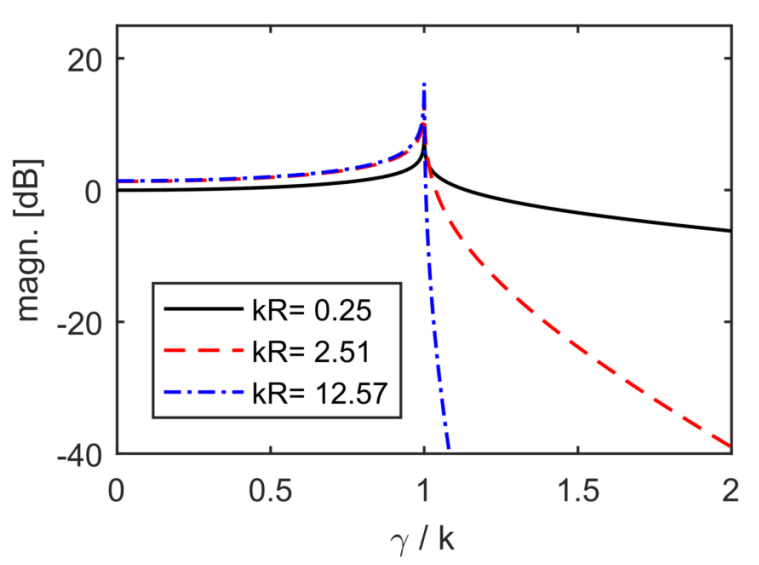

a) Source level, full scale

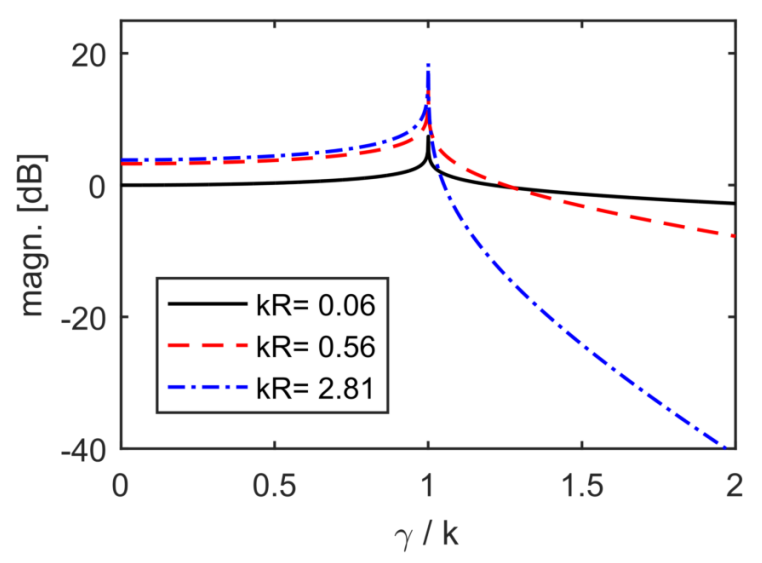

c) Source level, model scale

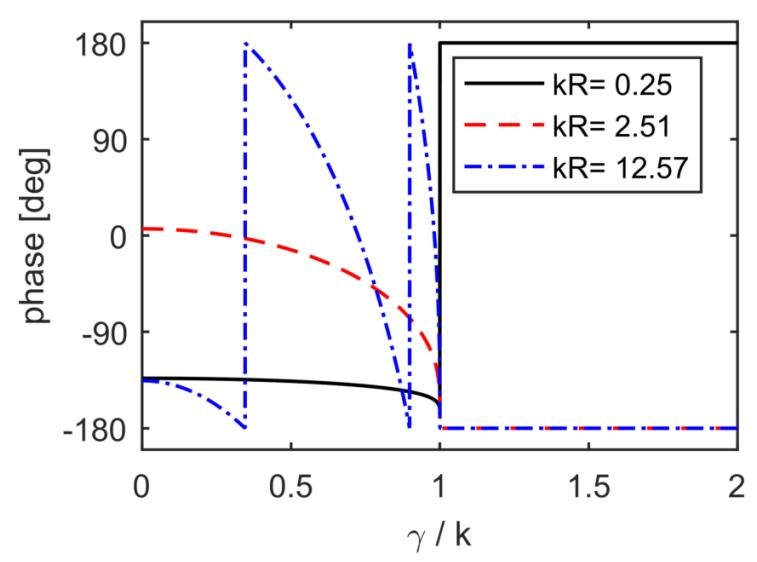

b) Phase, full scale

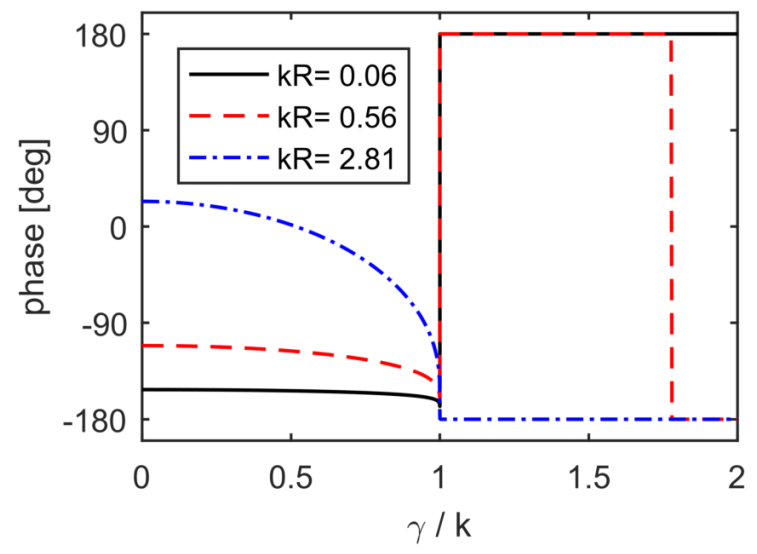

d) Phase, model scale

Figure 5.4: Normalized source level and phase showing the contribution of $\gamma$ to the radiated pressure for deformations on a finite-length cylinder for $k=0.2 \mathrm{~m}^{-1}$ at full scale and $k=0.9 \mathrm{~m}^{-1}$ at model scale.

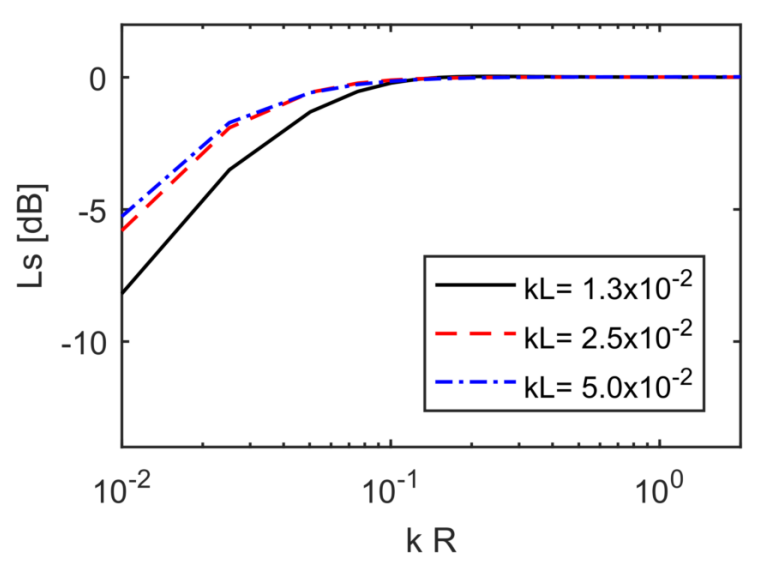

a) Full scale

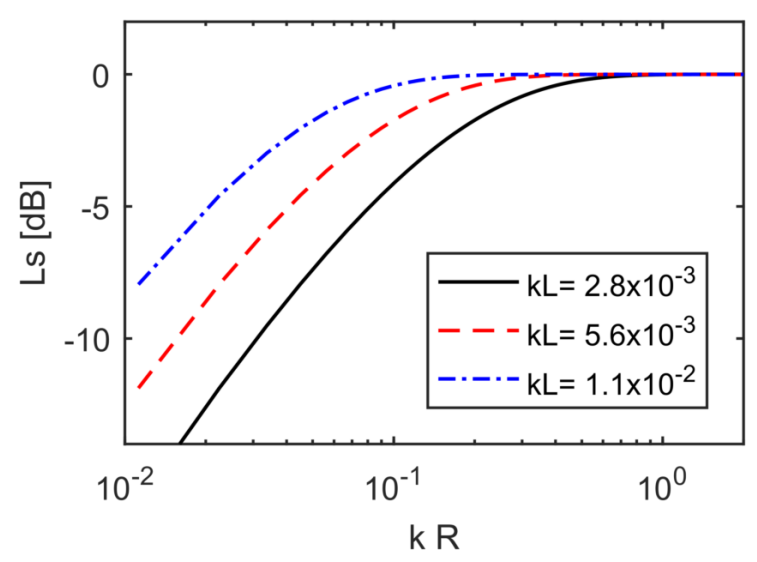

b) Model scale

Figure 5.5: Variation of normalized pressure level with distance $R$, subtracted by the level according to spherical spreading loss. Identical values for $k$ as Figure 5.4. 


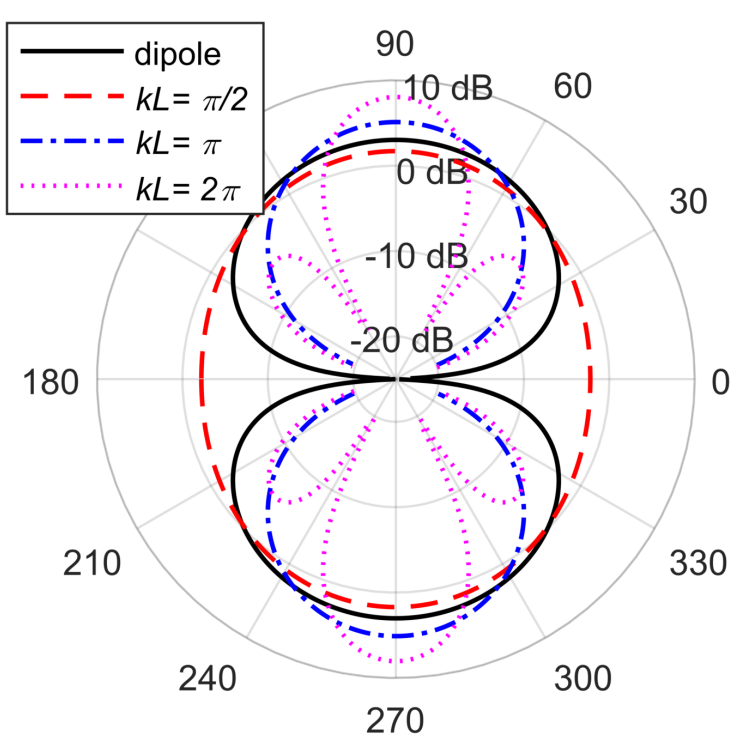

a) Formulation of Eq. (5.27)

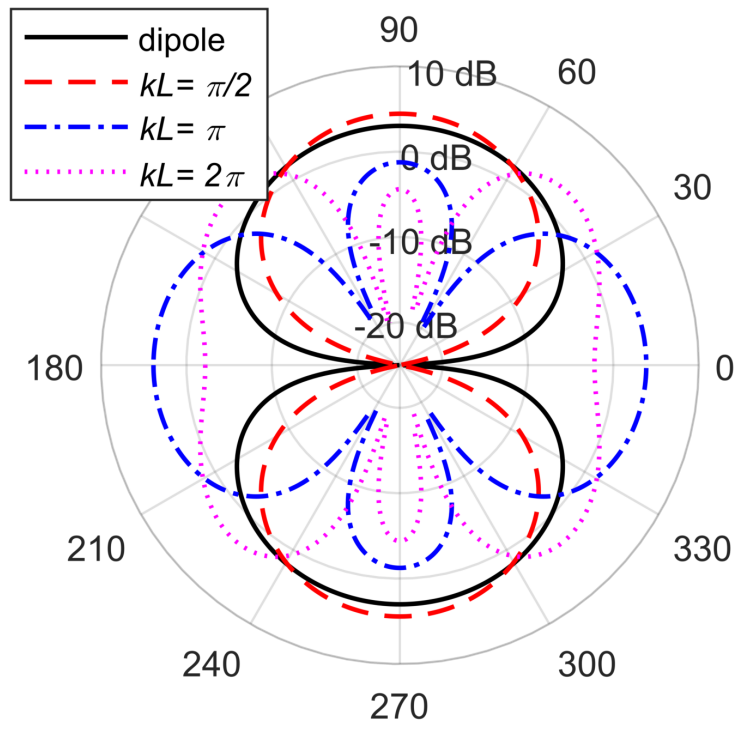

c) Formulation of Eq. (5.56), $k_{1} L=3 \pi / 2$

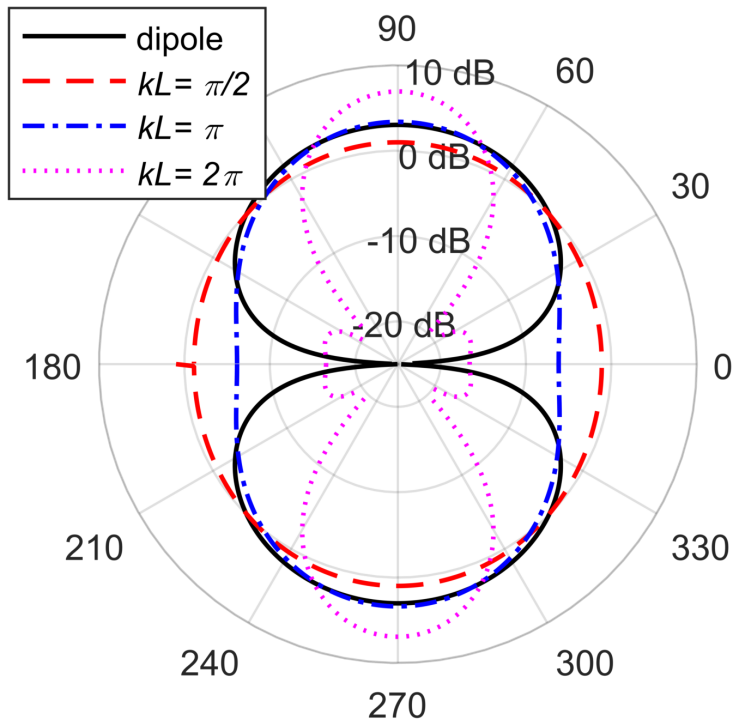

b) Formulation of Eq. (5.56), $k_{0} L=\pi / 2$

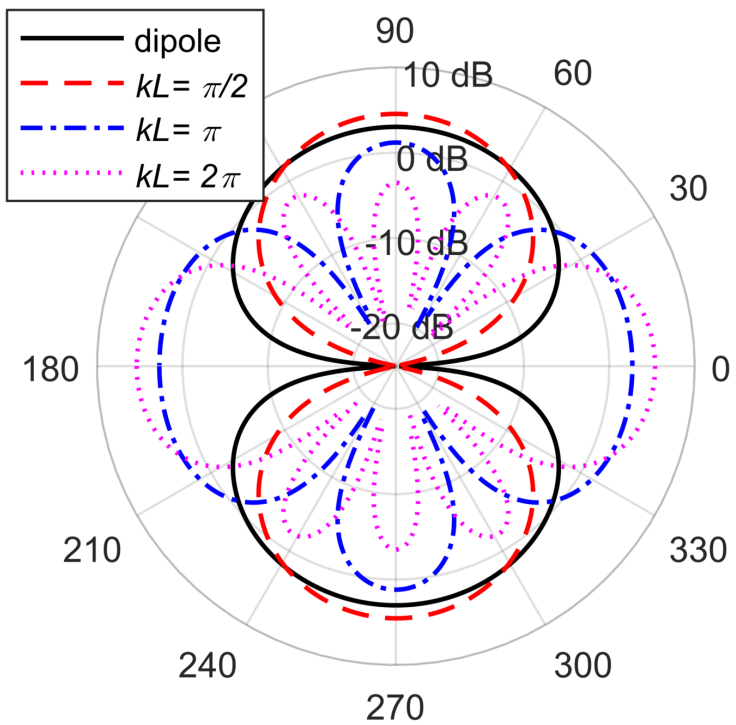

d) Formulation of Eq. (5.56), $k_{2} L=5 \pi / 2$

Figure 5.6: Comparison of directivity indices as function of $\vartheta$, given in degrees, between a dipole and two vortex formulations for three values of the acoustic wavenumber $k$. The formulation of Eq. (5.56) is presented for $m=0,1,2$ in $k_{m}$.

of the finite length vortex. The results are compared with the directivity of a dipole of which the formulation of the radiated pressure is given by Eq. (5.13). The directivity is presented by the directivity index DI defined as

$$
\mathrm{DI}(\vartheta)=10 \log _{10}\left(\frac{\hat{p}^{2}(R, \vartheta, \theta)}{\left\langle\hat{p}^{2}(R, \vartheta, \theta)\right\rangle_{\vartheta}}\right)
$$

where $\langle.\rangle_{\vartheta}$ denotes the mean value in $\vartheta$ direction, $\vartheta \in[0, \pi]$. The results for the directivity of Eq. (5.27), the finite length vortex with distributed sources, and Eq. (5.56), the stationary phase formulation for the finite length vortex, are presented in Figure 5.6. Both formulations 
are valid for the far field only. Note that with respect to Eq. (5.13) the dipole has been rotated over $90^{\circ}$ such that the maximum noise levels are at $\vartheta=90^{\circ}$. At low frequencies, $k L<\pi / 2$, both vortex formulations show that the directionality is negligible, while for high frequencies a directionality can be observed with the maximum noise levels located in the plane perpendicular to the vortex at $\vartheta=90^{\circ}$. If it is assumed that the deformation of the cavity extends over a length of $2 L=0.25 \mathrm{~m}$, the frequency for which $k L=\pi / 2$ corresponds to $f \approx 3 \mathrm{kHz}$. Hence, for the HPF at full scale the cavitating vortex can be considered as a monopole. The situation for the HPF in model tests is more complicated as discussed above. The URN levels are typically measured up to much higher frequencies and directionality then becomes relevant. At the higher frequencies, the noise will, however, not only be due to the collapse of the large scale cavity but also due to oscillating bubbles generated by the collapse process.

\subsection{Spectral analysis}

\subsubsection{Background}

Time series of HPF and URN are usually converted to frequency spectra using a discrete Fourier transform applied to a time window of finite length. Results for stationary deterministic signals, such as the low-frequency HPF due to the periodic passage of cavitating propeller blades, are usually presented by a peak-amplitude spectrum in units [Pa]. Stationary random signals, such as high-frequency cavitation noise, have their energy continuously distributed over frequency and the results are therefore presented in a power density spectrum, with units $\left[\mathrm{Pa}^{2} \mathrm{~Hz}^{-1}\right]$ or a power spectrum, with units $\left[\mathrm{Pa}^{2}\right]$. Power density spectra are usually obtained by a Fast Fourier Transform applied to overlapping time segments to improve accuracy on which a window, such as the Hann window, is applied to improve resolution and to reduce sidelobes (Randall, 1987; Marple, 1987; Smith, 1999). Power spectra can be obtained by integrating the power density spectrum over a desired frequency bandwidth. The power density spectra and power spectra are usually expressed in decibel values making use of a reference pressure, which for underwater noise is defined as $p_{\text {ref }}=1 \mu \mathrm{Pa}$,

$$
S P L(f)=10 \log _{10}\left[\frac{p_{\mathrm{rms}}^{2}(f)}{p_{\mathrm{ref}}^{2}}\right]=20 \log _{10}\left[\frac{p_{\mathrm{rms}}(f)}{p_{\mathrm{ref}}}\right]
$$

where SPL denotes Sound Pressure Level.

The collapse of a cavity is a transient process, which complicates the interpretation of Fourier spectra. For that reason, some simple stylistic examples of transient signals and their Fourier transform are presented in Section 5.3.2. These examples are useful for the interpretation of broadband hull pressure spectra as presented in Chapter 6.

The variation of the propeller blade loading is due to the ship wake and, therefore, the collapse of the cavity always occurs at the same blade position. This results in cavitation noise that is amplitude modulated by the blade passage frequency. However, the collapse of a cavitating vortex does not repeat perfectly due to stochastic variations in the flow related to for instance turbulence, water quality, sea state, ship motion and speed variations. In Section 5.3.3, it will be shown that these variations between blade passages cause the low-frequency broadband noise. 


\subsubsection{Examples of Fourier transform pairs}

The analysis of HPF and of URN is usually performed in the frequency domain. In this section some basic relations are presented that are helpful in understanding spectra discussed in following chapters. More details can be found in, amongst others, Randall (1987), Marple (1987) and Smith (1999).

For a continuous signal $g(t)$ defined in time, the continuous-time Fourier transform $G(\omega)$ of $g(t)$ is defined as

$$
G(\omega)=\int_{-\infty}^{\infty} g(t) \exp (-i \omega t) d t \quad \Leftrightarrow \quad g(t)=\frac{1}{2 \pi} \int_{-\infty}^{\infty} G(\omega) \exp (i \omega t) d \omega
$$

To keep the formulations short, use is made of the angular frequency $\omega$ whereas in practical applications use is made of the circular frequency $f$ with $\omega=2 \pi f$.

The first integral transform pair to be considered is for a single pressure peak due to a cavity collapse represented by a rectangular pulse ${ }^{5}$ of width $T$,

$$
g_{1}(t)=\operatorname{rect}(t / T) \Leftrightarrow G_{1}(\omega)=\frac{T \sin (\omega T / 2)}{\omega T / 2},
$$

where $G_{1}(\omega)$ is the sinc function of width $\omega T / 2$ multiplied with the pulse width $T$.

The second function is an oscillating pressure with frequency $\omega_{0}$ which is only present within a time window of width $T$, selected such that $\sin \left(\omega_{0} T / 2\right)=0$,

$$
g_{2}(t)=\operatorname{rect}(t / T) \sin \left(\omega_{0} t\right) \quad \Leftrightarrow \quad G_{2}(\omega)=\frac{2 i \omega_{0}}{\omega_{0}^{2}-\omega^{2}} \sin (\omega T / 2),
$$

where $G_{2}(\omega)$ now corresponds to the sum of a frequency shifted sinc function of $\left(\omega_{0}-\omega\right) T / 2$ and a frequency shifted sinc function of $\left(\omega_{0}+\omega\right) T / 2$.

The third function to be considered is a damped oscillating pressure signal with frequency $\omega_{0}$ that is present between $t=0$ and $t=\infty$ and with $\alpha>0$,

$$
g_{3}(t)=\exp (-\alpha t) \sin \left(\omega_{0} t\right) \quad \Leftrightarrow \quad G_{3}(\omega)=\frac{\omega_{0}}{(i \omega+\alpha)^{2}+\omega_{0}^{2}} .
$$

Examples of these three transform pairs are given in Figure 5.7. The spectral values are given in decibels. The single pulse $g_{1}$, that contains only positive values for the signal, leads to a spectrum with maximum value at zero frequency. Oscillating within $t \in[-T / 2, T / 2]$, the function $g_{2}$ gives a broadband peak centred at the oscillation frequency. The decaying oscillating function $g_{3}$ leads to a spectrum $G_{3}$ that is a combination of $G_{1}$ and $G_{2}$. The spectrum shows significant energy at zero frequency as the mean is different from zero and a broadband peak centred at the oscillation frequency. So it is seen that a transient oscillatory signal gives a broadband hump in the spectrum.

\footnotetext{
${ }^{5} \operatorname{rect}(x)=0$ if $|t|>1 / 2, \operatorname{rect}(x)=1 / 2$ if $|t|=1 / 2, \operatorname{rect}(x)=1$ if $|t|<1 / 2$
} 

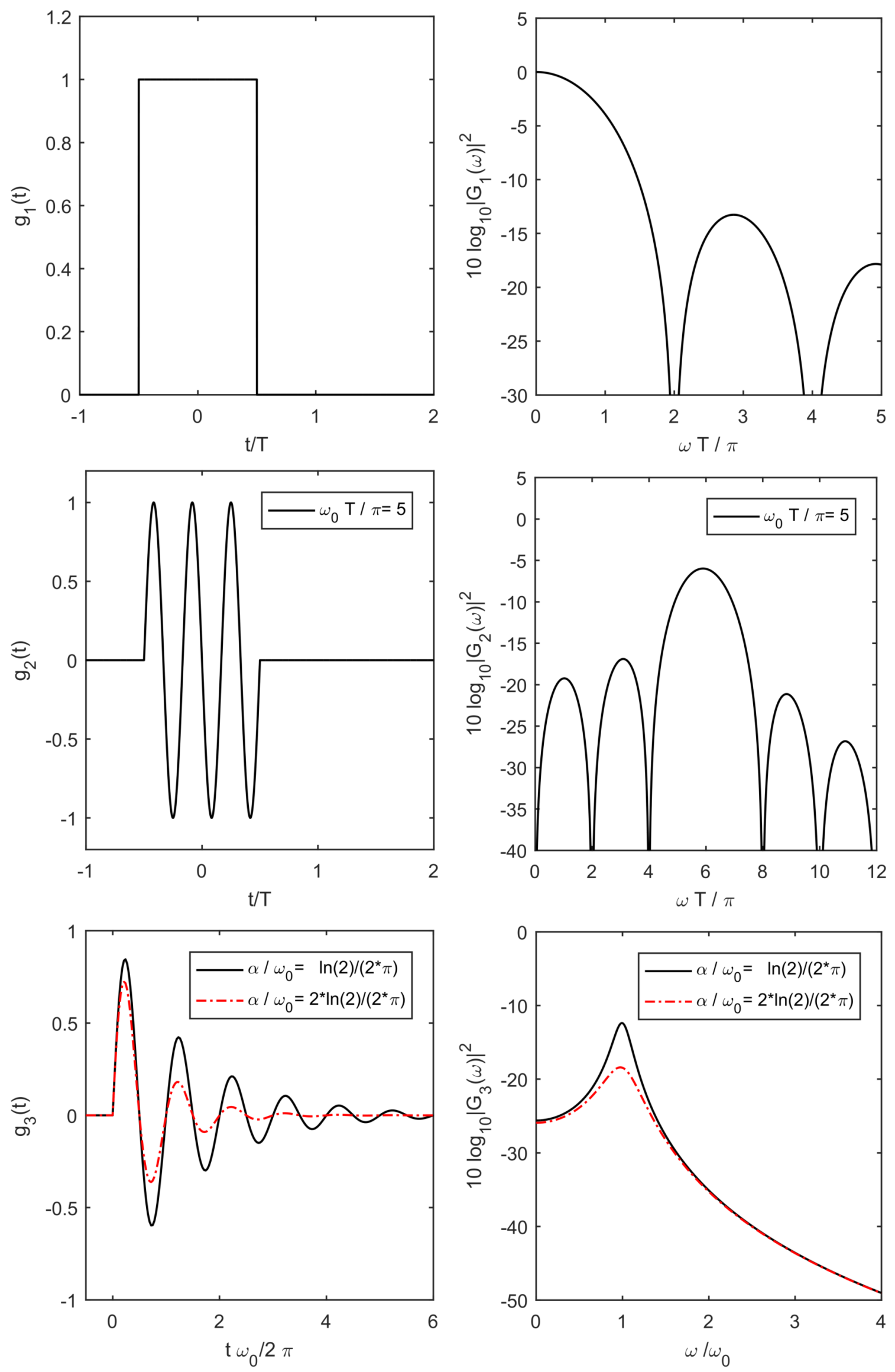

Figure 5.7: Integral transform pairs of Eq. (5.65), (5.66) and (5.67). 


\subsubsection{Formulation for effect of variability between blade passages}

Section 5.3.2 has shown some simplified functions that may represent the pressure signal by a cavity collapse of a single blade passage. As will be shown in the Section 6.3.3, the time trace of the radiated pressure generated by the dynamics of a vortex cavity varies significantly between blade passages which results in a variation of the spectral content from one blade passage to the next. The variability can be in amplitude, in frequency, in overall shape of the time trace, and in time of arrival with respect to the time between blade passages. For the resulting spectrum this can be interpreted as a variability in amplitude and phase.

The effect of the variability of a signal on the spectrum has been reviewed from a theoretical point of view by Baiter et al. (1982). An analytical formulation that shows the consequence of the variability of amplitude and phase angle of periodic events on the spectral content has been presented by MacFarlane (1949). It was shown by Baiter et al. (1982) and Bark (2000) that, in a qualitative manner, these formulations well explain the occurrence of low-frequency broadband HPF in relation to the blade rate tonals. MacFarlane (1949) has considered the effect of the variability of amplitude and phase angle separately. Here, the combination of the two will be considered and the method will be applied in Section 6.3.3 to reconstruct the broadband spectrum in a quantitative manner. It is noted that MacFarlane refers to the variability of the amplitude and phase angle as amplitude and phase angle modulation, respectively.

The root-mean-square (rms) amplitude density spectrum of a single pulse, due to the cavity collapse and possibly rebounds during a single blade passage, is denoted by $G(\omega)$. The spectrum of a similar pulse occurring at time $t$ later, corresponding to the following blade passage, is then given by $G(\omega) \exp (i \omega t)$. Hence, the shift in time $t$ represents a shift in phase $\omega t$ of the spectrum. Consider $2 N+1$ pulses spaced apart by the sum of a mean time difference $T$ and varying time $\tau_{n}$, and with an amplitude $a_{n}$ that also varies for each pulse $n$. The resulting spectrum is then given by

$$
S(\omega)=G(\omega) \sum_{-N}^{N} a_{n} \exp \left[i \omega\left(n T+\tau_{n}\right)\right]
$$

The variation in amplitude and phase ${ }^{6}$ are assumed independent and the probability functions are assumed to be Gaussian, i.e.

$$
\begin{aligned}
& q\left(a_{n}\right)=\frac{1}{\sigma_{a} \sqrt{2 \pi}} \exp \left[-\left(a_{n}-\bar{a}_{n}\right)^{2} / 2 \sigma_{a}^{2}\right] \\
& q\left(\tau_{n}\right)=\frac{1}{\sigma_{\tau} \sqrt{2 \pi}} \exp \left(-\tau_{n}^{2} / 2 \sigma_{\tau}^{2}\right)
\end{aligned}
$$

with $\sigma_{a}$ and $\sigma_{\tau}$ the standard deviation of the amplitude and the time variation, respectively, and

\footnotetext{
${ }^{6}$ In the following the variability in time will be referred to as variability in phase
} 
$\bar{a}_{n}$ the mean amplitude. The power spectral density is then given by

$$
\begin{aligned}
& R_{N}(\omega)=\frac{|S(\omega)|^{2}}{(2 N+1)} \\
&=|G(\omega)|^{2}\left\{\frac{1}{2 N+1}\left|\sum_{-N}^{N} a_{n} \cos \left[\omega\left(n T+\tau_{n}\right)\right]\right|^{2}+\right. \\
& \\
&\left.\frac{1}{2 N+1}\left|\sum_{-N}^{N} a_{n} \sin \left[\omega\left(n T+\tau_{n}\right)\right]\right|^{2}\right\}_{A V G} .
\end{aligned}
$$

where $A V G$ indicates that the average value is taken.

According to the central limit theorem, the properly normalized sum of independent random variables is described by a normal distribution. The mean square value of a parameter is then equal to the sum of the mean value squared and the standard deviation squared (Bendat and Piersol, 2010). For a general function $f(y)$ for which the parameter $y$ has a probability function $q(y)$, the mean $\bar{x}$ and standard deviation $\sigma$ are given by,

$$
\begin{aligned}
\bar{x} & =\int_{-\infty}^{\infty} f(y) q(y) \mathrm{d} y, \\
\sigma^{2} & =\int_{-\infty}^{\infty}[f(y)-\bar{x}]^{2} q(y) \mathrm{d} y .
\end{aligned}
$$

If the function $f$ is a function of multiple variables that are uncorrelated, its probability is given by the product of the probability functions of the individual variables.

The power density spectrum (5.71) can then be written as

$$
R(\omega)=\lim _{N \rightarrow \infty} \frac{1}{2 N+1}\left\{\left(\sum_{-N}^{N} \bar{x}_{n, \sin }\right)^{2}+\left(\sum_{-N}^{N} \bar{x}_{n, \cos }\right)^{2}+\sum_{-N}^{N}\left(\sigma_{n, \sin }^{2}+\sigma_{n, \cos }^{2}\right)\right\}|G(\omega)|^{2}
$$

The average mean square value of the sine term is then computed from

$$
\left\{\left|\sum_{-N}^{N} a_{n} \sin \omega\left(n T+\tau_{n}\right)\right|^{2}\right\}_{A V G}=\left(\sum_{-N}^{N} \bar{x}_{n, \sin }\right)^{2}+\sum_{-N}^{N} \sigma_{n, \sin }^{2}
$$


The mean value is given by

$$
\begin{aligned}
\bar{x}_{n, \sin }= & \frac{1}{\sigma_{a} \sqrt{2 \pi}} \frac{1}{\sigma_{\tau} \sqrt{2 \pi}} \int_{-\infty}^{\infty} \int_{-\infty}^{\infty} a_{n} \sin \omega\left(n T+\tau_{n}\right) \exp \left[-\frac{\left(a_{n}-\bar{a}_{n}\right)^{2}}{2 \sigma_{a}^{2}}\right] \times \\
& \exp \left(-\frac{\tau_{n}^{2}}{2 \sigma_{\tau}^{2}}\right) \mathrm{d} a_{n} \mathrm{~d} \tau_{n}, \\
= & \frac{1}{\sigma_{a} \sqrt{2 \pi}} \frac{1}{\sigma_{\tau} \sqrt{2 \pi}} \int_{-\infty}^{\infty}\left\{a _ { n } \operatorname { e x p } [ - \frac { ( a _ { n } - \overline { a } _ { n } ) ^ { 2 } } { 2 \sigma _ { a } ^ { 2 } } ] \int _ { - \infty } ^ { \infty } \left[\sin (\omega n T) \cos \left(\omega \tau_{n}\right)+\right.\right. \\
= & \left.\left.\sin \left(\omega \tau_{n}\right) \cos (\omega n T)\right] \exp \left(-\frac{\tau_{n}^{2}}{2 \sigma_{\tau}^{2}}\right) \mathrm{d} \tau_{n}\right\} \mathrm{d} a_{n}, \\
= & \bar{a}_{n} \sin (\omega n T) \exp \left(-\omega^{2} \sigma_{\tau}^{2} / 2\right) .
\end{aligned}
$$

Here, use is made of the expressions of the following definite integrals,

$$
\begin{aligned}
\int_{-\infty}^{\infty} \cos \left(\omega \tau_{n}\right) \exp \left(-\tau_{n}^{2} / 2 \sigma_{\tau}^{2}\right) \mathrm{d} \tau_{n} & =\sigma_{\tau} \sqrt{2 \pi} \exp \left(-\omega^{2} \sigma_{\tau}^{2} / 2\right) \\
\int_{-\infty}^{\infty} \sin \left(\omega \tau_{n}\right) \exp \left(-\tau_{n}^{2} / 2 \sigma_{\tau}^{2}\right) \mathrm{d} \tau_{n} & =0 \\
\int_{-\infty}^{\infty} a_{n} \exp \left[-\frac{\left(a_{n}-\bar{a}_{n}\right)^{2}}{2 \sigma_{a}^{2}}\right] \mathrm{d} a_{n} & =\int_{-\infty}^{\infty}\left(\bar{a}_{n}+b_{n}\right) \exp \left[-b_{n}^{2} / 2 \sigma_{a}^{2}\right] \mathrm{d} b_{n}, \text { where } b_{n}=a_{n}-\bar{a}_{n} \\
& =\bar{a}_{n} \sigma_{a} \sqrt{2 \pi}
\end{aligned}
$$

Equation (5.77) can be derived from the standard integral (Zeidler, 2003),

$$
\int_{0}^{\infty} \exp \left(-\alpha^{2} x^{2}\right) \cos (\beta x) \mathrm{d} x=\frac{\sqrt{\pi}}{2 \alpha} \exp \left(-\beta^{2} / 4 \alpha^{2}\right) .
$$

The integrand of Eq. (5.78) is an odd function of the integral variable, hence the integral equals zero. Equation (5.79) also contains a term that is an odd function of the integral variable, so that this integral equals zero. The remainder contains a term that corresponds to the standard integral (Zeidler, 2003),

$$
\int_{-\infty}^{\infty} \exp \left(-\alpha^{2} x^{2}\right) \mathrm{d} x=\frac{\sqrt{\pi}}{\alpha} .
$$

The mean value of the cosine term can be derived in a similar fashion,

$$
\bar{x}_{n, \mathrm{cos}}=\bar{a}_{n} \cos (\omega n T) \exp \left(-\omega^{2} \sigma_{\tau}^{2} / 2\right) .
$$


The formulation for the square of the standard deviations requires lengthy, but straightforward, algebraic manipulations which are not shown in detail here. The formulation for the sine term is given by

$$
\begin{aligned}
\sigma_{n, \sin }^{2}= & \frac{1}{\sigma_{a} \sqrt{2 \pi}} \frac{1}{\sigma_{\tau} \sqrt{2 \pi}} \int_{-\infty}^{\infty} \int_{-\infty}^{\infty}\left[a_{n} \sin \omega\left(n T+\tau_{n}\right)-\bar{x}_{n, \sin }\right]^{2} \times \\
& \exp \left[-\left(a_{n}-\bar{a}_{n}\right)^{2} / 2 \sigma_{a}^{2}\right] \exp \left(-\tau_{n}^{2} / 2 \sigma_{\tau}^{2}\right) \mathrm{d} a_{n} \mathrm{~d} \tau_{n}, \\
= & \frac{1}{2}\left(\sigma_{a}^{2}+\bar{a}_{n}^{2}\right)\left[1-\cos (2 \omega n T) \exp \left(-\omega^{2} \sigma_{\tau}^{2} / 2\right)\right]- \\
& \bar{a}_{n}^{2}\left[\sin (\omega n T) \exp \left(-\omega^{2} \sigma_{\tau}^{2} / 2\right)\right]^{2} .
\end{aligned}
$$

Here, use has been made of the standard integral (Zeidler, 2003),

$$
\int_{-\infty}^{\infty} x^{2} \exp \left(-\alpha^{2} x^{2}\right) \mathrm{d} x=\frac{\sqrt{\pi}}{2 \alpha^{3}} .
$$

The formulation for the square of the standard deviation of the cosine term reads

$$
\begin{aligned}
\sigma_{n, \cos }^{2}= & \frac{1}{\sigma_{a} \sqrt{2 \pi}} \frac{1}{\sigma_{\tau} \sqrt{2 \pi}} \int_{-\infty}^{\infty} \int_{-\infty}^{\infty}\left[a_{n} \cos \omega\left(n T+\tau_{n}\right)-\bar{x}_{n, \mathrm{cos}}\right]^{2} \times \\
= & \frac{1}{2}\left(\sigma_{a}^{2}+\bar{a}_{n}^{2}\right)\left[1+\cos (2 \omega n T) \exp \left(-\omega^{2} \sigma_{\tau}^{2} / 2\right)\right]- \\
& \bar{a}_{n}^{2}\left[\cos (\omega n T) \exp \left(-\omega^{2} \sigma_{\tau}^{2} / 2\right)\right]^{2}
\end{aligned}
$$

Combining all terms gives

$$
\begin{aligned}
\left(\sum_{-N}^{N} \bar{x}_{n, \sin }\right)^{2}+ & \left(\sum_{-N}^{N} \bar{x}_{n, \cos }\right)^{2}+\sum_{-N}^{N} \sigma_{n, \sin }^{2}+\sum_{-N}^{N} \sigma_{n, \cos }^{2}= \\
= & \exp \left(-\omega^{2} \sigma_{\tau}^{2}\right) \bar{a}_{n}^{2}\left\{\left[\sum_{-N}^{N} \sin (\omega n T)\right]^{2}+\left[\sum_{-N}^{N} \cos (\omega n T)\right]^{2}\right\}+ \\
& (2 N+1)\left[\left(\sigma_{a}^{2}+\bar{a}_{n}^{2}\right)-\bar{a}_{n}^{2} \exp \left(-\omega^{2} \sigma_{\tau}^{2}\right)\right]
\end{aligned}
$$


Use will now be made of the relations,

$$
\begin{aligned}
\lim _{N \rightarrow \infty} \frac{1}{2 N+1}\left[\sum_{-N}^{N} \sin (n \omega T)\right]^{2} & =0 \\
\lim _{N \rightarrow \infty} \frac{1}{2 N+1}\left[\sum_{-N}^{N} \cos (n \omega T)\right]^{2} & =\lim _{N \rightarrow \infty} \frac{1}{2 N+1}\left[\sum_{-N}^{N}[\cos (n \omega T)+i \sin (n \omega T)]\right]^{2}, \\
& =\lim _{N \rightarrow \infty} \frac{1}{2 N+1}\left[\sum_{-N}^{N} \exp (i n \omega T)\right]^{2} \\
& =\lim _{N \rightarrow \infty} \frac{1}{2 N+1}\left[(2 N+1) \Delta_{T}(\omega)\right]\left[\frac{2 \pi}{T} \Delta_{T}(\omega)\right] \\
& =\frac{2 \pi}{T} \Delta_{T}(\omega) \\
& =\omega_{r} \Delta_{T}(\omega)
\end{aligned}
$$

in which $\omega_{r}$ corresponds to the mean repetition rate of the event and in which both the discrete and the continuous formulation ${ }^{7}$ for the Dirac comb $\Delta_{T}(\omega)$ has been used,

$$
\Delta_{T}(\omega)=\frac{1}{2 N+1} \sum_{n=-N}^{N} e^{i n \omega T}=\frac{T}{2 \pi} \sum_{n=-\infty}^{\infty} e^{i n \omega T} .
$$

The Dirac comb is related to the Dirac delta function $\delta(\omega)$ through

$$
\Delta_{T}(\omega)=\sum_{m=-\infty}^{\infty} \delta\left(\omega-\frac{2 \pi m}{T}\right)
$$

with $m$ an integer.

Substituting Eq. (5.86) in Eq. (5.74) while using Eq. (5.87) and (5.88) gives

$$
R(\omega)=\left\{\sigma_{a}^{2}+\bar{a}_{n}^{2}\left[1-\exp \left(-\omega^{2} \sigma_{\tau}^{2}\right)\right]+\bar{a}_{n}^{2} \exp \left(-\omega^{2} \sigma_{\tau}^{2}\right) \omega_{r} \Delta_{T}(\omega)\right\}|G(\omega)|^{2} .
$$

Equation (5.91) shows the effect of the variability from blade passage to blade passage on the power density spectrum. The variability is expressed in terms of the standard deviation of amplitude and phase and the spectral shape corresponds to the mean spectrum $\bar{a}_{n}^{2} G(\omega)^{2}$ of all individual blade passages. This relation, which combines amplitude and phase variation, has not been presented before to the author's knowledge.

Similar to Bark (2000), the effect of the amplitude and phase variation separately is illustrated in Figure 5.8. A stylised spectrum is sketched of a single blade passage together with the spectrum of multiple blade passages. The latter corresponds to the spectrum that is normally considered. The frequency is non-dimensionalised by the blade passage frequency. If the signal repeats itself perfectly there is no variation in amplitude and phase and the spectrum only consists of blade rate tonals as shown in Figure 5.8a. Random variations between blade

\footnotetext{
${ }^{7}$ See e.g. http://dspillustrations.com/pages/posts/misc/the-dirac-comb-and-its-fourier-transform.html, visited August 14, 2018
} 


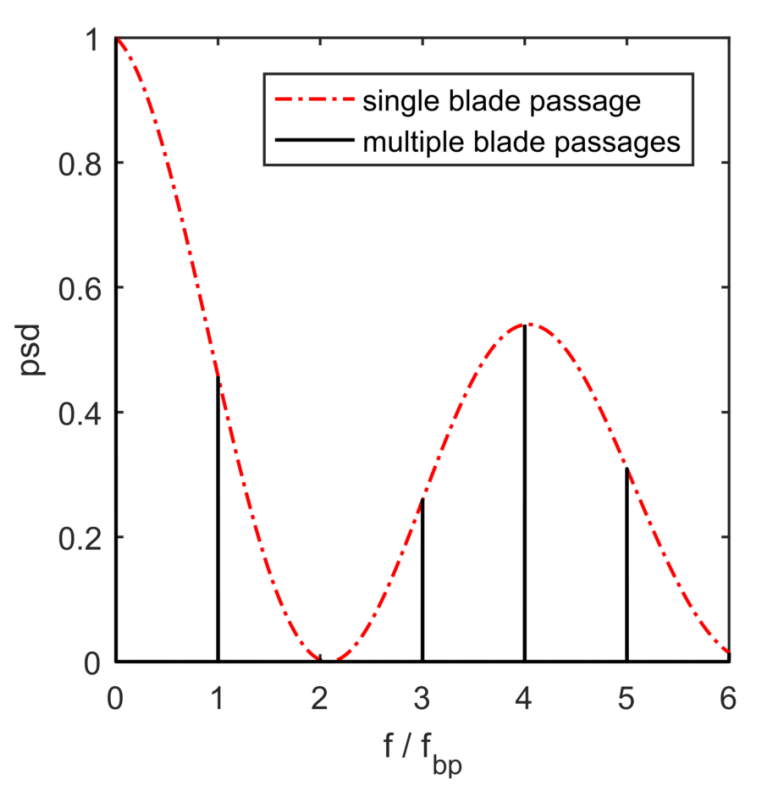

a) No variation

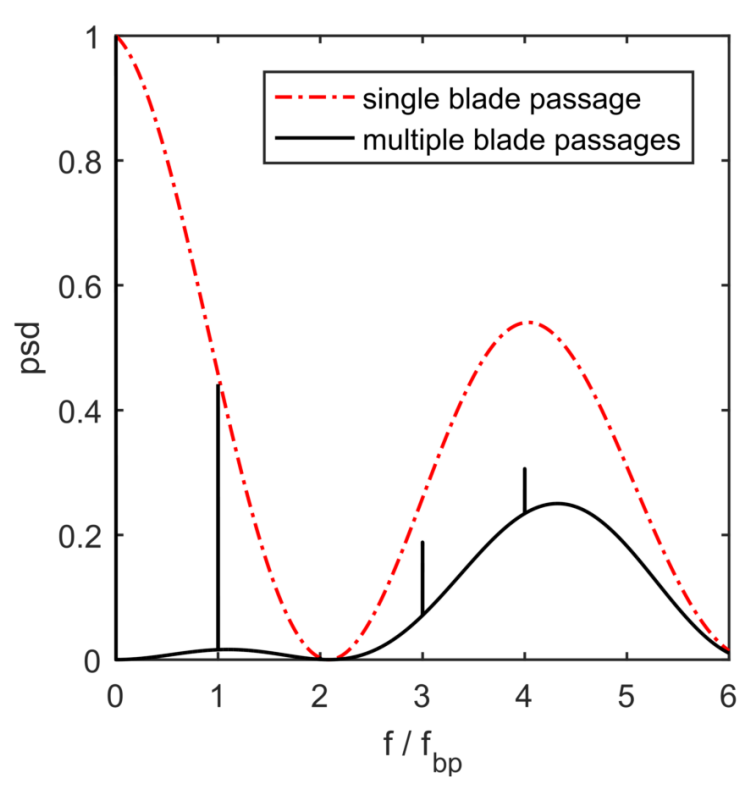

c) Arrival time (phase) variation

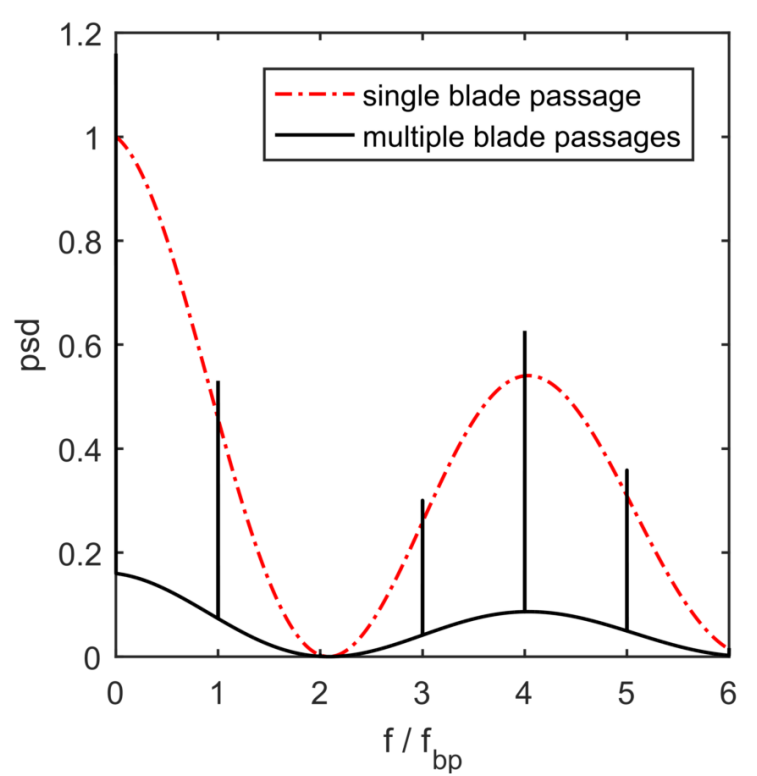

b) Amplitude variation

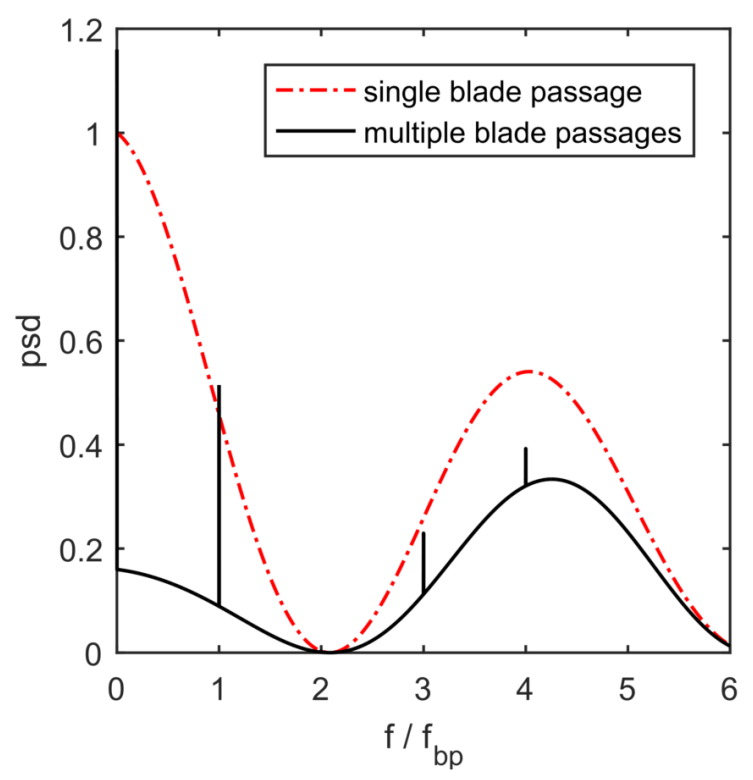

d) Amplitude and arrival time variation

Figure 5.8: Influence of variability of pulse amplitude and arrival time (phase) on the power spectral density (psd) of a number of blade passages with blade passage frequency $f_{b p}$.

passages of amplitude alone, $\sigma_{a}>0, \sigma_{\tau}=0$ in Eq. (5.91), lead to a small increase of the amplitudes of these tonals, see Figure 5.8b. However, the spectrum now also contains a broadband part that is proportional to the spectrum of the single blade passage. The magnitude equals the standard deviation of the amplitude variations as shown by Eq. (5.91). Random changes in time of arrival of the signal alone, $\sigma_{a}=0, \sigma_{\tau}>0$ in Eq. (5.91), lead to random phase angle variations of which the magnitude increases linearly with frequency. This random phase variation decreases the amplitude of the tonals and redistributes the power over a broadband region, see Figure 5.8c. The spreading is most pronounced at higher frequencies and may cause the com- 
plete disappearance of the higher harmonic tonals. The combination of amplitude and phase variation is a straightforward combination of both effects and is shown in Figure 5.8d.

For the interpretation of the magnitude of the tonals, we have to realize that the power spectral density of the tonal is scaled with frequency bandwidth $\omega_{r}$. The total power due to the tonals only, hence $\sigma_{a}=\sigma_{\tau}=0$, is then given by

$$
P=\int R(\omega) d \omega=\bar{a}_{n}^{2} \omega_{r} \sum_{m}\left|G\left(m \omega_{r}\right)\right|^{2},
$$

with $m$ is an integer that represents the harmonic. Hence, the factor $\omega_{r}$ can also be interpreted as the bin width of the tonal. Note that the Dirac comb can also be made non-dimensional by $\omega_{r} \Delta_{T}(\omega)=\Delta\left(\omega / \omega_{r}\right)$.

\subsection{Scaling of cavitation noise}

Measuring HPF and URN of cavitating propellers at model scale requires corrections for the prediction at ship scale. These scaling relations, given for pressures presented in the frequency domain, are briefly discussed here.

\subsubsection{Hull-pressure fluctuations at blade rate frequencies}

The amplitudes of the HPF at blade passage frequency and harmonics (blade rate tonals) scale with dynamic pressure using velocity proportional to propeller tip speed. The generally used non-dimensional pressure amplitude for harmonic $h$ is given by

$$
k_{p}(h)=\frac{p_{a}^{\prime}(h)}{\rho n^{2} D^{2}},
$$

where pressure $p_{a}^{\prime}$ is usually defined as a peak amplitude and has unit Pa, $\rho$ denotes density, $n$ the propeller rotation rate and $D$ the propeller diameter. The relation between ship-scale (subscript $s$ ) and model-scale (subscript $m$ ) pressure amplitudes then reads

$$
\frac{p_{a, s}^{\prime 2}(h)}{p_{a, m}^{\prime 2}(h)}=\left(\frac{\rho_{s}}{\rho_{m}}\right)^{2}\left(\frac{n_{s}}{n_{m}}\right)^{4}\left(\frac{D_{s}}{D_{m}}\right)^{4} .
$$

The pressure amplitudes scale independent of the blade passage frequency. The blade passage frequency scales with $Z n$ with $Z$ the number of blades.

\subsubsection{Broadband hull-pressure fluctuations}

The low frequency broadband HPF are usually presented by power spectral density values. The available scaling parameters are, similar as for the harmonics at the blade passage frequency, the dynamic pressure using a velocity associated with the propeller tip speed for the amplitude ${ }^{8}$

\footnotetext{
${ }^{8}$ The amplitude can be a peak value as well as an rms value as this is not relevant for the scaling relations presented here.
} 
and the blade passage frequency for the frequency bandwidth. The non-dimensional frequency $f_{p}$ is then defined as

$$
f_{p}=\frac{f}{f_{b p}}
$$

with $f_{b p}$ the blade passage frequency given by $f_{b p}=Z n$. The ratio between ship scale and model scale frequencies then yields

$$
\frac{f_{s}}{f_{m}}=\frac{n_{s}}{n_{m}}
$$

The scaling for the pressure amplitude density is then given by

$$
k_{p}\left(f_{p}\right)=\frac{p_{a}^{\prime}(f)}{\rho n^{2} D^{2}} \frac{1}{\sqrt{\Delta f / f_{b p}}},
$$

where $\Delta f$ corresponds here to the frequency resolution bandwidth of the spectrum which is made non-dimensional with the blade passage frequency ${ }^{9}$. The resulting value of $k_{p}$ is an amplitude spectral density value (in non-dimensional bandwidth) at non-dimensional frequency $f_{p}$. An rms amplitude density spectrum is equal to a power density spectrum when both are presented in decibels.

Because broadband pressure levels are usually presented by their power spectral density values, we introduce $p^{2}=p_{a}^{\prime 2} / \Delta f$, with unit of $p^{\prime 2}$ given by $\mathrm{Pa}^{2} / \mathrm{Hz}$. The ratio between shipscale and model-scale values then equals

$$
\frac{p_{s}^{\prime 2}\left(f_{s}\right)}{p_{m}^{\prime 2}\left(f_{m}\right)}=\left(\frac{\rho_{s}}{\rho_{m}}\right)^{2}\left(\frac{n_{s}}{n_{m}}\right)^{4}\left(\frac{D_{s}}{D_{m}}\right)^{4}\left(\frac{f_{b p, m}}{f_{b p, s}}\right)
$$

The levels for constant bandwidth, defined as $p_{1 H z}^{\prime 2}=p^{\prime 2}$, are then given by

$$
\frac{p_{s, 1 H z}^{\prime 2}\left(f_{s}\right)}{p_{m, 1 H z}^{\prime 2}\left(f_{m}\right)}=\left(\frac{\rho_{s}}{\rho_{m}}\right)^{2}\left(\frac{n_{s}}{n_{m}}\right)^{3}\left(\frac{D_{s}}{D_{m}}\right)^{4},
$$

and the levels in proportional bandwidth, given by $p_{1 / 3}^{\prime 2}=p^{\prime 2} \Delta f$, with $\Delta f_{s} \propto f_{b p, s}, \Delta f_{m} \propto f_{b p, m}$ read

$$
\frac{p_{s, 1 / 3}^{\prime 2}\left(f_{s}\right)}{p_{m, 1 / 3}^{\prime 2}\left(f_{m}\right)}=\left(\frac{\rho_{s}}{\rho_{m}}\right)^{2}\left(\frac{n_{s}}{n_{m}}\right)^{4}\left(\frac{D_{s}}{D_{m}}\right)^{4}
$$

where subscript $1 / 3$ refers to the $1 / 3$-octave band level spectrum, which is typically used for presenting URN levels. The unit of $p_{1 / 3}^{\prime 2}$ equals $\mathrm{Pa}^{2}$.

\footnotetext{
${ }^{9}$ This definition is introduced in the present thesis. The scaling with blade number that is thereby applied requires further attention.
} 


\subsubsection{Radiated noise: high-frequency formulation}

The formulation for high-frequency URN that is used at MARIN has been developed by de Bruijn and Ten Wolde (1974) and will be discussed in the following. The formulation is based on the original work of Levkovskii (1967).

The non-dimensional frequency $\Pi_{f}$ is obtained from the collapse time of a single bubble which can be derived from the Rayleigh-Plesset equation. It is assumed that the maximum bubble size $a$ has a linear relation with the propeller diameter $\mathrm{D}$. The non-dimensional frequency reads

$$
\Pi_{f}=f a \sqrt{\frac{\rho}{p_{e}}} \propto f D \sqrt{\frac{1}{n^{2} D^{2} \sigma}}=\frac{f}{n} \sqrt{\frac{1}{\sigma}},
$$

with the pressure reserve to cavitation $p_{e}$ defined by $p_{e}=p_{0}-p_{v}+\rho g h$ and the cavitation number is given by $\sigma=\left(p_{0}-p_{v}+\rho g h\right) / \frac{1}{2} \rho n^{2} D^{2}$. Parameter $p_{0}$ denotes the atmospheric pressure, $p_{v}$ the vapour pressure, $g$ the acceleration of gravity and $h$ the submersion depth of the cavity.

The radiated acoustic power $P_{a}$ of a cloud filled with cavitation bubbles is given by

$$
P_{a}=\frac{4 \pi R^{2} p^{\prime 2}}{\rho c} \Delta f
$$

where $p^{\prime 2}$, with unit $\mathrm{Pa}^{2} / \mathrm{Hz}$, corresponds to the mean-square sound pressure in power spectral density values at distance $R$, and $\Delta f$ corresponds to the total bandwidth of the cavitation noise. Parameter $c$ denotes the speed of sound.

The change in potential energy $P_{p o t}$ of the bubble cloud with bubbles of maximum radius $r_{b, \max }$ is given by

$$
\Delta P_{p o t} \propto r_{b, \max }^{3} p_{e} N
$$

where $N$ corresponds to the number of bubbles that implode per second. The product $r_{b \text { max }}^{3} N$ then corresponds to the volume of bubbles that implode per second. For similar cavitation extents at model scale and full scale, the cavity area scales with the propeller disc area and it is assumed that the cavity thickness scales with cavity length, and thus with propeller diameter. This leads to

$$
r_{b, \max }^{3} N \propto D^{3} n
$$

where $n$ corresponds to the propeller rotation rate. Remark that in this formulation the volume of cavitation does not depend on the pressure $p_{e}$ as it is required that the relative size of the cavity at model scale is identical to the one at full scale.

The radiated acoustic power $P_{a}$ is related to the rate of change in potential energy $P_{p o t}$ using the acoustic efficiency $\eta_{a c}{ }^{10}$,

$$
P_{a}=\eta_{a c} P_{p o t}
$$

For high frequencies one may assume that $\eta_{a c}$ of the collapsing bubbles is independent of the bubble size and becomes a constant which is identical at model scale and at full scale.

\footnotetext{
${ }^{10}$ If it is assumed that all potential energy is transferred into kinetic energy, this definition for the acoustic efficiency is identical as in Section 5.2.1 with $P_{p o t}=\omega E_{\text {kin }}$.
} 
This assumption is valid for a shock-wave spectrum according to Levkovskii (1967) and Baiter (1985). The non-dimensional acoustic power then reads

$$
\Pi_{p}=\frac{P_{a}}{\eta_{a c} P_{p o t}} \propto \frac{4 \pi R^{2} p^{\prime 2}}{\rho c} \Delta f \frac{1}{D^{3} n p_{e}} \propto \frac{R^{2} p^{\prime 2}}{\rho c D^{3} p_{e} n} \Delta f .
$$

Introducing the cavitation number gives the relation,

$$
\Pi_{p} \propto \frac{R^{2} p^{\prime 2}}{\rho^{2} c D^{5} \sigma n^{3}} \Delta f
$$

The ratio of frequency and pressure of model scale and full scale are then given by

$$
\begin{aligned}
\frac{f_{s}}{f_{m}} & =\sqrt{\frac{\sigma_{s}}{\sigma_{m}}} \frac{n_{s}}{n_{m}}, \\
\frac{p_{s}^{\prime 2}\left(f_{s}\right)}{p_{m}^{\prime 2}\left(f_{m}\right)} & =\left(\frac{\rho_{s}}{\rho_{m}}\right)^{2}\left(\frac{n_{s}}{n_{m}}\right)^{3}\left(\frac{D_{s}}{D_{m}}\right)^{5}\left(\frac{\sigma_{s}}{\sigma_{m}}\right)\left(\frac{c_{s}}{c_{m}}\right)\left(\frac{R_{m}}{R_{s}}\right)^{2}\left(\frac{\Delta f_{m}}{\Delta f_{s}}\right) .
\end{aligned}
$$

The pressure ratio in $1 \mathrm{~Hz}$ or constant bandwidth is obtained by using $\Delta f_{m} / \Delta f_{s}=f_{m} / f_{s}$,

$$
\frac{p_{s, 1 \mathrm{~Hz}}^{\prime 2}\left(f_{s}\right)}{p_{m, 1 \mathrm{~Hz}}^{\prime 2}\left(f_{m}\right)}=\left(\frac{\rho_{s}}{\rho_{m}}\right)^{2}\left(\frac{n_{s}}{n_{m}}\right)^{2}\left(\frac{D_{s}}{D_{m}}\right)^{5}\left(\frac{\sigma_{s}}{\sigma_{m}}\right)^{0.5}\left(\frac{c_{s}}{c_{m}}\right)\left(\frac{R_{m}}{R_{s}}\right)^{2} .
$$

For proportional bandwidth, the ratio of $p^{2} \Delta f$ is considered which gives

$$
\frac{\left(p^{\prime 2} \Delta f\right)_{s}}{\left(p^{\prime 2} \Delta f\right)_{m}}=\frac{p_{s, 1 / 3}^{\prime 2}\left(f_{s}\right)}{p_{m, 1 / 3}^{\prime 2}\left(f_{m}\right)}=\left(\frac{\rho_{s}}{\rho_{m}}\right)^{2}\left(\frac{n_{s}}{n_{m}}\right)^{3}\left(\frac{D_{s}}{D_{m}}\right)^{5}\left(\frac{\sigma_{s}}{\sigma_{m}}\right)\left(\frac{c_{s}}{c_{m}}\right)\left(\frac{R_{m}}{R_{s}}\right)^{2} .
$$

\subsubsection{Radiated noise: low-frequency formulation}

The formulation for low frequencies can be derived similarly as for high frequencies. However, at low frequencies the acoustic efficiency is assumed to be proportional to a Mach number ${ }^{11}$ (Baiter, 1985) as shown in e.g. Ross (1987),

$$
\eta_{a c} \propto \frac{V}{c} \propto \frac{\sqrt{p_{e} / \rho}}{c} \propto \frac{\sqrt{\sigma n^{2} D^{2}}}{c}=\frac{n D}{c} \sqrt{\sigma},
$$

where $V$ is the maximum velocity at the cavity interface. The non-dimensional acoustic power is then given by

$$
\begin{aligned}
\Pi_{p}=\frac{P_{a}}{\eta_{a c} P_{p o t}} & =\frac{c}{n D \sqrt{\sigma}} \frac{R^{2} p^{\prime 2}}{\rho^{2} c D^{5} \sigma n^{3}} \Delta f \\
& =\frac{R^{2} p^{\prime 2}}{\rho^{2} D^{6} \sigma^{1.5} n^{4}} \Delta f .
\end{aligned}
$$

\footnotetext{
${ }^{11}$ The acoustic efficiency for a pulsating sphere for $k a \ll 1$ was given in Section 5.2.1 as $\eta_{a c}=k a$. Using $k=\omega / c$ and assuming $\omega a \propto V$, we also arrive at $\eta_{a c} \propto V / c$.
} 
Similar as for the high-frequency formulation, the scaling of the frequency can be obtained from the Rayleigh equation for the time of the collapse of the largest cavities (Strasberg, 1977),

$$
T_{c}=0.915 r_{b, \max } \sqrt{\rho /\left(p_{0}-p_{v}\right)} .
$$

Assuming that the frequency scales with the inverse of $T_{c}$ and that the maximum bubble size scales with the propeller diameter we find

$$
f \propto \frac{1}{D} \sqrt{\frac{p_{0}-p_{v}}{\rho}} \propto n \sqrt{\sigma}
$$

from which it follows that

$$
\frac{f_{s}}{f_{m}}=\sqrt{\frac{\sigma_{s}}{\sigma_{m}}} \frac{n_{s}}{n_{m}} .
$$

The expression for the pressure ratios then read

$$
\begin{aligned}
\frac{p_{s, 1 \mathrm{~Hz}}^{\prime 2}\left(f_{s}\right)}{p_{m, 1 \mathrm{~Hz}}^{\prime 2}\left(f_{m}\right)} & =\left(\frac{\rho_{s}}{\rho_{m}}\right)^{2}\left(\frac{n_{s}}{n_{m}}\right)^{3}\left(\frac{D_{s}}{D_{m}}\right)^{6}\left(\frac{\sigma_{s}}{\sigma_{m}}\right)\left(\frac{R_{m}}{R_{s}}\right)^{2}, \\
\frac{p_{s, 1 / 3}^{\prime 2}\left(f_{s}\right)}{p_{m, 1 / 3}^{\prime 2}\left(f_{m}\right)} & =\left(\frac{\rho_{s}}{\rho_{m}}\right)^{2}\left(\frac{n_{s}}{n_{m}}\right)^{4}\left(\frac{D_{s}}{D_{m}}\right)^{6}\left(\frac{\sigma_{s}}{\sigma_{m}}\right)^{1.5}\left(\frac{R_{m}}{R_{s}}\right)^{2} .
\end{aligned}
$$

\subsubsection{Discussion}

The scaling of underwater radiated noise can also be applied to hull-pressure fluctuations. Due to geometrical scaling, the ratio of the propeller-hull clearance of model scale and full scale is identical to the ratio of the propeller diameters, so $R_{m} \propto D_{m}$ and $R_{s} \propto D_{s}$. Applying this to Eqs. (5.117) and (5.118) describing the low-frequency noise scaling, gives

$$
\begin{aligned}
{\left[\frac{p_{s, 1 / 3}^{\prime 2}\left(f_{s}\right)}{p_{m, 1 / 3}^{\prime 2}\left(f_{m}\right)}\right]_{h u l l} } & =\left(\frac{\rho_{s}}{\rho_{m}}\right)^{2}\left(\frac{n_{s}}{n_{m}}\right)^{4}\left(\frac{D_{s}}{D_{m}}\right)^{4}\left(\frac{\sigma_{s}}{\sigma_{m}}\right)^{1.5}, \\
{\left[\frac{p_{s, 1 H z}^{\prime 2}\left(f_{s}\right)}{p_{m, 1 H z}^{\prime 2}\left(f_{m}\right)}\right]_{h u l l} } & =\left(\frac{\rho_{s}}{\rho_{m}}\right)^{2}\left(\frac{n_{s}}{n_{m}}\right)^{3}\left(\frac{D_{s}}{D_{m}}\right)^{4}\left(\frac{\sigma_{s}}{\sigma_{m}}\right) .
\end{aligned}
$$

The scaling of hull pressure amplitudes at blade rate tonals as given by Eq. (5.94) is identical to the scaling of the pressure amplitudes in proportional bandwidth, except for the correction for differences in cavitation number. It is normal practice to perform cavitation tests at identical cavitation number to obtain similarity in cavitation extents and so for these conditions this difference is not relevant. Hence, if one wants to interpret tonals in a scaled spectrum, one should consider the spectrum in proportional bandwidth and not in constant bandwidth as the latter scales differently. Note that practice at MARIN is to treat the hull-pressure blade rate tonals separately from the broadband levels. The scaling of broadband HPF presented in Section 5.4.2 gives similar scaling relations as for the low-frequency URN, but the latter also includes a correction for differences in cavitation number. For tip vortex cavitation, the cavitation number at 
model scale can be different from the cavitation number at full scale, which is further discussed in Chapter 8.

Scaling relations for URN have also been developed by other authors. Strasberg (1977) has derived the scaling relation for the pressure level using dimension analysis assuming that inertial forces are dominating the collapse process. The inertial forces are obtained from the Rayleigh-Plesset equation. The resulting scaling relation is very similar to the low-frequency underwater noise relation Eq. (5.117) except for the scaling with the cavitation number. In the formulation of Strasberg the pressure ratio for constant bandwidth is proportional to $\left(\sigma_{s} / \sigma_{m}\right)^{3 / 2}$. The formulation derived by Bark (1985) is also identical to Eq. (5.117) but the scaling with cavitation number is not taken into account.

The scaling relations for high-frequency URN derived in Section 5.4.4 show some distinct differences with the low-frequency formulations given in Section 5.4.3. Most relevant is that the low-frequency formulation has an additional scaling to $n D$ which results in an additional scaling to propeller tip speed ratio between ship scale and model scale. For model tests performed at matching Froude number, this results in an additional correction factor of $5 \log _{10} \lambda$, with $\lambda$ the geometric scale factor. The differences in scaling of cavitation number are usually not relevant, as discussed above. The differences between the two formulations are due to the difference in acoustic efficiency, but the motivations for the formulations presented are not explained by e.g. Baiter (1985).

Unfortunately, the dividing frequency between the low-frequency and high-frequency formulation is not clear. Some discussion on this topic can be found in Baiter (1985) and Blake (1986).

\subsection{Scattering by hull and free surface}

\subsubsection{Analytical formulation for the solid boundary factor}

For the pressure fluctuations as measured on the hull we need to take the scattering effect of the hull into account. In maritime hydrodynamics, the resulting multiplication factor for the pressure is referred to as the solid boundary factor. The solid boundary factor does not take the effect of hull vibrations into account so the hull is assumed rigid. In acoustics, the pressure on the hull is referred to as blocked pressure. The scattering leads to a pressure doubling when the hull is a simple flat surface, infinite in extent. A pressure release boundary exists at the sea surface, so the pressure perturbation has to be equal to zero for the hull locations coinciding with the sea surface. This leads to a variation of the solid boundary factor over the breadth of the hull.

Hydrodynamic computations and experiments on systematic variations of hull geometries were performed by Huse and Guoqiang (1982) and simple empirical formulations were derived from these results. Nowadays, when the pressure on the hull or in the free field needs to be considered, acoustic computational tools solving for example the Helmholtz equations are applied to take the scattering effect of the hull and that of the free surface into account (van Wijngaarden, 2011). It is shown here that a simple analytical formulation can be derived to compute the 
solid boundary factor for a hull as a function of the hull radius of curvature and of frequency.

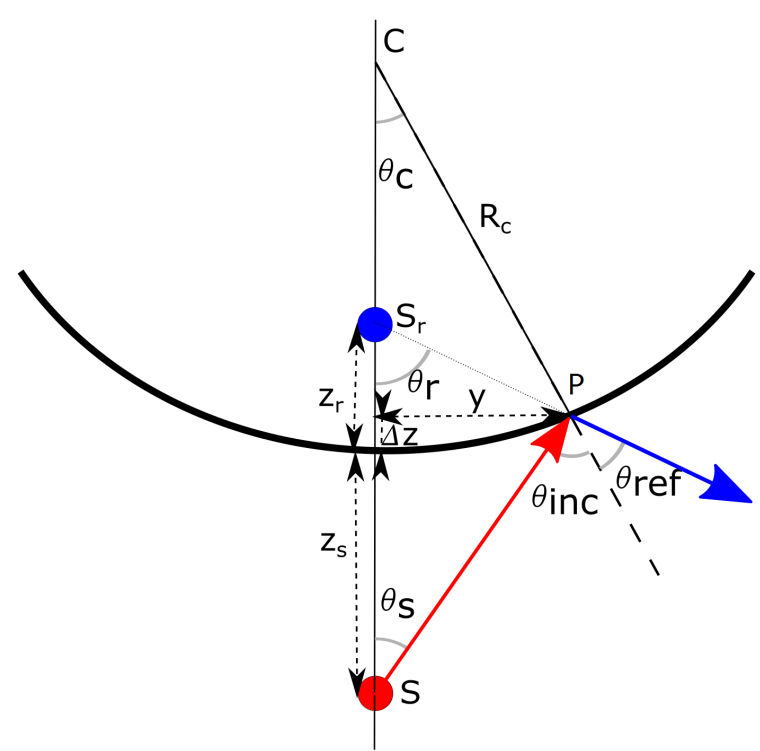

a)

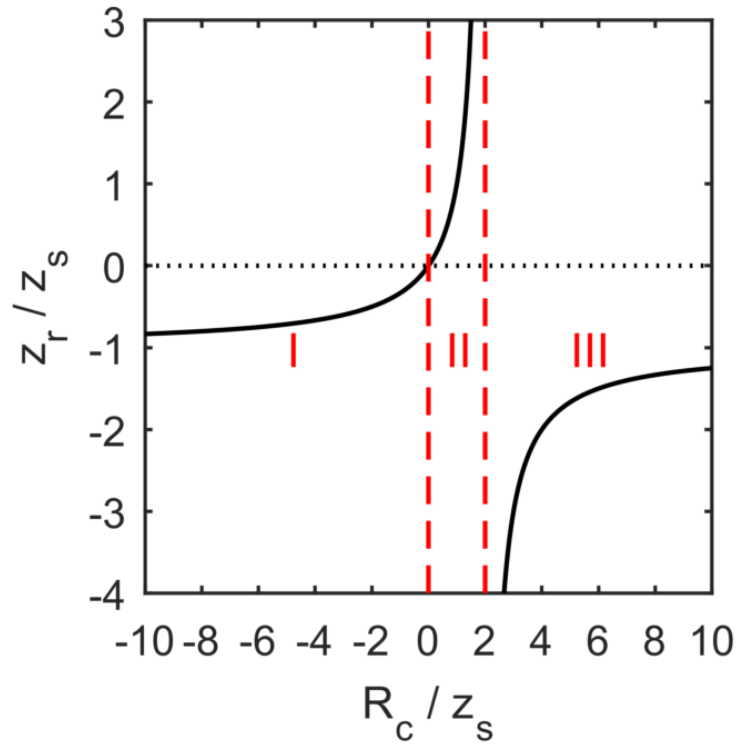

b)

Figure 5.9: Reflection of a pressure ray by a hull surface: a) diagram showing source $S$ and reflecting source $S_{r}$ for a convex hull $\left(R_{c}<0\right)$, b) relation between reflecting source distance $z_{r}$ and source distance $z_{s}$ as function of radius of curvature $R_{c}$, regimes I through III are also shown in Figure 5.10.

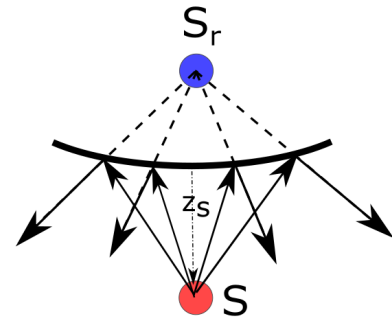

I

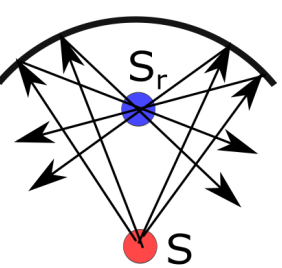

II

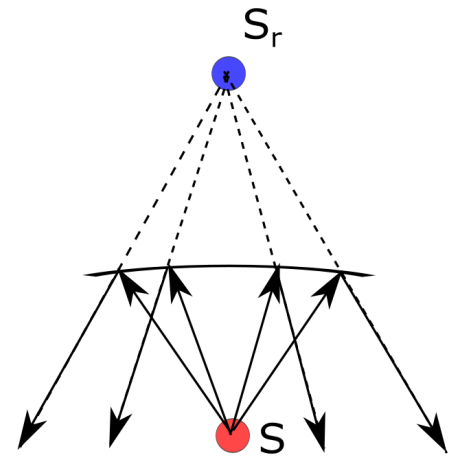

III

Figure 5.10: Various locations of the reflecting source $S_{r}$ for the thin lens formula, I: convex hull $\left(R_{c}<0\right)$, II: concave hull $\left(R_{c}>0\right), R_{c} / z_{s}<2$, III: concave hull $R_{c} / z_{s}>2$, regimes I through III are shown in Figure 5.9b. Figure adapted from Kuttruff (2009).

Consider an acoustic source and a rigid hull section with constant radius of curvature $R_{c}$ in the cross-sectional plane for which the distance between source and surface is $z_{s}$, see Figure 5.9. The $z$-axis is directed downwards such that $z_{s}$ is always positive and the origin is located on the hull surface. The radius of curvature is defined as $R_{c}=z_{c}$. Hence, $R_{c}$ has a negative value for the convex hull shown in Figure 5.9. The rays from the source $S$ are reflected by the hull 
surface with the reflections originating from a virtual source image $S_{r}$ that is located at distance $z_{r}$ from the hull surface. If the reflecting source is located on the opposite side of the hull as the source, as in Figure 5.9, $z_{r}$ has a negative value. It is assumed that specular reflection occurs, i.e. $\vartheta_{\text {inc }}=\vartheta_{\text {ref }}$, which results into

$$
\vartheta_{s}-\vartheta_{r}=-2 \vartheta_{c}
$$

The angles for location $P$ on the hull are defined by

$$
\begin{aligned}
\tan \vartheta_{s}(P) & =\frac{y_{p}}{z_{s}-z_{p}}, \\
\tan \vartheta_{r}(P) & =\frac{y_{p}}{-\left(z_{r}-z_{p}\right)}, \\
\tan \vartheta_{c}(P) & =\frac{y_{p}}{-\left(R_{c}-z_{p}\right)}, \\
z_{p} & =R_{c}\left(1-\cos \vartheta_{c} .\right)
\end{aligned}
$$

For small angles $\vartheta$, we find $\tan \vartheta \approx \vartheta$ and $\cos \vartheta \approx 1$ which results in

$$
\frac{1}{z_{s}}+\frac{1}{z_{r}}=\frac{2}{R_{c}}
$$

which is the thin lens formula. The reflecting source is a focal point of the hull surface. The distance $z_{r}$ can be computed from

$$
\frac{z_{r}}{z_{s}}=\frac{R_{c}}{2 z_{s}-R_{c}}=\frac{1}{2 \frac{z_{s}}{R_{c}}-1},
$$

where $z_{s}$, which is approximately equal to the propeller-hull clearance, has been used as a reference length. For large radius of curvature, thus approaching flat plate, the virtual focus becomes the mirror image with $z_{r}=-z_{s}$. A positive radius of curvature, i.e. a concave surface, resembles a twin-screw hull with tunnel shape. Starting from a large positive value for the radius of curvature, a decreasing value leads an increase of the distance between the focus and the hull surface with a value of $z_{r}=-\infty$ for $R_{c} / z_{s}=2$. For any radius of curvature for which $R_{c} / z_{s}<2$ and positive $R_{c}$, the focus switches side and is located on the same side with respect to the surface as the source. For negative radius of curvature, i.e. a convex surface which resembles a single-screw hull, the focus is located on the other side of the surface and slowly moves to the location of the focus for a flat plate when the radius of curvature becomes more negative. The locations of the focus are shown in Figure 5.10.

For the computation of the solid boundary factor, the radiated pressure $p^{\prime}$ and radially directed velocity $u^{\prime}$ for a monopole in the near field formulation are considered, see Section 5.2.1,

$$
\begin{aligned}
& p^{\prime}(R, t)=\hat{p}_{s} \frac{\exp [i(k R-\omega t)]}{R}, \\
& u^{\prime}(R, t)=\frac{\hat{p}_{s}}{\rho c} \frac{\exp [i(k R-\omega t)]}{R}\left(1+i \frac{1}{k R}\right) .
\end{aligned}
$$

Note that $R$ represents a distance and is always positive. The pressure induced by the reflecting source at distance $R_{r}$ from this source is given by multiplying Eq. (5.128) with a reflection coefficient $C_{r}$,

$$
p_{r}^{\prime}\left(R_{r}, t\right)=C_{r} \hat{p}_{s} \frac{\exp \left[i\left(k R_{r}-\omega t\right)\right]}{R_{r}}
$$




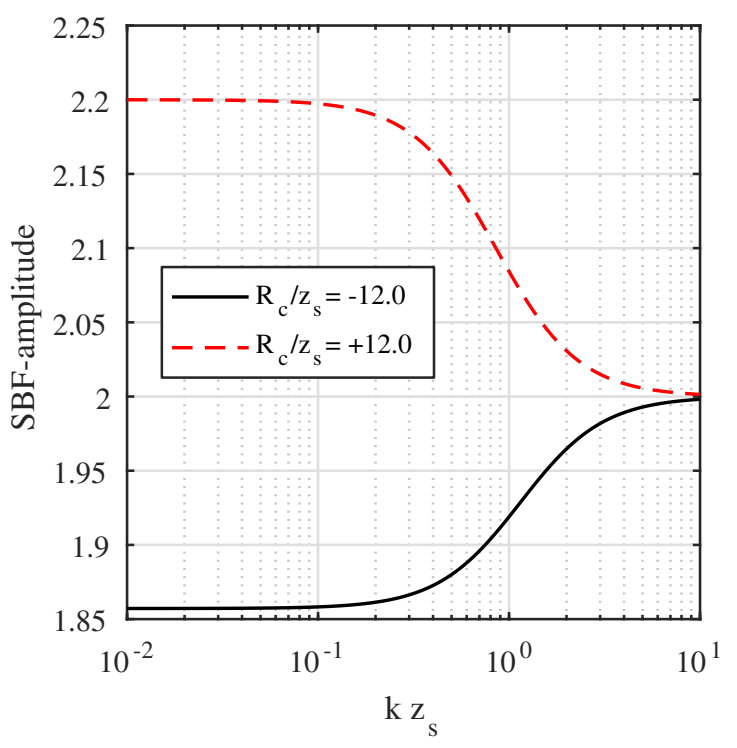

a)

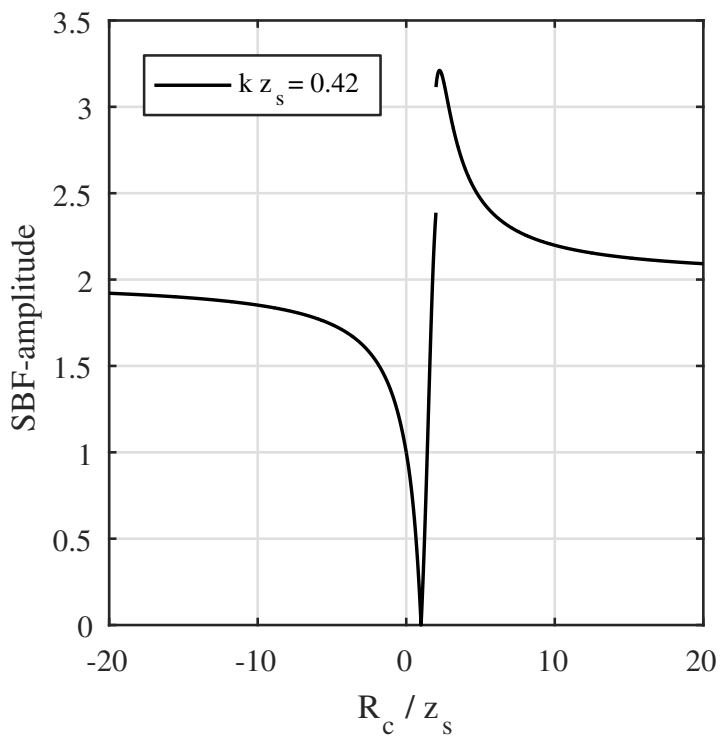

c)

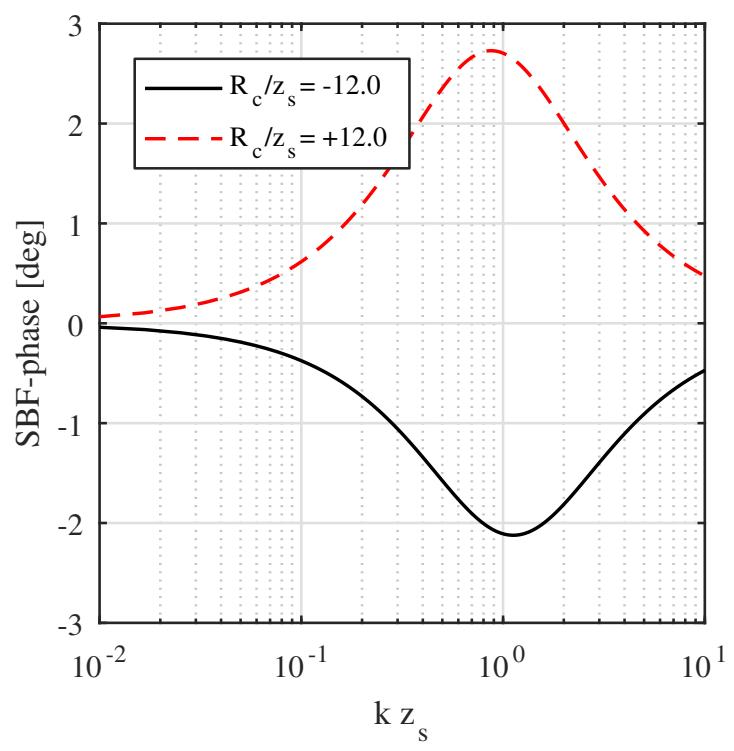

b)

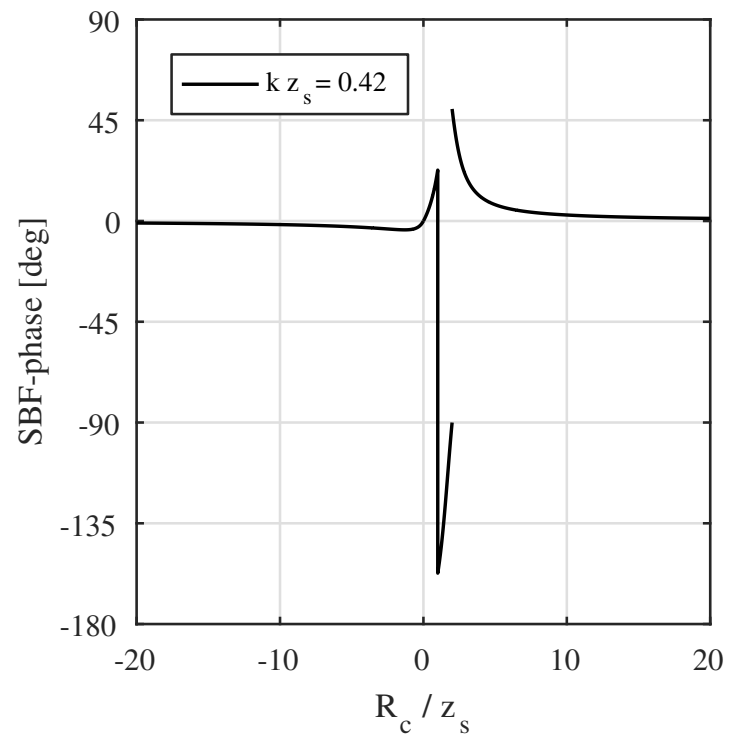

d)

Figure 5.11: Computation of the solid boundary factor from Eq. (5.135) and (5.136). Graph a) and $\mathrm{b}$ ) show the variation with acoustic wavenumber $k$, made non-dimensional by $z_{s}$, and graph c) and d) show the variation with radius of curvature $R_{c}$. A value of $k z_{s}=0.42$ corresponds to $f \approx 50 \mathrm{~Hz}$ for $z_{s}=2 \mathrm{~m}$.

and with the radial directed velocity from the reflecting source given by

$$
u_{r}^{\prime}\left(R_{r}, t\right)=C_{r} \frac{\hat{p}_{s}}{\rho c} \frac{\exp \left[i\left(k R_{r}-\omega t\right)\right]}{R_{r}}\left(1+i \frac{1}{k R_{r}}\right)
$$

At the wall we require that the velocity component normal to the wall equals zero. Hence, the transmitted velocity is zero and the effect of hull vibrations is neglected. The distance from a point on the hull to the source is $R_{s}$ and to the reflecting source is $R_{r}$. These distances are given 


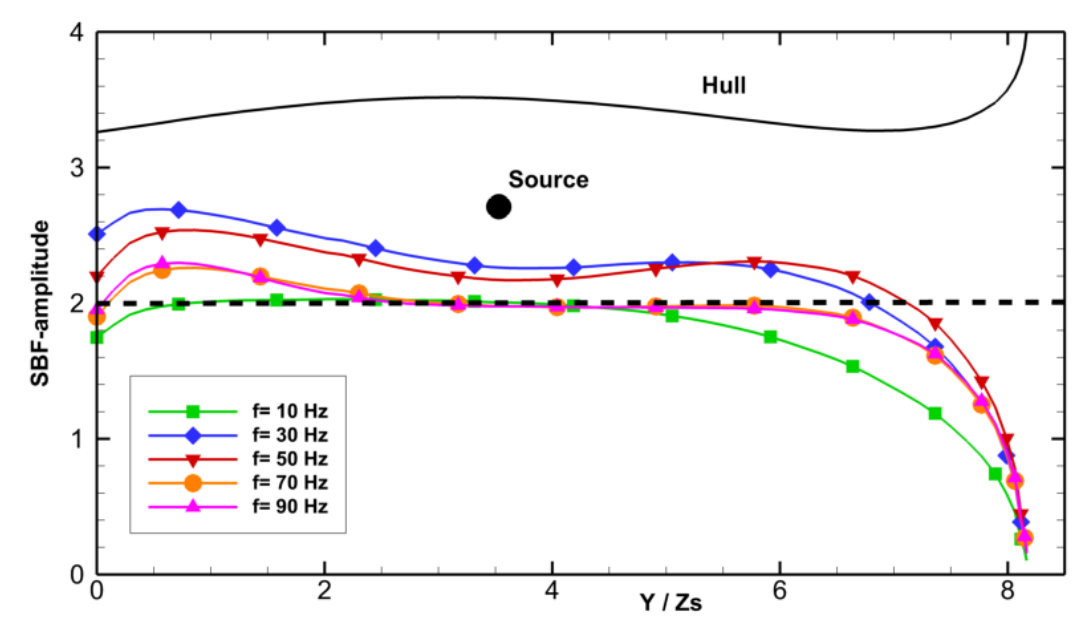

Figure 5.12: SBF computed by an acoustic boundary element method for a twin screw hull including the effect of the free surface. Results are presented for the starboard hull section in the propeller plane with $z_{s}=1.6 \mathrm{~m}$. The $S B F$-amplitude of the analytical model for the location on the hull directly above the source varies between 2.05 at $10 \mathrm{~Hz}$ to 2.04 at $90 \mathrm{~Hz}$. A frequency of $10 \mathrm{~Hz}$ corresponds to $k z_{s}=1.7 \times 10^{-3}$

by

$$
\begin{aligned}
& R_{s}=\sqrt{\left(z_{s}-z_{p}\right)^{2}+y_{p}^{2}}, \\
& R_{r}=\sqrt{\left(z_{r}-z_{p}\right)^{2}+y_{p}^{2}} .
\end{aligned}
$$

The boundary condition for the location on the hull directly above the source, where $R_{S}=$ $z_{s}, R_{r}=\left|z_{r}\right|$, then reads

$$
u^{\prime}+\operatorname{sign}\left(z_{r}\right) u_{r}^{\prime}=0 .
$$

The $\operatorname{sign}\left(z_{r}\right)$ is required as the direction of the reflected velocity depends on which side of the reflecting surface $S_{r}$ is located. Substituting Eqs. (5.129) and (5.131) in Eq. (5.134) gives the reflection coefficient,

$$
C_{r}\left(z_{s}, R_{c}, k\right)=-\operatorname{sign}\left(z_{r}\right) \frac{\exp \left(i k z_{s}\right)}{\exp \left(i k\left|z_{r}\right|\right)} \frac{\left|z_{r}\right|}{z_{s}} \frac{\left(1+i \frac{1}{k z_{s}}\right)}{\left(1+i \frac{1}{k\left|z_{r}\right|}\right)}
$$

The solid boundary factor $S B F$ is given by the ratio of the total pressure divided by the incident pressure,

$$
\operatorname{SBF}\left(z_{s}, R_{c}, k\right)=\frac{p^{\prime}+p_{r}^{\prime}}{p^{\prime}}=1+C_{r}=1-\operatorname{sign}\left(z_{r}\right) \frac{\exp \left(i k z_{s}\right)}{\exp \left(i k\left|z_{r}\right|\right)} \frac{\left|z_{r}\right|}{z_{s}} \frac{\left(1+i \frac{1}{k z_{s}}\right)}{\left(1+i \frac{1}{k\left|z_{r}\right|}\right)} .
$$

Because $z_{r}$ is a function $R_{c}$ and $z_{s}$, Eq. (5.127), $C_{r}$ and $S B F$ have been made a function of $R_{c}$ instead of $z_{r}$. It is reminded that the formulation was derived for $\vartheta_{s}(P)=0$. 
The variation of the amplitude and phase angle of the $S B F$ with frequency are presented in the upper graphs of Figure 5.11. Results are shown for a convex and for a concave hull form. The $S B F$-amplitude of the convex hull form is 1.85 at low frequencies and for the concave hull form it is 2.2. For both hull forms the $S B F$-amplitude becomes 2.0 at a frequency of $1 \mathrm{kHz}$. The phase angles are presented in Figure $5.11 \mathrm{~b}$, but these angles are very small. The lower graphs of Figure 5.11 show the variation of the amplitude and phase angle of the $S B F$ with radius of curvature. The $S B F$-amplitude is 2.0 for large positive as well as for large negative radius of curvature. For a convex hull the $S B F$-amplitude becomes smaller than 2.0 and for a concave hull the amplitude becomes larger than 2.0. Near $z_{s} / R_{c}=0.5$, hence $R_{c}=4.0 \mathrm{~m}$, a very large variation in amplitude is observed but such hull forms do not exist and this region is therefore of no significance here.

The presented relation for the $S B F$ is only valid if (i) the hull has constant radius of curvature, (ii) if the curvature in lengthwise direction is assumed small, and (iii) if the effect of the sea surface can be neglected. The latter condition is usually valid for the hull locations directly above the propeller and the second condition is also generally valid. The first condition is usually not satisfied. Therefore, the presented relation can only be applied locally if the acoustic wavelength is much smaller than the length scale of the hull considered. At the low frequencies at which hull pressures are considered, typically up to $200 \mathrm{~Hz}$ for ship scale, this condition is usually not satisfied and the local solution is therefore affected by the scattering of the whole hull and the sea surface as shown in Figure 5.12. The results in this graph were computed by the acoustic boundary element method EXCALIBUR (van Wijngaarden, 2011). Nevertheless, the presented relation for the $S B F$ explains some general trends and clearly shows that $S B F$ amplitudes different from 2.0 are caused by the near-field term of the velocity formulation, whereas the change in phase is expected to be negligible.

\subsubsection{Analytical formulations for Lloyd's mirror}

For a submerged noise source such as a cavitating propeller, the reflection of sound by the sea surface creates an interference pattern that is referred to as the Lloyd's mirror effect. For a monopole noise source a simple formulation can be derived for the total received pressure divided by the direct contribution of the source. This ratio is referred to as the propagation loss correction factor or transmission anomaly (Urick, 1983). The loss factor can be given as a function of distance $R$ between source and receiver or hydrophone location or as a function of frequency $f$,

$$
\begin{aligned}
P L_{L M} & =20 \log _{10}\left|1+K \frac{R_{S}}{R_{r}} \exp \left[-i k\left(R_{r}-R_{S}\right)\right]\right| \\
& \approx 20 \log _{10}\left|1+K \exp \left[-i \pi \frac{R_{0}}{R}\right]\right| \\
& \approx 20 \log _{10}\left|1+K \exp \left[-i \pi \frac{f}{f_{0}}\right]\right|
\end{aligned}
$$

with $R_{o}=4 z_{h} z_{s} f / c$ and $f_{0}=c R / 4 z_{h} z_{s}=c / 4 z_{s} \sin \theta$, with $z_{s}$ the source depth, $z_{h}$ the hydrophone depth, $c$ the speed of sound, and $\theta$ the depression angle, see Figure 5.13a. The parameter $K$ is the reflection coefficient with $K=-1$ for a flat sea surface. Equations (5.138) 
and (5.139) were derived using $\left(R_{r}-R_{S}\right)\left(R_{r}+R_{S}\right)=R_{r}^{2}-R_{s}^{2}=4 z_{h} z_{s}$, followed by the far field assumption $R_{s} \approx R_{r} \approx R$.

The propagation loss is presented in Figure 5.13b. Due to temporal averaging of radiated noise measurements, that are performed in a time window during which the distance between ship and hydrophone is varying, the interference pattern at high frequencies is less clearly observed in the radiated noise levels. With a rough sea surface the interference patterns at high frequencies also disappear. Clay and Medwin (1977) provide a formulation for the reflection coefficient that takes the free-surface wave height into account,

$$
\begin{aligned}
K & =\exp \left[-2 k^{2} \sigma^{2} \sin ^{2} \theta\right] \\
& =\exp \left[-2\left(\frac{f}{f_{0}}\right)^{2}\left(\frac{\pi \sigma}{2 z_{s}}\right)^{2}\right],
\end{aligned}
$$

with $k$ and $k_{0}$ the acoustic wavenumber for $f$ and $f_{0}$, respectively, and $\sigma$ the rms roughness height of the sea surface waves. Equation (5.140) is used in practical applications whereas Eq. (5.141) makes use of the frequency ratio $f / f_{0}$ used in Figure 5.13. At high frequencies $K$ approaches zero.

An alternative simple formula to correct for the propagation loss due to Lloyd's mirror is given by Ainslie (2010),

$$
\begin{aligned}
P L_{L M} & =-10 \log _{10}\left[\frac{1}{2}+\frac{1}{4 k^{2} z_{s}^{2} \sin ^{2} \theta}\right], \\
& =-10 \log _{10}\left[\frac{1}{2}+\left(\frac{f_{0}}{\pi f}\right)^{2}\right] .
\end{aligned}
$$

The most important difference with Eq. (5.141) is that the formula by Ainslie predicts a $3 \mathrm{~dB}$ increase at frequencies above $f_{0}$ and that it does not have a local maximum at $f_{0}$, see Figure 5.13b. Computations presented by Audoly and Meyer (2017) show that the propagation loss due to Lloyd's mirror equals zero $\mathrm{dB}$ at high frequencies for wind speeds above $5 \mathrm{~m} / \mathrm{s}$, corresponding to sea state 3 and higher, although there are some differences between the computational models applied. 


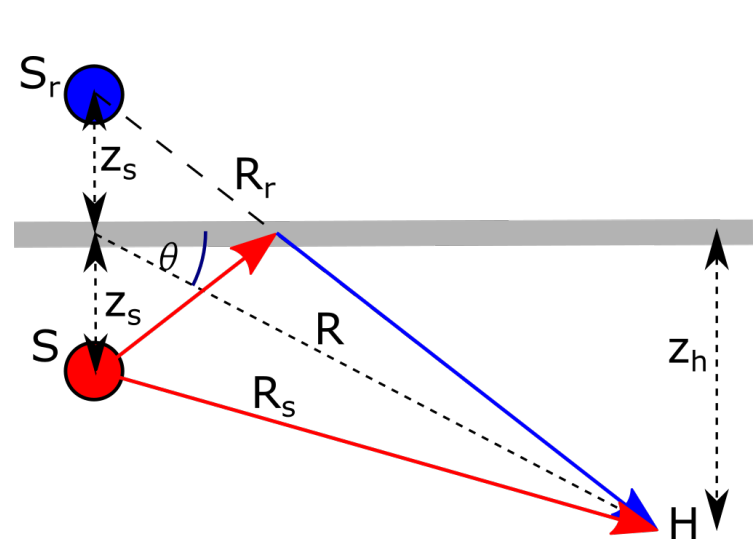

a)

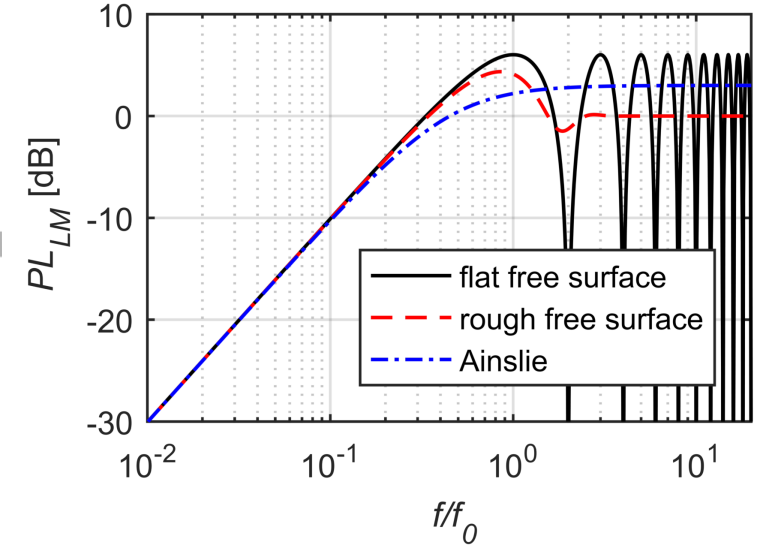

b)

Figure 5.13: Configuration and propagation loss by Lloyd's mirror. Graph a) shows location of a source $(S)$, the corresponding reflecting source $\left(S_{r}\right)$ and a receiving hydrophone $(H)$ with respect to a free surface. Graph b) shows the propagation loss for three models. The non-dimensional height of the rough surface is $\sigma / z_{s}=1 / \pi$.

\subsection{Conclusions}

Analytical formulations for the noise of a vibrating cylinder have been presented and applied to the case of a vortex cavity. At full scale, in the far field, and at low frequencies, the vibration of the vortex cavity over a finite length can be considered as a monopole noise source (Eq. 5.56 and Figure 5.6). At higher frequencies, some directivity can be expected. At low-frequencies, the theoretical results show that the higher order deformation modes of the cavity, i.e. bending and elliptical deformation, are much less efficient in the radiation of noise than the pulsating mode (Eq. 5.55). Therefore, it is expected that the contribution of these higher order modes to the radiated noise can be neglected, and that only the pulsating mode contributes to the farfield noise. These conclusions are also valid for model scale, except at low frequencies and in the near field where the hull-pressures are measured. For this condition, the assumption of spherical spreading loss may not be valid (Figure 5.5), whereas the directivity has not been analysed. However, detailed experimental data on the decay and directivity of the noise due to vibrations or collapse of a vortex cavity is not available, so results cannot be validated. The directivity of the cylindrical noise source is further discussed in Section 6.3.4.

The generation of a broadband hump in the spectrum of a cavitating propeller can well be explained from a signal analysis point of view: A broadband hump appears in the spectrum of a single event, so for a single blade passage, if the signal of that event is oscillatory and of finite extent (Figure 5.7). The frequency of the oscillation determines the centre frequency of the hump while the duration and decay rate of the signal affect the bandwidth of the hump as well as the amplitude. If all blade passages show fully identical pressure signals, the spectrum only consists of tonals at harmonics of the blade passage frequency. A broadband hump arises if variability in amplitude or phase is present between blade passages (Figure 5.8). An existing formulation that describes the effect of separate variation in amplitude and phase on a power density spectrum has been extended such the simultaneous variation of amplitude and phase is taken into account (Eq. 5.91). The formulation is applied to hull-pressure data in Section 6.3.3. 
The broadband hump of cavitating vortices can be non-dimensionalized and scaled with a formulation similar to the scaling of low-frequency underwater radiated noise (Eq. 5.97). Formulations to predict full-scale underwater radiated noise levels from model-scale measured values differ at high frequencies from low frequencies (Eqs. 5.110 and 5.117). The differences are due to the formulation for the acoustic efficiency. The frequency that divides these two ranges is not known and deserves further investigation.

An analytical formulation has been derived to predict the solid boundary factor of the ship hull directly above the propeller (Eq. 5.136). The formulation is useful for a quick assessment but should not be used for hull locations close to the free surface or in regions where the hull curvature changes rapidly.

Two existing formulations for the reflection of sound by the free surface have been presented. One of these models is used in the semi-empirical method for the prediction of the underwater radiated noise that is presented in Chapter 7 (Eq. 5.142). 


\section{Chapter 6}

\section{Mechanisms of vortex-cavitation noise}

This chapter presents the mechanisms that lead to the broadband noise by vortex cavitation, with broadband hull-pressure fluctuations in particular. First, narrowband noise by vortex cavitation is analysed with respect to the criteria for resonance defined in Section 4.3. Next, broadband hull-pressure fluctuations are analysed with respect to variability from one blade passage to the next, as well as the variability of the fluctuations on the ship hull using the formulations presented in Chapter 5. The cavitation patterns and the sources of excitation leading to the collapse and rebound of vortex-cavities are briefly discussed, followed by a review of the main mechanisms involved in broadband hull-pressure fluctuations induced by vortex cavitation.

\subsection{Introduction}

The literature review presented in Chapter 2 indicated that broadband HPF are related to vortex cavitation and to the variability of the hull-pressure signal with blade passages, but that the details of the mechanisms involved have not been investigated. The aim of this chapter is to investigate some mechanisms in more detail and to provide an overview of the main mechanisms making use of the knowledge gained from preceding chapters.

Propeller tip-vortex cavitation is usually associated with broadband noise levels. However, experiments in cavitation tunnels have shown that tip-vortex cavities of hydrofoils in stationary flow can also generate narrowband noise. This narrowband noise suggests the presence of resonance in the dynamics of the vortex cavity. In Section 6.2, the various criteria for resonance that were defined in Section 4.3 are compared to the experimental data on narrowband noise. Such resonance behaviour may then also be expected in the collapse and rebounds of propeller tip-vortex cavities. Propeller tip-vortex cavitation, however, is characterised by a collapse of the cavity that is periodic with blade passage frequency. The effect of this periodic behaviour has already been discussed from a theoretical point of view in Section 5.3. This theory will be applied in Section 6.3 to show how the variability of the hull pressure signal between blade passages in experimental datasets leads to a broadband hump in the spectrum. This section also shows the variability of the pressure on the hull in order to characterise the acoustic source.

A ship propeller may show an isolated tip-vortex cavity but very often this vortex cavity is 


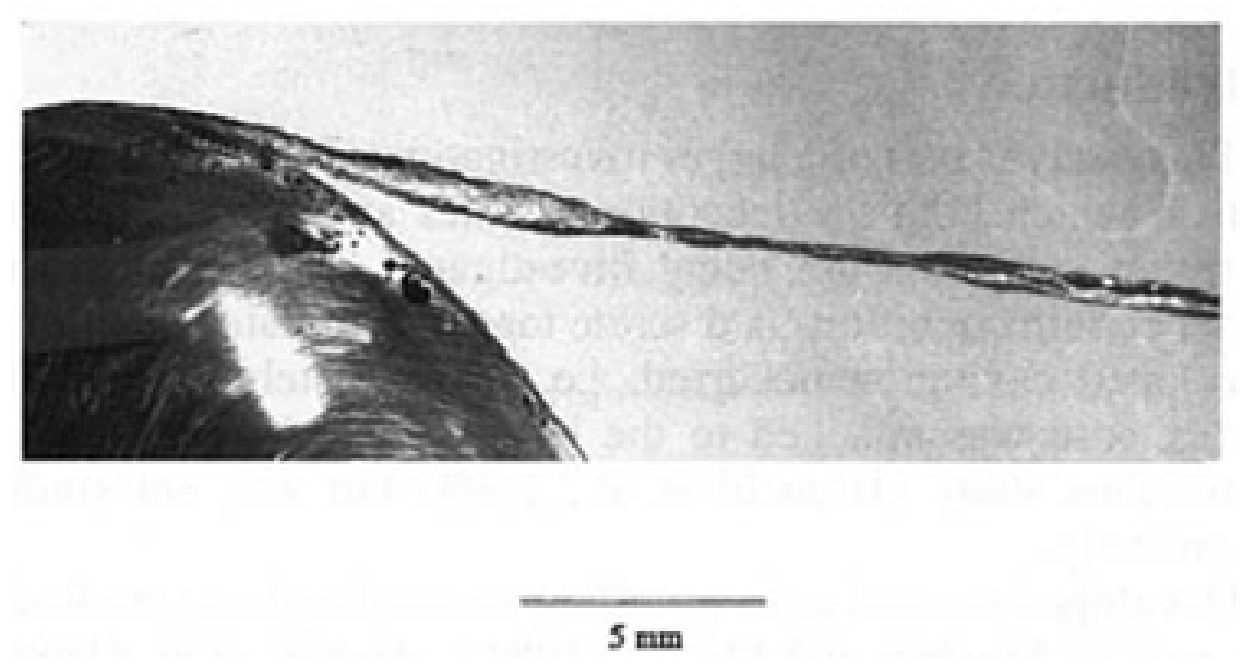

Figure 6.1: Image of the singing vortex (Maines and Arndt, 1997a). Reproduced with permission.

connected to a sheet cavity on the blade. The various cavitation patterns that have been observed on the tip of propeller blades are briefly discussed in Section 6.4. The sources of excitation for the collapses and rebounds of the tip-vortex cavity are discussed in Section 6.5. A review of the mechanisms involved in the broadband noise due to tip-vortex cavitation is provided in Section 6.6.

\subsection{Narrowband noise}

The occurrence of narrowband radiated noise due to a cavitating wing-tip vortex during a cavitation-tunnel test has been reported by Maines and Arndt (1997a) and Briançon-Marjollet and Merle (1996). In both situations, the wing was mounted at constant angle of attack and the vortex cavity did not collapse, see Figure 6.1. Maines and Arndt (1997a) relate the frequency of the narrowband noise levels to the mode $n= \pm 1$ of the dispersion relation as presented by Lord Kelvin (Thomson, 1880). Briançon-Marjollet and Merle (1996) analyse the dispersion relation presented by Morozov (1974) in the limit $k_{z} r_{c} \rightarrow 0$ with $k_{z}$ the axial wavenumber and $r_{c}$ the radius of the vortex cavity. A reasonable agreement is found with the measured frequencies of two narrowband noise levels for the modes $n=1$ and $n=2^{-}$. However, it was shown in Section 5.2.5 that the acoustic efficiency of a mode $n>0$ is much smaller than the efficiency of the mode $n=0$. Hence, in the present study, the criteria defined in Section 4.3.2, which involve the mode $n=0$, are applied to investigate if they can be related to the reported frequencies of the narrowband noise.

The experimental data of Maines and Arndt (1997a) is investigated first, as their results have been described in great detail. The test-case was first reported by Higuchi et al. (1989). Different hydrofoils of elliptical planform were tested in two different cavitation tunnels, one in Obernach, Germany and one at St. Anthony Falls Laboratory (SAFHL), Minneapolis, USA. In both tunnels, a very distinct frequency component was present in the radiated noise for a small range of cavitation numbers. The frequency component could be related to the cavitating vortex 


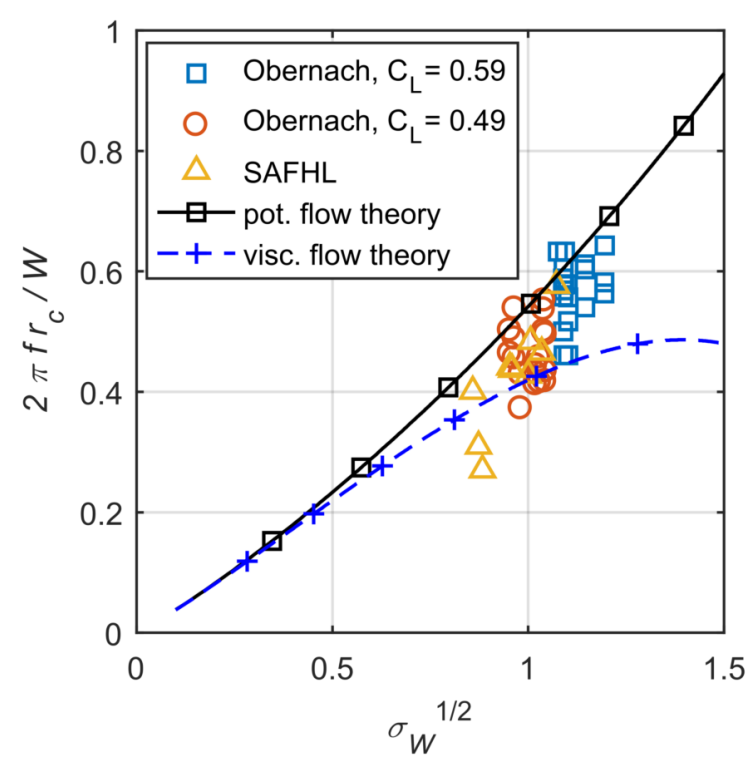

a) Prediction for zero group velocity (ZGV) of mode $n=0^{-}$.

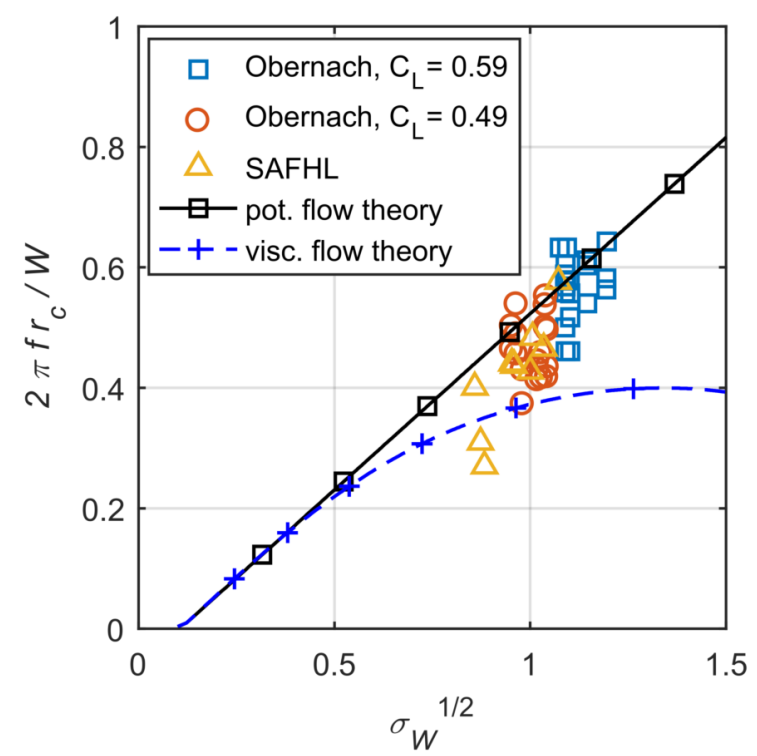

c) Prediction for crossing dispersion lines (CDL) of mode $n=0^{-}$ and $n=-2^{+}$.

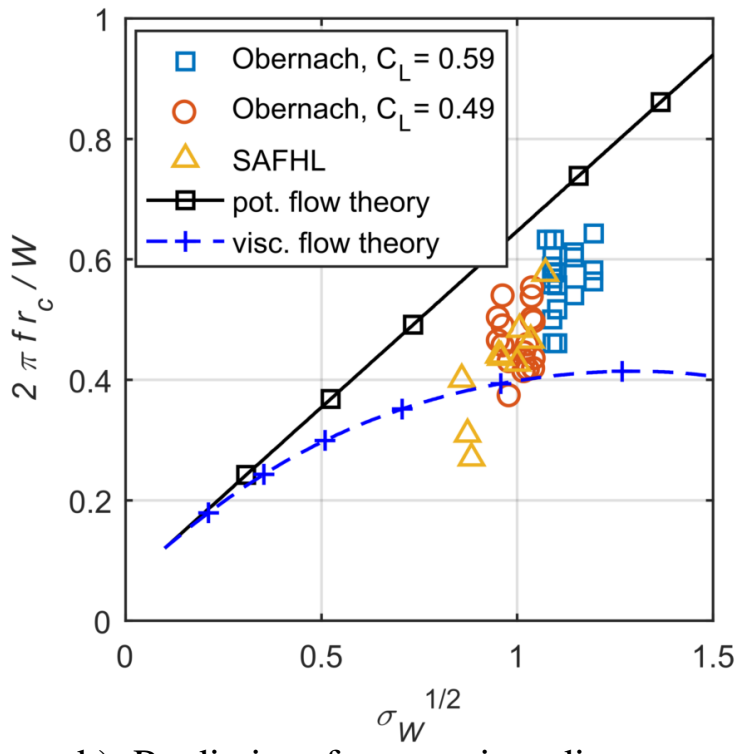

b) Prediction for crossing dispersion lines (CDL) of mode $n=0^{+}$ and $n=2^{-}$.

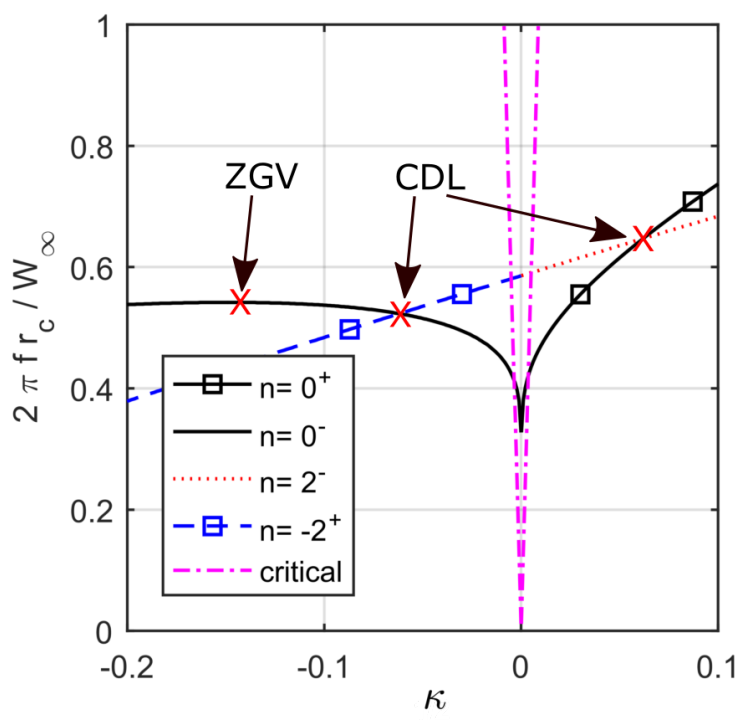

d) Dispersion diagram including critical frequency for $\sigma_{W}=1.0$, potential flow vortex.

Figure 6.2: Comparison between measured frequencies of narrowband noise levels (Maines and Arndt, 1997a) and predictions using different criteria for resonance.

through high-speed video. This 'singing' only occurred when the cavitating vortex was attached to the blade surface. The frequency was found to vary between $400 \mathrm{~Hz}$ and $1.1 \mathrm{kHz}$, depending on tunnel velocity and lift coefficient and, for the Obernach facility, on the air content of the water. Two different wave patterns were observed on the cavitating vortex, one wave showing stationary nodes, while the other wave oscillates in the axial direction. 
More recently, one of these foil geometries was tested in the cavitation tunnel of Delft University of Technology by Pennings (2016). Some of his results have already been presented in Section 3.3.2 and 4.3.3. The 'singing' of the vortex did not occur in this facility, but a narrowband peak was detected in the spectrum of the variation of the cavity diameter at a small distance from the tip. The results obtained in the cavitation tunnel of Delft University of Technology with respect to cavity dynamics have been reported by Arndt et al. (2015) and Pennings et al. (2015a). The same foil has also been tested in the small cavitation tunnel of CSSRC, Peng et al. (2017). They report the singing as a transient phenomenon that happened when the vortex cavity was attached to a sheet cavity on the foil. When the singing disappeared, the sheet cavity on the tip of the foil also disappeared. Peng et al. (2017) also show that, for identical cavitation number and angle of attack, the cavity radius decreased with increasing tunnel speed and that the non-dimensional frequency $2 \pi f r_{c} / W$ decreases with increasing tunnel speed. Results for the same foil and cavitation tunnel are also discussed by Song et al. (2018). They show that the air content has a significant effect on the cavitation number for which singing occurs, especially at low tunnel speeds. Their non-dimensional frequency, which has half the value of Peng et al. (2017) since their cavity radius is half the value, is in good agreement with the peak in the spectrum of the cavity diameter variation of Pennings et al. (2015a), and, on average, slightly smaller than the data of Maines and Arndt (1997a).

The values for the resonance frequencies reported by Maines and Arndt (1997a) are presented in Figure 6.2. Results are also presented for various criteria derived from the dispersion diagram as discussed in Section 4.3.2. These criteria are the zero group velocity for mode $n=0^{-}$, the crossing of the dispersion line for mode $n=0^{+}$and that of $n=2^{-}$and the crossing of the dispersion line for mode $n=0^{-}$and that of $n=-2^{+}$. The criteria are computed using Eq. (4.77) for a potential flow vortex and for a viscous flow vortex, both neglecting the contribution of surface tension. The formulation for the viscous flow vortex uses Eq. (4.71) for the, viscous-flow-corrected, stiffness coefficient. The ratio between cavity radius, $r_{c}$, and viscous core radius, $r_{v}$, is computed by making use of the relation between $r_{c} / r_{v}$ and $\sigma / \sigma_{i}$ for the cavitating Lamb-Oseen vortex, discussed in Section 3.2.2. The cavitation number at inception was taken as $\sigma_{i}=4$ as reported by Higuchi et al. (1989). Even though the cavitating LambOseen vortex does not give an accurate representation of the radial distribution of the azimuthal velocity, it does give a realistic trend for the effect of viscosity.

The results presented in Figure 6.2 indicate that, given the uncertainty of the predictions, all tested criteria are plausible. However, Maines and Arndt (1997a) and Peng et al. (2017) state that singing only occurred when the cavity was attached to the blade and the photograph in Figure 6.1 shows an elliptical shape of the cavity. This suggests that either a mode $n=2$ is involved or that there is an interaction between sheet cavity and vortex cavity. Mutual interaction between the sheet cavity and the vortex cavity would occur if a negative phase velocity is present. Such a negative phase velocity occurs for the zero group velocity condition and for the intersection of modes $n=0^{-}$and $n=-2^{+}$. The frequencies resulting from these two criteria are very close to each other, so it is also possible that both criteria are required.

Figure $6.2 \mathrm{~d}$ presents a zoom of the dispersion relation showing the locations of the three criteria for resonance at $\sigma_{W}=1.0$. The figure also shows the line for the critical frequency at which the axial phase velocity equals the speed of sound. The presented line has been generated for a tunnelspeed of $W=13 \mathrm{~m} / \mathrm{s}$ and a speed of sound $c=1500 \mathrm{~m} / \mathrm{s}$. In Section 5.2.5, it was shown that vibrations on a cylinder with absolute value of wavenumbers smaller than the 
critical wavenumber lead to noise radiation, whereas larger wavenumbers lead to hydrodynamic pressures, i.e. an evanescent wave is present instead of a propagating wave. The graph shows that the absolute value of the wavenumbers corresponding to the three criteria are all larger than the critical wavenumber. This suggests that, in the far field, these criteria do not correspond to efficient noise sources. Note that the frequency of mode $n=0$ approaches zero as the axial wave number approaches zero. Nevertheless, it is very well possible that, due to either viscous effects or to the finite length of the vortex cavity, the frequency of mode $n=0$ at $\kappa=0$ differs from the value predicted by potential flow vortex and that this location is the criterion for resonance. However, Sections 5.2.4 and 5.2.5 have also shown that if the deformation of the cavity only extends over a finite extent of the cavity and the spatial average is different from zero, the wavenumber spectrum has a contribution at wavenumber zero. This spatial-averaged amplitude is responsible for the radiated noise. Such deformations are not described by the expression used for the prediction. Therefore, the deformations of the cavity observed in the experiment need to be investigated in more detail in order to investigate the criterion for resonance.

It is remarkable that the singing vortex was only observed for a small range of cavitation numbers. In Section 3.3.2 it was shown that there is a cavitation number for which the frequency (and absolute value of wave number) of the zero group velocity condition can coincide with the frequency (and wave number) of the intersection of modes $n=0$ and $n=2$. If lock-in occurs, a small range of cavitation numbers will exist. An alternative explanation is that the singing requires a closed sheet cavity on the tip that is connected to the tip-vortex cavity. When the cavitation number is too high, sheet cavitation is not present, and when the cavitation number is too low, the structure of the sheet cavity changes from a closed structure to an open structure.

Another dataset in which narrowband noise was present was reported by Briançon-Marjollet and Merle (1996). They describe observations and measurements on tip vortex cavitation as occurring at the tip of a wing of elliptical plan form and NACA 0020 cross-section. The measurements were made in the large cavitation tunnel GTH. The noise spectrum was characterised by two distinct narrowband peaks with a factor three difference in frequency. Unfortunately, dimensionless frequencies are not directly given but for some conditions it could be determined from the data that was given. The smallest non-dimensional frequency of the two peaks for two conditions correspond to $2 \pi f r_{c} / W=1.13$ and 1.26 for $\sigma_{W}=3$ and 5 , respectively. These cavitation numbers are outside the range presented in Figure 6.2, but these numbers are in reasonable agreement with the analytical prediction for the potential flow vortex presented in Figure $4.13 \mathrm{c}$. The highest frequency of the two peaks can not be explained by the presented criteria.

Sponagle (1990) also identified two peaks in the noise spectrum of a propeller operating in bollard-pull condition of which the cavitation was dominated by tip-vortex cavitation. The measurements were made using a floating laboratory located in a part of a harbour. No estimates of the cavity radius are given, so the non-dimensional frequencies can not be determined. The ratio of the frequencies of the two peaks is 1.4. Figure 6.3 presents two ratios of criteria for resonance of the cavity deformation. The line for both ratios includes a value 1.4 , so the results presented by Sponagle could be explained by both criteria.

Criteria for resonance obtained from a dispersion relation have been obtained in other fields of application. The criterion of zero group velocity was identified by Tolstoy and Usdin (1957) in the theoretical analysis of the dispersion relation of Lamb waves in thin-walled structures 


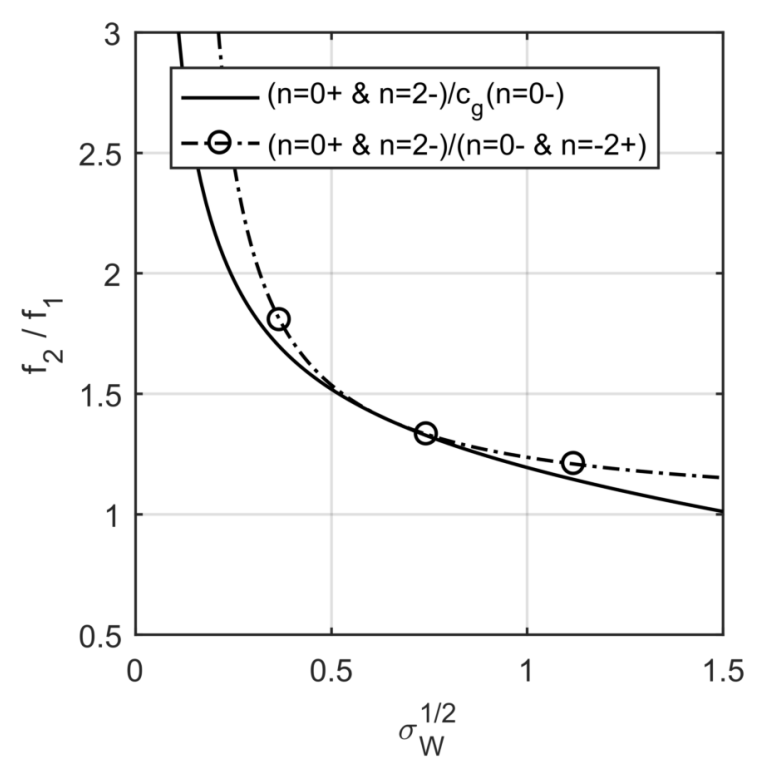

a) Potential flow vortex

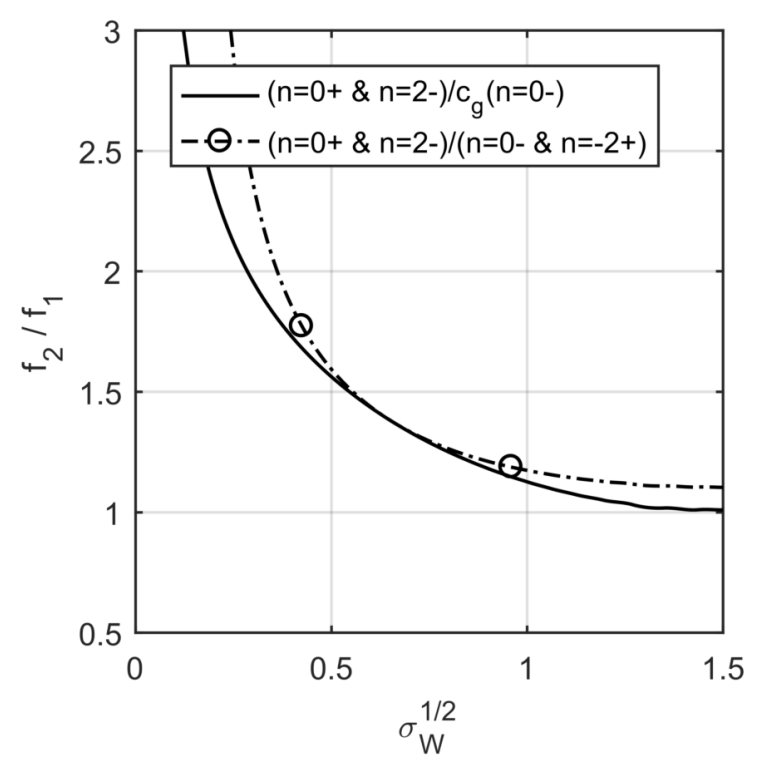

b) Viscous flow vortex, $\sigma_{i}=4$

Figure 6.3: Ratio of frequencies of mode $n=0^{+}$and $n=0^{-}$by combining two criteria for resonance.

of pipes ${ }^{1}$. Ultrasonic excitation of a thin-walled structure has shown a maximum response at the frequency corresponding to the zero group velocity condition (Holland and Chimenti, 2003; Prada et al., 2008; Tofeldt and Ryden, 2017). The ultrasonic excitation is applied for non-destructive local testing of the elastic properties and thickness of elastic plates.

In Section 2.2, the elliptic instability of Kelvin waves on the edge of the viscous core of noncavitating vortices was discussed. This elliptic instability occurs for a vortex in a strain field and is associated with the intersection of two modes of which the azimuthal wave number differs by two. For the tip-vortex cavity tested in a cavitation tunnel, such a strain field can be generated by the virtual image of the vortex in the walls of the tunnel. For a propeller, the strain field can be present in the ship wake or can be caused by the tip-vortices from the other blades. Lacaze et al. (2007) have shown that the combination of the modes $n=-2$ and $n=0$, describing deformations of the viscous core of a Batchelor vortex, becomes progressively unstable for increasing axial flow. Therefore, it is recommended to perform a similar analysis for a cavitating vortex to further investigate the relevance of the crossing dispersion lines. Another possibility is that there is an interaction between the deformations of the cavity interface and the deformations of the radius of the viscous core. This requires an analysis of the dispersion relation in viscous flow.

Finally, it is recalled that the goal of the investigation was to derive a criterion for the resonance frequency of a tip-vortex cavity. As will be shown in Section 6.5, such a resonance also occurs when the vortex cavity has a cylindrical shape. This leaves the criterion of the zero group velocity as the most likely hypothesis for resonance of the vortex cavity.

\footnotetext{
${ }^{1} \mathrm{~A}$ Lamb wave is an elastic wave in a solid plate a few wavelengths thick. The waves propagate parallel to the surface of the plate throughout its thickness with the particles moving in an elliptical orbit, www.nde-ed.org/ EducationResources/CommunityCollege/Ultrasonics/Physics/modepropagation.htm, visited June 20, 2018.
} 


\subsection{Broadband hull-pressure data}

\subsubsection{Background}

This section analyses the time traces of hull-pressure signals and the corresponding spectra in more detail.

Broadband HPF are usually presented by their power spectral density or by their power spectrum. However, the interpretation of such a spectrum requires information on the transient behaviour and on the variability of the signal between subsequent blade passages, as shown in Section 5.3. Information on the transient behaviour can be obtained from time-frequency analysis using, for instance, wavelets (Flandrin, 1999). In Section 6.3.2 the wavelet analysis will be applied for a time trace synchronised with high-speed video. The variability of the signal between subsequent blade passages has been investigated using two different methods. The first method considers the maximum peak-to-peak value in the time trace for each blade passage and the second method analyses the variability of amplitude and phase angle of the spectrum determined for each shaft revolution. The information on variability can be used to find the relation between the mean of the spectrum computed for each shaft revolution separately and the spectrum computed for all shaft revolutions. Section 6.3.3 presents the results of this study. The variability of the pressure amplitude and phase angle over the hull surface is shown in Section 6.3.4. Such an analysis gives an indication if the collapse of the vortex cavity behaves as a monopole or as cylindrical noise source.

The majority of the data presented in this section is for a two-bladed research propeller that was tested in the wake of a twin-screw ferry in an internal research project of MARIN. The propeller has diameter $D=0.233 \mathrm{~m}$ and is tip-unloaded. The pitch at $0.7 R$ was $P_{0.7} / D=1.2$ and the propeller was rotating inward over the top with the hull-pressure signals measured on the port side. The tests were performed in the Depressurized Wave Basin (DWB) at various advance ratios (around $J=1$ ) and various cavitation numbers with model speeds between 2.7 and $3.1 \mathrm{~m} / \mathrm{s}$. A photograph of the propeller and a contour plot of the ship wake are presented in Figure 6.4. The wake field shows in the upper part a velocity deficit caused by a vortex generated at the hull bossing where the shaft enters the ship hull.

The two-bladed propeller has the advantage that the view by a high-speed video camera on the vortex cavity is less obstructed by the following blade. Furthermore, the time trace due to the collapse and rebounds of the vortex cavity is also less affected by the arrival of the next blade and the growth of cavitation on that blade. The approach was copied from the CRS PRESS working group. However, it should be noted that the non-dimensional frequency, $f / f_{b p}$, of the broadband hump is higher than found for a typical ship propeller, because the frequency content of the cavity collapse is independent of blade number.

Another ship that will be considered in the present and following chapters is the DAMEN Combi Freighter, an $85 \mathrm{~m}$ single-screw general purpose cargo ship. The ship is equipped with a four-bladed controllable-pitch propeller (CPP) by Caterpillar Propulsion. The model has a length of about $7 \mathrm{~m}$ and was manufactured at a scale factor of about 1:12. Photographs of the model and propeller are presented in Figure 6.5. The sea trials for this vessel were performed within the CRS BROADBAND working group. The model tests were partly funded by the Top Consortium for Knowledge and Innovation (TKI) of the Dutch Ministry of Economic Affairs. 

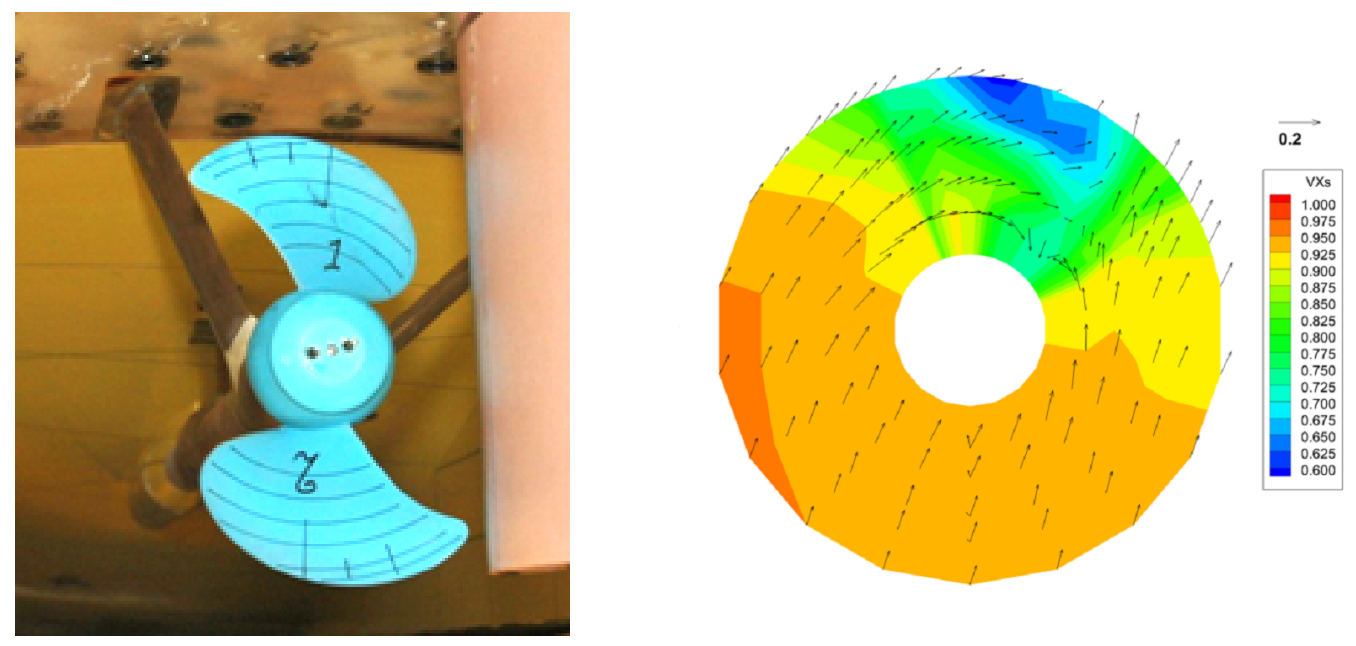

Figure 6.4: Photograph of two-bladed research propeller and contour plot of wake of twin-screw vessel.

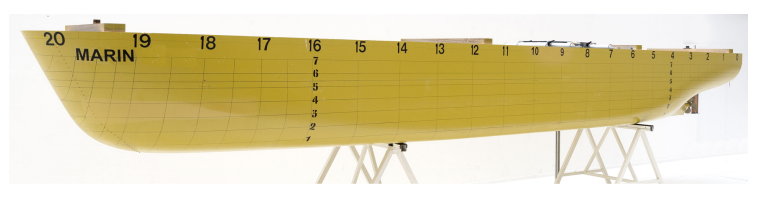

a) Ship hull

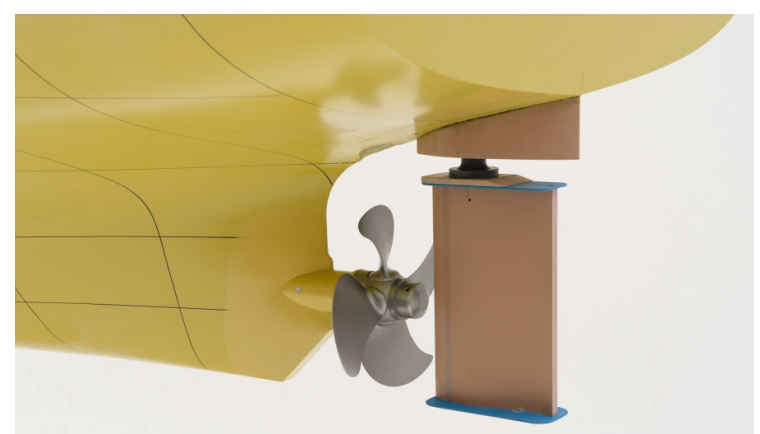

b) Aft ship and propeller

Figure 6.5: Photographs of ship hull and propeller of Combi Freighter.

Each of the following sections shows only one or two examples to illustrate a particular aspect and the data-analysis procedure used. As shown in Figure 1.2, various cavitation patterns can occur on ship propellers depending on ship and propeller geometry as well as on operating condition. The time traces and spectra due to the collapse of other cavities can differ from the examples shown.

\subsubsection{Hull-pressure data synchronized with high-speed video}

The variation of the pressure signal due to the collapse of a vortex cavity is investigated for the two-bladed skewed propeller operating in the wake field of a twin-screw vessel as described in Section 6.3.1 and shown in Figure 6.4. The pressure signal is for the transducer on the hull directly above the centre of the propeller.

Four high-speed video images showing the character of the vortex cavity are presented in Figure 6.6. The cavity pattern can be described as a small sheet with a side-entrant jet oriented parallel to the leading edge forming a cavity closure vortex. Such a cavity pattern is discussed in more detail in Section 6.4. The collapse of the cavity pattern behaves as a vortex cavity and 


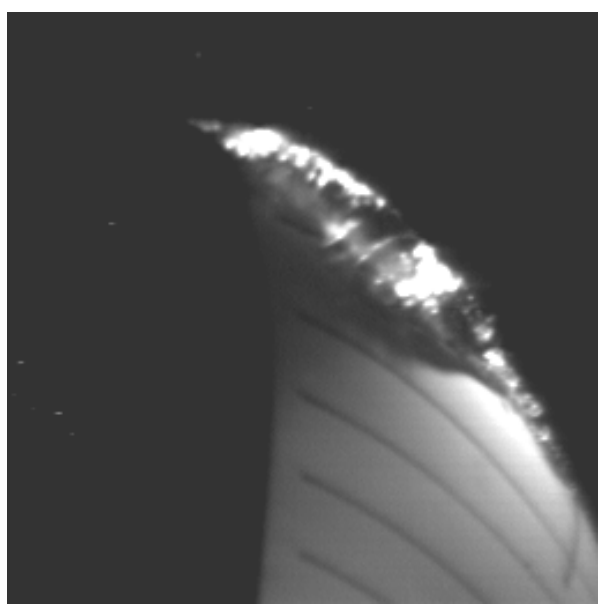

a) 54.5 deg blade position

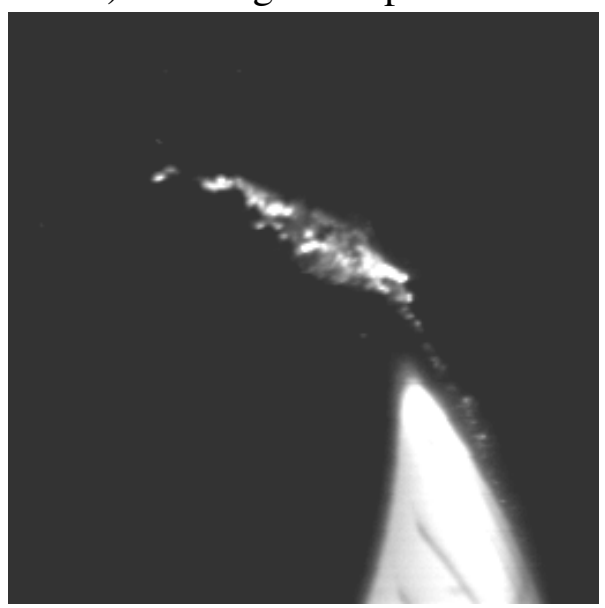

c) 85.7 deg blade position

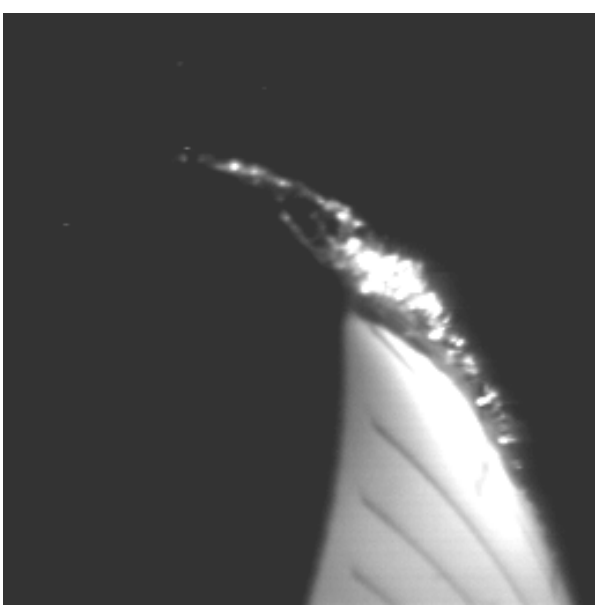

b) 71 deg blade position

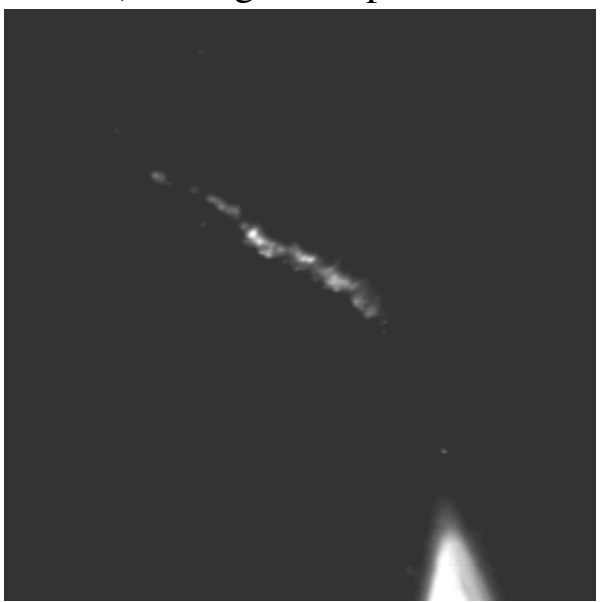

d) 100.5 deg blade position

Figure 6.6: High-speed video images of collapse of vortex cavity. Image (a) though (d) correspond to the annotations in the time trace in Figure 6.7.

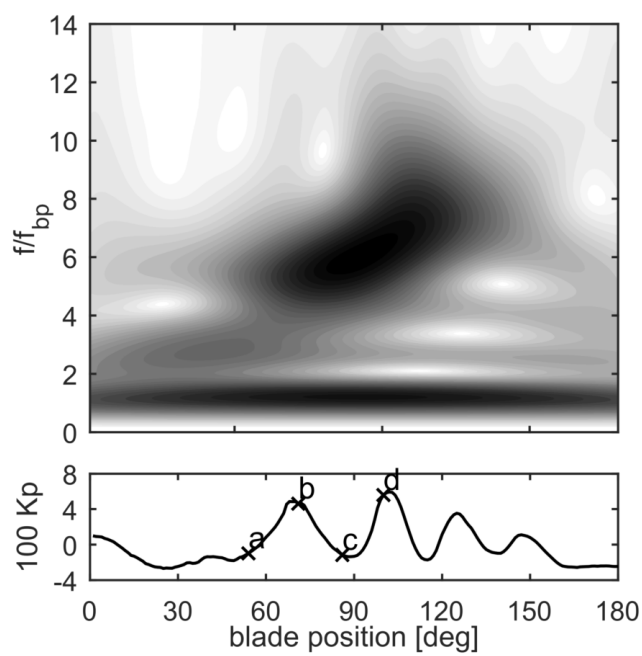

a) Time-frequency analysis

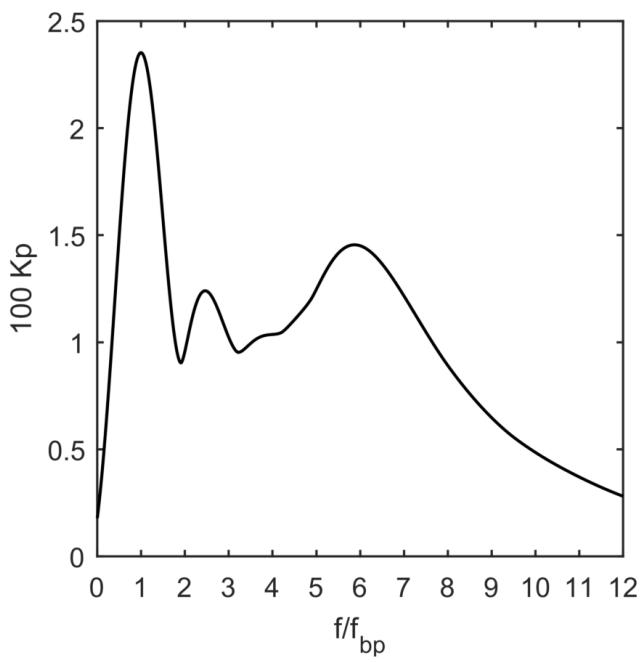

b) Blade-position-averaged spectrum

Figure 6.7: Wavelet analysis of hull pressure signal by collapse of vortex cavity. Positions (a) though $(d)$ correspond to images presented in Figure 6.6. Time is shown as blade position. 
is denoted a closure-vortex cavity. In addition to this closure-vortex cavity, also a tip-vortex cavity is present that can be observed in image (b). The rebound of the closure-vortex cavity is observed in image (c) as well as in image (d). The blade position is defined such that at $0 \mathrm{deg}$, the blade reference line is located at the 12 o'clock position as in Figure 6.4a.

The frequency content of the time trace of the hull-pressure signal is analysed using wavelets (Auger et al., 1996). Use is made of a Morlet (or Gabor) wavelet and results are presented as a scalogram with the scale of the wavelet converted to frequency. A scalogram gives the square of the magnitude of a continuous wavelet transform.

The time trace of the hull-pressure signal for a single blade passage and the resulting wavelet analysis are shown in Figure 6.7a. The presented time trace shows multiple rebounds of the vortex cavity and the maximum amplitude (taken as half of the peak-to-peak amplitude) equals $k_{p}=0.037$. Positions a) through d) correspond to the high-speed video images presented in Figure 6.6. The time-frequency diagram shows that the frequency of the pressure signal due to the collapse increases with time. At the first collapse, after the 60 deg blade position, the frequency is approximately 5.5 times the blade passage frequency and, in the rebounds, it increases to approximately 8 times the blade passage frequency at $120 \mathrm{deg}$ blade position. The blade-position-averaged spectrum of the wavelet transform is presented in Figure 6.7b. The spectrum shows a broadband hump centred at a frequency of six times the blade passage frequency.

\subsubsection{Variability between blade passages}

The variability of the measured hull-pressure signal between subsequent blade passages has been investigated using two different methods. First, the variability of the maximum peakto-peak amplitude and period has been investigated for a data set of the two-bladed research propeller. Next, the effect of variation in amplitude and phase between subsequent blade passages is investigated using the analytical formulation derived in Section 5.3.3. This analysis was performed for the two-bladed propeller as well as for a full-scale dataset.

The variability of the peak-to-peak amplitude, defined by a maximum in pressure and the nearest local minimum, was investigated by determining for each blade passage the maximum amplitude and corresponding time period. Figure 6.8 presents the data as a probability distribution. The probability distribution of the amplitude is well fitted by a lognormal distribution, but the distribution of the period could not be fitted satisfactory. An inverse lognormal distribution shows a reasonable fit, but, at large and small values for the period, this distribution does not have the correct behaviour. The simple analysis procedure has its flaws as the use of neighbouring extrema gives a bias to high frequency variations. In that respect, the procedure can be improved by analysing all extrema within a single blade passage. Nevertheless, the results clearly show the large variability of the cavity collapse between blade passages.

Broadband HPF data is usually presented as a spectrum as it can easily be computed and the variation with frequency is condensed into a single graph. The consequence on the spectrum of the variability of the pressure signal between blade passages is discussed next using the analytical formulation presented in Section 5.3.3. This formulation requires information on the mean and standard deviation of the amplitude, denoted by $\bar{a}(k)$ and $\sigma_{a}(k)$, respectively and the 


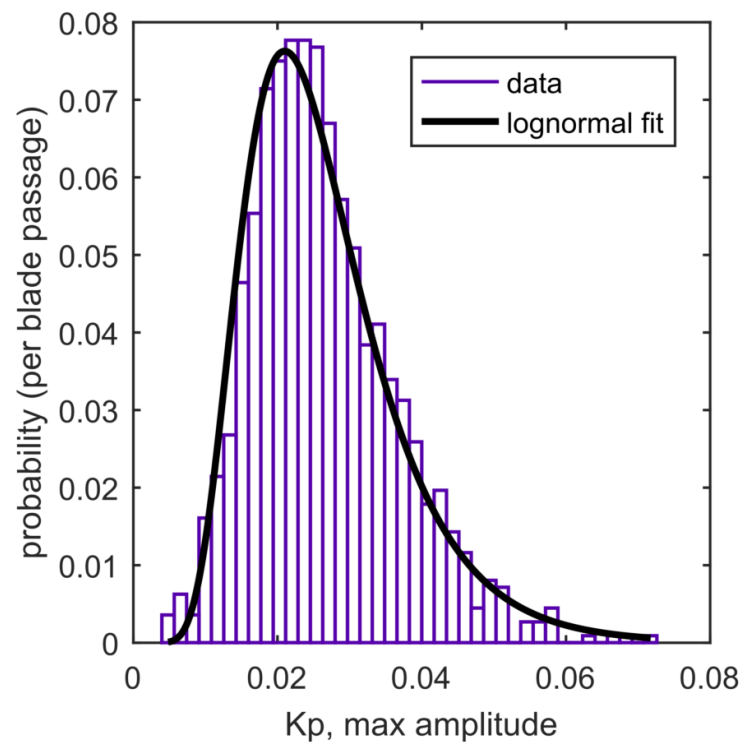

a) Amplitude

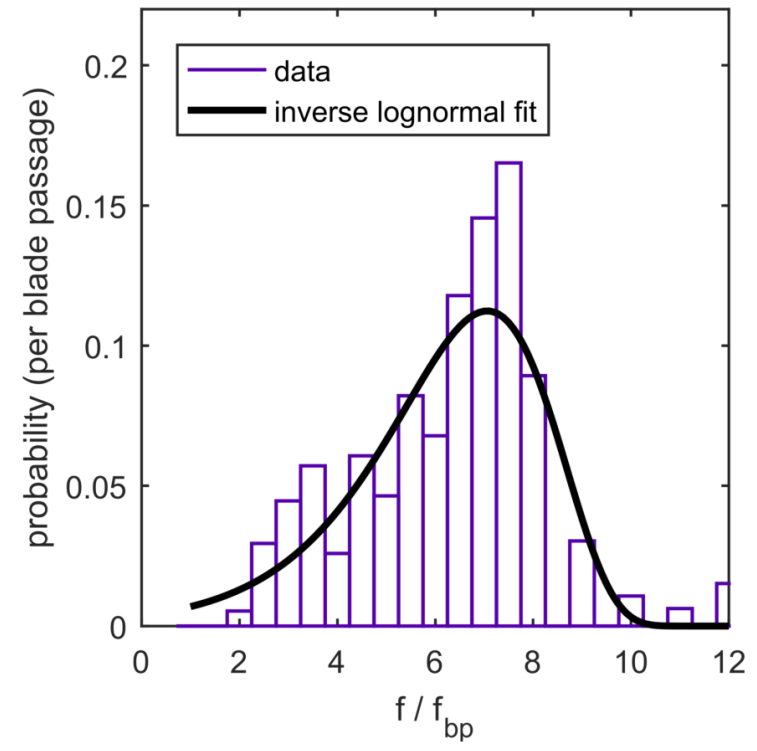

b) Frequency

Figure 6.8: Variability of maximum amplitude and corresponding time difference between neighbouring pressure extrema for each blade passage in the time trace of hull pressure of two-bladed propeller. The corresponding spectrum is presented in Figure 6.7.

standard deviation of the phase, denoted by $\sigma_{\theta}(k)$. Parameter $k$ is the harmonic of the shaft rate frequency.

The hull-pressure signal was analysed by first dividing the time trace into 'shaft revolutions' with a shaft revolution defined by a constant number of samples that does not change in time. This number of samples, that is representative of the mean revolution rate of the shaft, was determined from a colour-coded plot of the time trace with blade position and shaft revolution. The number of samples was defined as a real number if the data was time synchronized instead of shaft synchronized. Each shaft revolution was analysed with an FFT which provided the amplitude and phase for each harmonic of the shaft-rate frequency. The data of all shaft revolutions was then used to compute the mean and standard deviation. The value for $\sigma_{\theta}$ was computed using the theory of circular statistics (Fisher, 1993),

$$
\sigma_{\theta}=\sqrt{-2 \ln \rho}
$$

with $\rho$ corresponding to the length of the mean resultant. The mean resultant $\rho$ was computed from the variation of phase angle $\theta_{n}$ with revolution $n$ by

$$
\rho=\sqrt{\left(\frac{1}{N} \sum_{n=1}^{N} \cos \theta_{n}\right)^{2}+\left(\frac{1}{N} \sum_{n=1}^{N} \sin \theta_{n}\right)^{2}},
$$

with $N$ corresponding to the total number of shaft revolutions.

In sea trials, the HPF data is not always shaft-synchronised. Any variation in shaft rpm is then interpreted as a variation in blade position or in arrival time of the collapse. As discussed 


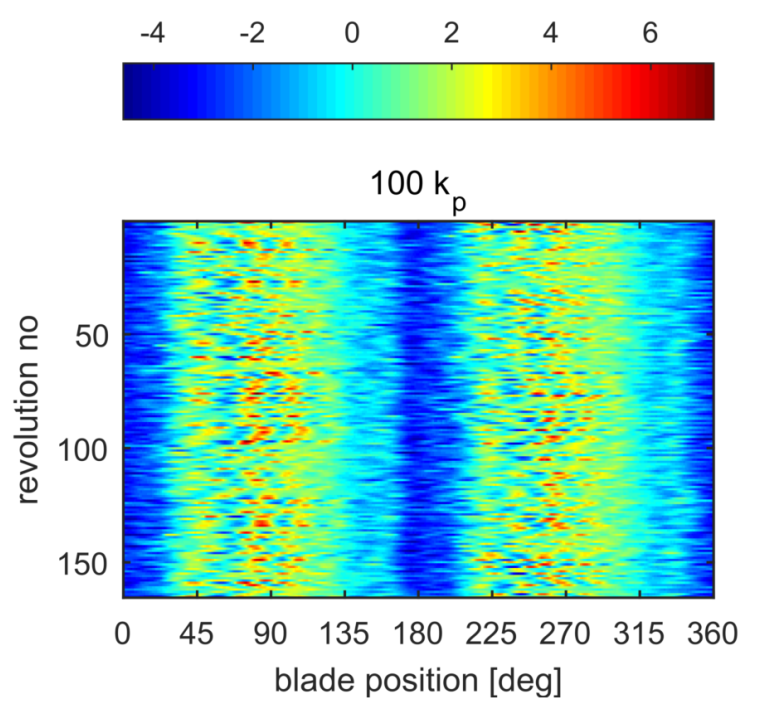

a) Model scale, $Z=2$

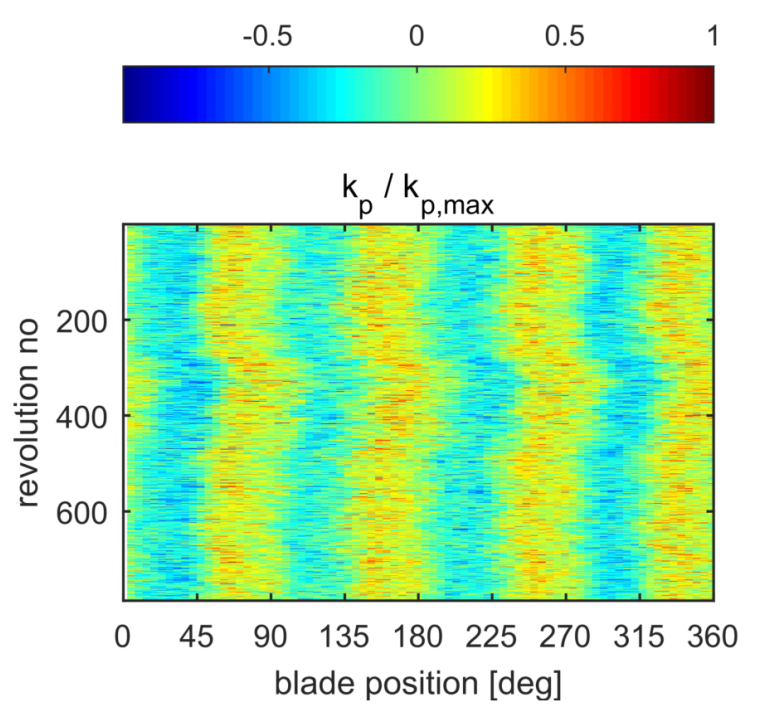

b) Full scale, $Z=4$

Figure 6.9: Time traces of pressure pulse presented as function of blade position and revolution.

in Section 5.3.3, this variation in arrival time results in a variation in phase. The data measured in model tests is usually shaft-synchronised, so any variation in shaft rpm has no effect on the variation in phase. Colour-coded contour plots of the division of the time trace in shaft revolutions and blade positions are presented in Figure 6.9 for the two-bladed propeller tested at model scale and for a data set obtained from full-scale tests.

The results of this spectral analysis for the two-bladed propeller are presented in Figure 6.10. A normalized amplitude spectrum as well as a normalized power density spectrum is presented. The line indicated with 'Ref' is the reference spectrum that is obtained from a standard spectrum analysis procedure using the whole time trace, the line indicated with 'Ampl' is the spectrum that is obtained using only $\bar{a}$ and $\sigma_{a}$, and the line indicated with 'Ampl \& Phase' is the spectrum that is obtained by correcting 'Ampl' for $\sigma_{\theta}$. The variation of $\sigma_{a}(k)$ is presented in Figure 6.10c, and the variation of $\sigma_{\theta}(k)$ is presented in Figure 6.10d.

In the analysis and presentation of results, a distinction was made between values at harmonics of the blade passage frequency, referred to as $h$ - $b p f$, and values at harmonics of the shaft rate frequency in between harmonics of the blade passage frequency, referred to here as $h$-srf. The values for $\sigma_{a}$ and $\sigma_{\theta}$ at h-srf do not change much with frequency and are much higher than the values at h-bpf. Due to the high value of $\sigma_{\theta}$ at h-srf, the spectrum of 'Ampl \& Phase' does not show tonal components at h-srf. To simplify the graphs of the spectra, the values for the mean of 'Ampl' are not shown at h-srf. The variation of $\sigma_{\theta}$ was fitted by a straight line using the values for all h-bpf only. The slope of this line gives the standard deviation in time of arrival by which the 'Ampl' spectrum is converted to the 'Ampl \& Phase' spectrum.

The value for $\sigma_{a}$ at h-bpf in Figure 6.10c is very low at the first bpf and increases with frequency until the fourth or fifth bpf after which some oscillation is observed. The first few harmonics of bpf show a small ratio of $\sigma_{a}$ and $\bar{a}$ because the amplitudes at these tonals are due to the thickness and loading of the blade. Thickness and loading do not vary much between blade passages and, therefore, the resulting pressure signal also does not vary much. For most ship 


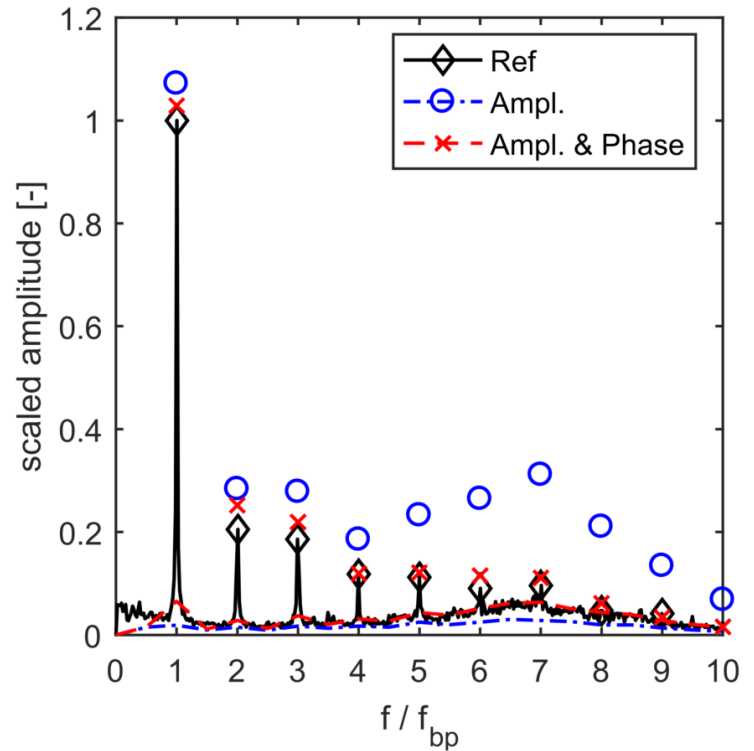

a) Amplitude spectrum

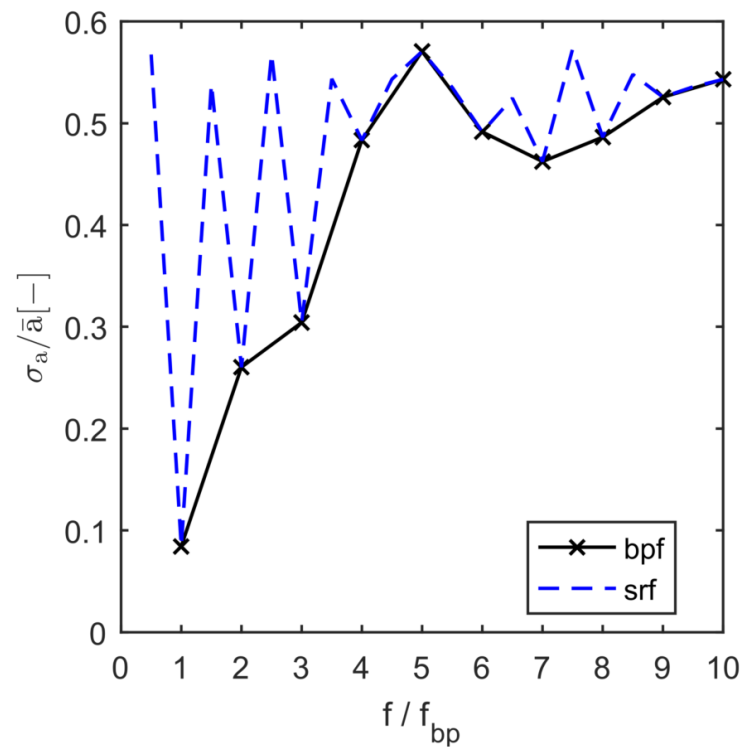

c) Ratio of standard deviation and mean of amplitude

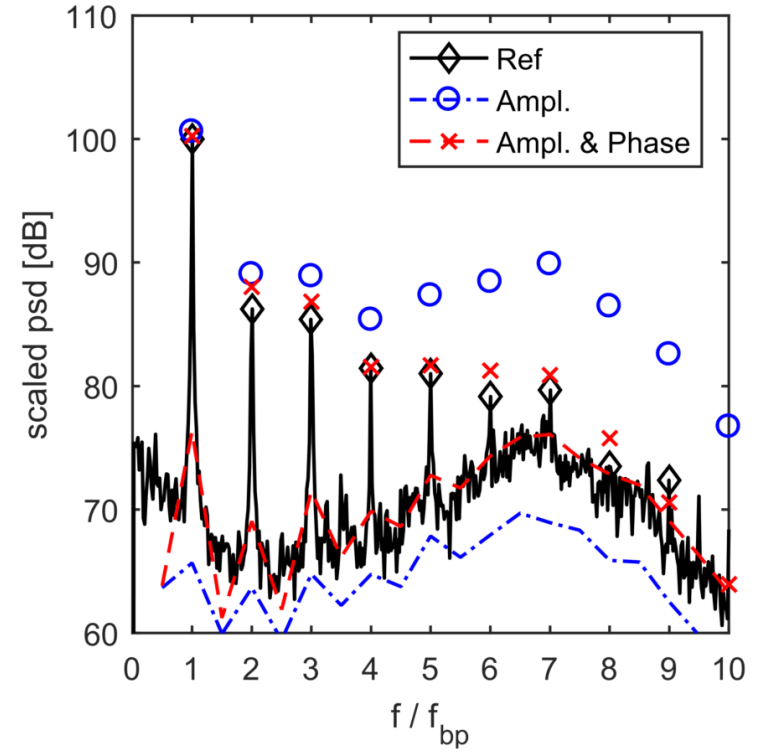

b) Power density spectrum

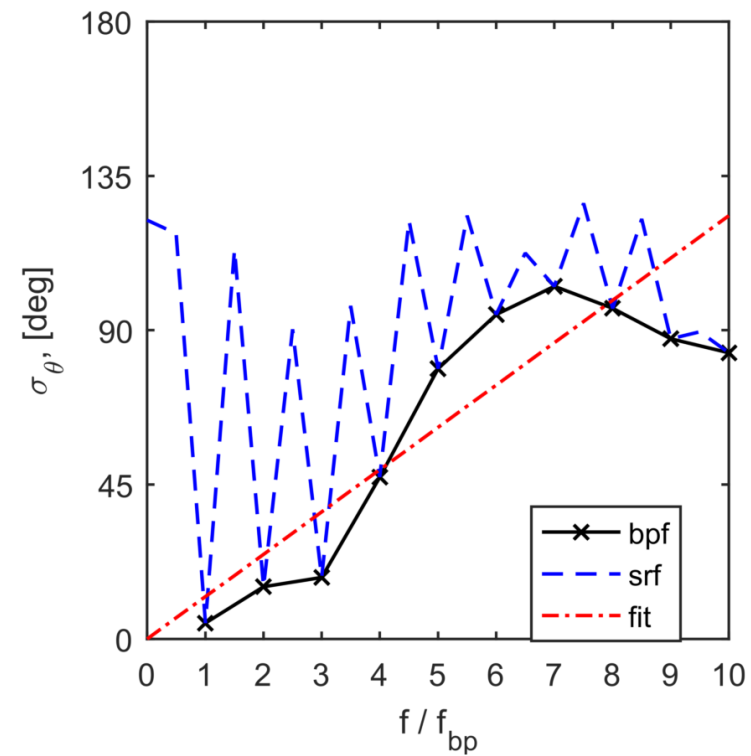

d) Standard deviation of phase

Figure 6.10: Analysis of HPF data of amplitude and phase variation between blade passages for two-bladed propeller, model-test data. Time trace shown in Figure 6.9a.

propellers, thickness and loading only contribute to the tonal at the bpf, but for a two-bladed propeller the contribution extends to higher harmonics as well.

The 'Ampl \& Phase' spectrum shows good agreement with the 'Ref' spectrum demonstrating the validity of Eq. (5.91). Therefore, it proves that the broadband part in the spectrum is caused by the variability of the pressure signal between blade passages. In comparison to the 'Ampl' spectrum, the 'Ref' spectrum shows smaller amplitudes at the higher harmonics of the bpf, which correspond to a factor three at the seventh bpf and it shows a six dB higher level of the broadband part. 


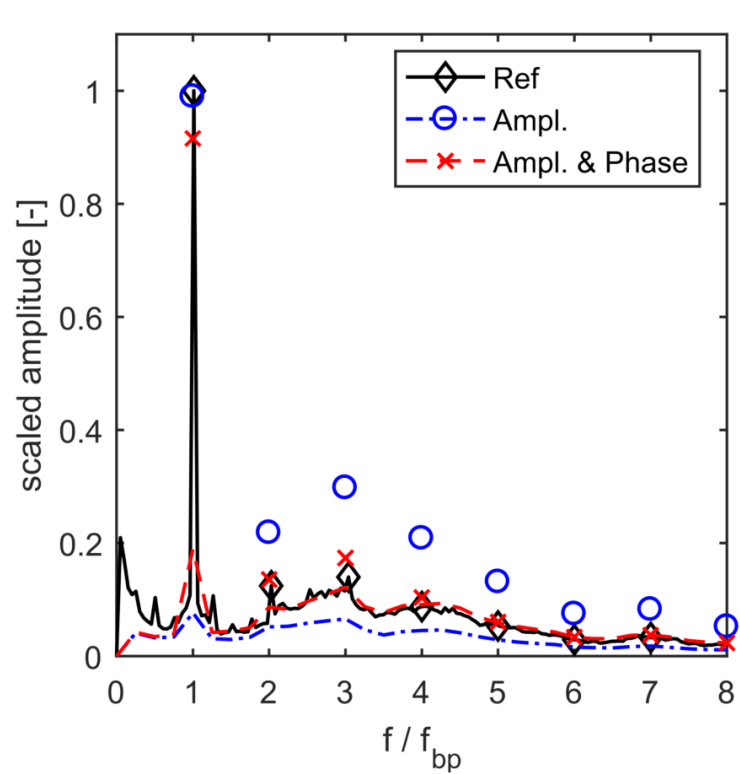

a) Amplitude spectrum

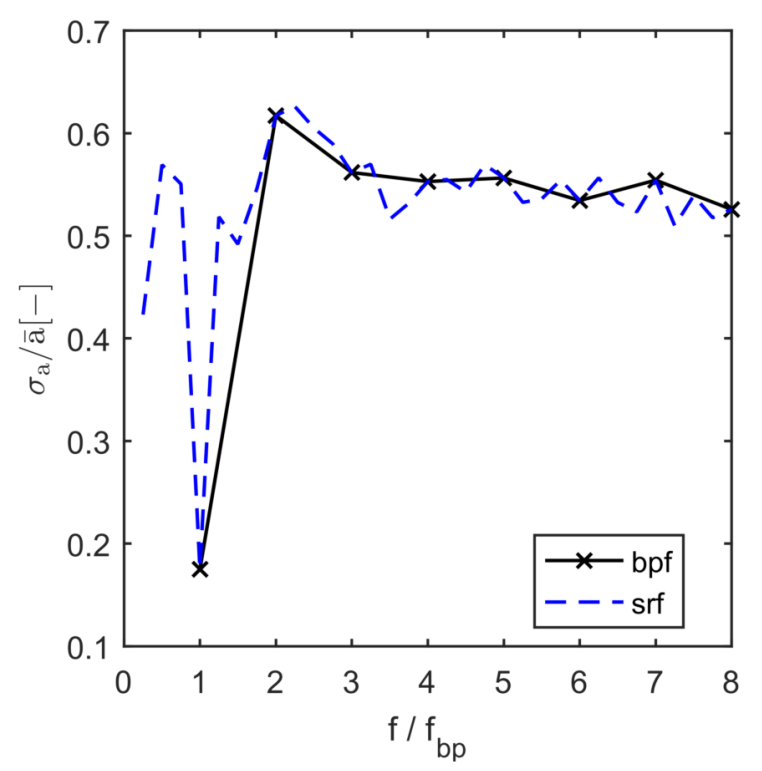

c) Ratio of standard deviation and mean of amplitude

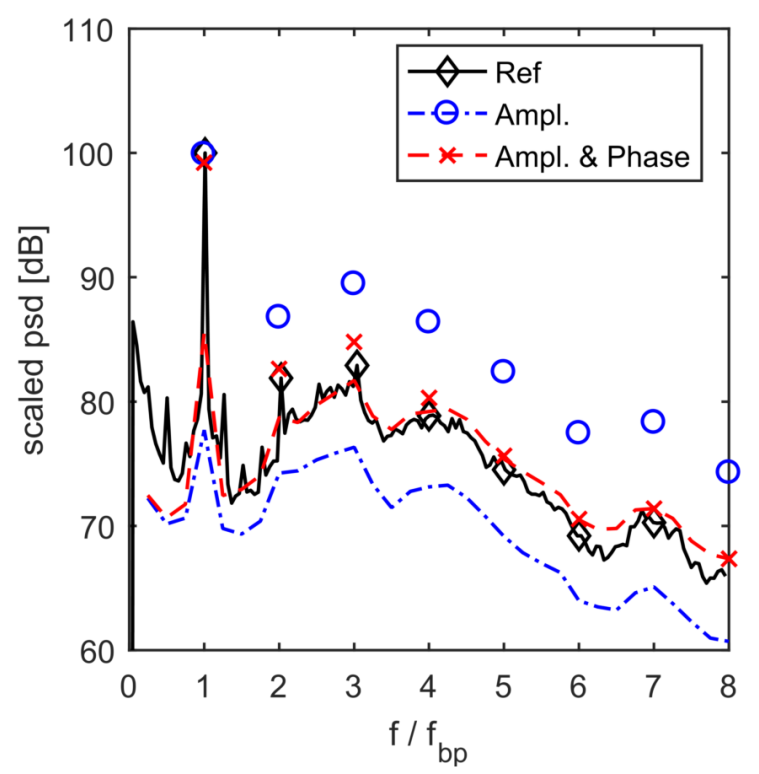

b) Power density spectrum

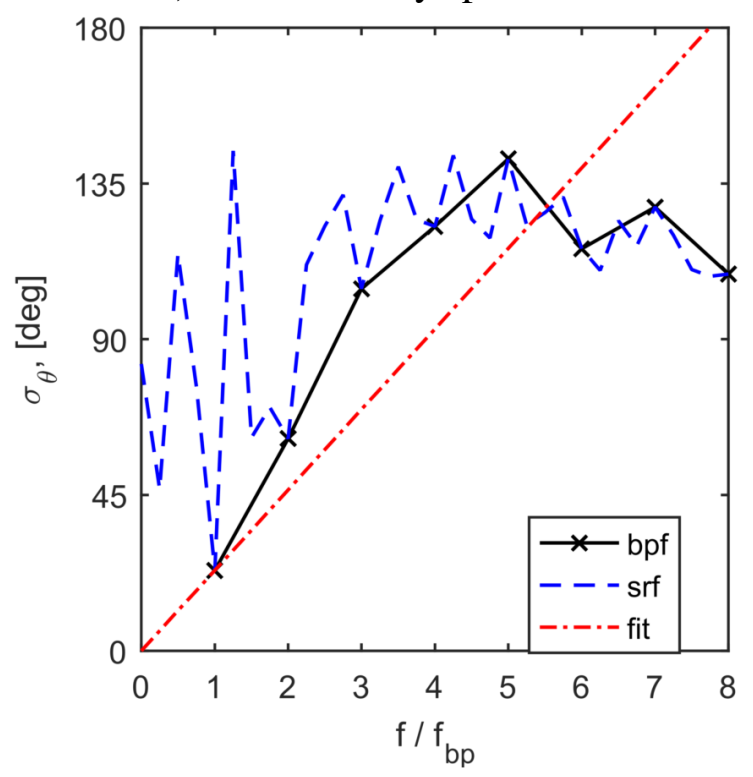

d) Standard deviation of phase

Figure 6.11: Analysis of HPF data of amplitude and phase variation between blade passages for a twin-screw vessel, full scale data. Time trace shown in Figure 6.9b.

The same exercise was also performed for a dataset measured at full scale on a twin-screw vessel with a four-bladed propeller. The results are presented in Figure 6.11. Results are very similar to the results for model scale presented in Figure 6.10, only the amplitudes at the harmonics of the bpf are much less pronounced. For this propeller, the non-cavitating blade contributes only to the first bpf. The difference between the 'Ampl' spectrum and 'Ref' spectrum is a factor two in amplitude at the third bpf and six $\mathrm{dB}$ for the maximum level of the broadband hump.

If the mean slope of $\sigma_{\theta}$ is presented as function of the harmonic, $k$, of shaft rate frequency, 
the value for the model test involving the two-bladed propeller and the value for the sea-trial involving a four-bladed propeller are both approximately $6 \mathrm{deg} / k$. The actual variation with frequency is, however, different for the two situations. Also, the slope should only be determined for the first few harmonics, as for higher harmonics $\sigma_{\theta}$ can be assumed constant. For the model tests, the variation of the pressure signal between blade passages is considered to be caused by the variability in cavitation extent between blade passages, while for the sea trials it is considered to be also caused by the variation in rpm and ship motions due to seaway and manoeuvring. The variation of $\sigma_{\theta}$ and $\sigma_{a}$ needs to be determined for a larger number of test-cases for model tests as well as for sea trials before more definite conclusions on trends can be drawn.

Bensow (2015) has shown that the instantaneous wake field of a single-screw chemical tanker computed by a Large Eddy Simulation (LES) for model scale conditions differs significantly between blade passages from the time averaged wake field. As a result, the distribution of blade force with blade rotation angle also varies significantly between blade number and revolution. These computational result show that the variability in cavitation extent and dynamics between blade passages at model scale is due to, among others, the variability of the ship wake field. Variability in nuclei content and therefore in cavitation inception is also considered to be a source of variability of cavitation.

The presented hull-pressure spectra in Chapters 7 and 8 of this thesis were generated by first converting the narrowband spectrum to a power spectrum in 1/6 octave band levels, followed by a conversion to a power density spectrum. Through this approach, the tonals at the higher harmonics of the blade passage frequency disappear, so that the focus is on the broadband part of the spectrum. The variability of the pressure signal between blade passages is therefore not so relevant for the analysis of the broadband part of the presented spectra as long as it is present. The cause and effect of the variability of the pressure signal on the amplitudes of the blade rate tonals should be further investigated.

\subsubsection{Spatial distribution over hull surface}

The spatial distribution over the hull surface of the measured pressures is discussed next. Use has been made of the dataset for the two-bladed propeller that was also used in preceding sections. Both amplitude and phase are presented using the values measured at the centre transducer as reference. The centre transducer is the transducer located directly above the centre of the propeller and the location of this transducer is also used as the origin of the coordinate system on the hull. The results have been corrected for the effect of vibrations using the method presented by van Wijngaarden (2011). In that method, the vibration-induced pressures on the ship hull are computed with an acoustic boundary element method using the measured accelerations as input. This correction had a negligible effect on the amplitudes and gave a small change in phase angle.

Results for the fifth and sixth harmonic of the blade rate frequency are presented in Figure 6.12. The maximum value of the amplitude is located above the downward going blade. The distribution the amplitude does not differ much from a circular distribution, but the distribution of the phase is completely different.

The distributions of amplitude and phase have also been computed using the analytical 
formulations for a monopole noise source, given by Eq. (5.8), and for a cylindrical noise source, given by Eq. (5.38). These acoustic sources were positioned at the tip of the propeller and below the maximum noise level observed in the experimental data. The data is again presented using the values for the centre transducer as reference. The frequency equals six times the blade rate frequency, corresponding to a non-dimensional acoustic wave number $k D=0.14$. Additional parameters for the cylinder are $r_{c} / D=0.03$ and $2 L / r_{c}=5$. The axis of the cylinder has been rotated by $15 \mathrm{deg}$ with respect to the $x$-axis. This angle corresponds to the pitch angle of the blade tip. The hull has a small tunnel shape that is taken into account in the computations. The solid boundary factor is taken constant over the hull, both in terms of amplitude and in terms of phase.

The results for the analytical formulations are presented in Figure 6.13. The distribution of the amplitude for the monopole has a circular shape but for the cylinder it becomes elliptical. Overall, the distribution of the amplitude observed in the experiments is reasonably well described by a monopole as well as a cylindrical noise source. The phase distribution of the monopole also shows a circular shape but the variation is very small. For the cylindrical source, however, a high variation in phase is observed along its longitudinal axis. This variation occurs because the hull surface is in the near field as $k z_{s}=0.05$ with $z_{s}$ the propeller-hull clearance.

The distribution of the phase of the cylindrical source shows some resemblance with the distribution observed in the experiments, but the variation is smaller and the orientation is also different. This suggests that the collapse of the vortex cavity behaves as a combination of a cylindrical and a spherical source. Therefore, the recommendation is to investigate other deformations of the cylinder than the adopted cosine function. 

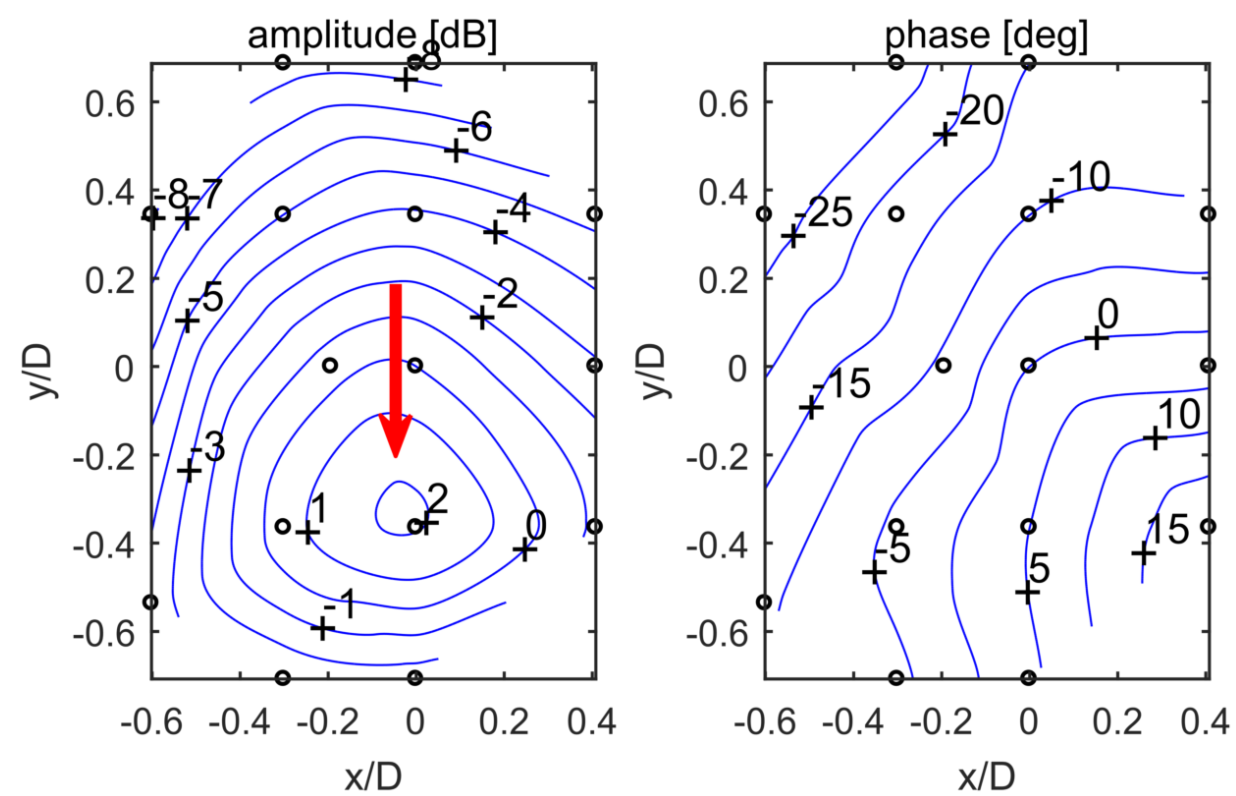

a) $f / f_{b p}=5$
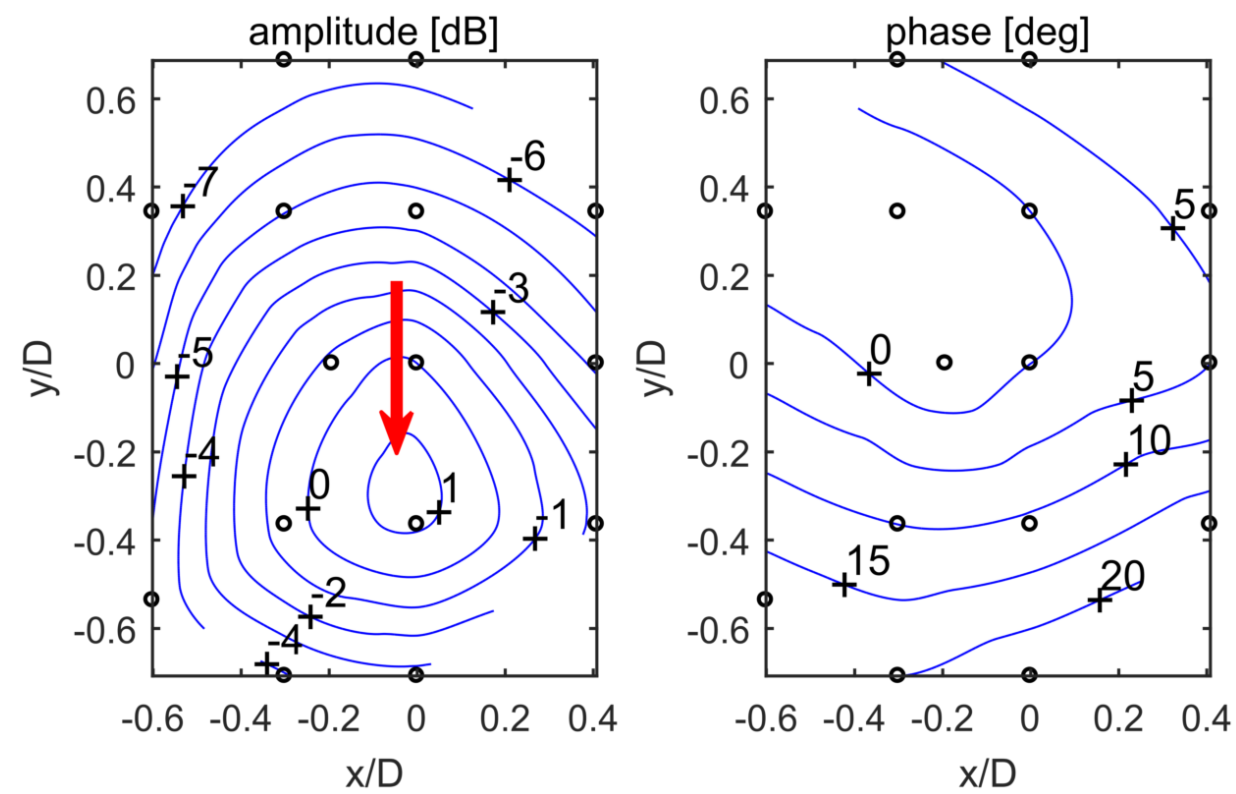

b) $f / f_{b p}=6$

Figure 6.12: Measured distribution of amplitude and phase of hull pressure for two-bladed propeller. The circles denote the locations of the pressure sensors. Amplitude and phase are presented with respect to the value of the sensor located at $(x / D, y / D)=(0,0)$. The $\mathrm{x}$-axis is directed upstream, the red arrow indicates the propeller rotation direction in top position. 

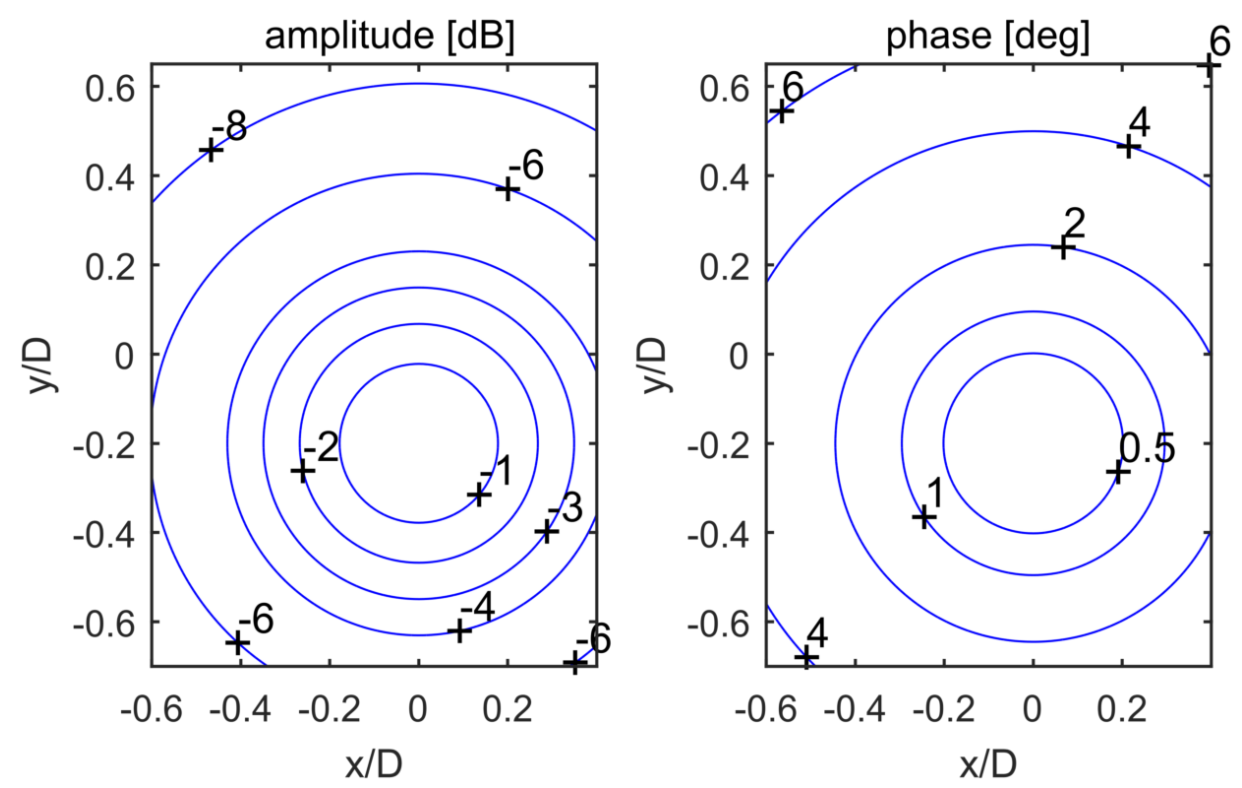

a) Monopole
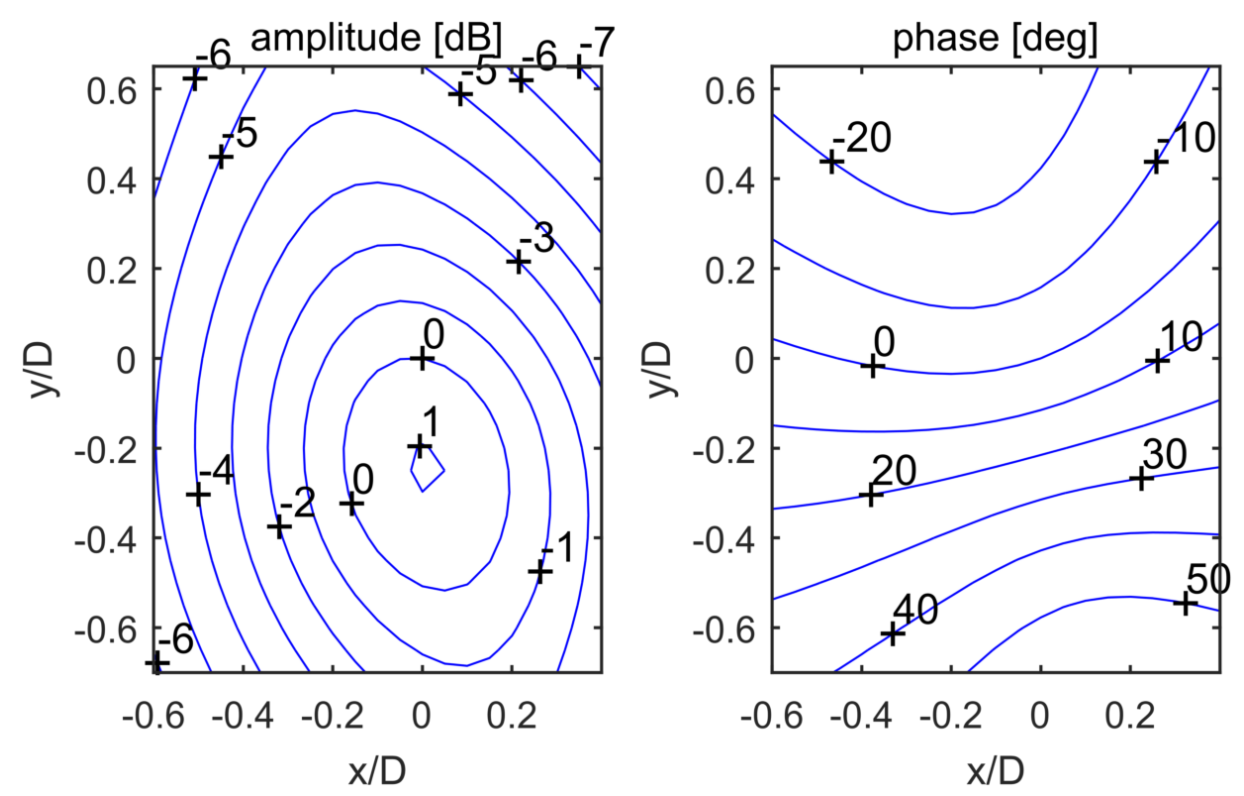

b) Cylinder

Figure 6.13: Distribution of amplitude and phase of hull-pressure for a noise source consisting of a monopole and of a finite length cylinder. Amplitude and phase are presented with respect to the value at $(x / D, y / D)=(0,0)$. The source is located at $(x / D, y / D, z / D)=(0,-0.2,-0.35)$. 


\subsection{Cavitation patterns}

This section discusses cavitation patterns as observed on hydrofoils and propeller blades, focussing on tip-vortex cavitation and on sheet cavitation in the tip area. It provides some information additional to that described in Section 2.4. This information is used in the subsequent section on excitation mechanisms.

\section{Tip-vortex cavitation}

A detached tip-vortex cavity refers to the situation that the inception of cavitation occurs downstream of the tip of the hydrofoil or of the propeller. This vortex cavity has a cylindrical shape with cavity radius initially increasing with downstream direction, followed by a decrease of the cavity radius along the vortex core, see Case A in Figures 4.15 and 4.16, and Case 1 in Figure 6.15. The increase of the cavity radius is related to the increase in vortex strength due to the roll-up of vorticity. The decrease of the cavity radius is related to the growth of the viscous core radius in downstream direction. The pressure in the vortex centre is mainly due to the radial distribution of the azimuthal velocity component, and the axial velocity component does not have a significant effect on this pressure. Just downstream of the tip, the axial velocity in the centre of the vortex has a small value due to the boundary layer on the blade that is ingested in the vortex. The location of inception of the detached vortex cavity may be stationary, which suggests that the axial velocity of the liquid reduces to almost zero in front of the cavity.

When the vortex cavity attaches to the blade, it first forms a small, probably closed, sheet cavity on the blade, possibly in combination with an elliptical cross-section of the vortex cavity. Examples are shown in Figure 4.15, Case B, and in Figure 6.15, Case 2. This cavity pattern is sketched in Figure 6.14a. The closed cavity on the blade that turns into the vortex cavity shows a resemblance with the Chaplygin-Lamb vortex dipole (Meleshko and van Heijst, 1994), which describes a 2-D circular vortex consisting of two regions with opposite vorticity while the vorticity equals zero outside these regions. Such a vortex also describes a semi-cylindrical inviscid vortex on a flat rigid wall. For the vortex cavity, we can assume that vorticity is present within the cavity and, therefore, that this vorticity is also present in the cavity that is attached to the blade. Because of the solid-wall boundary condition, this vorticity is mirrored in the blade surface leading to a cavity interface that has a semi-cylindrical shape that downstream of the blade turns into the elliptical vortex cavity.

\section{Sheet cavity and closure-vortex cavity}

With further increase in blade loading by increasing angle of attack or reduction in cavitation number, the sheet cavity grows in size with the detachment point moving upstream along the leading edge. When the location of the closure line of this sheet cavity does not vary in chordwise direction when moving towards the tip, a re-entrant jet is formed leading to the formation of a cloud of cavity bubbles and vortices, see Figure 1.2. When the chordwise location of the closure line of the sheet cavity moves downstream when moving towards the tip, the reentrant jet has a spanwise component and is denoted a side-entrant jet (Foeth et al., 2008). The chordwise-directed velocity component of the side-entrant jet can be directed in downstream 
direction. The re-entrant jet and side-entrant jet lead to an open structure of the cavity at its closure region. Within the re-entrant jet and side-entrant jet, the liquid evaporates.

Single-screw ships often show a very significant amount of sheet cavitation, of which the chordwise extent can rapidly increase in radial direction. During the growth of the sheet, a side-entrant jet is developed of which the axis can even be oriented in chordwise direction. The high curvature of the sheet-cavity closure region leads to a cavity-closure vortex, defined by Bark and Bensow (2013) in their description of cavity structures in relation to erosion. We will refer here to the cavity-closure vortex as the vortical structure inside the sheet cavity. The circulation, $\Gamma$, of the cavity-closure vortex can be computed from a surface integral over the vorticity vector, $\omega$, by $\Gamma=\iint_{S} \omega \cdot d \mathbf{S}$. When the blade leaves the wake peak ${ }^{2}$, the cavity reduces in size and may collapse. The cavity-closure vortex may form a secondary cavity structure when the side-entrant jet hits the cavity interface. As there is circulation present around this cavity structure, the collapse process becomes similar to the collapse process of a vortex cavity. This cavity structure is denoted a closure-vortex cavity. Analysis of high-speed video images suggests that a closure-vortex cavity also occurs in case the side-entrant jet does not protrude the cavity interface, but this requires further investigation. When the sheet cavity extends into the wake of the blade and forms a supercavity, the collapse and rebound of the closure-vortex cavity occurs downstream of the blade trailing edge. In that case, it may interact with the tip-vortex cavity. This was already discussed in Section 2.4.

The high curvature of the sheet also leads to a vorticity layer on the liquid side of the cavity interface, similar as for the vortex cavity presented in Section 3.2.1. The thickness of this vorticity layer is expected to be affected by the Reynolds number, but its effect on the size and on the dynamics of the sheet cavity is unknown. This vorticity layer increases the value for the circulation of the cavity-closure vortex. Because sheet cavitation is reasonably well described by potential-flow theory, and the collapse of cavitation is inertia driven, we assume here that the effect can be neglected. This is to be further investigated.

When the propeller blade has more skew and tip-unloading, the cavity-closure vortex can be located close to the leading edge of the propeller. The direction of rotation of this closure vortex is identical to that of the tip vortex so that the sheet cavity can directly merge and turn into the tip-vortex cavity. Downstream of the trailing edge, the cavity needs to be in equilibrium with the pressure due to the radial distribution of the azimuthal velocity within the tip vortex. This may result in a reduction in cavity radius downstream of the blade trailing edge. An example of such a cavity pattern is seen in Figure 1.1 for the blade position in the lower part of the propeller disc, and in Figure 2.5b.

The sheet with cavity-closure vortex can also form a cavity structure that is separated from the tip-vortex cavity. The downstream part of the sheet is then formed by a closure-vortex cavity, and due to the small vortex strength, the cavity radius will rapidly decrease with downstream distance. Such as sheet with cavity-closure vortex that is separated from the tip-vortex cavity can, for instance, be generated when the blade moves through a peak in the wake of the ship. The sheet cavity will then grow forming the cavity closure vortex and will collapse as a closurevortex cavity. This cavity pattern is sketched in Figure 6.14b. Examples of such a cavity pattern are shown in Figure 6.6 for the two-bladed propeller.

\footnotetext{
${ }^{2}$ The wake peak is the peak in the deficit of the axial velocity in the ship wake. At this location, the blade has its highest loading.
} 


\section{Leading-edge vortex cavity}

At the leading edge of a highly-skewed propeller blade, the flow may separate forming a cavitating leading-edge vortex, as shown in Figure 2.5a. For a leading-edge vortex, the cavity size is directly related to the vortex strength and, therefore, it is a persistent flow structure, similar to the cavitating tip vortex. This cavity pattern is sketched in Figure 6.14c.

For propellers, a leading-edge vortex cavity may be difficult to distinguish from, what we call here, a closure-vortex cavity, but there are some distinct differences. The closure-vortex cavity is formed by sheet cavitation detaching from the leading edge and the inception of cavitation occurs on the surface of the propeller blade. The sheet can not carry any vorticity, except when it is highly curved. A leading-edge vortex is formed by a vortex layer detaching from the leading edge. This free shear layer rolls-up into the leading-edge vortex with the minimum pressure in the centre of the vortex. The inception of cavitation occurs within the flow field. Cavitation may also occur in the vortex layer but will present itself by several small vortex cavities within that layer. The radius of a cavity within a leading-edge vortex may increase in downstream direction due to the further roll-up of the vorticity layer. This does not occur for a closure-vortex cavity. Due to the growth of the radius of the viscous core, the radius of the cavity will decrease in downstream direction for both vortex cavities. Note that the initial size of the closure-vortex cavity is related to the sheet cavity, and the strength of the closure-vortex cavity is probably too small to maintain this cavity size. The cavity size thus decreases once the closure-vortex cavity is generated. 

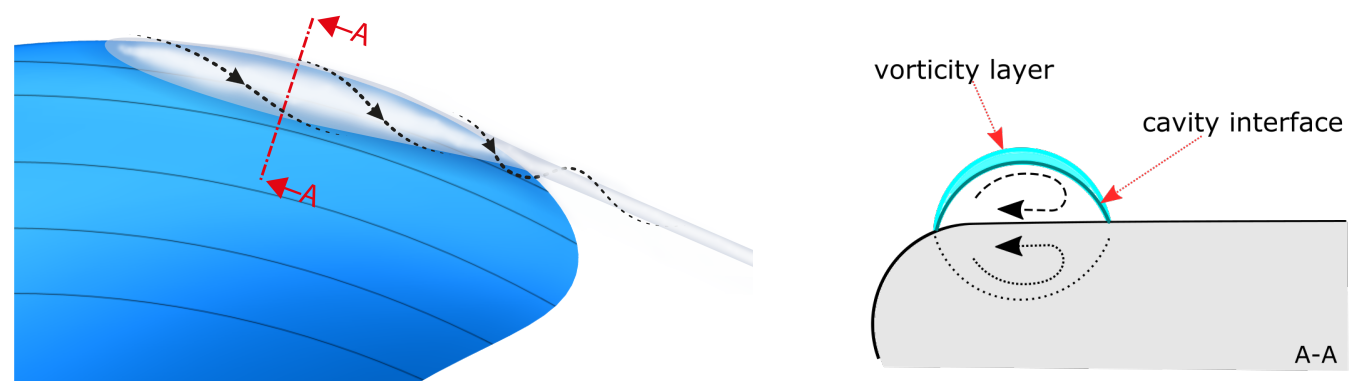

a) Tip-vortex cavity attached to a closed-sheet cavity with an enclosed vortex
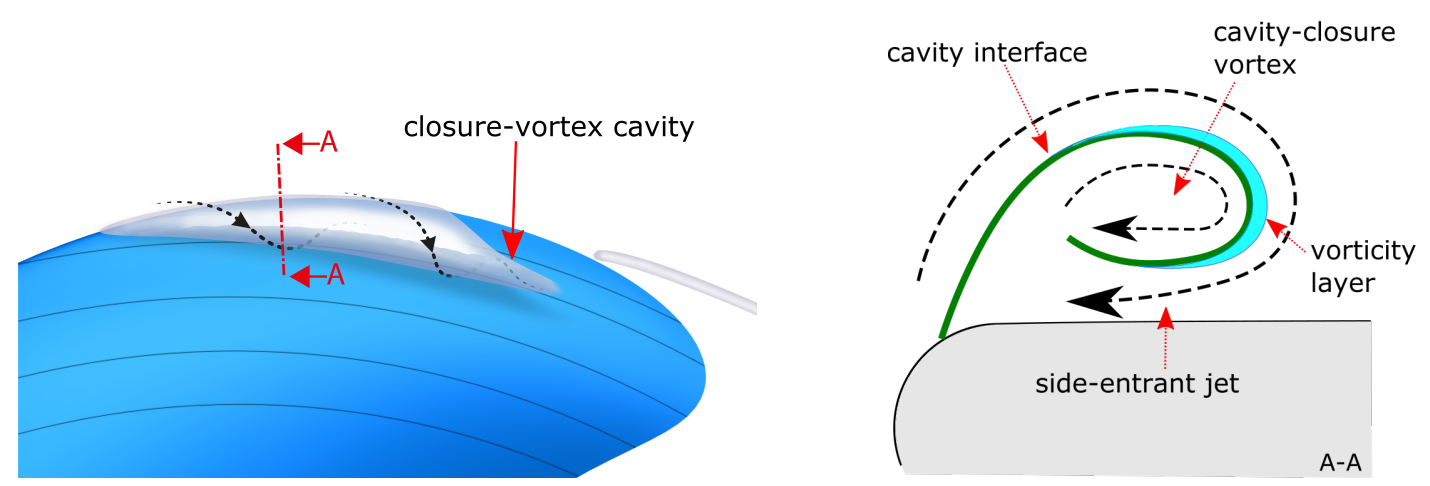

b) Open-sheet cavity with cavity-closure vortex, not connected to the tip-vortex cavity mirror image of vortex in solid surface not shown.
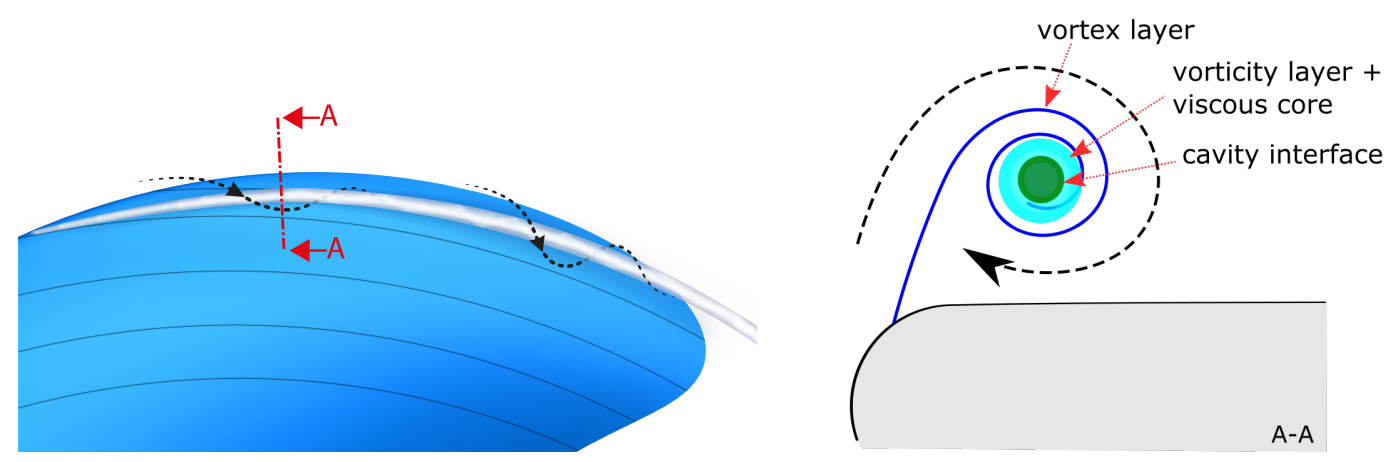

c) Leading-edge vortex cavity, mirror image of vortex in solid surface not shown.

Figure 6.14: Schematic patterns of vortex cavities. The boundary layer on the blade that is convected from the blade to the cavity interface is not shown. 


\subsection{Excitation of vortex-cavity dynamics}

The various possible excitation mechanisms for the dynamics of the cavity of a tip-vortex are briefly discussed next. The interaction of the tip vortex with, for example, the rudder is not considered here.

\section{Ship wake field}

The rotation of the propeller blade through the ship wake leads to a variation in blade loading and, therefore, also to a variation of the tip-vortex strength. Furthermore, due to the variation of the hydrostatic pressure in the propeller disc, the cavitation number at the blade tip varies with blade position. These two effects lead to a variation in radius of the vortex-cavity with blade position and, therefore, to a variation of this radius in downstream direction. In addition, the roll-up of the vorticity layer leads to a small increase in radius of the vortex cavity immediately aft of the blade tip, followed by a decrease of that radius due to the growth of the viscous core, see Figure 4.16, top figure, Case A. These variations in cavity radius occur very gradually and do not lead to significant dynamics of the cavity.

In addition, the vortex cavity will deform under influence of the spatial and temporal velocity fluctuations due to the non-uniformity and turbulence in the ship wake, respectively. The interaction with turbulence also occurs for a tip-vortex cavity of a wing or propeller blade that is tested in a cavitation tunnel in open water conditions, but due to the, in this case, smaller velocity fluctuations the cavity dynamics will be less than for a propeller operating in a ship wake. The experiments discussed in Section 6.2 have shown that the velocity fluctuations of the tunnel lead to dynamic behaviour of the vortex cavity, but the corresponding amplitudes of the cavity deformations are small. Significant noise levels are only generated under special conditions.

Even though these mechanisms do not lead to a significant increase in noise level, they can still be measured as shown in Figure 6.15. Case 1 corresponds to a detached vortex cavity. Hence, only excitation mechanisms as discussed above are present. The HPF spectrum shows that for this case the broadband noise levels are approximately $10 \mathrm{~dB}$ above the background noise levels. The latter are defined as the spectrum measured for non-cavitating conditions.

\section{Interaction with a sheet cavity on the blade}

In most situations, the vortex cavity is attached to the blade and a sheet cavity is present, as discussed in Sections 2.4 and 6.4. We will present here a few examples of the resulting cavity dynamics.

In the first example, the vortex-cavity is just attached to the blade, forming a small closed cavity on the blade surface, see Case 2 in Figure 6.15. In comparison to the situation with the detached vortex cavity, i.e. Case 1, the broadband noise levels have increased by $6 \mathrm{~dB}$. These cases are for a podded propeller where, at the top position, the blade tip moves through the boundary layer of the hull and through the decelerated flow in front of the strut of the torpedo. A sequence of images from the high-speed video recordings is presented in Figure 6.16, showing the collapse of the sheet cavity. Image 1 shows the maximum extent of the sheet cavity. The 


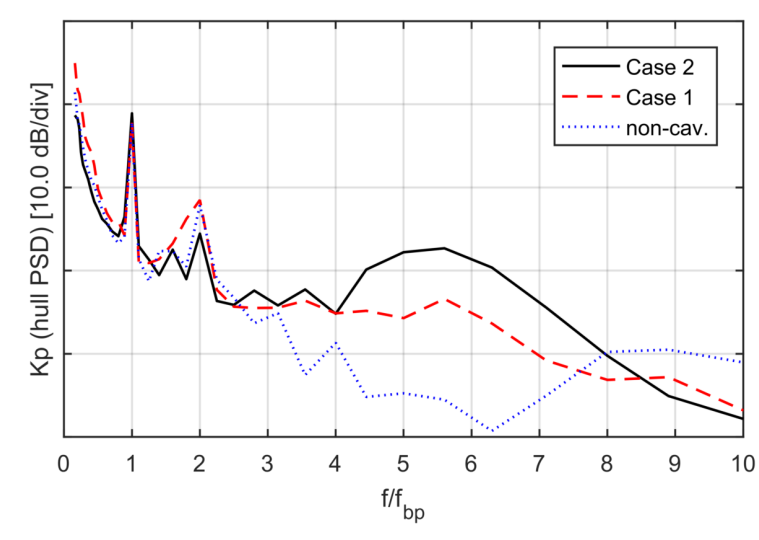

a) Spectra

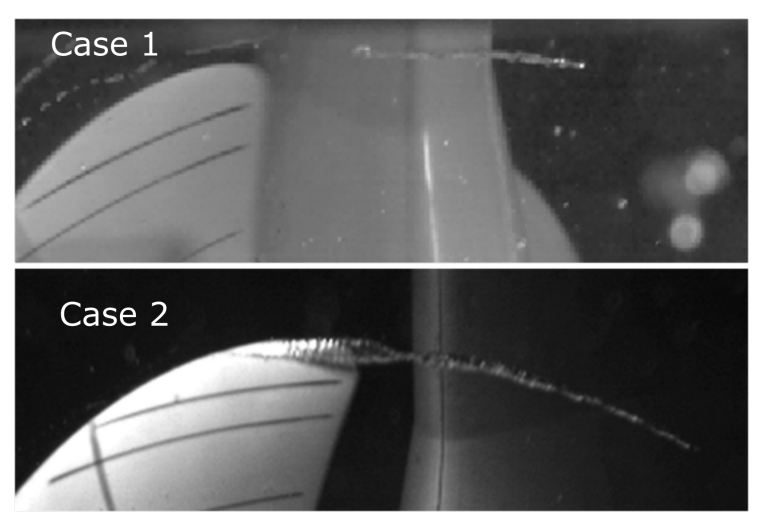

b) Cavitation observations

Figure 6.15: Two cases of a podded propeller showing difference in spectrum of hull pressure for detached (Case 1) and attached (Case 2) tip-vortex cavity. The spectrum of the non-cavitating propeller is also shown. The angle of the pod is different for Case 1 and Case 2.

subsequent images show that the detachment point of the sheet cavity moves aft towards the blade tip. At the same time, the vapour volume of the closed sheet cavity is shed into the vortex cavity thereby increasing the thickness of the vortex cavity. In fact, the vapour volume of the sheet cavity remains almost stationary with respect to the ship wake field. The vortex cavity is, therefore, no longer in equilibrium resulting in a collapse and rebound of the vortex cavity as observed in images 3 through 6.

If the sheet cavity is more developed in the tip area, a side-entrant jet is formed leading to the formation of a cavity-closure vortex, as discussed in Section 6.4. Figure 6.17 presents a series of images from a high-speed video that show the collapse of a sheet cavity that is directly connected to the tip-vortex cavity. It concerns the case of the single-screw Combi Freighter, shown in Figure 6.5. The ship-wake field has a narrow peak which leads to a local increase in cavity thickness that is already observed in image 1 . This cavity bulge remains at the location of the wake peak and thereby moves aft with respect to the blade as the blade rotates through the wake. When the bulge enters the vortex cavity, it collapses and rebounds as observed in images 4 through 8. The resulting broadband HPF for this case are presented in Section 8.4.4.

An example for which the sheet cavity is more developed and has formed a closure vortex cavity, is the two-bladed research propeller of which results were presented in Section 6.3.3. For this case, the sheet cavity is not connected to the tip-vortex cavity. In the peak of the ship wake field, the sheet cavity develops at the leading edge forming a side-entrant jet and a cavity closure vortex. When the blade tip moves out of the wake peak, neither the blade loading nor the circulation generated by the flow around the curved closure region can sustain the cavity volume and a collapse and rebound of the closure-vortex cavity occurs.

These examples clearly show that, if the sheet cavity is directly connected to the tip-vortex cavity, the collapses and rebounds of the tip-vortex cavity are strongly affected by the shedding of vapour volume from the sheet cavity into the vortex cavity. 

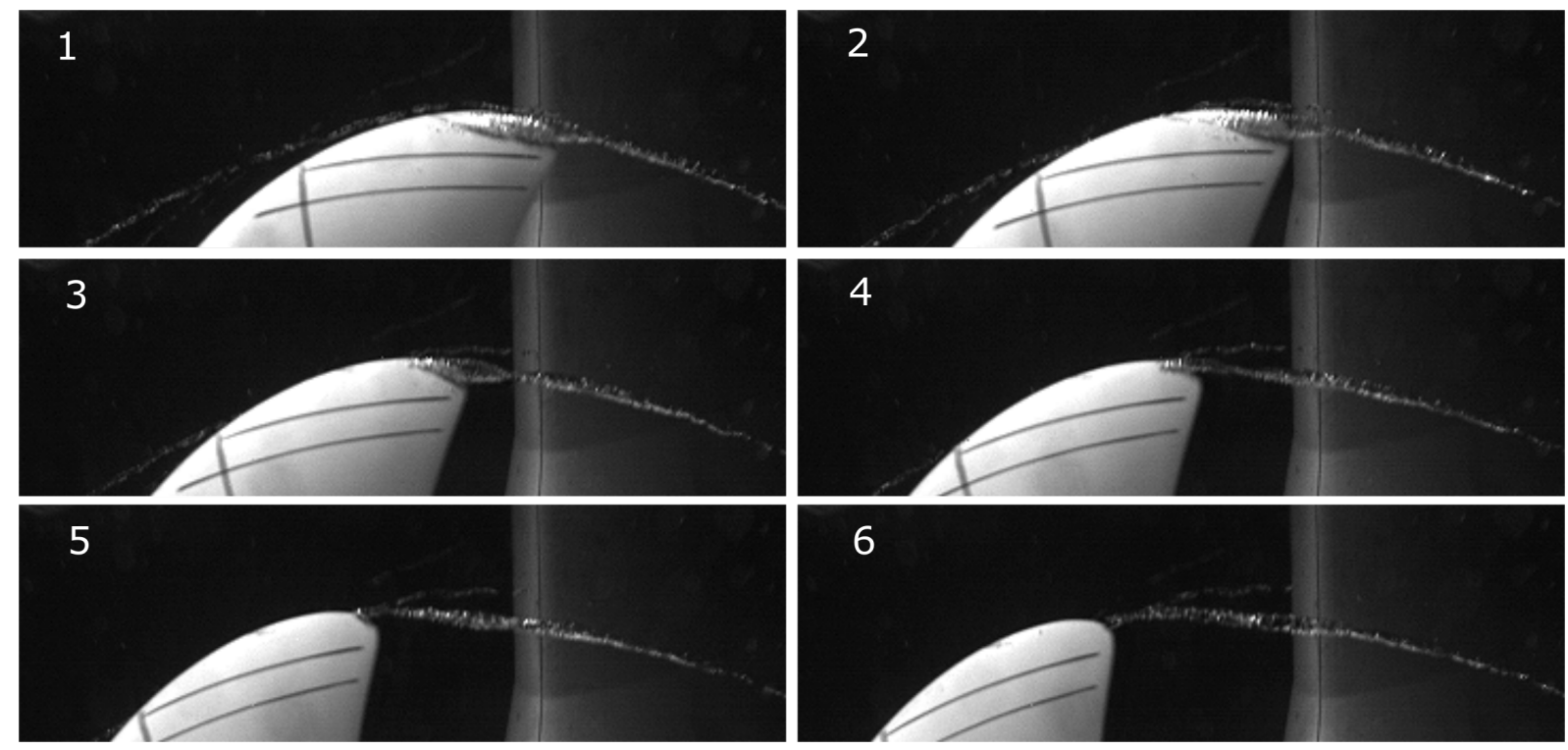

Figure 6.16: Series of high-speed video images showing collapse of an attached tip-vortex cavity corresponding to Case 2 in Figure 6.15. Time between frames corresponds to an increment in blade position of $3.8 \mathrm{deg}$. The blade is located just beyond the 12 o'clock position.

\section{Interaction with other propeller vortices}

The interaction of a tip-vortex cavity with other propeller generated vortices is known to lead to significant broadband noise levels. Two cases can be distinguished: (i) the interaction of the vortices generated by different blades, possibly in combination with the hub vortex, and (ii) the interaction of two vortices generated by the same blade. In the first case the vortices are at relative large distance from each other whereas in the second case the distance between the vortices is small.

The interaction of two counter-rotating vortices is referred to as Crow instability, as discussed in Section 2.2. The interaction leads to bending of the centreline of the vortex which brings the vortices closer to each other. This ultimately leads to break-up of the vortex structure. Examples of this process are shown in Figure 2.4. For most ship propellers, the helical structure of the tip vortex cavitation is already disrupted due to the interaction with the rudder positioned directly aft of the propeller. Any interaction further downstream does not affect the HPF as it occurs downstream of the stern of the ship.

The interaction of vortices generated by the same blade occurs for example for a skewed controllable-pitch propeller of a ship sailing at low speed. The pitch is then reduced, while the propeller-tip speed is still relatively high. This situation is known to generate significant broadband noise levels, as discussed in Section 2.5. Two images of such a cavitation pattern are shown in Figure 6.18. The pitch reduction leads to a large suction peak in the pressure at the leading edge on the face side of the blade at the outer radii through which a leading-edge vortex is generated. As the overall tip loading is still positive, a tip vortex is generated on the back of the propeller. The circulation of these two vortices has opposite sign showing the characteristics of the Crow instability but, due to the unequal vortex strength, the weakest vortex will spiral 
around the stronger vortex and ultimately break up forming ring vortex cavities. The resulting broadband HPF are presented in Section 8.4.4. 


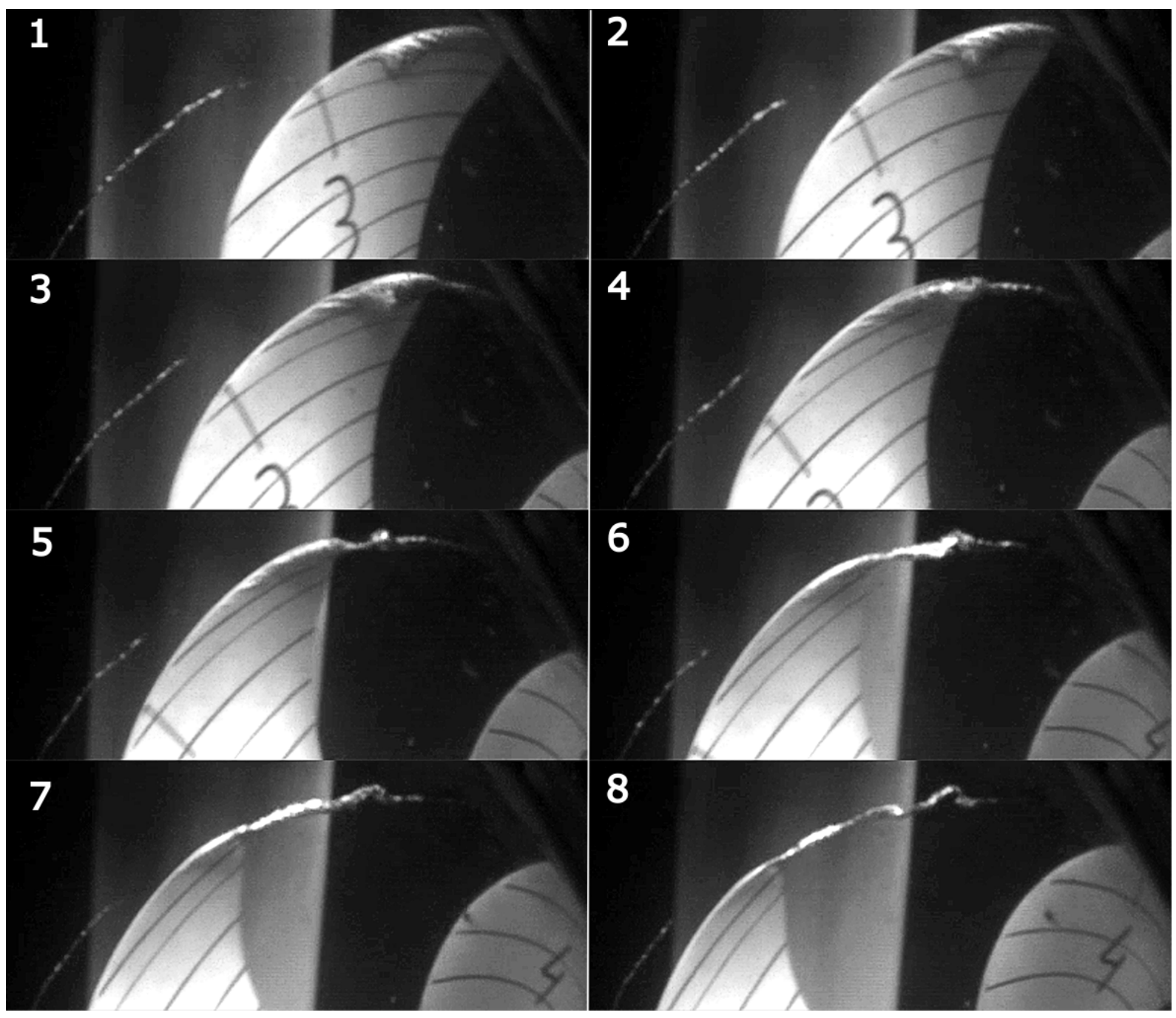

Figure 6.17: Series of high-speed video images showing collapsing sheet cavity with cavityclosure vortex, directly connected to tip-vortex cavity. Time between frames correspond to an increment in blade position of $5.7 \mathrm{deg}$. The test-case is the high-pitch condition of the Combi Freighter.

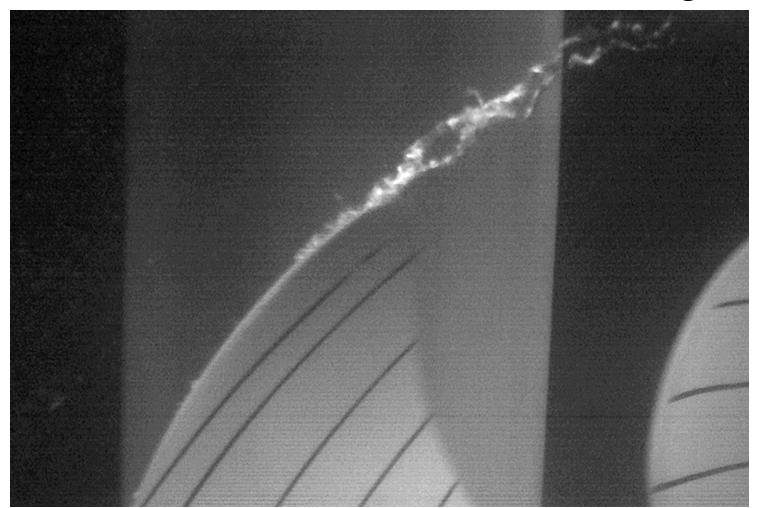

a

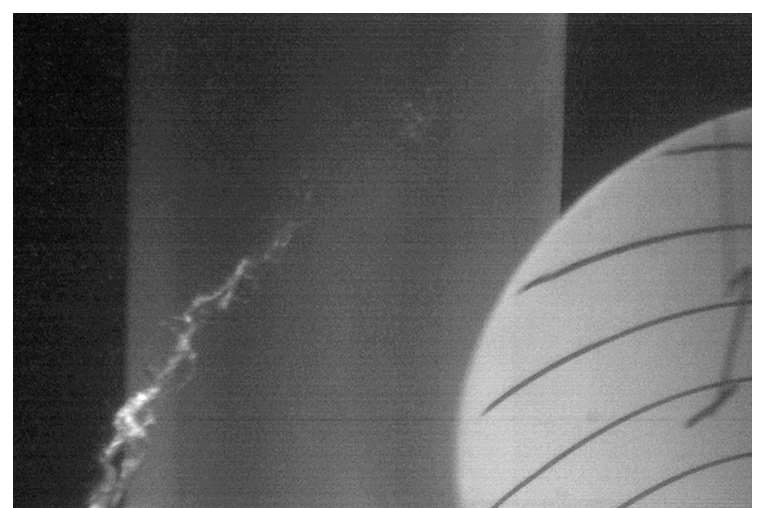

$\mathrm{b}$

Figure 6.18: Two images of interaction between leading-edge vortex cavity generated on face and tip-vortex cavity on back of CPP. The test-case is the low-pitch condition of the Combi Freighter. 


\subsection{Review of mechanisms of broadband cavitation noise}

This section reviews the mechanisms of broadband noise by vortex cavitation. It makes use of the results presented in preceding sections and in preceding chapters.

Cavity pattern and excitation: An important mechanism for the excitation of the tip-vortex cavity is through the transport of vapour of sheet cavitation into the vortex cavity. Excitation of the tip-vortex cavity through the variation of the blade loading and the velocity fluctuations in the ship wake field is also expected, but the noise levels are smaller than for cases in which the tip-vortex cavity is attached to a sheet cavity on the blade. Vortex-vortex interaction also leads to very significant broadband noise levels but this situation is less common. This situation is most relevant for controllable-pitch propellers operating at high rpm and low pitch to achieve low ship speed. When the sheet cavity becomes larger on the blade, a sheet-cavity closure vortex may be formed by the side-entrant jet. The collapse of the sheet is through a, here called, closure-vortex cavity, that also generates broadband noise. The vortex-cavity patterns and excitation mechanisms have been discussed in Sections 6.4 and 6.5, respectively.

Collapse and rebound: Computations in 2-D viscous flow showed that the collapse is inertia driven although a large collapse up to small cavity radius could not be obtained due to lack of numerical stability. Because viscous effects are small during the collapse, the azimuthal velocity increases at the cavity interface, leading to a decrease in pressure which dampens the collapse and leads to a rebound. As the collapse is inertia driven, it is expected that a full collapse can not occur. Whether this also holds for 3-D flow needs to further investigated. Also, the effect of the flow inside the cavity on these high azimuthal velocities requires further attention. Results have been presented in Section 4.2.

Resonance frequency: Resonance manifests itself in the rebounds after the cavity collapse. A formulation for the resonance frequency of a vortex cavity can be derived for 2-D viscous flow. The formulation consists of the added mass of the vortex cavity and a stiffness term containing the cavitation number or, in an alternative formulation, the azimuthal velocity at the cavity interface. However, a formulation for the resonance frequency for 3-D flow could not be obtained. A dispersion relation has been derived for the propagation of waves on the cavity interface in 3-D inviscid flow, but all perturbations result in neutrally stable waves. Therefore, several criteria for resonance were proposed, among which the criterion of zero group velocity of the mode that involves a cavity volume variation. Results have been presented in Chapter 4 and in Section 6.2.

Viscous effects: Viscosity, and thereby Reynolds number, has an effect on the radius of a vortex cavity when this radius is smaller than or of the same size as the radius of the viscous core in non-cavitating flow. This conclusion is drawn from an analytical vortex model which has been validated, but not all aspects in the comparison between predictions and measurements were understood. Results have been presented in Chapter 3. The effect of the Reynolds number on the vortex cavity size is further discussed in Chapter 8 because of its relevance for model tests. The resonance frequency of the 2-D vortex cavity and the dispersion relation for the 3-D vortex cavity were also noticeable affected by viscosity when the radius of the vortex cavity became smaller than the radius of the viscous core in non-cavitating flow. Results have been presented in Chapter 4. 
Noise source: Analytical formulations, in which the vortex cavity is considered as a cylindrical noise source, show that volume variations are the dominant noise source and that the deformations of the centreline or the rotation of an elliptical cross-section are much less efficient with respect to the radiation of noise. The cylindrical noise source behaves in the far field and at low frequencies as a monopole noise source. In the near field, where the HPF are measured, the situation is more complicated. Results of the analytical formulation have been presented in Section 5.2. A limited comparison with experimental data in Section 6.3.4 showed that the distribution of phase over the hull surface is in between the distribution of a monopole and of cylindrical noise source. Therefore, the employed formulation for the cylindrical noise source requires further research.

Spectrum: Analytical formulations show that a transient oscillatory signal gives a broadband spectrum when analysed by a Fourier transform, see Section 5.3.2. When that signal is repetitive, as occurring for multiple blade passages, the spectrum only shows blade rate tonals. The broadband hump in the spectrum only shows if there is variability between blade passages, as shown by an analytical formulation in Section 5.3.3. The effect of the variability between blade passages on the spectrum was confirmed by applying this formulation to two experimental datasets, one obtained from a model test and one obtained from a sea trial, see Section 6.3.3. Further investigation is required to check for consistent differences between model tests and sea trials as the variability also affects the pressure amplitudes of the higher-order blade rate tonals.

SBF and Lloyd's mirror: The solid boundary factor (SBF) of the hull also has an effect on the amplitudes of the pressure fluctuation on the hull and, to a small extent, on its phase. A simple analytical prediction formula was presented in Section 5.5.1 that shows how the SBF changes with curvature of the hull and with frequency. The spectrum of the radiated URN is affected by the Lloyd mirror effect for which two formulations were presented that are available from literature, see Section 5.5.2.

All these mechanisms need to be considered when predicting HPF and URN due to vortex cavitation. For several mechanisms, it can be expected that in a model test they differ from those at full scale. These mechanisms are (i) the measurement of the HPF in the near field of the vortex cavity, (ii) the effect of Reynolds number, and (iii) the effect of variability between blade passages. These mechanisms should be considered in addition to the aspects discussed in Section 2.5 for measuring HPF and URN in model tests. For the present thesis, the effect of a lower Reynolds number in the model test compared to the Reynolds number at full scale is considered in detail in Chapter 8. Available measurements did not indicate that the propagation loss in the model test for HPF is different from that at full scale, so this was given low priority. The same holds for the effect on broadband noise of variability between blade passages. 
Page intentionally left blank 


\section{Chapter 7}

\section{Semi-empirical prediction of broadband noise}

This chapter presents a semi-empirical method for the prediction of broadband hull-pressure fluctuations (HPF) and underwater radiated noise (URN) due to vortex cavities on marine propellers including validation studies ${ }^{1}$. The method is used in combination with a boundary element method and can easily be applied in the propeller design process. The engineering method makes use of the results presented in Chapters 3 through 6.

\subsection{Introduction}

For the prediction of broadband HPF and URN use can be made of empirical and computational methods and of model tests. In recent years, significant progress has been made in the analysis of cavitating propellers by CFD. Using CFD to predict broadband noise emitted by cavitating propellers has become possible by scale-resolving simulations of turbulent flow (Li et al., 2015; Fujiyama and Nakashima, 2017). However, the methods are computationally very expensive, while accurately capturing the dynamics of the cavitating vortex is still an issue. Therefore, the propeller design process still heavily relies on the use of potential flow methods, possibly in combination with CFD simulations solving the RANS-equations. These methods are only capable of predicting the pressure amplitudes at harmonics of the blade-passage frequency.

Potential flow methods are capable of predicting sheet cavitation and have been coupled to semi-empirical methods to predict the resulting URN (Matusiak, 1992; Brown, 1999). Because in potential flow methods vortices are fitted rather than captured, alternative methods are required to predict developed vortex cavitation and its dynamics. For instance, use can be made of the 2-D Rayleigh-Plesset type of equation for a vortex cavity as presented in Section 4.2. Application of this equation to segments of a propeller tip vortex has been presented by Ligneul (1988); Koronowicz and Szantyr (2006); Berger et al. (2016). Use can also be made of semi-empirical methods (Raestad, 1996; Yamada et al., 2015). The method of Raestad (1996)

\footnotetext{
${ }^{1}$ The method has been published as Bosschers, J. (2018). A semi-empirical prediction method for broadband hull-pressure fluctuations and underwater radiated noise by propeller tip-vortex cavitation. Journal of Marine Science and Engineering, Vol. 6, 49. Section 7.5.3 is not part of that publication.
} 


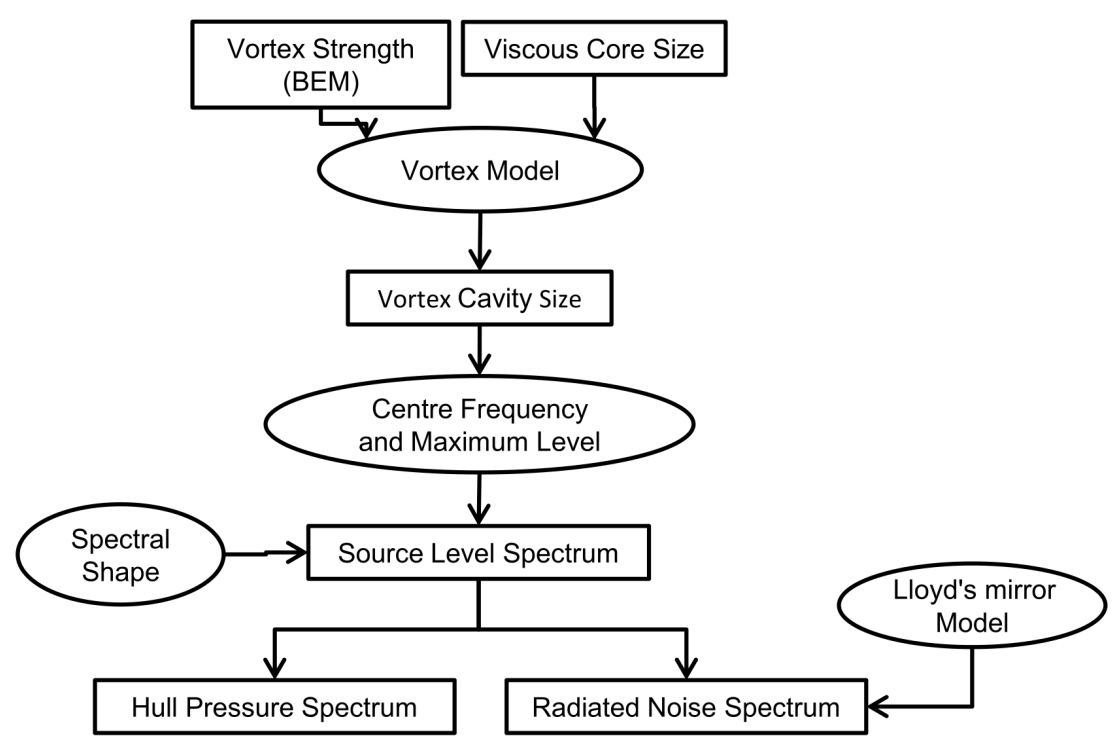

Figure 7.1: Flow chart of the Empirical model for Tip Vortex cavitation noise (ETV-model).

predicts the inboard noise while the method of Yamada et al. (2015) predicts the URN.

It has been shown that the source level of URN due to propeller cavitation has a direct relation with the pressure levels on the hull (Newman and Abrahamsen, 2007; Foeth and Bosschers, 2016). By correcting the hull pressures for the solid boundary factor on the hull as well as for the distance between the location of the cavity collapse and the pressure sensor, the source levels can directly be obtained from the measured hull pressures. These source levels are in reasonable agreement with source levels obtained by hydrophone measurements in the far field of the ship. This shows that the method for predicting broadband HPF and URN can employ the same model to predict the source levels.

The adopted methodology for the semi-empirical prediction method is based on the method to predict the noise inboard ships related to cavitating tip vortices as developed by Raestad (1996). Raestad's method relates measured inboard noise levels to the predicted size of the vortex cavity using a computed circulation distribution on the propeller blade determined by a potential flow method. The present method, that has been designated the Empirical cavitating Tip Vortex method, or ETV-method, follows a similar approach, but with the difference that it predicts the broadband spectrum of HPF and URN.

The overall approach of the ETV-method is sketched in the flow chart shown in Figure 7.1. The vortex cavity size is predicted using a vortex model, such as the ones discussed in Chapter 3 , that requires as input the vortex strength and the size of the viscous core. The vortex strength is obtained from results obtained by a boundary element method (BEM), and the size of the viscous core is obtained from reference data that is corrected for Reynolds number and propeller size. The prediction of the cavity size is discussed in Section 7.2. The cavity size is used in a semi-empirical relation to predict the centre frequency and level of the broadband hump of the source level spectrum. These semi-empirical relations are presented in Section 7.3. The shape of the source level spectrum is prescribed which is discussed in Section 7.4. From the spectrum of the source level, the HPF spectrum is obtained by correcting for propeller-hull clearance 
and for the solid boundary factor, and the URN spectrum is obtained by correcting for the Lloyd's mirror effect. The equations used for this purpose have been presented in Chapter 5 . The comparison between measured spectra and predicted spectra is shown in Section 7.5. The presented model is the second version of the ETV-method, and, therefore, some figures refer to the method as ETV-2.

\subsection{Estimation of cavity size}

The estimation of the vortex cavity size is the core of the ETV-model. The size of the vortex cavity can be predicted from a vortex model for the distribution of the azimuthal velocity with radius. From this distribution, the pressure distribution, and thus cavitation number, can be computed. This has been discussed in Section 3.3. Input parameters for the vortex model are the vortex strength and the viscous core size. The strength of the vortex is estimated from the radial circulation distribution on the propeller blade as obtained by a BEM that solves the potential flow equation. The viscous core size is obtained by a semi-empirical relation. This section discusses these aspects in more detail and presents a comparison between predictions and measurements of the size of the cavity.

Several vortex models were presented in Chapter 3. A modified Lamb-Oseen vortex model has been developed and extended to cavitating flow. Measured azimuthal velocity distributions around a vortex cavity are well described by this vortex model if the viscous core size was corrected for the presence of cavitation. However, this correction should not be applied for the prediction of the relation between cavity size and cavitation number in case the vortex cavity is attached to the foil. This is not understood and requires further research. In case the vortex cavity is attached to the foil, the relation between cavity size and cavitation number is also predicted well if the vortex model for non-cavitating flow is used. To keep the ETV-model as simple as possible, it has been decided to only consider vortex models for non-cavitating flow. Because during the development of the ETV-model the modified Lamb-Oseen vortex was still in development, the Proctor vortex, described by Eq. (3.30), was selected as the principal vortex model. But, as the modified Lamb-Oseen vortex model for non-cavitating flow gives very similar results as the Proctor vortex model, these models can easily be exchanged.

The streamwise variation of the strength of the vortex, denoted by $\Gamma_{\infty}$ in Eq. (3.30), has been measured by Jessup (1989) for the three-bladed propeller P4119 using Laser Doppler Velocimetry (LDV). Near the blade tip, the vortex strength was approximately $10 \%$ of the maximum value of the distribution of the bound circulation on the blade. Downstream of the blade tip, the vortex strength rapidly increases and, at a downstream distance of about half a propeller diameter, the vortex strength obtained its maximum value, which was approximately $90 \%$ of the maximum value of the bound circulation. The inception of tip vortex cavitation typically occurs on the blade or within a short distance downstream of the tip (Astolfi et al., 1999; Arndt, 2002). Therefore, the vortex strength used in the ETV-model should correspond to the strength of the vortex close to the tip.

For the estimation of the strength of the vortex, use has been made of the BEM PROCAL, which solves for the potential flow around propellers operating in a ship wake, and which was developed within CRS working groups. PROCAL is capable of predicting sheet cavitation on 
the propeller, but for the present study only non-cavitating flow has been considered. PROCAL has been coupled with an acoustic boundary element method to predict HPF and with RANS solvers to predict effective wake fields. More information can be found in Vaz (2005); Vaz and Bosschers (2006); Bosschers et al. (2008b) and Bosschers et al. (2015).

A BEM does not capture the tip vortex and vortex-sheet roll-up is not represented. Instead, the strength of the trailing vortices is directly related to the distribution of the bound circulation and remains constant in downstream direction. Experience with PROCAL has learned that the solution is reliable up to, typically, $95 \%$ of the tip radius $R$. Further towards the tip, the limitations of potential flow as well as modeling issues for a BEM become apparent. Therefore, the value of the bound circulation calculated at $0.95 \mathrm{R}$ has been used for the strength of the tip vortex.

Vortex models for viscous flow, such as the Proctor vortex model, require a value for the size $r_{v}$ of the viscous core. Use can be made of, again, the LDV measurements of Jessup (1989) for the three-bladed propeller P4119. The viscous core size of the tip vortex shows, on average, a linear increase with distance to the blade tip and at a distance of half a propeller diameter the value is three times as large as near the blade tip. The value for the size of the viscous core near the blade tip, taken as reference value, equals $100\left(r_{v} / R\right)_{\text {ref }}=0.6$. This viscous core size is then to be scaled to the test-case being analysed using the chord length $c$ at $0.95 \mathrm{R}$ and the Reynolds number Re based on this chord length. However, for marine propellers, there is very little information available on the dependency of the size of the viscous core with Reynolds number.

Information is available on the effect of Reynolds number on cavitation inception. For cavitation test facilities, the ratio of full-scale and model-scale cavitation inception is traditionally scaled with the ratio of Reynolds numbers to the power $m$ following the formulation of McCormick (1962). The value of $m$ varies from facility to facility depending on experience (21st ITTC Cavitation Committee, 1996). Shen et al. (2009) have proposed a scaling formula for cavitation inception in which $m$ depends on Reynolds number by considering the Reynolds number dependency of the skin friction. This formula can also be used to scale the size of the viscous core by making two assumptions. First, cavitation inception is taken as the condition for which the minimum pressure equals vapour pressure. Second, it is assumed that the minimum pressure coefficient scales with the inverse of the square of the size of the viscous core, as shown by Eq. (3.23). The Reynolds-number scaling for the viscous core size then reads

$$
\frac{r_{v}}{c}=\left(\frac{r_{v}}{c}\right)_{\mathrm{ref}}\left(\frac{\mathrm{Re}}{\operatorname{Re}_{\mathrm{ref}}}\right)^{-m / 2},
$$

with the value for $m$ given by (Shen et al., 2009)

$$
m=5.16 \frac{\log _{10}\left(\log _{10} \mathrm{Re} / \log _{10} \mathrm{Re}_{\mathrm{ref}}\right)}{\log _{10}\left(\mathrm{Re} / \mathrm{Re}_{\mathrm{ref}}\right)} .
$$

Through this procedure, the cavitation inception prediction of the vortex model is consistent with practical experience, although it is noted that the effect of tensile strength on cavitation inception is not included in the model.

The method to predict the cavity radius of a propeller tip vortex was validated using experimental data by Kuiper (1981). A propeller with tip vortex cavitation was tested in the cavitation 


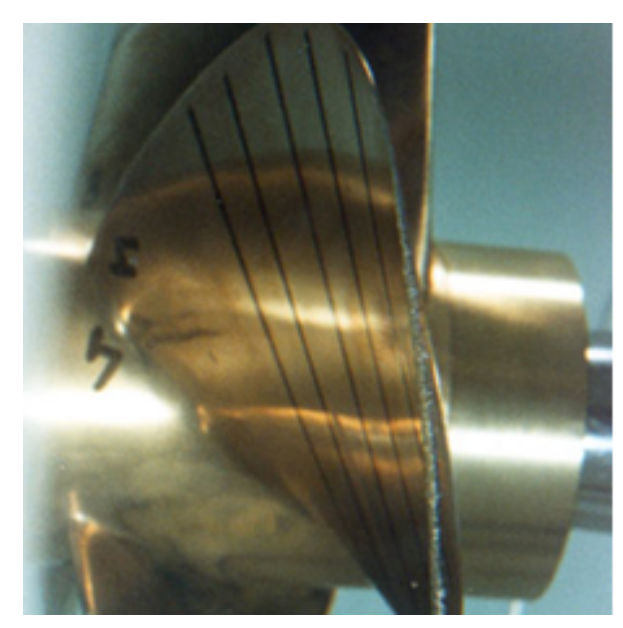

a) $J=0.4, \sigma_{n}=3.90, \operatorname{Re}_{n}=2.76 \times 10^{6}$

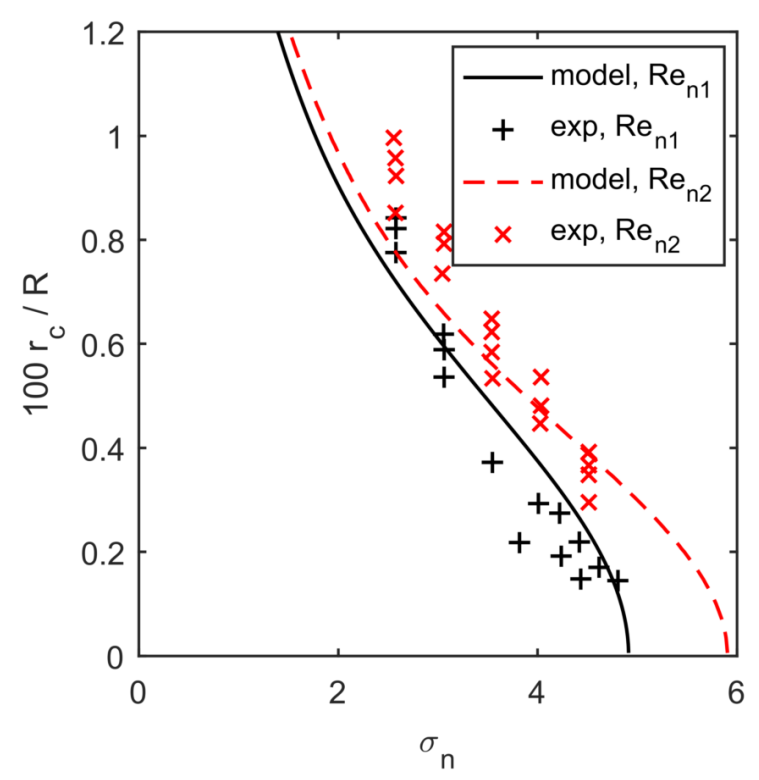

c) $J=0.4$

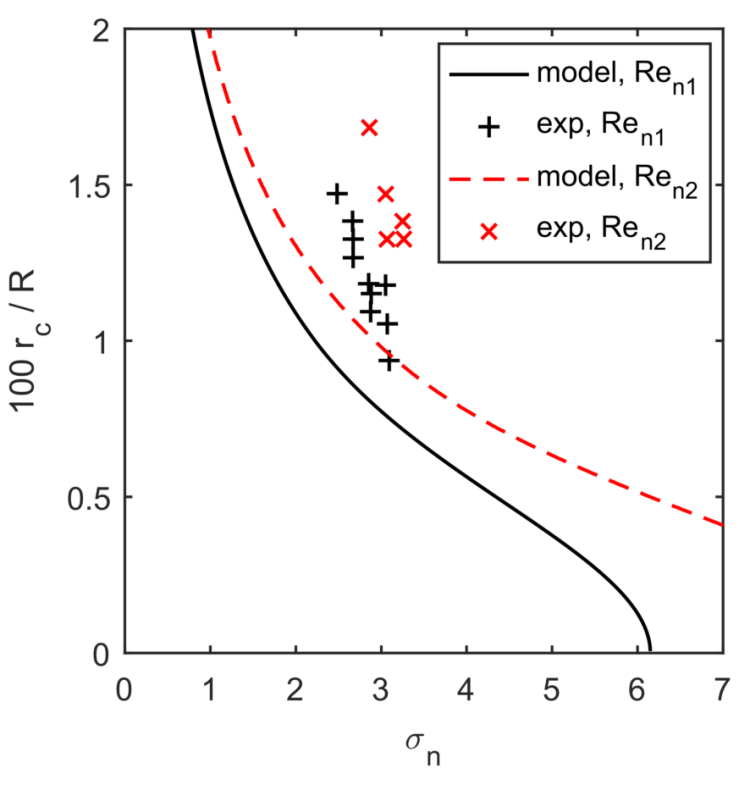

b) $J=0.3$

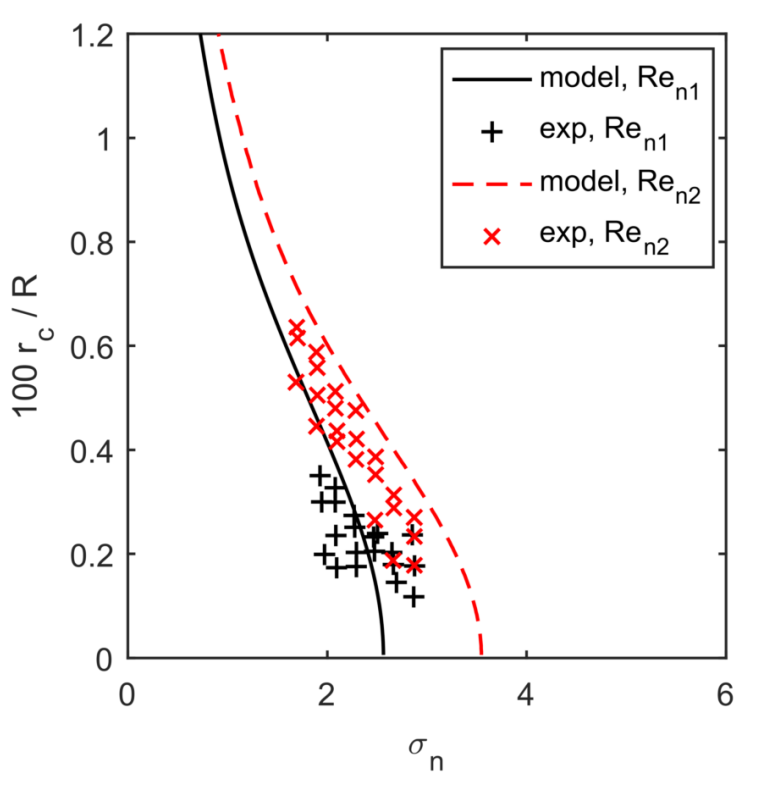

d) $J=0.5$

Figure 7.2: Predicted and measured (Kuiper, 1981) relation between cavity size and cavitation number, Reynolds numbers correspond to $\mathrm{Re}_{\mathrm{n} 1}=1.38 \times 10^{6}$ and $\mathrm{Re}_{\mathrm{n} 2}=$ $2.76 \times 10^{6}$. The estimated uncertainty of the experimental data is $100 r_{c} / R=0.06$.

tunnel at MARIN in open water conditions at various advance ratios and cavitation numbers. Each condition was tested at two shaft rotation rates to investigate the effect of Reynolds number $\mathrm{Re}_{\mathrm{n}}$, with this Reynolds number defined as $\operatorname{Re}_{\mathrm{n}}=n D^{2} / v$ with $n$ the shaft rotation rate, $D$ the propeller diameter, $D=0.34 \mathrm{~m}$, and $v$ the kinematic viscosity. The propeller, denoted S5280 or 'propeller V', has four blades of conventional planform with no skew or rake, and is tip-loaded with pitch ratio $P_{t i p} / P_{0.7}=1.02$. The pitch at $0.7 R$ equals $P_{0.7} / D=0.74$. The cavity size was obtained by analysing photographs of the cavity diameter at an angle of 45 deg shaft 
Table 7.1: Effective open water advance ratios for the propeller tests in the cavitation tunnel by Kuiper (1981).

\begin{tabular}{l|ccc}
\hline & $J=0.3$ & $J=0.4$ & $J=0.5$ \\
\hline $\mathrm{Re}_{n}=1.38 \times 10^{6}$ & 0.28 & 0.37 & 0.48 \\
$\mathrm{Re}_{n}=2.76 \times 10^{6}$ & 0.33 & 0.39 & 0.52 \\
\hline
\end{tabular}

rotation behind the tip. Comparing these results with photographs taken after one revolution showed that the cavity size decreased with downstream distance. The cavity size increased a little when increasing the air content in the facility. The effect of roughness on the blade surface was investigated by applying $60 \mu \mathrm{m}$ carborundum on the blade surface between $0.9 \mathrm{R}$ and the tip. The roughness was applied along the whole chord length except for the edge of the blade. The roughness was applied on the back of one blade and on the face of another blade. Two blades were smooth but the geometry of one of these blades differed from the other blades causing differences in cavitation inception and cavity size. The results for this blade have been excluded from the present analysis. Overall, the effect of air content and roughness was within the measurement uncertainty so the effect was not very significant and is not discussed further.

The measurements were performed for advance ratios $J=0.3,0.4$ and 0.5 and varying cavitation numbers $\sigma_{n}$, with $J$ and $\sigma_{n}$ defined as

$$
\begin{array}{r}
J=\frac{W}{n D} \\
\sigma_{n}=\frac{p_{s}-p_{v}}{\frac{1}{2} \rho n^{2} D^{2}}
\end{array}
$$

with $W$ the tunnel speed and $p_{s}$ the pressure at the propeller centre. However, due to tunnel wall effects, the thrust-coefficient of the propeller in the tunnel was different from the thrustcoefficient of the open water test performed in a towing tank. Therefore, for each shaft rotation rate an effective advance ratio was determined for which the measured thrust-coefficient in the tunnel was equal to that of the open water test. These effective advance ratios, given in Table 7.1, were also used in the PROCAL computations.

The parameters of the Proctor vortex model were tuned using data for $J=0.4$ and $J=0.5$. The size of the viscous core as obtained from the data of Jessup (1989) had to be multiplied with a factor 1.7 to get good results. A comparison between the predictions for cavity radius $r_{c}$ and the experimental data is presented in Figure 7.2. The agreement for $J=0.4$ and $J=0.5$ is reasonable but for $J=0.3$ the model predicts too small values for $r_{c} / R$. Most likely, for this advance ratio the vortex cavity is connected to a sheet cavity on the blade, which leads to an increase of the radius of the vortex cavity, as also discussed in Section 3.3.2. As shown in Table 7.1, the equivalent open water advance ratios were slightly different for the two Reynolds numbers which leads to a slightly different non-dimensional loading. The effect of this change in loading leads to a difference in cavity size of less than 5\% which is considered negligible in the presented comparison.

The effect of the change in Reynolds number is reasonably well predicted. The predictions also suggest that with decreasing $\sigma_{n}$, or increasing $r_{c}$, the effect of the difference in Reynolds number decreases, but in the experimental data only the results for $J=0.4$ give some support 
to this trend.

\subsection{Estimation of the centre frequency and level}

The spectrum is modelled by assuming a spectral shape and by a prediction formula for the frequency and level of the centre of the hump. The spectral shape is discussed in Section 7.4. The prediction formula for the frequency and the level was obtained by a curve fit of measured values of frequency and level in terms of parameters like cavitation number and cavity size. Use was made of a combination of model-scale, measured in the Depressurized Wave Basin (DWB), and full-scale experimental data for hull pressures obtained for twin-screw vessels. The curve-fit was performed using non-dimensional parameters. The rms pressure amplitudes were converted into $k_{p}$-values according to Eq. (7.5) in which $\Delta f$ corresponds to the resolution bandwidth of the amplitude spectrum and $f_{b p}$ to the blade passage frequency, see also Section 5.4.2. The levels were converted to decibel values according to Eq. (7.6). The frequencies were made non-dimensional using $f_{b p}$.

$$
\begin{aligned}
k_{p} & =\frac{p_{r m s}}{\rho n^{2} D^{2} \sqrt{\Delta f / f_{b p}}}, \\
k_{p}[d B] & =120+20 \log _{10}\left(k_{p}\right) .
\end{aligned}
$$

The resulting spectrum can then be interpreted as a non-dimensional power density spectrum or non-dimensional rms amplitude density spectrum. The hull-pressure spectra were converted to source levels by correcting for the propeller-hull clearance and for the solid boundary factor. The solid boundary factor was computed using the formulation described in Section 5.5.1. Only the centre pressure sensor, located directly above the 12 o'clock position of the propeller, was considered, since measurements at this location were available for all data sets.

To focus on the broadband character, the spectrum was converted to 1/6 octave band levels and scaled back to a power density spectrum, an example of which is given in Figure 7.3. The resulting spectrum then becomes a smoothened power density spectrum. Next, the centre frequency, the maximum level and the bandwidth of the hump were determined by a curve fit. In Figure 7.3, the centre frequency of the curve fit was determined at $f_{c} / f_{b p}=7.3$.

The parameters for the semi-empirical model are the cavity size $r_{c}$ made non-dimensional with propeller diameter $D$, the cavitation number $\sigma_{n}$ and the number of blades $Z$. The empirical relation for the maximum level, $k_{p \text {, max }}$, which equals the level of the centre of the hump, is given by

$$
k_{p, \max }=a_{p}+20 \log _{10}\left[\left(\frac{r_{c}}{D}\right)^{\kappa_{1}} \sqrt{Z}\right]
$$

in which the non-dimensional empirical constants $a_{p}$ and $\kappa_{1}$ were obtained by curve fitting of Eq. 7.7 to the measured values for $k_{p \text {,max }}$. Raestad (1996) has used $\kappa_{1}=2$ in his formulation but the value $\kappa_{1}=3$ is used in the present method, as this is closer to the trend seen in the datasets. The contribution from all $Z$ blades is summed as a set of incoherent sources.

The formulation for the centre frequency, $f_{c}$, is based on theoretical considerations and is 


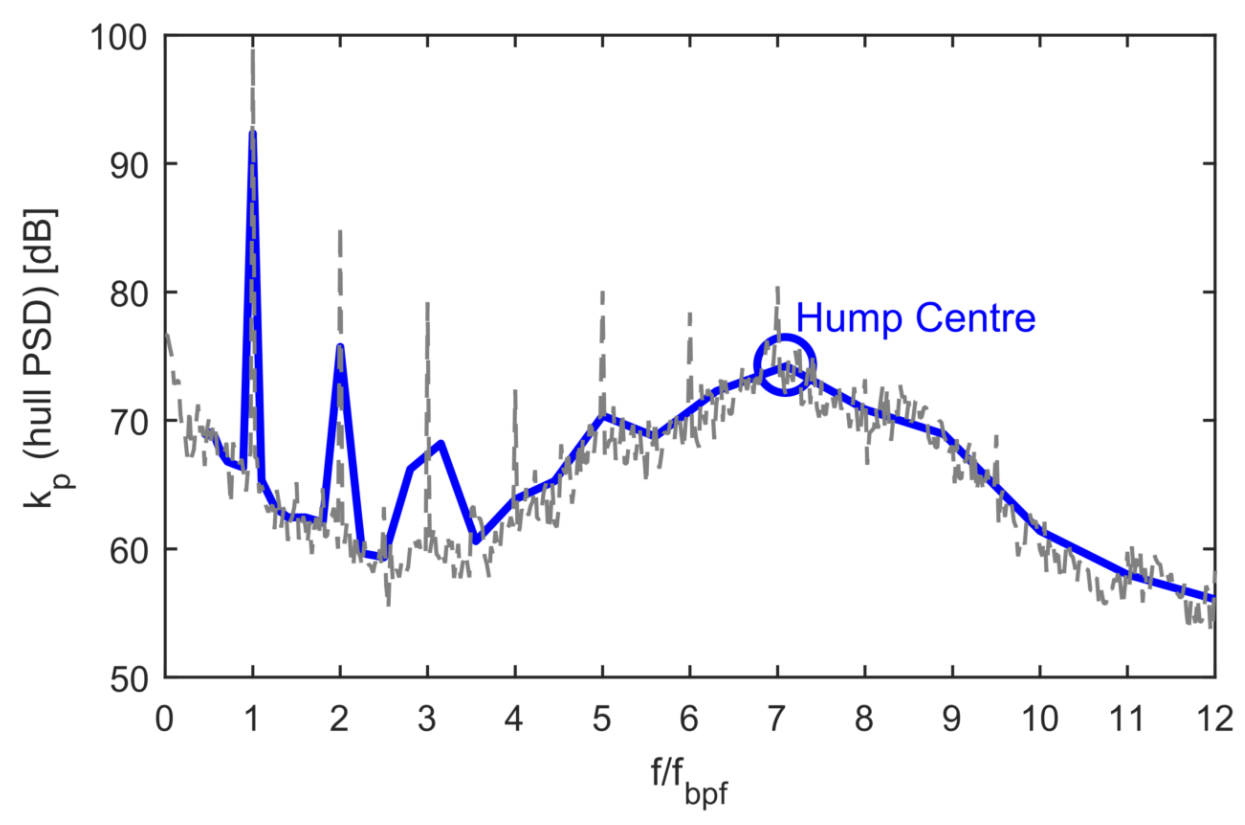

Figure 7.3: Example of a non-dimensional hull pressure power density spectrum of the twobladed research propeller. The solid line is the $1 / 6$ octave band smoothened spectrum.

given by

$$
\frac{f_{c}}{f_{b p}}=b_{f} \frac{1}{r_{c} / D} \frac{\sqrt{\sigma_{n}}}{Z}
$$

with $b_{f}$ a non-dimensional empirical constant. Raestad derived this relation from the resonance frequency of a bubble, but the relation can also be derived from the dispersion relation for a cavitating vortex as discussed in Section 6.2 and derived in Section 4.3.

The vortex strength used in the vortex model to compute the cavity radius was obtained from the maximum value of the circulation at $0.95 \mathrm{R}$ for all blade positions computed by PROCAL, using model-scale measured wake fields. For the analysis of sea-trial conditions, these wake fields were scaled using a method developed in a CRS working group (Hally, 2002). This method decomposes the ship wake into the contribution from the hull boundary layer and from the wake of struts and shaft, and scales these components separately using semi-empirical formulations.

The ships in the experimental dataset used to check the validity of Eqs. (7.7) and (7.8) and to determine the empirical constants $a_{p}, \kappa$ and $b_{f}$, were twin-screw vessels equipped with fixed-pitch podded propellers, and with fixed or controllable pitch propellers with an openshaft arrangement. The test conditions were close to or in the design condition for varying ship speeds. During the process of determining the empirical constants, it became apparent that the experimental datasets had to be divided in separate groups. A distinction was made between cases for which tip-vortex cavitation was dominant and cases for which, close to the tip, sheet cavitation was also present. Figure 7.4 shows examples of these two different situations. These two situations were also expected in the fit of the cavity size for the propeller tip vortex cavitation as discussed in Section 7.2. 


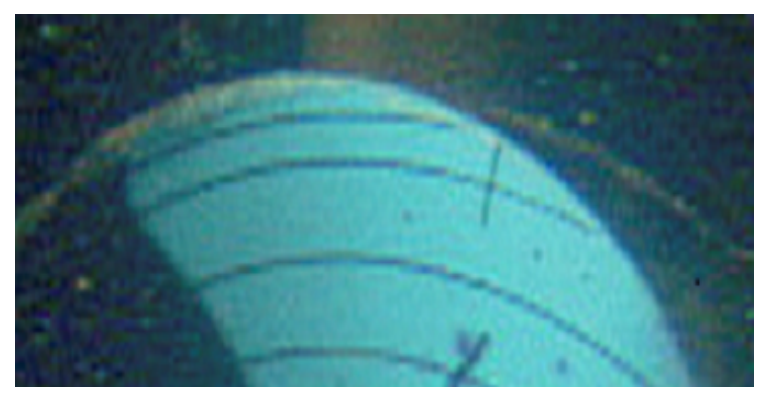

a) Tip vortex cavitation

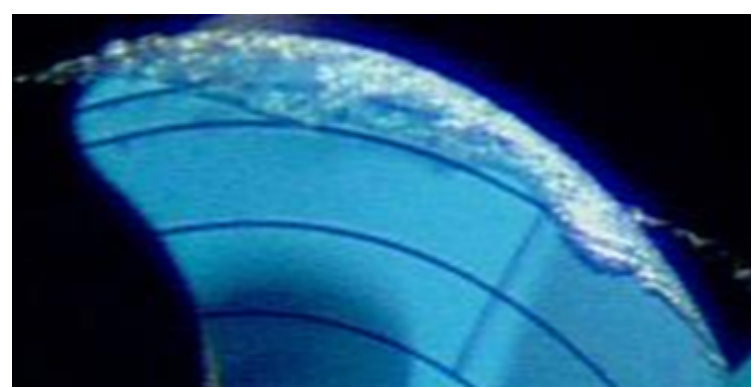

b) Tip-sheet and tip-vortex cavitation

Figure 7.4: Examples of cavitation patterns on the propellers used for the development of the method.

The accuracy of the fit for $k_{p, \max }$ and for $f_{c}$ for the two groups is presented in Figure $7.5 \mathrm{a}$ and Figure 7.5b, respectively. An example of the fit of the noise level for an individual data set, not included in Figure 7.5, is presented in Figure 7.6. The propeller is the two-bladed research propeller with skew and tip-unloading tested for a range of cavitation numbers and thrust coefficients, described in Section 6.3.1. The cavitation pattern on this propeller is a cavitating vortex structure that is generated at the leading edge. All results presented in Figure 7.5 and 7.6 are for the Proctor vortex model. Similar results could also be obtained with an inviscid-flow vortex models, but with different empirical constants. This shows that, for the test-cases considered, the cavity size is in general much larger than the viscous core size.

The results in Figure 7.5 are quite reasonable for the propellers with vortex cavitation although the standard deviation of the frequency is rather high. In particular, one condition is poorly predicted. The standard deviation for the propellers with tip-sheet and vortex cavitation is significantly higher than for the group with tip-vortex cavitation only. However, Figure 7.6 shows that the trend for a single propeller with tip-sheet and vortex cavitation is well predicted employing the tuned parameters. Remarkable was that a good fit for the variation of the centre frequency for this two-bladed propeller could only be obtained if a constant was added to the right-hand-side of Eq. (7.8). This constant was not required for the curve fit for the results presented for actual ship propellers in Figure 7.5b. This aspect was not understood and is left open for further research. 


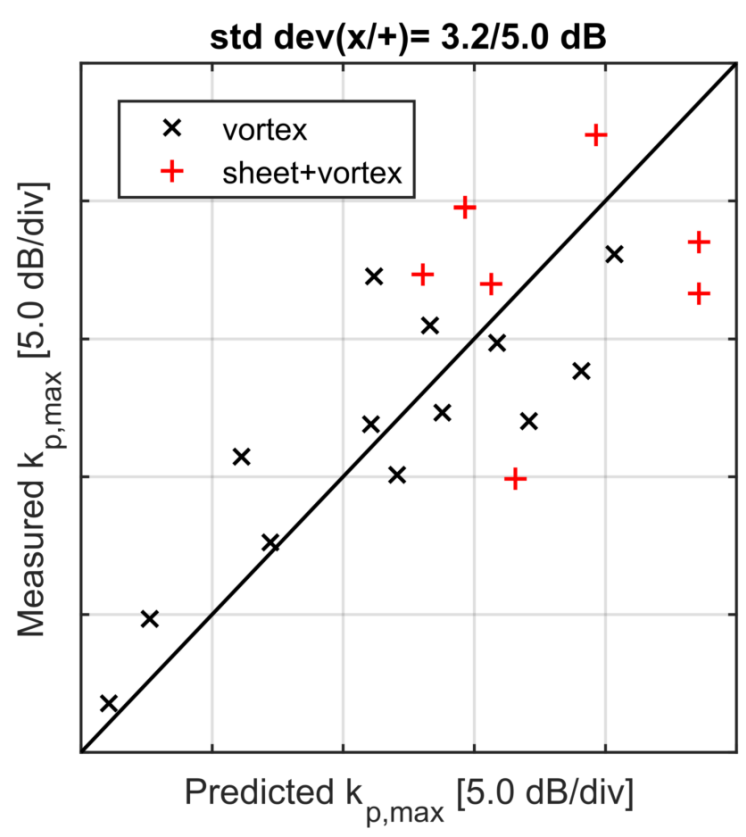

a) Fit of maximum level

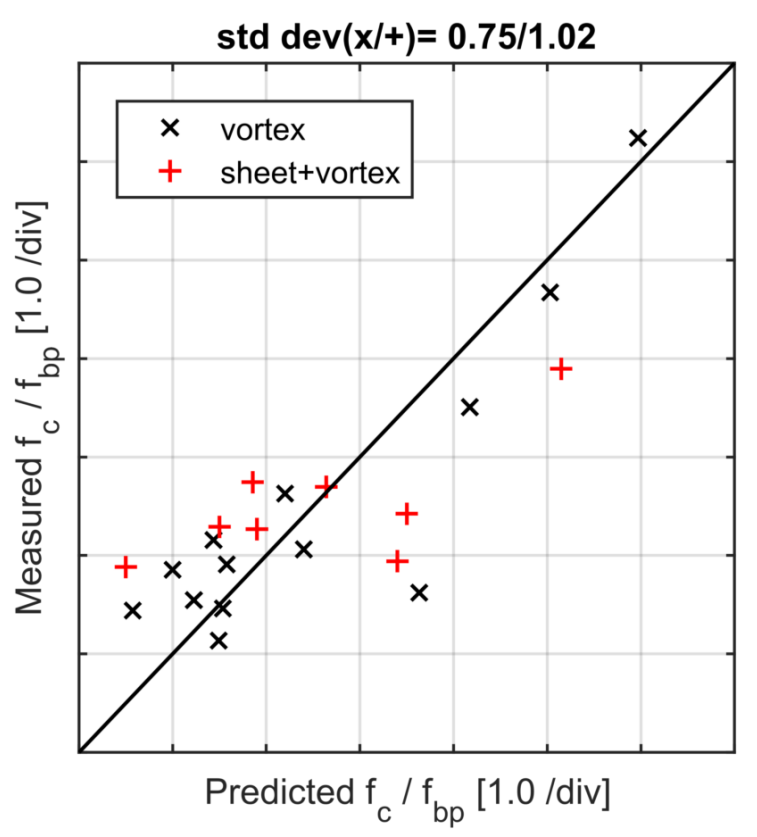

b) Fit of centre frequency

Figure 7.5: Comparison between measured and predicted levels and frequency of the centre of the hump for the cases used in the development of the method.

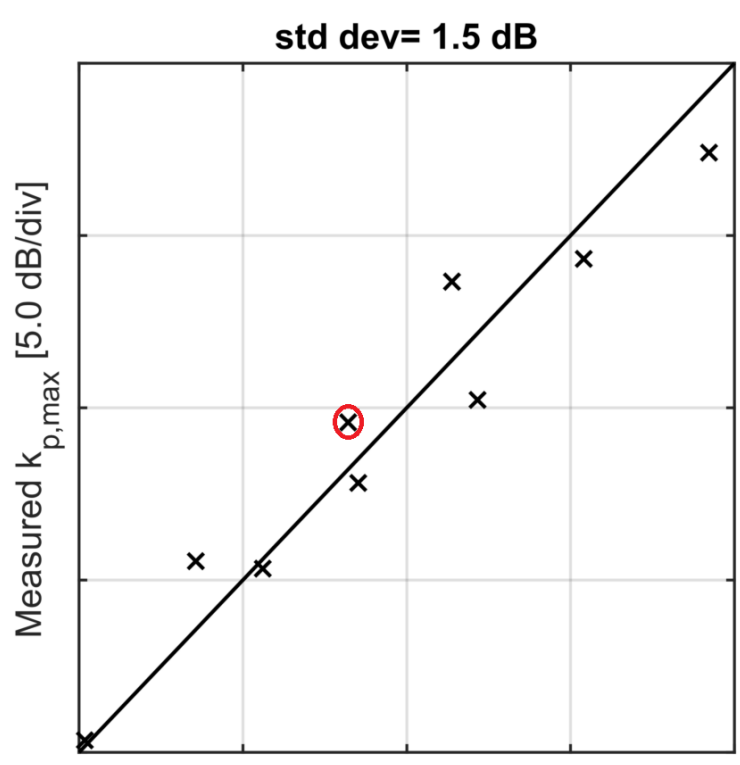

Predicted $k_{p, \max }[5.0 \mathrm{~dB} / \mathrm{div}]$

a) Fit of maximum level

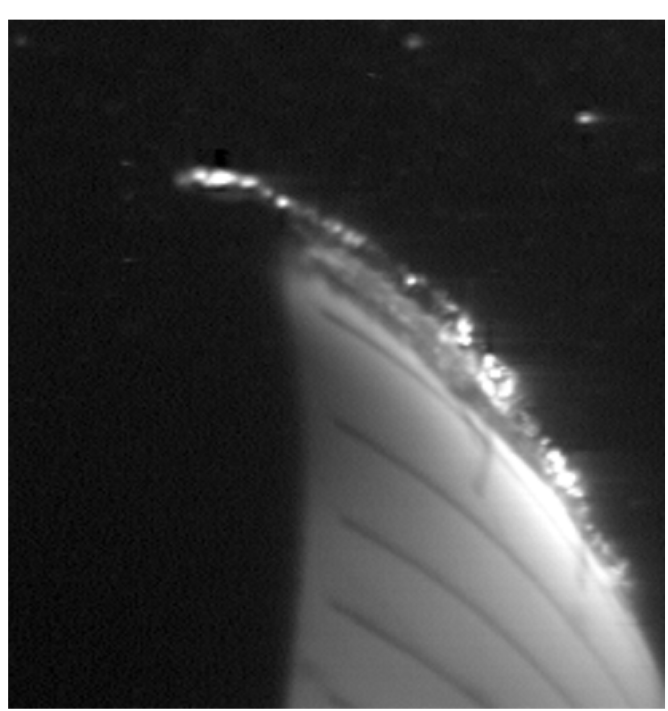

b) Typical cavitation pattern

Figure 7.6: (Left:) Comparison of measured and predicted maximum level of the hump for a two-bladed research propeller tested at various advance ratios and cavitation numbers. The cavitation pattern corresponding to the encircled symbol is shown on the right. 


\subsection{Spectral shape}

The shape of the spectrum of cavitation noise is described by e.g. Fitzpatrick and Strasberg (1956), Brown (1976), Lövik (1981) and Blake (1986). The spectrum can be divided in a low-frequency part and a high-frequency part. The low-frequency part is characterized by a hump due to the overall growth, collapse and rebounds of the cavity. The high-frequency part is related to the final phase of the collapse process during which velocities may approach or exceed the speed of sound, and compressibility effects become important. The collapse and rebounds of the smaller size bubbles generated by the collapse of the large scale structure may also contribute to the high-frequency part.

In the present method, it is assumed that $f_{c}$ corresponds to a resonance frequency of the vortex cavity. The related pressure signal is a damped oscillatory signal and the corresponding spectrum is presented in Section 5.3.2. However, the hump can also be described well by a shifted sinc function, corresponding to a spectrum of an oscillatory signal multiplied with a rectangular window. This sinc function is preferred from an implementation point of view as the bandwidth does not affect the amplitude and the amplitude is already normalized. Therefore, the shape of the spectrum, presented in decibel values, has been defined as

$$
H_{h}(f)=20 \log _{10}\left\{\operatorname{sinc}\left(\frac{f-f_{c}}{0.830 \Delta f_{-6 \mathrm{~dB}}}\right)\right\}
$$

where $\Delta f_{-6 \mathrm{~dB}}$ corresponds to the bandwidth of the hump for which the pressure amplitude is equal to half the maximum. A small value of $\Delta f_{-6 \mathrm{~dB}}$ corresponds to a time trace with multiple rebounds (small damping) resulting in a narrow hump in the spectrum. A large value of $\Delta f_{-6 \mathrm{~dB}}$ corresponds to a highly damped system resulting in a wide hump in the spectrum. Analysis of experimental data suggested that $\Delta f_{-6 \mathrm{~dB}}$ is proportional to $f_{c}$, though the scatter was high.

The shape of the spectrum is modelled differently at frequencies much lower and much higher than the centre frequency of the hump. The simple model used here consists of prescribed slopes at low and high frequency in a power density spectrum,

$$
H_{S}(f)=10 \log _{10}\left\{\frac{2\left(f / f_{c}\right)^{\alpha_{l}}}{1+\left(f / f_{c}\right)^{\alpha_{l}-\alpha_{h}}}\right\}
$$

in which $\alpha_{h}$ corresponds to the slope for high frequency, with a typical value $\alpha_{h}=-2$, and $\alpha_{l}$ corresponds to the slope for low frequency, for which a value of $\alpha_{l}=4$ is used, as suggested by Fitzpatrick and Strasberg (1956). For different values of $\alpha_{l}$ and $\alpha_{h}$, the maximum value of $H_{s}$ is different from $0 \mathrm{~dB}$, which needs to be corrected for.

The resulting spectrum is then taken as a weighted sum of powers of the two spectral functions,

$$
H(f)=10 \log _{10}\left\{\alpha 10^{H_{h}(f) / 10}+(1-\alpha) 10^{H_{s}(f) / 10}\right\}
$$

where $\alpha$ is a user-defined parameter. For practical applications, this parameter is computed from

$$
\Delta L_{\alpha}=10 \log _{10}(1-\alpha)
$$

with $\Delta L_{\alpha}$ the difference in noise level between the maximum of the two-slope function and the maximum of the hump. 


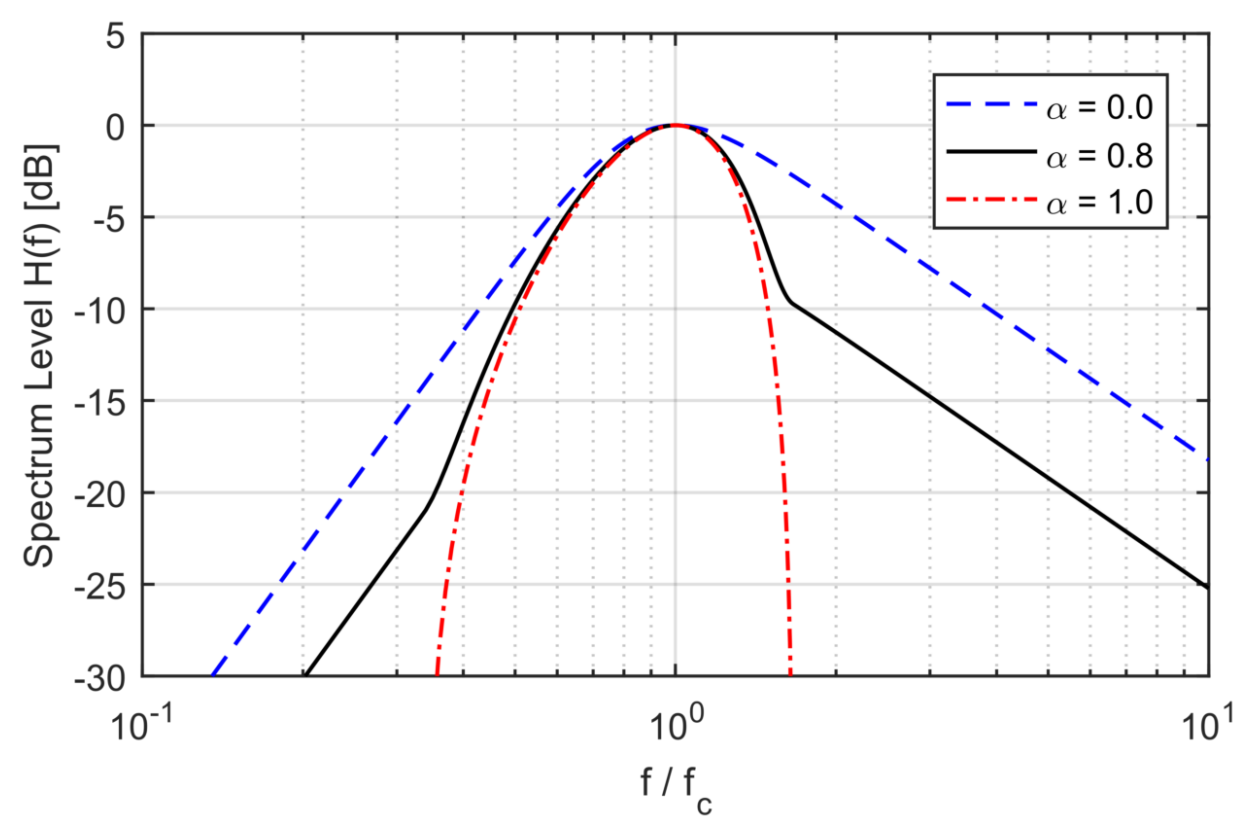

Figure 7.7: Shape of the spectrum for different values of $\alpha$.

Examples of the shape of the spectrum of the source level are given in Figure 7.7 for three values of $\alpha$. The curve for $\alpha=0.0$ corresponds to the spectrum of the two-slope function and the curve for $\alpha=1.0$ corresponds to the spectrum of the sinc function. In its current version, the default value is $\alpha=0.8$. For some cases, however, the default values do not give a good representation of the measured spectrum. It is remarked that the maximum absolute value of the argument of the sinc function has been limited to avoid the presence of 'sidelobes' in the spectrum.

The resulting source-level spectrum $(S L)$ can now be defined as

$$
S L(f)=L_{p, \max }+H(f) \quad\left[\mathrm{dB}, \mathrm{re} 1 \mu \mathrm{Pa}^{2} \mathrm{~m}^{2} / \mathrm{Hz}\right]
$$

with $L_{p, \max }$ the maximum level of the hump in the power density spectrum, which is the dimensional value of $k_{p \text {, max }}$.

The spectrum of the underwater radiated noise level $(R N L)$ is computed from the $S L$ by correcting for the interference of the underwater radiated noise with the sea surface, referred to as the propagation loss due to Lloyd's mirror $\left(P L_{L M}\right)$,

$$
R N L(f)=S L(f)+P L_{L M}(f)\left[\mathrm{dB}, \mathrm{re} 1 \mu \mathrm{Pa}^{2} \mathrm{~m}^{2} / \mathrm{Hz}\right]
$$

Formulations for $P L_{L M}$ have been presented in Section 5.5.2. For the results presented in the next section, Eq. (5.142) by Ainslie (2010) has been used. The RNL levels, or URN levels in general, are often presented in one-third-octave band levels denoted by $R N L_{1 / 3}$ and $U R N_{1 / 3}$, respectively.

The shape of the spectrum and the centre frequency of the HPF is identical to that of the source level. The maximum level of the HPF is computed from the maximum source level by correcting for the solid boundary factor and the propeller-hull clearance. 


\subsection{Results}

\subsubsection{Hull-pressure fluctuations}

An example of the resulting spectrum of hull pressures predicted by the ETV model for the two-bladed research propeller is presented in Figure 7.8. Overall, the hump in the spectrum is well represented by the default spectral shape. The values of the empirical parameters for the maximum level and for the centre frequency were obtained from a dedicated fit to the test series for this propeller, with the accuracy of the fit for the maximum level for various testcases given in Figure 7.6a. Examples of spectra for ship configurations used in the database are presented for model scale and full scale in Figure 7.9a and Figure 7.9b, respectively. Both cases are part of the vortex-cavitation group for which the accuracies of the fits are presented in Figure 7.5. The results of the model test show some disturbance at the eighth harmonic of the blade passage frequency generated by the propeller drive train. However, this frequency is located at a much higher frequency than the broadband hump due to the cavitating tip vortex, and it does, therefore, not contribute to the broadband hump. Both figures are examples of cases for which the spectrum is well predicted by the model. As shown in Figure 7.5, there are also cases for which the maximum level and centre frequency are predicted less satisfactorily.

The model- and full-scale data are all made non-dimensional using Eq. (7.5). Since no significant differences in the trend of the maximum level and centre frequency with scale could be discerned, it is concluded that Eq. (7.5) can be used to scale the broadband pressure spectra from model scale to full scale. In Section 5.4, it was already shown that this relation is identical to the low-frequency scaling relation for URN.

The ETV-model has also been applied to predict the broadband hull pressure levels for the $85 \mathrm{~m}$ Combi Freighter equipped with a single controllable pitch propeller. This ship was not in the database and, being a single screw vessel, it also does not resemble any ship in the data base. The sea trials for the ship, which included URN measurements in deep water, were performed by DAMEN, DNV and MARIN collaboratively and were financed by the CRS BROADBAND2 working group. The effective wake field for the PROCAL computation was obtained from a coupled RANS-BEM procedure (Starke and Bosschers, 2012; Rijpkema et al., 2013). Details of this data set are given in Lafeber et al. (2017) and Lloyd et al. (2018) which show a comparison of the full-scale URN levels with predictions using data from model tests in the DWB. The cavitation pattern on the propeller as observed in the model test for this condition is presented in Figure 6.17. The values for the empirical constants derived using the complete database were used in the ETV method.

The comparison of broadband HPF predicted by the ETV method and measured during the sea trial at 10 knots is reasonable, as illustrated in Figure 7.10a. It is notable that the hump in the spectrum for this single-screw ship is overpredicted by the ETV method. The URN of this vessel is dominated by a very pronounced hump that will be discussed later. This hump is not so obvious in the pressures measured by the centre transducer, P1. However, it can clearly be seen in the spectrum of the pressure transducer, P2, located closer to the collapse of the vortex, although it is expected that the spectrum for this transducer is affected by vibrations of the hull plating.

In general, the results were considered to be quite acceptable for a range of pitch settings 


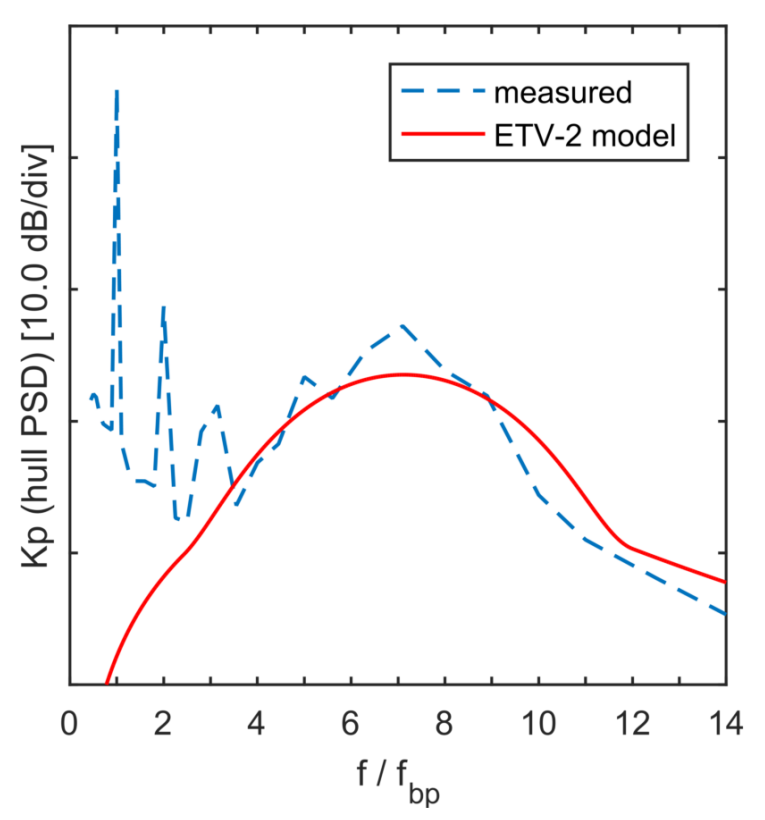

a) Case 1

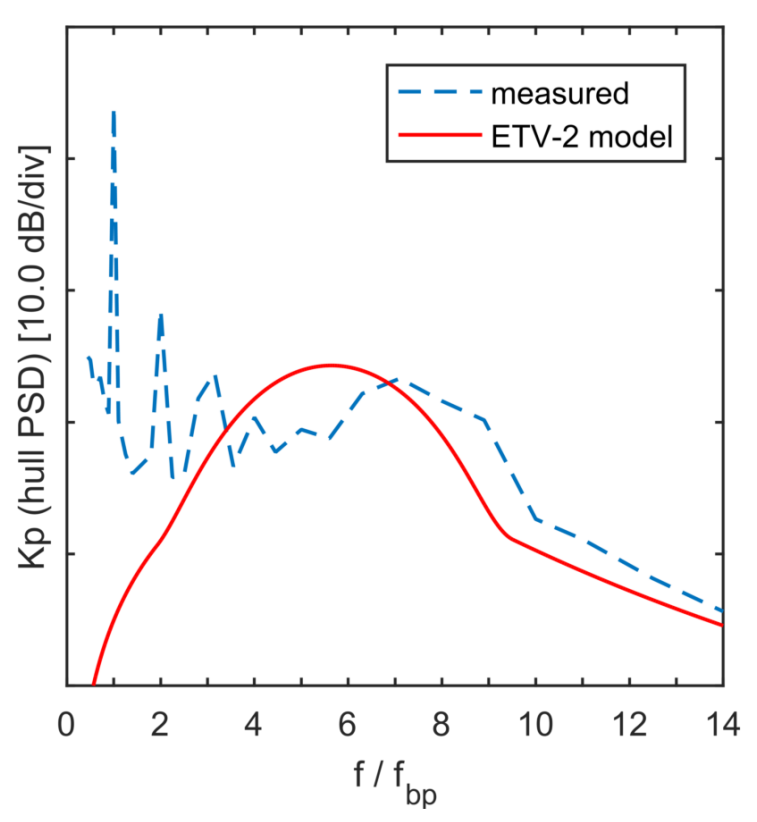

b) Case 2

Figure 7.8: Example of the measured and predicted HPF spectrum for the two-bladed research propeller.

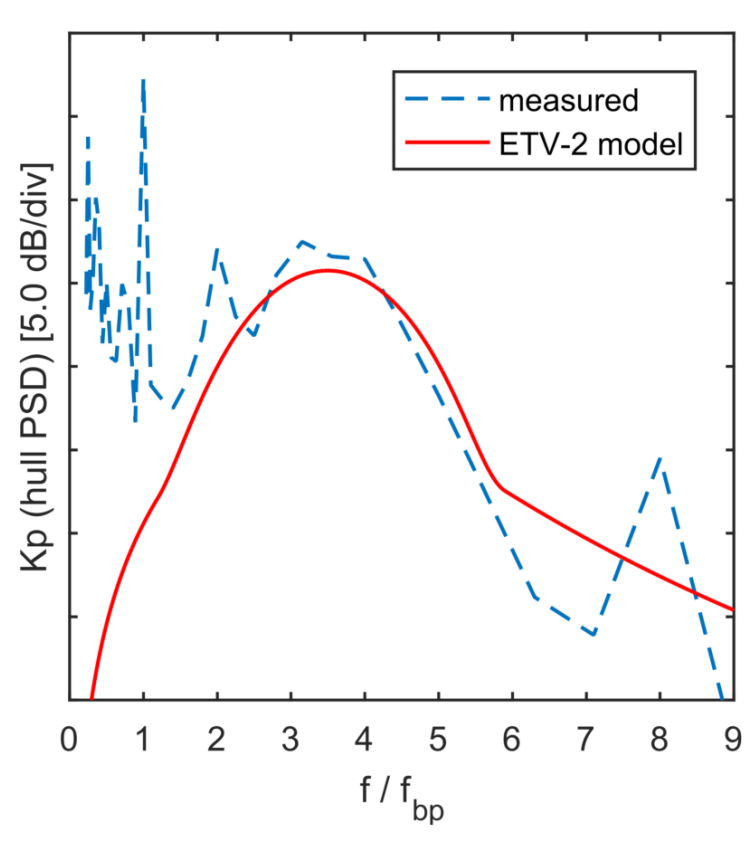

a) Model scale

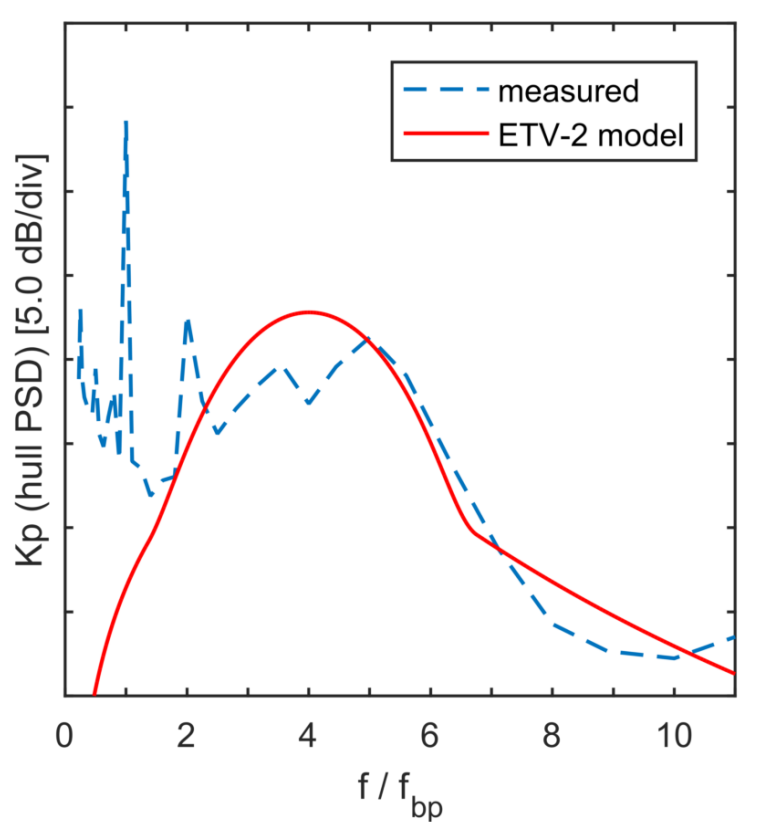

b) Full scale

Figure 7.9: Examples of the measured and predicted HPF spectrum for ship configurations of the database.

and shaft rotation rates as long as the cavitation was present on the back side of the blade. In its present form, the model was not capable of predicting the broadband HPF spectrum due to face side cavitation that occurs for a low pitch setting such as presented in Figure 6.18. 


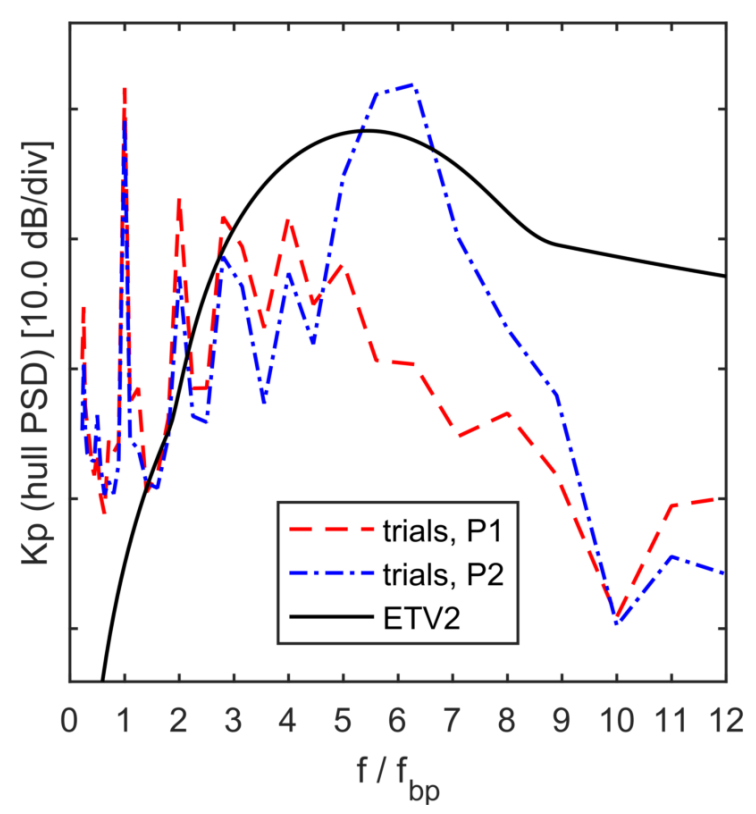

a) Default, $\Delta L_{\alpha}=-7 \mathrm{~dB}$

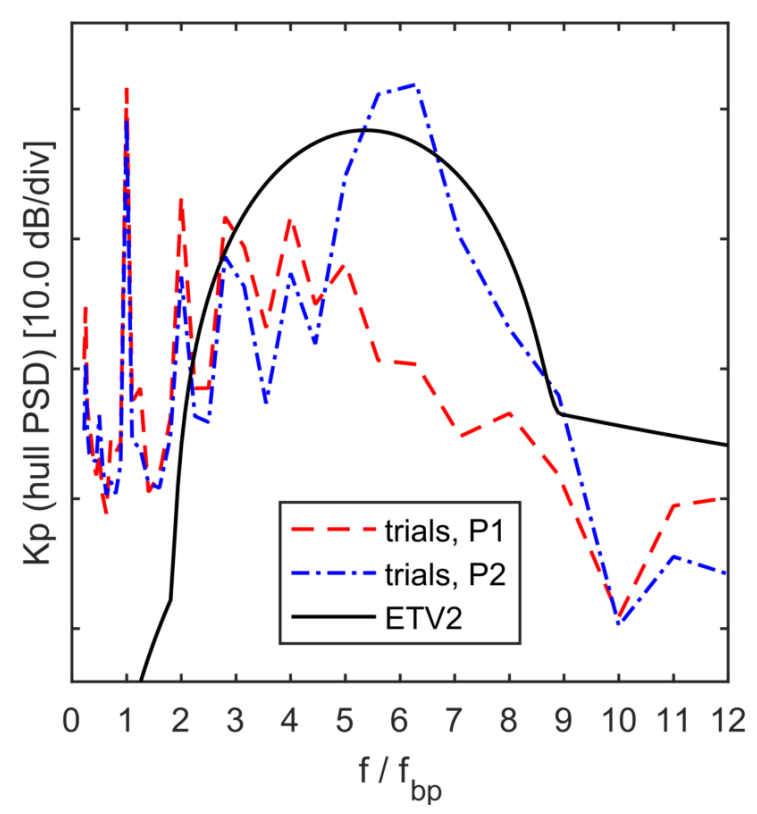

b) $\Delta L_{\alpha}=-20 \mathrm{~dB}$

Figure 7.10: Measured and predicted HPF spectrum for the Combi Freighter at a ship speed of 10 knots. P1 corresponds to the centre pressure transducer and P2 is located closer to the cavity collapse. Sea-trial data by DAMEN and MARIN.

\subsubsection{Underwater radiated noise}

The model that was developed using data for hull pressures has also been applied to predict the URN due to propeller cavitation. The first test case considered for the prediction of URN is the single-screw Combi Freighter also discussed above. The noise measurements were performed in deep water by DNV-GL according to the procedure for their Silent Class notation. The noise levels for the default values of the method are presented in Figure 7.11a. It is seen that the hump in the spectrum is very pronounced. The centre of the hump is well predicted in terms of level and frequency, but, at high frequencies, the predicted noise levels are approximately $10 \mathrm{~dB}$ too high. The difference in noise levels of the hump and noise levels in the high-frequency region can easily be adjusted for with the parameter $\Delta L_{\alpha}$. Results for $\Delta L_{\alpha}=-20 \mathrm{~dB}$ are presented in Figure 7.11b. The default value for the slope of the spectrum at high frequencies is in good agreement with the sea trial data. The hull-pressure prediction for this setting of $\Delta L_{\alpha}$ is also in better agreement with the experimental data, see Figure 7.10b.

The second data set analysed is that of the twin-screw cruise vessel MS Statendam, sailing at 18 knots as reported by Kipple (2002). The length between perpendiculars is $182 \mathrm{~m}$ and the ship is driven by controllable pitch propellers. The URN measurements were performed in deep water. The measured SL are directly taken from Kipple and the RNL are computed from the received noise levels at 500 yards as reported by Kipple. All measured levels are converted to levels at $1 \mathrm{~m}$ distance assuming spherical spreading loss. The applied formulation by Kipple (2002) to compute the source levels from the measured radiated noise levels is unknown but the difference in results of SL and RNL is similar to those given by Eq. (5.137) for a rough sea surface. Results with default values are presented in Figure 7.12a. The maximum level in the 


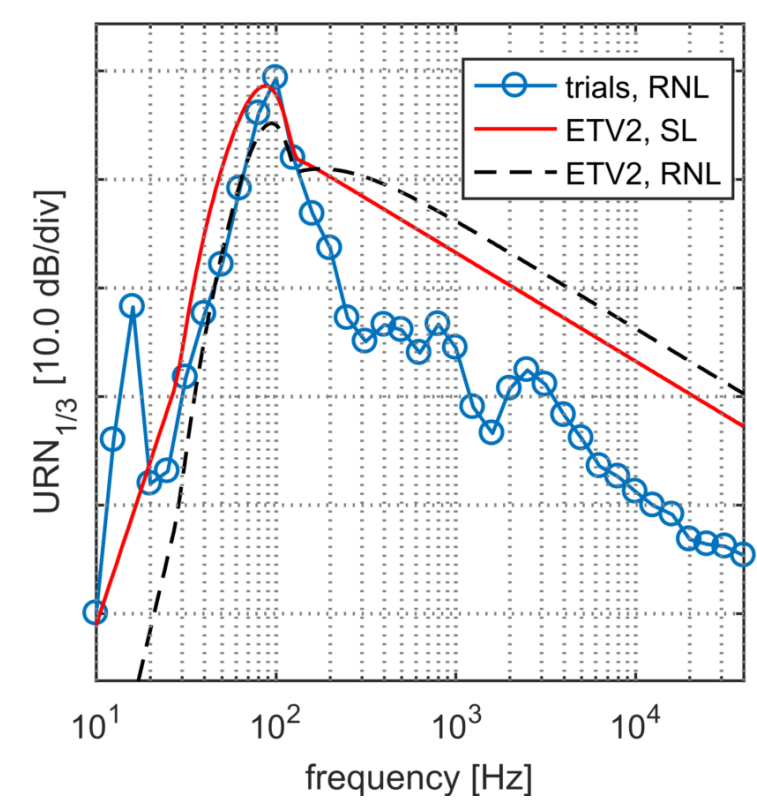

a) Default, $\Delta L_{\alpha}=-7 \mathrm{~dB}$

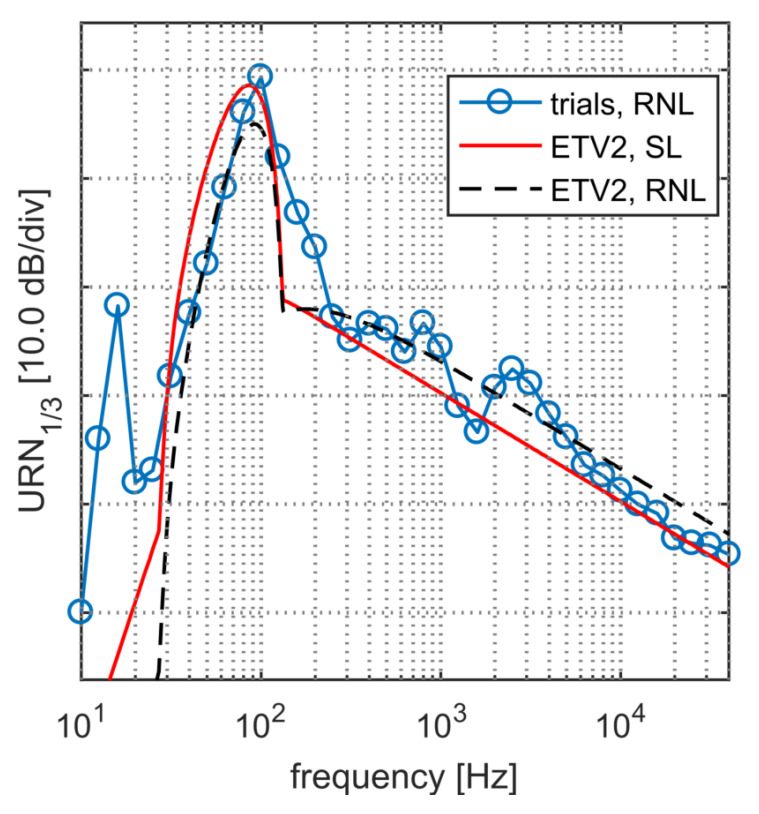

b) $\Delta L_{\alpha}=-20 \mathrm{~dB}$

Figure 7.11: Measured and predicted radiated noise spectrum in one-third octave band levels for the Combi-Freighter at a ship speed of 10 knots. Sea-trial data by DNV-GL.

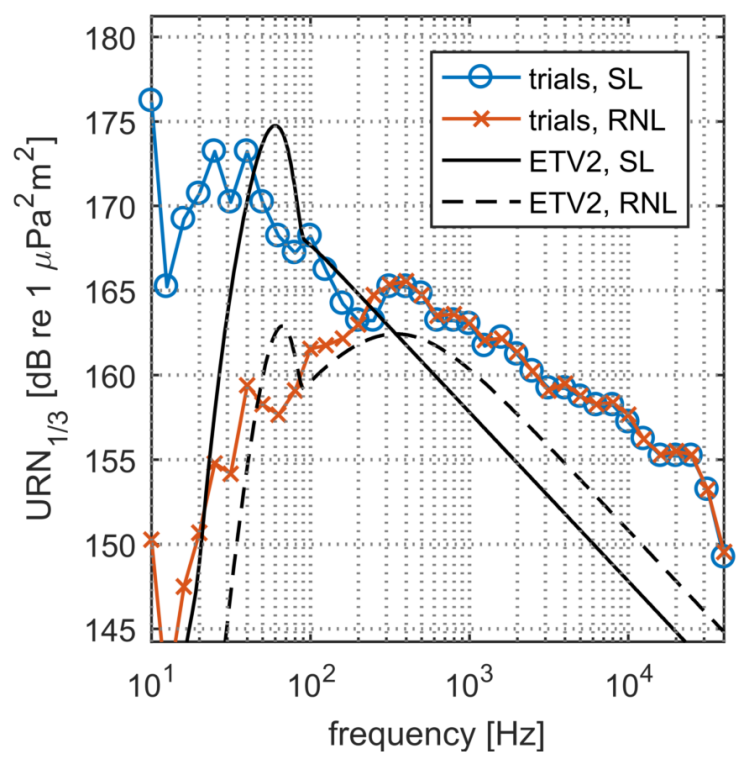

a) Default, $\alpha_{h}=-2.0$

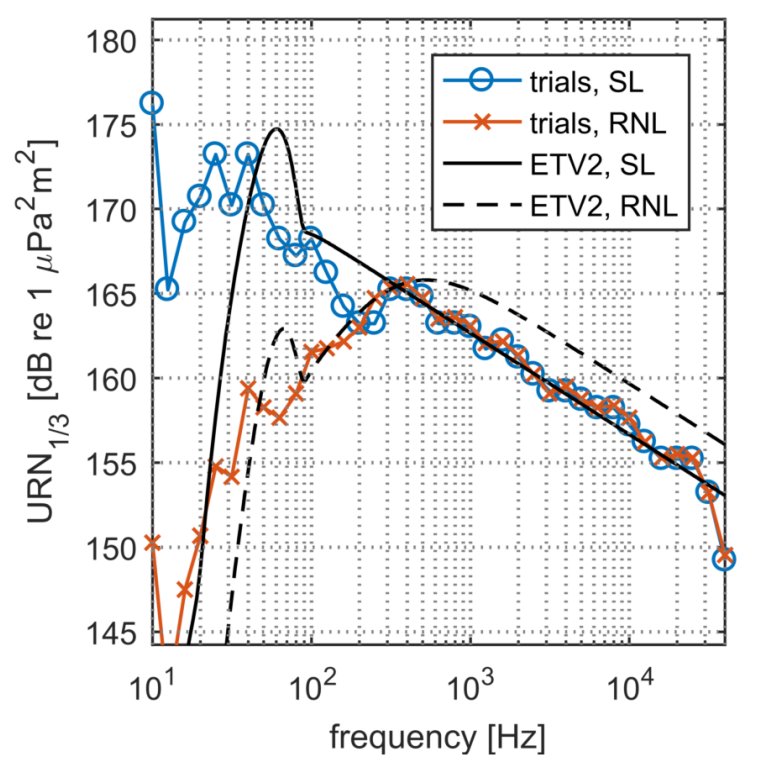

b) $\alpha_{h}=-1.6$

Figure 7.12: Measured and predicted URN spectrum in one-third octave band levels for a cruise vessel at a ship speed of 18 knots. Sea-trial data by Kipple (2002).

hump is very well predicted but the measured values in that frequency region are reported to be mainly due to machinery noise. At high frequencies, the agreement is not as good because the slope of the spectrum in the prediction is smaller than in the experiments. Adjustment of the high-frequency slope to $\alpha_{h}=-1.6$ gives good agreement, see Figure 7.12b. 


\subsubsection{Scaling with ship speed}

Whereas in the preceding sections a comparison has been made between measured and predicted spectra of HPF and URN, in this section we investigate the scaling of URN with ship speed. Use is made of the data by Strasberg (1977) for a World War II class submarine for which the cavitation pattern is dominated by tip-vortex cavitation.

The relations in the ETV-method of maximum level, centre frequency, and cavity size can easily be converted to a relation in terms of propeller thrust coefficient if the vortex model for inviscid-flow is used. This vortex model is considered to be valid when the vortex cavity is fully developed and vorticity roll-up is completed. The relation between cavity size, cavitation number and circulation is then given by

$$
\frac{r_{c}}{D}=\frac{1}{2 \pi} \frac{\Gamma_{\infty}}{n D} \frac{1}{\sqrt{\sigma_{n}}}
$$

The non-dimensional vortex strength can be written as the product of the propeller blade thrust coefficient $K_{T} / Z$ and a tip loading parameter $\tau$. The relations for the maximum level and centre frequency of the hump then read

$$
\begin{array}{r}
k_{p, \max } \propto\left(\frac{r_{c}}{D}\right)^{\kappa_{1}} \sqrt{Z} \propto\left(\frac{\tau K_{T}}{Z \sqrt{\sigma_{n}}}\right)^{\kappa_{1}} \sqrt{Z} \propto n^{\kappa_{1}}, \\
\frac{f_{c}}{f_{b p}} \propto \frac{1}{r_{c} / D} \frac{\sqrt{\sigma_{n}}}{Z} \propto \frac{\sigma_{n}}{\tau K_{T}} \propto n^{-2} .
\end{array}
$$

Here, it is assumed that $K_{T}$ and $\tau$ do not change with varying ship speed $V_{s}$. If $J$ also remains constant with ship speed, we find $n \propto V_{s}$. The speed scaling of the maximum noise level for a well developed vortex cavity, expressed in values of power spectral density with unit $\left[\mathrm{Pa}^{2} / \mathrm{Hz}\right]$, is then obtained from Eq. (7.5), with $\Delta f=1 \mathrm{~Hz}$,

$$
L_{p, \max , 1 \mathrm{~Hz}} \propto 10 \log _{10}\left(n^{3} r_{c}^{2 \kappa_{1}}\right) \propto 10 \log _{10}\left(n^{3} n^{2 \kappa_{1}}\right) .
$$

For constant $J$ and $\kappa_{1}=3$, we find $L_{p, \max , 1 \mathrm{~Hz}} \propto 10 \log _{10} V_{s}^{9}$. The maximum level in proportional bandwidth, or power, in unit $\left[\mathrm{Pa}^{2}\right]$ for a well-developed vortex cavity is obtained from Eq. (7.5) with $\Delta f \propto f_{b p}$ and is given by

$$
L_{p, \max , 1 / 3} \propto 10 \log _{10}\left(n^{4} r_{c}^{2 \kappa_{1}}\right) \propto 10 \log _{10}\left(n^{4} n^{2 \kappa_{1}}\right) .
$$

For constant $J$ and $\kappa_{1}=3$, we find $L_{p, \max , 1 / 3} \propto 10 \log _{10} V_{s}^{10}$.

The overall sound pressure level (OASPL) with unit $\left[\mathrm{Pa}^{2}\right]$ is obtained by integrating the power spectral density levels over the whole frequency range. If we assume that the frequency range scales with $\Delta f_{-6 \mathrm{~dB}} \propto f_{c} \propto n^{-1}$, we find for a well-developed vortex cavity

$$
L_{\text {oaspl }} \propto 10 \log _{10}\left(n^{2} r_{c}^{2 \kappa_{1}}\right) \propto 10 \log _{10}\left(n^{2} n^{2 \kappa_{1}}\right) .
$$

For constant $J$ and $\kappa_{1}=3$, we find $L_{\text {oaspl }} \propto 10 \log _{10} V_{s}^{8}$.

The $L_{\text {oaspl }}$ can also be obtained by summing the values of $L_{p, \max , 1 / 3}$. If the number of proportional frequency bands is independent of ship speed, we find

$$
L_{\text {oaspl }} \propto L_{p, \max , 1 / 3} \propto 10 \log _{10}\left(n^{4} r_{c}^{2 \kappa_{1}}\right) .
$$




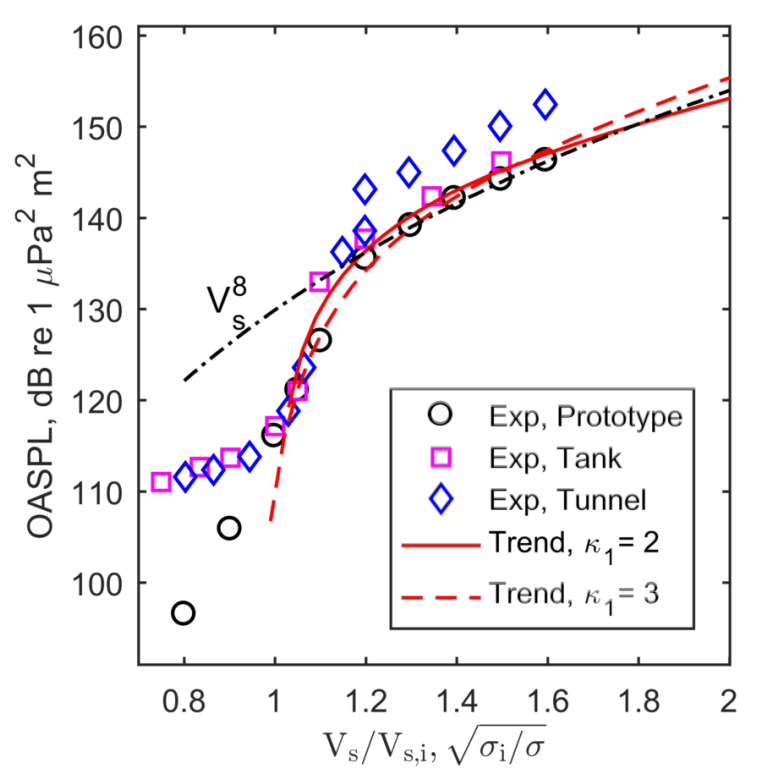

a) Eq. (7.20), $L_{\mathrm{oaspl}} \propto 10 \log _{10}\left(V_{s}^{2} r_{c}^{2 \kappa_{1}}\right)$

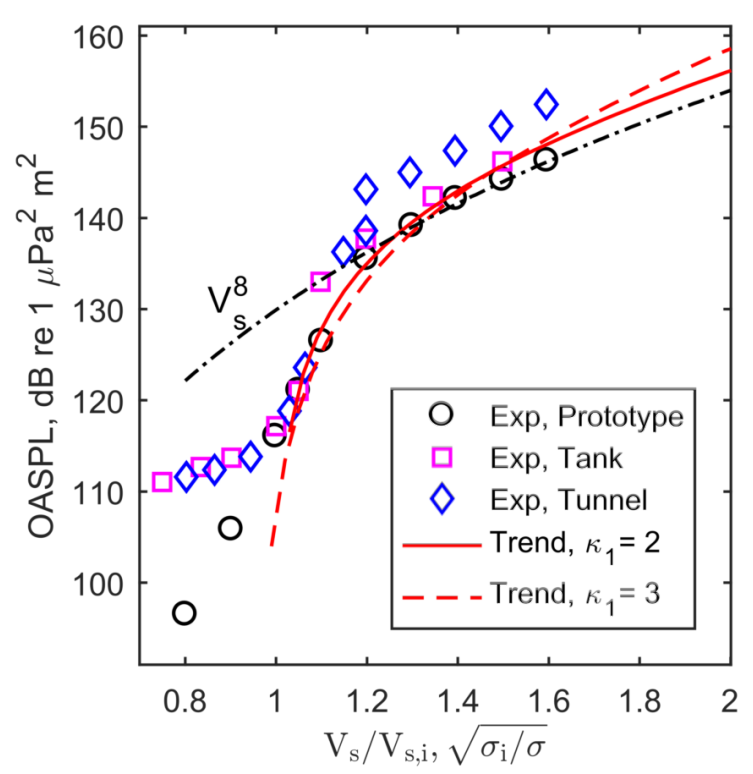

b) Eq. (7.21), $L_{\text {oaspl }} \propto 10 \log _{10}\left(V_{s}^{4} r_{c}^{2} \kappa_{1}\right)$

Figure 7.13: Speed scaling of tip-vortex cavitation on a submarine propeller, experimental data by Strasberg (1977). The predicted trend line is computed employing the Proctor vortex model using a fit for the noise level.

This results in a different scaling with ship speed than Eq. (7.20).

The speed scaling of tip-vortex cavitation noise has been presented by Strasberg (1977) for a World War II class submarine for prototype measurements and for model tests in a water tunnel and in a towing tank. The spectra show a pronounced hump and are of similar shape as adopted in the ETV-method. The data was also presented as OASPL as function of ship speed $V_{s}$ divided by ship speed at inception $V_{s, i}$. These OASPL are presented in Figure 7.13 with the values obtained from Blake (1986). Good agreement is obtained between the data set measured in the tank and as measured for the prototype, but the data measured in the tunnel are higher than in the tank and for the prototype, which might be related to reverberation. For developed cavitation, the noise levels scale, on average, as $L_{\mathrm{oaspl}} \propto 10 \log _{10} V_{s}^{8}$. At inception, a higher speed dependency is obtained. The noise levels at speeds below cavitation inception have different origin and therefore scale differently for prototype and model tests.

For the analysis of the trend lines, we will use both Eq. (7.20) and Eq. (7.21) to investigate the assumptions made. For both relations, also the sensitivity to the value of $\kappa_{1}$ is investigated by using $\kappa_{1}=2$ in addition to the default $\kappa_{1}=3$. The variation in cavity size with cavitation number, or ship speed, is computed using the Proctor vortex. As the data is presented as a ratio of $\sigma_{i} / \sigma$, the vortex strength has no effect on the trend line. Only a single value for the offset of the noise levels is required, which is selected by fitting the trend line to the average data for developed cavitation. An offset of $\Delta\left(V_{s} / V_{s, i}\right)=-0.04$ was also applied to the velocity to fit the trend line for $\kappa_{1}=3$ with the results of the experiments at cavitation inception. This offset was not required for the trend line for $\kappa_{1}=2$. The effect of the variation in Reynolds number with ship speed on the viscous core was taken into account using Eq. (7.1). The trend line is not so sensitive to the empirical parameters of the vortex model. Actually, rather similar results are 
obtained when using the Lamb-Oseen vortex.

The predictions of the various trend lines show that all investigated options show acceptable to good agreement with the experimental data due to the small range of ship speeds and the tuned offset. The scaling with $V_{s}^{8}$ can be obtained for $\kappa_{1}=2$ as well as for $\kappa_{1}=3$, depending on the formulation used. Larger variations in ship speed would be required to accurately determine the speed scaling of tip-vortex cavitation. However, this is in general not possible due to the inception of sheet cavitation at higher ship speeds. It should also be kept in mind that the speed scaling of OASPL levels for ships with cavitating propellers has been reported by Ross (1987) to behave as $L_{\text {oaspl }} \propto 10 \log _{10} V_{s}^{6}$. However, this scaling might also be affected by machinery noise levels at low frequencies. It is also not clear if the speed scaling for sheet cavitation noise is different from the speed scaling for tip-vortex cavitation noise. This topic has not been further pursued in the present study.

\subsection{Discussion}

The ETV-method has been developed to predict the broadband noise by propeller tip-vortex cavitation as present on propellers of twin-screw vessels. Both model- and full-scale hullpressure data were used for this purpose.

The majority of the test conditions were at, or close to, the design condition of the propeller. This restriction in variability of conditions was deemed necessary to avoid the effect of sheet cavitation on the measured hull-pressure data. Nevertheless, the cavitation observations showed that, for some cases, a combination of tip vortex cavitation and sheet cavitation was present. This sheet cavity detaches from the leading edge upstream of the blade tip. Due to the skew of the blade, the re-entrant jet at the cavity closure has a strong radial component, which is why it is sometimes called a side-entrant jet (Foeth et al., 2008). This side-entrant jet forms a cavity closure vortex (Bark and Bensow, 2012) that is chordwise oriented in the tip region and that collapses as a closure-vortex cavity when the blade leaves the wake peak as discussed in Section 6.4. The propellers with such a cavitation pattern have been considered as a separate group.

The group of propellers with tip-vortex cavitation has a cavitation pattern that is similar to Figure 6.14a, hence a tip-vortex cavity that is attached to the blade and connected to a small sheet cavity. The group of propellers with sheet and tip-vortex cavitation has a cavitation pattern that is similar to Figure $6.14 \mathrm{~b}$, although the sheet is often connected to the tip-vortex cavity.

The broadband noise levels for the propellers with such sheet and vortex cavitation were significantly higher than for propellers with tip-vortex cavitation alone. This required adjustment of the empirical parameter for the maximum level of the hump. Surprisingly, the adopted methodology still appears to apply to such a cavitation pattern indicating that the noise due to the collapse of the closure-vortex cavity follows a similar trend as the tip-vortex cavity. Another possibility is that the collapse of the closure vortex excites the cavitating tip-vortex and that the tip-vortex cavity is still responsible for the broadband hump. The standard deviation of the fit for this small group of propellers with sheet cavitation was higher than for the group with tip-vortex cavitation alone. Because the value of the tuning parameter for the maximum level was different for the two groups, some information on the propeller cavitation pattern is 
required in the application of the method. This information can for instance be obtained from a computation of sheet cavitation by the applied boundary element method. Such information can probably also be used to improve the fit for the group of propellers with sheet cavitation.

The test-cases used for the development of the method were all twin-screw vessels. Despite the limited variability of the wake fields for these ships, the broadband hump was also reasonably well predicted for a single-screw vessel equipped with skewed propeller blades. This gives some confidence that the method is applicable to a wider range of wake fields. The most important limitation of the method is that it only describes the noise due to tip-vortex cavitation and, as discussed above, due to a closure-vortex cavity in the tip region. The method is therefore most suited for the analysis of tip-unloaded (and skewed) propeller blades and is not expected to work for heavy-loaded propellers with extensive sheet cavitation.

Even though the choice of the vortex model is critical to predict the relation between cavity size and cavitation number, it is much less critical for the prediction of the noise levels. This is explained by the fact that the measurement data for the relation between cavity size and cavitation number was obtained for relative small cavity radii with respect to the viscous core radius. Apparently, the relative cavity radius was higher for the test cases used to tune the noise levels. Also, the introduction of the empirical coefficients to tune the centre frequency and maximum level of the hump in the spectrum makes up for some of the deficiencies of the vortex models. Nevertheless, it was seen that a vortex model with a viscous core radius is required to predict speed scaling of noise levels near cavitation inception.

In the present study, the shape of the spectrum was not studied in much detail, and thus requires further research. This not only holds for the high-frequency part of the spectrum, as shown by the URN prediction for the two test-cases shown, but also for the width of the broadband hump.

The scaling relation for the centre frequency does have some theoretical background although it is only based on hypotheses as there is still no theoretical criterion for resonance of a 3-D vortex cavity. The scaling relation for the noise levels does not have any theoretical background, which is a weak point. There is especially a need for a theoretical value for the parameter $\kappa_{1}$. The fit showed a slightly better results for a value of $\kappa_{1}=3$ than using $\kappa_{1}=2$ but it should be kept in mind that other parameters may be required in the relation for the maximum noise levels. A better fit for $\kappa_{1}$ might be obtained from large variations in ship speed, or in cavitation number, but such variations are often hindered by the inception of sheet cavitation. The selection of $\kappa_{1}$ is considered further in Section 8.3.

\subsection{Conclusions}

Broadband HPF and URN generated by cavitating tip vortices on tip-unloaded propeller blades can be modelled by a semi-empirical prediction method (Figure 7.1). The method, denoted ETV-method, makes use of results obtained by a boundary element method and can, therefore, very easily be used for evaluation of tip-vortex cavitation noise, for instance during the propeller design process. The principal parameter in the method is the radius of the vortex cavity from which the maximum noise level and the centre frequency of the broadband hump is determined, similar to the method presented by Raestad (1996). 
The standard deviation of the fit of the ETV-method was about $3 \mathrm{~dB}$ for the maximum level and 0.75 for the non-dimensional centre frequency, as long as the extent of the, considered closed, sheet cavity on the blade was small (Figure 7.5). For a larger extent of the, considered open, sheet cavity in the tip area that forms a closure-vortex cavity, the methodology could still be used but the empirical parameter to predict the maximum of the hump in the spectrum had to be adjusted. The standard deviation of the fit of the model for this group was higher than for the group with only tip-vortex cavitation.

The ETV-method gives an acceptable to good prediction of HPF and URN of two vessels that were not used to develop the empirical relations. However, the high-frequency region of the URN required adjustment of the empirical parameters that describe the shape of the spectrum (Figures 7.10 through 7.12).

The scaling of the noise level with ship speed is well predicted by the method (Figure 7.13). Accurate determination of the empirical value for $\kappa_{1}$ was shown to be difficult due to the small range of ship speeds available.

During the development and application of the method, a number of issues were identified that require further investigation. An important aspect is the contribution to the broadband noise of, what is interpreted here as, a closure-vortex cavity generated by the sheet. Another aspect is the shape of the spectrum, including the relation between the levels of the hump and the levels in the high-frequency part. Physical aspects that affect the shape of the hump and the slope of the high-frequency noise also require further investigation. 
Page intentionally left blank 


\section{Chapter 8}

\section{Experimental prediction of broadband noise}

This chapter presents a novel method for the experimental prediction of broadband hull-pressure fluctuations (HPF) and underwater radiated noise (URN) due to tip-vortex cavities on marine propellers. The method accounts for the dissimilarity in Reynolds number of model tests and sea trials that can affect the cavity size. Use is made of results presented in Chapters 3 and 7.

\subsection{Introduction}

Model tests for the purpose of the prediction of HPF and URN have been reviewed in Sections 2.5 and 2.6, respectively. It was shown that many aspects should be taken into account when performing such model tests. A specific aspect that is relevant for vortex cavitation is the dissimilarity in Reynolds number. The lower Reynolds number in the model test leads to a delay of the inception of vortex cavitation (McCormick, 1962). Whereas the delay in inception is well known, the effect of this difference in Reynolds number on the cavity size is unknown, which complicates the model test as described by the 28th ITTC Specalist Committee on Hydrodynamic Noise (2017). At present, there is no generally established procedure for noise measurements at model-scale on propellers with a vortex cavity to account for this difference in Reynolds number.

The problem for the model tests is illustrated by the cavitation inception diagram sketched in Figure 8.1. The various curves in the diagram show for each cavity pattern how the cavitation number $\sigma_{n}$ at inception varies with mean propeller thrust coefficient $K_{T}$. The vertically oriented solid line shows the operational curve of the propeller with the numbers representing the ship speed in knots. In general, the model test is performed at identical cavitation number and identical thrust coefficient as for the ship propeller. It can also be decided to perform the cavitation test for identical thrust coefficient at the blade position for which the cavity has its largest extent instead of at identical mean thrust coefficient. If the Froude number in the model test is different from full scale, care should be taken that similarity of cavitation number occurs at the location of the cavity.

Figure 8.1 shows the inception diagram of sheet cavitation and of vortex cavitation, both on 


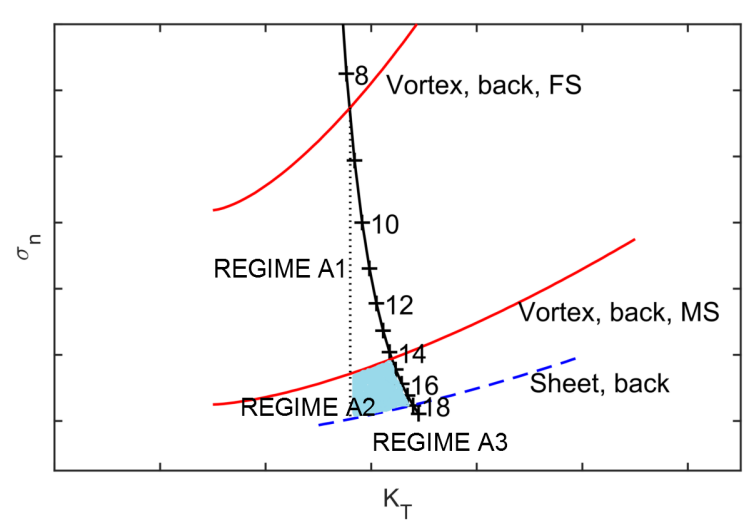

a) Isolated vortex cavity present at MS

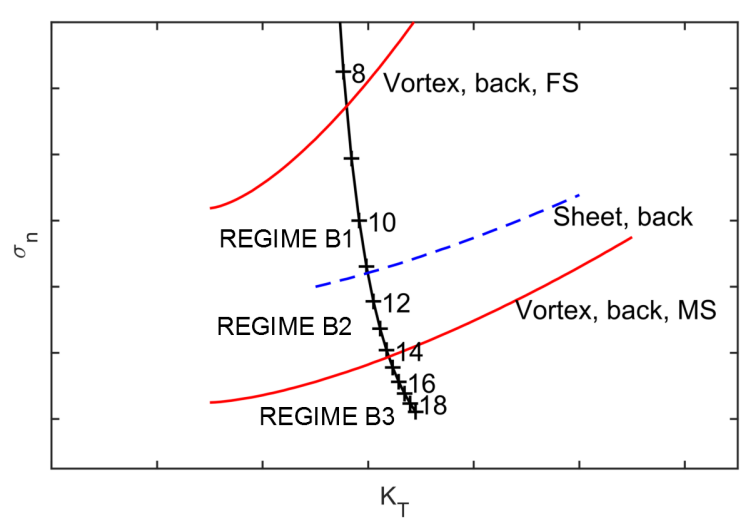

b) No isolated vortex cavity present at MS

Figure 8.1: Diagram showing inception curves of tip vortex cavitation at model scale (MS) and full scale (FS). Inception of sheet cavitation is assumed identical for model and full scale. The propeller operational curve is presented with ship speed in knots. Figure adapted from 28th ITTC Specalist Committee on Hydrodynamic Noise (2017).

the back of the propeller. For vortex cavitation, the inception curve is shown for the model test as well as for the ship, whereas for sheet cavitation the inception number is identical for both situations. Due to the lower Reynolds number of the propeller in the model test, the viscous core of the vortex is larger and, as shown by Eq. (3.23), this results in a delay in cavitation inception. For this example, the inception of the tip vortex cavity occurs just above 14 knots in the model test, but for the ship the inception occurs already at about 8.5 knots. If we do not consider the non-cavitating regime for the ship, two situations can be discerned: Situation A, for which, at model scale, inception of sheet cavitation occurs at higher ship speed than inception of vortex cavitation at model scale, and Situation B, for which inception of sheet cavitation occurs before inception of vortex cavitation at model scale. It is remarked that the presented inception diagram shows only sheet cavitation and tip-vortex cavitation on the back of propeller. The inception of other cavitation patterns, such as face-side cavitation, blade root cavitation, hub vortex cavitation, etc. are not considered here, this in order to simplify the problem.

For Situation A, presented in Figure 8.1a, the following speed regimes can be distinguished:

Regime A1 Tip vortex cavitation is present at full scale but not in the model test. The cavitation number in the model test needs to be reduced to Regime A2 to obtain a vortex cavity.

Regime A2 Tip vortex cavitation is present in the model test, but its size may not agree with the size of the vortex cavity at full scale. Additional scaling of the HPF and URN levels may need to be applied, possibly in combination with a reduction of the cavitation number while staying in Regime A2.

Regime A3 Sheet cavitation is present, the cavitation number can not be adapted. Usually, it is assumed that the noise from sheet cavitation is dominant over tip vortex cavitation, so it is expected that additional scaling for differences in vortex cavity size is not necessary. The model test can be performed at identical cavitation number as at full scale.

For Situation B, presented in Figure 8.1b, the speed regimes are: 
Regime B1 Tip vortex cavitation is present at full scale but not in the model test. As no isolated vortex cavity can be generated in the model test, the HPF and URN levels need to be predicted by computational or semi-empirical methods, such as for instance the method presented in Chapter 7.

Regime B2 Sheet cavitation is present so the cavitation number cannot be adapted. The model test is performed at identical cavitation number as at full scale. Often, it is assumed that sheet cavitation dominates the noise levels. The noise levels emitted by the vortex cavity can be computed from prediction methods such as presented in Chapter 7 and can be compared to the measured levels. If necessary, these computed levels can be added to the measured levels.

Regime B3 The cavitation pattern in the model test is similar to the ship although the vortex cavity size may be different. The regime is identical to Regime A3.

Performing model scale tests for the URN of cavitating vortices near inception has been discussed by various authors. Strasberg (1977) presents data as function of $\sigma_{i} / \sigma$. Blake (1986) discusses scaling rules for cavitation noise including corrections for differences in inception of vortex cavitation. He proposes ratios $\sigma_{i} / \sigma$ and $\sqrt{\sigma_{i} / \sigma-1}$ for the scaling of noise levels. For dissimilarity in cavitation inception, Baiter (1989) provides an extensive review of possible length- and time-scale relations for sheet and vortex cavitation from a theoretical point of view. Oshima (1994) shows that high-frequency HPF levels of a propeller with a cavitating vortex arising at the face of the propeller are well predicted if the cavitation number in the cavitation tunnel is selected smaller than the cavitation number at full scale. The ratio of the modelscale and full-scale cavitation number is written as the ratio of Reynolds numbers, making use of Eq. (2.2), resulting in a value for the exponent $m$ that is smaller than that for cavitation inception. Shen and Strasberg (2003) present a similar analysis and show that the value for $m$ is smaller for developed vortex cavitation than for vortex cavitation near inception. Park and Seong (2017) present a relation to scale model-test URN levels to full scale that also includes a correction for the dissimilarity in Reynolds number. However, all these corrections to $m$ do not vary with cavity size and the behaviour of the scaling relation for large cavity size is not addressed.

We propose here a new method to account for the viscous-scale effect on vortex cavitation noise that can occur for a model test. The method can also be used to reduce the cavitation number in the model test such that the relative vortex cavity size at model scale is equal to the size at full scale. The method consists of two parts. The first part, discussed in Section 8.2, is the computation of the ratio between ship-scale and model-scale cavity size. The second part, discussed in Section 8.3, concerns the scaling of vortex cavitation noise to account for a difference in ship-scale and model-scale cavity size. The method has been applied to a few cases for which sea trial data is available and these results are briefly described in Section 8.4. 


\subsection{Scaling of vortex-cavity size with Reynolds number}

The variation of the cavity size with Reynolds number is investigated first by analysing the limiting behaviour for small and that for large cavity size of the cavitating Lamb-Oseen vortex. The analytical formulations are presented as function of cavitation number and cavitation inception number. Then, the formulation for arbitrary cavity size is presented using an additional expression for the effect of Reynolds number on viscous core size and on cavitation inception number.

The theoretical analysis of the limiting situations is performed for the non-cavitating LambOseen vortex. The cavity radius is defined as the radius where pressure equals vapour pressure. For small cavity size, $r_{c} \ll r_{v}$, the distribution of the azimuthal velocity is given by a solid body rotation, Eq. (3.9), while for large cavity size, $r_{c} \gg r_{v}$, the distribution of the azimuthal velocity is described by a potential-flow vortex, Eq. (3.7). Using Eq. (3.4), the azimuthal velocity distribution for the two limiting conditions has been integrated to derive relations for cavity size, $r_{c}$, as function of the cavitation number and the cavitation inception number. The relation for very small cavity size reads

$$
r_{c}\left(\sigma, \sigma_{i}\right)=\frac{\sqrt{\sigma_{i}-\sigma}}{\sigma_{i}}\left(\frac{\Gamma_{\infty} \ln 2}{\pi V_{\mathrm{ref}}}\right)
$$

which was also given by Baiter (1989). The relation for large cavity size is given by

$$
r_{c}(\sigma)=\left(\frac{\Gamma_{\infty}}{2 \pi V_{\text {ref }}}\right) \frac{1}{\sqrt{\sigma}},
$$

which corresponds to the cavity thickness of a potential flow vortex.

The variation of cavity size with cavitation number is presented in Figure 8.2 for the cavitating Lamb-Oseen vortex for three values of the viscous core size with $V_{\text {ref }}=\Gamma_{\infty} / 2 \pi r_{\text {ref }}$. The parameter $r_{\text {ref }}$ is an arbitrary reference value used to non-dimensionalize $r_{c}$ and $r_{v}$. Results are presented as function of $\sqrt{\sigma_{i}-\sigma} / \sigma_{i}$ and as function of $1 / \sqrt{\sigma}$, showing a good collapse of the data for small and for large cavity size, respectively. The formula for small cavity size is valid when $r_{c}$ is smaller than approximately $50 \%$ of $r_{v}$, and the relation for large cavity size is valid when $r_{c}$ is larger than approximately $150 \%$ of $r_{v}$. The relation for small cavity size depends on the cavitation inception number and therefore on the propeller Reynolds number. The formulation for large cavity size is independent of the cavitation inception number and therefore independent of Reynolds number. Results for the cavitating modified Lamb-Oseen vortex are very similar.

It should be kept in mind that the effects of surface tension and nuclei distribution are not included in the formulation for small cavity size. Also, it has been assumed that the viscous core does not change for cavitating flow, which is in agreement with Figure 3.9, but which is not understood as discussed in Section 3.3.

For the non-cavitating Lamb-Oseen vortex, it was shown that the relation between $r_{c}$ and $\sigma$ can be transformed into a single curve, independent of $\Gamma_{\infty}$ and $r_{v}$, by presenting $r_{c} / r_{v}$ as a function of $\sigma / \sigma_{i}$, see Figure 3.9. The function can be given as $\sigma / \sigma_{i}=F\left(r_{c} / r_{v}\right)$, keeping in mind that the cavitation numbers are defined at the location of the vortex cavity in the propeller disc. This single curve $F$ is also obtained for the cavitating vortex models, but only if $r_{v}$ does 


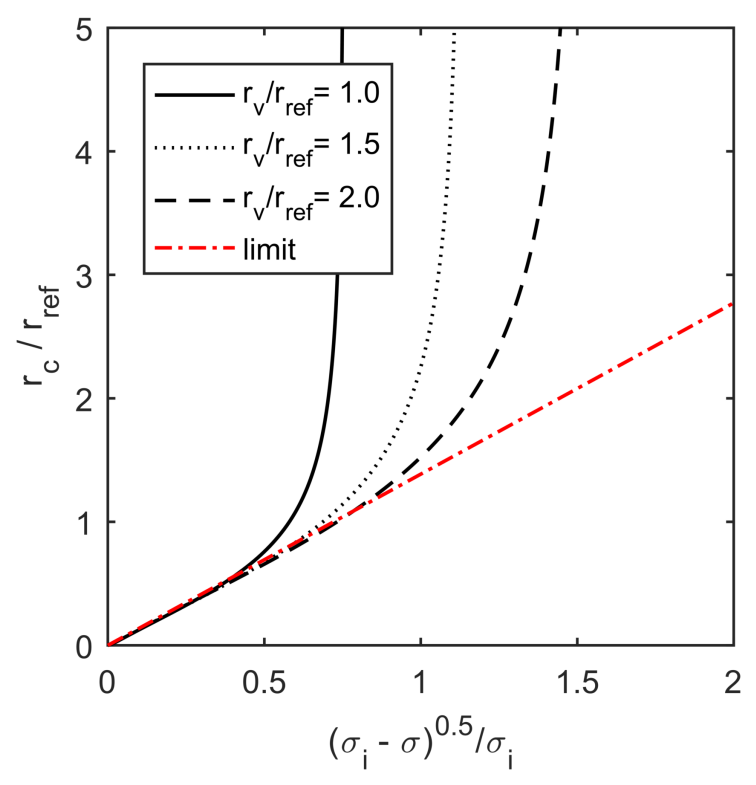

a) Small cavity size

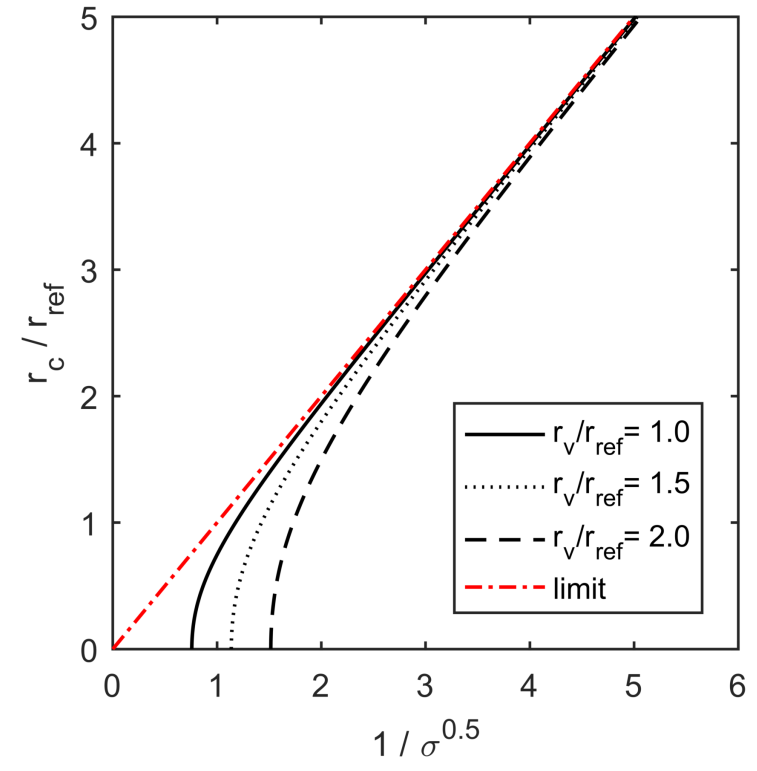

b) Large cavity size

Figure 8.2: Scaling relations for small and for large cavity size with cavitation number and cavitation inception number, using the cavitating Lamb-Oseen vortex. Results are shown for three values of the viscous core size $r_{v}$, and for Eqs. (8.1) and (8.2) for small and large cavity size, respectively.

not change with $r_{c}$. As we are interested in the value for $r_{c}$, we consider the inverse of $F$ and write the relation between $r_{c}$ and $\sigma$ as

$$
\frac{r_{c}}{r_{v}}=f\left(\frac{\sigma}{\sigma_{i}}\right) .
$$

The function $F$ has an analytical expression for the (cavitating) Lamb-Oseen vortex as shown in Section 3.2.1, but there is no analytical expression for $f$ so the function is obtained numerically.

We now consider two situations for $r_{v}$. In the first situation, the change in $\sigma$ is through a change in operational pressure such as in a cavitation-test facility. In this situation the Reynolds number is constant and $r_{v}$ does not change with $\sigma$. In the second situation, the change in $\sigma$ is through a change in velocity as for ship propellers. In this situation the Reynolds number is varying with $\sigma$ which also leads to a change in $r_{v}$.

For the first situation, for which $r_{v}$ is constant and denoted by $r_{v, i}$ as we take the value at inception, we can use Eq. (8.3) to define the ratio of cavity size between model scale, subscript $m$, and full scale, subscript $s$,

$$
\left(\frac{r_{c}}{D}\right)_{m}\left(\frac{D}{r_{c}}\right)_{s}\left(\frac{r_{v, i}}{D}\right)_{s}\left(\frac{D}{r_{v, i}}\right)_{m}=\frac{f\left[\left(\sigma / \sigma_{i}\right)_{m}\right]}{f\left[\left(\sigma / \sigma_{i}\right)_{s}\right]}
$$

If the non-dimensional vortex strength is identical at model and full scale, Eq. (3.23) for the Lamb-Oseen vortex shows that

$$
\frac{\left(r_{v, i} / D\right)_{s}}{\left(r_{v, i} / D\right)_{m}}=\left(\frac{\sigma_{i, s}}{\sigma_{i, m}}\right)^{-1 / 2}
$$


It is assumed that this relation is generally valid. Substituting Eq. (8.5) in Eq. (8.4) gives

$$
\left(\frac{r_{c}}{D}\right)_{m}=\left(\frac{r_{c}}{D}\right)_{s}\left(\frac{\sigma_{i, s}}{\sigma_{i, m}}\right)^{1 / 2} \frac{f\left[\left(\sigma / \sigma_{i}\right)_{m}\right]}{f\left[\left(\sigma / \sigma_{i}\right)_{s}\right]}
$$

which is the relation from which the ratio between $\left(r_{c} / D\right)_{m}$ and $\left(r_{c} / D\right)_{s}$ can be computed if $r_{v}$ does not change with $\sigma$. The relation requires the cavitation inception number to be determined in the model test from which the full-scale value can be estimated according to standard procedure of the specific model basin, as discussed in Sections 2.3 and 7.2.

In the second situation, $r_{v}$ is varying with $\sigma$ and denoted by $r_{v}(\sigma)$. We should keep in mind that in the function $\sigma / \sigma_{i}=F\left(r_{c} / r_{v}\right)$, the values for $r_{v}$ at $\sigma_{i}$ and $\sigma$ are identical, so this function needs to be modified. We consider the ratio of $r_{v}(\sigma)$ to $r_{v}\left(\sigma_{i}\right)=r_{v, i}$, and a new relation $r_{c} / r_{v, i}=f_{\operatorname{Re}}\left(\sigma / \sigma_{i}, r_{v} / r_{v, i}\right)$ is computed. Use can then be made of a formulation similar to Eq. (7.1), assuming that the non-dimensional vortex strength does not change with $\sigma$,

$$
\frac{r_{v}(\sigma)}{r_{v}\left(\sigma_{i}\right)}=\left(\frac{\operatorname{Re}}{\operatorname{Re}_{i}}\right)^{-m / 2}=\left(\frac{\sigma}{\sigma_{i}}\right)^{m / 4},
$$

in which the variation in speed has been expressed by the variation in $\sigma$. If the value for the Reynolds number is available, use can be made of Eq. (7.2) to determine $m$, otherwise, a default value for $m$ can be used. At MARIN, good experience has been obtained with $m=0.35$. The ratio of cavity sizes is now written as

$$
\left(\frac{r_{c}}{D}\right)_{m}\left(\frac{D}{r_{c}}\right)_{s}\left(\frac{r_{v, i}}{D}\right)_{s}\left(\frac{D}{r_{v, i}}\right)_{m}=\frac{f_{\operatorname{Re}}\left[\left(\sigma / \sigma_{i}\right)_{m}\right]}{f_{\operatorname{Re}}\left[\left(\sigma / \sigma_{i}\right)_{s}\right]} .
$$

The dependency on $r_{v, i} / D$ can be replaced by a dependency on $\sigma_{i}$, as given by Eq. (8.5), leading to

$$
\left(\frac{r_{c}}{D}\right)_{m}=\left(\frac{r_{c}}{D}\right)_{s}\left(\frac{\sigma_{i, s}}{\sigma_{i, m}}\right)^{1 / 2} \frac{f_{\operatorname{Re}}\left[\left(\sigma / \sigma_{i}\right)_{m}\right]}{f_{\operatorname{Re}}\left[\left(\sigma / \sigma_{i}\right)_{s}\right]},
$$

which is the relation from which the ratio between the cavity size in the model test and that at full scale can be computed if $r_{v}$ does change with $\sigma$.

The cavitation inception number needs to be corrected for the tensile strength of water such that it is equivalent to the minimum pressure in the vortex. Even though such correction procedures have been proposed, it is not yet standard practise in cavitation tests. The electrolysis process applied in the cavitation tests in the DWB generates relatively large-size nuclei (Birvalski and van Rijsbergen, 2018), and it is thus assumed that the effect of the tensile strength on cavitation inception can be neglected.

The vortex model has an effect on the scaling relation for the cavity size. In Chapter 3 it was shown that the effect of cavitation on Eq. (8.3) is very small and that the relation for a noncavitating vortex could be used to predict this relation, although it is not understood why this is possible, considering the significant differences in azimuthal velocity distribution. In the present scaling procedure, use is made of the vortex model of Proctor et al. (2010), given by Eq. (3.30), that was also used for the semi-empirical prediction method presented in Chapter 7 . The effect of the tuning factor $\zeta_{1}$, with default value $\zeta_{1}=10$, on the scaling is presented in Figure 8.3 


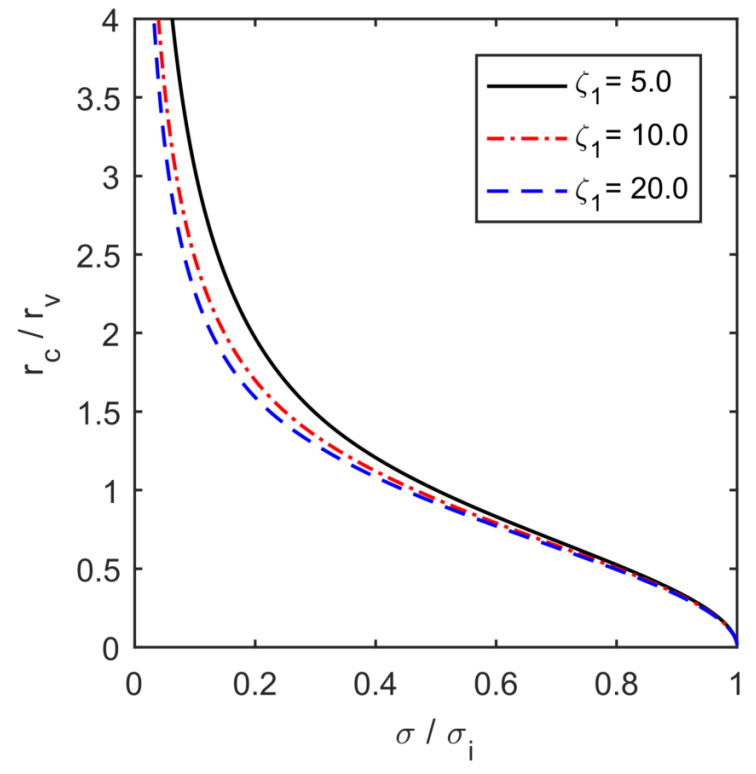

a) Eq. (8.3)

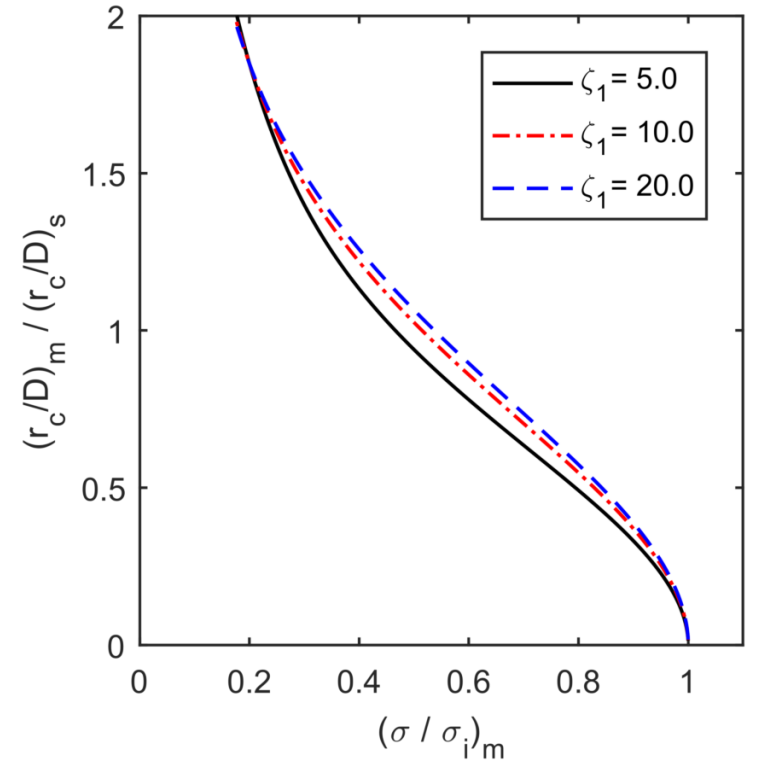

b) Eq. (8.6), $\left(\sigma / \sigma_{i}\right)_{s}=0.2, \sigma_{i, s} / \sigma_{i, m}=3.4$

Figure 8.3: Effect of parameter $\zeta_{1}$ in the Proctor vortex model, Eq. (3.30) on the scaling relation of cavity size with cavitation number. Parameter $r_{v}$ does not change with $\sigma$.

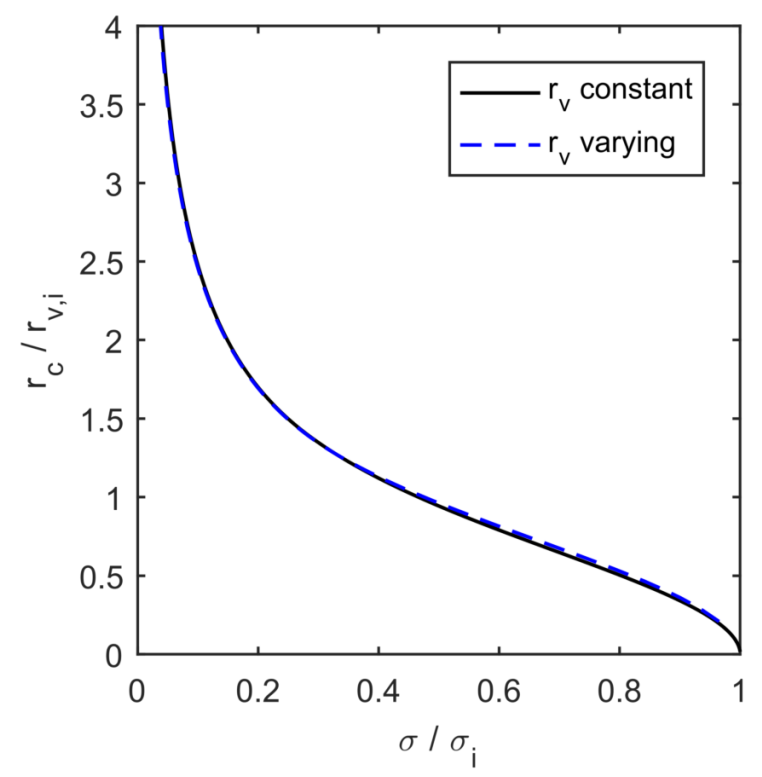

a) $r_{c} / r_{v, i}=f_{\operatorname{Re}}\left(\sigma / \sigma_{i}\right)$

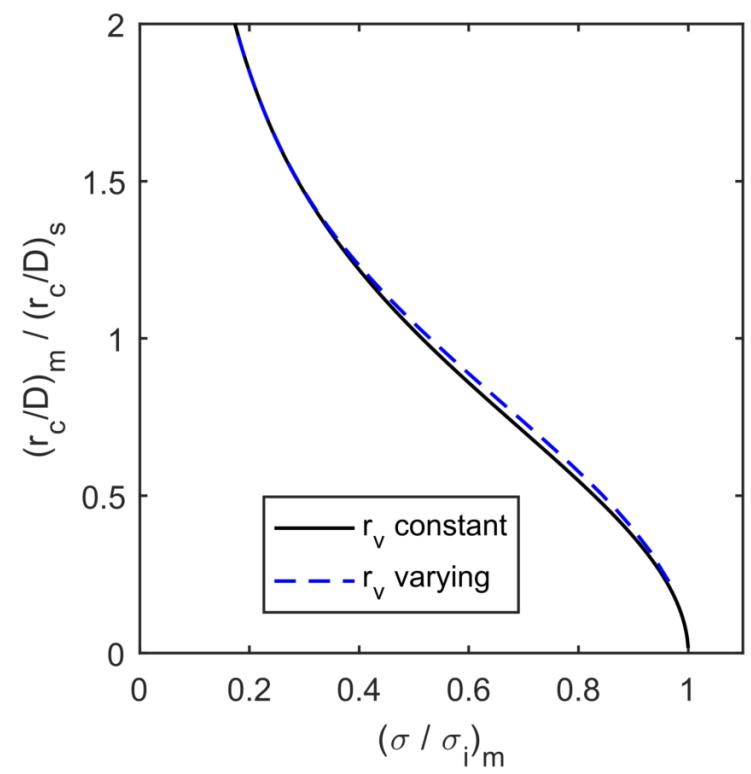

b) Eq. (8.9), $\left(\sigma / \sigma_{i}\right)_{s}=0.2, \sigma_{i, s} / \sigma_{i, m}=3.4$

Figure 8.4: Effect of variation of $r_{v}$ with $\sigma$ for the Proctor vortex, $\zeta_{1}=10$.

using $B / r_{v}=12$. At $\sigma_{m}$ for which $\left(r_{c} / D\right)_{m}=\left(r_{c} / D\right)_{s}$ for $\zeta_{1}=10,\left(r_{c} / D\right)_{m} /\left(r_{c} / D\right)_{s}$ varies between $+2 \%$ and $-8 \%$ for the variation in $\zeta_{1}$ considered.

The effect of the variation of $r_{v}$ with $\sigma$ is compared to the situation of constant $r_{v}$ in Figure 8.4. It is seen that with varying $r_{v}$ the cavity size increases by a small amount as long as $r_{c}$ is smaller than $r_{v, i}$. For larger cavity size, the effect of the variation of $r_{v}$ can no longer be 
discerned. As the effect of the variation of $r_{v}$ is rather small, it is not taken into account in the following sections and Eq. (8.6) is used instead of (8.9).

Equations (8.6) and (8.9) can be used in three approaches to correct for the Reynolds scale effect on vortex cavity size:

(i) The model test is performed for $\sigma_{m}$ for which $\left(r_{c} / D\right)_{m}=\left(r_{c} / D\right)_{s}$ by reducing the pressure in the facility. This is for instance required when operating in Regime A1 in Figure 8.1.

(ii) If a vortex cavity is present in the model test at $\sigma_{m}=\sigma_{s}$, the size of this cavity can be related to its full-scale value. The ratio of $\left(r_{c} / D\right)_{m} /\left(r_{c} / D\right)_{s}$ can be used in a noise scaling relation, such as presented in the next section, to scale model-test results to full scale. This can for instance be applied when operating in Regime A2.

(iii) A combination of adjusting $\sigma_{m}$ and applying the noise scaling relation is to be applied if the $\sigma_{m}$ for (i) can not be obtained due to, for instance, the occurrence of sheet cavitation.

All three approaches require the determination of the cavitation inception number in the model test.

\subsection{Scaling of vortex-cavity noise with cavity size}

The difference in relative cavity size $r_{c} / D$ of model scale and that of full scale needs to be accounted for when scaling the model-test results to full-scale values. Here, two relations are presented to scale the noise levels. The first relation makes use of the empirical formulation of the ETV-method, presented in Section 7.3. The second relation is derived using a similar approach as used for low-frequency URN levels, presented in Section 5.4.4. The formulation for low frequencies is preferred as the focus is on the broadband hump of the spectrum. To keep the model simple, the high-frequency part of the measured spectrum is considered to scale similar as the low-frequency part.

Both relations use the same relation for the frequency. The frequency scales with the centre frequency of the ETV-method, Eq. (7.8), which results into

$$
\frac{f_{s}}{f_{m}}=\frac{\left(r_{c} / D\right)_{m}}{\left(r_{c} / D\right)_{s}} \frac{n_{s}}{n_{m}} \sqrt{\frac{\sigma_{s}}{\sigma_{m}}}
$$

The various possible criteria for the resonance frequency, discussed in Section 6.2, give some theoretical support to this relation.

The first relation for the scaling of noise levels generated by a vortex cavity makes use of the empirical relation (7.7) for the scaling of the maximum level of the broadband hump. The combination with Eq. (5.99) for the scaling for broadband HPF gives

$$
\frac{p_{s, 1 \mathrm{~Hz}}^{\prime}{ }^{2}}{p_{m, 1 \mathrm{~Hz}}^{\prime}{ }^{2}}=\left(\frac{\rho_{s}}{\rho_{m}}\right)^{2}\left(\frac{n_{s}}{n_{m}}\right)^{3}\left[\frac{\left(r_{c} / D\right)_{s}}{\left(r_{c} / D\right)_{m}}\right]^{2 \kappa_{1}}\left(\frac{D_{s}}{D_{m}}\right)^{6}\left(\frac{R_{m}}{R_{s}}\right)^{2}
$$

It is recalled that a value of $\kappa_{1}=3$ was used in the ETV-method. 
The second relation to scale noise levels is derived using a similar approach as in Section 5.4.4. The acoustic power $P_{a}$ is defined in Eq. (5.102) and is repeated here for convenience,

$$
P_{a}=\frac{4 \pi R^{2} p^{\prime 2}}{\rho c} \Delta f
$$

The parameter $\Delta f$ corresponds to the total frequency band over which the acoustic power is distributed.

The rate of change in potential energy of a 3-D vortex cavity is obtained from Eq. (4.36) derived for a 2-D vortex cavity, which shows that the potential energy per unit length equals $2 \pi r_{c}^{2}\left(p_{\infty}-p_{v}\right)$. For the 3-D situation, an effective length $L$ of the vortex cavity over which the collapse occurs is introduced. The power due to the rate of change in potential energy then reads

$$
P_{p o t} \propto r_{c}^{2} L\left(p_{\infty}-p_{v}\right) N, \propto r_{c}^{2} L \sigma \rho n^{3} D^{2},
$$

in which $N$ corresponds to the number of collapses per second, hence $N=Z n$. It is remarked that this potential energy is also converted into an increase in kinetic energy in azimuthal direction that does not contribute to the radiated noise as shown by Eq. (4.36). Furthermore, it is seen that in Eq. (8.13) the actual cavity size is used, whereas in Section 5.4.3 similarity of cavitation volume was assumed.

For the acoustic efficiency, we use Eq. (5.112) as applied in Section 5.4.4 for the scaling of low-frequency cavitation noise,

$$
\eta_{a c} \propto \frac{n D}{c} \sqrt{\sigma}
$$

The formulation for the non-dimensional acoustic power then reads

$$
\begin{aligned}
\Pi_{p}=\frac{P_{a}}{\eta_{a c} P_{p o t}} & \approx \frac{c}{n D \sqrt{\sigma}} \frac{R^{2} p^{\prime 2}}{\rho c} \Delta f \frac{1}{r_{c}^{2} L \sigma \rho n^{3} D^{2}} \\
& \approx \frac{R^{2} p^{\prime 2}}{\rho^{2} n^{3} r_{c}^{3} L D^{2} \sigma},
\end{aligned}
$$

in which $\Delta f$ is assumed to scale with the bandwidth of the hump, for which it was assumed in Section 7.3 that it is proportional to the centre frequency of the hump. The scaling of the frequency bandwidth is then given by $\Delta f \propto\left(n D / r_{c}\right) \sqrt{\sigma}$. Remark that in Sections 5.4.3 and 5.4.4 it was assumed that $\Delta f \propto n$.

We will now also assume that $L \propto r_{c}$. Using $r_{c}=\left(r_{c} / D\right) D$, the ratio of ship-scale and model-scale noise levels, in constant bandwidth, reads

$$
\frac{p_{s, 1 H z}^{\prime}{ }^{2}}{p_{m, 1 H z}^{\prime}{ }^{2}}=\left(\frac{\rho_{s}}{\rho_{m}}\right)^{2}\left(\frac{n_{s}}{n_{m}}\right)^{3}\left[\frac{\left(r_{c} / D\right)_{s}}{\left(r_{c} / D\right)_{m}}\right]^{4}\left(\frac{D_{s}}{D_{m}}\right)^{6}\left(\frac{\sigma_{s}}{\sigma_{m}}\right)\left(\frac{R_{m}}{R_{s}}\right)^{2}
$$

Equation (8.16) shows two differences with respect to Eq. (8.11):

(i) The scaling with cavity size differs for the two models if the default value $\kappa_{1}=3$ is used, whereas it becomes identical if we use $\kappa_{1}=2$. In the development of the ETV-method, the values for $\kappa_{1}$ were found to vary between 2.5 and 3 depending on the test-case and a value of $\kappa_{1}=3$ was selected. The analysis of the speed scaling presented in Section 7.5.3 showed that $\kappa_{1}=2$ as well as $\kappa_{1}=3$ gave acceptable agreement with experimental data. 
(ii) The correction for differences in cavitation number is not included in the relation for the semi-empirical model, as the cavitation number has a direct effect on the cavity size. If the ratio of cavitation numbers in Eq. (8.16) is written as a ratio of cavity size using Eq. (8.2), it leads to a reduction of the scaling with $r_{c}$. This further increases the difference in scaling with $r_{c}$ when comparing Eqs. (8.16) and (8.11).

It is therefore concluded that the scaling relation based on the ETV-method cannot be retrieved from theory.

In the following sections, we will analyse several datasets for URN and broadband HPF measured at model scale for which also full-scale data is available. The model-test results were already scaled using formulations presented in Sections 5.4.2 through 5.4.4, so differences in $\rho, n, D$ and $R$ are not considered. The focus is on the correction for differences in vortex cavity size, if present. Because the effect of $\sigma$ can not be studied separately from the effect of $r_{c}$, it is also not further considered here. The correction for differences in cavity size is then obtained from Eq. (8.11) and reads

$$
\frac{p_{s, 1 \mathrm{~Hz}}^{\prime}{ }^{2}}{p_{m c, 1 \mathrm{~Hz}}^{\prime}{ }^{2}}=\left[\frac{\left(r_{c} / D\right)_{s}}{\left(r_{c} / D\right)_{m}}\right]^{2 \kappa_{1}},
$$

in which subscript $m c$ corresponds to a conventional scaled URN or HPF level. Some of the results will be presented for $\kappa_{1}=2$ as well as for $\kappa_{1}=3$ to investigate the uncertainty of the scaling relation, similarly as performed for the analysis of the speed scaling in Section 7.5.3.

\subsection{Results}

\subsubsection{Published hull-pressure data of a face-side vortex cavity}

One of the first publications on the scaling of noise from tip-vortex cavitation is by Oshima (1990, 1994). It concerns a twin-screw vessel equipped with four-bladed controllable pitch propellers. Full-scale hull-pressure spectra, measured up to approximately $40 \mathrm{kHz}$ with a hydrophone mounted on the hull at a location above the propeller for $\sigma_{s}=3.56$ showed good agreement with model-scale measurements as performed in a cavitation tunnel at $\sigma_{m}=2.25$. Oshima stated that the ratio of this full-scale and model-scale cavitation number equals the ratio of the corresponding Reynolds numbers raised to the power 0.15 . The results of his measurements, performed at various cavitation numbers at model scale, are presented in Figure $8.5 \mathrm{~b}$. The model-scale data is scaled according to Eq. (5.117), although it is not clear whether or not the ratio of full-scale and model-scale cavitation number is accounted for. The full-scale data is presented for the starboard and for the port-side propeller showing small differences for frequencies above $7 \mathrm{kHz}$. The cavity type was tip-vortex cavitation occurring on the face of the propeller.

The full-scale and model-scale cavitation inception numbers were not given, but sketches of the cavitation pattern for model-scale conditions showed that at $\sigma_{n}=3.07$ the cavity extent was very small, see Figure $8.5 \mathrm{a}$. Hence, inception was occurring at a somewhat higher cavitation number. The ratio of full-scale and model-scale Reynolds numbers could be determined from the data given by Oshima (1990) and the full-scale cavitation inception number could then be 

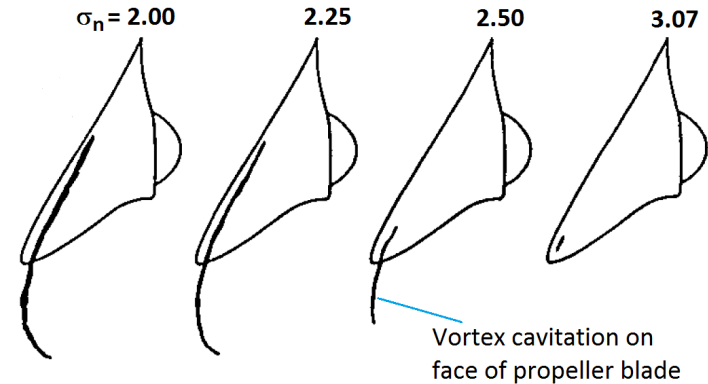

a) Cavity patterns

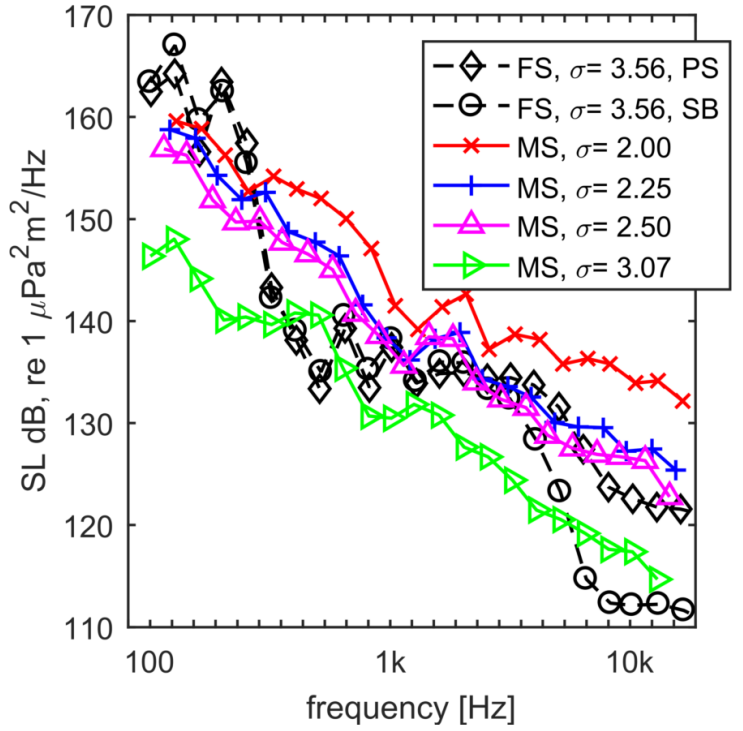

b) HPF spectra

Figure 8.5: Model-scale cavitation patterns and HPF data for full scale (FS) and model scale (MS) by Oshima (1990), uncorrected for difference in cavity size. PS: port side; SB: starboard side propeller.

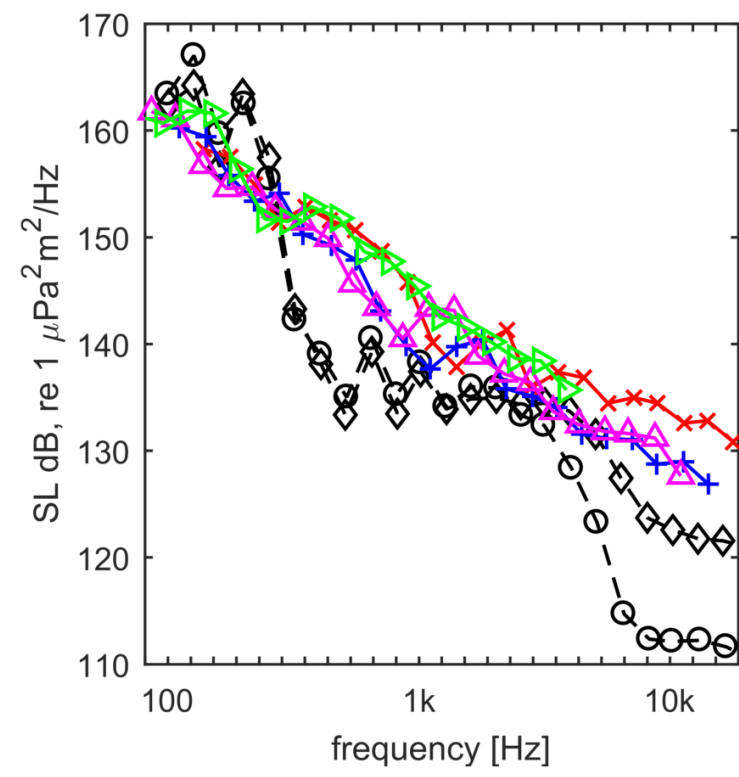

a) $\kappa_{1}=2.0, \sigma_{i, m}=3.2$

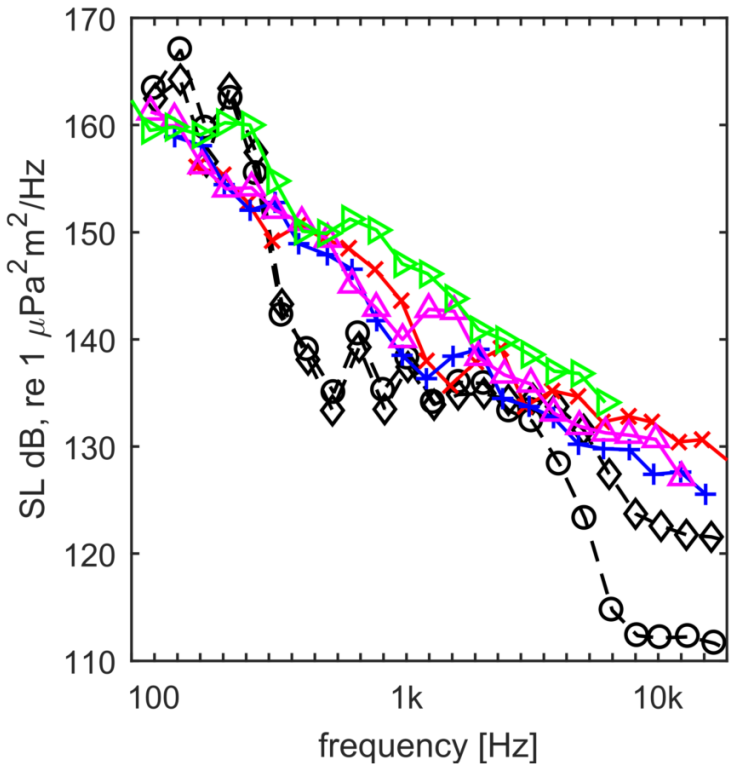

b) $\kappa_{1}=3.0, \sigma_{i, m}=3.4$

Figure 8.6: Measurement data by Oshima (1990), corrected for difference in cavity size, legend given in Figure $8.5 \mathrm{~b}$.

computed from the estimated model-scale value using the McCormick scaling formula with $m=$ 0.35 . The ratio of full-scale and model-scale cavity size was computed using the Proctor-vortex with $\zeta_{1}=10$. The frequencies of the model-scale spectra were corrected for the difference in cavity size using Eq. (8.10). It is assumed here that the cavitation numbers given by Oshima are defined at the location of the tip vortex cavity. 

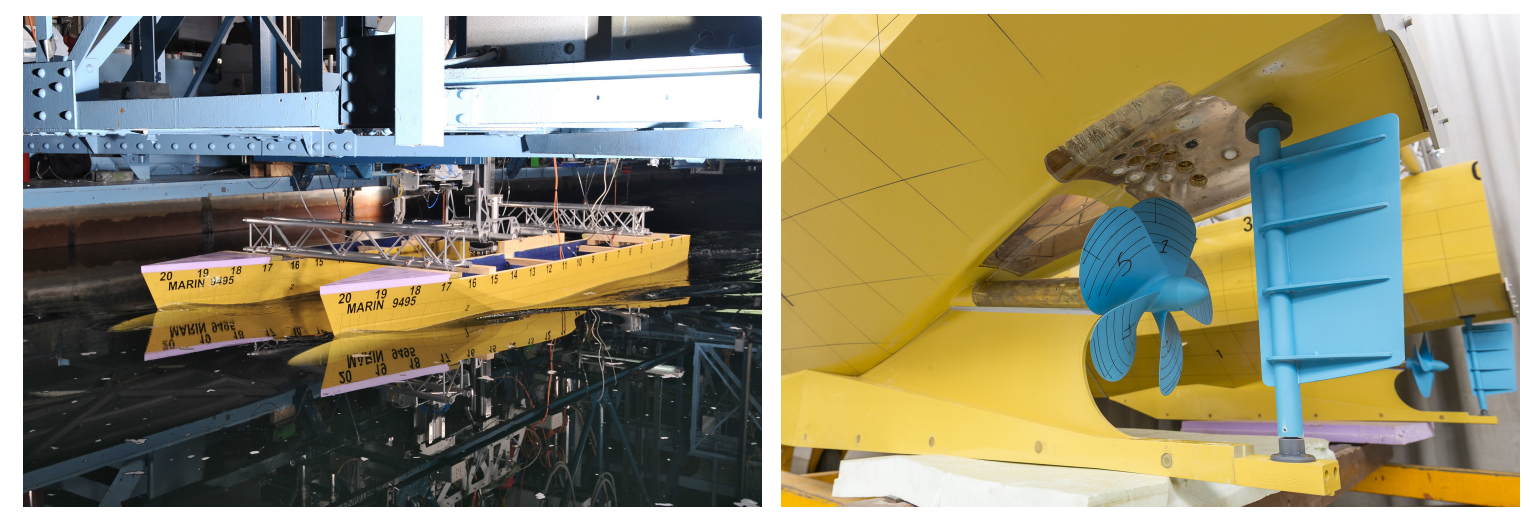

Figure 8.7: Ship model (sailing at 6 knots) and propeller of The Princess Royal.

The resulting spectra are presented in Figure 8.6 for two values of $\kappa_{1}$ for which also the used values for $\sigma_{i, m}$ are given. The values for $\sigma_{i, m}$ were selected such that a reasonable collapse of the model-scale data was obtained. The effect of tensile strength of the water in the cavitation tunnel on $\sigma_{i, m}$ is unknown and is therefore not accounted for. It is seen that, after tuning $\sigma_{i, m}$, the model-scale data obtained for a range of cavitation numbers collapse well for both values of $\kappa_{1}$ and that these data show acceptable agreement with the data from full-scale measurements. The tuned value of $\sigma_{i, m}$ was different for the two values of $\kappa_{1}$ considered, showing the importance of accurately determining $\sigma_{i, m}$. Due to the uncertainty of $\sigma_{i, m}$ in this data set, a best fit for $\kappa_{1}$ in Eq. (8.17) could not be determined.

\subsubsection{Radiated noise of a small research vessel}

Another example to show the effect of tip-vortex scaling is the small research vessel The Princess Royal of which the URN was measured at model scale and at full scale in the EU FP7 SONIC project (Prins et al., 2016). The vessel is a $19 \mathrm{~m}$ catamaran equipped with two propellers of $0.75 \mathrm{~m}$ diameter that is designed and operated by Newcastle University (Atlar et al., 2013). The full-scale measurements of the URN were simultaneously performed by CETENA and the University of Southampton (SOTON), for ship speeds between 5 knots and 15 knots (Humphrey et al., 2015). The model tests were, amongst others, performed in the DWB of MARIN and are described in Lafeber and Bosschers (2016). The geometric scale factor of the model is 1:3. Photographs of the model and propeller are shown in Figure 8.7. The shaft rotation rates and basin pressure in the model tests were determined from the sea trials using Froude scaling while the model speed was adapted such that the (extrapolated) torque of the propeller matches the value measured during the sea trials.

Here, we will only discuss the effect of correcting the noise levels measured in the DWB for the difference in inception of tip vortex cavitation. At model scale, the inception of tip vortex cavitation occurred at $\sigma_{n m, i}=5.30$ which was determined through observations. Parameter $\sigma_{n}$ is defined here as the cavitation number at the tip of the blade in the 12 o'clock position. Scaling according to the McCormick rule with $m=0.35$ results in a full-scale inception number of $\sigma_{n s, i}=8.6$, equivalent to a propeller-shaft rotation rate of $393 \mathrm{RPM}$. This is in good agreement with full-scale values reported by Turkmen et al. (2015), who estimated cavitation inception to 


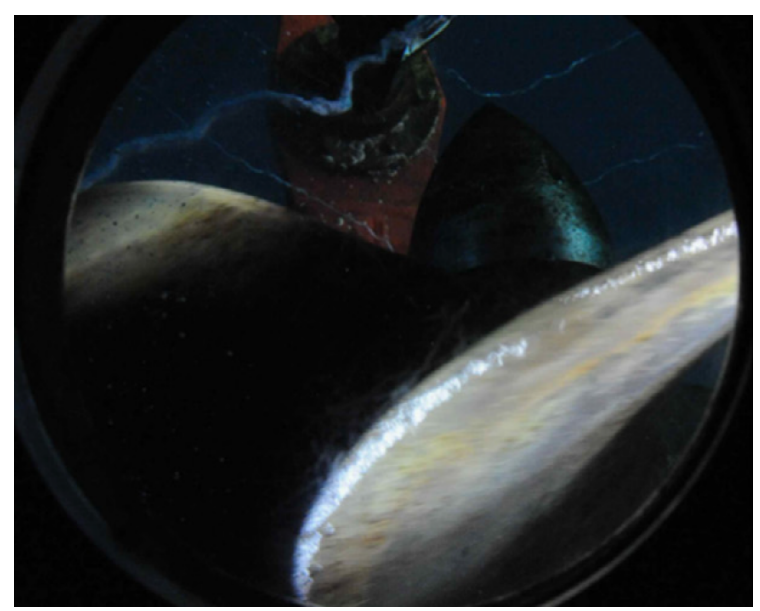

a) Full scale

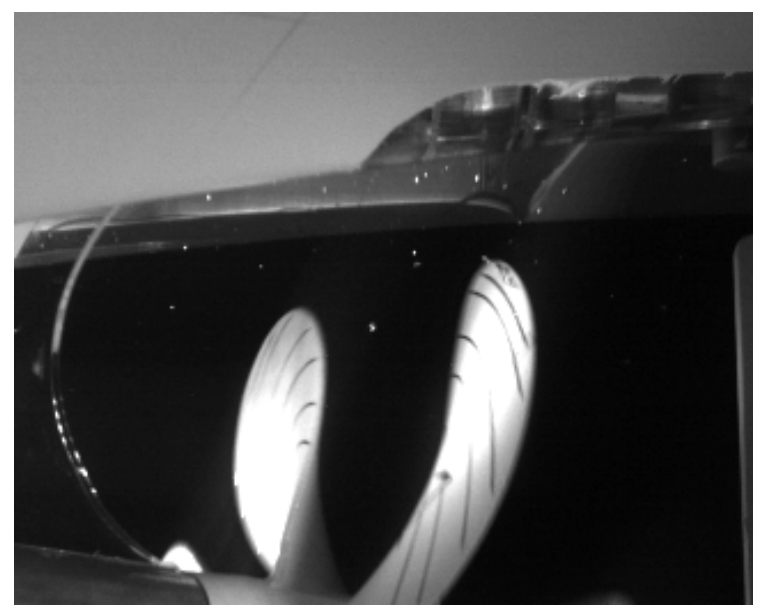

b) Model scale

Figure 8.8: Image of the cavity extent on the propeller of The Princess Royal, 7 knots ship speed. Full scale image by Aktas et al. (2016), published with permission.

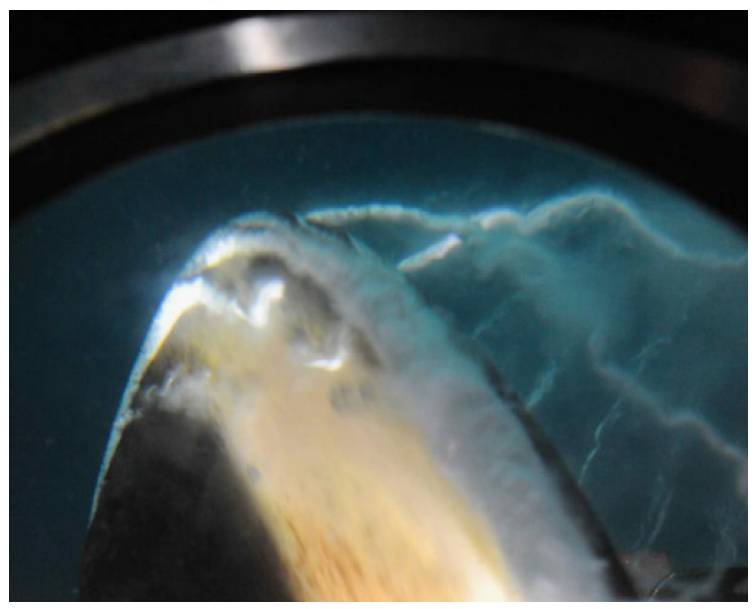

a) Full Scale

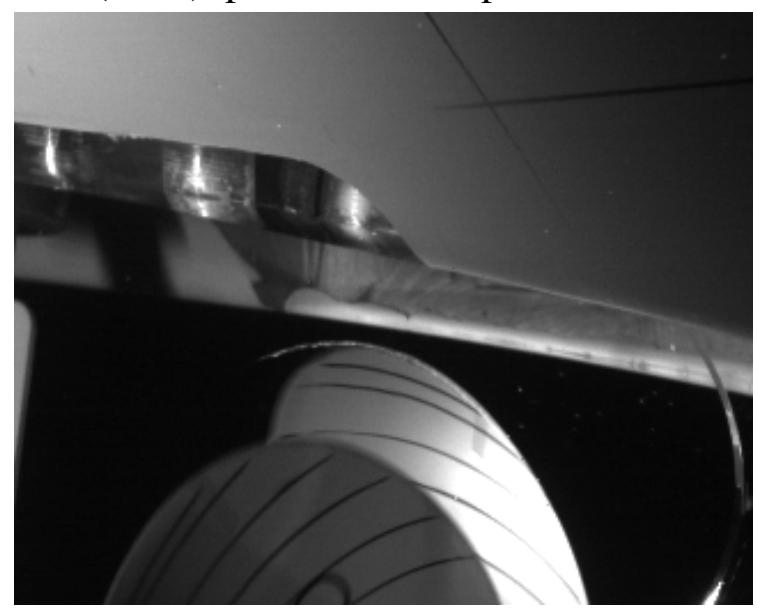

b) Model Scale

Figure 8.9: Image of the cavity extent on the propeller of The Princess Royal, 9 knots ship speed. Full scale image by Aktas et al. (2016), published with permission.

occur between 372 and 400 RPM, corresponding to 650 and 700 RPM of the shaft of the diesel engine.

The cavitation observations for 7 knots ship speed, corresponding to an engine RPM of 900 with propeller RPM of 514, show at model scale a small vortex cavity as this condition was just beyond cavitation inception at model scale, see Figure 8.8. The observations at full scale, presented by Aktas et al. (2016), show that the vortex cavity is much more developed, while there is also a small amount of sheet cavitation present. The model tests were performed at identical cavitation number as full scale, with $\sigma_{n s}=\sigma_{n m}=4.99$, and the URN levels were scaled according the Eq. (5.111). The model-scale values, uncorrected for difference in cavity size, are much smaller than the full-scale values. However, applying the correction for difference in size of the vortex cavity leads to an acceptable prediction of the model test, see Figure 8.10. The two values for $\kappa_{1}$ give $7 \mathrm{~dB}$ difference in noise levels, but the results for both values for $\kappa_{1}$ show 


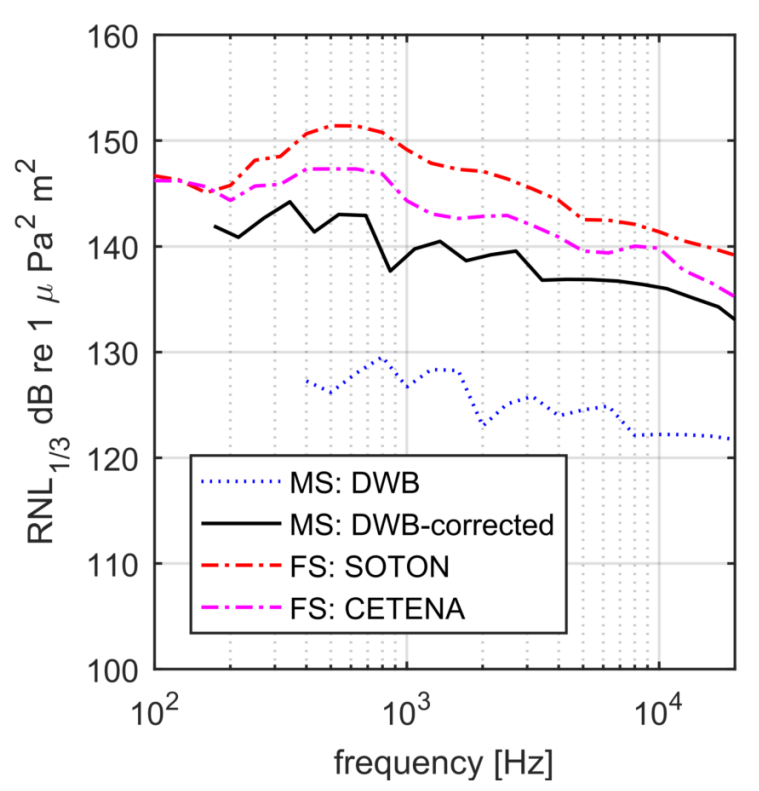

a) $\kappa_{1}=2.0$

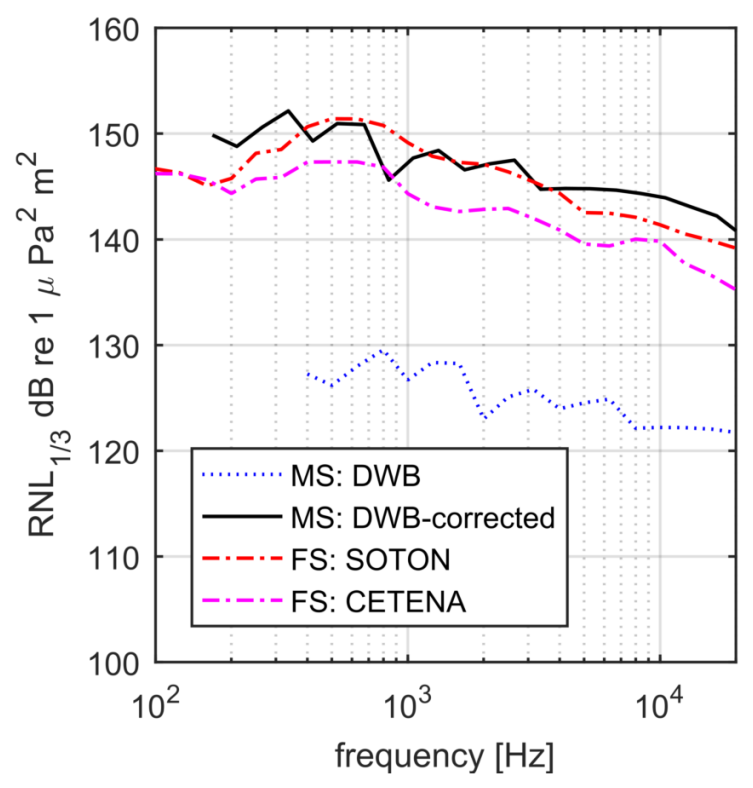

b) $\kappa_{1}=3.0$

Figure 8.10: Influence of cavity size correction on URN levels of The Princess Royal, 7 knots ship speed.

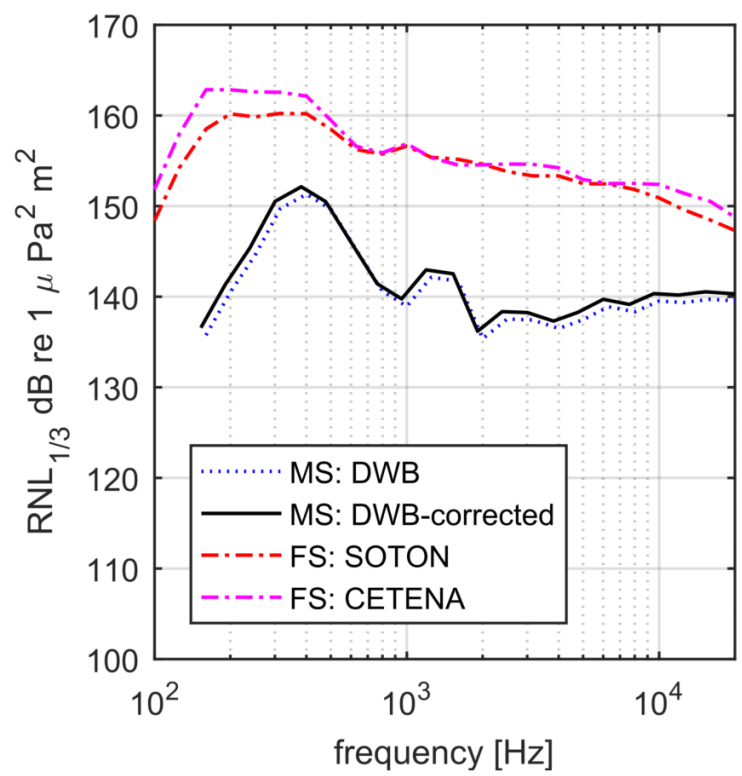

a) $\kappa_{1}=2.0$

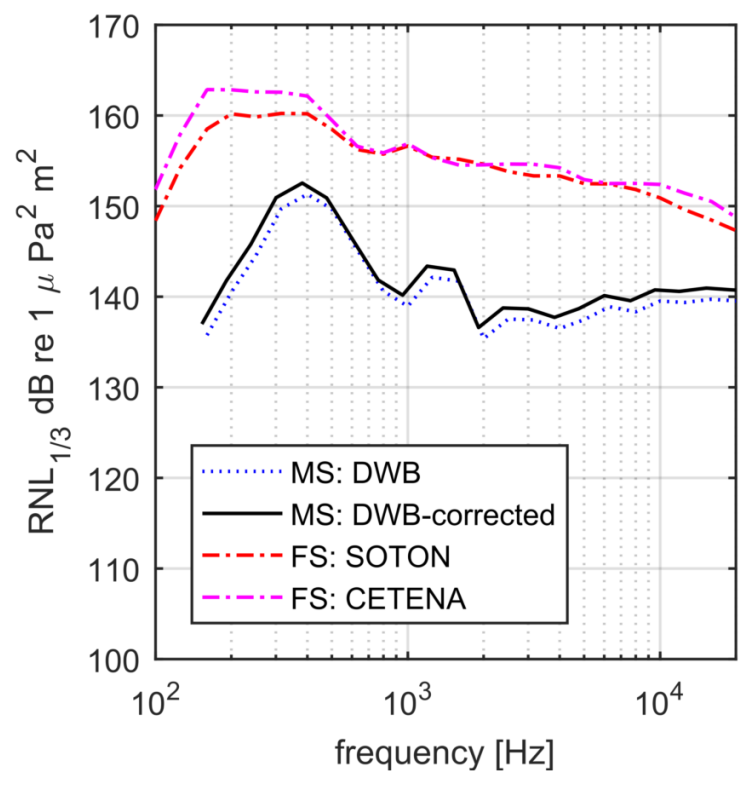

b) $\kappa_{1}=3.0$

Figure 8.11: Influence of cavity size correction on URN levels of The Princess Royal, 9 knots ship speed

satisfactory agreement with the full-scale data. Considering the overall uncertainty of the data presented, it cannot be judged which value for $\kappa_{1}$ is to be preferred.

The URN levels for 9 knots ship speed, corresponding to an engine RPM of 1200 with propeller RPM of 682 and $\sigma_{n s}=2.84$, are presented in Figure 8.11. The model tests were again performed at identical cavitation number as at full scale. At this condition, the vortex cavity is 
much more developed and the correction to the URN levels due to difference in cavity size is therefore much smaller than for the 7 knots condition. The two values for $\kappa_{1}$ give very similar results, which are much smaller than the URN levels for full-scale. However, the cavitation observations at full scale also show the presence of a small sheet cavity that was not observed in the model test, see Figure 8.9. The reason for this discrepancy in cavitation extent was not clear but it can explain the difference in the noise levels for full scale and those for model scale. It is also not clear if the cause for the difference in the extent of the sheet cavitation is of importance for the URN levels at the 7 knots condition.

\subsubsection{Hull pressures of a twin-screw vessel}

The next example is a twin-screw vessel with podded propellers for which data for both model scale and full scale is available. Unfortunately, the model tests, performed in the DWB, were performed at an operating condition of the propeller slightly different from the one during the sea trials. The ship speed was almost identical but the advance coefficient was notably different leading to a $4 \%$ lower cavitation number in the model test and a $7 \%$ higher thrust coefficient. The model tests were performed according to the standard procedures for HPF tests in the DWB, hence a Froude-scaled condition with a correction on the model speed to account for the scale effect on the ship wake. The cavitation extent for the model test is shown in Figure 8.12b and consists of a well developed tip-vortex cavity. Information was not available on the inception speed of the vortex cavity, but, as the vortex cavity is well developed, it was asssumed that a Reynolds-number correction was not necessary.

The comparison of the broadband HPF of the model test and full scale is presented in Figure 8.12a. The model test predicts a higher noise level than measured at full scale with the centre frequency of the hump at a lower value. The difference in propeller operating condition can be corrected for by using the ETV-method. Both model- and full-scale conditions were analysed with PROCAL and with the ETV-method. At model scale, a measured nominal wake field was used and the effect of torpedo and strut of the pod was taken into account in the PROCAL computation. The wake field at full scale was obtained by correcting the model-scale measured wake field for the Reynolds number effect on the boundary layer of the hull. The CRS wake scaling tool was used for this purpose (Hally, 2002). The results indicated that the $r_{c} / D$ for model scale was $14 \%$ larger than for full scale which leads to a difference of $3.5 \mathrm{~dB}$ in $k_{p, \max }$. The differences in cavity size and cavitation number lead to a centre frequency of the hump that is $17 \%$ lower at model scale.

The corrections obtained from the ETV-method were applied to the model-test results for $f / f_{b p}>2$ to avoid a shift of the first two blade rate tonals. These results are also presented in Figure 8.12a. The corrected model-test data show very good agreement with the results from the sea trial. The perfect agreement of the two smaller humps on top of the broadband hump is considered a coincidence. The broadband part of the spectrum at frequencies smaller than $f / f_{b p}=2$ is also present for non-cavitating conditions and is therefore not related to propeller cavitation. 


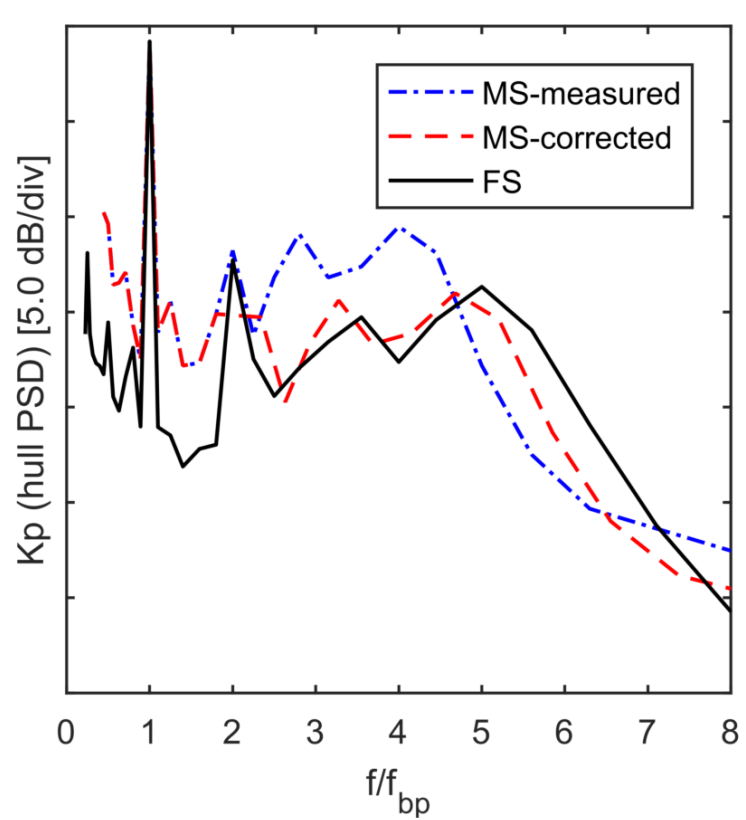

a) HPF data

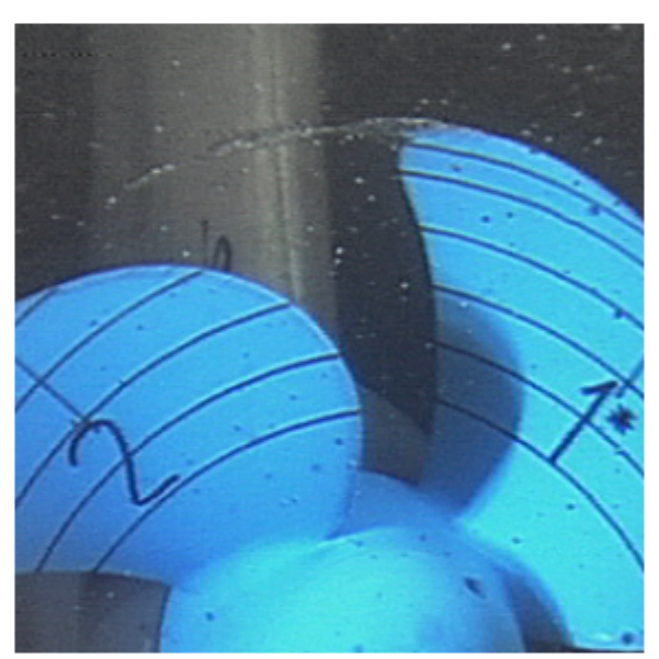

b) Image of cavitation extent in the model test

Figure 8.12: Comparison of broadband HPF spectrum measured at model-scale (MS) and fullscale (FS) on a twin-screw vessel. The model-scale data has also been corrected for the difference in propeller loading to full scale.

\subsubsection{Hull pressures of the Combi Freighter}

The last example that is presented concerns the Combi Freighter shown in Figure 6.5. The comparisons between the predictions of the ETV-method and sea-trial data for HPF and URN were shown in Section 7.5. Here, we present a comparison between broadband HPF obtained by model tests in the DWB and as obtained by sea trials. The model tests were performed after the sea trials, hence ship draft, ship speed, shaft rotation rate, propeller pitch setting and locations of pressure transducers were taken from the sea trial conditions. The model speed was increased to account for the viscous scale effect on the mean wake fraction. The comparison of the URN levels between model tests and sea trials is presented by Lafeber et al. (2017) and Lloyd et al. (2018). Here, we show the comparison of the HPF for the centre transducer that was located directly above the top position of the propeller. Analysis of the HPF measured in the sea trials indicated that the spectra of the other transducers were affected by vibrations of the hull plating. The centre transducer was located very close to the centre girder and was therefore not so much affected by the hull-plate vibrations. Results are presented for two conditions.

The first condition corresponds to a high blade-pitch condition with the ship sailing at 10.2 knots and a shaft rotation rate of $224.2 \mathrm{rpm}$. The cavitation pattern consists of a sheet cavity that merges with the tip-vortex cavity of which a series of images by high-speed video camera is shown in Figure 6.17. During the sea trials, the propeller blade was observed with a boroscope. These observations show a similar cavitation pattern as observed in the model tests, but the weather conditions were not good enough to quantify the cavitation extents and to analyse the cavitation dynamics. The model tests were performed at identical cavitation number as at full scale, being $\sigma_{n}=2.89$. Inception of tip-vortex cavitation was measured at $\sigma_{n}=7.3$ in the 


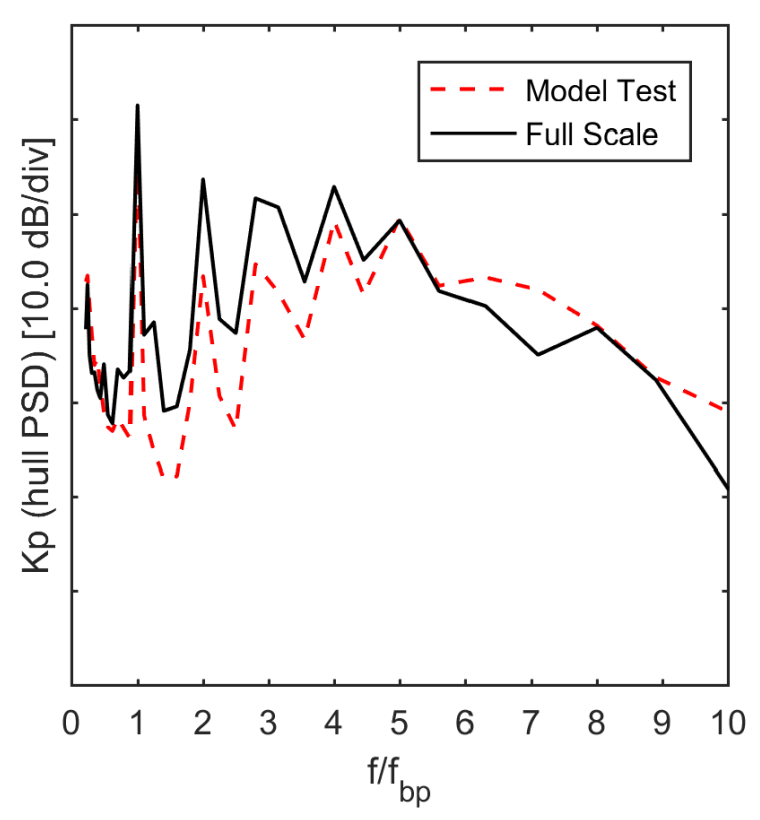

a) High pitch, $V_{s}=10.2$ knots

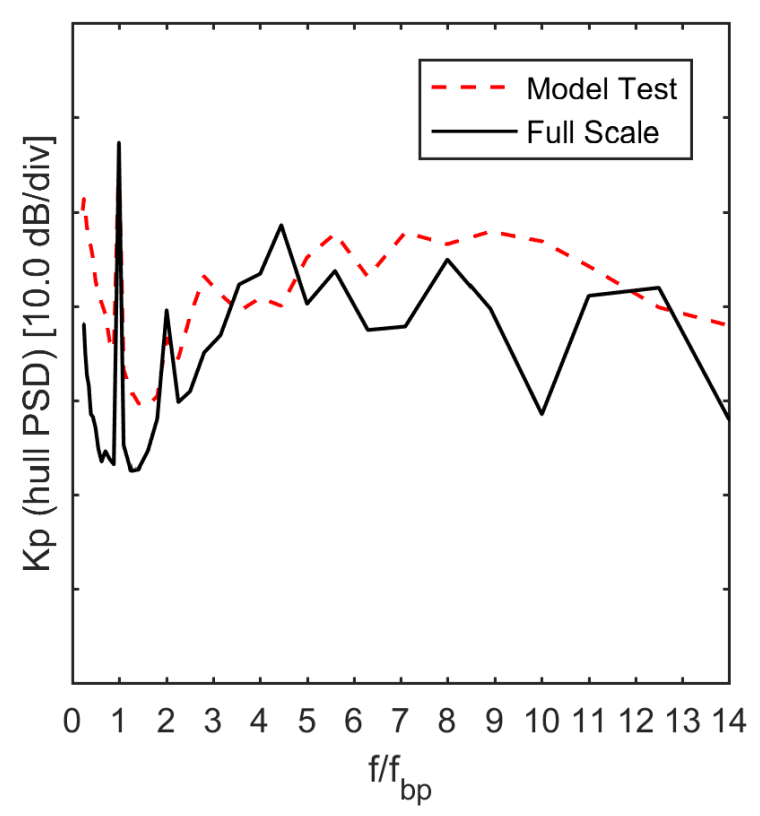

b) Low pitch, $V_{s}=3.1$ knots

Figure 8.13: Comparison of a broadband HPF spectrum measured at model scale and full scale for the Combi Freighter.

model tests. As the propeller is a CPP, the inception of the propeller at this pitch setting could not be determined during the sea trials. Analysis of the viscous scale effect on the tip-vortex cavity size suggested that the broadband noise by the tip-vortex would be underpredicted by approximately $3 \mathrm{~dB}$ and that the ratio between full-scale and model-scale centre frequency equals 0.86 . Because of the presence of sheet cavity on the blade, the comparison is shown for a cavitation number in the model test that is identical as during the sea trials and no correction to the broadband levels has been made.

The comparison of the broadband HPF for the high blade-pitch condition is presented in Figure 8.13a. Overall, the model-test results show reasonable agreement with the sea-trial data, but the model-scale values for frequencies below the centre frequency are smaller than the fullscale values, also resulting in an underprediction of the levels at the second and third harmonic of the blade passage frequency.

The second condition corresponds to a low blade-pitch condition with the ship sailing at 3.1 knots and identical shaft rotation rate as the first condition. The cavitation pattern consists of a leading-edge cavity on the face of the blade and a tip-vortex cavity on the back and is presented in Figure 6.18. The model tests were performed at various shaft rotation rates to check for the dependency on Reynolds number but the effect on the broadband HPF was very small. Because the leading-edge vortex cavity was very well developed while the tip-vortex cavity was not too far from inception, it is concluded that the broadband levels are caused by the leading-edge vortex cavity. The comparison between model tests and sea trials is presented in Figure 8.13b and the results show acceptable agreement. 


\subsection{Discussion}

A new method has been proposed for model tests to account for the effect of Reynolds number on the size of a vortex cavity and thereby on HPF and URN. The method makes use of an analytical vortex model discussed in Section 3.3.2, through which the ratio of cavity and viscous core size can be related to the ratio of cavitation number and cavitation inception number. Unfortunately, experimental validation of this relation could not be performed as no dataset was available for which both the viscous core size and cavitation inception was measured. Furthermore, it needs to be noticed that the analytical relation assumes that the cavitation inception number equals the, absolute value of the, minimum pressure coefficient in the vortex, so the inception number obtained in an experiment needs to be corrected for the effect of the tensile strength of water. As already discussed in Section 3.3.3, this complicates the experimental validation. The analytical relation is also somewhat dependent on the empirical parameters of the vortex model which adds to the uncertainty of the results of the method but the dependency is not so large. Confidence in the relation is obtained from the observation that it gives the expected behaviour in the limit of very small and very large cavity size.

The change in noise levels due to a difference in model-scale and full-scale cavity size can be computed from the empirical relations used in the ETV-method which were presented in Chapter 7. Theoretical relations for the noise scaling were also investigated, but two issues were encountered that require further research.

The first issue is that the empirical relation for the scaling of noise with cavity radius could not be matched with theoretical relations. However, the derivation of the theoretical relation required a number of assumptions. These assumptions are related to the formulation for the acoustic efficiency, and the formulation for the total frequency band in which noise is radiated. For the acoustic efficiency, the formulation for the low-frequency noise of a bubble was used, although it was discussed in Chapter 4.2 that the collapse of a cavitation bubble is different from the collapse of a vortex cavity. Therefore, it is recommended to analyse the acoustic efficiency of a vortex cavity by detailed experiments. A first impression of the scaling of the acoustic efficiency can also be obtained using the computational method for the 2-D vortex cavity presented in Section 4.2, but, as discussed in that section, the relation between the 2$\mathrm{D}$ vortex cavity and the 3-D vortex cavity requires further research. The scaling of the total frequency band could also be obtained from such computations, but only for the low frequency part of the spectrum: The high frequency part is considered to be caused by the last phase of the collapse where compressibility is also of importance, and by the smaller cavitation bubbles generated in the collapse process. Both are not included in the computational method described.

The second issue is that the theoretical relation shows a dependency on the ratio of the cavitation number at full scale and at model scale. This ratio was not included in the semi-empirical ETV-method as the effect of the cavitation number is difficult to separate from the effect of the cavity size. Because the ratio of cavitation numbers is also included in the formulation for the acoustic efficiency, further investigation of this efficiency may provide such information. Because model tests are traditionally performed at identical cavitation number as at full scale to obtain identical cavitation patterns, this topic has not been investigated as far as known.

The application of the scaling method improved the prediction of the model tests when the test condition was close to inception. However, as discussed in Sections 2.5 and 2.6, many 
aspects affect the HPF and URN measured in model tests and sea trials. These aspects include the difference in ship wake field, the effect of non-condensable gas, the effect of hull plate vibrations, and uncertainties in the sea trial on operational conditions and propeller pitch. These aspects were not considered here to limit the scope of the thesis, for some cases confidentiality reasons, and, for other cases due to lack of information. The descriptions of the experiments are therefore incomplete, but the experiments in the DWB were performed according to standard procedures at MARIN which are described in the references provided in Sections 2.5 and 2.6. Due to the uncertainties for some of these aspects for either model tests or sea trials, the presented data could not be used to further improve the scaling method with respect to the assumptions made.

The high pitch condition for the Combi Freighter illustrates a practical problem encountered in applying the scaling method. The cavitation pattern consists of a sheet cavity that directly merges with the tip-vortex cavity. Such a cavitation pattern was also used in the fitting process of the ETV-method, where the cavity size was assumed to scale with tip-vortex strength, although the uncertainty of the prediction for this cavitation pattern was higher than for tip-vortex cavitation. The boundary layer on the blade below the side-entrant jet and the formation of the vorticity layer by the curvature of the sheet cavity are both affected by viscosity, but the effect on the size of the sheet cavity is unknown and it is generally assumed that viscosity does not affect the sheet cavity. One may argue that, for such a cavitation pattern, the magnitude of the level is related to the volume of the sheet cavity that is shed into the vortex cavity whereas the centre frequency of the hump is related to the tip-vortex strength. It is then only the frequency of the hump that needs to be corrected for viscous effects. This would improve the correlation between model tests and sea trials for this example, but such a correction was not applied due to the large uncertainty involved. This topic requires further investigation.

\subsection{Conclusions}

The effect of the difference in model-scale and full-scale Reynolds number on the size of the tipvortex cavity and, therefore, on broadband noise, can accounted for by a novel scaling method. The method uses a vortex model in which a change in viscous core radius, due to a change in Reynolds number, can be related to a change in cavity radius without knowing the actual viscous core radius (Eq. 8.9). Instead, the method requires the measurement of cavitation inception in the model test. The effect of a change in cavity size on broadband noise levels is then predicted using semi-empirical relations employed by the ETV-method (Eq. 8.17). The method shows that for small cavity size the effect of the cavitation inception number, and therefore Reynolds number, is very relevant, whereas for large cavity size the effect of Reynolds number becomes very small (Figure 8.2).

Predictions of broadband noise by model tests were improved by the method when the test condition is close to inception (Figures 8.6 and 8.10). It was also shown that the ETVmethod can be used to correct results of model tests for a difference in operating point to full scale (Figure 8.12). After the correction, a good agreement in broadband HPF was obtained. Cases were also shown for which the correction could not be applied because of the presence of sheet cavitation or of multiple vortices. For these cases, however, the model tests showed an acceptable prediction of the broadband HPF (Figure 8.13). 
The scaling method requires further development and validation as several issues need to be resolved. The applied vortex model has been validated for wings and propellers (Figures 3.9 and 7.2), but, as discussed in Section 3.3.3, there are still some aspects that need to be investigated. More experimental studies to validate the vortex model are required, especially showing the effect of a change in Reynolds number on cavity size and viscous core size. Another issue is that the scaling of broadband noise with cavity size is empirically determined and has no theoretical background. A practical issue is how to deal with situations in which both a sheet cavity and a vortex cavity are present, or in which two vortex cavities are present. 


\section{Chapter 9}

\section{Concluding remarks}

This chapter presents the concluding remarks of the thesis. It summarises the conclusions, it provides the practical implications of the studies performed, and it gives the recommendations for further research.

\subsection{Introduction}

There is a lack of both fundamental and applied knowledge on developed tip-vortex cavities and its related broadband noise as shown by literature reviews. This leads to uncertainties in the application of, for instance, model tests to predict hull-pressure fluctuations (HPF) and underwater radiated noise (URN) involving vortex cavitation. Therefore, the objectives of the present thesis were (i) to investigate the mechanisms of broadband noise by propeller tip-vortex cavitation, and (ii) to develop prediction methods for HPF and URN.

The first part of the thesis consists of theoretical studies on the kinematics, dynamics and acoustics of vortex cavitation. With respect to the kinematics, the developed analytical vortex model for the radial distribution of the azimuthal velocity describes the experimental data of Pennings (2016) well. With respect to the dynamics, the developed analytical formulation for the dispersion relation showed an acceptable match with experimental data of, again, Pennings (2016). To our knowledge, both comparisons were first to provide a detailed quantitative assessment of the kinematic and dynamic aspects of developed tip-vortex cavitation. The acoustics of vortex cavitation were considered by analysing, among others, formulations for a cylindrical noise source.

The results obtained from the first part of the thesis were used in the second part to elucidate the mechanisms of broadband HPF measured on the hull above the propeller. These results were also used for the development of a semi-empirical prediction method for the broadband noise by propeller tip-vortex cavitation. The method predicts both the broadband HPF and URN. It employs results of a boundary element method that computes the flow on the propeller operating in the ship wake field. Furthermore, a new methodology for model tests has been proposed and evaluated to correct for the scale effect on the radius of the vortex-cavity due to the difference in Reynolds number between model scale and full scale. 
The following sections of this chapter will summarise the conclusions from the studies performed from the perspective of the two main objectives. The prediction of broadband noise by the semi-empirical method is discussed separately from the prediction using model tests. Detailed conclusions on the separate topics of the theoretical studies have been provided in the corresponding chapters. The practical implications of the studies performed are presented in Section 9.5. Finally, some recommendations for further research are presented in Section 9.6.

\subsection{Conclusions on mechanisms of vortex-cavitation noise}

The conclusions on the mechanisms of noise generated by vortex-cavitation can be summarised as follows:

Cavity pattern and excitation: The cavity pattern on the propeller of a twin-screw vessel often consists of a tip-vortex cavity that is connected to a closed or open sheet cavity on the propeller blade. When the tip-vortex cavity is connected to a closed sheet cavity, it was shown that, when the blade moves out of the wake peak, the vapour volume of the sheet cavity moves into the vortex cavity. As the vortex cavity is then not in equilibrium, a collapse and rebound occurs. When the sheet cavity is open, a side-entrant jet may develop which may result into a, here called, closure-vortex cavity when the blade moves out of the wake peak. This closurevortex cavity shows a collapse and rebound similar to that of a tip-vortex cavity.

Viscous effects: Computations in 2-D viscous flow showed that the collapse of the vortex cavity is inertia driven, so the effect of viscosity on the collapse is considered small. However, viscosity does affect the radius of the vortex cavity, especially when it is smaller than or approximately equal to the radius of the viscous core.

Resonance: An analytical formulation for the resonance frequency of a vortex cavity has been derived for 2-D viscous flow. The formulation consists of the added mass of the vortex cavity and a stiffness term containing the cavitation number or, in an alternative formulation, the azimuthal velocity at the cavity interface. A formulation for the resonance frequency for 3-D flow could not be obtained. A dispersion relation has been derived for the propagation of waves on the cavity interface in 3-D inviscid flow with an ad-hoc correction for viscous flow, but all perturbations result in neutrally stable waves. Therefore, several criteria for resonance were proposed, among which the criterion of zero group velocity of the mode that involves a cavity volume variation. The dispersion relation showed an acceptable agreement with experimental data of Pennings (2016).

Noise source: Analytical formulations, in which the vortex cavity is considered as a cylindrical noise source, show that volume variations are the dominant noise source and that the deformations of the centreline or the rotation of an elliptical cross-section are much less efficient with respect to the radiation of noise. The, finite-length, cylindrical noise source behaves in the far field and at low frequencies as a monopole noise source. In the near field, where the HPF are measured, the situation is more complicated. A limited comparison with experimental data showed differences in the distribution of phase over the hull surface from which it was concluded that the employed formulation for the cylindrical noise source requires further research. 
Spectrum: Analytical formulations and analysis of experimental data show that the transient oscillatory behaviour of the collapse and rebound of the vortex cavity, in combination with the variability of the cavity collapse with blade passage, leads to a spectrum featuring a broadband hump. It was shown that this variability not only leads to the broadband hump but also affects the amplitudes of the higher harmonics of the blade passage frequency.

\subsection{Conclusions on the semi-empirical prediction method}

The conclusions regarding the development of the semi-empirical prediction method can be summarised as follows:

Prediction of the cavity radius: An analytical solution for the distribution of the azimuthal velocity of a vortex cavity, extended with an empirical term to account for the effect of vorticity roll-up, shows a good match with experimental data of Pennings (2016). The analytical solution was derived for 2-D axisymmetric incompressible viscous flow. The experimental data also showed agreement with the derived boundary condition for the azimuthal velocity at the cavity interface that corresponds to a shear-stress continuity condition. By approximation, this corresponds to a zero-shear stress condition for the flow on the liquid side of the cavity interface. From the distribution of the azimuthal velocity, the relation between cavity radius and cavitation number can be determined. For this relation, Pennings (2016) has also provided experimental data. Remarkable is that the fitted formulation for cavitating flow only predicts the cavity radius correctly when the vortex cavity is detached from the surface of the wing. When the vortex cavity is attached to the wing, the additional corrections for cavitating flow were not required. In fact, it was concluded that the formulation for non-cavitating flow was also well suited to predict the cavity radius, which was defined by the radius where pressure equals vapour pressure. Even though this aspect was not understood, a vortex model for non-cavitating flow was used for the prediction of the cavity radius in the semi-empirical prediction method. Such a vortex model well captures the variation of the radius of a vortex cavity with cavitation number for a propeller, except for high propeller loading.

Prediction of the centre of the hump: The maximum level and centre frequency of the broadband hump of HPF and URN by vortex cavitation were well predicted using semi-empirical formulations that include cavity radius and cavitation number. This approach was adopted from Raestad (1996). The empirical coefficients were obtained using experimental data of HPF for twin-screw vessels, originating from model tests as well as from sea trials. Two groups of cases had to be distinguished for determining the empirical parameters, one group of cases for which the cavitation pattern was dominated by vortex cavitation and one group of cases which also featured a closure-vortex cavity generated by the sheet cavity. The standard deviation of the maximum noise levels was 3.2 and $5.0 \mathrm{~dB}$ for the cases with a vortex cavity and with a closurevortex cavity, respectively, and the standard deviation for the non-dimensional centre frequency was 0.75 and 1.0 , respectively.

Prediction of the spectrum: The shape of the spectrum was well described by two functions, one function describing the broadband hump and one function describing the very low and high-frequency part. The validation cases for the URN required different empirical factors for the high-frequency part. The physical background of these different factors is not understood 
and requires further investigation. Overall, the spectrum for the HPF and URN was predicted satisfactorily.

Limitations: Even though the method was developed for twin-screw vessels, it was also successfully applied to a single-screw vessel. The main limitation is that it only gives a satisfactory prediction for cases where cavitation is limited to the tip area, so there should not be too much sheet cavitation on the propeller blade.

\subsection{Conclusions on the experimental prediction method}

The conclusions regarding the use of model tests to predict broadband noise can be summarised as follows:

Model tests in general: In principle, model tests are well suited to predict broadband HPF and URN by propeller cavitation, but there are many aspects that need to be taken into account. One particular aspect investigated in the present study is the effect of viscosity on the radius of the vortex-cavity. This is relevant as the Reynolds number in the model test is much smaller than at full scale.

Viscous-scale effect on the radius of the vortex cavity: The analytical vortex model suggests that the viscous-scale effect on the radius of the vortex cavity becomes small when the cavity radius becomes larger than approximately $150 \%$ of the viscous-core radius of the non-cavitating vortex. When the radius of the cavity becomes smaller than the radius of the viscous core, the viscous-scale effect is very significant. A scaling rule was developed from the analytical vortex model by presenting the ratio between cavity radius and viscous core radius as a function of the ratio between cavitation number and cavitation number at inception. This scaling rule provides the ratio between model-scale and full-scale non-dimensional cavity radius for given model-scale and full-scale cavitation number, without knowing the radius of the viscous core. However, it does require the measurement of the cavitation inception number in the model test.

Selecting the cavitation number: The scaling rule for cavity radius can be used to select the cavitation number in the model test such that the non-dimensional cavity radius in the model test is equal to the full-scale value for that operational condition. This approach is different from the classical approach in which the cavitation number in the model test is equal to the value of the cavitation number at full-scale.

Scaling noise levels: The semi-empirical relation to predict the noise level utilizing the cavity radius can also be used to relate the ratio of (non-dimensional) model-scale and full-scale cavity radius to a difference in noise level. However, the semi-empirical relation applied cannot be derived from existing scaling rules for broadband cavitation noise. Predictions from model tests were improved when the test-condition was close to inception showing the feasibility of the applied method.

Limitations: The most important limitation of the method is that it does require the presence of an isolated tip-vortex cavity in the model test. If sheet cavitation is present on the propeller, the cavitation number cannot be reduced because the extent of sheet cavitation is considered to be correctly modelled when the cavitation number in the model test equals the cavitation number at full scale. Also, measured noise levels in the presence of sheet cavitation include the 
contribution of sheet cavitation, so the correction of the noise level due to the Reynolds-scale effect on vortex cavitation can not be applied.

\subsection{Practical implications of the studies performed}

The studies described in the present thesis lead to the following practical implications:

Propeller design: The semi-empirical method allows tip-vortex noise to be included as a criterion in the propeller design. In propeller design, the blade-tip loading needs to be selected such that there is a balance between efficiency and cavitation noise. The boundary element method used to evaluate the strength of the tip-vortex is already being used to evaluate the propeller efficiency, so both aspects can now be taken into account directly. The method is already used for that purpose at MARIN and by members of the CRS-organisation.

Model tests: For model tests, the proposed methodology improves the noise predictions for situations in which, due to viscous-scale effects, the non-dimensional radius of the tip-vortex cavity in the model test is much smaller than at full-scale. Nowadays, such situations occur more often than in the past due to the improvement in the designs of ship hulls and of propellers. These improvements have led to a reduction of sheet cavitation on the blade and to an increase in inception speed of tip-vortex cavitation. The methodology is already used in cavitation tests in the DWB of MARIN.

Broadband noise: The improvements in the prediction of broadband noise by the methods described in the thesis result in a reduction of the risks when designing a ship that needs to comply with requirements on comfort or on underwater radiated noise. The improvements in knowledge on the mechanisms involved lead to a better understanding of broadband-noise spectra obtained by sea trials, model tests, CFD computations, semi-empirical prediction methods, and their comparison.

\subsection{Recommendations}

The recommendations for further research are as follows:

Kinematics: The kinematics of a vortex cavity needs to be further investigated with respect to the prediction of the radius of the cavity using the radial distribution of the azimuthal velocity, especially when the vortex cavity is attached to the blade. In particular, the increase of the vorticity layer around the vortex cavity with increasing cavity radius as well as the contribution of non-condensable gas require further research. The relation between the ratio of cavity radius and viscous core radius and the ratio of cavitation number and cavitation inception number requires further validation with respect to its expected independence of vortex strength and viscous core size.

Dynamics: The dynamics of a vortex cavity requires further research with respect to the final phase of the collapse, including the analysis of the forces exerted by the vapour part of the cavity. Also, the analysis of the dispersion relation needs to be extended from potential flow to full-viscous flow, either analytically or numerically. It is especially the criterion for 
resonance that needs to be investigated, as well as the behaviour of the breathing mode at small wavenumber. The criterion for resonance can also be investigated experimentally by highspeed video observations of the vortex cavity at and near the wing tip from which the dispersion relation at small wavenumbers can be analysed in detail.

Acoustics: The acoustics of a vortex cavity needs to be further investigated experimentally with respect to its directionality and spreading loss to obtain validation material for the noisesource model. The effect of other deformations of the cylindrical noise source than those applied need to be evaluated. Furthermore, the scaling of cavitation noise requires further research, for instance using geometrically similar models of different size in a cavitation-test facility. Specifically, there is a lack of information on the formulation for the acoustic efficiency to be used in the scaling formula for cavitation noise.

Mechanisms: The mechanisms of broadband noise require further investigation with respect to the variability between blade passages, for model tests as well as at full scale, for instance using the approach presented in this thesis. This variability is expected to be of more importance for the amplitudes of the higher order blade rate tonals. Also, the similarity and dissimilarity between the collapse of the tip-vortex cavity and the collapse of the closure-vortex cavity require further investigation. The contribution of non-condensable gas to the size and dynamics of vortex cavities needs to be further assessed.

Prediction: The semi-empirical relation to predict the broadband noise levels cannot be explained from a theoretical point of view. Hence, more research is required on both data analysis procedures and on theoretical aspects. The methodology to correct for the effect of the smaller Reynolds number in the model-test than at full scale requires more detailed validation studies: This concerns the effect on vortex cavity size as well as on noise levels. 


\section{Bibliography}

21st ITTC Cavitation Committee (1996). Final report and recommendations to the 21st ITTC. In 21 st International Towing Tank Conference, Bergen, Norway.

23rd ITTC Specialist Committee on Cavitation Induced Pressures (2002). Final report and recommendations to the 23rd ITTC. In 23rd International Towing Tank Conference, Venice, Italy.

23rd ITTC Specialist Committee on Water Quality and Cavitation (2002). Final Report and Recommendations to the 23rd ITTC. In 23rd International Towing Tank Conference, Venice, Italy.

27th ITTC Specialist Committee on Hydrodynamic Noise (2012). Final report and recommendations to the 27th ITTC. In 27th International Towing Tank Conference, Copenhagen, Denmark.

28th ITTC Specalist Committee on Hydrodynamic Noise (2017). Final report and recomendations to the 28th ITTC. In 28th International Towing Tank Conference, Wuxi, China.

Abramowitz, M. and Stegun, I. A. (1972). Handbook of Mathematical Functions With Formulas, Graphs and Mathematical Tables. National Bureau of Standards, 10th edition.

Ackeret, J. (1930). Über stationäre Hohlwirbel. Ing. -Archiv, I Band, pages 399-402.

Ainslie, M. (2010). Principles of Sonar Performance. Springer-Verlag.

Aktas, B., Atlar, M., Turkmen, S., Shi, W., Sampson, R., Korkut, E., and Fitzsimmons, P. (2016). Propeller cavitation noise investigations of a research vessel using medium size cavitation tunnel tests and full-scale trials. Ocean Engineering, 120:122-135.

Arakeri, V. H., Higuchi, H., and Arndt, R. E. A. (1988). A model for predicting tip vortex cavitation characteristics. J. of Fluids Engineering, Vol. 110(2):190-193.

Arndt, R., Pennings, P., Bosschers, J., and van Terwisga, T. (2015). The singing vortex. Interface Focus, 5:2015.0025.

Arndt, R. E. A. (1981). Cavitation in fluid machinery and hydraulic structures. Annual Review of Fluid Mechanics, 13:273-328.

Arndt, R. E. A. (2002). Cavitation in vortical flows. Ann. Rev. Fluid Mech., 34:143-179.

Arndt, R. E. A. and Keller, A. P. (1992). Water quality effects on cavitation inception in a trailing vortex. Journal of Fluids Engineering, 114(3):430-438.

Ash, R. L. and Khorrami, M. R. (1995). Vortex stability. In Green, S. I., editor, Fluid Vortices, pages 317-372. Kluwer Academic Publishers.

ASME V\&V 20-2009 (2009). Standard for Verification and Validation in Computational Fluid Dynamics and Heat Transfer. Technical report, The American Society of Mechanical Engineers.

Astolfi, J.-A., Fruman, D., and Billard, J.-Y. (1999). A model for tip vortex roll-up in the near field region of three-dimensional foils and the prediction of cavitation onset. European 
Journal of Mechanics - B/Fluids, 18(4):757-775.

Atlar, M., Aktas, B., Sampson, R., Seo, K.-C., Viola, I. M., Fitzsimmons, P. A., and Fetherstonhaugh, C. (2013). A multi-purpose marine science and technology research vessel for full-scale observations and measurements. In 3rd International Conference on Advanced Measurement Technology for the maritime industry, AMT'13, Gdansk, Poland.

Audoly, C. and Meyer, V. (2017). Measurement of radiated noise from surface ships - Influence of the sea surface reflection coefficient on the Lloyd 's mirror effect. In Acoustics 2017, Perth, Australia.

Auger, F., Flandrin, P., Gonçalvès, P., and Lemoine, O. (1996). Time-Frequency Toolbox. For Use with MATLAB. CNRS (France) and Rice University (USA).

Baiter, H.-J. (1985). On the Theoretical Background of Cavitation Noise Scaling. Technical Report IHAK-TN 218/85, Fraunhofer Institute for Hydroacoustics, Ottobrunn, Germany.

Baiter, H.-J. (1989). On cavitation noise scaling with the implication of dissimilarity in cavitation inception. In ASME International Symposium on Cavitation Noise and Erosion in Fluid Systems.

Baiter, H.-J., Gruneis, F., and Tilmann, P. (1982). An extended base for the statistical description of cavitation noise. In ASME International Symposium on Cavitation Noise, Phoenix, Arizona, USA, pages 93-108.

Bark, G. (1985). Prediction of propeller cavitation noise from model tests and its comparison with full scale data. Journal of Fluids Engineering, 107(1):112-120.

Bark, G. (2000). Selected problems about scaling of cavitation noise at low and medium high frequencies. In Developments in the Design of Propulsors and Propulsion Systems. 34th WEGEMT School, Delft University of Technology, the Netherlands.

Bark, G. and Bensow, R. E. (2012). Hydrodynamic mechanisms controlling cavitation erosion. In 29th Symposium on Naval Hydrodynamics, Gothenburg, Sweden.

Bark, G. and Bensow, R. E. (2013). Hydrodynamic mechanisms controlling cavitation erosion. International Shipbuilding Progress, 60:345-374.

Batchelor, G. K. (1964). Axial flow in trailing line vortices. J. Fluid Mech., 20(4):645-658.

Batchelor, G. K. (1967). An Introduction to Fluid Dynamics. Cambridge University Press.

Baudin, E. and Mumm, H. (2015). Guidelines for Regulation on UW Noise from Commercial Vessels. Technical report, AQUO and SONIC EU FP7 projects, Bureau Veritas, DNV-GL.

Bendat, J. S. and Piersol, A. G. (2010). Random Data - Analysis and Measurement Procedures. John Wiley \& Sons, Inc., fourth edition.

Bensow, R. E. (2015). Large eddy simulation of a cavitating propeller operating in behind conditions with and without pre-swirl stators. In 4th International Symposium on Marine Propulsors (smp'15), Austin, Texas, USA.

Berger, S., Gosda, R., Scharf, M., Klose, R., Greitsch, L., and Abdel-Maksoud, M. (2016). Efficient numerical investigation of propeller cavitation phenomena causing higher-order hull pressure fluctuations. In 31st Symposium on Naval Hydrodynamics, Monterey, California, USA.

Berghult, L. (2000). Propeller induced tip vortex noise as function of blade area and blade-tip loading. In Int. Conf. on Propeller Cavitation, NCT'50, Newcastle upon Tyne, UK.

Billant, P. and Gallaire, F. (2005). Generalized Rayleigh criterion for non-axisymmetric centrifugal instabilities. Journal of Fluid Mechanics, 542:365-379.

Billet, M. L. and Holl, J. W. (1981). Scale effects on various types of limited cavitation. Journal of Fluids Engineering, 103(3):405 - 414. 
Birch, D. M. (2012). Self-similarity of trailing vortices. Physics of Fluids, 24(2).

Birvalski, M. and van Rijsbergen, M. X. (2018). Size and concentration measurements of cavitation nuclei in the wake of a ship model. In 10th International Cavitation Symposium CAV2018, Baltimore, Maryland, USA.

Blackstock, D. T. (2000). Fundamentals of Physical Acoustics. John Wiley \& Sons, Inc.

Blake, W. K. (1986). Mechanics of Flow-Induced Sound and Vibration. Academic Press Inc.

Bosschers, J. (2008). Analysis of inertial waves on inviscid cavitating vortices in relation to low-frequency radiated noise. In WIMRC Cavitation Forum, Warwick University, UK.

Bosschers, J. (2009a). Investigation of hull pressure fluctuations generated by cavitating vortices. In 1st International Symposium on Marine Propulsors (smp'09), Trondheim, Norway.

Bosschers, J. (2009b). Investigation of the resonance frequency of a cavitating vortex. In NAG/DAGA International Conference on Acoustics, Rotterdam, The Netherlands.

Bosschers, J. (2009c). Modeling and analysis of a cavitating vortex in 2D unsteady viscous flow. In 7th International Symposium on Cavitation CAV2009, Ann Arbor, Michigan, USA.

Bosschers, J. (2010). On the influence of viscous effects on 2-D cavitating vortices. In 9th International Conference on Hydrodynamics, Shanghai, China.

Bosschers, J., Janssen, A., and Hoeijmakers, H. W. M. (2008a). Similarity solutions for viscous cavitating vortex cores. Journal of Hydrodynamics, Ser. B, 20(6):679-688.

Bosschers, J., Lafeber, F. H., de Boer, J., Bosman, R., and Bouvy, A. (2013). Underwater radiated noise measurements with a silent towing carriage in the Depressurized Wave Basin. In 3rd International Conference on Advanced Measurement Technology for the Maritime Industry, AMT'13, Gdansk, Poland.

Bosschers, J. and van Wijngaarden, E. (2012). Scale effects on hull pressure fluctuations due to cavitating propellers. In 10th International Conference on Hydrodynamics, volume 2, St. Petersburg, Russia.

Bosschers, J., Vaz, G., Starke, A. R., and Wijngaarden, E. V. (2008b). Computational analysis of propeller sheet cavitation and propeller-ship interaction. In RINA MARINE CFD Conference, Southampton, UK.

Bosschers, J., Willemsen, C., Peddle, A., and Rijpkema, D. (2015). Analysis of ducted propellers by combining potential flow and RANS methods. In 4th International Symposium on Marine Propulsors (smp'15), Austin, Texas, USA.

Bradshaw, P. (1969). The analogy between streamline curvature and buoyancy in turbulent shear flow. J. Fluid Mech., 36:177-191.

Brandner, P. A. and Walker, G. J. (2003). Hydrodynamic performance of a vortex generator. Experimental Thermal and Fluid Science, 27(5):573-582.

Brennen, C. (2009). Cavitation and Bubble Dynamics. Oxford University Press.

Breslin, J. P. (1970). Theoretical and experimental techniques for practical estimation of propeller-induced vibratory forces. In SNAME Symposium on Ship Vibration, pages 23-40, New York, USA.

Breslin, J. P., van Houten, R. J., Kerwin, J. E., and Johnsson, C.-A. (1982). Theoretical and experimental propeller-induced hull pressures arising from intermittent blade cavitation, loading, and thickness. SNAME Transactions, 90:111-151.

Briançon-Marjollet, L. and Merle, L. (1996). Inception, development, and noise of a tip vortex cavitation. In 21st Symposium on Naval Hydrodynamics, Trondheim, Norway.

Brown, N. A. (1976). Cavitation noise problems and solutions. In International Symposium on Shipboard Acoustics, Noordwijkerhout, Netherlands. 
Brown, N. A. (1999). Thruster noise. In Dynamic Positioning Conference.

Brubakk, E. and Smogeli, H. (1988). QE2 from turbine to diesel - consequences for noise and vibration. In IMAS Conference, The Design and Development of Passenger Ships.

Burnham, D. C. and Hallock, J. N. (1982). Chicago Monostatic Acoustic Vortex Sensing System. Vol. IV: Wake Vortex Decay. Technical report, U.S. Department of Transportation, Research and Special Programs Administraton, Cambridge, MA, USA.

Burrill, L. C. (1951). Sir Charles Parsons and cavitation. The Institute of Marine Engineers, Transactions, LXIII(8):149-167.

Butcher, J. C. (2008). Numerical Methods for Ordinary Differential Equations. John Wiley \& Sons Ltd, 2nd edition.

Carlton, J. (2007). Marine Propellers and Propulsion. Butterworth-Heineman, 2nd edition.

Carlton, J. S. (2015). Broadband cavitation excitation in ships. Ships and Offshore Structures, 10(3):302-307.

Carlton, J. S. and Fitzsimmons, P. A. (2006). Full scale cavitation observations relating to propellers. In Sixth International Symposium on Cavitation, CAV2006, Wageningen, The Netherlands.

Chahine, G. L. (1995). Bubble interaction with vortices. In Green, S. I., editor, Fluid Vortices, pages 783-827. Kluwer Academic Publishers.

Chahine, G. L., Frederick, G. F., and Bateman, R. D. (1993). Propeller tip vortex cavitation suppression using selective polymer injection. Journal of Fluids Engineering, 115(3):497503.

Chang, N., Ganesh, H., Yakushiji, R., and Ceccio, S. L. (2011). Tip vortex cavitation suppression by active mass injection. Journal of Fluids Engineering, 133(11).

Chang, N. a. and Ceccio, S. L. (2011). The acoustic emissions of cavitation bubbles in stretched vortices. The Journal of the Acoustical Society of America, 130(5):3209-3219.

Chesnakas, C. and Jessup, S. (1998). Experimental characterization of propeller tip flow. In 22nd Symposium on Naval Hydrodynamics, Washington D.C., U.S.A.

Choi, J. and Ceccio, S. L. (2007). Dynamics and noise emission of vortex cavitation bubbles. Journal of Fluid Mechanics, 575:1-26.

Choi, J., Hsiao, C.-T., Chahine, G. L., and Ceccio, S. (2009). Growth, oscillation and collapse of vortex cavitation bubbles. Journal of Fluid Mechanics, 624:255.

Choi, J.-K., Hsiao, C.-T., and Chahine, G. L. (2004). Tip vortex cavitation inception study using the surface averaged pressure (SAP) model combined with a bubble splitting model. In 25th Syposium of Naval Hydrodynamics, St John's, Newfoundland and Labrador, Canada.

Chow, J. S., Zilliac, G. G., and Bradshaw, P. (1997). Mean and turbulence measurements in the near wake of wingtip vortex. AIAA Journal, 35(10):1561-1567.

Clay, C. C. and Medwin, H. (1977). Acoustical Oceanography: Principles and Applications. John Wiley \& Sons Ltd.

Cotel, A. J. (2002). Turbulence inside a vortex: Take two. Physics of Fluids, 14(8):2933-2934.

Crow, S. C. (1970). Stability theory for a pair of trailing vortices. AIAA Journal, 8(12):21722179.

de Bruijn, A. and Ten Wolde, T. (1974). Measurement and prediction of sound inboard and outboard of ships as generated by cavitating propellers. In Symposium on High Powered Propulsion of Large Ships, Wageningen, the Netherlands.

de Lorenzo, D. and Biot, M. (2006). Noise and vibration: Comfort standards evolving in the wrong direction? The Naval Architect. 
del Pino, C., Parras, L., Felli, M., and Fernandez-Feria, R. (2011). Structure of trailing vortices: comparison between particle image velocimetry measurements and theoretical models. Physics of Fluids, 23.

Delery, J. M. (1994). Aspects of vortex breakdown. Prog Aerospace Sci, 30:1-59.

Denny, S. (1967). Comparisons of Experimentally Determined and Theoretically Predicted Pressures in the Vicinity of a Marine Propeller. Technical report, NSRDC Report 2349, Bethesda, MD, USA.

Devenport, W. J., Rife, M. C., Liapis, S. I., and Follin, G. J. (1996). The structure and development of a wing-tip vortex. J. Fluid Mech, 312:67-106.

Di Felice, F., Di Florio, D., Felli, M., and Romano, G. P. (2004). Experimental investigation of the propeller wake at different loading conditions by Particle Image Velocimetry. Journal of Ship Research, 48(2):168-190.

Di Felice, F., Romano, G., and Elefante, M. (2000). Propeller wake analysis by means of PIV. In 23rd Symposium on Naval Hydrodynamics, Val-de-Reuil, France.

Dreyer, M. (2015). Mind the Gap - Tip Leakage Vortex Dynamics and Cavitation in Axial Turbines. PhD thesis, École Polytechnique Fédérale de Lausanne, Switzerland.

Eça, L. and Hoekstra, M. (2002). An evaluation of verification procedures for CFD applications. In 24th Symposium on Naval Hydrodynamics Fukuoka, Japan.

English, J. W. (1979). Cavitation induced hull surface pressures - measurements in a water tunnel. In Symposium on Propeller Induced Ship Vibration, pages 55-72. RINA.

Escudier, M. (1988). Vortex breakdown: Observations and explanations. Progress in Aerospace Sciences, 25(2):189-229.

Fabre, D., Sipp, D., and Jacquin, L. (2006). Kelvin waves and the singular modes of the LambOseen vortex. Journal of Fluid Mechanics, 551:235-274.

Fahy, F. and Gardonio, P. (2007). Sound and Structural Vibration. Academic Press, Oxford, $\mathrm{UK}, 2$ 2nd edition.

Falçao De Campos, J. A. C. (1992). Laser Dopler Velocimetry measurements on tip vortices in non-cavitating and cavitating conditions. In ASME FED-Vol. 135, Cavitation and Multiphase Flow Forum.

Felli, M., Camussi, R., and Di Felice, F. (2011). Mechanisms of evolution of the propeller wake in the transition and far fields. Journal of Fluid Mechanics, 682:5-53.

Felli, M., Falchi, M., Pereira, F., and Di Felice, F. (2010). Dynamics of the propeller wake structures interacting with a rudder. In 28th Symposium on Naval Hydrodynamics, Pasadena, CA, USA.

FfowcsWilliams, J. E. and O'Shea, S. (1970). Sound generation by hydrodynamic sources near a cavitated line vortex. J. Fluid Mech, 43(4):675-688.

Fisher, N. I. (1993). Statistical Analysis of Circular Data. Cambridge University Press.

Fitzpatrick, H. M. and Strasberg, M. (1956). Hydrodynamic sources of sound. In First Symposium on Naval Hydrodynamics, pages 241-280, Washington, DC, USA.

Flandrin, P. (1999). Time-Frequency / Time-Scale Analysis. Academic Press.

Foeth, E. J. and Bosschers, J. (2016). Localization and source-strength estimation of propeller cavitation noise using hull-mounted pressure transducers. In 31st Symposium on Naval Hydrodynamics, Monterey, California, USA.

Foeth, E.-J., van Terwisga, T., and van Doorne, C. (2008). On the collapse structure of an attached cavity on a three-dimensional hydrofoil. Journal of Fluids Engineering, 130(7).

Franc, J.-P. and Michel, J.-M. (2004). Fundamentals of Cavitation. Kluwer Academic Publish- 
ers.

Fréchou, D., Dugué, C., Briançon-Marjollet, L., Fournier, P., Darquier, M., Descotte, L., and Merle, L. (2000). Marine propulsor noise investigations in the hydroacoustic water tunnel GTH. In 23rd Symposium on Naval Hydrodynamics, Val-de-Reuil, France.

Friesch, J. (1998). Correlation investigations for higher order pressure fluctuations and noise for ship propellers. In Third International Symposium on Cavitation, Grenoble, France.

Fruman, D., Dugue, C., Pauchet, A., Cerruti, P., and Briançon-Marjollet, L. (1992). Tip vortex roll-up and cavitation. In 19th Symposium on Naval Hydrodynamics, Washington D.C., U.S.A.

Fruman, D. H. and Aflalo, S. S. (1989). Tip vortex cavitation inhibition by drag-reducing polymer solutions. Journal of Fluids Engineering, 111(2):211-215.

Fruman, D. H., Cerrutti, P., Pichon, T., and Dupont, P. (1995). Effect of hydrofoil planform on tip vortex roll-up and cavitation. Journal of Fluids Engineering, 117(1):162-169.

Fujiyama, K. and Nakashima, Y. (2017). Numerical prediction of acoustic noise level induced by cavitation on ship propeller at behind-hull condition. In Fifth International Symposium on Marine Propulsors (smp'17), Espoo, Finland.

Ganesh, H., Mäkiharju, S., and Ceccio, S. L. (2014). Partial cavity shedding due to the propagation of shock waves in bubbly flows. In 30th Symposium on Naval Hydrodynamics, Hobart, Tasmania, Australia.

Gerz, T., Holzäpfel, F., and Darracq, D. (2002). Commercial aircraft wake vortices. Progress in Aerospace Sciences, 38:181-208.

Gindroz, B., Bailo, G. M., Matera, F., and Elefante, M. (1996). Influence of the cavitation nuclei on the cavitation bucket, when predicting the full-scale behavior of a marine propeller. In 21st Symposium on Naval Hydrodynamics, Trondheim, Norway.

Giuni, M. and Benard, E. (2011). Analytical / experimental comparison of the axial velocity in trailing vortices. In AIAA 2011-990, 49th AIAA Aerospace Sciences Meeting, Orlando, Florida, USA.

Götz, T., Hastie, G., Hatch, L., Raustein, O., Southall, B., Tasker, M., Thomsen, F., Campbell, J., and Fredheim, B. (2009). Overview of the impacts of anthropogenic underwater sound in the marine environment. In OSPAR Convention.

Gowing, S. and Shen, Y. (2001). Nuclei effects on tip vortex cavitation scaling. In Fourth International Symposium on Cavitation, CAV2001, Pasadena, California, USA.

Green, S. I. (1995). Wing tip vortices. In Green, S. I., editor, Fluid Vortices, pages 427-470. Kluwer Academic Publishers.

Hally, D. (2002). User's guide for PIF-WAKE : the CRS PIF Wake Scaling Program for Single and Twin Screw Forms. Technical report, DRDC Atlantic ECR 2002-053, Canada.

Hämäläinen, R., Lönnberg, B., Aren, P., and Petterson, G. (2005). Highest comfort class design for M/S Color Fantasy, the world's largest ever cruise-liner with a car-deck. In 1st International Ship Noise and Vibration Conference, London, UK.

Hämäläinen, R. and van Heerd, J. (1998). Hydrodynamic development for a large fast monohull passenger ferry. In SNAME annual meeting.

Helmholtz, H. (1858). Ueber integrale der hydrodynamischen gleichungen, welche den wirbelbewegungen entsprechen. Journal für die Reine und Angewandte Mathematik, 55:25-55.

Higuchi, H., Arndt, R. E. A., and Rogers, M. F. (1989). Characteristics of tip vortex cavitation noise. Journal of Fluids Engineering, 111(4):495-501.

Higuchi, H., Quadrelli, J. C., and Farell, C. (1987). Vortex roll-up from an elliptical wing at 
moderately low Reynolds numbers. AIAA Journal, 25(12):1537-1542.

Hildebrand, J. A. (2009). Anthropogenic and natural sources of ambient noise in the ocean. Marine Ecology Progress Series, 395:5-20.

Hoffmann, E. R. and Joubert, P. N. (1963). Turbulent line vortices. J. Fluid Mech., 16:395-411.

Holland, S. D. and Chimenti, D. E. (2003). Air-coupled acoustic imaging with zero-groupvelocity Lamb modes. Applied Physics Letters, 83(13):2704-2706.

Holtrop, J. and Kuiper, G. (2003). Cavitation and vibration research in the new Depressurised Towing Tank. In STG Sprechtag Kavitation, Hamburg, Germany.

Holzäpfel, F., Hofbauer, T., Gerz, T., and Schumann, U. (2002). Aircraft wake vortex evolution and decay in idealized and real environments: Methodologies, benefits and limitations. In Friedrich, R. and Rodi, W., editors, Advances in LES of Complex Flows, pages 293-309. Kluwer Academic Publishers.

Hommes, T., Bosschers, J., and Hoeijmakers, H. W. M. (2015). Evaluation of the radial pressure distribution of vortex models and comparison with experimental data. In 9th International Symposium on Cavitation (CAV2015); Journal of Physics: Conference Series 656.

Hsiao, C.-T. and Chahine, G. L. (2005). Scaling of tip vortex cavitation inception noise with a bubble dynamics model accounting for nuclei size distribution. Journal of Fluids Engineering, 127(1):55-65.

Hsiao, C.-T. and Chahine, G. L. (2008). Scaling of tip vortex cavitation inception for a marine open propeller. In 27th Symposium on Naval Hydrodynamics, Seoul, Korea.

Humphrey, V., Brooker, A., Dambra, R., and Firenze, E. (2015). Variability of underwater radiated ship noise measured using two hydrophone arrays. In MTS/IEEE Conference OCEANS 2015, Genova, Italy.

Huse, E. and Guoqiang, W. (1982). Cavitation-induced excitation forces on the hull. SNAME Transactions, 90:85-109.

ISO 18405 (2017). Underwater Acoustics - Terminology.

Jacobsen, F. and Juhl, P. (2011). Radiation of Sound. Technical University of Denmark \& University of Southern Denmark.

Jacquin, L., Fabre, D., Sipp, D., Theofilis, V., and Vollmers, H. (2003). Instability and unsteadiness of aircraft wake vortices. Aerospace Science and Technology, 7:577-593.

James, J. H. and Skelton, E. A. (1997). Theoretical Acoustics of Underwater Structures. World Scientific.

Jessup, S. D. (1989). An Experimental Investigation of Viscous Aspects of Propeller Blade Flow. PhD thesis, The Catholic University of America, USA.

Johannsen, C. (1998). Investigation of propeller-induced pressure pulses by means of highspeed video recording in the three- dimensional wake of a complete ship model. In 22nd Symposium on Naval Hydrodynamics, Washington D.C., U.S.A.

Johannsen, C., van Wijngaarden, E., Lücke, T., Streckwall, H., and Bosschers, J. (2012). Investigation of hull pressure pulses, making use of two large scale cavitation test facilities. In 8 th International Symposium on Cavitation CAV2012, Singapore.

Junger, M. C. (1981). Extended sound sources. In Modern Data Analysis Techniques in Noise and Vibration Probems. AGARD R700.

Junger, M. C. and Feit, D. (1986). Sound, Structures, and their Interaction. The MIT Press, 2nd edition.

Keller, J. J. and Escudier, M. P. (1980). Theory and observations of waves on hollow-core vortices. Journal of Fluid Mechanics, 99(3):495-511. 
Kerswell, R. R. (2002). Elliptical instability. Ann. Rev. Fluid Mech., 34:83-113.

Kipple, B. (2002). Southeast Alaska Cruise Ship Underwater Acoustic Noise. Technical report, Technical Report NSWCCD-71-TR-2002/S74, Naval Surface Warfare Center - Detachment Bremerton, Bremerton, Washington, USA.

Konno, A., Wakabayashi, K., Yamaguchi, H., Maeda, M., Ishii, N., Soejima, S., and Kimura, K. (2002). On the mechanism of the bursting phenomena of propeller tip vortex cavitation. Journal of Marine Science and Technology, 6:181-192.

Koop, B. and Krohn, J. (1978). Studies on Tip Vortex Cavitation as Source of Sound. Technical report, Ac 1/78, HSVA, Hamburg, Germany.

Koronowicz, T. and Szantyr, J. A. (2006). Vortex cavitation as a source of high level acoustic pressure generated by ship propellers. Acta Acustica United with Acustica, 92:175-177.

Kuiper, G. (1974). Cavitation testing of marine propellers in the NSMB Depressurized Towing Tank. In IMECH Conference on Cavitation, Edinburgh, UK.

Kuiper, G. (1981). Cavitation Inception on Ship Propeller Models. PhD thesis, Delft University of Technology, the Netherlands.

Kuiper, G. (2001). New developments around sheet and tip vortex cavitation on ships' propeller. In 4th International symposium on Cavitation, Pasadena, California, USA.

Kuiper, G. (2010). Cavitation on Ship Propellers. Delft University of Technology, the Netherlands.

Kuiper, G., van Terwisga, T. J. C., Zondervan, G.-J., Jessup, S. D., and Krikke, E. M. (2006). Cavitation inception tests on a systematic series of two-bladed propellers. In 26th Symposium on Naval Hydrodynamics, Rome, Italy.

Kuttruff, H. (2009). Room Acoustics. Spon Press, 5th edition.

Lacaze, L., Ryan, K., and Le Dizès, S. (2007). Elliptic instability in a strained Batchelor vortex. Journal of Fluid Mechanics, 577:341.

Lafeber, F. H. and Bosschers, J. (2016). Validation of computational and experimental prediction methods for the underwater radiated noise of a small research vessel. In PRADS2016, Copenhagen, Denmark.

Lafeber, F. H., Bosschers, J., de Jong, C., and Graafland, F. (2015). Acoustic reverberation measurements in the Depressurized Wave Basin. In 4th International Conference on Advanced Measurement Technology for the maritime industry, AMT'15, Istanbul, Turkey.

Lafeber, F. H., Lloyd, T. P., and Bosschers, J. (2017). Validation of underwater radiated noise predictions for a merchant vessel using full-scale measurements. In Inter.noise, Hong Kong.

Lafeber, F. H., Wijngaarden, E. V., and Bosschers, J. (2009). Computation of hull-pressure fluctuations due to non-cavitating propellers. In First International Symposium on Marine Propulsors (smp'09), Trondheim, Norway.

Lamb, H. (1932). Hydrodynamics. Cambridge University Press, 6th edition.

Lauterborn, W. and Ohl, C.-D. (1997). Cavitation bubble dynamics. Ultrasonics Sonochemistry, $4: 65-75$.

Le Dizès, S. and Laporte, F. (2002). Theoretical predictions for the elliptical instability in a two-vortex flow. J. Fluid Mech, 471:169-201.

Leibovich, S. (1978). The structure of vortex breakdown. Annual Review of Fluid Mechanics, 10:221-246.

Leighton, T. G. (1992). The Acoustic Bubble. Academic Press Inc.

Levkovskii, Y. L. (1967). Modeling of cavitation noise. Soviet Physics Acoustics, 13(3).

Leweke, T., Le Dizés, S., and Williamson, C. H. K. (2016). Dynamics and instabilities of vortex 
pairs. Ann. Rev. Fluid Mech., 48:507-541.

Leweke, T. and Williamson, C. H. K. (1998). Cooperative elliptic instability of a vortex pair. Journal of Fluid Mechanics, 360:85-119.

Li, D.-q., Hallander, J., and Karlsson, R. (2015). Progress in predicting pressure pulses and underwater radiated noise induced by a propeller with pressure side cavitation. Proceedings of 19th Numerical Towing Tank Symposium, pages 1-6.

Lighthill, J. (1978). Waves in Fluids. Cambridge University Press.

Ligneul, P. (1988). Theory of tip vortex cavitation noise of a screw propeller operating in a wake. In 17th Symposium on Naval Hydrodynamics, The Hague, The Netherlands.

Ligneul, P., Crance, C., and Bovis, A. (1983). Tip vortex cavitation noise of a screw propeller theory and experiments. In Proceedings of the Second Conference on Cavitation, Edinburgh, UK.

Ligtelijn, J., van Wijngaarden, H., Moulijn, J., and Verkuyl, J. (2004). Correlation of cavitation; comparison of full-scale data with results of model tests and computations. In SNAME Annual Meeting, Washington D.C., U.S.A.

Ligtelijn, J. T., van der Kooij, J., Kuiper, G., and van Gent, V. (1992). Research on propeller-hull interaction in the Depressurized Towing Tank. In Boom, H. J. J. V. D., editor, Hydrodynamics: Computations, Model tests and Reality, pages 539-555, Wageningen, The Netherlands. Elsevier Science Publishers.

Lloyd, T., Lafeber, F. H., and Bosschers, J. (2018). Investigation and validation of procedures for cavitation noise prediction from model-scale measurements. In 32nd Symposium on Naval Hydrodynamics, Hamburg, Germany.

Lövik, A. (1981). Scaling of propeller cavitation noise. In Noise Sources in Ships. Nordforsk, Stockholm, Sweden.

Lucca-Negro, O. and O'Doherty, T. (2001). Vortex breakdown: A review. Progress in Energy and Combustion Science, 27(4):431-481.

Lücke, T. (2006). Investigations of propeller tip vortex bursting. In NAV2006, International Conference on Ship and Shipping Research, Genova, Italy.

MacFarlane, G. G. (1949). On the energy spectrum of an almost periodic succession of pulses. Proceedings of the Institute of Radio Engineering, 37(10):1139-1142.

Maines, B. and Arndt, R. E. A. (1997a). The case of the singing vortex. Journal of Fluids Engineering, 119(2):271-276.

Maines, B. H. and Arndt, R. E. A. (1997b). Tip vortex formation and cavitation. Journal of Fluids Engineering, 119(2):413-419.

Marple, S. L. (1987). Digital Spectral Analysis. Prentice-Hall.

Matusiak, J. (1992). Pressure and Noise Induced by a Cavitating Marine Screw Propeller. Phd thesis, Helsinki University of Technology, Espoo, Finland.

McCormick, B. W. (1962). On cavitation produced by a vortex trailing from a lifting surface. Journal of Basic Engineering, 84(September):369-379.

Meleshko, V. V. and van Heijst, G. J. F. (1994). On Chaplygin's investigations of twodimensional vortex structures in an inviscid fluid. Journal of Fluid Mechanics, 272:157-182.

Meunier, P., Ledizes, S., and Leweke, T. (2005). Physics of vortex merging. Comptes Rendus Physique, 6:431-450.

Milligan, T. A. (1995). Modern Antenna Design. John Wiley \& Sons Ltd, 2nd edition.

Moet, H., Laporte, F., Chevalier, G., and Poinsot, T. (2005). Wave propagation in vortices and vortex bursting. Physics of Fluids, 17(5). 
Moore, D. W. and Saffman, P. G. (1973). Axial flow in laminar trailing vortices. Proceedings of the Royal Society A, 333:491-508.

Moore, D. W. and Saffman, P. G. (1975). The instability of a straight vortex filament in a strain field. Proceedings of the Royal Society A, 346:413-25.

Morozov, V. P. (1974). Theoretical analysis of the acoustic emission from cavitation line vortices. Sov. Phys. Acoust., 19(5):468-471.

Narain, J. P. and Uberoi, M. S. (1973). Nonlinear stability of cylindical vortex enclosing a central jet of light or dense fluid. The Physics of Fluids, 16(9):1406-1417.

Nethercote, W. C. E., Hally, D., M, M., Noble, D. J., and Sponagle, N. C. (1992). DREA's propeller design and analysis experience. In van den Boom, H. J. J., editor, Hydrodynamics: Computations, Model tests and Reality, pages 579-589. Elsevier Science Publishers.

Newman, B. G. (1959). Flow in a viscous trailing vortex. Aeronautical Quarterly, 10(2):167188.

Newman, M. and Abrahamsen, K. (2007). Measurement of underwater noise. In Ship Noise and Vibration Conference, London, UK.

Nhieu, M. T. V. and Ywanne, F. (1994). Sound scattering by slender bodies of arbitrary shape. Journal of the Acoustical Society of America, 95(4):1726-1733.

Nijland, M. A. (2006). Modeling of Low Frequeny Radiated Noise from Tip Vortex Cavity Deformations. Msc thesis, Delft University of Technology, the Netherlands.

Noordzij, L. (1977). A note on the scaling of tip vortex cavitation inception. International Shipbuilding Progress, 24(277).

Okamura, N., Fujino, R., and Tanaka, T. (1994). An experimental investigation of the mechanism and the pressure of counter-rotating vortices on a CPP at the off-design condition. In 20th Symposium on Naval Hydrodynamics, Santa Barbara, California, USA.

Okulov, V. L. and Sørensen, J. N. (2007). Stability of helical tip vortices in a rotor far wake. Journal of Fluid Mechanics, 576:1-25.

Oshima, A. (1990). A study on correlation of vortex cavitation noise of propeller measured in model experiments and full scale. Journal of the Society of Naval Architects of Japan, 168(1):89-96.

Oshima, A. (1994). Scaling of Tip Vortex Cavitation Noise of Propeller. Technical report, Vol. 31, No. 3, Mitsubishi Heavy Industries, Ltd., Nagasaki, Japan.

Oshima, A., Sasajima, T., and Chiba, N. (1986). Study on propeller tip vortex bursting and its effect on pressure fluctuations on ship hull. In International Symposium on Cavitation, Sendai, Japan.

Oweis, G. F., Fry, D., Chesnakas, C. J., Jessup, S. D., and Ceccio, S. L. (2006). Development of a tip-leakage flow - part 1: the flow over a range of Reynolds numbers. Journal of Fluids Engineering, 128(4):751-764.

Oweis, G. F., van der Hout, I. E., Iyer, C., Tryggvason, G., and Ceccio, S. L. (2005). Capture and inception of bubbles near line vortices. Physics of Fluids, 17(2).

Park, J. and Seong, W. (2017). Novel scaling law for estimating propeller tip vortex cavitation noise from model experiment. Journal of Hydrodynamics, 29(6):962-971.

Park, S.-I., Lee, S.-J., You, G.-S., and Suh, J.-C. (2014). An experimental study on tip vortex cavitation suppression in a marine propeller. Journal of Ship Research, 58(3):157-167.

Peng, X., Wang, B., Li, H., Xu, L., and Song, M. (2017). Generation of abnormal acoustic noise: singing of a cavitating tip vortex. Physical Review Fluids, 2.

Pennings, P., Westerweel, J., and van Terwisga, T. (2016). Cavitation tunnel analysis of radiated 
sound from the resonance of a propeller tip vortex cavity. International Journal of Multiphase Flow, 83:1-11.

Pennings, P. C. (2016). Dynamics of Vortex Cavitation. PhD thesis, Delft University of Technology, the Netherlands.

Pennings, P. C., Bosschers, J., Westerweel, J., and van Terwisga, T. J. C. (2015a). Dynamics of isolated vortex cavitation. J. Fluid Mech., 778:288-313.

Pennings, P. C., Westerweel, J., and van Terwisga, T. J. C. (2015b). Flow field measurement around vortex cavitation. Experiments in Fluids, 56:206:1-13.

Phillips, W. R. C. (1981). The turbulent trailing vortex during roll-up. J. Fluid Mech., 105:451467.

Platzer, G. P. and Souders, W. G. (1979). Tip Vortex Cavitation Inception Delay with Application to Marine Lifting Surfaces, a Literature Survey. Technical report, DTNSRDC 79-051, Bethesda, MD, USA.

Platzer, G. P. and Souders, W. G. (1980). Tip vortex cavitation characteristics and delay on a three-dimensional hydrofoil. In 19th American Towing Tank Conference, Ann Arbor, Michigan, USA.

Pond, H. L. (1966). Low-frequency sound radiation from slender bodies of revolution. Journal of the Acoustical Society of America, 40(3):711-720.

Prada, C., Clorennec, D., and Royer, D. (2008). Local vibration of an elastic plate and zerogroup velocity Lamb modes. The Journal of the Acoustical Society of America, 124(1):203212.

Prins, H. J., Flikkema, M. B., Bosschers, J., Koldenhof, Y., De Jong, C. A., Pestelli, C., Mumm, H., Bretschneider, H., Humphrey, V., and Hyensjö, M. (2016). Suppression of underwater noise induced by cavitation: SONIC. Transportation Research Procedia, 14:2668-2677.

Proctor, F., Ahmad, N., Switzer, G., and Limon Duparcmeur, F. (2010). Three-phased wake vortex decay. In AIAA 2010-7991: AIAA Atmospheric and Space Environments Conference, Toronto, Ontario, Canada.

Pylkkänen, J. V. (1981). Stability of a vortex enclosing a cavity. In Euromech Colloquium 146, Villard de Lans, France.

Pylkkänen, J. V. (1982). Viscous effects on the stability of cavitating line vortices. In 14th Symposium on Naval Hydrodynamics.

Raestad, A. E. (1996). Tip vortex index - an engineering approach to propeller noise prediction. The Naval Architect, pages 11-16.

Randall, R. B. (1987). Frequency Analysis. Bruel \& Kjaer, 3rd edition.

Rayleigh, L. (1917). On the dynamics of revolving fluids. Proc. R. Soc. Lond. Ser. A, 93(648):148-154.

Rienstra, S. W. and Hirschberg, A. (2006). An Introduction to Acoustics. Eindhoven University of Technology, the Netherlands.

Rijpkema, D., Starke, B., and Bosschers, J. (2013). Numerical simulation of propeller-hull interaction and determination of the effective wake field using a hybrid RANS-BEM approach. In Third International Symposium on Marine Propulsors, smp'13, Launceston, Tasmania, Australia.

Roberts, P. H. (2003). On vortex waves in compressible fluids. I. The hollow-core vortex. Proceedings of the Royal Society A, 459(2030):331-352.

Rood, E. P. (1991). Review - Mechanisms of cavitation inception. Journal of Fluids Engineering, 113(2):163-175. 
Rosenhead, L. (1931). The formation of vortices from a surface of discontinuity. Proc. R. Soc. Lond. Ser. A, 134.

Ross, D. (1987). Mechanics of Underwater Noise. Peninsula Publishing.

Rossi, M. (2000). Of vortices and vortical layers: an overview. In Maurel, A. and Petitjeans, P., editors, Vortex Structure and Dynamics. Lecture Notes in Physics, vol 555, pages 40-123. Springer Berlin Heidelberg.

Rule, J. A. and Bliss, D. B. (1998). Prediction of viscous trailing vortex structure from basic loading parameters. AIAA Journal, 36(2):208-218.

Saffman, P. G. (1992). Vortex Dynamics. Cambridge University Press.

Schuiling, B., Lafeber, F. H., van der Ploeg, A., and van Wijngaarden, E. (2011). The Influence of the wake scale effect on the prediction of hull pressures due to cavitating propellers. In Second International Symposium on Marine Propulsors, (smp'11), Hamburg, Germany.

Seol, H., Paik, B.-G., Park, Y.-H., Kim, K.-Y., Ahn, J.-W., Park, C., Kim, G.-D., and Kim, K.-S. (2015). Propeller cavitation noise model test in KRISO large cavitation tunnel and its comparison with full-scale results. In 4rd International Conference on Advanced Measurement Technology for the maritime industry, AMT'15, Istanbul, Turkey.

Shen, Y., Gowing, S., and Jessup, S. (2009). Tip vortex cavitation inception scaling for high Reynolds number applications. Journal of Fluids Engineering, 131(7).

Shen, Y. T. and Strasberg, M. (2003). The Effect of Scale on Propeller Tip-Vortex Cavitation Noise. Technical report, NSWCCD-50-TR-2003/057, West Bethesda, MD, USA.

Smith, S. W. (1999). The Scientist and Engineer's Guide to Digital Signal Processing. California Technical Publishing, San Diego, California.

Song, M., Xu, L., Peng, X., Tang, D., and Chen, Y. (2018). Acoustic modeling of the singing vortex and its sound signatures. International Journal of Multiphase Flow, 99:205-212.

Sponagle, N. C. (1990). Noise from Tip Vortex and Bubble Cavitation. Technical report, Memorandum 90/202, Defence Research Establishment Atlantic, Canada.

Starke, B. and Bosschers, J. (2012). Analysis of scale effects in ship powering performance using a hybrid RANS-BEM approach. In 26th Symposium on Naval Hydrodynamics, Gothenburg, Sweden.

Staufenbiel, R. W. (1984). Structure of lift-generated rolled-up vortices. J. Aircraft, 21(10):737744.

Steijl, R. (2001). Computational Study of Vortex Pair Dynamics. PhD thesis, University of Twente, Enschede, the Netherlands.

Stepanishen, P. R. (1997). Acoustic axisymmetric radiation and scattering from bodies of revolution using the internal source density and Fourier methods. Journal of the Acoustical Society of America, 102(2):726-732.

Stepanishen, P. R. and Chen, H.-w. (1992). Surface pressure and harmonic loading on shells of revolution using an internal source density method. Journal of the Acoustical Society of America, 92(4):2248-2259.

Stinebring, D. R., Farrell, K. J., and Billet, M. L. (1991). The structure of a three-dimensional tip vortex at high Reynolds number. Journal of Fluids Engineering, 113(3):496-503.

Strasberg, M. (1977). Propeller cavitation noise after 35 years of study. In ASME Noise and Fluids Engineering.

Szantyr, J. A. (2006). A computational model of the propeller cavitating tip vortex interacting with the rudder. In Sixth International Symposium on Cavitation, CAV2006, Wageningen, The Netherlands. 
Takahashi, H. and Ueda, T. (1969). An experimental investigation into the effect of cavitation on fluctuating pressures around a marine propeller. In 12th International Towing Tank Conference, written contribution to the Propeller Session, pages 315-317, Rome, Italy.

Tani, G., Viviani, M., Hallander, J., Johansson, T., and Rizzuto, E. (2016). Propeller underwater radiated noise: A comparison between model scale measurements in two different facilities and full scale measurements. Applied Ocean Research, 56:48-66.

Thomson, W. L. K. (1880). Vibrations of a columnar vortex. Philosophical Magazine, X.

Tofeldt, O. and Ryden, N. (2017). Zero-group velocity modes in plates with continuous material variation through the thickness. The Journal of the Acoustical Society of America, 141(5):3302-3311.

Tolstoy, I. and Usdin, E. (1957). Wave propagation in elastic plates: low and high mode dispersion. Journal of the Acoustical Society of America, 29(1):37-42.

Tsai, C.-Y. and Widnall, S. E. (1976). The stability of short waves on a straight vortex filament in a weak externally imposed strain field. J. Fluid Mech, 73:721-33.

Tung, C., Pucci, S. L., Caradonna, F. X., and Morse, H. A. (1981). The structure of trailing vortices generated by model rotor blades. In 7th European Rotorcraft and Powered Lift Aircraft Forum, Garmisch-Partenkirchen, Germany.

Turkmen, S., Aktas, B., Atlar, M., Sasaki, N., Sampson, R., Shi, W., and Fitzsimmons, P. A. (2015). On-board measurement techniques to quantify underwater radiated noise level. In $4 r d$ International Conference on Advanced Measurement Technology for the maritime industry, AMT'15, Istanbul, Turkey.

Uberoi, M. S., Chow, C.-Y., and Narain, J. P. (1972). Stability of coaxial rotating jet and vortex of different densities. The Physics of Fluids, 15(10):1718-1727.

Urick, R. J. (1983). Principles of Underwater Sound. McGraw-Hill.

van der Kooij, J. (1979). Experimental determination of propeller-induced hydrodynamic hull forces in the NSMB Depressurised Towing Tank. In Symposium on Propeller Induced Ship Vibration. The Royal Institution of Naval Architects.

van der Kooij, J. and de Bruijn, A. (1984). Acoustic measurements in the NSMB Depressurized Towing Tank. International Shipbuilding Progress, 31(353).

van Manen, J. D. (1972). The effect of cavitation on the interaction between propeller and ship's hull. International Shipbuilding Progress, 19(209).

van Oossanen, P. and van der Kooij, J. (1973). Vibratory hull forces induced by cavitating propellers. RINA Transactions, 115:111-144.

van Rijsbergen, M. X. (2016). A review of sheet cavitation inception mechanisms. In International Symposium on Transport Phenomena and Dynamics of Rotating Machinery, Honolulu, Hawaii, USA.

van Rijsbergen, M. X. and Kuiper, G. (1997). Modeling a cavitating vortex. In ASME FEDSM97-3266, ASME Fluids Engineering Division Summer Meeting.

van Terwisga, T., Kuiper, G., and van Rijsbergen, M. X. (1999). On experimental techniques for the determination of tip vortex cavitation on ship propellers. In Proceedings of the 1999 ASME/JSME, FEDSM99-7302, San Francisco, California, USA.

van Terwisga, T., van Wijngaarden, E., Bosschers, J., and Kuiper, G. (2007). Achievements and challenges in cavitation research on ship propellers. International Shipbuilding Progress, 54(2-3):165-187.

van Wijngaarden, E., Bosschers, J., and Kuiper, G. (2005). Aspects of the cavitating propeller tip vortex as a source of inboard noise and vibration. In ASME Fluids Engineering Division 
Summer Meeting and Exhibition, Houston, Texas, USA.

van Wijngaarden, H. C. J. (2011). Prediction of Propeller-Induced Hull-Pressure Fluctuations. PhD thesis, Delft University of Technology, the Netherlands.

Vaz, G. (2005). Modelling of Sheet Cavitation on Hydrofoils and Propellers using BoundaryElement-Methods. PhD thesis, Technical University of Lisbon IST, Portugal.

Vaz, G. and Bosschers, J. (2006). Modeling three dimensional sheet cavitation on marine propellers using a boundary element method. In 6th International Symposium on Cavitation CAV2006, Wageningen, The Netherlands.

Vaz, G., Hally, D., Huuva, T., Bulten, N., Muller, P., Becchi, P., Herrer, J. L., Whitworth, S., Mace, R., and Korsström, A. (2015). Cavitating flow calculations for the E779A propeller in open water and behind conditions: code comparison and solution validation. In 4th International Symposium on Marine Propulsors (smp'15), Austin, Texas, USA.

Weitendorf, E. A. (1976). Kavitationseinflüsse auf die vom Propeller induzierten Druckschwankungen. PhD thesis, Technische Universitat Hamburg-Harburg, Germany.

Widnall, S. E., Bliss, D. B., and Tsai, C.-Y. (1974). The instability of short waves on a vortex ring. J. Fluid Mech, 66:35-47.

Wu, J.-Z., Ma, H.-Y., and Zhou, M.-D. (2005). Vorticity and Vortex Dynamics. Springer-Verlag. Yamada, T., Sato, K., Kawakita, C., and Oshima, A. (2015). Study on prediction of underwater radiated noise from propeller tip vortex cavitation. In 9th International Conference on Cavitation (CAV2015), Lausanne, Switzerland. Journal of Physics: Conference Series 656.

Young, F. (1989). Cavitation. McGraw-Hill.

Zeidler, E., editor (2003). Oxford User's Guide to Mathematics. Oxford University Press.

Zeman, O. (1995). The persistence of trailing vortices: a modeling study. Physics of Fluids, 7(1):135-143. 


\section{Acknowledgements}

The present thesis was partly funded by the background research programme of the Maritime Research Institute Netherlands (MARIN), and I thank MARIN management for their support. The development of the ETV-method was sponsored by the Broadband and Broadband 2 working group of the Cooperative Research Ships (CRS). I much appreciate the permission of CRS to publish the method and its results. Use has been made of results of model tests for the Princess Royal performed within the EU FP7 SONIC project, and of model tests for the Combi Freighter sponsored by the Top Consortium for Knowledge and Innovation (TKI) of the Dutch Ministry of Economic Affairs. I also like to acknowledge the permission by the concerned companies and persons to show results of their model tests at MARIN.

I like to thank Tom van Terwisga for talking me into starting a $\mathrm{PhD}$, his continuous support, and for motivating me to also finish it. I thank Harry Hoeijmakers for his patience and time, his tireless correction of my 'engineering' writing style, his willingness to check some of the analytical formulations, and for showing how to be conscientious. The final 'sprint' would not have been possible without his support.

The findings presented here are selected results of the research performed over the years. In this process, the students Maarten Nijland, Bram Janssen, Simon Glazenborg and Thomas Hommes have contributed through their Master thesis or training period at MARIN and their work was of great benefit. Tuomas Sipilä spent some time at MARIN during which we analysed vortex-cavity deformations using high-speed video observations that were made by Arne van der Hout in the Delft Cavitation Tunnel. Unfortunately, results were not good enough to be published but it showed the feasibility of such an analysis. The measurement data of Pepijn Pennings are crucial elements in the studies reported in Chapters 3 and 4 of this thesis. The development and acceptance of analytical formulations requires detailed and accurate measurements, and I am happy that the diligence on both sides was rewarded. I also thank Pepijn for his support and discussions on his data after his PhD was finished.

Being educated in aerodynamics, it takes some time to get familiar with hydroacoustics, including hull-pressure fluctuations, of cavitating propellers. It requires measurements in modelscale facilities, measurements at sea, computations, and theory, involving many different disciplines. I like to thank my colleagues and former colleagues of MARIN for their cooperation. The people most closely involved in building up the knowledge and results in relation to model tests and sea trials discussed in the present thesis are: Bas Boerrigter, Arthur Bouvy, Evert-Jan Foeth, Wouter van Kampen, Jos Koning, Frans Hendrik Lafeber, Do Ligtelijn, Thomas Lloyd, Luigi Minerva, Martijn van Rijsbergen, Erik van Wijngaarden, and of course the personnel of the DWB. I also like to thank the many colleagues from other institutions for the cooperation in (inter)national projects such as CRS, HTA, HTF, ITTC, SONIC, as well as naval projects. These projects have certainly shown that progress goes much faster when working together. Also, the 
discussions with Alex de Bruijn and Christ de Jong were most helpful for my knowledge on acoustics.

The present thesis was written in LaTeX for which use has been made of files kindly provided by Serge Toxopeus. The comments by Alex de Bruijn, Christ de Jong, Thomas Lloyd, and especially Erik van Wijngaarden on parts of the manuscript are much appreciated. Some of the photos of vortex cavitation in Chapter 2 were taken from the legacy picture database of Gert Kuiper. Thijs Hasselaar provided Figure 2.3a. Ina Louwrink designed the cover and made the nice 3-D visualisations of cavitation shown in Figure 6.14. The time trace on the cover was inspired by the logo of the CRS Broadband working group by Layton Gilroy. I also would like to thank my brothers Arno and René for their moral support by accepting the role of paranymph.

Writing a $\mathrm{PhD}$ thesis affects your private life as well. I thank Soo for taking care of my horse for several days a week. Most importantly, the support of my wife Ina was crucial in writing this thesis, especially during the last year, and I am very grateful for that.

Johan Bosschers

Renkum, August 2018 


\section{About the author}

Johan Bosschers was born on April $5^{\text {th }} 1967$, in Holten, the Netherlands. He finished secondary school at the Ichthus College in Enschede in 1985, and subsequently started to study Aeronautical Engineering at Delft University of Technology. Johan graduated in 1991 with a Master's thesis on CFD computations to capture leading-edge vortices above delta wings. The following year, he attended the Diploma Course at the Von Karman Institute for Fluid Dynamics in Sint-Genesius-Rode, Belgium, where he graduated with honours. His research topic was the implementation and evaluation of a rotated Riemann solver and of multi-dimensional upwind schemes.

In 1992, he joined the department of Unsteady Aerodynamics and Aeroelasticity of the National Aerospace Laboratory (NLR) in Amsterdam, the Netherlands. There, he developed computational models to predict aerodynamic loads on helicopter rotor and windturbine blades. The collapse of Fokker Aircraft led to a change in type of rotating systems to be modelled.

In 1996, he became project manager at the Ships Powering department of the Maritime Research Institute Netherlands (MARIN) in Wageningen, the Netherlands. His activities were related to experimental and computational prediction of propulsor hydrodynamics including cavitation and the resulting noise and vibrations. In 2007, these activities were continued in the Research and Development department of MARIN. One of his main tasks has been the development of the boundary element method PROCAL within the Cooperative Research Ships (CRS) organization. Another main task has been related to hydroacoustic predictions of propulsors, including the measurement of cavitation noise in the, nowadays called, Depressurized Wave Basin of MARIN. He has been involved in various international research projects within the naval community, CRS and the European Union, and he currently chairs the Specialist Committee on Hydrodynamic Noise of the 29th International Towing Tank Conference. Johan's present position is Senior Researcher.

The topic of tip-vortex cavitation and its broadband noise combines several of his research interests and has been studied intermittently while working in the R\&D department of MARIN. 
Page intentionally left blank 


\section{List of Publications}

\section{Journal papers}

- Bosschers, J., Janssen, A.A., and Hoeijmakers, H.W.M. (2008). Similarity solutions for viscous cavitating vortex cores. Journal of Hydrodynamics, Vol. 20, No. 6.

- Arndt, R., Pennings, P., Bosschers, J., and van Terwisga, T. (2015). The singing vortex. Interface Focus, 5:2015.0025.

- Pennings, P. C., Bosschers, J., Westerweel, J., and van Terwisga, T. J. C. (2015). Dynamics of isolated vortex cavitation. Journal of Fluid Mechanics, 778:288-313.

- Bosschers, J. (2018). An analytical and semi-empirical model for the viscous flow around a vortex cavity. International Journal of Multiphase Flow, 105:122-133.

- Bosschers, J. (2018). A semi-empirical prediction method for broadband hull pressure fluctuations and underwater radiated noise by propeller tip vortex cavitation. Journal of Marine Science and Engineering 6(49).

\section{Selected Conference Papers}

- van Wijngaarden, E., Bosschers, J., and Kuiper, G. (2005). Aspects of the cavitating propeller tip vortex as a source of inboard noise and vibration. In ASME Fluids Engineering Division Summer Meeting and Exhibition, Houston, Texas, USA.

- Bosschers, J. (2008). Analysis of inertial waves on inviscid cavitating vortices in relation to low-frequency radiated noise. In WIMRC Cavitation Forum, Warwick University, UK.

- Bosschers, J. (2009). Investigation of hull pressure fluctuations generated by cavitating vortices. In 1st International Symposium on Marine Propulsors (smp'09), Trondheim, Norway.

- Bosschers, J. (2009). Modeling and analysis of a cavitating vortex in 2D unsteady viscous flow. In 7th International Symposium on Cavitation CAV2009, Ann Arbor, Michigan, USA.

- Bosschers, J. (2009). Investigation of the resonance frequency of a cavitating vortex. In NAG/DAGA International Symposium on Acoustics, Rotterdam, Netherlands.

- Bosschers, J. and van Wijngaarden, E. (2012). Scale effects on hull pressure fluctuations due to cavitating propellers. In 10th International Conference on Hydrodynamics (ICHD), St. Petersburg, Russia.

- Bosschers, J. (2015). On the relation between tonal and broadband content of hull pressure spectra due to cavitating ship propellers. In 9th International Conference on Cavitation (CAV2015), Journal of Physics: Conference Series 656 
- Hommes, T., Bosschers, J., and Hoeijmakers, H.W. M. (2015). Evaluation of the radial pressure distribution of vortex models and comparison with experimental data. In 9th International Symposium on Cavitation (CAV2015); Journal of Physics: Conference Series 656.

- Lafeber, F. H. and Bosschers, J. (2016). Validation of computational and experimental prediction methods for the underwater radiated noise of a small research vessel. In PRADS2016, Copenhagen, Denmark.

- Bosschers, J. (2018). On the dispersion relation of a vortex cavity. In 10th International Conference on Cavitation (CAV2018), Baltimore, USA

- Lloyd, T., Lafeber, F. H., and Bosschers, J. (2018). Investigation and validation of procedures for cavitation noise prediction from model-scale measurements. In 32nd Symposium on Naval Hydrodynamics, Hamburg, Germany. 



\section{Propeller Tip-Vortex Cavitation and its Broadband Noise}

\section{- JOHAN BOSSCHERS -}

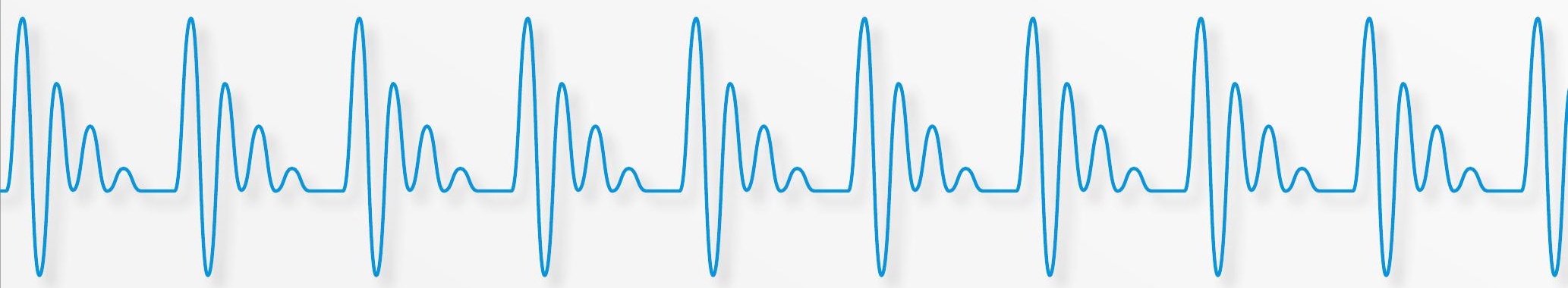


Stellingen behorend bij het proefschrift

\title{
"Propeller TIP-VORTEX CAVITATION AND ITS BROADBAND NOISE"
}

\author{
Johan Bosschers, 21 september 2018
}

1. De voorwaarde voor de azimuthale snelheid van de vloeistof op de rand van een wervelcaviteit is, bij benadering, de conditie dat de schuifspanning gelijk is aan nul. (Dit proefschrift, sectie 3.2.1.)

2. Na een implosie van een wervelcaviteit volgt altijd een rebound. (Dit proefschrift, sectie 4.2 en hoofdstuk 6.)

3. De wervel die wordt gegenereerd door de kromming van stroomlijnen bij de achterrand of zijrand van een vliescaviteit bepaalt het uitgestraalde geluid van de vliescaviteit. (Dit proefschrift, hoofdstuk 6 en 7 .)

4. De voorspelling van door wervelcavitatie veroorzaakte drukfluctuaties en uitgestraald geluid op ware grootte, door middel van een modelproef, vereist de bepaling van het cavitatiegetal bij inceptie. (Dit proefschrift, sectie 8.2.)

5. Bij referenda kan rationaliteit in het stemhokje worden gebracht door de stemkeuze te wegen met de uitkomst van een beperkt aantal, vooraf bekendgemaakte, meerkeuze vragen over de voor- en nadelen van de keuze, waarbij de juiste antwoorden op de vragen vooraf uitgebreid door wetenschap, politiek en pers zijn besproken. (Kristof Jacobs (redactie), Het Oekraïne-referendum, Nationaal Referendum Onderzoek 2016. Stichting KiezersOnderzoek Nederland.)

6. De randelementenmethode is zeer geschikt voor de analyse van scheepsschroeven in de ontwerpconditie vanwege de korte doorlooptijd door gebruik van het effectieve volgstroomveld, vanwege de bereikbare nauwkeurigheid door de lage invalshoeken en vanwege de beschikbaarheid van cavitatiemodellen.

7. Een effectief volgstroomveld kan alleen nauwkeurig worden berekend als een methode voor de berekening van de visceuze omstroming van de scheepromp wordt gecombineerd met een methode voor de berekening van de potentiaalstroming om de schroef. (Rijpkema, D., Starke, B., and Bosschers, J. Numerical simulation of propeller-hull interaction and determination of the effective wake field using a hybrid RANS-BEM approach. Third International Symposium on Marine Propulsors, 2013)

8. Met een technisch rapport kan men voldoen aan de 20-80 regel (Pareto principe) als relatie tussen inspanning en waarde of kwaliteit in percentages. Een proefschrift vereist een hogere kwaliteit en vraagt daarom significant meer inspanning. (Pareto, V., Cours d'économie politique. Vol. 1. Librairie Droz, 1964.)

9. Met de opkomst van het recreatie- en sportpaard werkt de mens voor het paard in plaats van dat het paard voor de mens werkt.

10. Na de Brexit kan 'Europees Engels' worden ingevoerd als voertaal in het Europees Parlement.

Deze stellingen worden opponeerbaar en verdedigbaar geacht en zijn als zodanig goedgekeurd door de promotor, prof. dr. ir. H.W.M. Hoeijmakers. 
Propositions accompanying the $\mathrm{PhD}$ thesis

\title{
“Propeller Tip-Vortex CAVITATION AND ITS BROADBAND NOISE"
}

\author{
Johan Bosschers, 21 September 2018
}

1. The boundary condition for the azimuthal velocity of the liquid at the interface of a vortex cavity is, by approximation, the condition that the shear stress equals zero. (This thesis, Section 3.2.1.)

2. The collapse of a vortex cavity is always followed by a rebound. (This thesis, Section 4.2 and Chapter 6.)

3. The vortex generated by the re-entrant or side-entrant jet of a sheet cavity determines the radiated noise of the sheet cavity. (This thesis, Chapter 6 and 7.)

4. The prediction of vortex-cavity-induced pressure fluctuations and radiated noise for full scale, by means of a model test, requires the determination of the cavitation-inception number. (This thesis, Section 8.2.)

5. For referendums, rationality can be brought in the voting booth by weighting the choice of vote with the outcome of a limited number of, pre-announced, multiple-choice questions that address the pros and cons of the vote, and of which the correct answers have been extensively discussed beforehand by science, politics and press. (Kristof Jacobs (redactie), Het Oekrä̈ne-referendum, Nationaal Referendum Onderzoek 2016. Stichting KiezersOnderzoek Nederland.)

6. The boundary-element method is well suited for the analysis of marine propellers at design condition because of its short turnaround time due to the use of an effective wake field, because of the achievable accuracy due to the low angles of attack, and because of the availability of cavitation models.

7. An effective wake field of a ship can only be computed accurately if a viscous-flow solver for the ship hull is combined with a potential-flow solver for the propeller. (Rijpkema, D., Starke, B., and Bosschers, J. Numerical simulation of propeller-hull interaction and determination of the effective wake field using a hybrid RANS-BEM approach. In Third International Symposium on Marine Propulsors, 2013)

8. With a technical report one can meet the 20-80 rule (Pareto principle) as a relation between effort and value or quality in percentages. $\mathrm{A} \mathrm{PhD} \mathrm{thesis} \mathrm{requires} \mathrm{a} \mathrm{higher} \mathrm{quality}$ and therefore requires significantly more effort. (Pareto, V., Cours d'économie politique. Vol. 1. Librairie Droz, 1964.)

9. With the rise of the recreational and sport horse, man works for the horse instead of the horse working for man.

10. After the Brexit, 'European English' can be introduced as the main language in the European Parliament.

These propositions are regarded as opposable and defendable and have been approved as such by the supervisor prof. dr. ir. H.W.M. Hoeijmakers. 